ORNL/M-6627

\title{
Proceedings of the IEA Workshop/Working Group Meeting on Ferritic/Martensitic Steels ${ }^{*}$
}

\author{
ECN Nuclear \\ Petten, Netherlands \\ October 1-2, 1998
}

- Prepared by: R. L. Klueh

IEA WORKING GROUP - TASK ANNEX II

\author{
Implementing Agreement \\ for a program of research \\ and development on fusion materials
}

"Research sponsored by the Office of Fusion Energy Sciences, U. S. Department of Energy, under contract DE-AC05-960R22464 with Lockheed Martin Energy Research Corp. 


\section{DISCLAIMER}

This report was prepared as an account of work sponsored by an agency of the United States Government. Neither the United States Government nor any agency thereof, nor any of their employees, make any warranty, express or implied, or assumes any legal liability or responsibility for the accuracy, completeness, or usefulness of any information, apparatus, product, or process disclosed, or represents that its use would not infringe privately owned rights. Reference herein to any specific commercial product, process, or service by trade name, trademark, manufacturer, or otherwise does not necessarily constitute or imply its endorsement, recommendation, or favoring by the United States Government or any agency thereof. The views and opinions of authors expressed herein do not necessarily state or reflect those of the United States Government or any agency thereof. 


\section{DISCLAIMER}

Portions of this document may be illegible in electronic image products. Images are produced from the best available original document. 


\section{Contents}

Working Group Participants

Agenda

Executive Summary

Appendix: Viewgraphs of Presentations and Other Handouts

Ferromagnetic Effects and Steel Properties

Effects of Ferromagnetic Structural Material on the Electromagnetic Behaviour of Fusion Reactor Components - P. Ruatto

Present and Past Research on the Effect of Ferromagnetic Materials in Fusion Reactors:

A Brief Review - R. L. Klueh

Fatigue Properties of Low Activation Ferritic Steel; JF-1 and Its Welded Joints -

A. Kohyama

Mechanical Behaviour After Thermal Ageing of RAFM Steels and F82H Weldments -

A. Alamo

Unirradiated Properties of F82H IEA Heat-Status and Recent Results-K. Shiba

Development of SPS Bonding Procedure for RAF Steel - A. Hishinuma

Irradiated Properties and Composition Effects

Present Status of JAERI Irradiation Program For LAM - K. Shiba

Post Irradiation Properties of F82H Plate, TIG and EB Welds - E. V. van Osch

Japanese University Program of RAFS R \& D for Fusion Reactor - A. Kohyama

Ferritic Isotopic Tailoring (FIST) Experiment - A. Kohyama

Effect of Tantalum on Properties of 9Cr-1MoVNb Steel - R. L. Klueh 
ODS Alloys and Alloy Development Schaaf

Oxide Dispersion Strengthened RAFM Steels: Review and Prospects - B. van der Development of 9Cr Ferritic-Martensitic Steels Strengthened by Oxide Dispersion A. Alamo

Preliminary Studies for ODS/RAF Development - A. Hishinuma

Integrating Modeling, Experiment and Data Base Development: A Mechanism Based Approach to Developing Advanced Ferritic Steels for Fusion Applications - G. R. Odette

Helium Effects Studies

Electron Microscopy of RAFM Steels With Helium — R. Schäublin

Tensile and Charpy Properties of B-Doped F82H After Irradiation - K. Shiba

Helium Effects on Mechanical Properties and Microstructure of RAFM Steels After Irradiation - E. Materna-Morris

Post Irradiation.Welding and Helium Generation with ${ }^{10} \mathrm{~B}$ in RAFM Steel - E. V. van Osch

Strategy for Development of Ferritic/Martensitic Steels

EU Strategy for RAFM Development - B. van der Schaaf

Fusion Materials Programs in Japan - A. Hishinuma

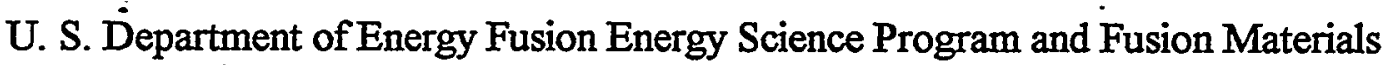
Program - F. W. Wiffen

A comment for RISO Meeting: Materials R \& D Strategy Proposal - A. Kohyama 


\section{Workshop Participants}

European Union
A. Alamo, CEA Saclay, France
E. Materna-Morris, FZK, Germany
R. Schäublin, PSI, Switzerland
B. van der Schaaf, ECN Petten, Netherlands
E. V. van Osch, ECN Petten, Netherlands

Japan

A. Hishinuma, JAERI, Japan

A. Kimura, Kyoto University, Japan

A. Kohyama, Kyoto University, Japan

K. Shiba, JAERI, Japan

\section{United States}

R. L. Klueh, Oak Ridge National Laboratory, USA

G. R. Odette, University of California at Santa Barbara, USA

F. W. Wiffen, U. S. Department of Energy, USA 


\section{EA WORKSHOP ON REDUCED-ACTIVATION FERRITIC/MARTENSITIC STEELS \\ ECN, Petten, The Netherlands}

1-2 October 1998

1 October

9:00

Welcome and Introduction

1. Ferromagnetic Effects and Stéel Properties

(Ch: G. R. Odette)

09:15 M. Ruatto

Magnetization of RAFM Steels

09:45 A. Kohyama

Fatigue Data on JLF-1 and the Future Plan

10:15 A. Alamo

Thermal Ageing Behaviour of RAFM Steels and F82H Weldments

$10: 45$

Break

11:00 K. Shiba

Unirradiated Properties of F82H IEA Heat-Status and Recent Results

11:30 A. Hishinuma

Development of Spark Plasma Sintering (SPS) Joining Techniques for RAM Steels

11:40

Discussion of Ferromagnetic Effects and Steel Properties

$12: 00$

Lunch

2. Irradiated Properties and Composition Effects

(Ch: A. Alamo)

13:00 K. Shiba

Present Status of JAERT Irradiation Program for LAM

13:30 E. V. van Osch

Post-Irradiation Mechanical Properties of F82H plate, TIG, and EB Welds

14:00 A. Kohyama

Present Status of JUPITER Program

14:30 R. L. Klueh

Effect of Tantalum on Properties of Irradiated 9Cr-2WVTa Steel

15:00

Break

3. ODS Alloys and Alloy Development

(Ch: A. Kohyama)

15:15 B. van der Schaaf ODS RAFM Steels: Review and Prospects

15:45 A. Alamo Development of Ferritic/Martensitic ODS alloys

16:15 A. Hishinuma Preliminary Studies for Developing ODS RAM Steels 
16:30 G. R. Odette

17:00

$17: 30$

2 October

\section{Helium Effects Studies}

09:00 R. Schäublin

09:30 K. Shiba

10:00 E. Materna-Morris

$10: 30$

10:45 A. Kimura

11:15 E.V. van Osch

11:45

12:15
Integrating Modeling, Experiment and Data Base Development: A MechanismBased Approach to Developing Martensitic Steels for Fusion Applications

Discussion of Irradiation Effects, Composition Effects, ODS Steels and Alloy Development

Adjourn

\section{Strategy For Development of Ferritic/Martensitic Steels}

(Ch: F. W. Wiffen)

Electron Microscopy of RAFM Steels With Helium

Tensile and Charpy Properties of B-Doped F82H After Irradiation

Microstructural Investigations of He-Generation Effects.

Break

Effects of Helium Implantation on the Ductile-Brittle Transition Behavior of Reduced Activation 9Cr-2W Martensitic Steel

Post-Irradiation Welding and Helium Generation With ${ }^{10} \mathrm{~B}$ in RAFM Steel

Discussion of Helium Effects Studies

Lunch
13:30 A. Hishinuma
Summary of Japanese Strategy
13:50 $\quad$ B. van der Schaaf
Summary of European Union Strategy
14:10 F. W. Wiffen
Summary of U. S. Strategy
$14: 30$
Discussion to Develop a Proposed Strategy for Riso Meeting
6. Progress and Future Cooperation
(Ch: B. van der Shaaf and R. L. Klueh)
15:15
Discussion on Progress and Status of Action Items
Recommendations for Future Cooperation
$16: 00$
Adjourn 


\title{
REPORT ON THE IEA WORKSHOP/WORKING GROUP MEETING ON FERRITIC/MARTENSITIC STEELS FOR FUSION
}

\author{
ECN Nuclear Research, Petten, The Netherlands, 1-2 October 1998
}

R. L. Klueh

Executive Summary

The International Energy Agency (IEA) Working Group on Ferritic/Martensitic Steels for Fusion held a workshop at ECN Nuclear Research, Petten, The Netherlands, 1-2 October 1998. The Working Group, consisting of researchers from Japan, the European Union, the United States, and Switzerland, met to review research that has been completed since the previous meeting and to continue planning and coordinating an international collaborative test program on reducedactivation ferritic/martensitic steels for fusion applications. At the workshop, data were presented from the continuing research on the IEA heats of steel that are being studied in the collaboration. Data on these and other reduced-activation steels in the irradiated and unirradiated condition were presented. Other subjects that were discussed included effects of a ferromagnetic steel in a fusion machine, the effect of helium on properties, and the development and application of oxide dispersion-strengthened steels for fusion. A Working Group status-review meeting is planned in conjunction with the International Conference on Fusion Reactor Materials (ICFRM-9) in Colorado Springs, Colorado, USA, 10-15 October 1999, at which time plans for a workshop to be held in 2000 will be finalized.

\section{Introduction}

The IEA Working Group on Ferritic/Martensitic Steels for Fusion under the auspices of the IEA Executive Committee for the Implementing Agreement on Fusion Materials conducted a workshop at ECN Nuclear Research, Petten, The Netherlands, 1-2 October 1998. Researchers from Japan (4), the European Union (5), the United States (3), and Switzerland (1) participated. Russian Federation participation was invited, but no one from there attended the meeting. The objective of the Working Group is the establishment and coordination of an international collaborative test program to determine the feasibility of using ferritic/martensitic steels for fusion.

This workshop was the ninth meeting of the Working Group, which was formed as a result of a workshop on ferritic/martensitic.steels in Tokyo in October 1992. At the first meeting following the Tokyo workshop, the Working Group developed specifications for large heats of reducedactivation steels and outlined a collaborative research program. Two 5-ton heats of the IEAmodified F82H steel and two 1-ton heats of JLF-1 steel were produced, fabricated into plates, and distributed to the participants of the collaboration. Subsequent meetings have been used to plan a test program and to coordinate the acquisition of the data needed to prove feasibility for the steels for fusion. 
The Petten meeting was a follow up to the meeting at Tokyo, Japan, 3-4 November, 1997, in which information was presented that indicated helium has an embrittling effect on ferriticl martensitic steels irradiated at $250-400^{\circ} \mathrm{C}$. At the Tokyo meeting and at ICFRM- 8 at Sendai, Japan, 27-31 October 1997, several investigators presented data on oxide dispersion-strengthened (ODS) steels as possible structural materials that will allow higher operating temperatures. At Petten, information was presented on both of these subjects, along with recently developed information on the properties of the IEA heats of reduced-activation steels and other reducedactivation steels. Information was also presented on work designed to determine the effects produced by ferritic/martensitic steels in the high magnetic fields of a magnetically confined fusion reactor.

\section{Research and Development Activities}

The following is a brief description of the information presented at the Petten workshop. Copies of viewgraphs and other information presented at the workshop are appended to this summary.

\section{Ferromagnetic Effects}

At one time it was believed that the expected strong interaction of a ferromagnetic material with the magnetic fields of a magnetically confined fusion system would make it impossible to use the ferritic/martensitic steels as structural materials. Calculations in the early 1980s indicated that the effect could be taken into account in the design of the reactor. Because these early studies were but cursory analyses of the problem, questions as to the magnitude of the effect persist, and detailed design studies as well as experimental investigations are required to eliminate this uncertainty.

P. Ruatto has been involved in a program at FZK Karlsruhe to study the transient eddy current problems and magnetic fields and forces that can develop with the use of ferritic/martensitic steels. Ruatto's presentation (given by E. Materna-Morris) discussed some of the information derived with the three-dimensional finite element method program AENEAS that was developed at FZK. Results were presented for calculations that examined the effect of plasma disruptions on the outboard blanket segment of the DEMO helium-cooled pebble bed outboard blanket segment and the European Helium cooled pebble bed test blanket module in ITER. A centered plasma disruption was considered for the DEMO, and the forces on the components were calculated and described for the MANET steel. The force calculations for a plasma disruption in ITER were also summarized. The conclusion was that for a correct mechanical design of a fusion power plant it is necessary to include an electromagnetic analysis, and the AENEAS is an appropriate tool for this task.

K. Shiba discussed the Japan Atomic Energy Research Institute (JAERI) research effort on the effect of a ferromagnetic material (ferritic/martensitic steel) on the operation of a fusion machine. (Note that this presentation was part of Shiba's presentation in the section on Steel PropertiesIrradiated, and the viewgraphs on the ferromagnetic effects are included in his presentation in that 
section.) Three subject areas are being pursued by JAERI: (1) an experimental study of the use of a ferritic steel for plasma ripple reduction in ITER by the installation of a "ferritic board" on the JFT-2M tokamak, (2) an experimental study of the possibility of producing rippleless plasma operation with a reduced-activation martensitic steel as the vacuum vessel by the installation of a reduced-activation ( $\mathrm{F} 82 \mathrm{H}$ ) ferritic steel liner in a small tokamak, and (3) using this lined tokamak to conduct research on possible undesirable effects due to ferritic/martensitic steels on plasma production and control. Work began on (1) this past year and involved a computer simulation and preliminary experiment using JFT-2M. The results indicated a reduction in ripple magnitude and a modification of the magnetic field over the whole plasma region due to the insertion of the ferritic board.

R. Klueh has attempted to determine what work has been conducted and what work is ongoing throughout the world on ferromagnetic effects, and the results of that have been summarized. A copy of that summary is included in the appended material following Ruatto's presentation.

\section{Steel Properties-Unirradiated}

The work of the Monbusho fatigue test program in Japan to develop mini-sized test techniques, study size effects, develop a strain-control test technique without contacting the specimen, and determine the fatigue behavior of JF-1 steel was described by A. Kohyama. A hydraulic servocontrolled testing machine using laser measurements has been developed that should be applicable to hot-lab testing. Testing of full-sized hour-glass specimens ( $100 \mathrm{~mm}$ long, $9 \mathrm{~mm}$ at the center of the specimen) and miniature specimens ( $25.4 \mathrm{~mm}$ long, $1.25 \mathrm{~mm}$ at the center) measured comparable properties except under the condition of very low cycle fatigue. The machine was used to test JLF-1 base metal and TIG weldments, and the results indicated that fatigue strength (S-N curve) of the base metal was less than that of the weld metal. A correlation was developed between the fatigue limit and Vicker hardness that was related to the tensile strength, which was shown to provide good predictions for the fatigue limit.

A. Alamo presented data on the effect of thermal aging on the tensile and Charpy behavior of six European Union (EU) reduced-activation ferritic/martensitic steels and F82H and JFF-1. Aging was for up to 13400 hours at $250,350,400,450$, and $550^{\circ} \mathrm{C}$. The EU steels included steels with high carbon and nitrogen (LA12Ta), low C with (LA12TaLC) and without (LA12LC) tantalum and low nitrogen (LA12TaLN). There was also high (11\%) chromium (LA4Ta) and high (3\%) tungsten (LA13Ta).

There was little effect of aging on the yield stress of the F82H after aging $13400 \mathrm{~h}$, but the reduction of area was significantly reduced above $400^{\circ} \mathrm{C}$. The Charpy results for the EU steels indicated that there were chemical composition effects. For example, aging the high-tungsten steel to $10000 \mathrm{~h}$ at $350,400,450$, and $550^{\circ} \mathrm{C}$, resulted in a reduction of upper-shelf energy (USE) and an increase of transition temperature for the higher temperatures, which was associated with Laves phase. There were also indications of chromium effects, especially at $400^{\circ} \mathrm{C}$ aging, which may be due to chromium-rich $\alpha^{\prime}$ formation. The F82H and JLF-1 showed a reduction in USE and 
an increase in transition temperature after thermal aging $13400 \mathrm{~h}$ at $550^{\circ} \mathrm{C}$, but little effect after aging at $250,350,400$, and $450^{\circ} \mathrm{C}$. Laves phase formation may play a role in this behavior. Tensile, creep, and Charpy tests were also made on thermally aged $\mathrm{F} 82 \mathrm{H}$ weldments produced by the TIG and electron beam (EB) processes. TIG welds, which were post-weld heat treated (PWHT), displayed a similar strength but a slightly lower ductility and USE compared to the base metal. In particular, some degradation of impact properties was found after aging at $550^{\circ} \mathrm{C}$. However, the results for EB welds without a post-weld heat treatment still need to be compared with steels that have a PWHT to fully evaluate the properties of the EB weldments.

$\mathrm{K}$. Shiba reported on the continued progress on the JAERI round robin tests that are generating a range of mechanical and physical properties data for the IEA heat of F82H. Mechanical property tests that have been made or that are in progress include hardness, tensile, Charpy impact, fatigue, fracture toughness, and creep. The range of physical properties include density, specific heat, thermal expansion, thermal and electrical conductivity, melting point, Young's modulus, Poisson's ratio, modulus of rigidity, and magnetic hysteresis. Other measurements include the determination of a continuous-cooling-transformation diagram, water corrosion, hydrogen permeability, and hydrogen cracking. Some of the mechanical property tests have also been conducted on aged steel and on weldments. Shiba presented recent tensile, Charpy, fatigue, and fracture toughness results on thermally aged and unaged F82H steel. Analysis of the extracted precipitates from the aged steel indicated a tendency toward the production of Laves phase for steel aged at 550,600 , and $650^{\circ} \mathrm{C}$. Mechanical properties of the weldments were generally comparable to the base metal.

Fabrication of the blanket structure of a fusion power plant presents many difficulties, especially welding and joining, and A. Hishinuma presented information on a potential joining technique. Hot Isostatic Pressing (HIP) bonding is a potential technique for certain geometries. However, the optimum conditions for $\mathrm{HIP}$ bonding are $150 \mathrm{MPa}$ at $1040^{\circ} \mathrm{C}$ for $2 \mathrm{~h}$ followed by tempering. Such a high temperature and long hold time can have negative effects on the properties due to austenite grain growth. Spark plasma sintering (SPS) bonding, which involves the formation of a plasma between the parts being joined, is being studied by JAERI as an alternative to HIP bonding. SPS conditions are $20-50 \mathrm{MPa}$ at $800-900^{\circ} \mathrm{C}$ with hold times of $0.08-1 \mathrm{~h}$. Excellent joints have been obtained with this technique; the joints are improvements over HIP-bonded material in metallographic appearance and strength.

\section{Steel Properties-Irradiated}

The status of the JAERI irradiation program on F82H was reviewed by $\mathrm{K}$. Shiba. Irradiations are being carried out in the High Flux Isotope Reactor (HFIR) in the U.S./JAERI collaboration and in the Japan Materials Test Reactor (JMTR) and the Japan Research Reactor (JRR-2/JRR-3/JRR-4). Accelerator (dual/triple beam) irradiations are also being conducted. The program involves tensile, Charpy, and fracture toughness measurements and microstructural studies of the irradiated steel. 
E. V. van Osch reported on results of work at ECN at Petten on post-irradiation properties of the IEA F82H plate and welds. Irradiation was in the High Flux Reactor (HFR) to 2-3 dpa and the testing (tensile, impact, and static fracture toughness) is in progress. A $65 \mathrm{~kg}$ heat of steel (ECNBS) was obtained and irradiated with $\mathrm{F} 82 \mathrm{H}$. ECN-BS contained somewhat more $\mathrm{Cr}, \mathrm{C}$, and $\mathrm{Ta}$ and less B than the F82H. The ECN-BS steel showed improved Charpy properties over the F82H after irradiation to $2.5 \mathrm{dpa}$ at $300^{\circ} \mathrm{C}$. Comparison was made between EB and TIG weldments of the IEA F82H. Before irradiation, the EB welds had a higher strength and ductility; testing of the irradiated welds is in progress. Irradiations to $10 \mathrm{dpa}$ at $300^{\circ} \mathrm{C}$ are in progress, with the testing to be performed under the next EU Framework Program (1999-2002). The F82H is included in this experiment, but emphasis of this framework program will be on the new EUROFER steels. This experiment will also include work on B-doped steels to investigate the effect of helium on properties, to investigate the distribution of the boron in the steel, and to measure the helium content.

The Japanese universities (Monbusho) program on the properties of irradiated reduced-activation ferritic steels for fusion reactors was reviewed by A. Kohyama. Most of this work was on the JLF-1. The first irradiations were carried out in FFTF. The results included: tensile studies conducted on steels irradiated to $60 \mathrm{dpa}$ at $365-600^{\circ} \mathrm{C}$, swelling data obtained after irradiation to $70 \mathrm{dpa}$ at $420^{\circ} \mathrm{C}, \triangle \mathrm{DBTT}$ (change in ductile-brittle transition temperature) data obtained from irradiations to $50^{\circ} \mathrm{C}$ at $\approx 400^{\circ} \mathrm{C}$, and pressurized-tube irradiation creep tests for specimens irradiated to $35 \mathrm{dpa}$ at $520^{\circ} \mathrm{C}$. Current work involves experiments in HFIR, JOYO, and JMTR. At present a dual-beam ion-irradiation facility (DuET) is being constructed at Kyoto University that will be used for future in-beam studies. The facility is expected to begin operation in FY 1999.

Another Monbusho effort is the Ferritic Isotopic Tailoring (FIST) experiment in which isotopictailored F82H disks were irradiated in HFIR to simulate the fusion environment effects of producing hydrogen and helium in the steel. Preliminary results from TEM and shear-punch tests have been obtained and are being evaluated.

The effect of tantalum in the ORNL 9Cr-2WVTa steel on Charpy and tensile properties after irradiation was discussed by R. L. Klueh. The steel has excellent strength and impact toughness before and after irradiation in the Fast Flux Test Facility (FFTF) and the High Flux Reactor (HFR). The ductile-brittle transition temperature (DBTT) increased only $32^{\circ} \mathrm{C}$ after $28 \mathrm{dpa}$ at $365^{\circ} \mathrm{C}$ in FFTF, compared to a shift of $\approx 60^{\circ} \mathrm{C}$ for a $9 \mathrm{Cr}-2 \mathrm{WV}$ steel-the same as the $9 \mathrm{Cr}$ 2 WVTa steel but without tantalum. This difference occurred despite the two steels having similar tensile properties before and after irradiation. The $9 \mathrm{Cr}-2 \mathrm{WVTa}$ steel has a smaller prior-austenite grain size, but otherwise microstructures are similar before irradiation and show similar changes during irradiation. The irradiation behavior of the $9 \mathrm{Cr}-2 \mathrm{WVTa}$ steel differs from the $9 \mathrm{Cr}-2 \mathrm{WV}$ steel and other similar steels in two ways: (1) the shift in DBTT of the 9Cr-2WVTa steel irradiated in FFTF does not saturate with fluence by $\approx 28 \mathrm{dpa}$, whereas for the $9 \mathrm{Cr}-2 \mathrm{WV}$ steel and most similar steels, saturation occurs by $<10 \mathrm{dpa}$ and (2) the shift in DBTT for $9 \mathrm{Cr}-2 \mathrm{WVTa}$ steel irradiated in FFTF and HFR increased with irradiation temperature, whereas it decreased for the 
9Cr-2WV steel, as it does for most similar steels. The improved properties of the $9 \mathrm{Cr}-2 \mathrm{WVTa}$ - steel and the differences with other steels were attributed to tantalum in solution and the loss of that tantalum during irradiation by precipitation. The precipitation still needs to be confirmed.

\section{ODS Steels and Alloy Development}

B. van der Schaaf reviewed the possibility of oxide dispersion-strengthened (ODS) steels for fusion applications. These steels contain a high number density of $\left(\mathrm{TiO}_{2}\right.$ or $\left.\mathrm{Y}_{2} \mathrm{O}_{3}\right)$ oxide particles that provide enhanced creep strength. One problem with the conventional and reduced-activation ferritic/martensitic steels being investigated for fusion is that the upper operating temperature will be limited to $\approx 550^{\circ} \mathrm{C}$, and this limits the systems in which they can be used (e.g. water-cooled system). ODS steels with their improved creep properties offer the possibility of extending that temperature to $600^{\circ} \mathrm{C}$ and higher. Because they are strengthened by a high number density of small oxide particles, the oxide particles could provide sites for defect recombination and helium trapping and thus reduce swelling and suppress helium bubble effects. Most of the prior work on these materials for nuclear applications were for fuel canning for fast breeder reactors. The results for that application indicated significant improvement in creep strength over conventional steels with the helium effects suppressed. The major problems involved the anisotropy due to the powder metallurgy fabrication techniques used to make the tubes. There is limited experience on thick-wall parts, and although reduced-activation ODS steels are being developed, there is as yet no literature information available on them.

Van der Schaaf concluded that the ODS reduced-activation steels being developed show considerable promise that indicates they could, if developed, extend operating temperatures above $600^{\circ} \mathrm{C}$ (assuming creep controls and not corrosion) and reduce helium effects. However, the fabrication route needs to be developed for the larger sections needed in a fusion reactor blanket. Joining may present some difficulty and should be addressed early in the development stage.

Work on the development of ODS steels was described by A. Alamo. The high-chromium ferritic steels (MA 956 and MA 957) had elongated grains (recrystallized grain size $d>1 \mathrm{~mm}$, recrystallization temperature $>1300^{\circ} \mathrm{C}$ ) with a high texture, anisotropic properties, and low ductility. The creep and aging behavior of these steels was studied. The MA 957 with an . optimized grain size showed excellent creep resistance at $650^{\circ} \mathrm{C}$ relative to a $15-15$ austenitic stainless steel, especially for longer rupture times $\left(>10^{4}\right)$. Precipitation of intermetallic phases $(\chi$, Laves, and $\alpha^{\prime}$ phases) was detected in the thermal aging studies and irradiation experiments.

The development of a $9 \mathrm{Cr}$ ODS steel which can transform to martensite is being pursued. The objective is to avoid intermetallic phase precipitation and reduce the anisotropy of the properties compared to the fully ferritic materials. $9 \mathrm{Cr}-\mathrm{Mo}$ and $9 \mathrm{Cr}-\mathrm{W}$ steels containing $\mathrm{Y}_{2} \mathrm{O}_{3}$ are being examined. These steels developed an eqiaxed grain structure when normalized and tempered, and there was no grain growth in the range $1000-1250^{\circ} \mathrm{C}$. The yield stress for each of these steels was higher than that for MA 957 with a somewhat reduced, but still high, reduction of area. This development study is continuing. 
A. Hishinuma reported on the JAERI efforts to produce an ODS reduced-activation ferritic/ martensitic steel. The compositions that have been investigated were variations on the $\mathrm{F} 82 \mathrm{H}$ with $8 \% \mathrm{Cr}, 0-1.75 \% \mathrm{~W}, 0.1-0.28 \% \mathrm{Ti}, 0.15 \% \mathrm{O}, 0.1-0.23 \% \mathrm{Y}, 0.12 \% \mathrm{C}$. The manufacturing process involved mechanical alloying the powders followed by hot extrusion at $1050^{\circ} \mathrm{C}$ to fabricate the steel, after which it was normalized and tempered. Microstructures have been produced that have a fine-grain structure that appears relatively equiaxed. Excellent Charpy and tensile properties were obtained from several of the experimental steels. The compositional variations indicated that the tensile properties depended on the $\mathrm{Y}_{2} \mathrm{O}_{3}$ and tungsten content, but much less on the titanium content.

G. R. Odette discussed the recent review of the fusion materials program in the U.S. by The Fusion Energy Systems Advisory Committee of which Odette was a member. They recommended that the fusion materials program seek to integrate modeling, experiment, and data-base development to develop advanced materials for fusion. This means bringing more modeling into the materials program, as the committee viewed the program as being deficient in this area. Odette feels that one area where such an approach can be applied is the study of fracture of fusion reactor components. Since fracture behavior of irradiated materials is of critical importance for fusion, micromechanical-based local fracture models need to be applied with small specimen measurement of fracture resistance on unirradiated and irradiated material to provide the resultant properties necessary to predict limits for fusion structures. These results need to be further combined with microstructure-property models that reflect the effect of alloy composition, processing variables, and irradiation. The implementation of such an integrated approach was discussed in terms of work being conducted at the University of California at Santa Barbara. As one example, work on reactor pressure vessel embrittlement was cited and discussed.

\section{Helium Effects Studies}

Electron microscopy studies of the reduced-activation ferritic/martensitic steels IEA F82H and OPTIMAX A were discussed by $R$. Schaüblin. The F82H was irradiated to 0.5 and $1.7 \mathrm{dpa}$ with $590 \mathrm{MeV}$ protons at PSI in Switzerland, and F82H and OPTIMAX A were irradiated to $2.5 \mathrm{dpa}$ at $250^{\circ} \mathrm{C}$ in HFR in Petten. The dislocation structure, carbide composition and size distribution, and grain/lath boundary chemistry of the F82H irradiated with protons were determined for the unirradiated (before and after tensile deformation) and irradiated steels. The results for the proton irradiation of the IEA F82H generally indicated that there was essentially no difference in the microstructural defects in the as-received (unirradiated), deformed (material taken outside the necked region), and irradiated conditions. The $\mathrm{M}_{23} \mathrm{C}_{6}$ particles, which constituted the majority of the precipitate, were found to be coherent with the matrix. Chromium enrichment at prior austenite grain boundaries was detected for the normalized-and-tempered steel, but after irradiation, chromium depletion was observed.

Neutron irradiation of OPTIMAX A at $250^{\circ} \mathrm{C}$ produced no defects, but faceted cavities were observed. For the F82H, on the other hand, no cavities were present, but black dot (loops) damage was observed. 
Helium effects studies using boron-doped F82H steel irradiated in HFIR and JMTR were reported by K. Shiba. Standard F82H, which contains a small amount of natural boron, $\mathrm{F} 82 \mathrm{H}$ to which natural boron was added, and $\mathrm{F} 82 \mathrm{H}$ to which ${ }^{10} \mathrm{~B}$ was added were compared. The ${ }^{10} \mathrm{~B}$ is transmuted to helium; natural boron contains $\approx 20 \%{ }^{10} \mathrm{~B}$. Irradiation in HFIR at $300-500^{\circ} \mathrm{C}$ up to $\approx 30 \mathrm{dpa}$ produced very little effect on the tensile properties (yield stress and total elongation). Tensile specimens irradiated in JMTR to $0.7 \mathrm{dpa}$ and 120 appm He for the ${ }^{10} \mathrm{~B}$-doped steel had little effect on the yield stress, but there was an indication of a slight reduction in total elongation and reduction of area. Although the standard F82H and the $\mathrm{F} 82 \mathrm{H}$ containing the ${ }^{10} \mathrm{~B}$ addition had similar Charpy impact properties in the unirradiated condition, irradiation to $0.2-0.6 \mathrm{dpa}$ at 250$350^{\circ} \mathrm{C}$ in JMTR produced a much larger shitt in the Charpy transition temperature for the ${ }^{10} \mathrm{~B}-$ doped $\left(\approx 100\right.$ appm He) steel. At temperatures above $\approx 400^{\circ} \mathrm{C}$, there was only a small difference in the Charpy behavior of the steels with and without ${ }^{10} \mathrm{~B}$. Microstructural examination of steels irradiated to $57 \mathrm{dpa}$ in HFIR indicated $2 \times 10^{21} \mathrm{~m}^{-3}(3 \mathrm{~nm})$ cavities present in the ${ }^{10} \mathrm{~B}$-doped steel but none in the non-doped steel.

E. Materna-Morris reported on the effect of helium on steels after dual-beam irradiation and neutron irradiation in the HFR. MANET I hardened more than the F82H did during dual-beam irradiation to $0.3 \mathrm{dpa}$ and $500 \mathrm{appm} \mathrm{He}$. For irradiations in HFR at $300^{\circ} \mathrm{C}$, a larger shift in Charpy transition temperature was observed for MANET I and OPTIFER II than for the ORNL 9Cr-2WVTa and F82H. Dual-beam irradiation of the F82H to $0.8 \mathrm{dpa}$ and $300 \mathrm{appm} \mathrm{He}$ at $250^{\circ} \mathrm{C}$ produced a larger shift in the transition temperature than for a similar irradiation in HFR. The excess shift was attributed to helium. Likewise, to explain the relative Charpy transition temperature behavior of MANET I, OPTIFER II, F82H, and 9Cr-2WVTa (listed in order of decreasing transition temperature shift) after irradiation in HFR, the results were correlated with ${ }^{10} \mathrm{~B}$ content, which transmuted to helium, although it was stated that the helium contribution to the shift in transition temperature cannot be determined quantitatively because it is not possible to separate helium and alloying effects. Scanning electron microscopy observations of relative amounts of cleavage and intergranular fracture on the fracture surfaces were correlated with the Charpy results (change in transition temperature).

A. Kimura discussed a small punch test procedure used to evaluate the effect of helium on the DBTT of $9 \mathrm{Cr}-2 \mathrm{~W}$ steels. Disk specimens 3- $\mathrm{mm}$ in diameter and $0.22-\mathrm{mm}$ thick were irradiated in a $36 \mathrm{MeV} \alpha$-particle beam from a cyclotron. An energy degrader was used to uniformly implant 120 and $580 \mathrm{appm} \mathrm{He}(0.048$ and $0.23 \mathrm{dpa})$ in the disk. Irradiation was at $<150^{\circ} \mathrm{C}$. Hardness data were used to estimate a yield stress and yield stress increase $\left(\Delta \sigma_{y}\right)$ during irradiation. Data from JMTR irradiations where no helium was present indicated that the shift in yield stress fit a $\mathrm{dpa}^{1 / 4}$ law, which agreed with the results for the cyclotron-irradiated specimens, indicating no helium effect on hardening (just the effect of displacement damage). The data for the cyclotronirradiated material fit the linear correlation between $\Delta \sigma_{\mathrm{y}}$ and $\triangle \mathrm{DBTT}$ obtained from the JMTR data, indicating that helium did not affect the shift in DBTT. From hardening changes during annealing, it was found that helium reduced the rate of recovery of the irradiation hardening, suggesting that helium stabilizes the defect clusters. TEM indicated that helium decreased the 
size of the clusters but increased the number density. In this experiment, irradiation to $0.23 \mathrm{dpa}$ and $580 \mathrm{appm} \mathrm{He}$ at $<150^{\circ} \mathrm{C}$ did not affect irradiation hardening and embrittlement .

E. V. van Osch reported on work being started to study the post-irradiation welding of heliumcontaining steel at ECN in Petten. Neutron irradiated F82H plates of 1,3 , and $5 \mathrm{~mm}$ thickness that were irradiated in HFR to $2 \mathrm{dpa}(\approx 5 \mathrm{appm} \mathrm{He})$ and some $1 \mathrm{~mm}$ plates that were irradiated to 2.5 dpa were available for the study. The irradiated 1,3 , and $5 \mathrm{~mm}$ plates were successfully TIG welded to unirradiated plates with no external defects detected by SEM. The 1 and $3 \mathrm{~mm}$ plates were welded in a single pass with no filler metal, and the $5 \mathrm{~mm}$ plate contained a $Y$-groove and was welded with 4-6 passes. Further inspection of the welds is planned. Heats of steel have been ordered with ${ }^{10} \mathrm{~B},{ }^{11} \mathrm{~B}$, and natural boron, so that it will be possible to generate various amounts of helium up to 250 appm He and higher.

\section{Strategy for the Development of Ferritic/Martensitic Steels for Fusion}

Presentations were made on the strategy for the development of ferritic/martensitic steels in Japan, EU, and the U.S. by A. Hishinuma, B. van der Schaaf, and F. W. Wiffen, respectively. The stated goal of this session was the development of a united strategy that could be presented by representatives from the Working Group (van der Schaaf and Hishinuma) to an IEA panel that was meeting in Copenhagen the following week, 5-9 October 1998, to consider a coordinated strategy for fusion materials development.

The strategies for Japan and the EU are pointed toward a DEMO using a martensitic steel, and this gives rise to dates for selecting a given material for the construction of the plant. Japan has a potential date of 2015 for selecting a material for DEMO, and the EU has a date for a DEMOrelevant design by 2009 based on conventional-type ferritic/martensitic steels. Should ODS steels be successfully developed, the date for a DEMO-relevant design for this material would be 2015 .

In contrast to Japan and the EU, the U.S. has no plans for a DEMO and instead is involved in a science-based approach in which the technical program will emphasize, "enabling technologies for plasma experiments, domestic and internationally." The materials work will be targeted at developing materials that will support economically attractive, environmentally attractive, and safe fusion energy source designs.

A. Kohyama presented some further views on the Japanese strategy. He expressed concern about what should be done beyond the work presently being carried on the large heats of the IEA F82H and JLF-1 that are being studied in the IEA collaboration. He emphasized the need for a clear strategy for ferritic/martensitic steel development to be presented to the fusion community.

The discussion on the strategies of the various programs indicated that at present it appears there are common features in the strategies of the three programs, starting with the need to coordinate the materials development with the design and engineering community. The question of whether ferromagnetic structural materials are acceptable for magnetically confined fusion still needs to be 
answered, as do questions on the effect of the simultaneous helium and displacement damage on properties (embrittlement). An expansion of the design window for ferritic/martensitic steels is desirable, and it is agreed that the ODS steels offer the best approach to achieve that goal by raising the operating temperature. This development needs to be pursued.

Other questions vital to the application of ferritic/martensitic steels to fusion include nuclear transmutations that will burn out elements of the steel (e.g., W, Ta, etc.), the effect of tungsten on the breeding ratio, compatibility issues and the need for barriers or other coatings for the steel. The urgent need for a $14 \mathrm{MeV}$ neutron source was again emphasized.

Despite different objectives of the European Union, Japan, and the United States and given the time and financial constraints on the programs, the complexity of the common problems standing in the way of the three programs meeting their respective goals makes a coordinated effort of international collaboration by the three programs essential if their goals are to be achieved.

\section{Action Items}

No formal action items were set forth at this meeting. However, the following action item from the Tokyo meeting has not yet been completed:

Considerable work has now been completed on the IEA heats of ferritic/ martensitic steels. Compilations of the work on the IEA heat of F82H by the Japanese and European Union are being prepared by K. Shiba and R. Lindau, respectively, who will consult on an exchange of reports and a distribution of the reports to other members involved in the IEA collaboration. In the future, a report summarizing the work being carried out in the European Union, Japan, and the United States will be prepared.

$\mathrm{K}$. Shiba has agreed to continue this cooperative effort with $\mathrm{R}$. Lindau. In addition, E. van Osch has expressed his interest to Shiba in participating in the effort.

On an informal basis, the EU approached Shiba requesting the JAERI irradiation matrix for F82H, so they can avoid duplication in their program. Shiba has this information in his data base, but he also agreed to prepare a hard copy and distribute it.

\section{Other Information}

Although it was not discussed formally in the meeting, the EU has ordered a $4000 \mathrm{~kg}$ heat of EUROFER 97, the EU reduced-activation reference steel for DEMO. Delivery is expected in the spring of 1999. Most of the ingot will be processed into plate to be used for the EU testing program for wrought and weld products. Tens of meters of tubes will be produced that will be used for welding trials and component mock-ups. Welds for testing will be made by fusion and HIP processes. There will be a limited number of forged bars, some of which will be atomized for 
powder products that will be made by the HIP process for qualification of the process. There are plans to offer material to participants in the IEA program for evaluation.

\section{Next Meeting}

The next meeting of the Working Group will occur on one evening of the ICFRM-9 Conference in Colorado Springs, Colorado, USA, during the week of 10-15 October 1999. This meeting will serve as the planning meeting for the next workshop, which will be held in the fall of 2000 . 
Appendix

Viewgraphs and Handouts 


\section{Ferromagnetic Effects and Steel Properties}




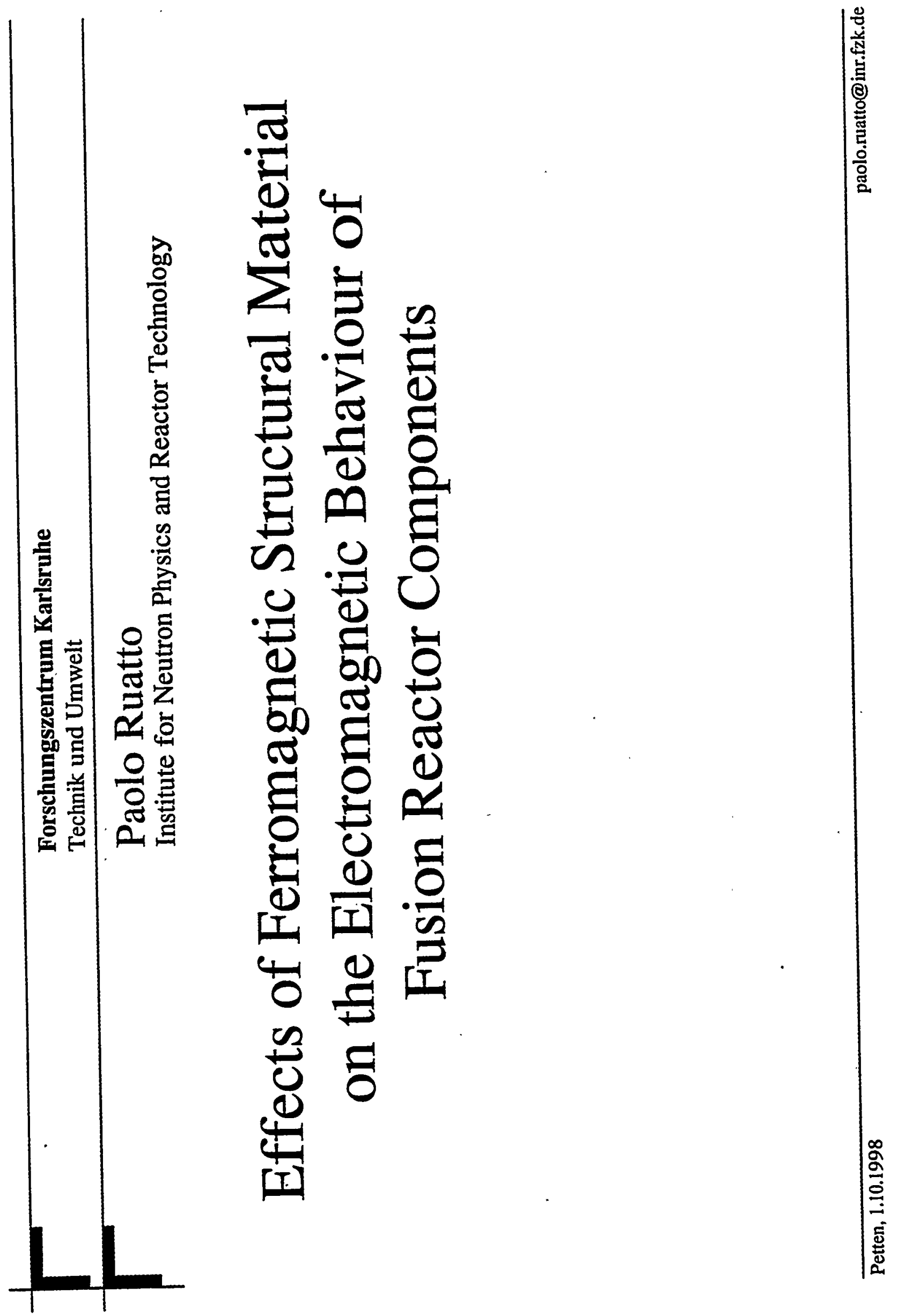




\section{Introduction}

The Finite Element Method program AENEAS, developed at FZK to study transient eddy current problems as well as magnetic fields and forces in non-linear magnetic materials, has been applied to investigate the electromagnetic behaviour during a reference plasma disruption for

(1) the DEMO Helium cooled Pebble Bed (HCPB) outboard Blanket segment,

(2) the European Helium Cooled Pebble Bed (HCPB) Test Blanket Module in ITER 


\section{Plasma Disruption}

When a plasma disruption occurs, eddy currents are induced in the conducting structures surrounding the plasma region.

Such currents interact with the magnetic field and cause an electromagnetic load (Lorentz forces) acting on the structure.

If the material is ferromagnetic - to determine the effective loading of the structure - attention has to be paid to:

(1) changes in the eddy current distribution,

(2) changes in the magnetic field in the structure,

(3) additional contributions to the load due to direct interaction between the magnetic field and the magnetized material. 


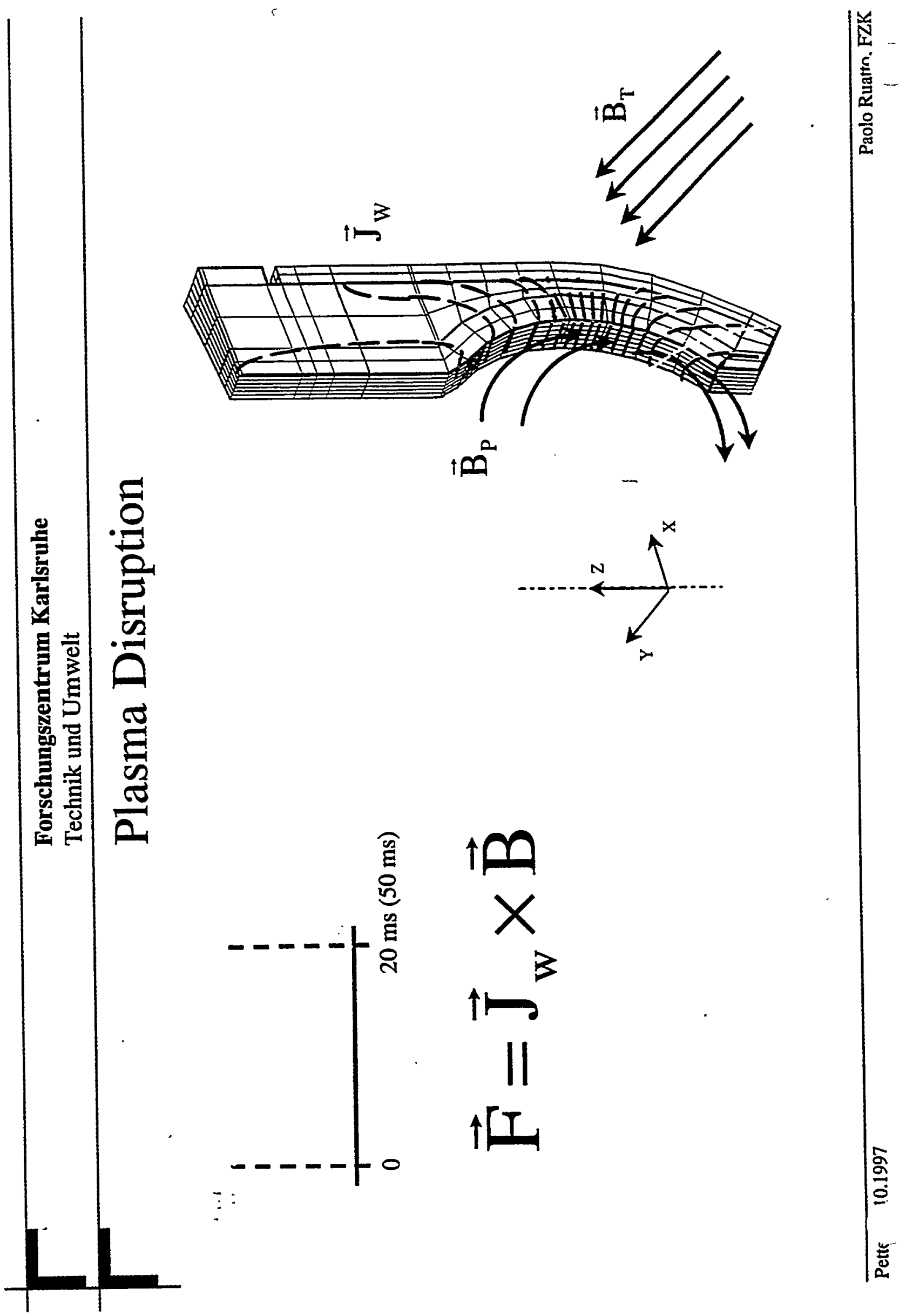




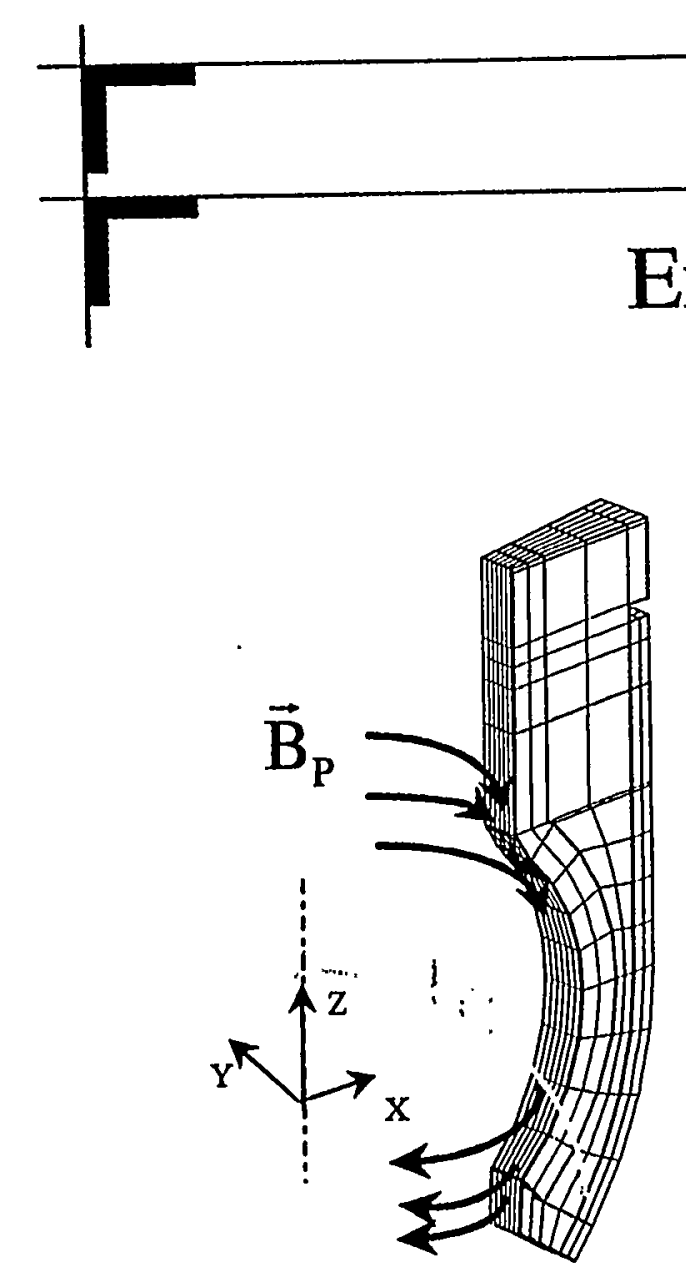

Forschungszentrum Karlsruhe

Technik und Umwelt

\section{Effect of Ferromagnetic Properties}

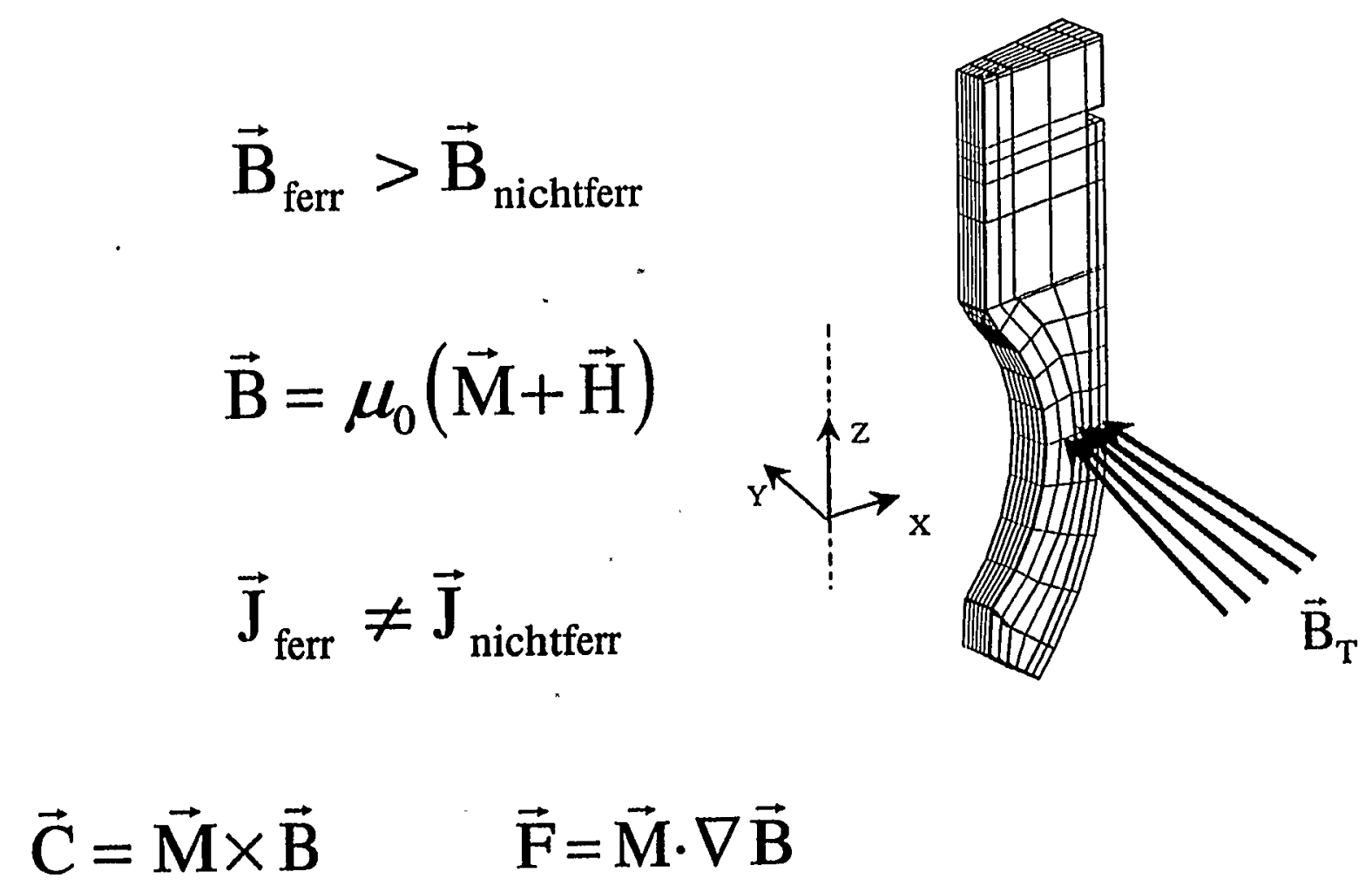




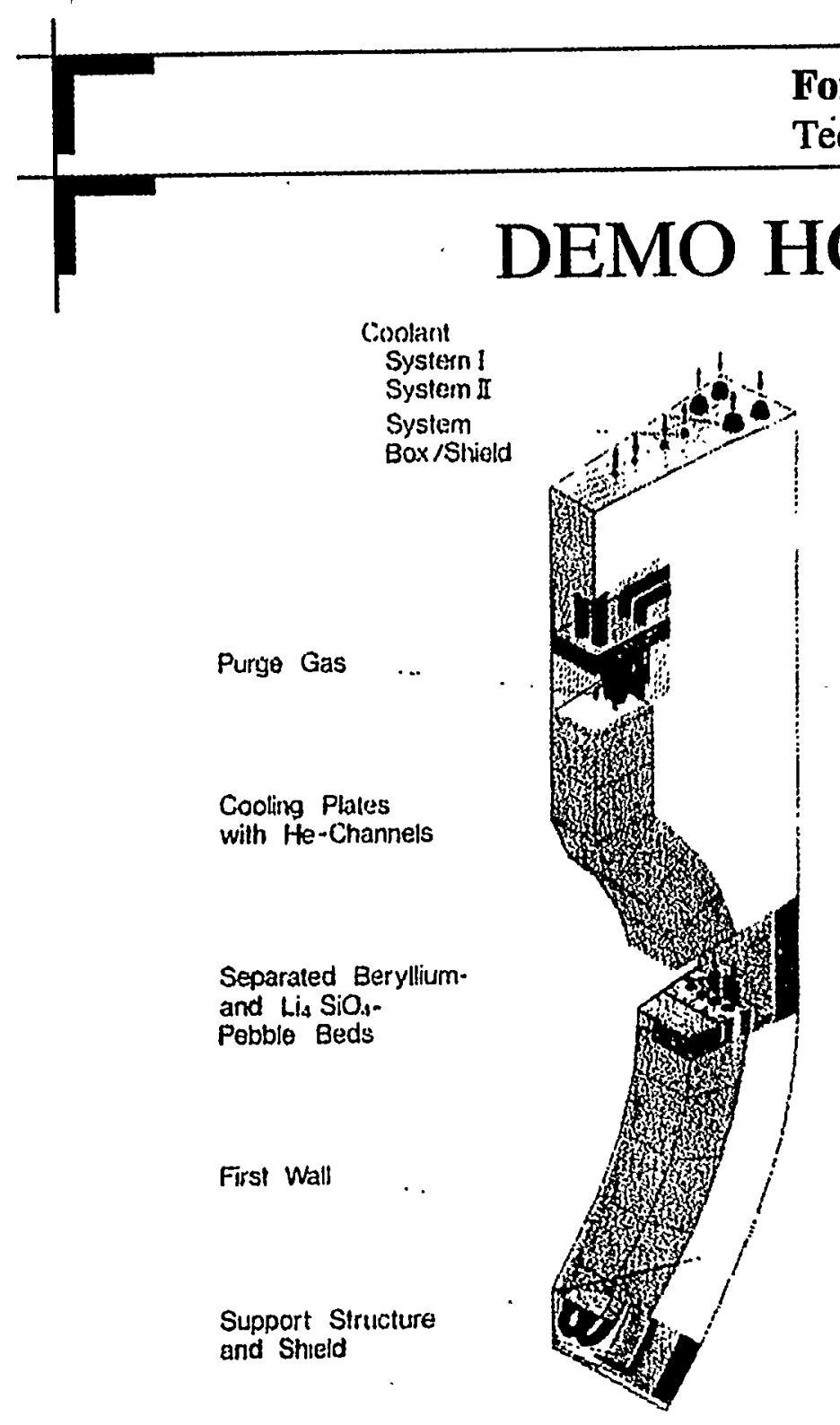

Forschungszentrum Karlsruhe

Technik und Umwelt

\section{DEMO HCPB Solid Breeder Blanket}

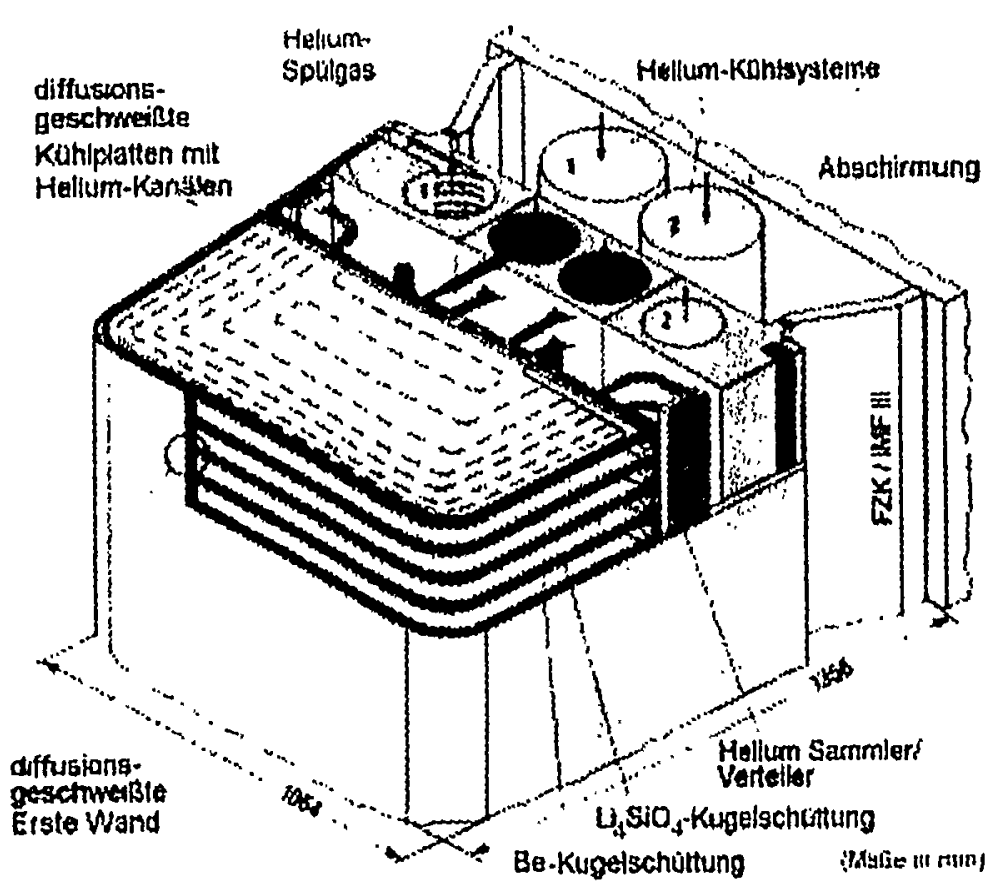

Outboard Segment 


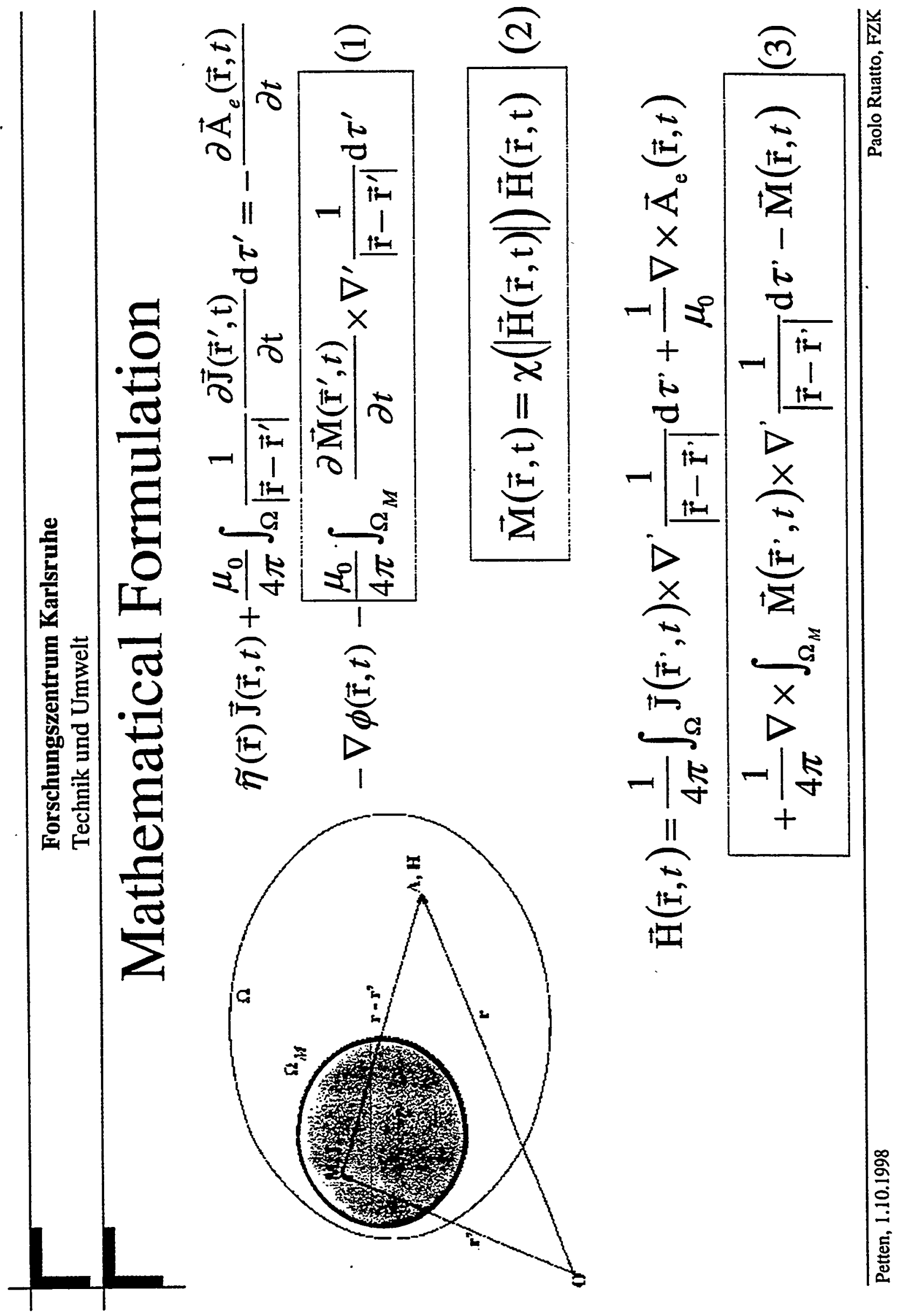




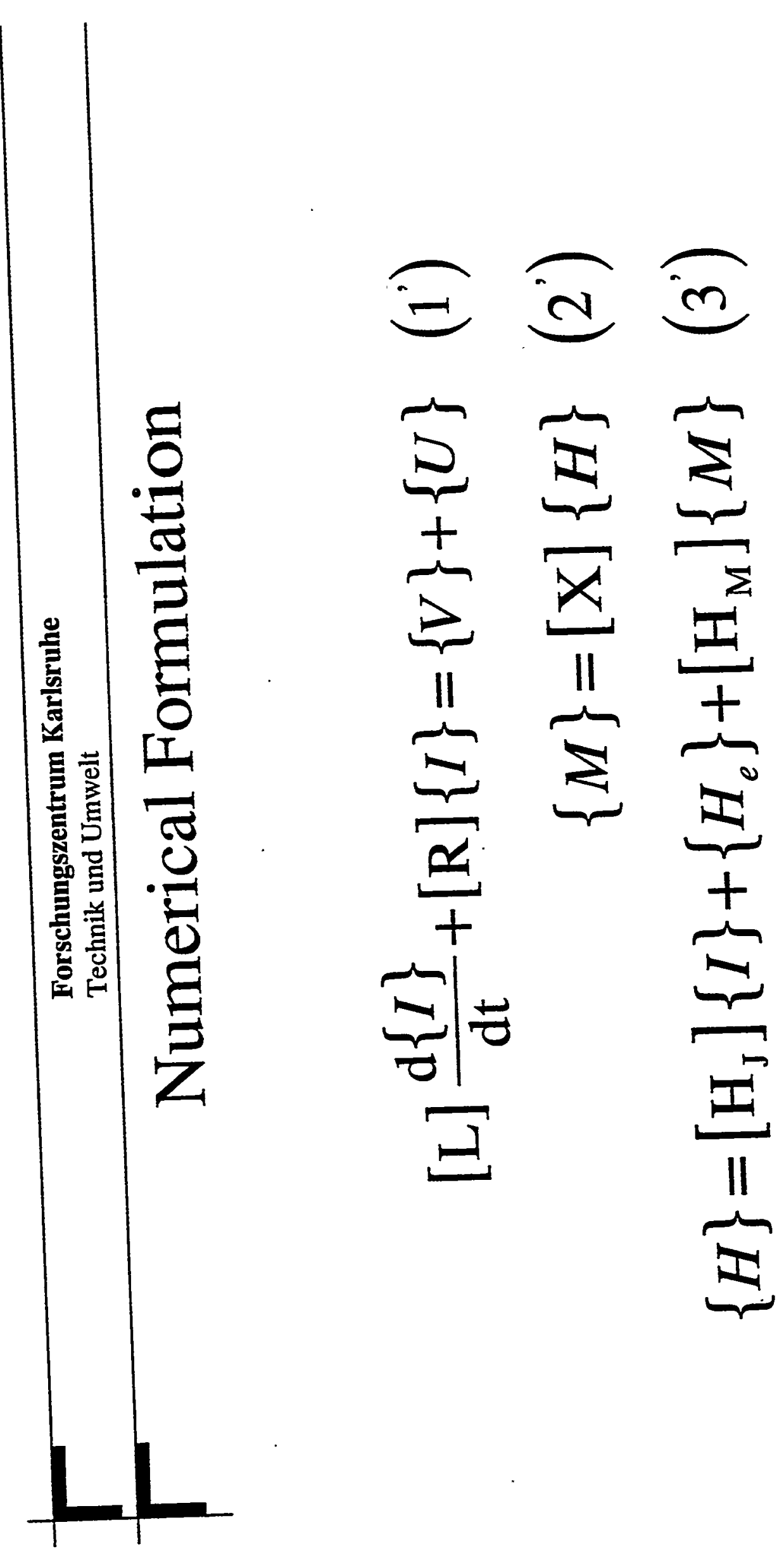




\section{Time integration}

$$
\begin{aligned}
& {[\mathrm{D}]\{I\}_{\mathrm{w}+1}=[\mathrm{D}]\{I\}_{\mathrm{w}}-\Delta t[\mathrm{R}]\{I\}+\int_{\mathrm{t}_{\mathrm{w}}}^{\mathrm{t}_{\mathrm{w}+1}}\{V\} \mathrm{dt}+\int_{\mathrm{t}_{\mathrm{w}}}^{\mathrm{t}_{\mathrm{w}+1}}\{U\} \mathrm{dt}} \\
& {[\mathrm{D}]=[\mathrm{L}]+\omega \Delta t[\mathrm{R}]} \\
& \Delta t=t_{\mathrm{w}+1}-t_{\mathrm{w}}, \quad \text { integration time-step } \\
& 0 \leq \omega \leq 1, \quad \text { Crank-Nicolson-Parameter }
\end{aligned}
$$

$$
\begin{gathered}
\{I\}_{\mathrm{w}+1}=([1]-[\mathrm{A}])\{I\}_{\mathrm{w}}+[\mathrm{B}]\left(\{f\}_{\mathrm{w}+1}-\{f\}_{\mathrm{w}}\right)+[\mathrm{C}]\left(\{M\}_{\mathrm{w}+1}-\{M\}_{\mathrm{w}}\right) \\
{[\mathrm{A}]=\Delta t[\mathrm{D}]^{-1}[\mathrm{R}], \quad[\mathrm{B}]=[\mathrm{D}]^{-1}\left[\mathrm{~V}_{\mathrm{koe}}\right], \quad[\mathrm{C}]=[\mathrm{D}]^{-1}\left[\mathrm{U}_{\mathrm{koe}}\right]}
\end{gathered}
$$




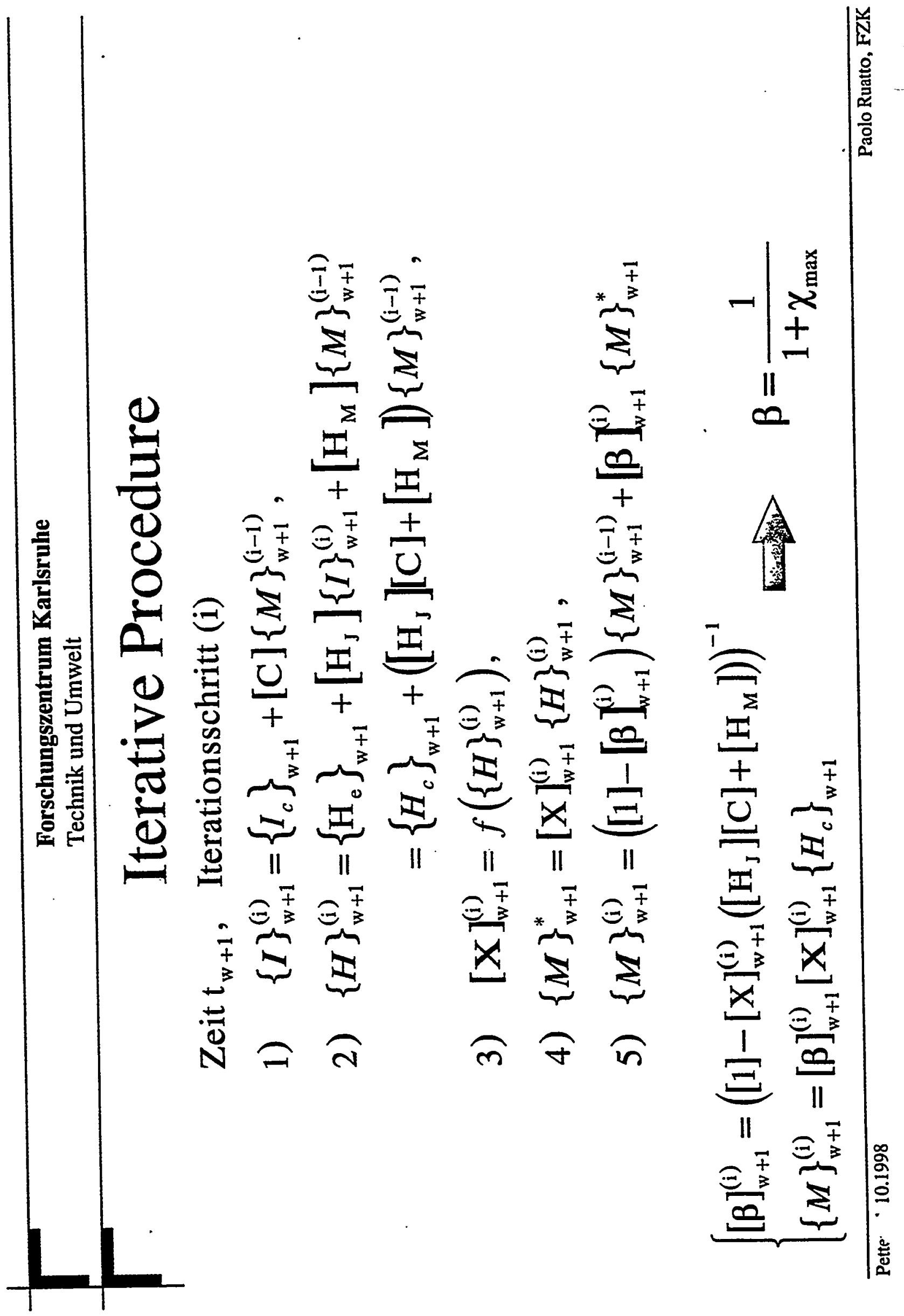




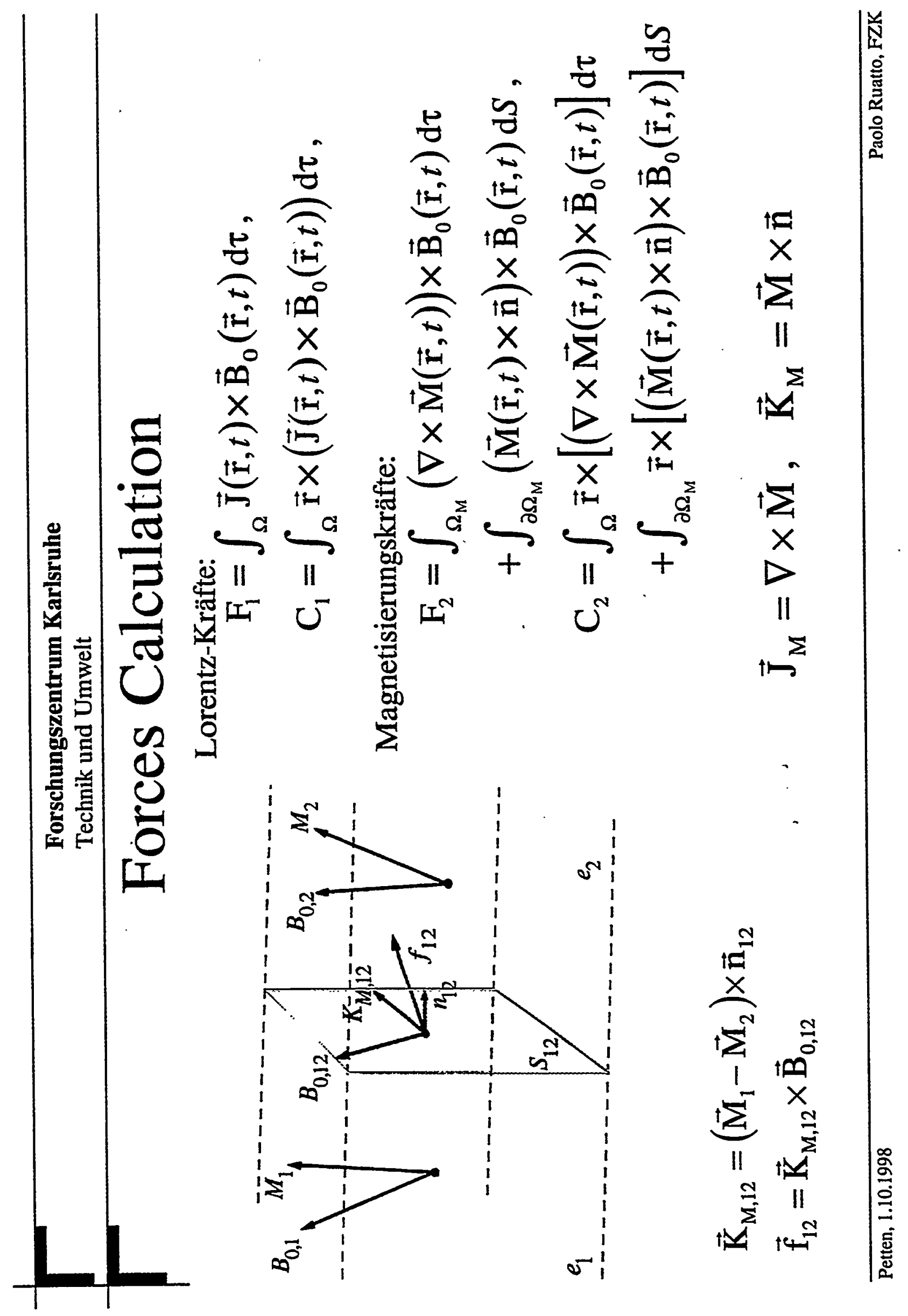




\section{AENEAS}

- 3D Finite-Element-Method Program

- Integral-Volume-Method

- Symmetry

- Magnetization Curve

- S.O.R. iterative Method

- Analytical Algorithms for magnetic field calculations

- Magnetization forces (EMC-Method) 
Forschungszentrum Karlsruhe

Technik und Umwelt

\section{Electromagnetic Analysis for DEMO}

- A centered plasma disruption with a linear decay of the plasma current from 20MA to zero in $20 \mathrm{~ms}$ has been considered.

- The structure is fully saturated by the strong toroidal magnetic field (6 tesla) and the magnetization $\mathbf{M}$ is directed almost entirely toroidally causing a thickening of the toroidal component of the magnetic flux density $\mathbf{B}$, whereas the influence of $\mathbf{M}$ on the poloidal component of $\mathbf{B}$ is slight.

- It means that when a plasma disruption occurs, the varying poloidal magnetic field of the plasma "sees" a similar situation as the structure would be nonmagnetic, so that behaviour and distribution of the eddy currents induced in the structure are approximately the same with or without ferromagnetic structural material. 


\section{Measurement of the Magnetization Curve for MANET Steel}
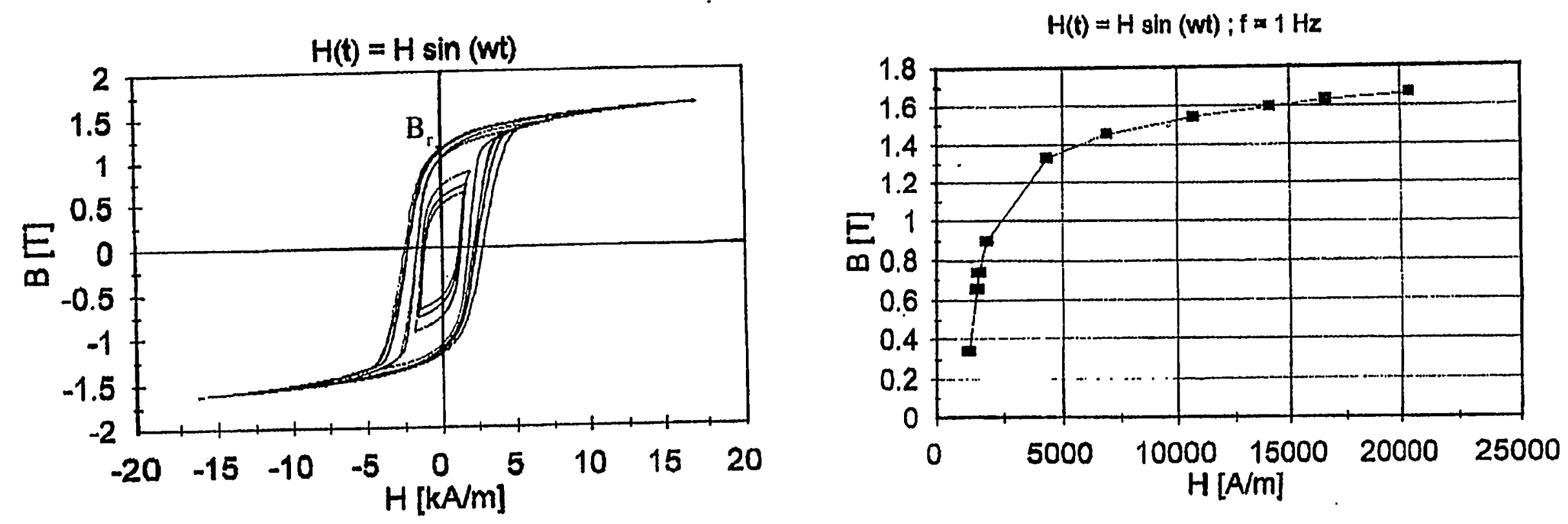

$$
\begin{gathered}
\mathrm{H}_{\text {sat }}=24 \mathrm{kA} / \mathrm{m}, \quad \mathrm{B}_{\text {sat }}=1.64 \mathrm{~T}, \quad \mathrm{~B}_{\mathrm{r}}=1.15 \mathrm{~T} \\
\mathrm{DEMO} \rightarrow 4800 \mathrm{kA} / \mathrm{m} !
\end{gathered}
$$




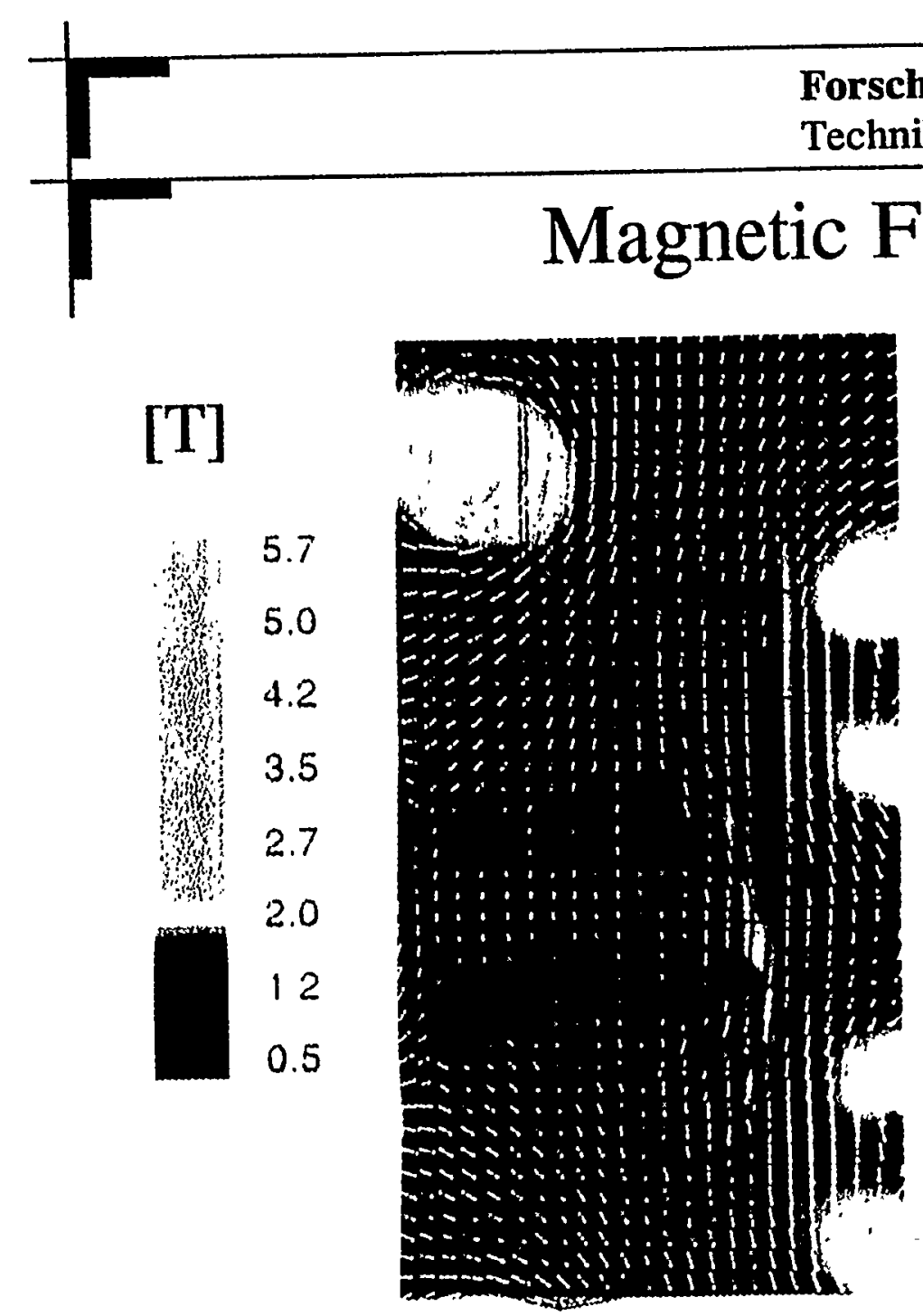

Poloidal Component $\mathbf{B}_{\mathbf{P}}$

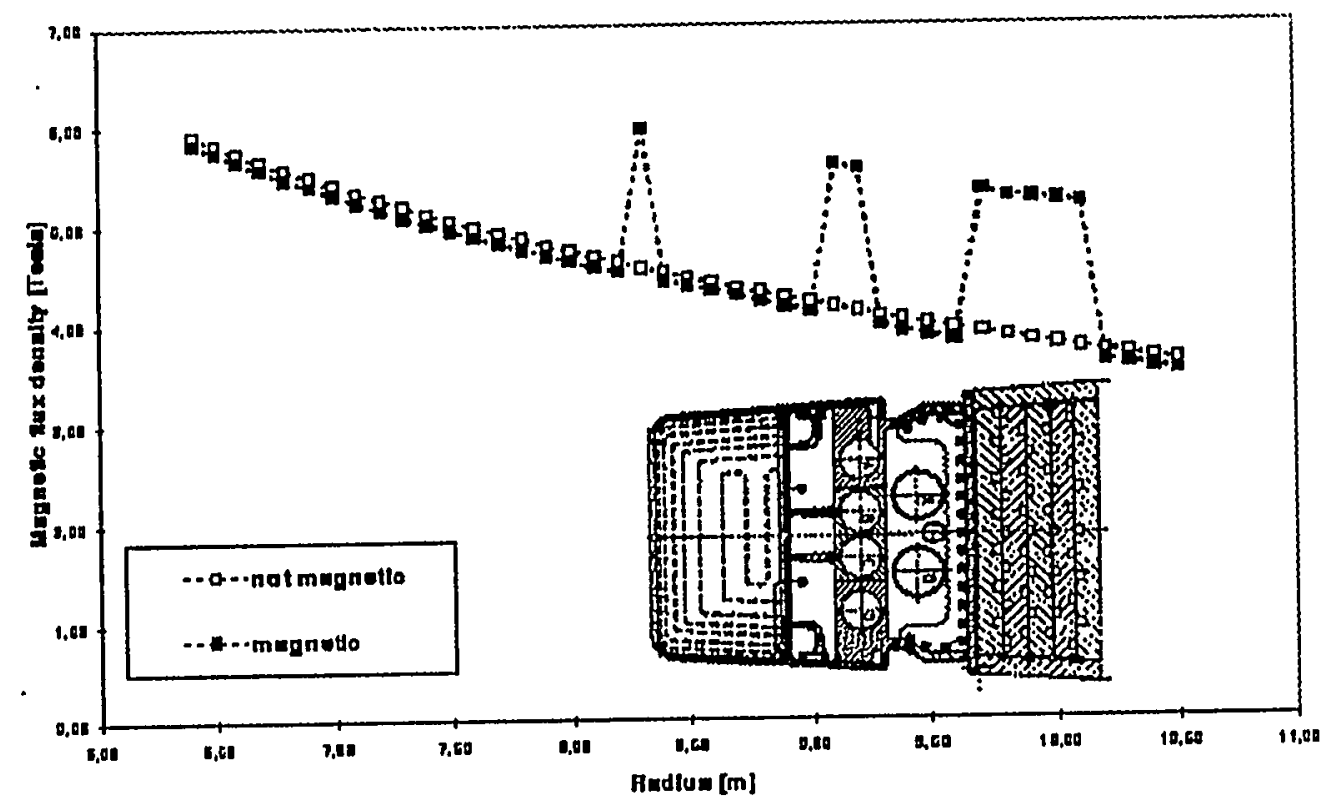

Toroidal Component $\mathbf{B}_{\mathbf{T}}$ 


\section{Electromagnetic Analysis for DEMO}

(continued)

- The electromagnetic forces (and couples) caused by interaction of the eddy currents $(\mathbf{J})$ with the magnetic field $\mathbf{B}$ (Lorentz forces $\mathbf{J x B}$ ) increase only their magnitude due to the contribution of the magnetization $\mathbf{M}$ to the toroidal component of $\mathbf{B}$, whereas their direction remains practically unchanged. In the most stressed part of the outboard segment box, like the side walls, the increase is about $11 \%$. Considering the resultant forces and couples, we observe that a large torque acts on $\mathrm{X}$-axis and a torsion in Z-direction.

- Additional forces and couples are present in the structure caused by direct interaction of $\mathbf{M}$ with $\mathbf{B}$. The resultant of such forces produces a stretching of the structure. This contribution, which is present only in a ferromagnetic structure even during normal operation, determines in case of a plasma disruption a completely different electromagnetic load for the DEMO outboard segment as in the case with nos:-magnetic structural material. 
Forschungszentrum Karlsruhe

Technik und Umwelt

\section{Electromagnetic Forces}

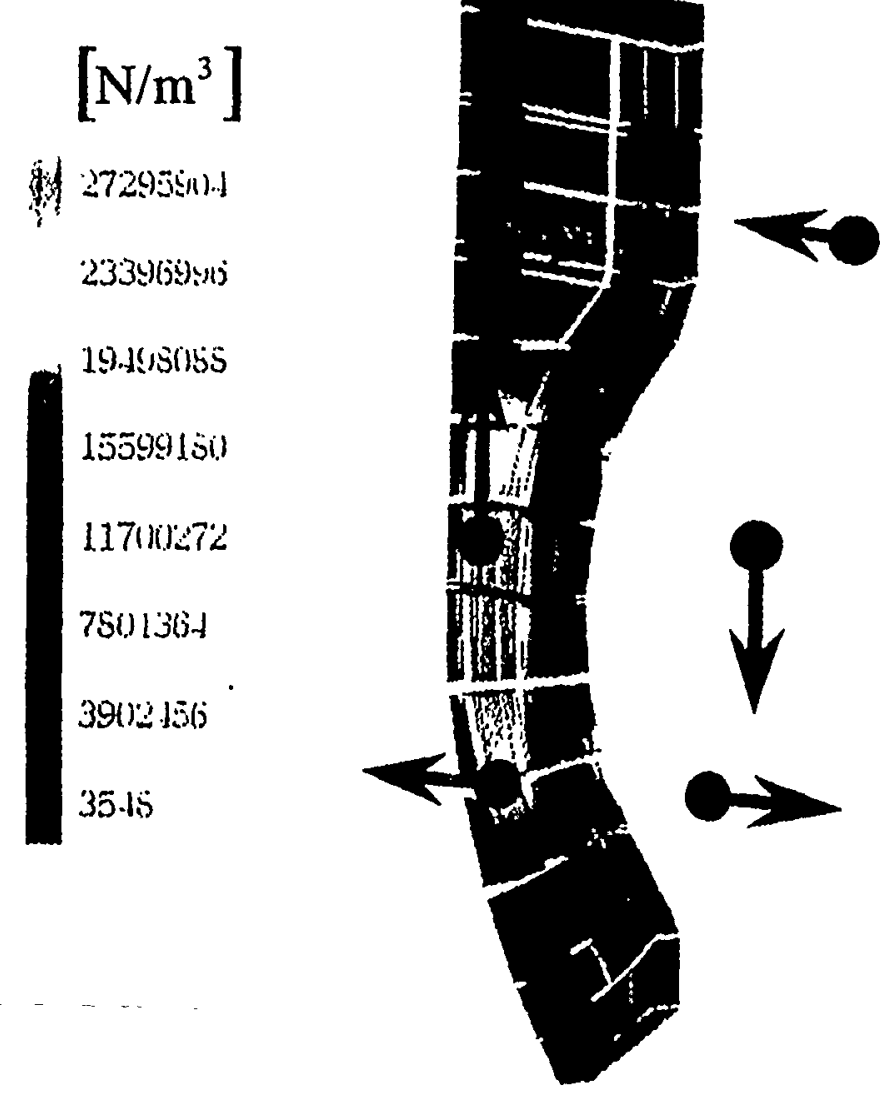

Distribution of the Lorentz forces

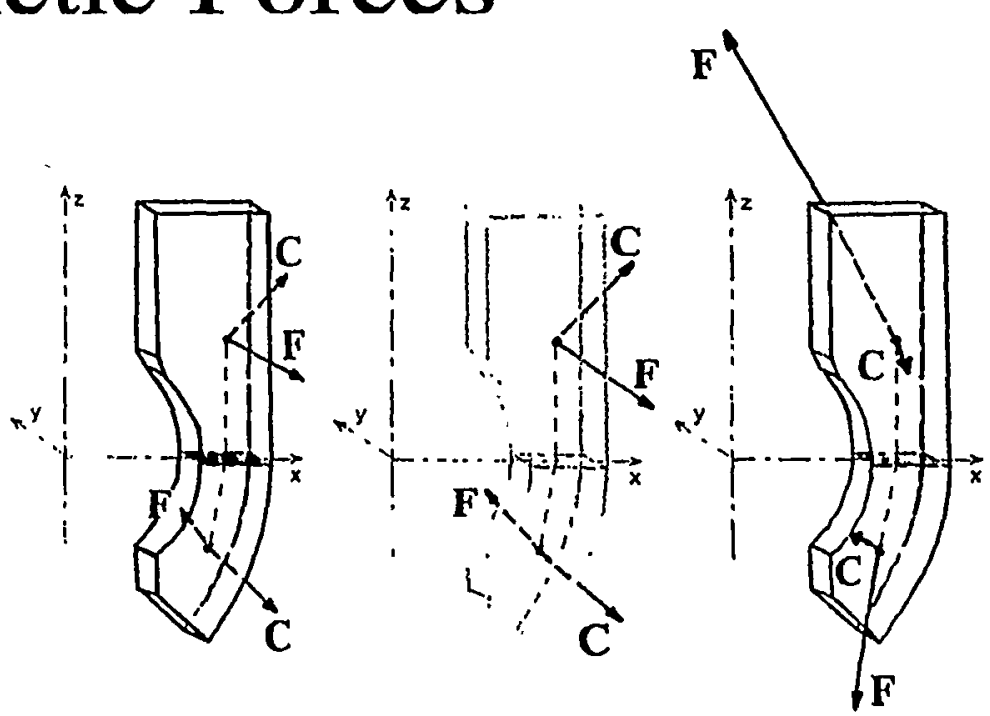

\begin{tabular}{|c|c|c|c|c|c|c|}
\hline & \multirow{2}{*}{\multicolumn{2}{|c|}{$\frac{\text { Non-magnetic Steel }}{\text { Lorentz Forces }}$}} & \multicolumn{4}{|c|}{ MANET } \\
\hline & & & \multicolumn{2}{|c|}{ Lorentz Forces } & \multicolumn{2}{|c|}{ Magnetization Forces } \\
\hline \multirow{3}{*}{0} & $f_{x}=1.1$ & $C_{x}=30.4$ & $F_{x}=0.8$ & $c_{x}=38.0$ & $F_{x}=-3.9$ & $c_{x}=-10.4$ \\
\hline & $F_{r}=-2.4$ & $c_{p}=7.1$ & $F_{r}=-3.6$ & $c_{r}=10.2$ & $F_{r}=3.8$ & $c_{\mathrm{r}}=-16.1$ \\
\hline & $f_{2}=-0.2$ & $C_{2}=22.6$ & $F_{2}=0.7$ & $C_{z}=25.8$ & $F_{L}=13.7$ & $C_{2}=-0.6$ \\
\hline \multirow{3}{*}{$\mathbf{u}$} & $F_{x}=1.0$ & $C_{x}=24.0$ & $F_{8}=0.7$ & $c_{y}=27.5$ & $F_{X}=-2.4$ & $C_{x}=0.5$ \\
\hline & $F_{r}=2.5$ & $c_{r}=.49$ & $F_{y}=2.8$ & $c_{r}+.6 .1$ & $F_{r}=-1.5$ & $C_{r}=0.8$ \\
\hline & $F_{2}=-0.1$ & $c_{t}=.23 .0$ & $F_{2}=0.9$ & $c_{z}=-25.1$ & $F_{2}=-5.7$ & $C_{z}=\cdot 0.8$ \\
\hline
\end{tabular}

Resultants of Forces (MN) and Couples (MNm) 


\section{Electromagnetic Analysis for TBM in ITER}

Following critical issues have to be studied by means of an electromagnetic analysis:

(1) magnetized matter interacts directly with the external magnetic field causing a magnetic loading (Magnetization Force, MF) on the structure even during normal operation which has to be considered for the mechanical design of the TBMs;

(2) when a plasma disruption occurs, an electromagnetic load (Lorentz force, LF) due to interaction between eddy currents and the magnetic field is added to the structure; the value of this load and the effect of the magnetization on it have to be investigated to assess the capability of the TBM structure to withstand the mechanical effects of plasma disruptions;

(3) the magnetized structure of the TBMs produces a non-axisymmetrical magnetic field in the plasma region which can affect plasma stability and lead to disruption. 


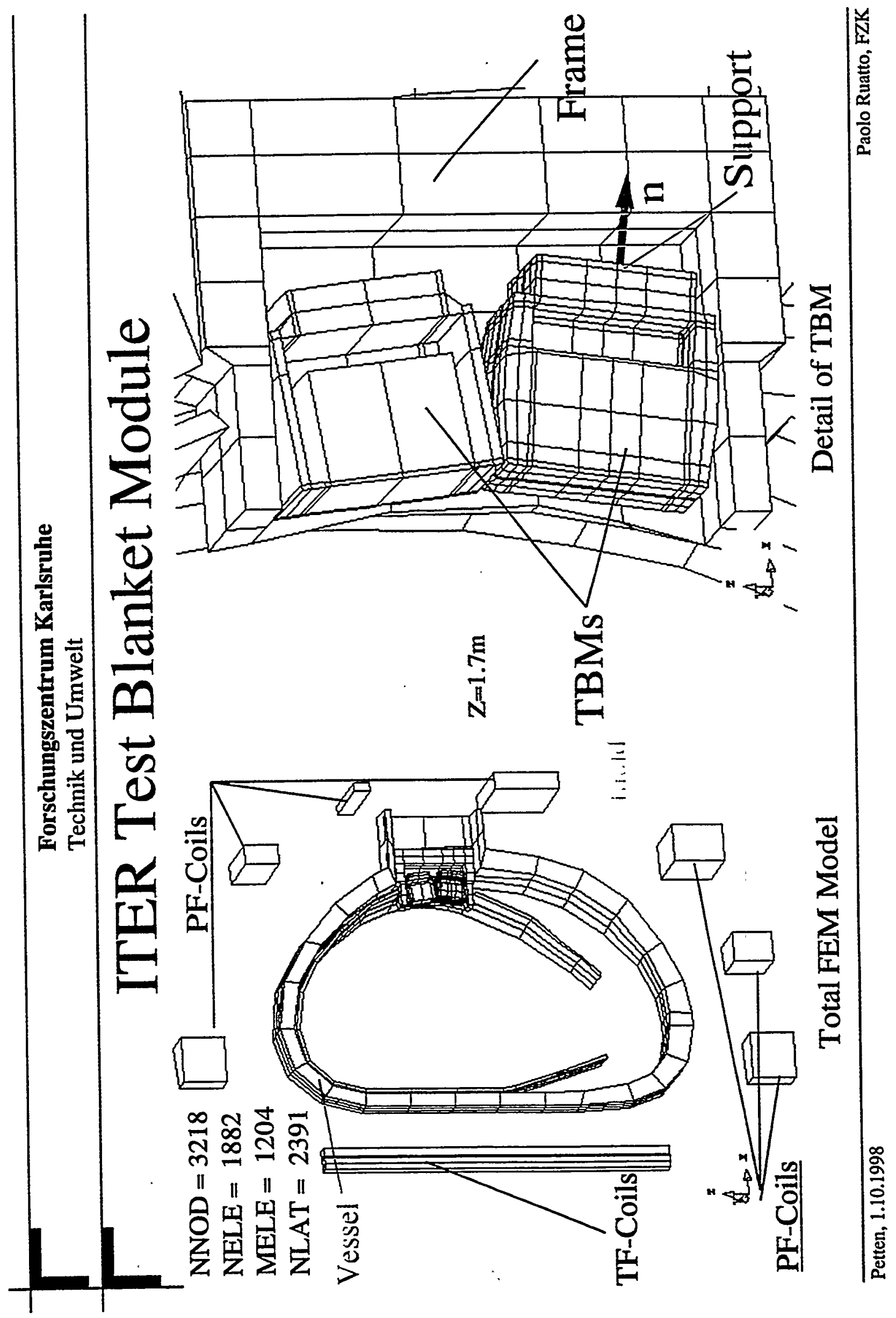




\section{Force Calculation for TBM in ITER}

Electromagnetic force distributions have been calculated for the reference centered disruption (CD, 50ms) and for upward and downward VDEs (50ms).

Resultant forces and torques have been derived at the TBM support. If we consider normal components of force and torques for the $50 \mathrm{~ms} \mathrm{CD}$, we have that:

- A pulling force of $0.2 \mathrm{MN}$ in the direction of the plasma center acts on the TBM even during normal operation;

- the resultant $F_{N}$ of the $L F$ distribution originating during the disruption achieves a maximum value of $0.12 \mathrm{MN}$ in positive normal direction, contributing to a reduction of the total $\mathrm{F}_{\mathrm{N}}$ acting on the support. However, if we consider the spatial distribution of the forces on the TBM structure, we can observe that whereas the MFs act prevalently in the radial direction, the LFs are differently directed depending on the eddy current patterns in the structure. Therefore, during a disruption the loading of the structure can be locally higher than during normal operation (for example on the first wall).

- Considering the $T_{N}$ we note that the only significant contribution is given from the LF (max. 0.8 MNm). 
Forschungszentrum Karlsruhe

Technik und Umwelt

\section{Force Calculation for TBM in ITER (continue)}

- The effect of the magnetization on the LF (stronger magnetic flux density) has been evaluated carrying out the same electromagnetic analyses for a non-magnetic structural material. For the reference $\mathrm{CD}(50 \mathrm{~ms})$ peak values of the $\mathrm{LF}$ contribution have been calculated as $\mathrm{F}_{\mathrm{N}}=0.07 \mathrm{MN}$ and $\mathrm{T}_{\mathrm{N}}=0.65 \mathrm{MNm}$.

- Upward and downward VDE have been also analysed, but from the table below - showing the maximum values of the total resultant forces and torques acting on the support for the reference CD (50ms) and upward and downward VDEs - it is evident that the most critical event for the TBM is represented by a centered plasma disruption.

\begin{tabular}{|l|c|c|c|c|}
\hline \multicolumn{1}{|c|}{ Max Values } & $\mathrm{F}_{\mathrm{N}}(\mathrm{MN})$ & ${ }_{\mathrm{F}} \mathrm{F} \mid(\mathrm{MN})$ & $\mathrm{T}_{\mathrm{N}}(\mathrm{MNm})$ & $\mid \mathrm{T}_{\mathrm{T}} \mathrm{I}(\mathrm{MNm})$ \\
\hline $\mathrm{CD}(50 \mathrm{~ms})$ & $-\mathbf{0 . 2 0}$ & 1.14 & 0.72 & 0.32 \\
Up. VDE (50ms) & $-\mathbf{0 . 2 0}$ & 0.08 & 0.47 & 0.25 \\
Down. VDE (50ms) & $-\mathbf{0 . 2 0}$ & 0.12 & 0.49 & 0.22 \\
\hline
\end{tabular}


Forschungszentrum Karlsruhe

Technik und Umwelt

\section{Force Calculation for TBM in ITER}

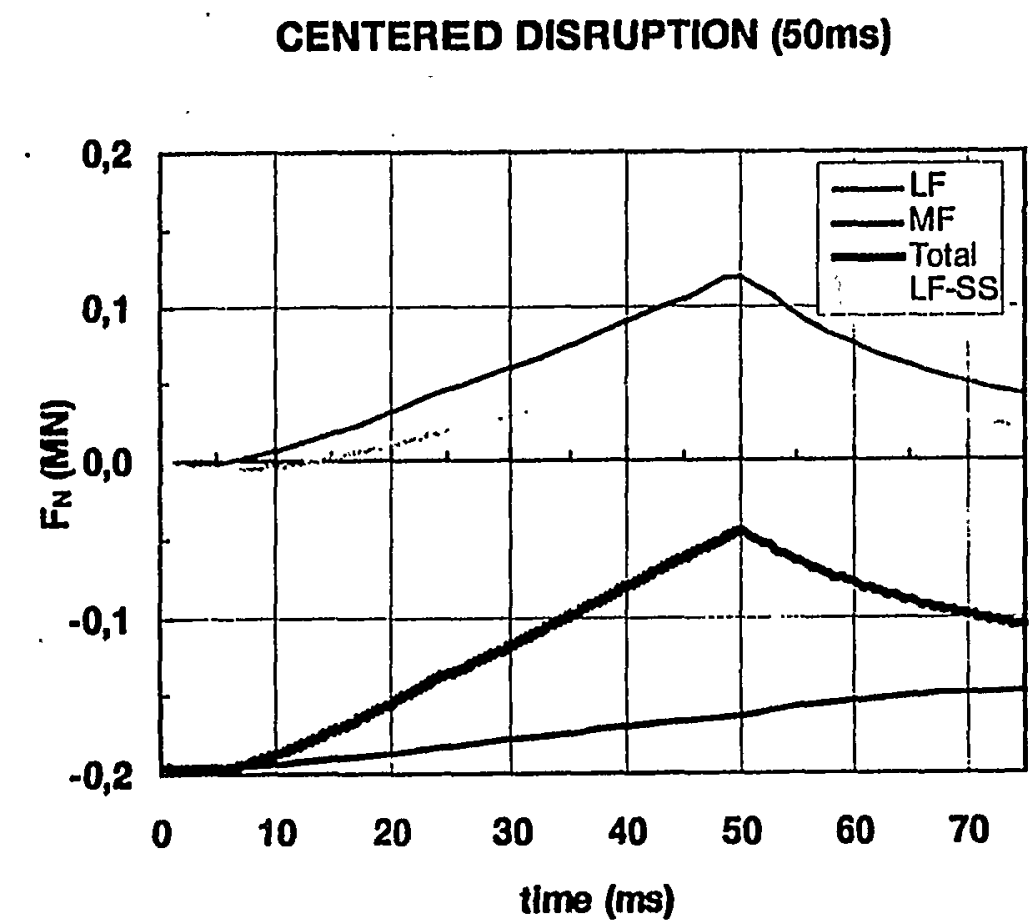

CENTERED DISRUPTION (50ms)

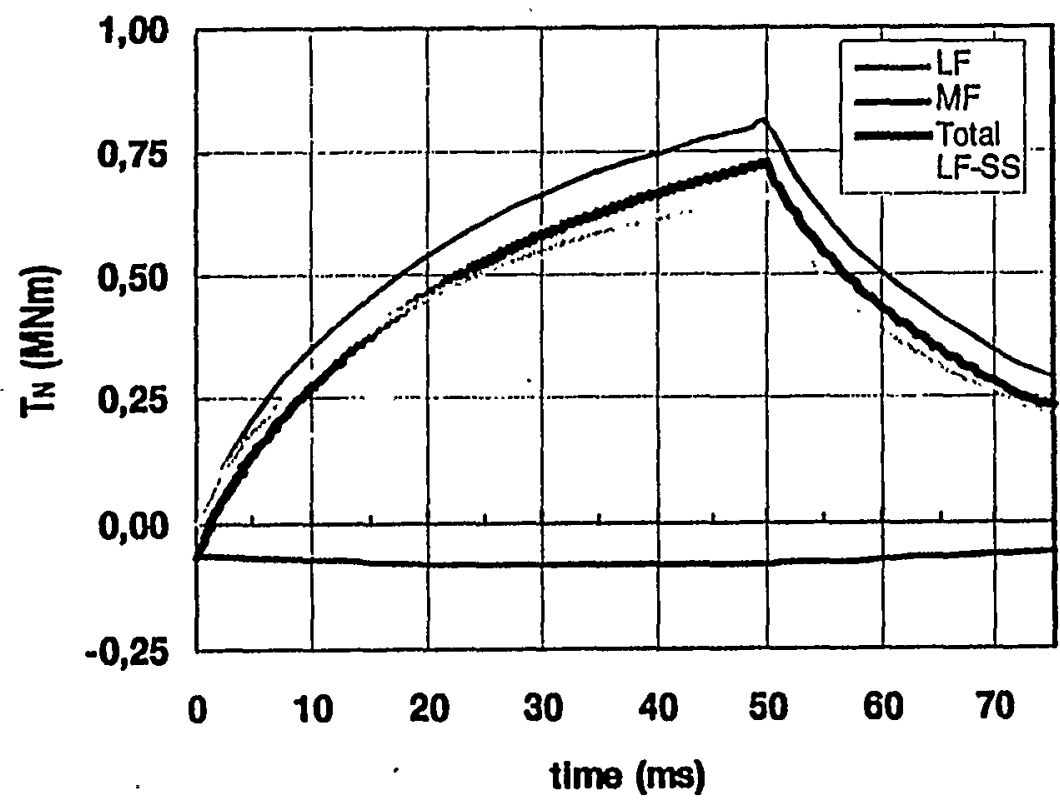




\section{Error Field Calculation for TBM in ITER}

The magnetic field produced by the magnetized matter of the TBM (error field) has been calculated to evaluate the effect of the TBMs on the toroidal magnetic field in the plasma region. Results are reported on the next slide:

- On the left, the magnitude of the error field is shown as a function of the toroidal angle in the plane $\mathrm{Z}=1.7 \mathrm{~m}$ and for different radius values.

- Toroidal magnetic field ripple values $\delta_{\mathrm{Fe}}$ for the same plane are reported on the right and have been calculated following the expression

$$
\delta_{\mathrm{Fe}}(\mathrm{R}, \mathrm{Z}=1.7)=\frac{\mathrm{B}_{\varphi}^{(\text {max })}\left(\mathrm{R}, \varphi^{(\text {max })}\right)-\mathrm{B}_{\varphi}^{(\text {min) }}\left(\mathrm{R}, \varphi^{(\text {min) }}\right)}{2 \mathrm{~B}_{\varphi}(\mathrm{R})} \cdot 100 \%
$$

- At $\mathrm{R}=10.947 \mathrm{~m}$ - outer edge of the plasma $-\delta_{\mathrm{Fe}} \cong 0.85 \%$, but it can be observed that near the module, $\delta_{\mathrm{Fe}}$ changes very rapidly with $\mathrm{R}$. Changes in $\delta_{\mathrm{Fe}}$ have also been obtained by varying the $\mathrm{Z}$ coordinate. 
Forschungszentrum Karlsruhe

Technik und Umwelt

\section{Error Field Calculation for TBM in ITER}
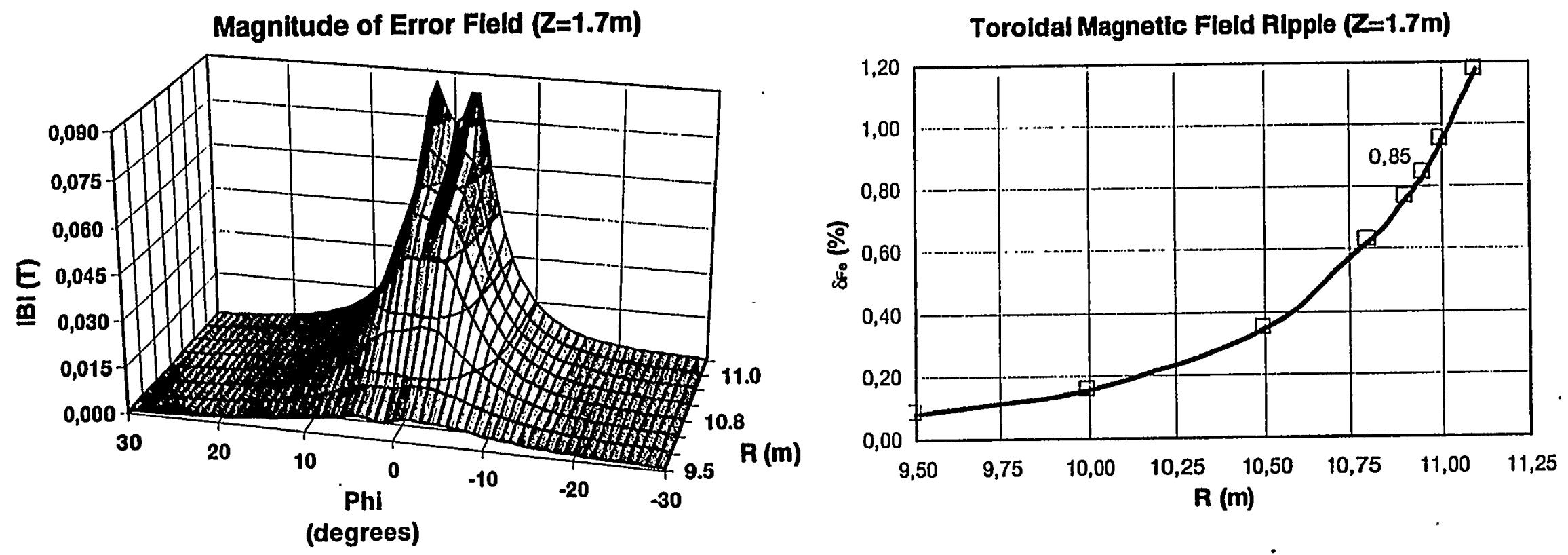


\section{Conclusions}

- An electromagnetic analysis taking into account the ferromagnetic properties of the structural material is necessary for a correct mechanical design of the DEMO outboard blanket segment as well as of the TBMs in ITER.

- Furthermore, the real structure of the TBMs has to be included in the calculations to evaluate accurately the error field effect on the plasma.

- The Finite Element program AENEAS is an appropriate tool to fulfil these requirements. 


\title{
PRESENT AND PAST RESEARCH ON THE EFFECT OF FERROMAGNETIC MATERIALS IN FUSION REACTORS: A BRIEF REVIEW
}

\author{
R. L. Klueh \\ Oak Ridge National Laboratory \\ Oak Ridge, Tennessee
}




\title{
A STATUS REPORT ON THE REVIEW OF RESEARCH ON THE EFFECT OF FERROMAGNETIC MATERIALS IN FUSION REACTORS
}

\author{
R. L. Klueh
}

People who are familiar with this research area were contacted in the United States, Europe, and Japan. In our query to those experts, we asked for their assessment of the problem, what had been done in their organizations in the past, and what was being done at present. We have received four replies from Japan, two from the U.S., and one from Europe. A brief summary of what was obtained is given below.

\section{Work in the United States}

The first work in the world in this area appears to have been done in the United States in the early 1980s. Attaya et al. [1] considered the effect of a ferritic steel on the first wall, blanket, and coolant circuit of a tandem mirror machine. The field perturbations were found to be small and confined to the end region and on the same order of magnitude as the field ripples produced by the central cell magnets. Based on the calculations of the magnetostatic forces on a ferritic stzel pipe in the magnetic field of the machine, the stresses were found to be small, but not negligibie, and they must be incorporated in the stress analysis of the design.

Lechtenberg et al. [2], considered the ferromagnetic stresses on a coolant pipe in a tokamak (Starfire) from the toroidal field magnets. They concluded that the stresses were at such a level that they had to be accounted for, but they were not of the level that they were unmanageable.

Earlier work at GA [3], which was summarized by Lechtenberg et al. [2], considered the effect of a ferromagnetic material (Sandvik HT9) on the toroidal field ripple for three conceptual designs (GA TNS, ETF, and Starfire); the concern was that the ferromagnetic steel could introduce an additional ripple resulting in a loss of plasma energy or a plasma disruption. These studies concluded that due to the high level of saturation, the ferromagnetic blankets have an effective magnetic permeability close to unity and so do not cause any significant changes to the toroidal field ripple. Perturbations of up to $3 \%$. were found possible for the polodial fields (calculated for Starfire), but these could be handled by normal plasma control techniques. From a simple force calculation based on simple cylinders in the magnetic fields, it was concluded that a "simple 'keystoned' blanket structure could be designed to accommodate this minor parasitic force."

The references for that early work are:

1. H. Attaya, K. Y. Yuan, W. G. Wolfer, and G. L. Kulcinski, in: Proceedings of Topical Conference on Ferritic Steels for use in Nuclear Energy Technologies, Eds. J. W. Davis and D. J. Michel (The Metallurgical Society of AIME, Warrendale, PA, 1984) p. 169. 
2. T. Lechtenberg, C. Dahms, and H. Attaya, ibid, p. 179.

3. J. Rawis, W. Chen, E. Chung, J. Dillassandro, P. Miller, S. Rosenwasser and L. Thompson, Assessment of Martensitic Steels as Structural Materials in Magnetic Fusion Devices, General Atomic Report GA-A15749, January 1980.

In one of the replies to our queries to U.S. researchers, J. Blanchard indicated that not much has been done in the U.S. beyond the work of Attaya, Lechtenberg, and the workers at GA in the 1980 s. Blanchard bases that on "a quick review of ferromagnetic effects for a system design study (ARIES)" that he carried out. His review resulted in a recommendation that a further study be carried out.

The only other information I received from the U.S. was a copy of an ITER Memo, "The Study of Ferromagnetic Inserts in the Vacuum Vessel (No. 1)," sent by J. Schmidt of PPL. The inserts were meant to reduce toroidal field ripple, and the report showed how this could be accomplished with the ferritic 430 stainless steel.

Both Blanchard and Schmidt expressed the importance of the problem and indicated that their organizations have the tools and personnel to carry out a detailed analysis of the problem should funds for such a project become available.

\section{Work in Japan}

Japan has an ongoing program, which is being carried out at JAERI and Hitachi (in an early e-mail message from Kohyama, he mentioned that work might be going on at the universities, but $I$ have not received anything yet on that work). The Japanese work includes the installation of an F82H liner in a small tokamak. Some of this work was reported in the following papers: .

1. S. Takeji et al., "Error Field Analysis of Steady State Tokamak Reactor with Ferromagnetic (F82H) Blanket," in: Proc. 16 ${ }^{\text {th }}$ IEEE/NPSS SOFE, Vol. 2, 1995, p. 1214.

2. M. Sato et al., "Analysis of Error Field due to Ferritic Steel in the Advanced Material Testing Program of JFT-2M," J. Nucl. Mater., to be published.

3. T. Nakayama, et al., "Evaluation of Magnetic Fields due to Ferromagnetic Vacuum Vessel And their Influence on Plasma Discharge in Tokamak Devices," J. Nucl. Mater., to be published.

The first of these papers analyzes the error fields generated by a ferromagnetic steel blanket for the conceptual design of a steady state tokamak reactor. The second paper is a computational comparison of the case of a vacuum vessel of non-magnetic material with a "ferritic board" [which I think means ferritic steel liner] and one made entirely of ferritic steel. The analysis involves the ripple amplitude reduction, and they show that the results are the same for both types of vacuum vessels. 
For the third paper, the magnetic fields due to the ferromagnetic vacuum vessel in the Hitachi Tokamak HT-2 were calculated and determined experimentally to verify that plasma discharge is possible with a ferritic steel vacuum vessel. The results were extrapolated to JFT-2M and ITER. Plasma discharge was possible for the HT-2, and the effect was concluded to be smaller for the larger tokamak devices, indicating plasma discharge should be possible for the larger vessels.'

\section{Work in Europe}

A list of sixteen papers/reports was received from $\mathrm{P}$. Ruatto of Karlsruhe, who is working with $\mathrm{L}$. Boccaccini. Ruatto later sent me five papers-the most important ones-that contained the main substance of their work. Their goal appears to be an assessment of the mechanical loading on the DEMO reactor components during plasma disruption. The following are the titles of the publications Ruatto sent:

1. L. V. Boccaccini et al., "Analysis of Mechanical Effects Caused by Plasma Disruptions in the European BOT Solid Breeder Blanket Design with MANET as Structural Material, "Third International Symposium on Fusion Nuclear Technology, Los Angeles, CA, June 27-July 1, 1994, Fusion Engineering and Design 27 (1995) Part A, 407.

2. P. Ruatto, "Computation of the Generation and Impact of Electromagnetic Fields on Ferromagnetic Structures Surrounding the Plasma in a DEMO Fusion Reactor," ISEM 95 International Symposium on Non-Linear Electromagnetic Systems, Cardiff, UK, September 17-20, 1995, in: Nonlinear Electromagnetic Systems, A. J. Moses and A. Basak, eds. (IOS Press, Amsterdam, 1996) 835.

3. L. V. Boccaccini and P. Ruatto, "Effect of the Presence of Ferromagnetic Structural Material on the DEMO Helium Cooled Pebble Bed Blanket During Plasma Disruptions," Fusion Technology (1997) 1519.

4. T. Takagi, J. Tani, P. Ruatto, B. Krevet, and L. V. Boccaccini, "Numerical Evaluation of Natural Frequency Change of a Ferromagnetic Plate Due to Magnetic Stiffness Effect," IEEE Transactions on Magnetics, 32 (3) (1996) 1054.

5. P. Ruatto, "Development of a Numerical of a Numerical Method to Compute Electromagnetic Forces Generated by Magnetic Field Changes in Magnetic Structures and Application of this Method to.a Plasma Disruption in a Tokamak Fusion Reactor," Forschungszentrum Karlsruhe, Wissenschaftliche Berichte, FZKA 5683, March 1996 (in German)

According to Ruatto, the early European work involved a code that could not consider nonlinear magnetic materials. A new code was developed by Ruatto that has been used and is being used for the analyses discussed in the publications. Until now they have not considered the influence of the ferromagnetic material on the error field of the plasma, but they are presently getting ready to study that problem. 


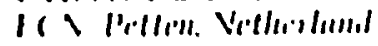

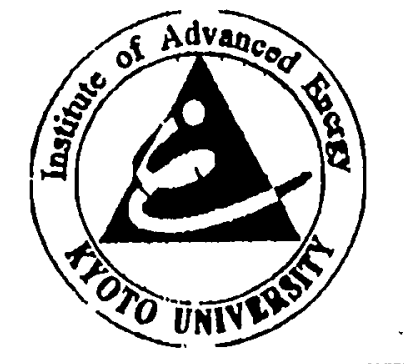

\section{Fatigue Properties of Low Activation Ferritic Steel; JLF-1 and Its Welded Joints}

Institute of Advanced Energy

A. Kohyama, T. Hirose and Y. Katoh

Institute of Advanced Energy, Kyoto University

Gokasho, Uji, Kyoto 611, Japan

Kohyama@iae.kyoto-u.acjip

http://infosrv.iae.kyoto-u.ac.jp 


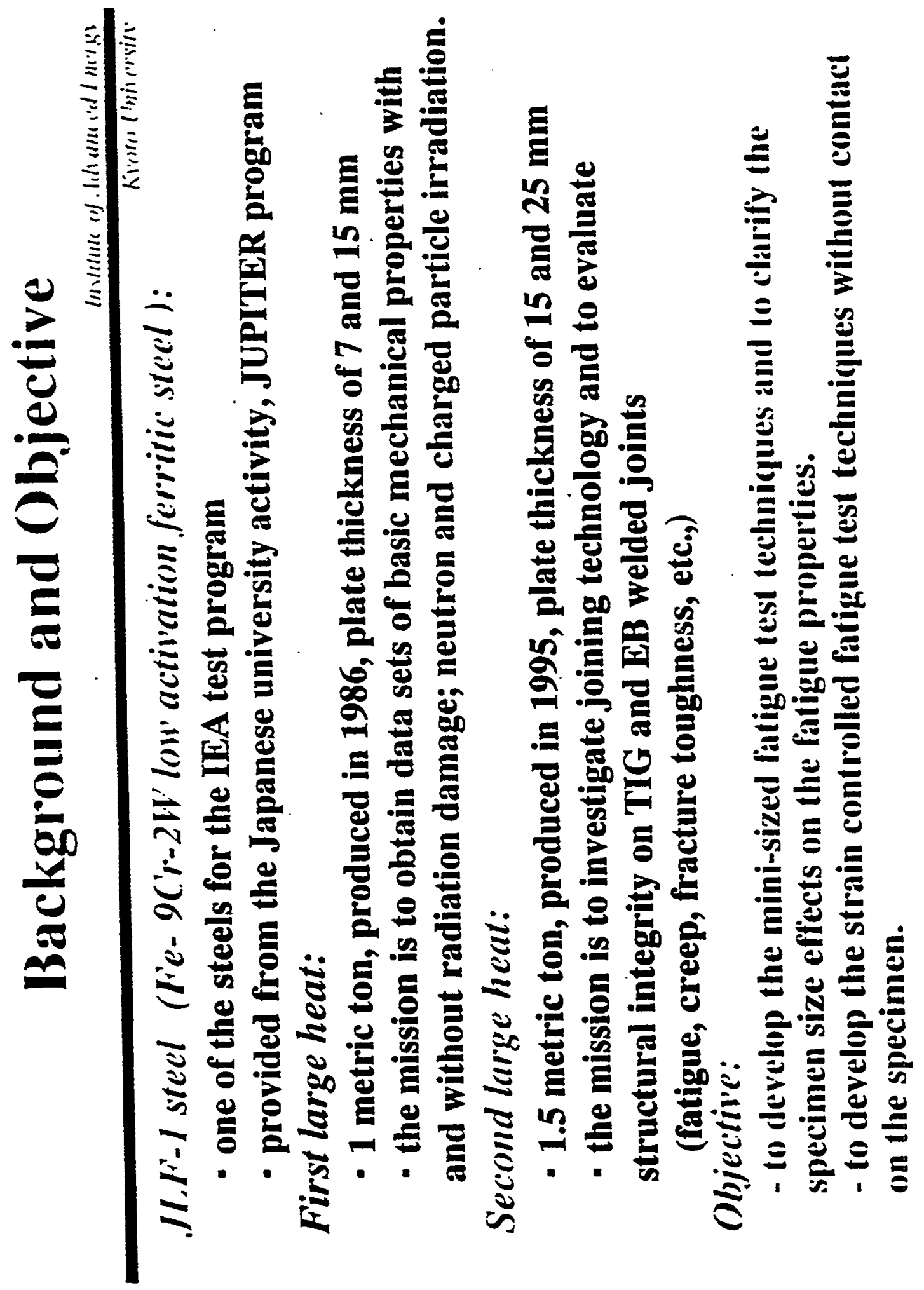




\section{Fabrication of Specimen for Fatigue Test}

\section{Instiutu of Adivanced tincrgy}
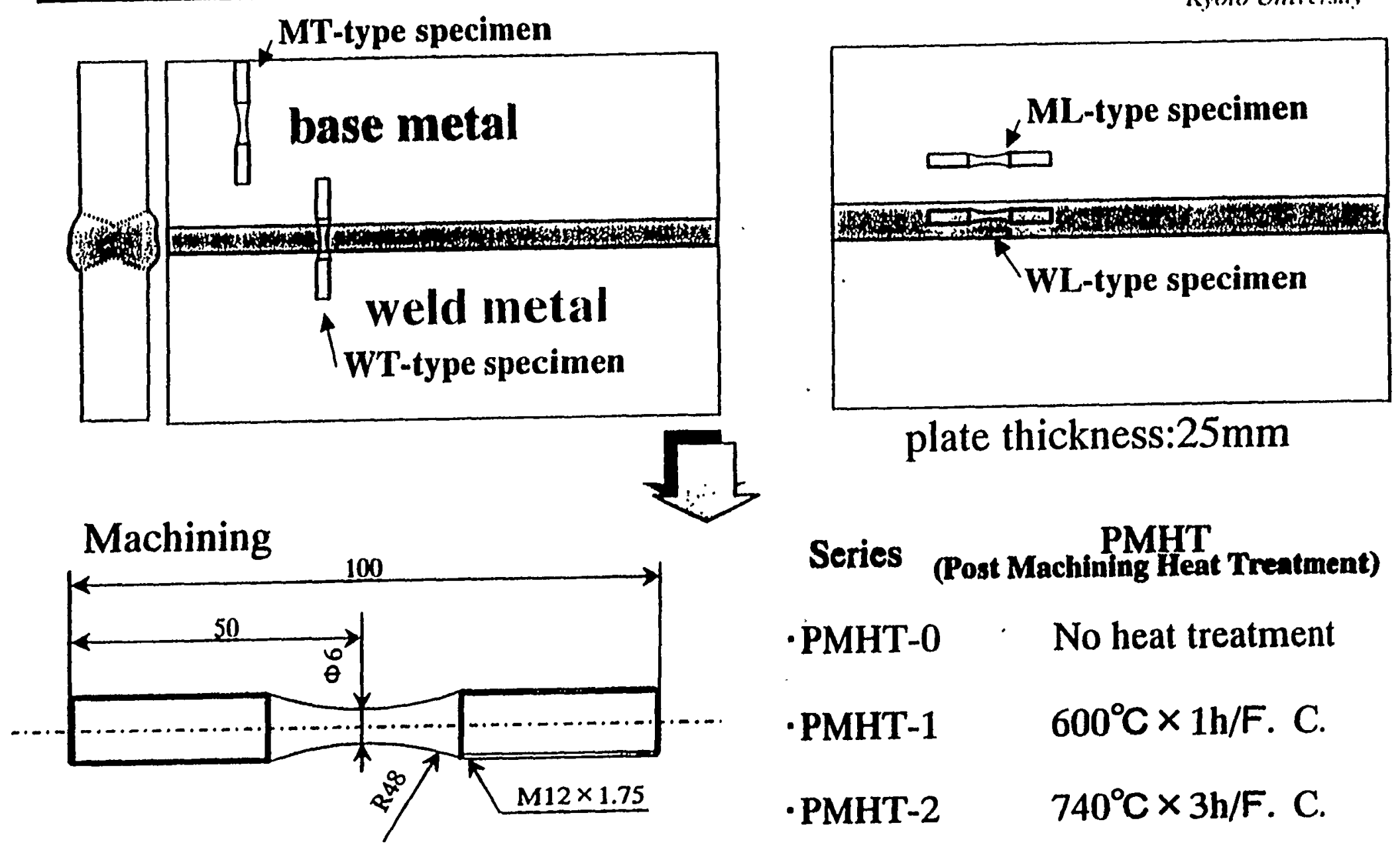

-PMHT-0 ' No heat treatment

-PMHT-1 $\quad 600^{\circ} \mathrm{C} \times 1 \mathrm{~h} / \mathrm{F} . \mathrm{C}$.

-PMHT-2 $\quad 740^{\circ} \mathrm{C} \times 3 \mathrm{~h} / \mathrm{F} . \mathrm{C}$.

Dimension:mm 


\section{Strategy to Evaluate Fatigue Properties under irradiation \\ Institute of Advanced Energy}

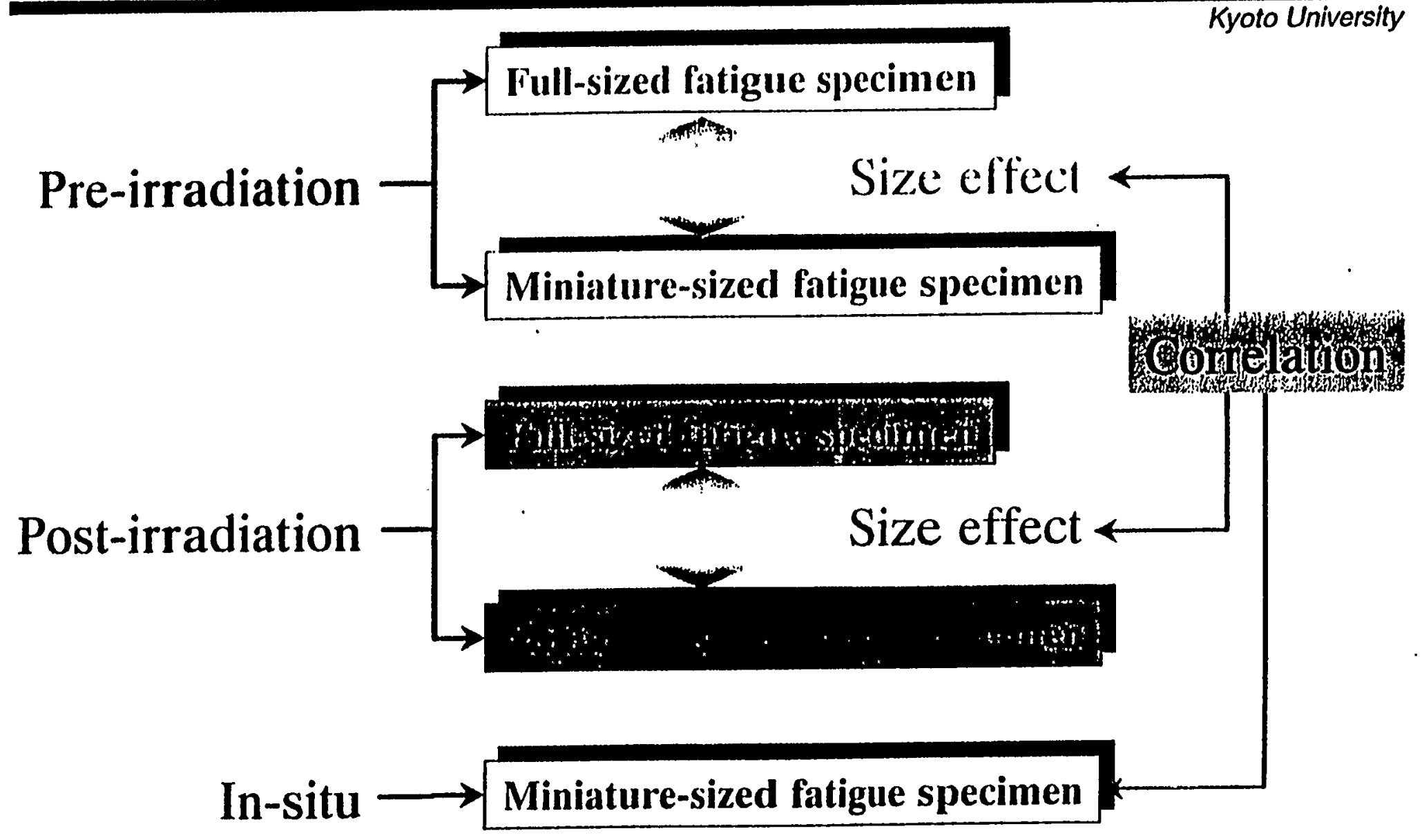




\section{Fatigue Specimen Used}

Institute of Advanced Energy

Kyoto University

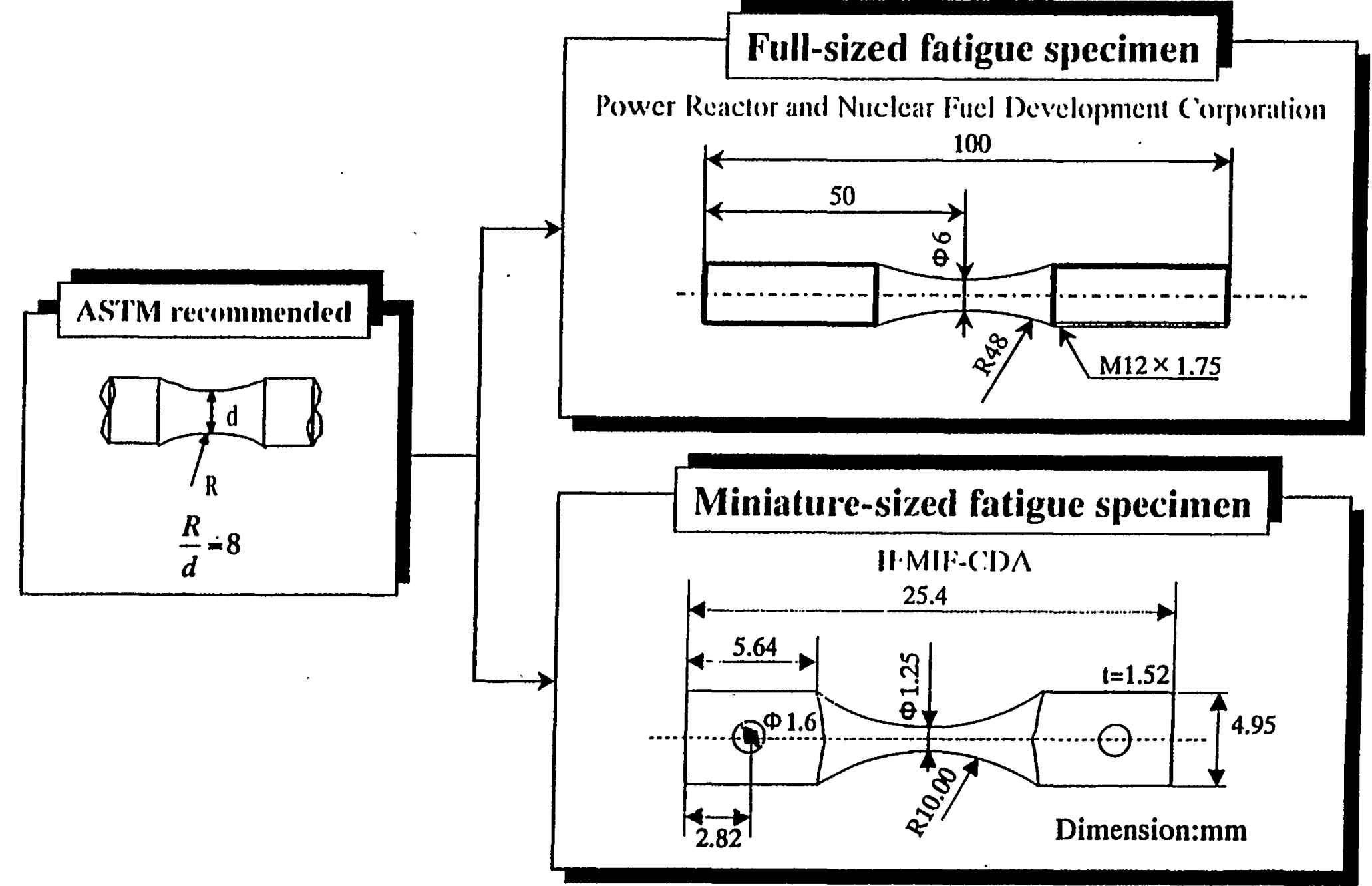




\section{Experimental Procedure (2)}

Institute of Advanted lincregy'

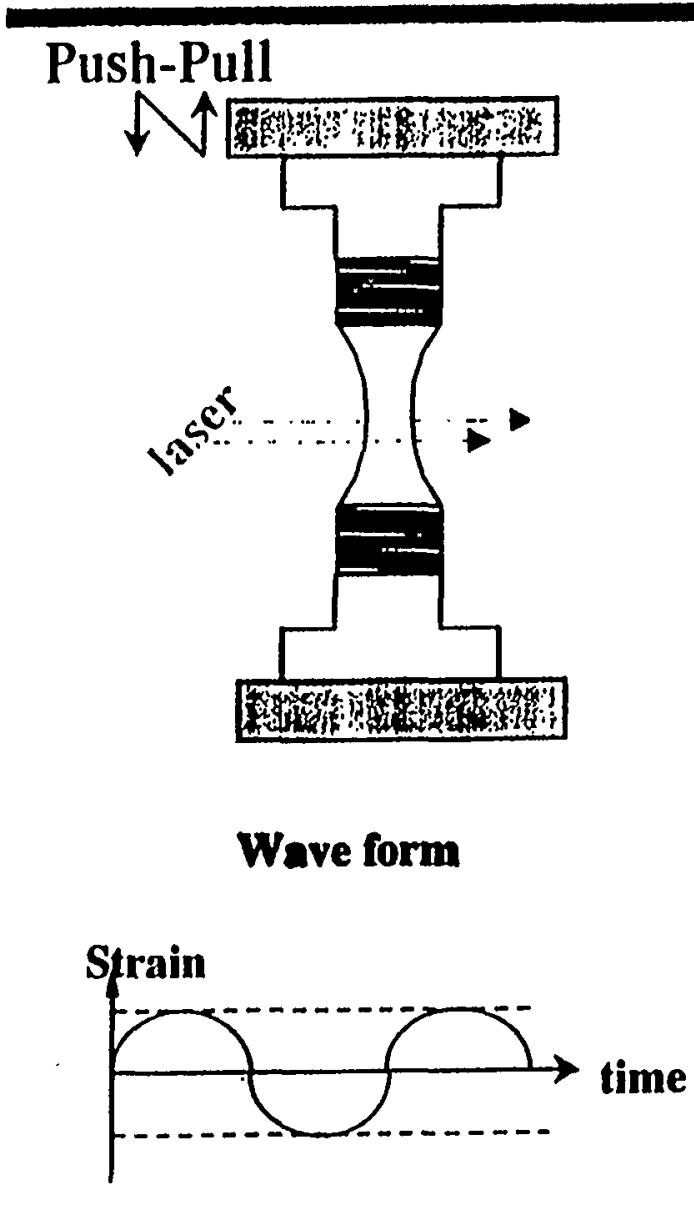

Sroheme of radius strain mectsureme'nt spystem

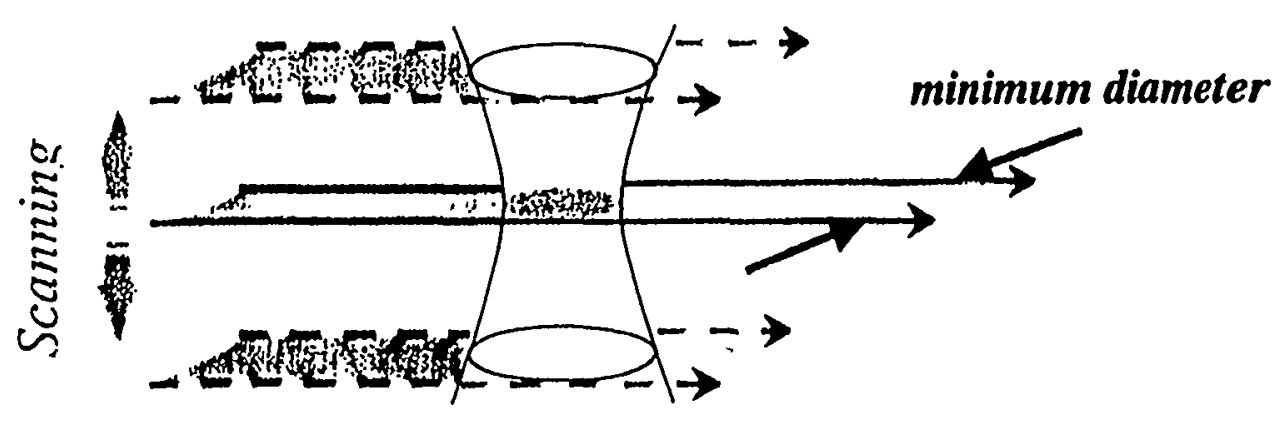

- Hydraulic servo controlled testing machine Maximum load:10tf

- Wave form : sine

- Stress ratio : $R=-1$

- Frequency : $1 \mathrm{~Hz}$

- Total radius strain controlled 


\section{Experimental Procedure}

Institute of Advanced Energy

\section{Facilities}

Pre-irradiation

Institute of Advanced Energy,

Kyoto University

Post-irradiation

In-situ : TBD

\section{Test Machine}

full-sized fatigue specimen

Mini-sized fatigue specimen

- Hydraulic servo controlled testing machine Maximum load:10tf

- Electrical testing machine Maximum load:200kgf 


\section{Welding Conditions}

Institute of Advanced Energy

Kyoto University

Tungsten insert gas (TIG) welding

\section{Conditions}

\begin{tabular}{|l|c|}
\hline CURRENT & $230 \sim 250 \mathrm{~A}$ \\
\hline Voltage & $10.5 \mathrm{~V}$ \\
\hline Travel speed & $10 \mathrm{~cm} / \mathrm{min}$ \\
\hline Heat input & $14.5 \sim 15.8 \mathrm{~kJ} / \mathrm{cm}$ \\
\hline Preheat temp & $\leqq 200^{\circ} \mathrm{C}$ \\
\hline Interlayer temp & $\leqq 200^{\circ} \mathrm{C}$ \\
\hline Number of passes & $\fallingdotseq 20$ \\
\hline Wire diameter & $1.2 \mathrm{~mm}$ \\
\hline
\end{tabular}

PWHT $740^{\circ} \mathrm{C} \times 3 \mathrm{~h} / \mathrm{F}$. C.

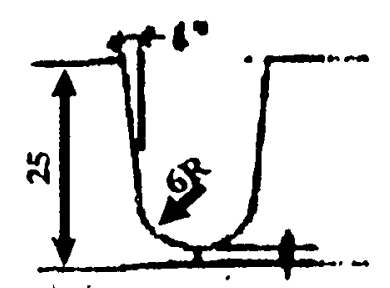

Groove

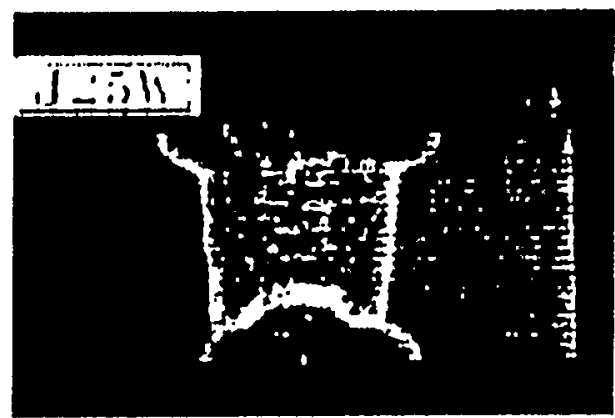

Macrostructure

(Post Welding Heat Treatment) 


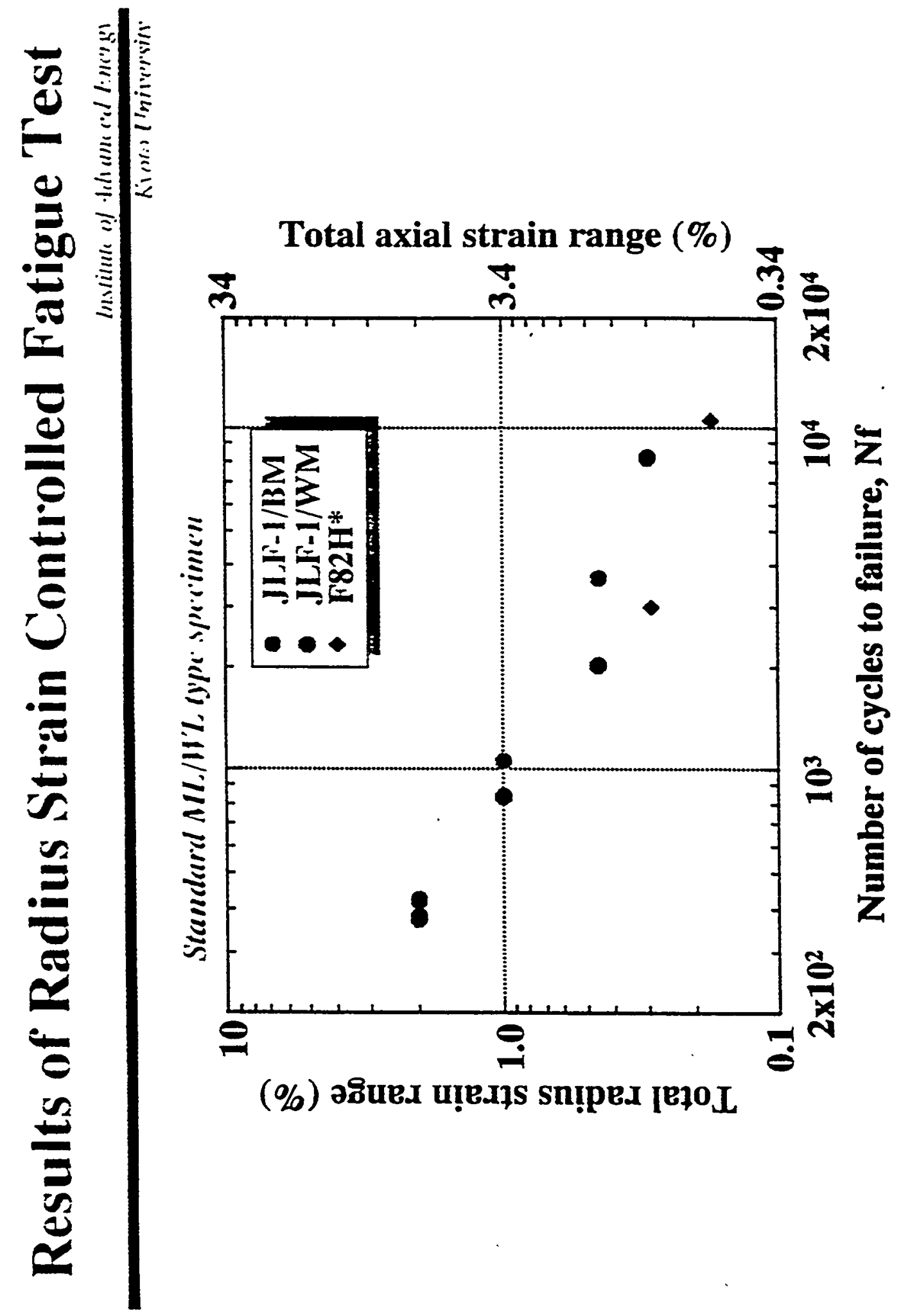



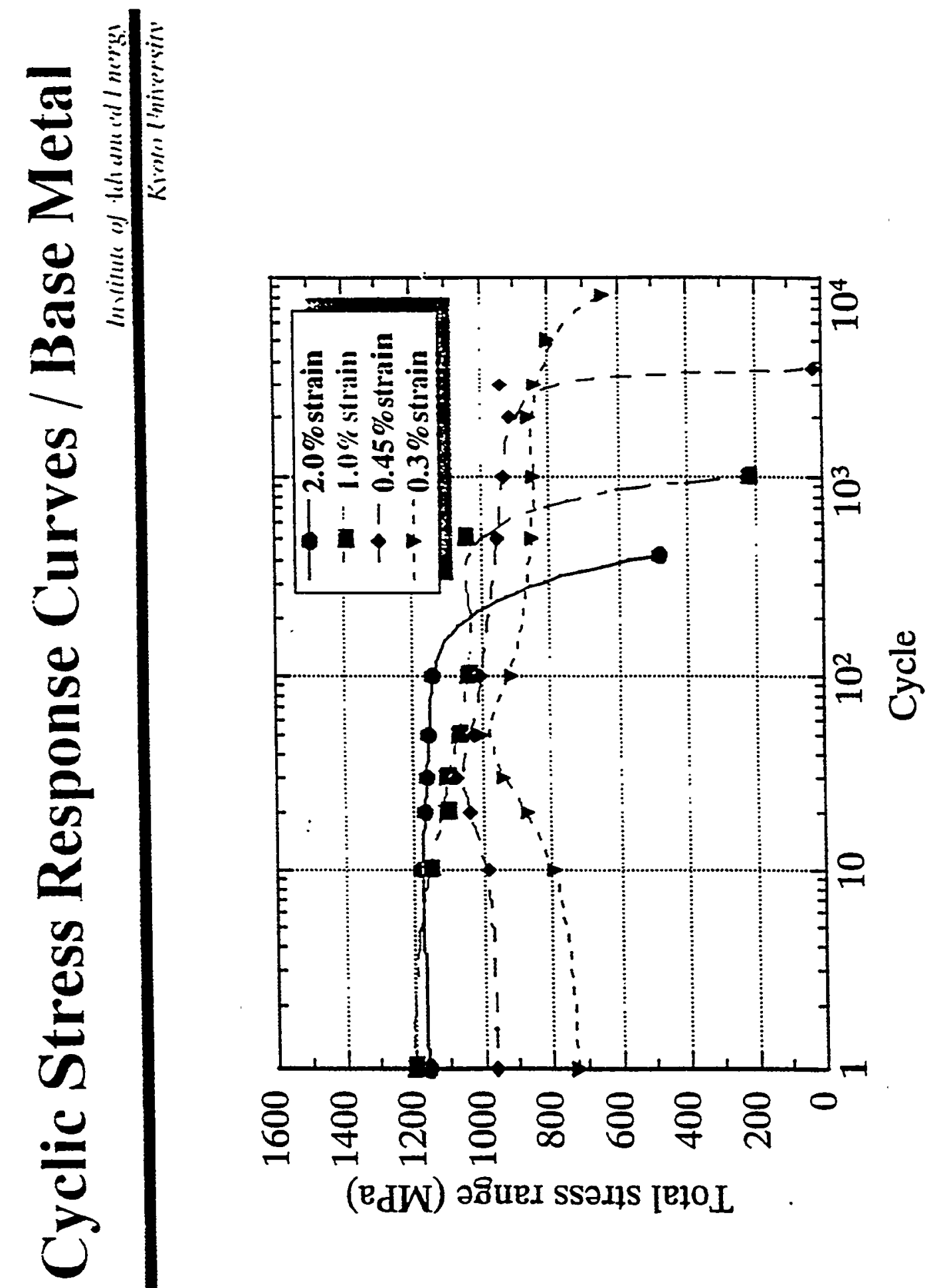


\section{Cyclic Stress Response Curves / Weld Metal}

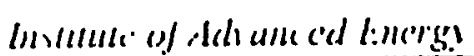

Kiontr l'nincesine

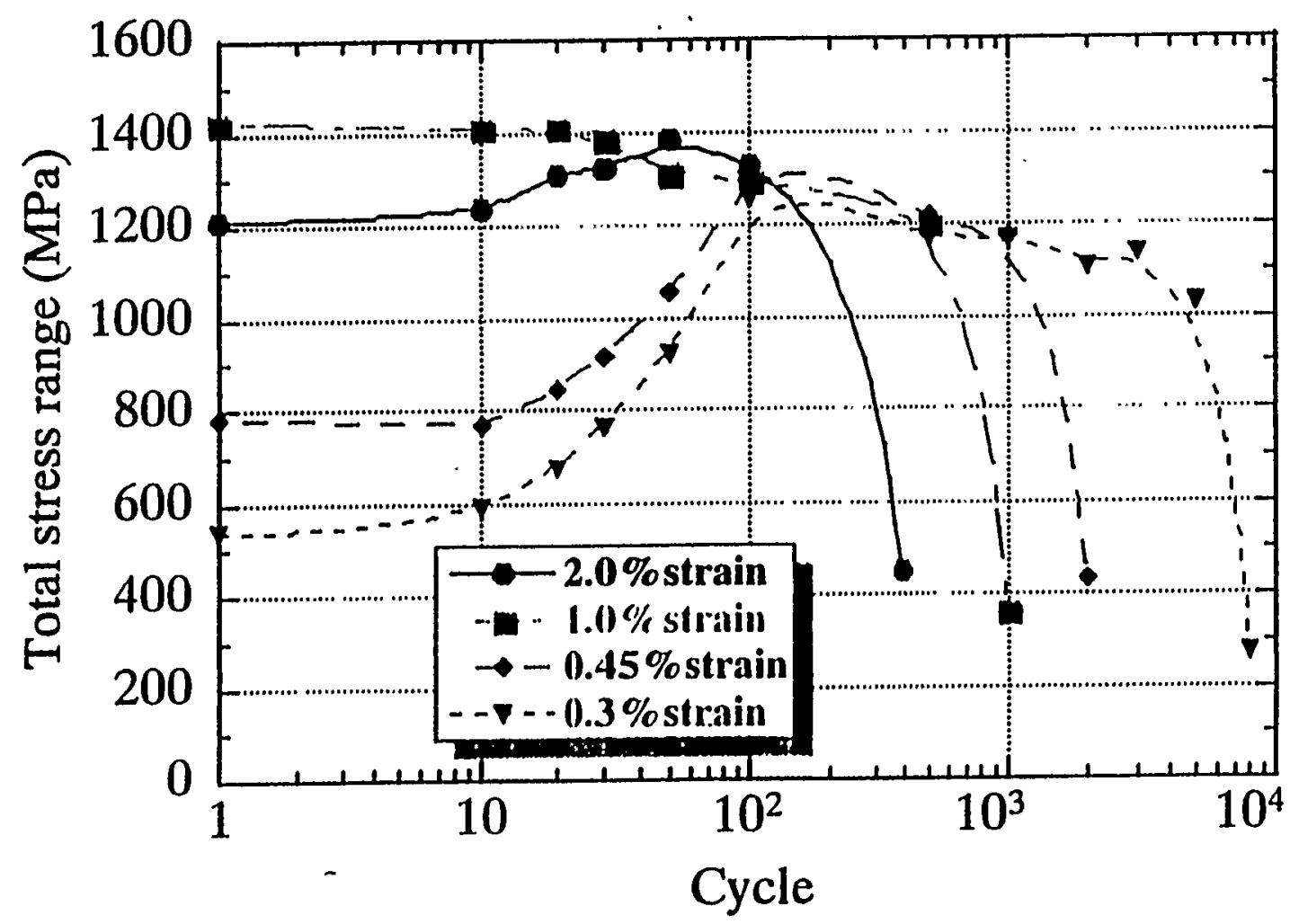




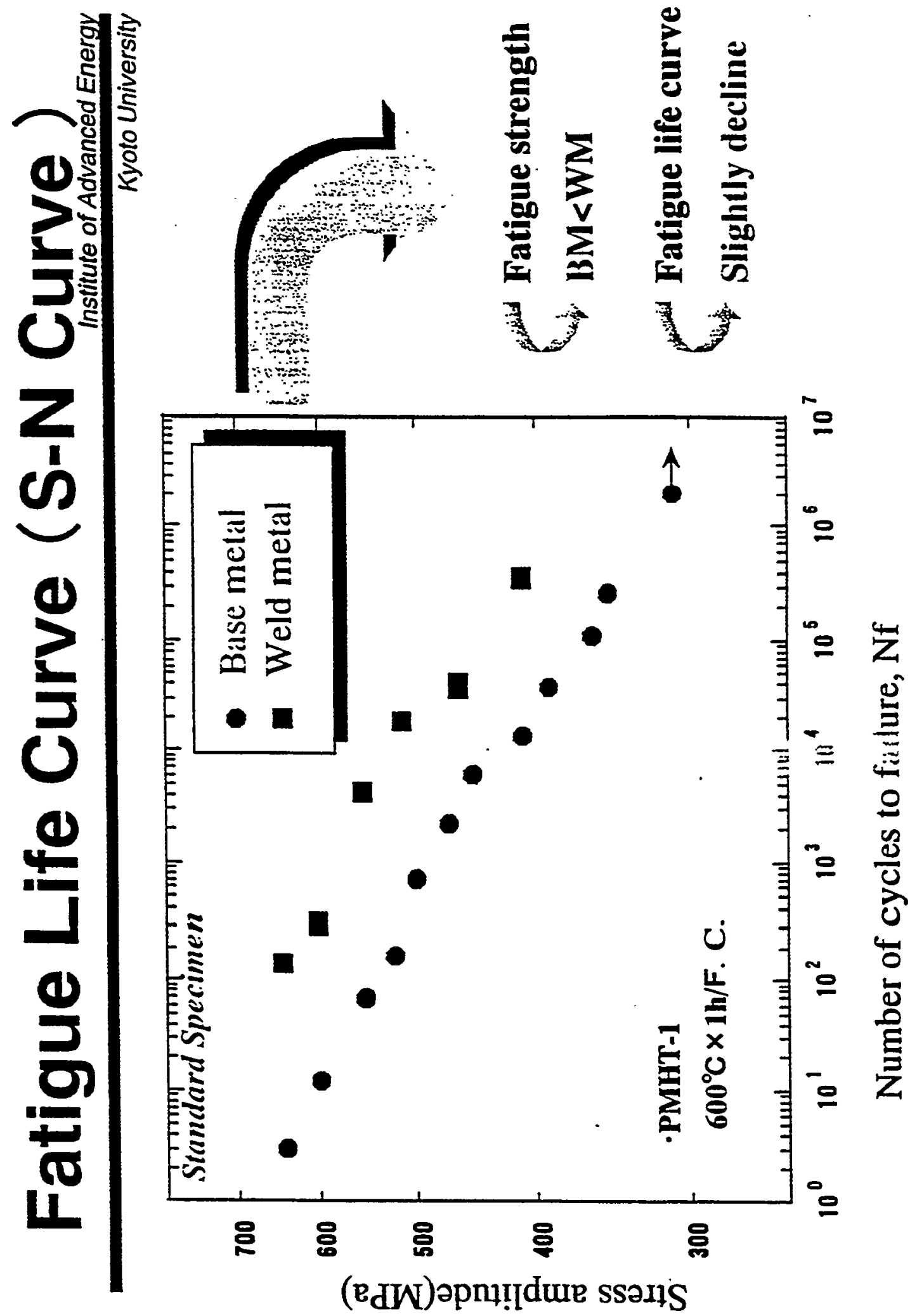




\section{Correlation between \\ Fatigue Limit and Tensile properties}

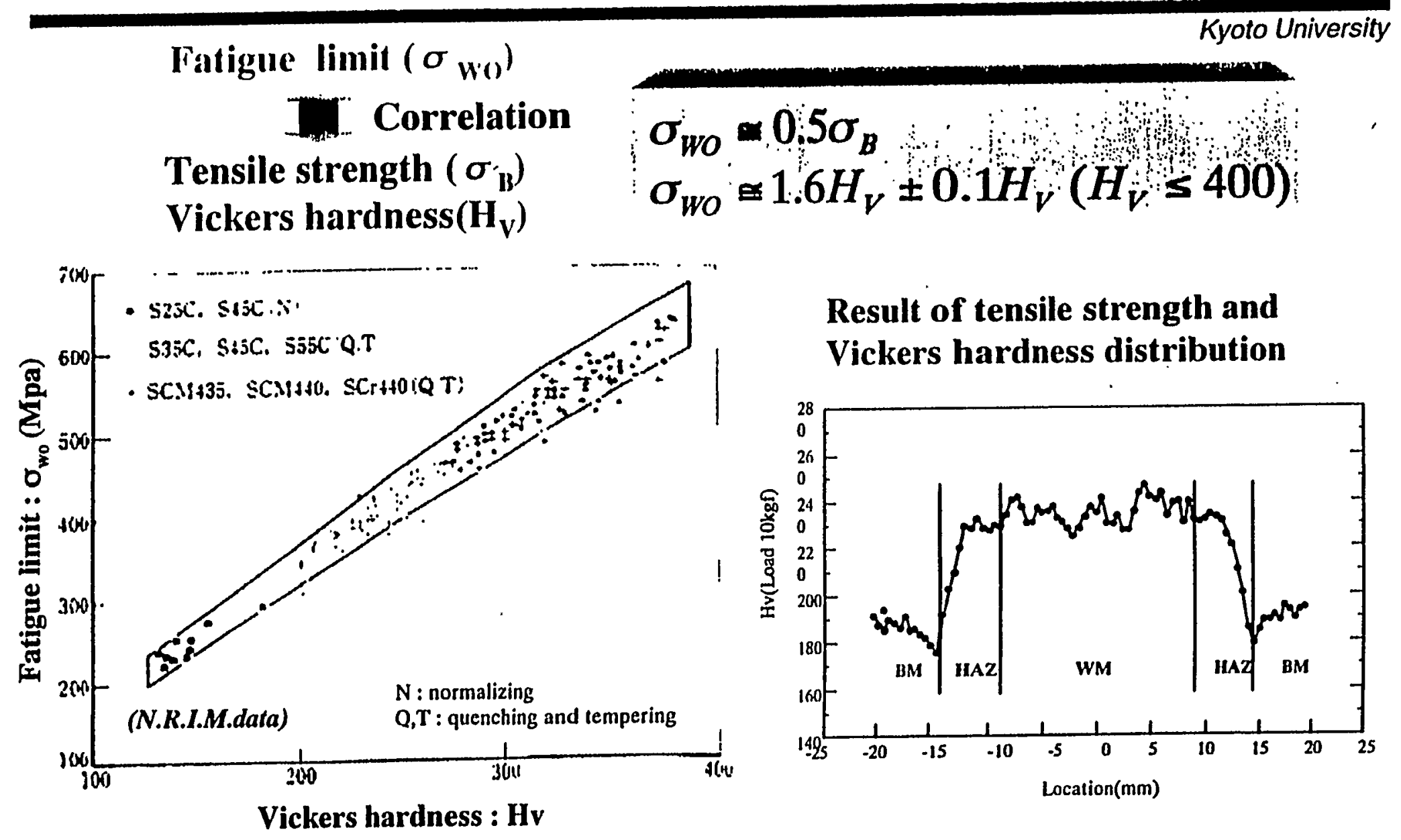




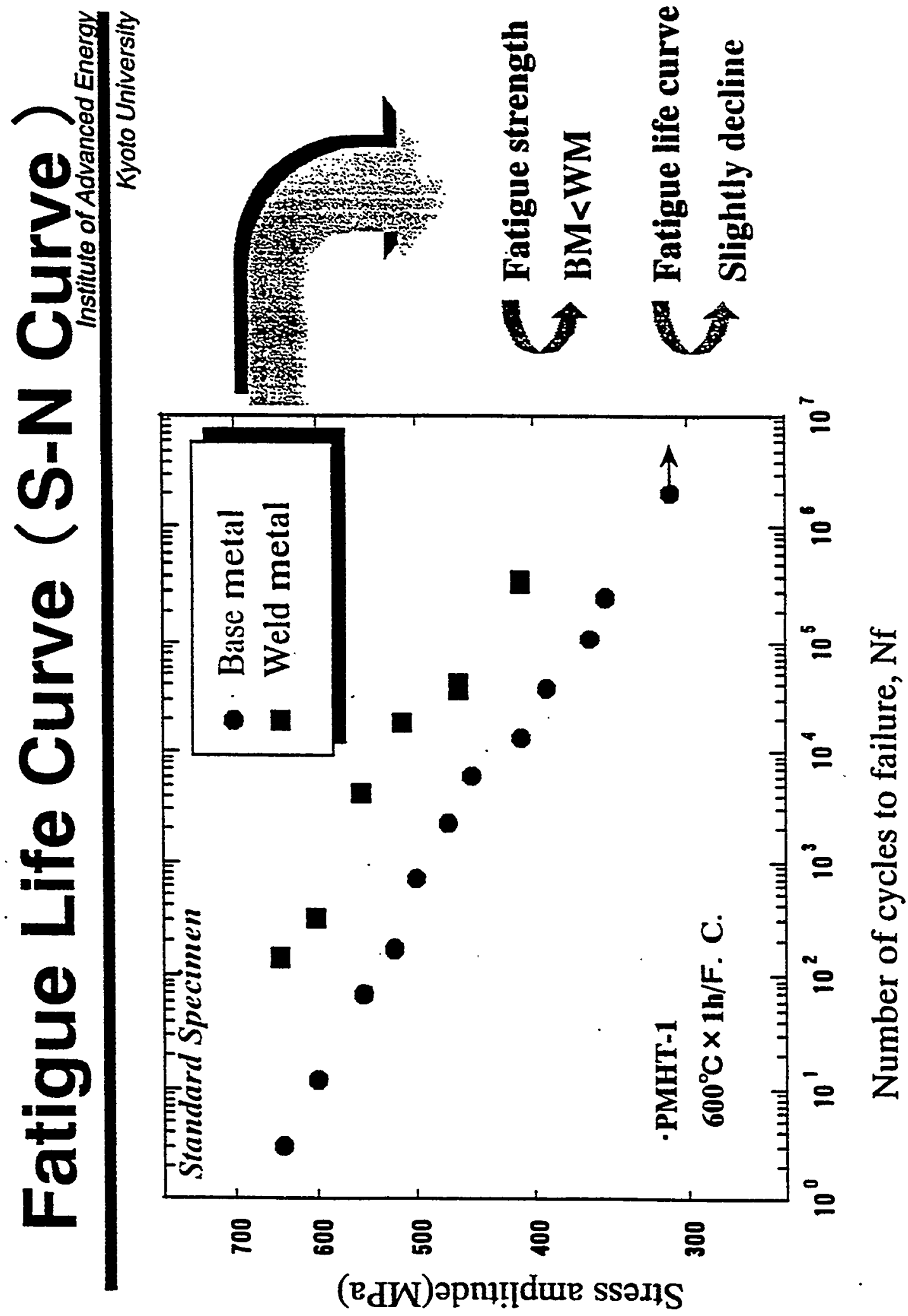




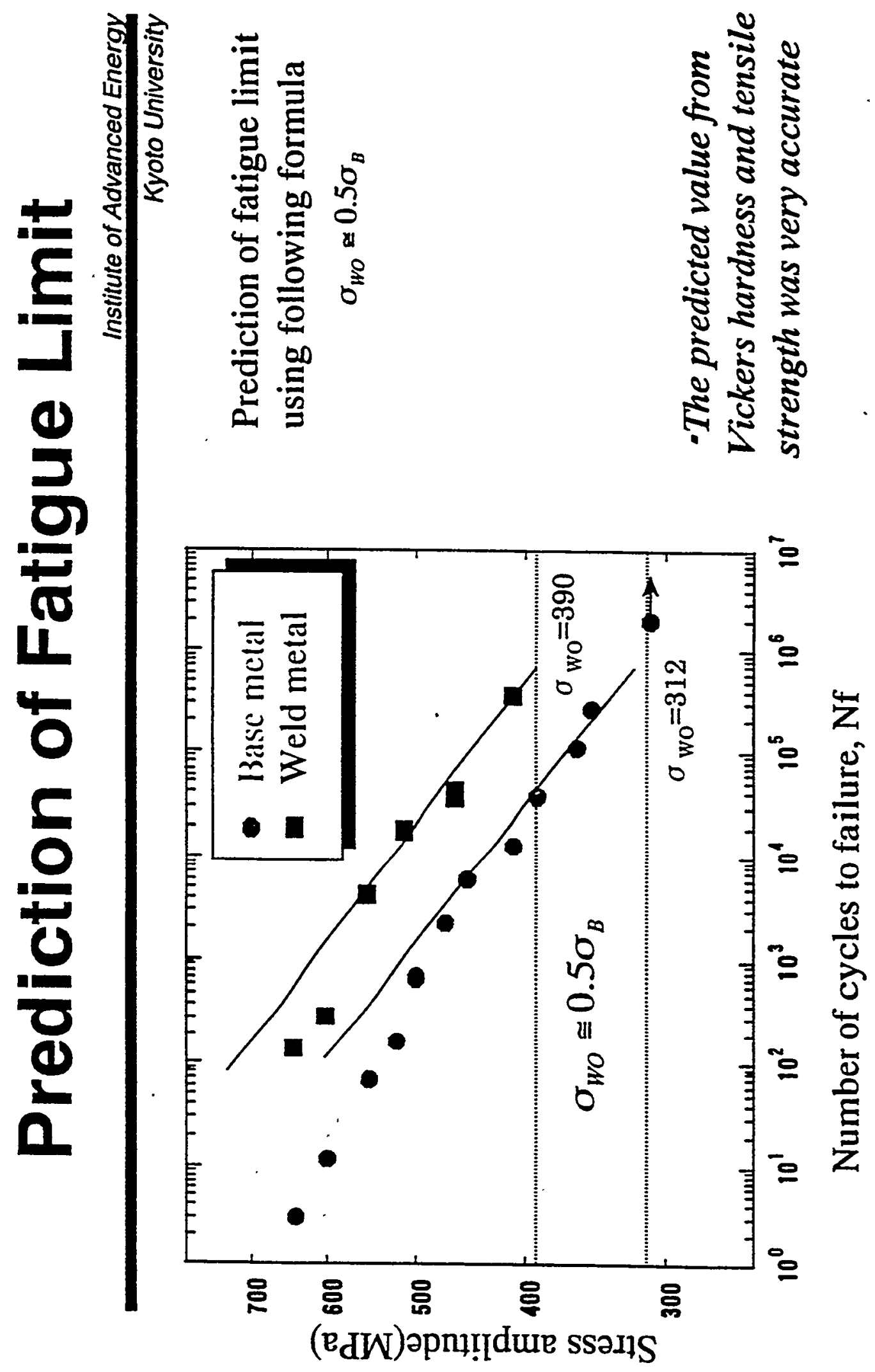




\section{Effect of Specimen Size on Fatigue Properties}

Kyoto University

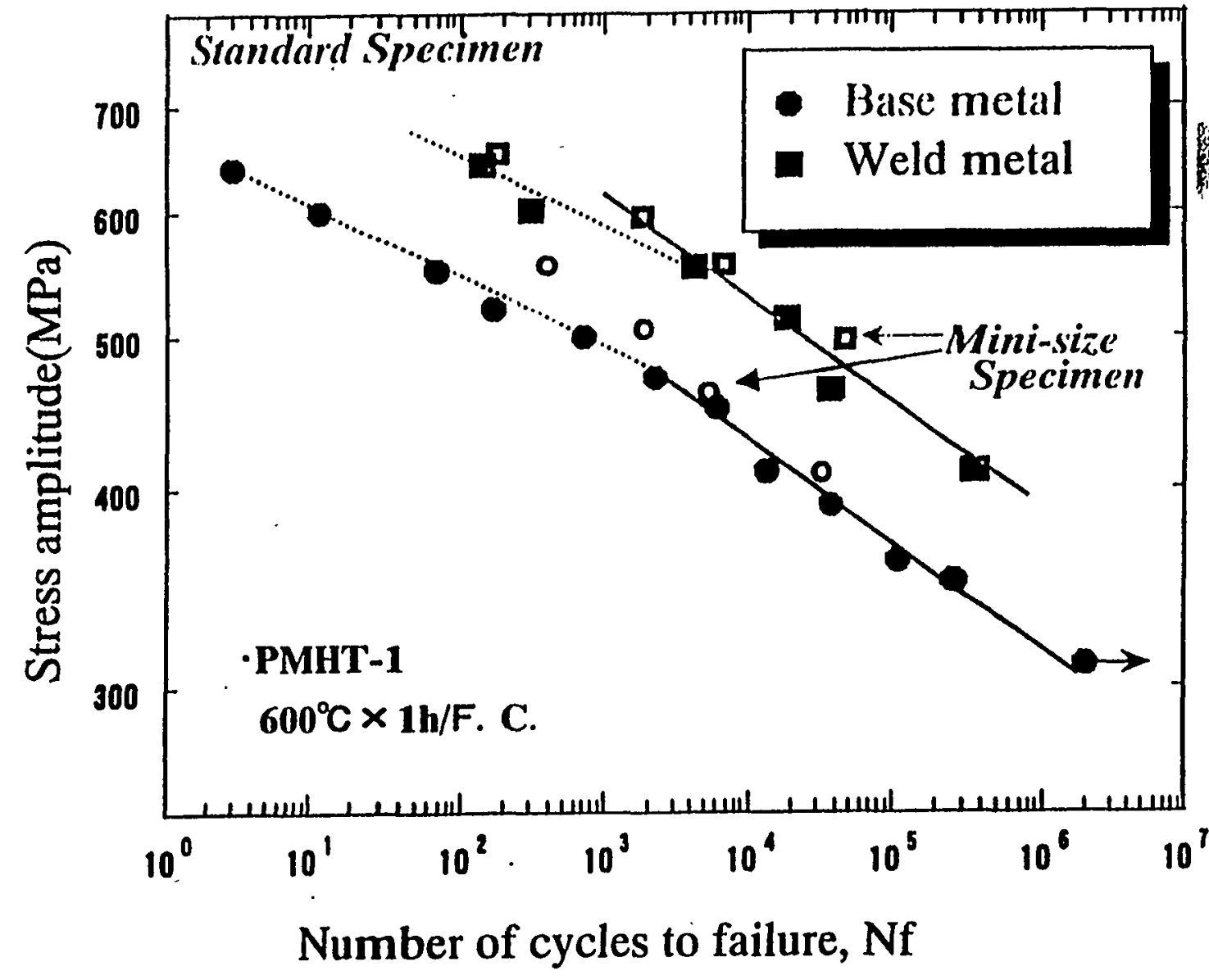

Speci. Size effect

small

Slightly decline of Fafigue life curve Slight shift to higher stress

Number of cycles to failure, Nf 


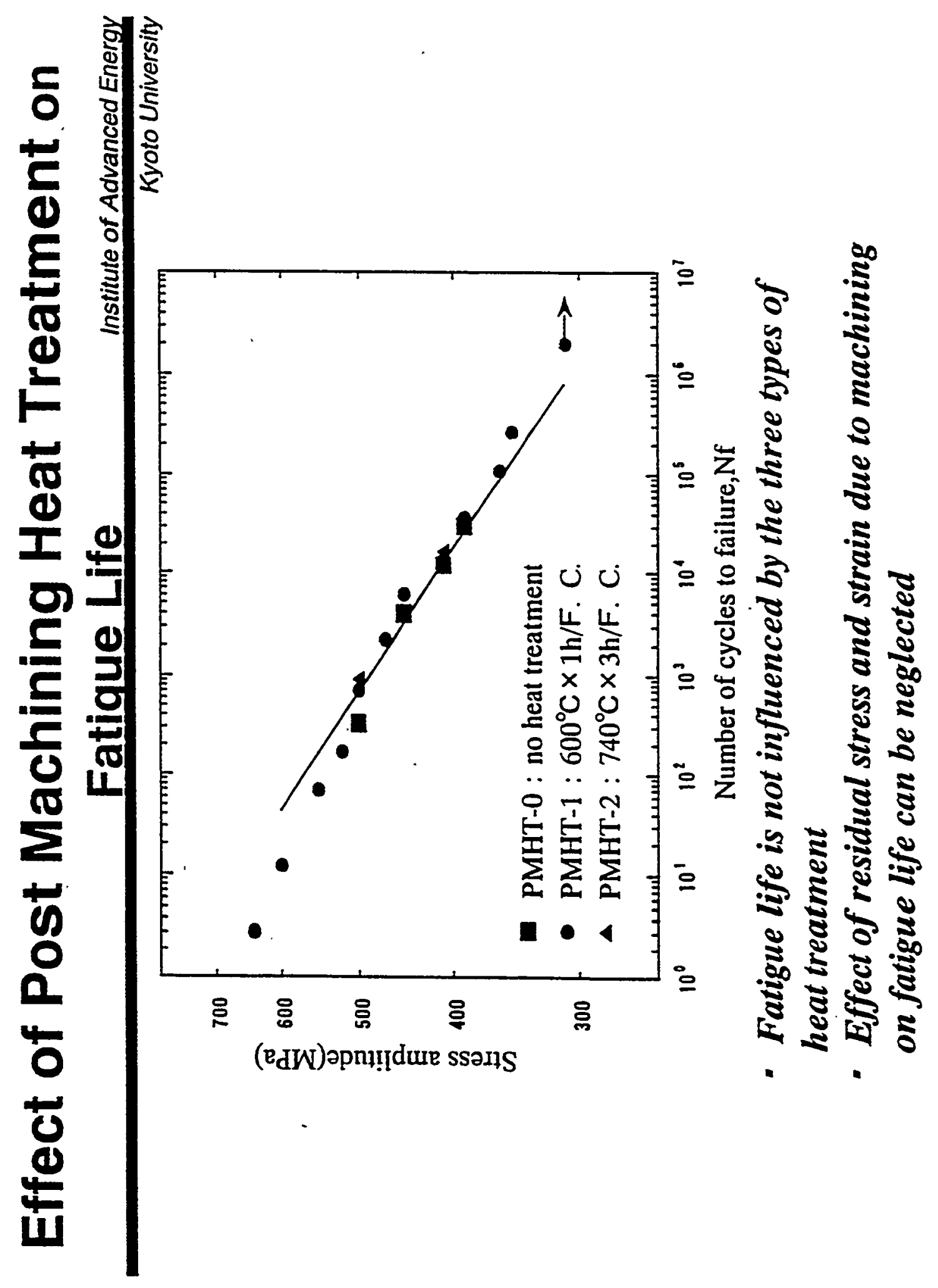




\section{Conclusion}

Insirule ol dels am ced l:meng!

K'rolul Iniveresiry

- A miniature fatigue specimen testing machine for a hot-laboratory has been successfully developed and the specimen size effect was studied. So far, there is no significant specimen size effect on fatigue properties except at very low cycle fatigue region.

- Fatigue life of JLF-1, TIG weld metal was longer and the fatigue strength was larger than those of base metal. 
IEA WORKSHOP ON REDUCED-ACTIVATION FIM STEELS

ECN, Petten, The Netherlands

1-2 October 1998

\title{
MECHANICAL BEHAVIOUR AFTER THERMAL AGEING OF RAFM STEELS AND F82H WELDMENTS
}

\author{
A. ALAMO, Y. de CARLAN \\ A. CASTAING, P. WIDENT \\ Commissariat à l'Energie Atomique \\ Service de Recherches Métallurgiques Appliquées \\ CEA - Saciay, France
}




\section{MATERIALS}

Chemical composition of LA martensitic steels (in wt\%).

\begin{tabular}{|c|c|c|c|c|c|c|c|c|}
\hline Steel & C & Si & Mn & Cr & V & W & N & Ta \\
\hline LA12Ta & 0.155 & 0.03 & 0.88 & 9.86 & 0.28 & 0.84 & 0.0430 & 0.10 \\
\hline LA12TaLN & 0.165 & 0.02 & 0.84 & 9.04 & 0.24 & 0.75 & 0.0048 & 0.10 \\
\hline LA12TaLC & $\mathbf{0 . 0 9 0}$ & 0.03 & 1.13 & 8.80 & 0.30 & 0.73 & 0.0190 & 0.10 \\
\hline LA12LC & $\mathbf{0 . 0 8 9}$ & 0.03 & 1.13 & 8.92 & 0.30 & 0.73 & 0.0350 & 0.010 \\
\hline LA4Ta & 0.142 & 0.03 & 0.78 & 11.08 & 0.23 & 0.72 & 0.0410 & 0.07 \\
\hline LA13Ta & 0.179 & 0.04 & 0.79 & 8.39 & 0.24 & 2.79 & 0.0480 & 0.09 \\
\hline F82H & 0.087 & 0.10 & 0.21 & 7.46 & 0.15 & $\mathbf{1 . 9 6}$ & $\mathbf{0 . 0 0 5 9}$ & 0.023 \\
\hline JLF-1 & $\mathbf{0 . 1 0 6}$ & 0.05 & 0.52 & 8.70 & 0.18 & $\mathbf{1 . 9 1}$ & 0.0280 & 0.08 \\
\hline
\end{tabular}

LA.... alloys, F82H and JLF-1 steels have been supplied respectively by AEA-Culham (U.K.), JAEAl and Tokyo University (Japan).

Types of materials :

- $9 \mathrm{Cr}-0.8 \mathrm{~W}-\mathrm{V}-\mathrm{Ta}:$ LA12Ta (high C and N), LA12TaLC (low C, Ta), LA12LC (low C, no Ta), LA12TaLN (low N).

- $11 \mathrm{Cr}-0.8 \mathrm{~W}-\mathrm{V}-\mathrm{Ta}$ : LA4Ta (high $\mathrm{Cr}$ )

- $9 \mathrm{Cr}-3 \mathrm{~W}-\mathrm{V}-\mathrm{Ta}$ : LA13Ta (high W)

- 7.5/9Cr-2W-V-Ta : F82H (low Cr, low Ta), JLF-1 


\section{METALLURGICAL CONDITION}

Experimental LA-steels have been produced as plates of $3.5 \mathrm{~mm}$ thick. The last steps of the fabrication route consisted of normalisation and tempering treatments followed by a final coldworking pass. This condition is denoted « N\&T - CW ».

Normalisation: $\quad 1030^{\circ} \mathrm{C}-40$ minutes

Tempering: $\quad 750 / 790^{\circ} \mathrm{C}-1 \mathrm{~h}$

Final $\mathrm{CW}: \quad 10 \%$

Large-scale heats, $\mathrm{F} 82 \mathrm{H}$ and JLF-1, have been supplied as plates of $7.5 \mathrm{~mm}$ and $15 \mathrm{~mm}$ thick in the normalised and tempered condition, denoted " N\&T ».

Normalisation: $\quad \mathrm{F} 82 \mathrm{H}: 1040^{\circ} \mathrm{C}-40$ minutes

JLF-1: $\quad 1050^{\circ} \mathrm{C}-1 \mathrm{~h}$

Tempering: $\quad \mathrm{F} 82 \mathrm{H}: \quad 750^{\circ} \mathrm{C}-1 \mathrm{~h}$

JLF-1: $\quad 780^{\circ} \mathrm{C}-1 \mathrm{~h}$ 
F82H - TENSILE PROPERTIES AFTER AGEING
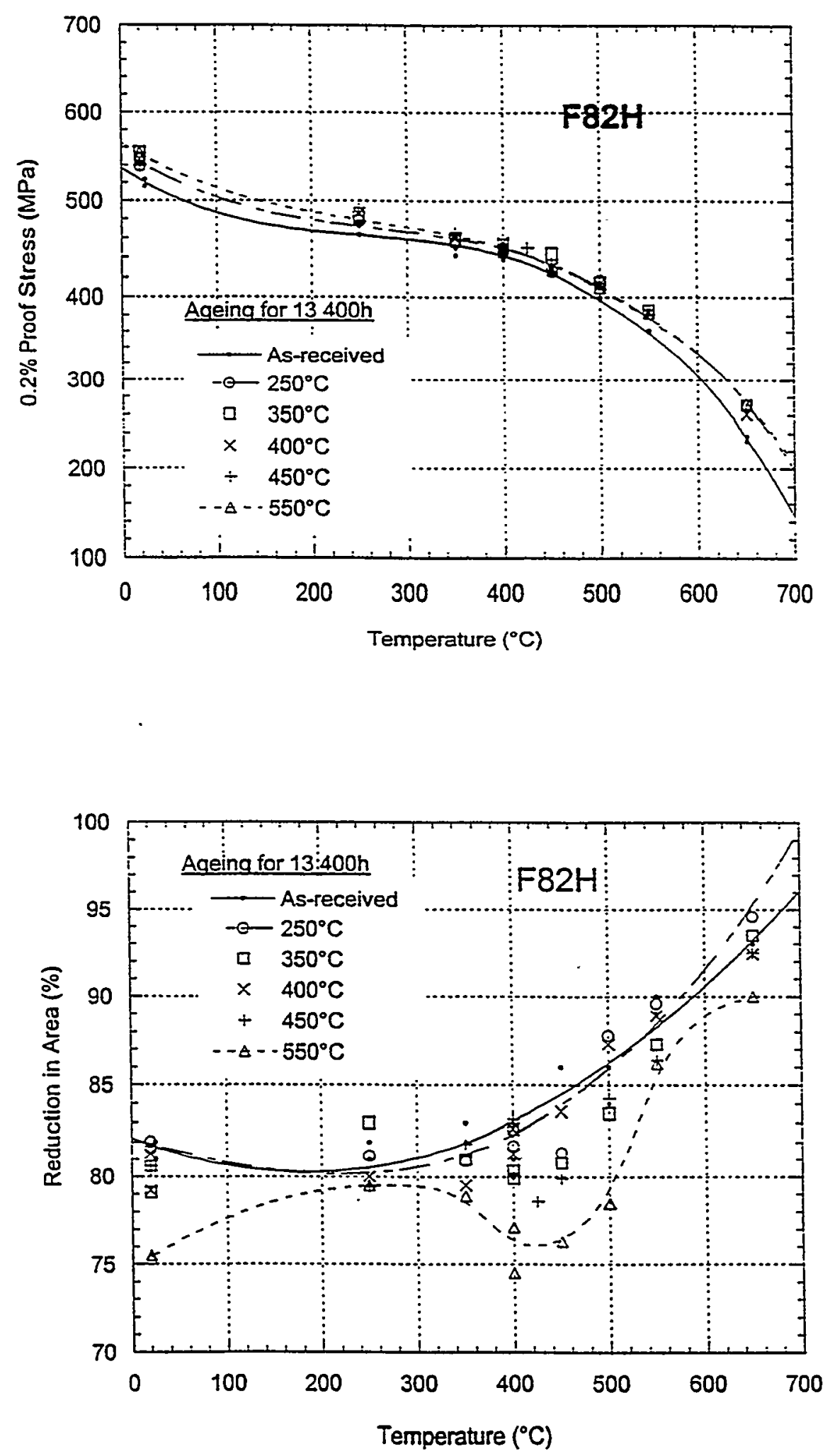


\section{cen}

IMPACT PROPERTIES AFTER AGEING - EFFECTS OF CHEMICAL COMPOSITION
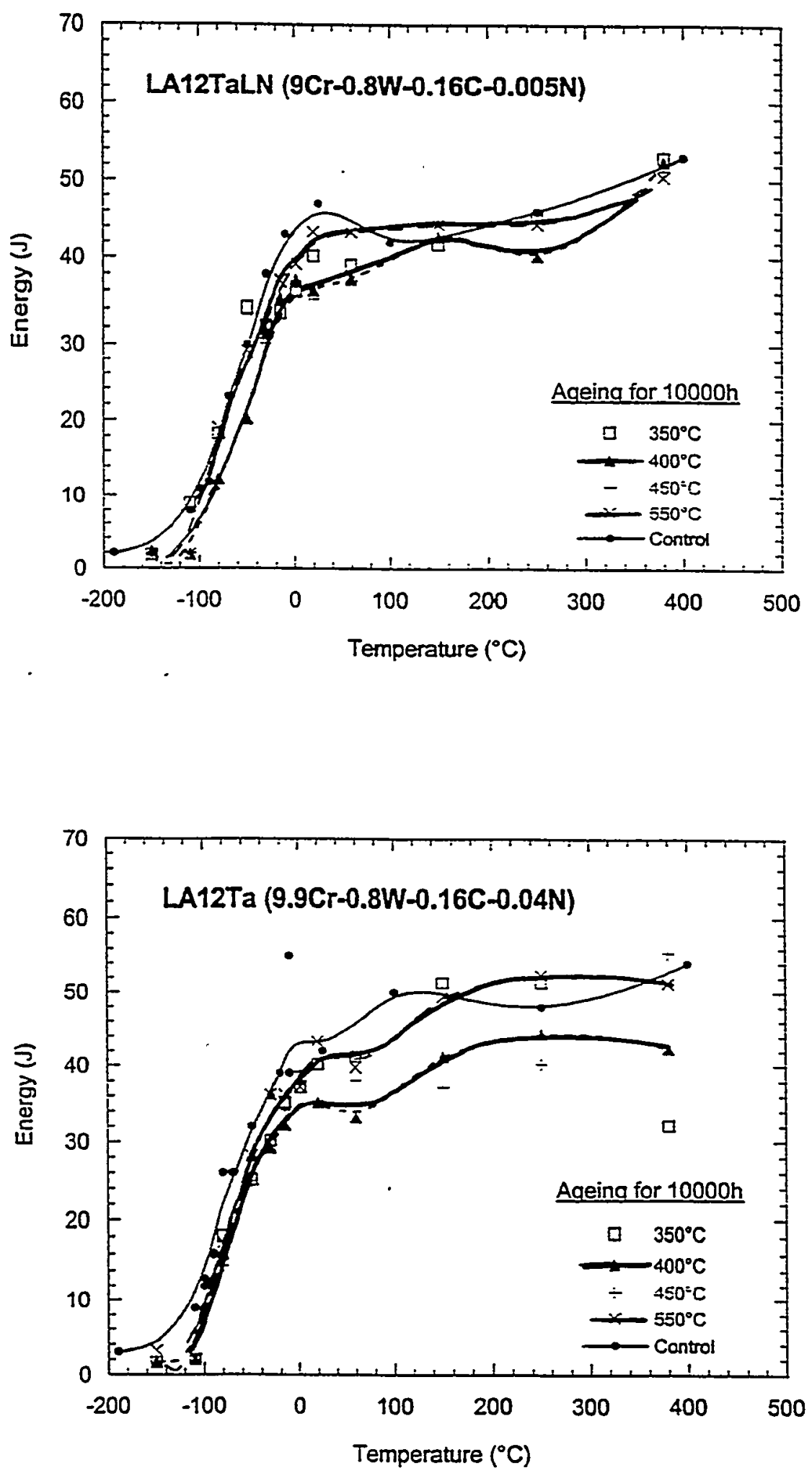


\section{cea}

IMPACT PROPERTIES AFTER AGEING - EFFECTS OF CHEMICAL COMPOSITION
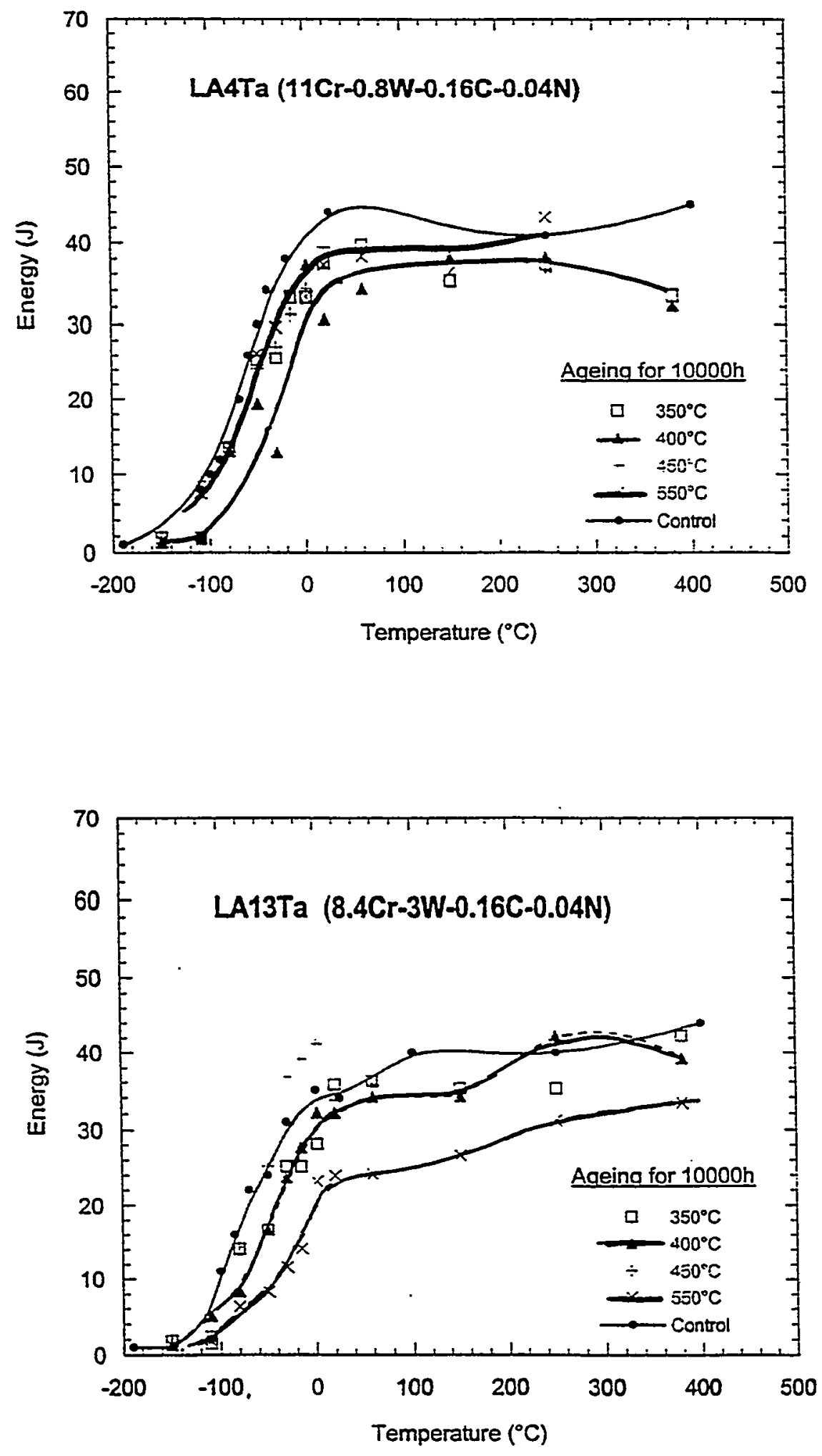


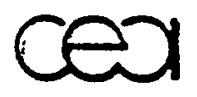

IMPACT PROPERTIES AFTER AGEING
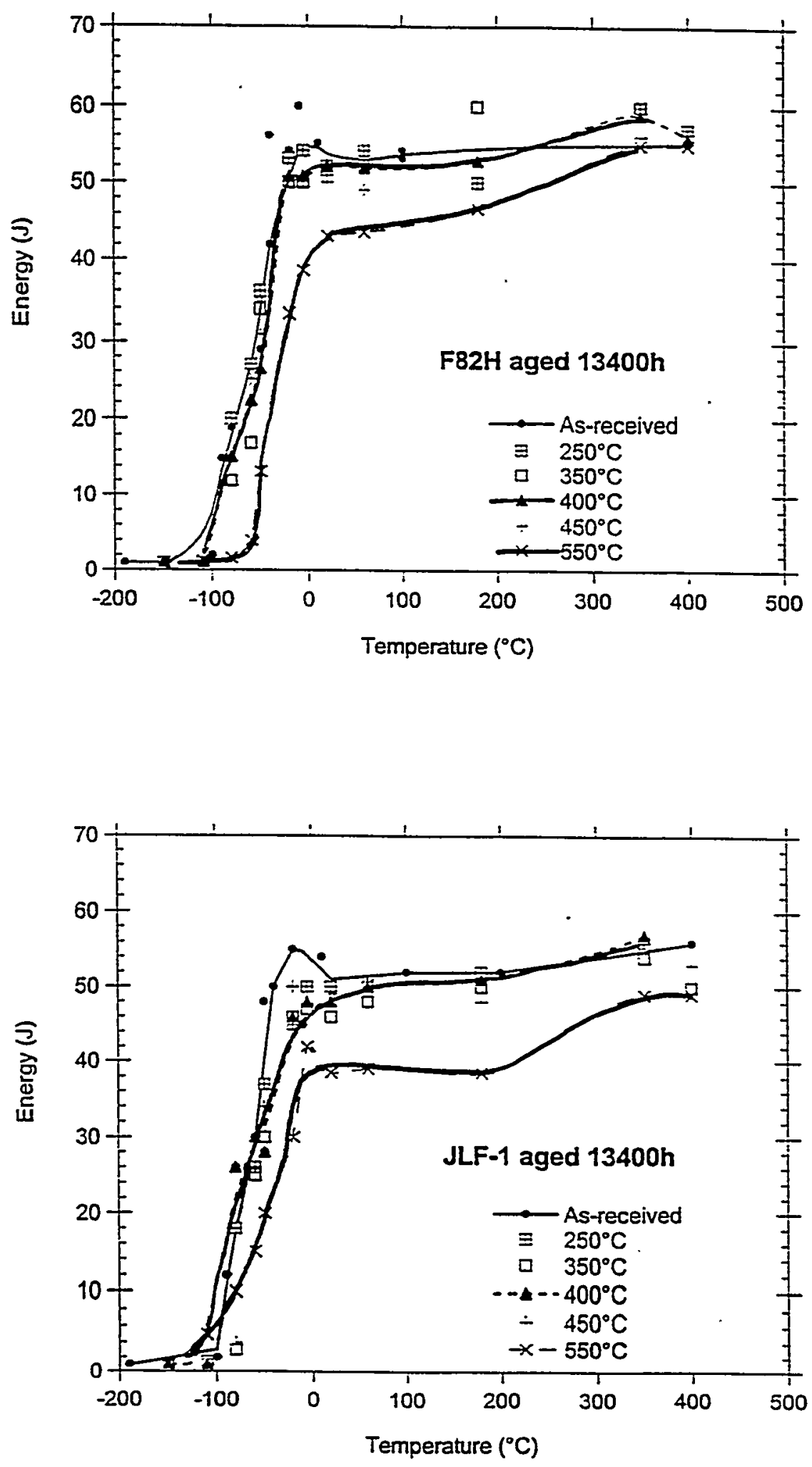
F82H WELDMENTS
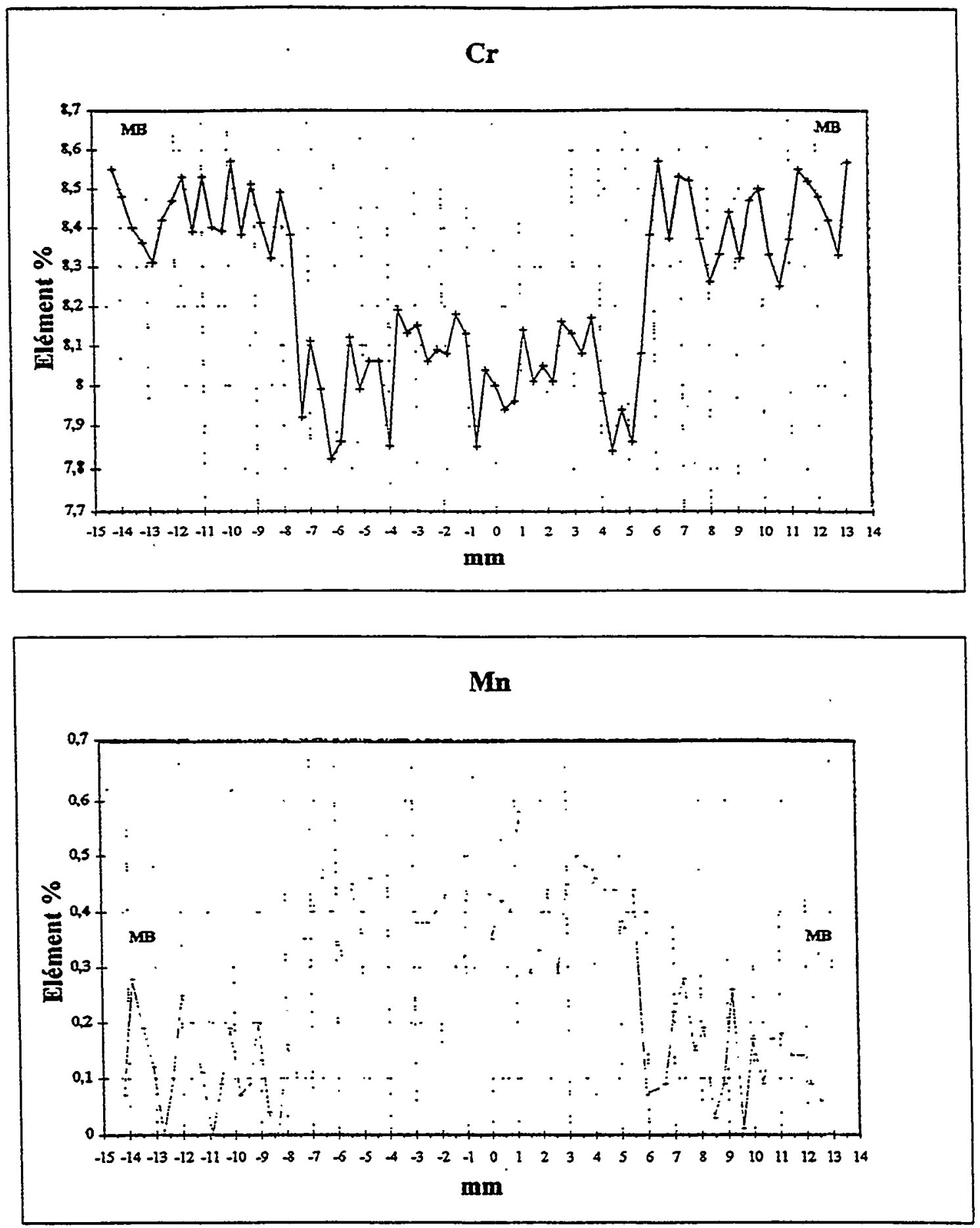

Profiles of $\mathrm{Cr}$ and $\mathrm{Mn}$ concentrations measured on the weld cross-section (mid-thickness). TIG specimen KG820-1-31W-37 (heat $\mathrm{n}^{\circ}$ 9753) of $25 \mathrm{~mm}$ thick. 


\section{F82H WELDMENTS}

\section{Hardness values}

$\left(H V_{10}\right.$, average of 10 measurements)

\begin{tabular}{|c|c|c|c|}
\hline Welds & Welded Metal & $\begin{array}{c}\text { Heat Affected } \\
\text { Zone }\end{array}$ & Base Metal \\
\hline TIG & 221 & 223 & 210 \\
\hline EB & 390 & 400 & 214 \\
\hline
\end{tabular}

Soudage FE monopasse de l'acier F82H JAERI (4-5)

Duretés dans l'axe transverse à la zone fondue

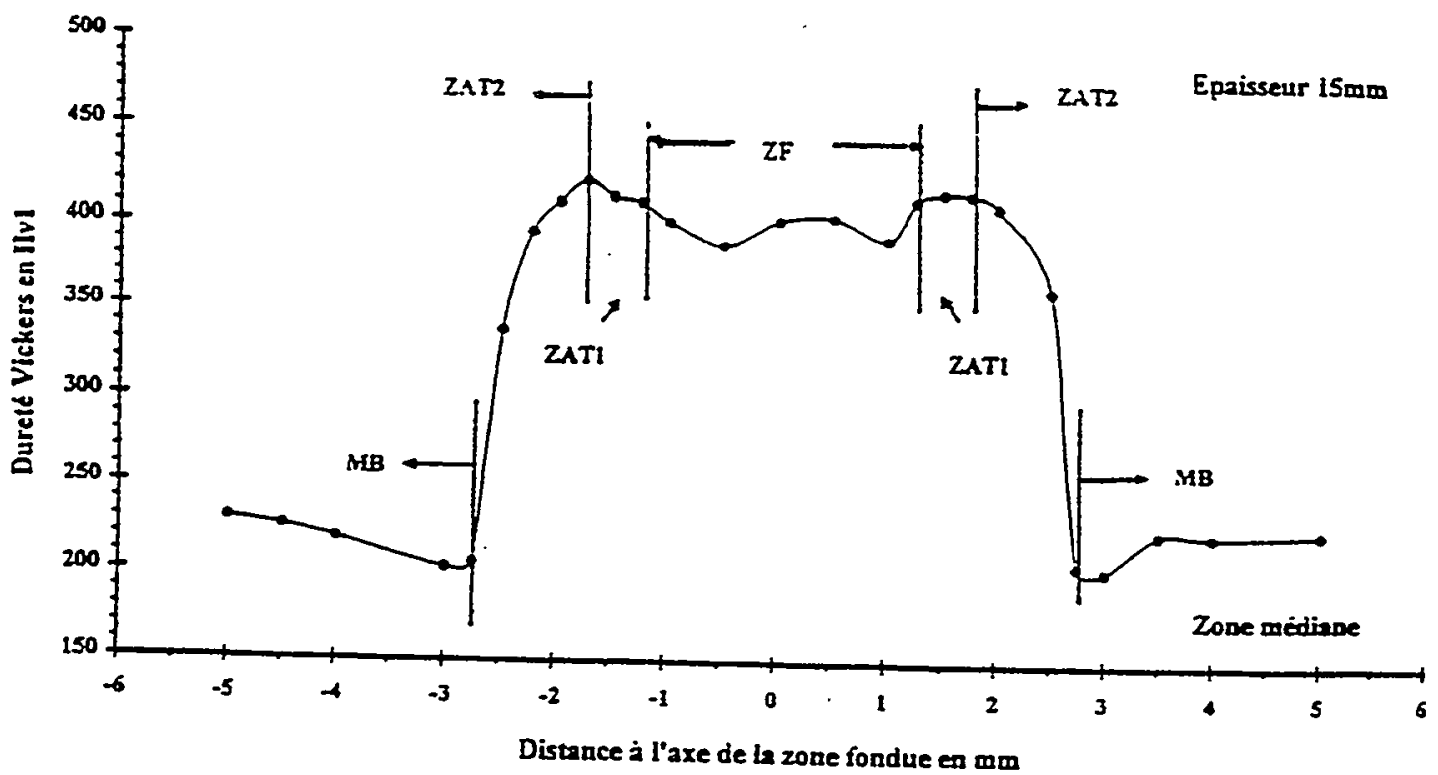




\section{Creep and tensile specimens}

creep : $4 \mathrm{~mm}$ dia., $\mathrm{lo}=20 / 25 \mathrm{~mm}$

tensile : $2 \mathrm{~mm}$ dia., $10=12 \mathrm{~mm}$
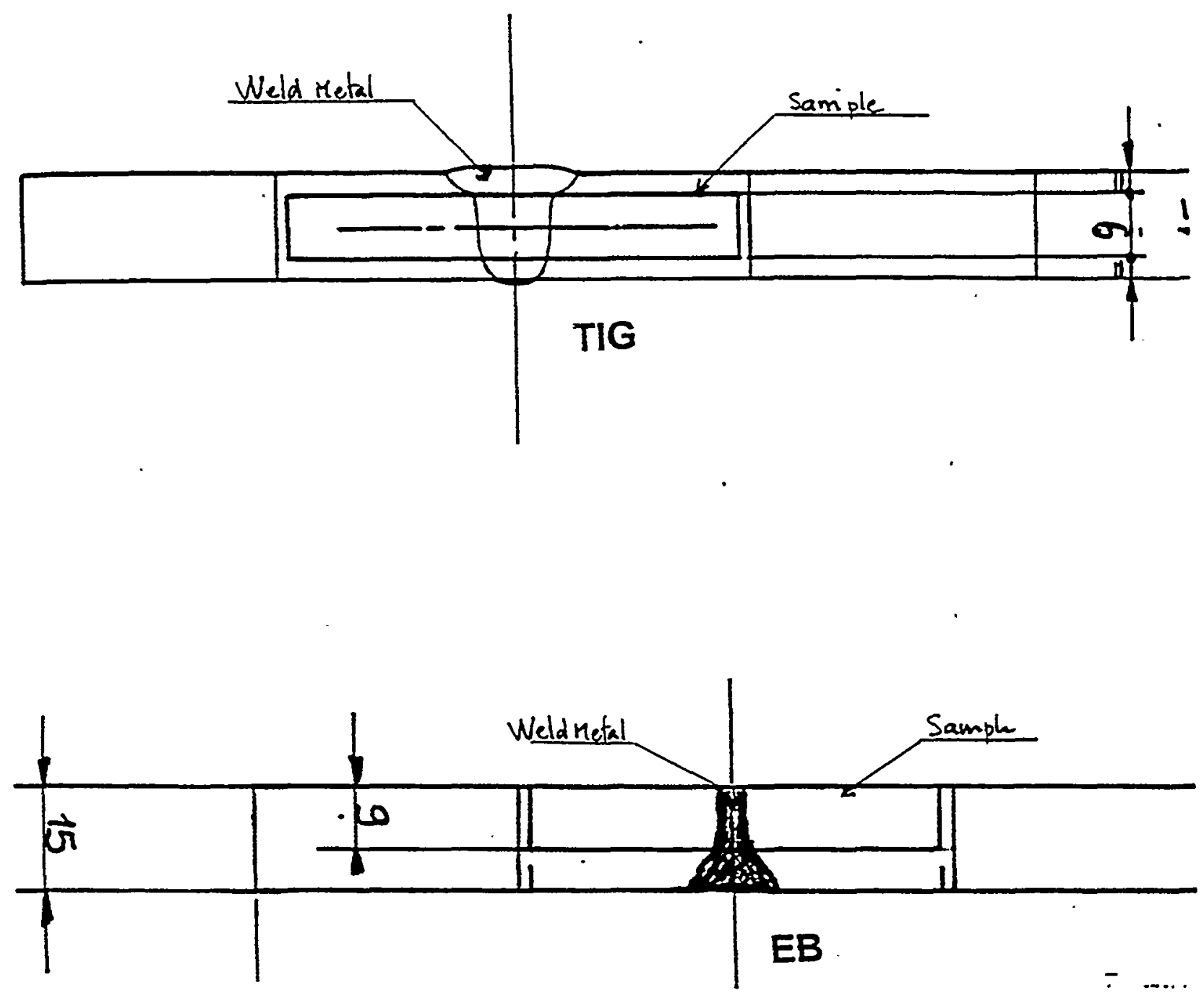


\section{F82H WELDMENTS}

\section{Creep properties - Time to rupture}

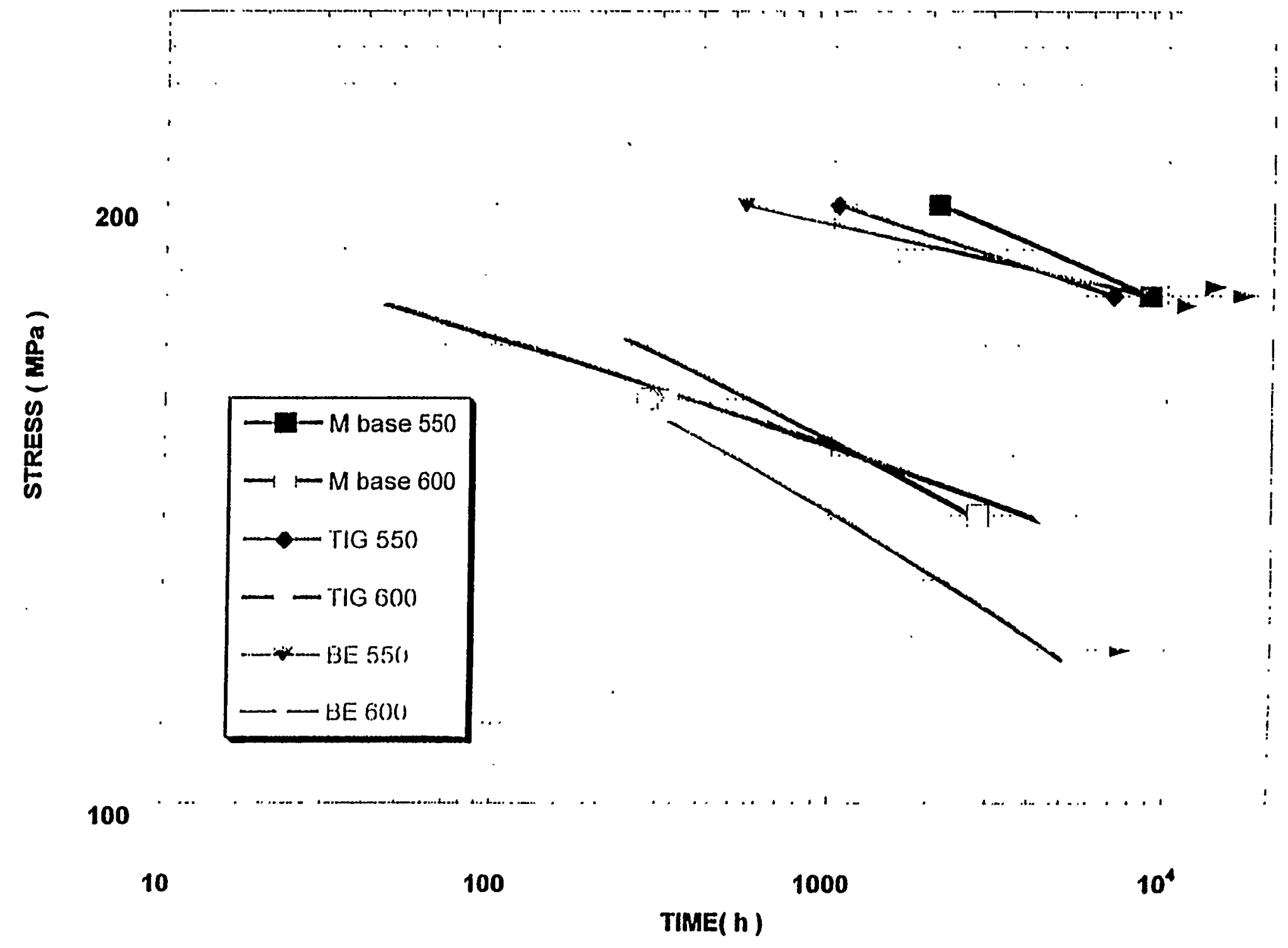




\title{
F82H WELDMENTS
}

\author{
(15 $\mathrm{mm}$ thick)
}

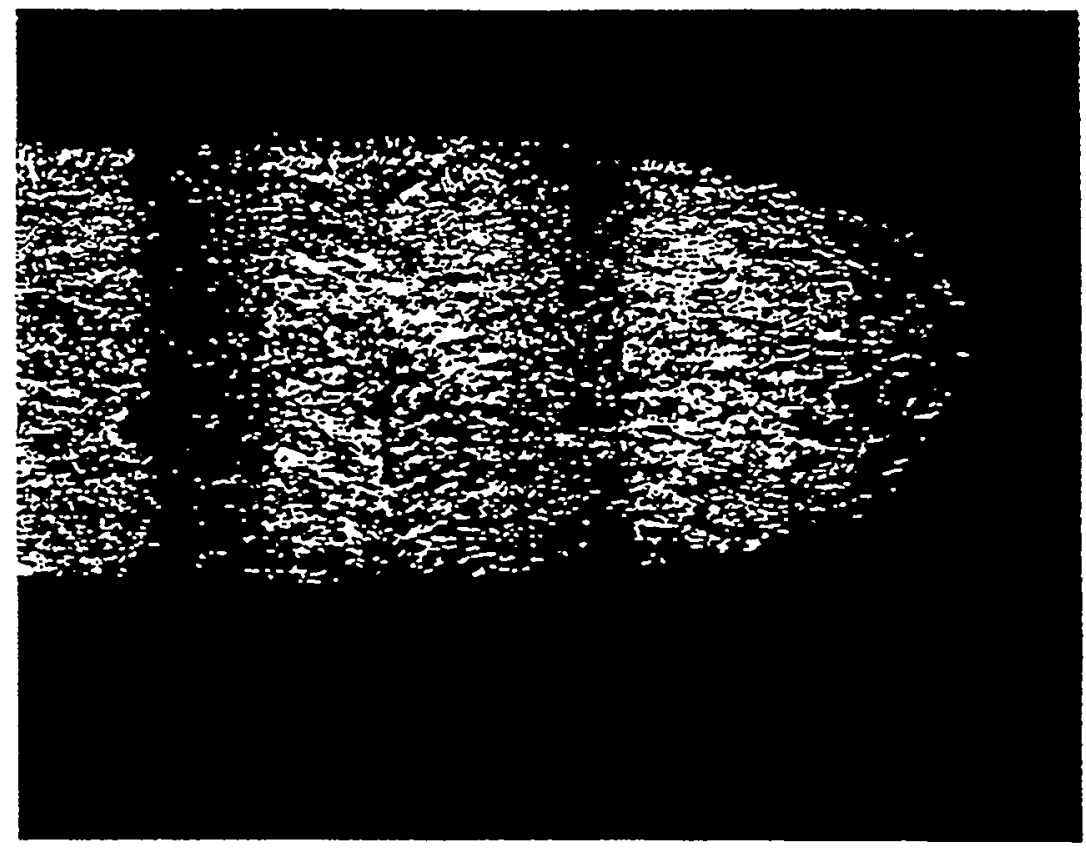

$1 \mathrm{~mm}$

longitudinal metallography showing the rupture location for TIG weld 
TENSILE PROPERTIES OF. F82H WELDMENTS
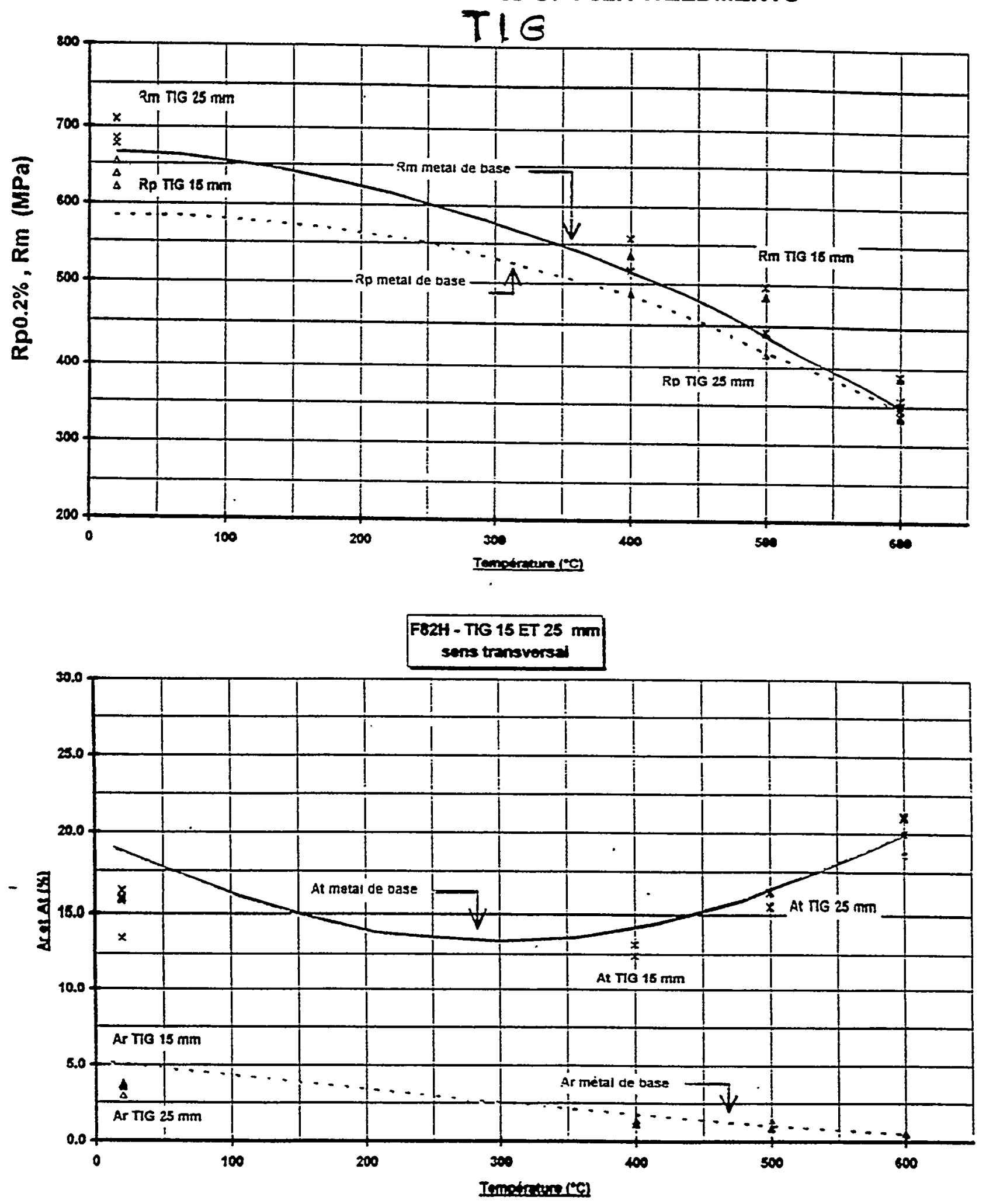


\section{F82H WELDMENTS}

\section{Charpy V specimens - TL orientation}

(55 mm long, $10 \mathrm{~mm}$ wide, $2.5 \mathrm{~mm}$ thick)

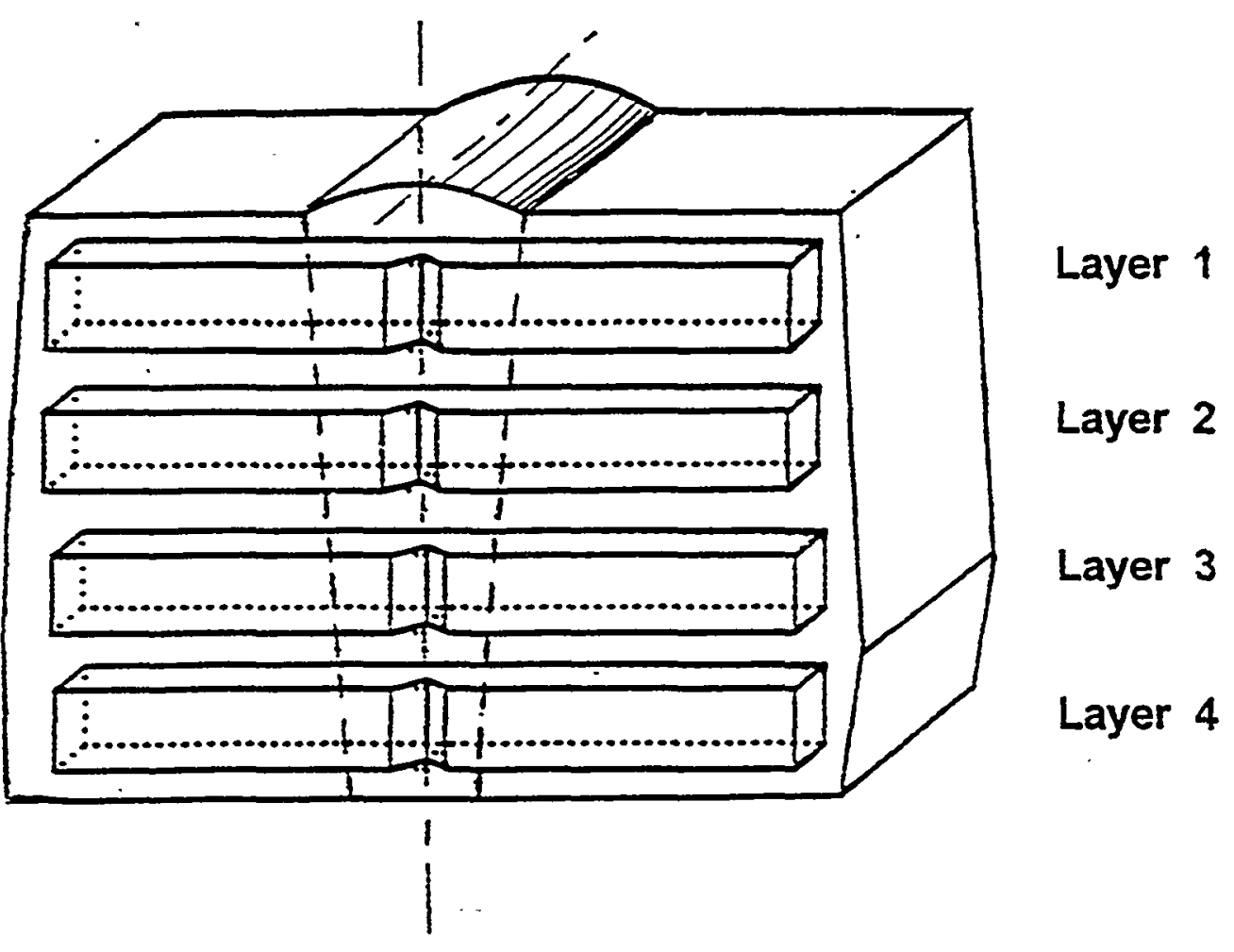




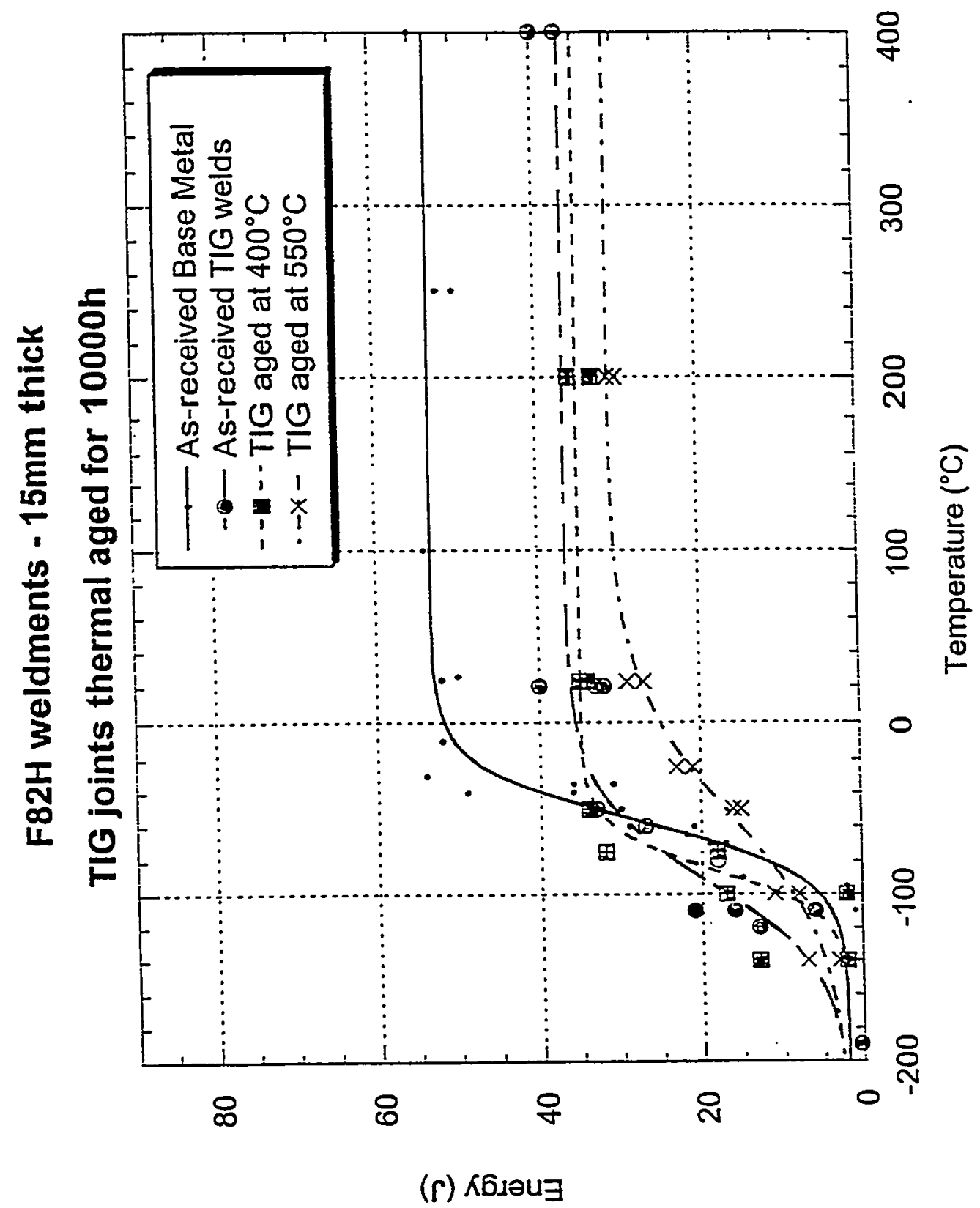

$\overline{8}$ 


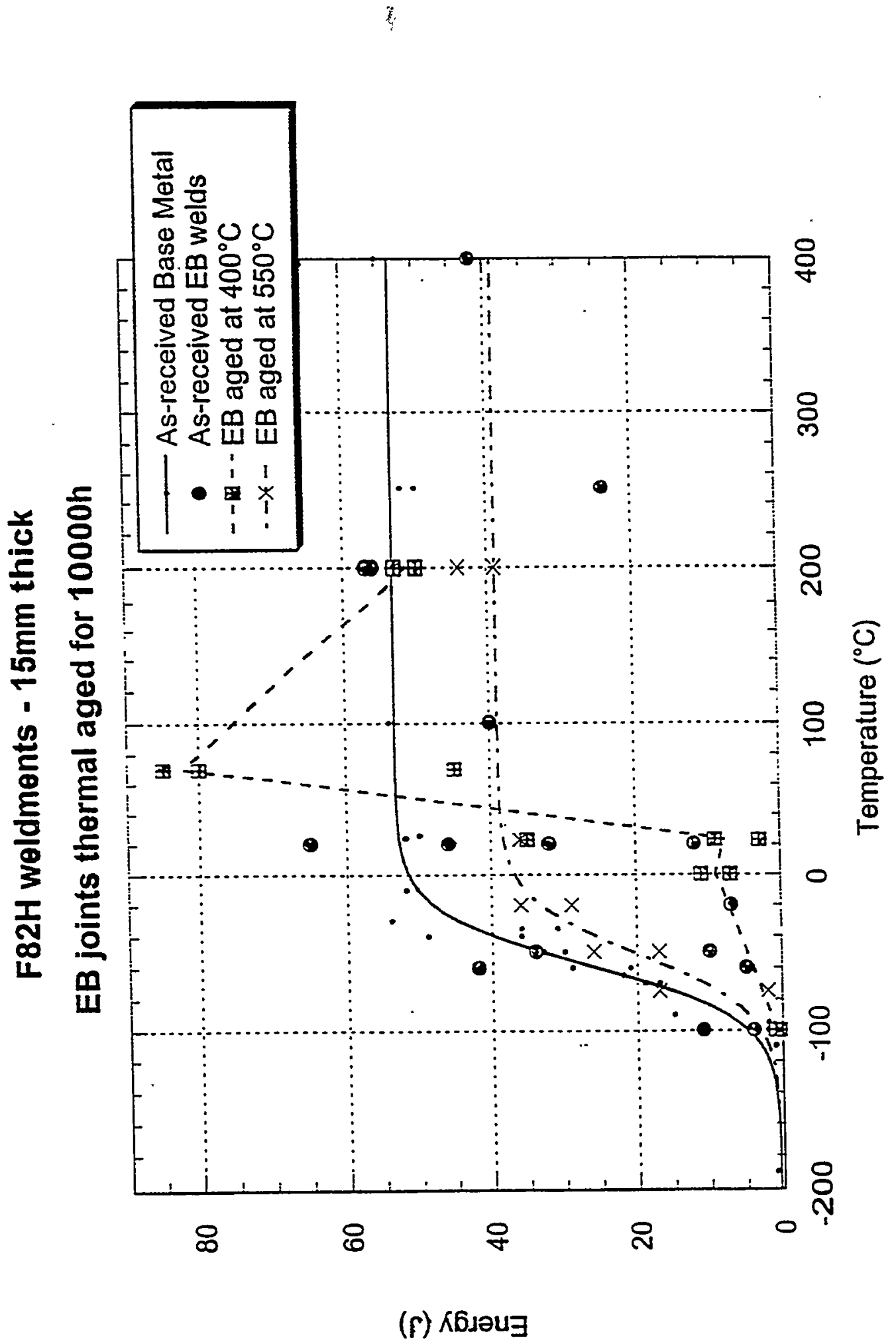

$\overline{8}$ 


\section{UNIRRADIATED PROPERTIES OF F82H IEA HEAT}

- Status and Recent Results -

K. Shiba/JAERI

IEA Working Group Meeting on

Reduced Activation Ferritic/Martensitic Steels

1-2, October, 1998

ECN Petten, The Netheriands 


\section{JAPANESE IEA ROUND ROBIN TEST PROGRAM ON F82H}

1. Metallurgical tests (Base Metal) (1995 - 1996) .

\begin{tabular}{|c|c|c|}
\hline Test & Schedule & Remarks \\
\hline 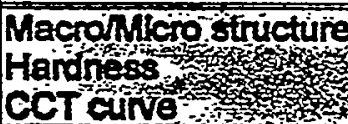 & 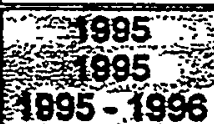 & Optical SEM TeM microscopy \\
\hline
\end{tabular}

2. Mechanical properties (Base Metal) (1995 - 1997)

\begin{tabular}{|c|c|c|c|}
\hline Property & Test condition & Schedule & Remarks \\
\hline 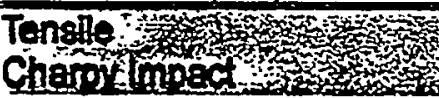 & S $600^{\circ} \mathrm{C}$ & $\begin{array}{r}1995-1096 \\
095-1996\end{array}$ & 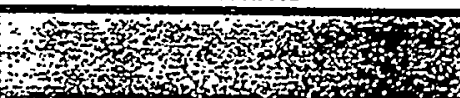 \\
\hline $\begin{array}{l}\text { Fracture toughness }\left(K_{\mathrm{lc}}, \mathrm{J}_{\mathrm{lc}}\right) \\
\text { Creep }\end{array}$ & RT, $100,-30^{\circ} \mathrm{C}$ & $1995-1996$ & \\
\hline $\begin{array}{ll} & \begin{array}{l}\text { Creep Rapture } \\
\text { Creep Rate } \\
\text { Creep Curve } \\
\text { Creep Fatigue }\end{array} \\
\text { Fatigue } & \\
\end{array}$ & $\begin{array}{l}500-650^{\circ} \mathrm{C} \\
500-650^{\circ} \mathrm{C} \\
500-650^{\circ} \mathrm{C} \\
500-650^{\circ} \mathrm{C} \\
\text { RT }-600^{\circ} \mathrm{C}\end{array}$ & $\begin{array}{l}1995-1997 \\
1995-1997 \\
1995-1997 \\
1995-1997 \\
1995-1997\end{array}$ & $\begin{array}{l}\max : 50000 \mathrm{~h} \\
\max : 3000 \mathrm{~h} \\
\text { max: } 3000 \mathrm{~h} \\
\text { holding time: } 0,1,3,10,30 \mathrm{~min} \\
\varepsilon: 0.5-1.5 \% \text { (push/pull) }\end{array}$ \\
\hline
\end{tabular}

3. Aging tests

Aging condition: $(400),(500), 550,600,650 \mathrm{C} ; 1000,3000,10000,(30000) \mathrm{h}$

\begin{tabular}{|c|c|c|c|}
\hline & Property & Test condition & Schedule \\
\hline $\begin{array}{l}\text { Metallurgical tests } \\
\text { Mechanical properties }\end{array}$ & $\begin{array}{l}\text { Tensile } \\
\text { Charpy Impact } \\
\text { Fracture toughness }\left(K_{19}, J_{1 c}\right)\end{array}$ & \begin{tabular}{|c|}
$\mathrm{RT}, 550^{\circ} \mathrm{C}$ \\
Full curve \\
$\mathrm{RT}, 100,-30^{\circ} \mathrm{C}$
\end{tabular} & $\begin{array}{l}1995 \text { - } \\
1995 \text { - } \\
1995 \text { - } \\
1995 \text { - }\end{array}$ \\
\hline
\end{tabular}


4. Weldments (TIG/EB)

Metallurgical tests

\begin{tabular}{|l|c|c|}
\hline \multicolumn{1}{|c|}{ Test } & Schedule & Remarks \\
\hline Macro/Micro structure & 1995 & Optical, SEM, TEM microscopy \\
Hardness & 1995 & \\
CCT curve & $1995-1996$ & \\
\hline
\end{tabular}

Mechanical properties

\begin{tabular}{|l|c|c|c|}
\hline \multicolumn{1}{|c|}{ Property } & Test condition & Schedule & Remarks \\
\hline Tensile & RT-600 & $1995-1996$ & \\
Charpy Impact & Full curve & $1995-1996$ & \\
Fracture toughness $\left(\mathrm{K}_{\mathrm{I}}, \mathrm{J}_{\mathrm{IC}}\right)$ & $\mathrm{RT}, 100,-30^{\circ} \mathrm{C}$ & $1995-1996$ & \\
Creep Rapture & $500-600^{\circ} \mathrm{C}$ & $1996-1998$ & $\max : 10000 \mathrm{~h}$ \\
Fatigue & $550^{\circ} \mathrm{C}$ & $1995-1997$ & $-: 0.5-1.0 \%$ (push/pull) \\
\hline
\end{tabular}

\section{Aging tests}

Aging condition: $550,600,650 \mathrm{C} ; 1000,3000,10000 \mathrm{~h}$

\begin{tabular}{|l|l|l|c|}
\hline & \multicolumn{1}{|c|}{ Property } & Test condition & Schedule \\
\hline Metallurgical tests & \multicolumn{1}{|c|}{} & $1995-$ \\
Mechanical properties & Tensile & RT, $550^{\circ} \mathrm{C}$ & $1995-$ \\
& Charpy impact & Full curve & $1995-$ \\
& Fracture toughness $\left(\mathrm{K}_{1}, J_{1 \mathrm{C}}\right)$ & $\mathrm{RT}, 100,-30^{\circ} \mathrm{C}$ & $1995-$ \\
\hline
\end{tabular}

5. Physical properties (1995 - 1996)

\begin{tabular}{|c|c|c|}
\hline Property & Temperature & Schedule \\
\hline Density & & \\
\hline Spocicheal & & \\
\hline Thomar expansion & & 95 \\
\hline Thermal conductuty & II $=$ & 199. \\
\hline Electrical conductivity & RT & 199 \\
\hline Mellna point & & \\
\hline Young s modutes & & \\
\hline $\begin{array}{l}\text { Poisson ratio } \\
\text { Modules of rioidit }\end{array}$ & & 0 \\
\hline onetehystere & on & \\
\hline
\end{tabular}

6. Other properties

\begin{tabular}{|l|l|l|l|}
\hline Property & Measurment & Schedule & Remarks \\
\hline Vacuum Properties & Released gas & $1995-1996$ & \\
& measurement & $\cdot$ & \\
Corrosion & Corrosion test & $1995-1996$ & Corrosion loss \\
Resistance & In high temperature water & & $\begin{array}{l}\text { SSRT test } \\
\text { Hydrogen solubility } \\
\text { Hydrogen Test }\end{array}$ \\
& Tritium permeability & $1995-1996$ & $\begin{array}{l}\text { after aging } \\
\text { SSRT test }\end{array}$ \\
\hline
\end{tabular}

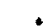




\section{PROGESS IN ROUND-ROBIN TEST}

- TOPICS -

1. Base Metal Properties

- as N\&T

- Fatigue, Fracture Toughness, Tensile (low temp)

- Aging

- Tensile, Charpy, Fracture Toughness

2. Welded Joints (TIG/EB)

- as Weld (PWHT: $720^{\circ} \mathrm{Cx} 1 \mathrm{~h}$ )

- Hardness, Tensile, Charpy, Fracture Toughness

- Aging in progress $\left(500-650^{\circ} \mathrm{C} / \mathrm{up}\right.$ to $\left.100000 \mathrm{~h}\right)$

- Hardness, Tensile, Charpy, Fracture Toughness

(Optional) Magnetic Properties (as N\&T)

- presented previously 
Fracture Toghness of F82H IEA heat

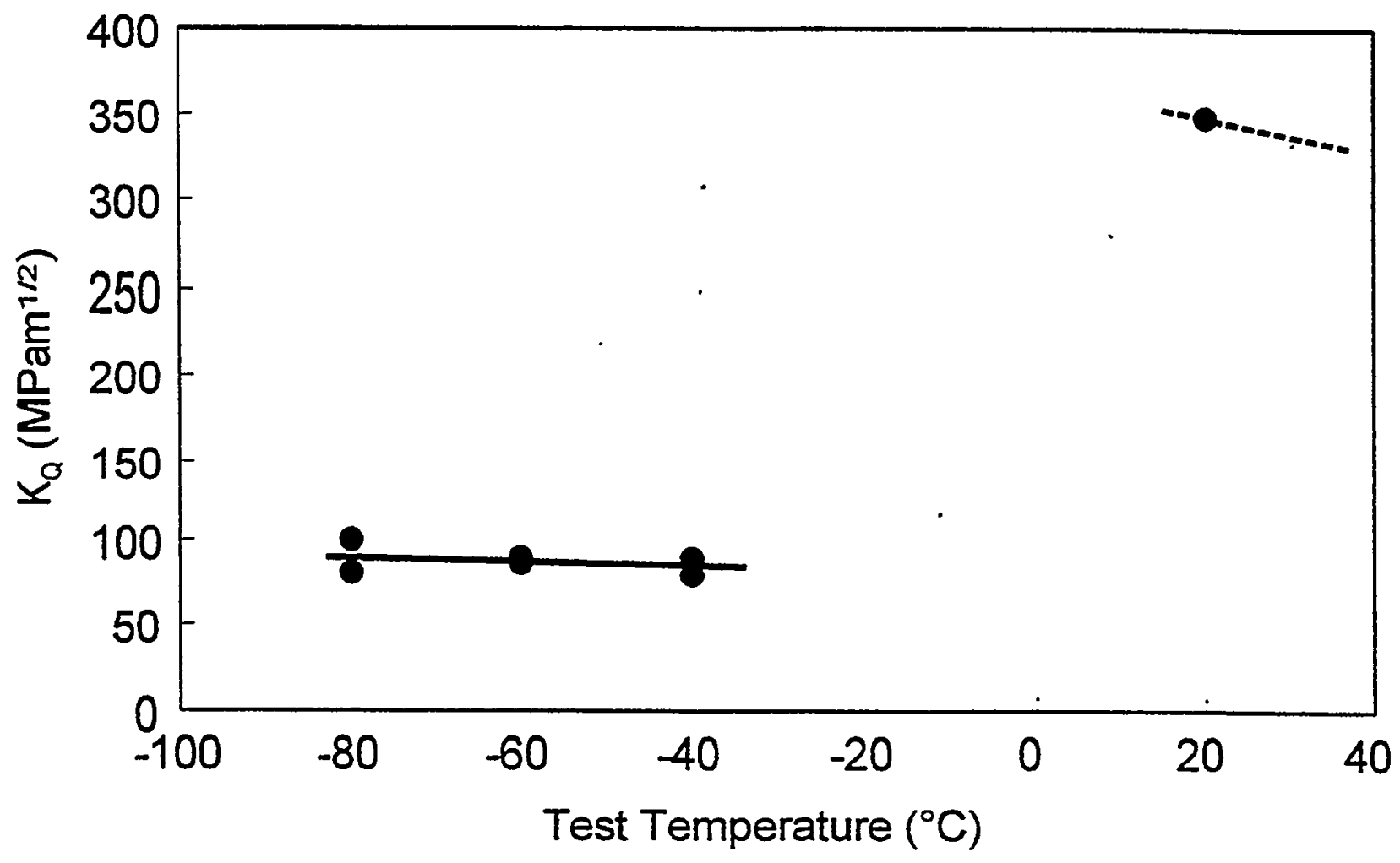




\section{Tensile Properties of F82H IEA heat}

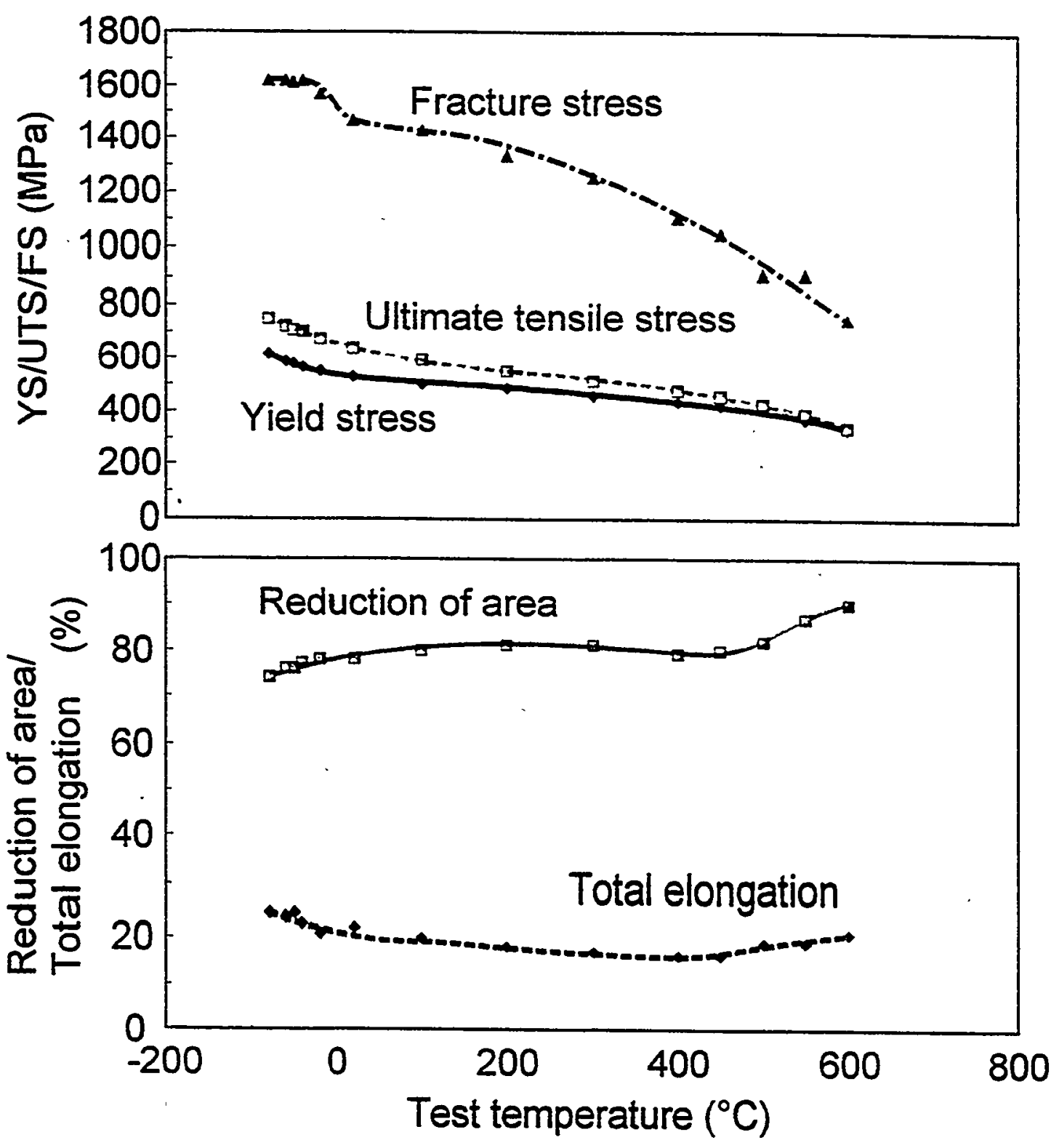



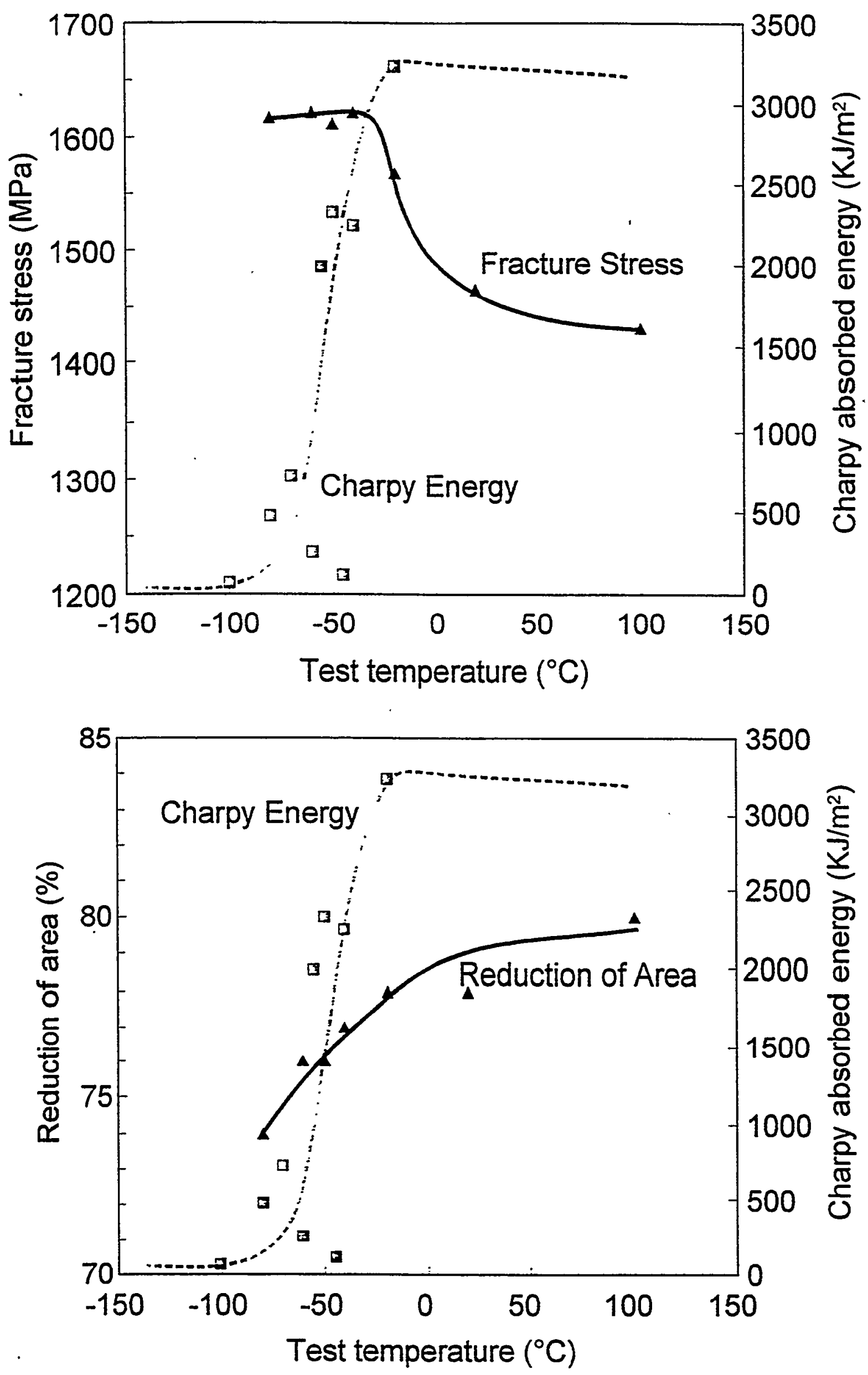


\section{BASE METAL PROPERTIES (as N\&T)}

- FATIGUE

Test Conditions

Strain rate: $0.1 \% / s$ (saw wave)

\begin{tabular}{|c|c|c|l|}
\hline $\begin{array}{c}\text { Test } \\
\text { Temp } \\
\left({ }^{\circ} \mathrm{C}\right)\end{array}$ & $\begin{array}{c}\text { Total Strain } \\
\text { Range } \\
(\%)\end{array}$ & $\begin{array}{c}\text { Cycles to } \\
\text { Rupture }\end{array}$ & Remarks \\
\hline \multirow{3}{*}{ RT } & 0.6 & 10504 & \\
\cline { 2 - 4 } & 1.0 & 3028 & \\
\cline { 2 - 4 } & 1.5 & 923 & \\
\hline \multirow{4}{*}{300} & 0.5 & 14726 & Buckled \\
\cline { 2 - 4 } & 0.5 & 9875 & Retest \\
\cline { 2 - 4 } & 0.75 & 2814 & \\
\cline { 2 - 4 } & 0.75 & 4831 & Retest \\
\cline { 2 - 4 } & 1.0 & 1597 & \\
\cline { 2 - 4 } & 1.5 & 746 & Buckled \\
\hline \multirow{4}{*}{550} & 0.5 & 3850 & \\
\cline { 2 - 4 } & 0.75 & 1860 & \\
\cline { 2 - 4 } & 1.0 & 880 & \\
\cline { 2 - 4 } & 1.0 & 987 & Retest \\
\cline { 2 - 4 } & 1.5 & 247 & \\
\hline
\end{tabular}




\section{FATIGUE TESTT RESULTS OF F82H IEA HEAT (RT Test)}

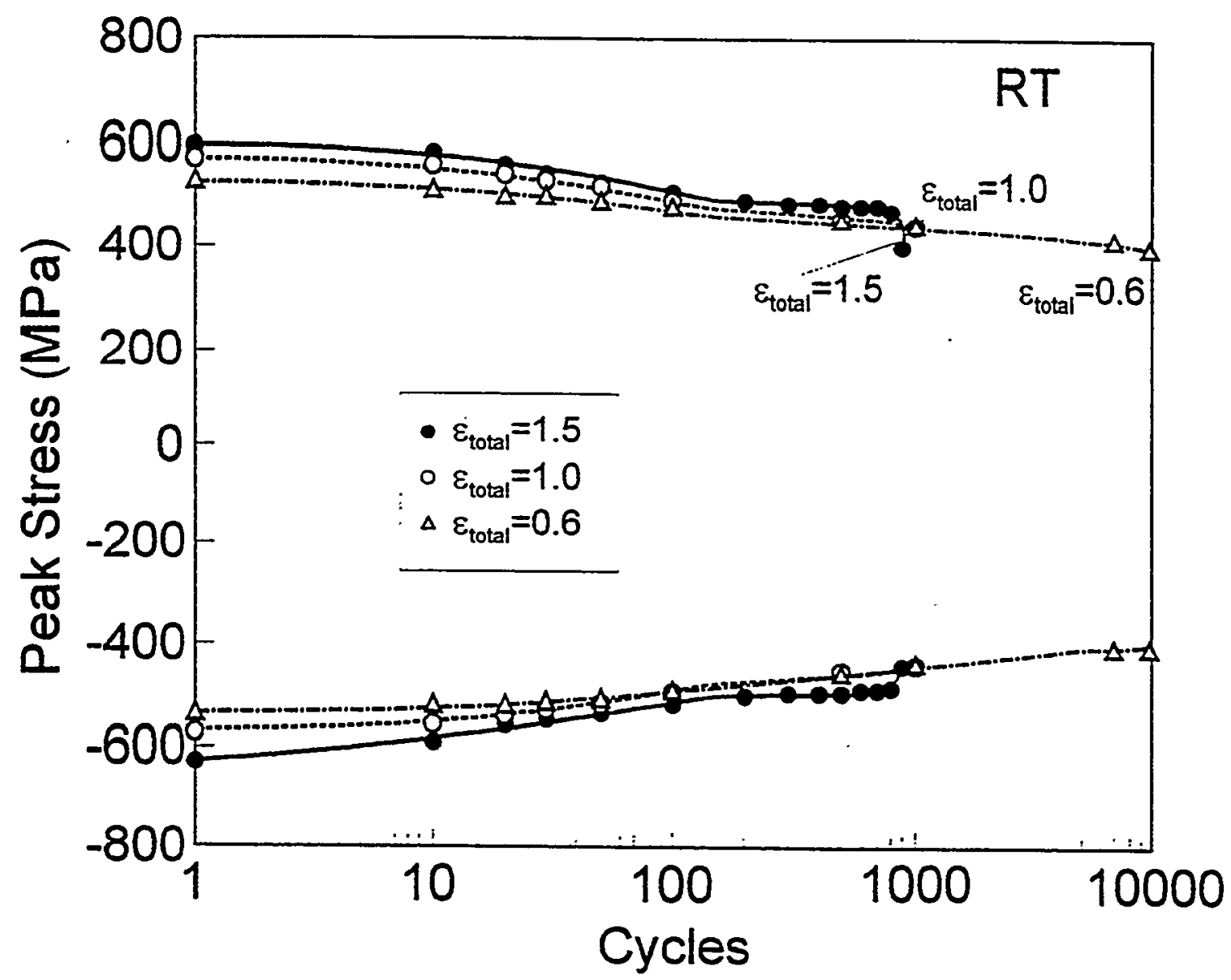


FATIGUE TEST RESULTS OF F82H IEA HEAT $\left(300^{\circ} \mathrm{C}\right.$ Test)

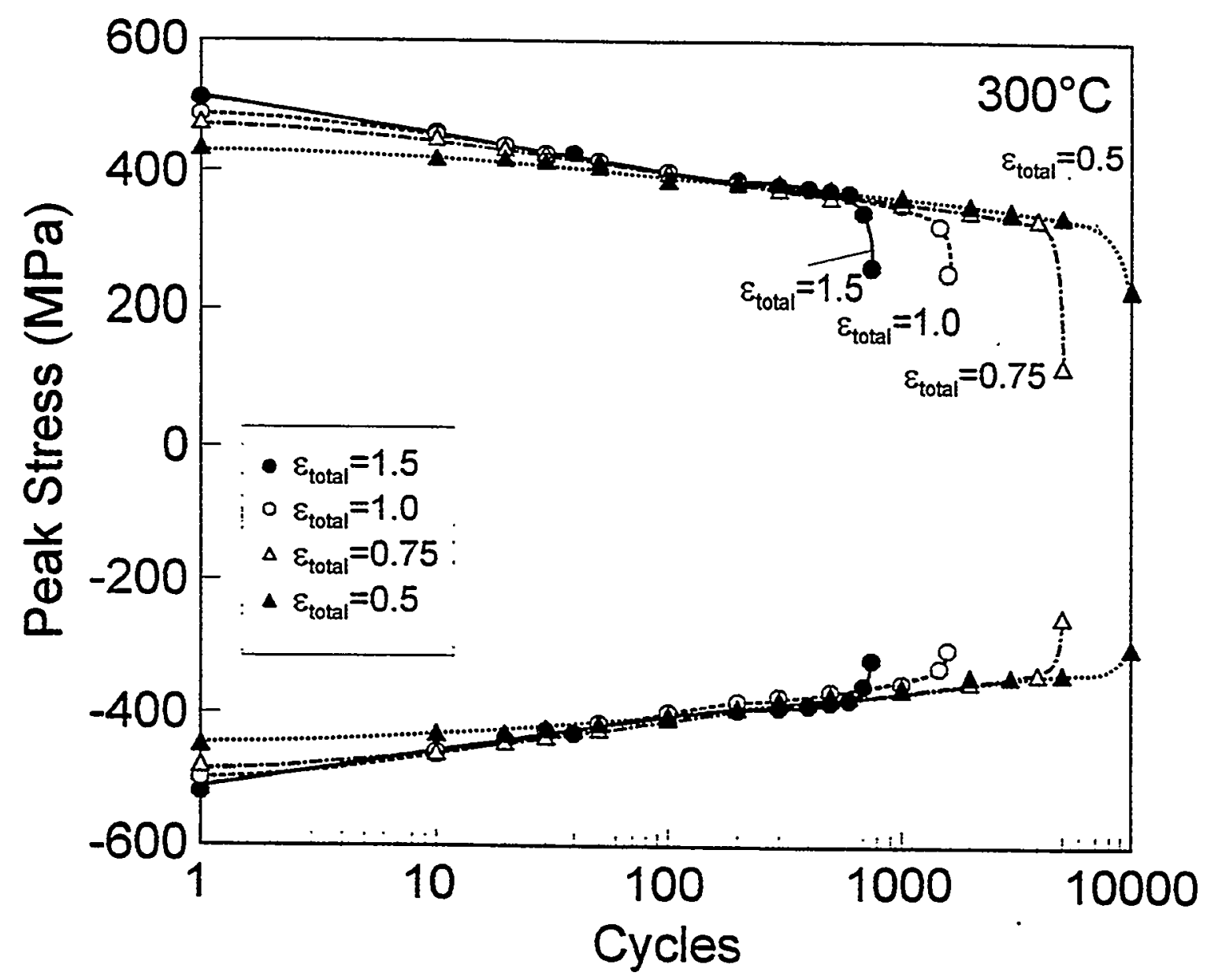


FATIGUE TEST RESULTS OF F82H IEA HEAT $\left(550^{\circ} \mathrm{C}\right.$ Test)

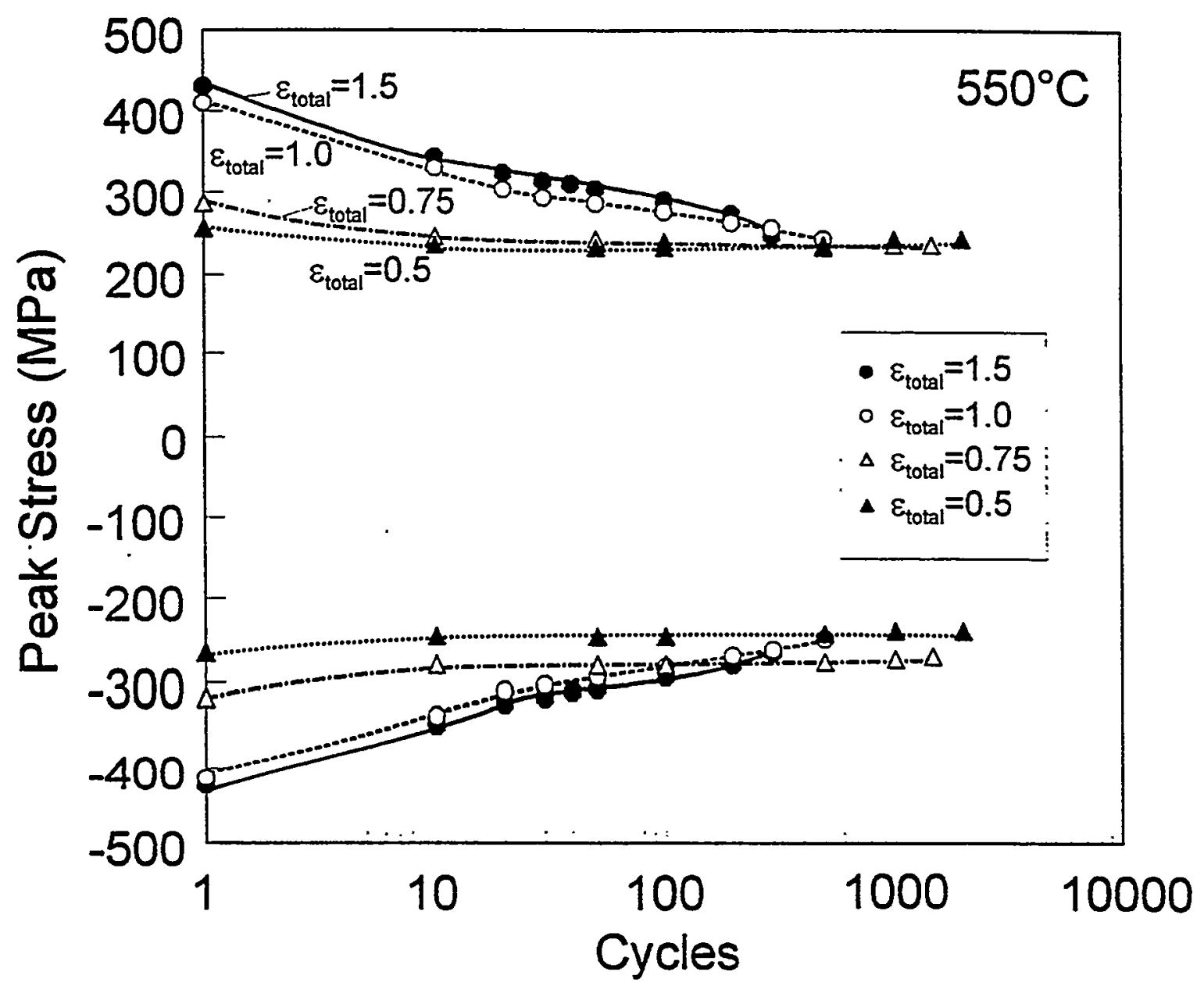




\section{FATIGUE TEST RESULTS OF F82H IEA HEAT}

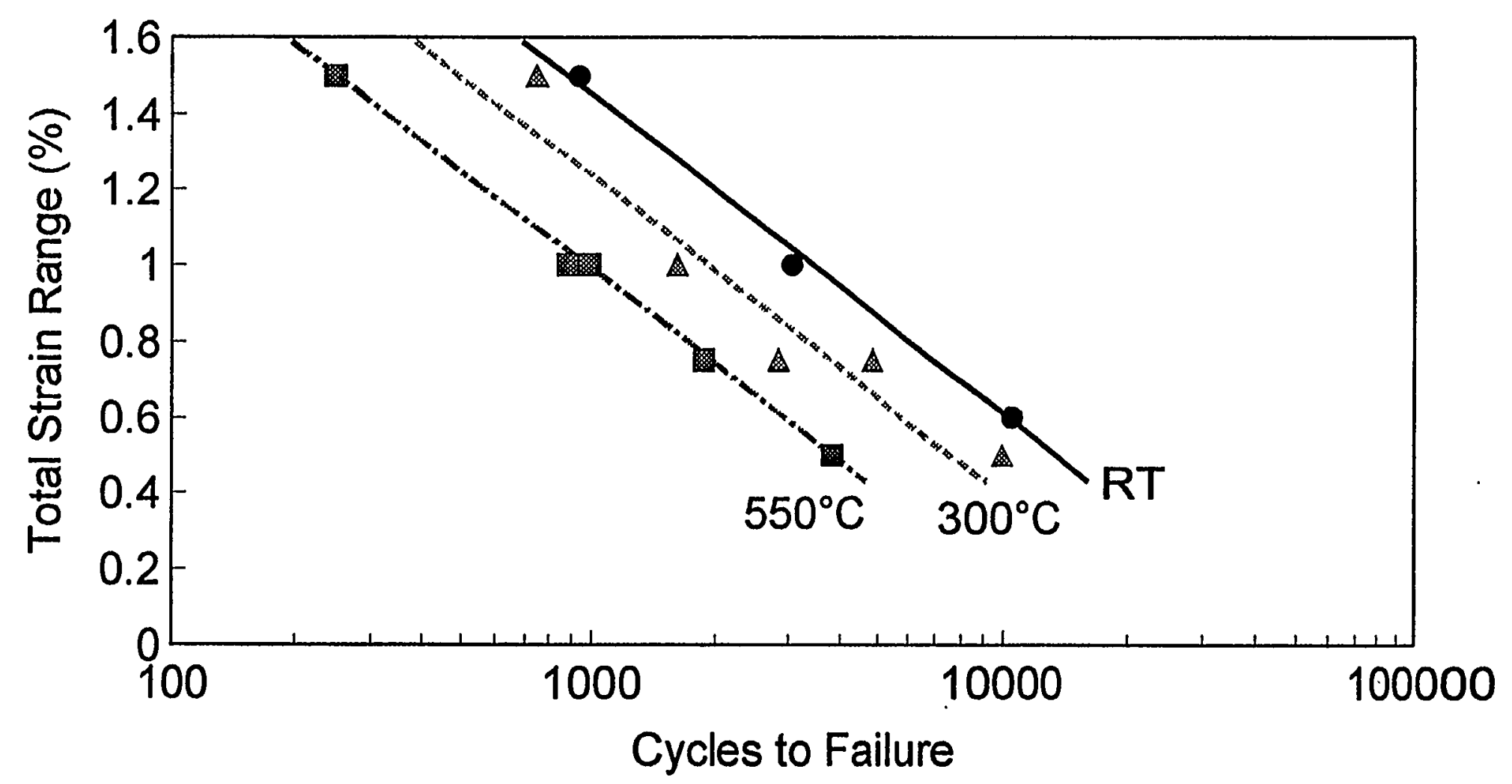

- Detailed data on fatigue results are available from WWW homepage (Database) http://realab01.tokai.jaeri.go.jp/lafdb01/fmain.htm 


\section{Magnetic Property of F82H IEA heat}
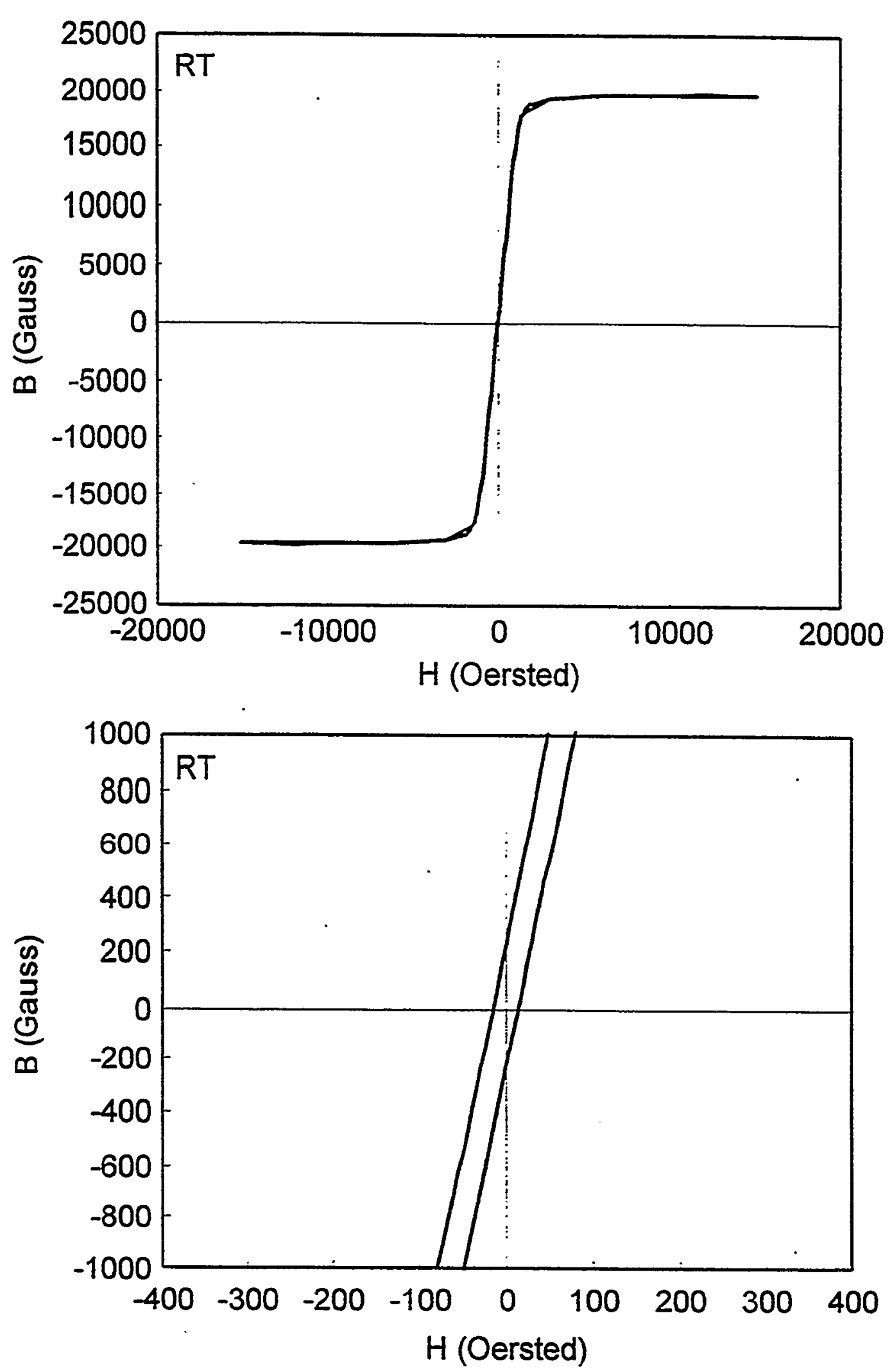


\section{BASE METAL PROPERTIES (Aging)}

\section{Current Status of Aging Tests}

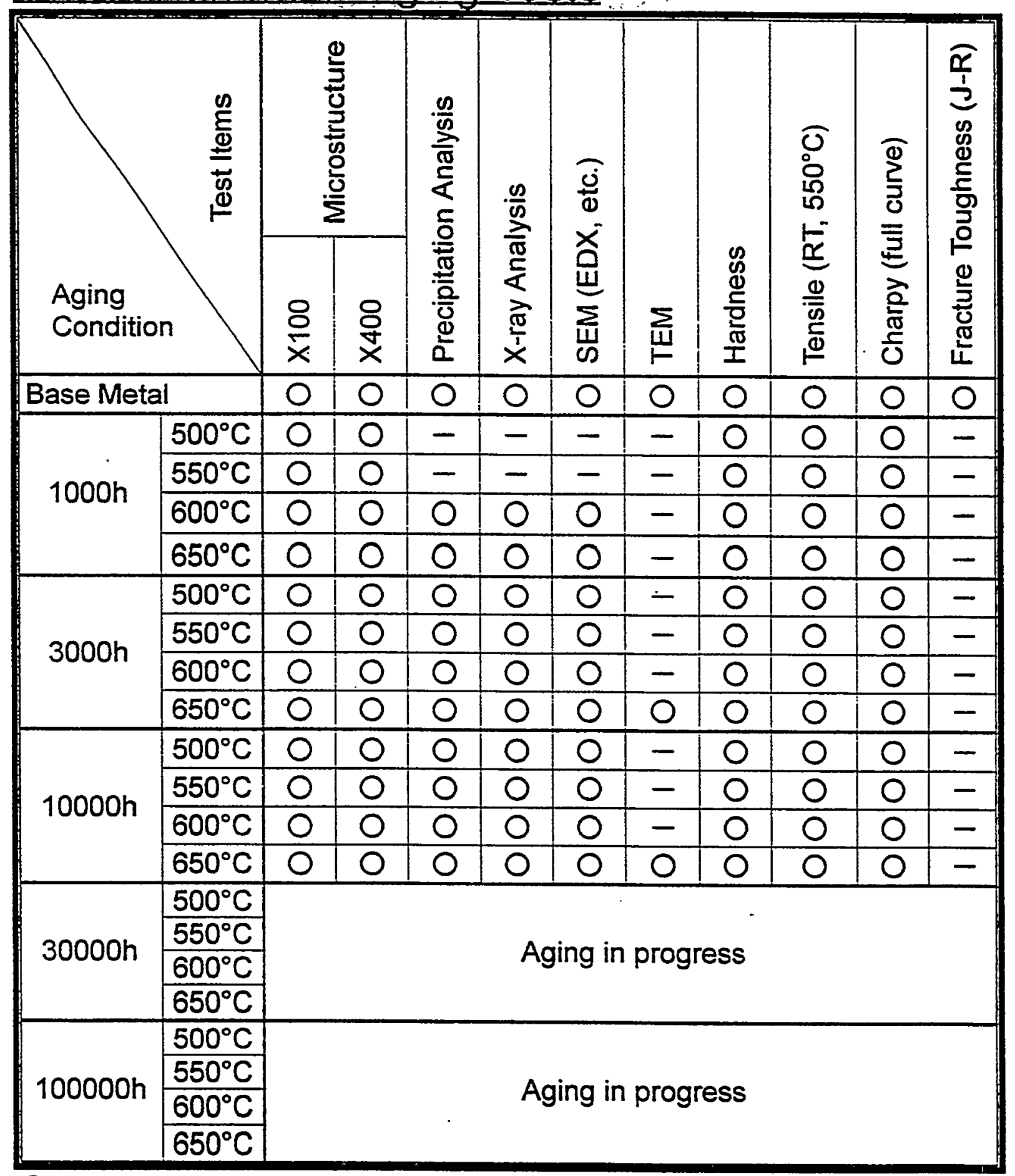

$\mathrm{O}$ : finished, $\Delta$ : in progress, - : not scheduled 


\section{Tensile Properties of Aged F82H IEA heat}
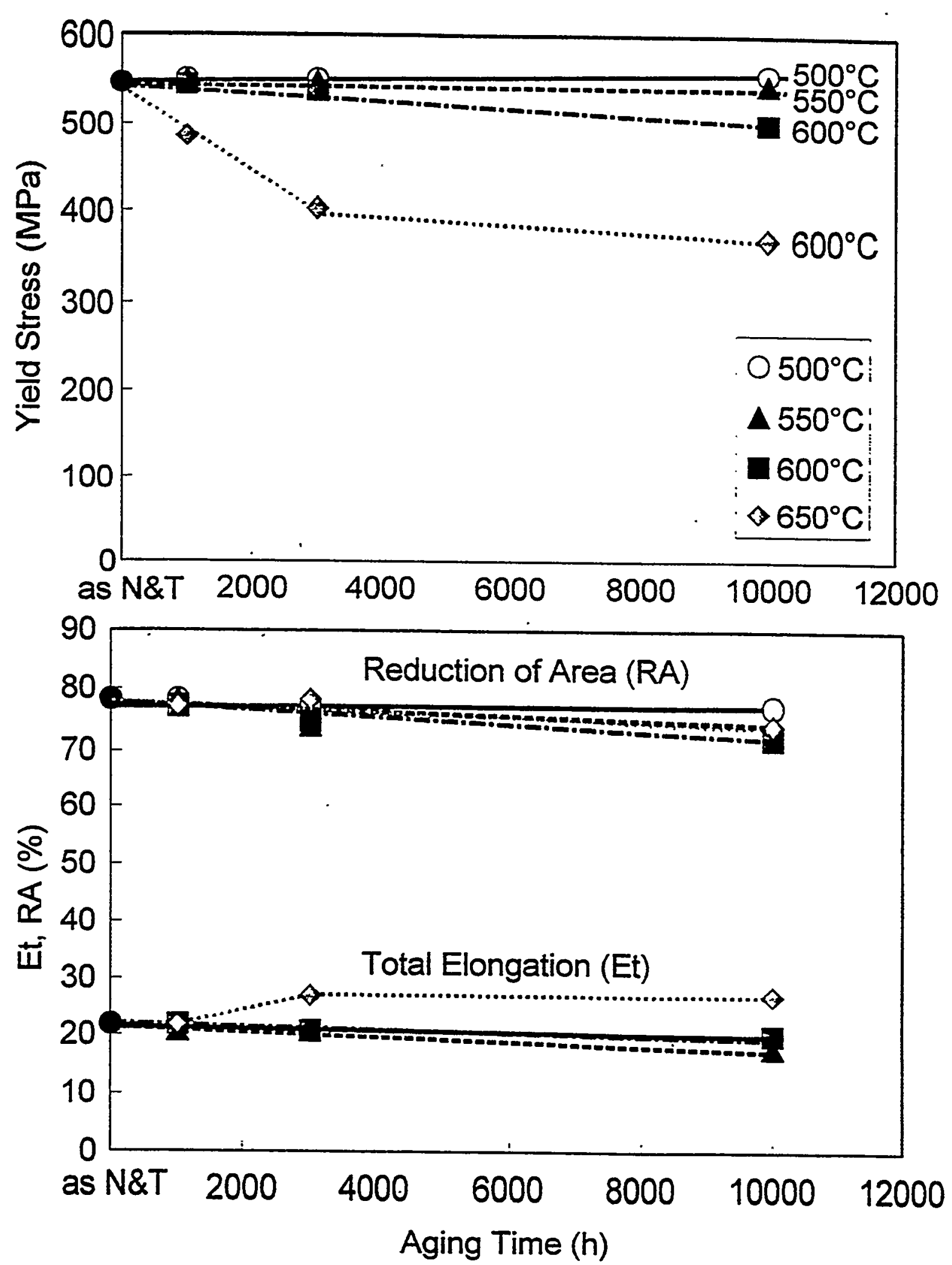
F82H IEA Heat after $10000 \mathrm{H}$ Aging

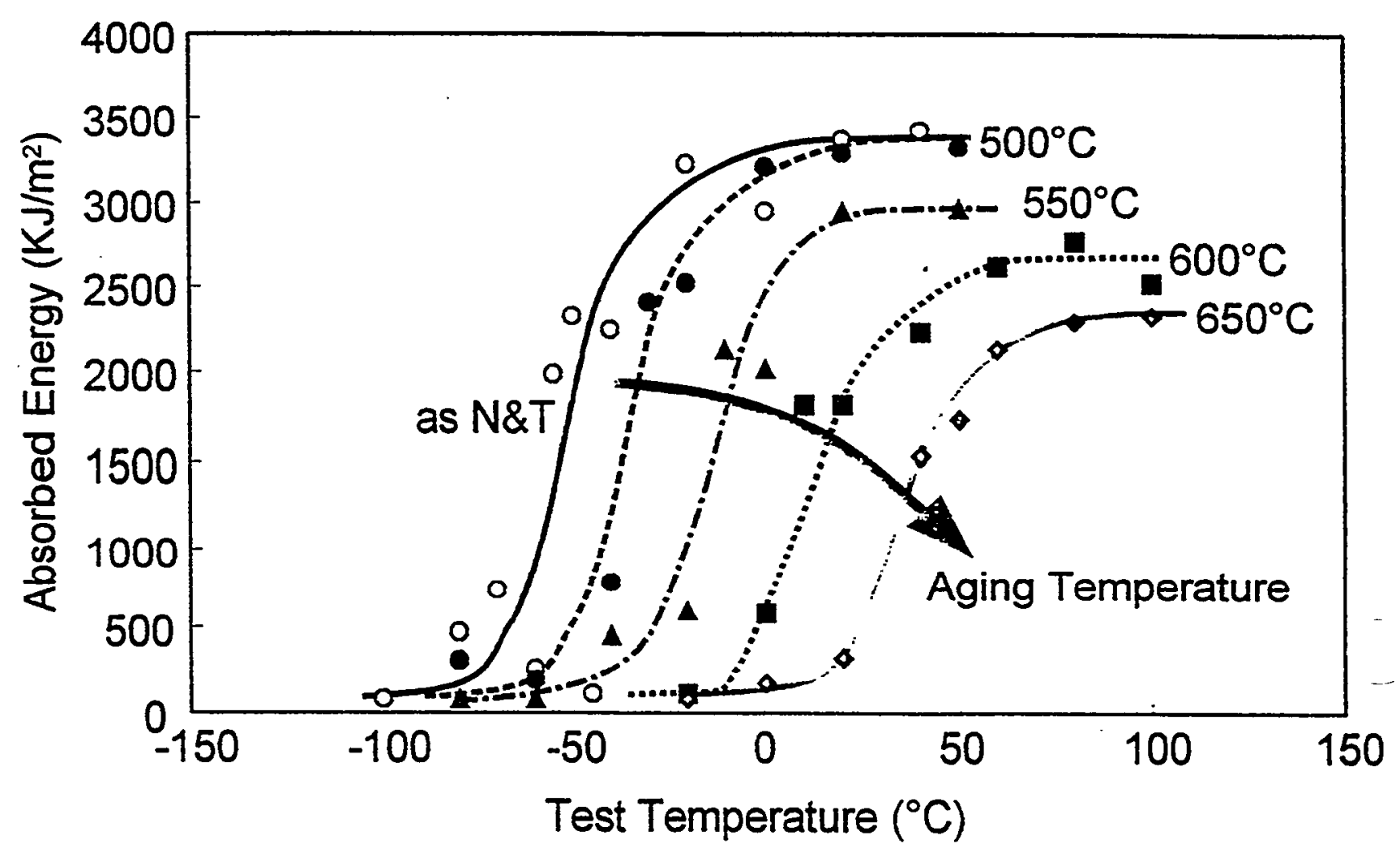



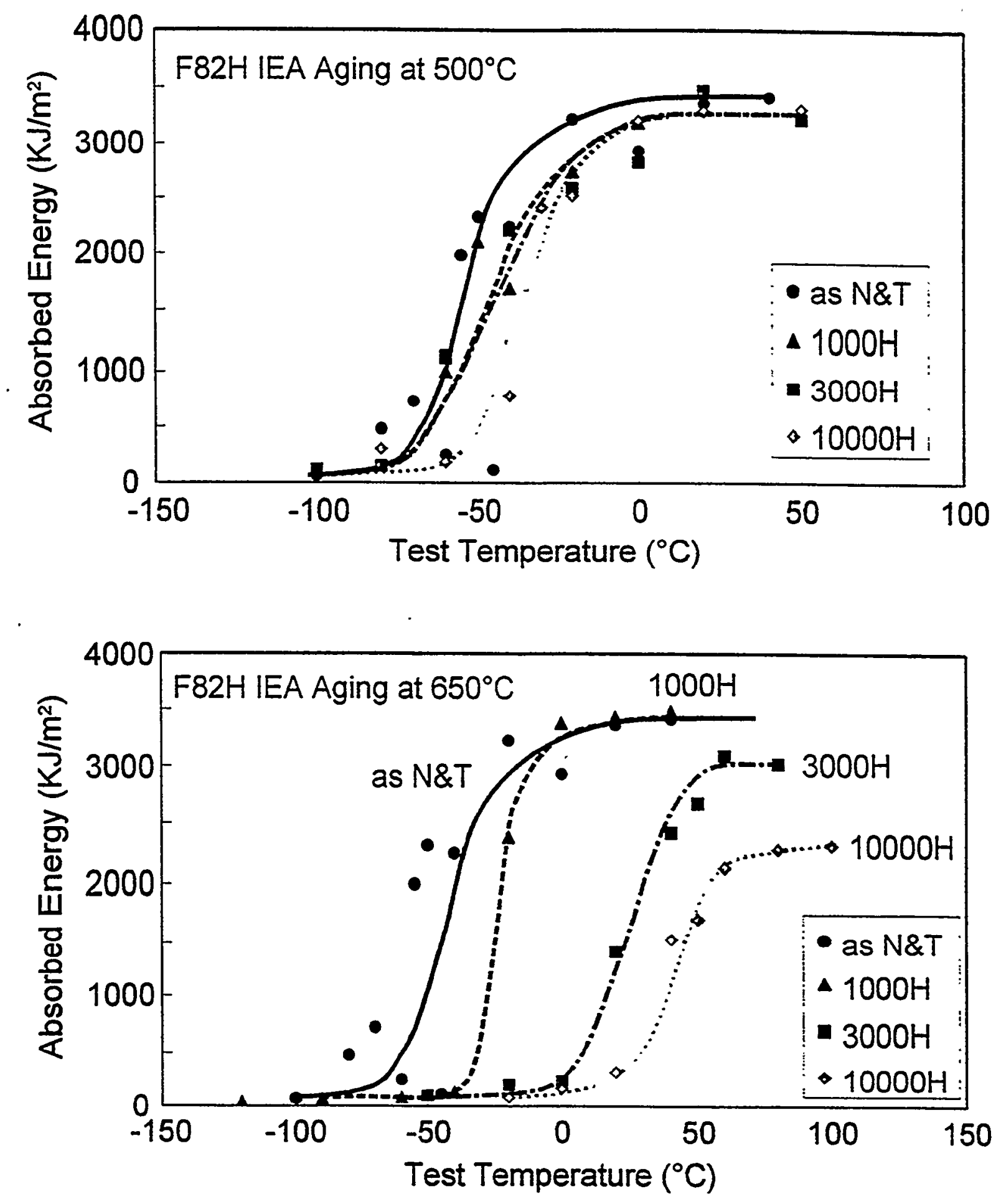


\section{Charpy Impact Test Results of Thermal Aged F82H IEA Heat}
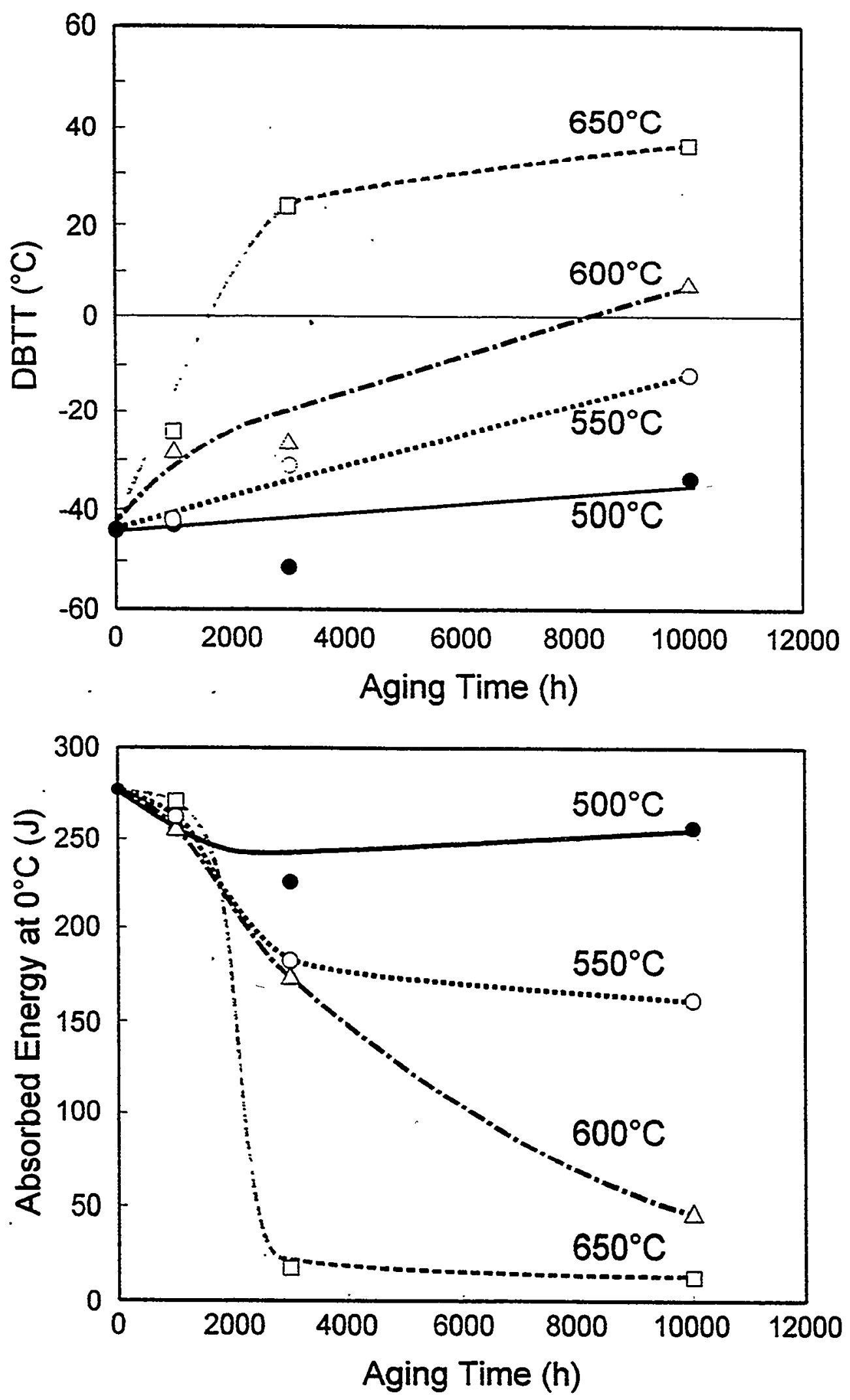


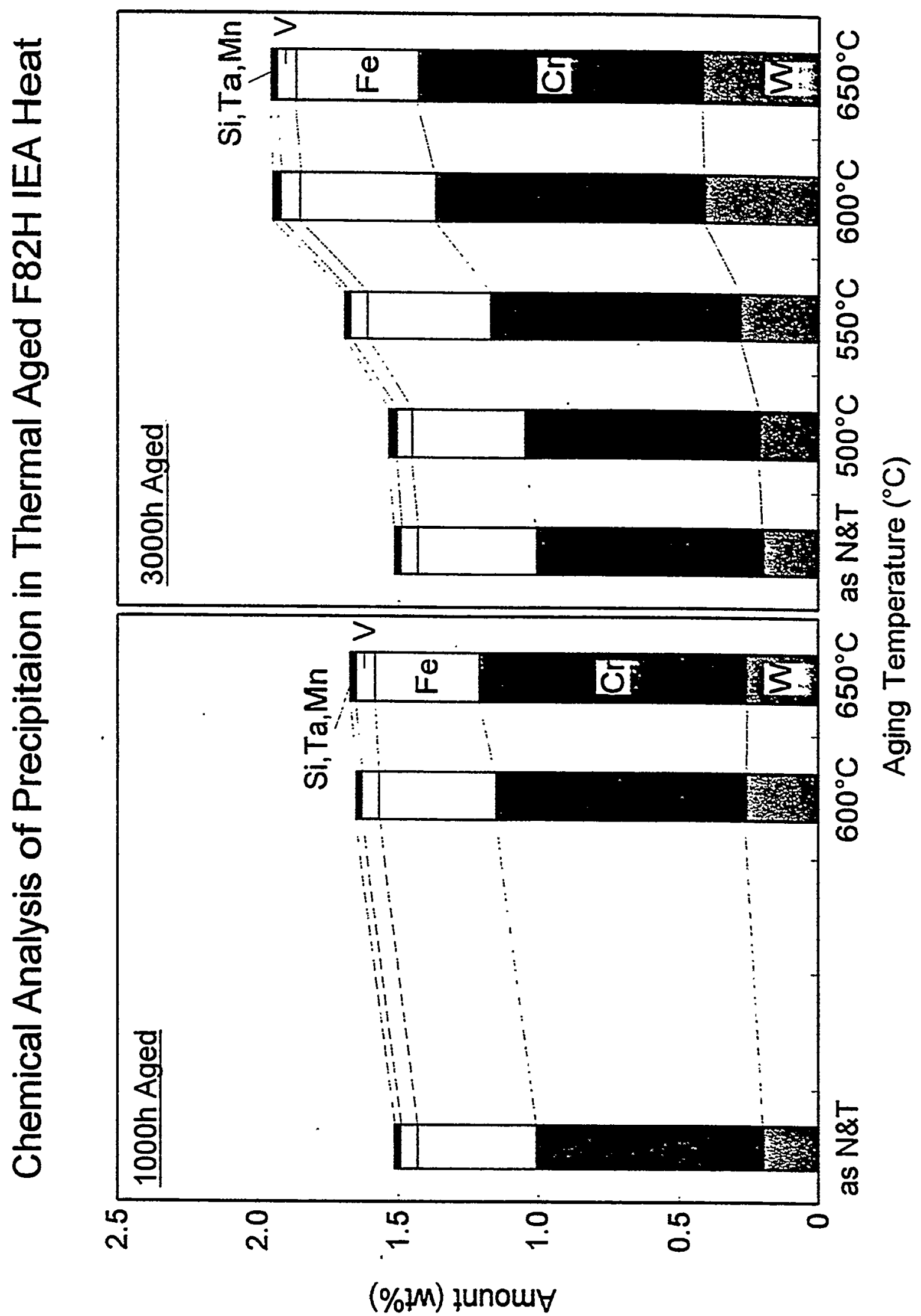




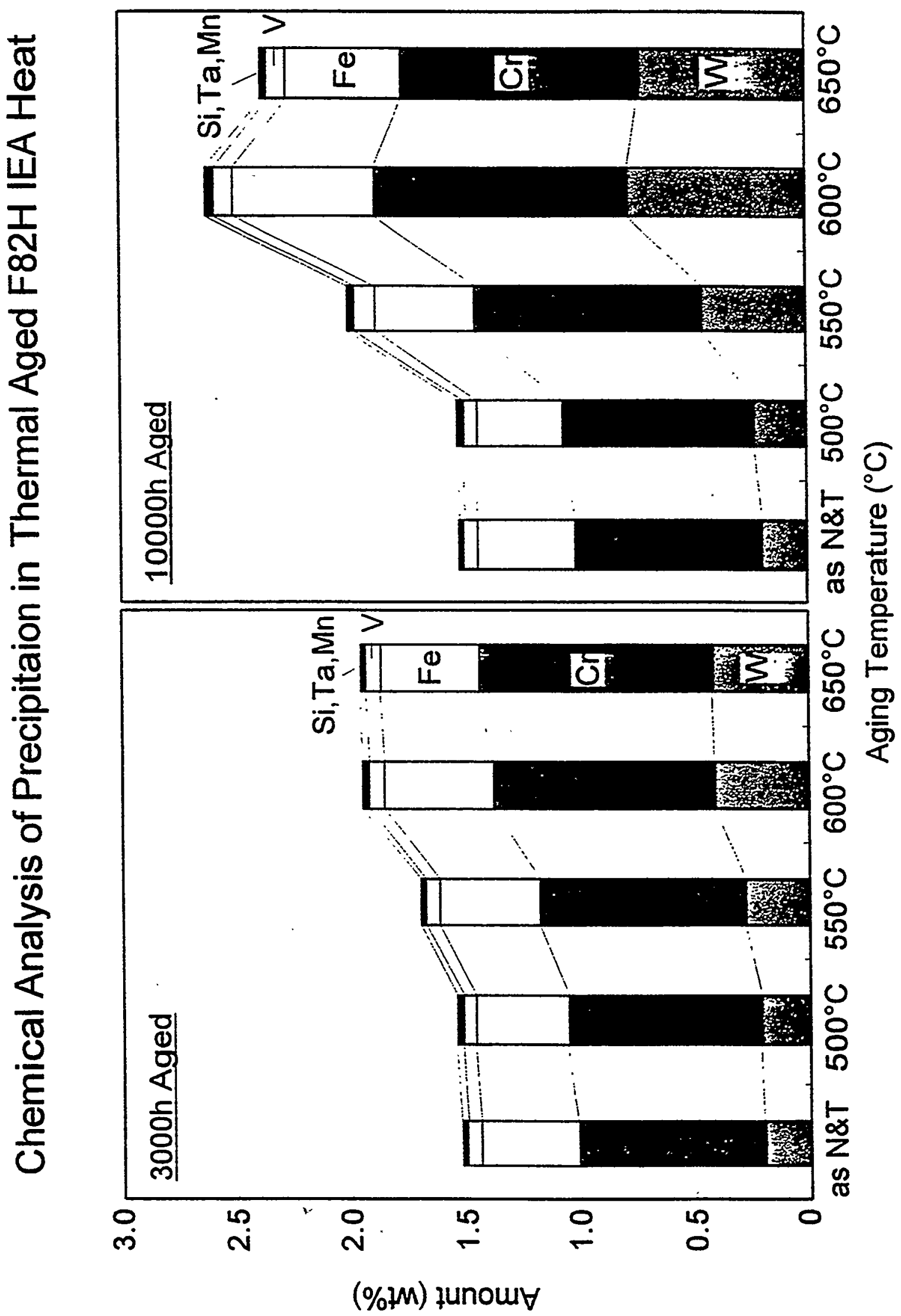




\section{Thermal Aging Condition of F82H IEA Heat (TTP Diagram)}

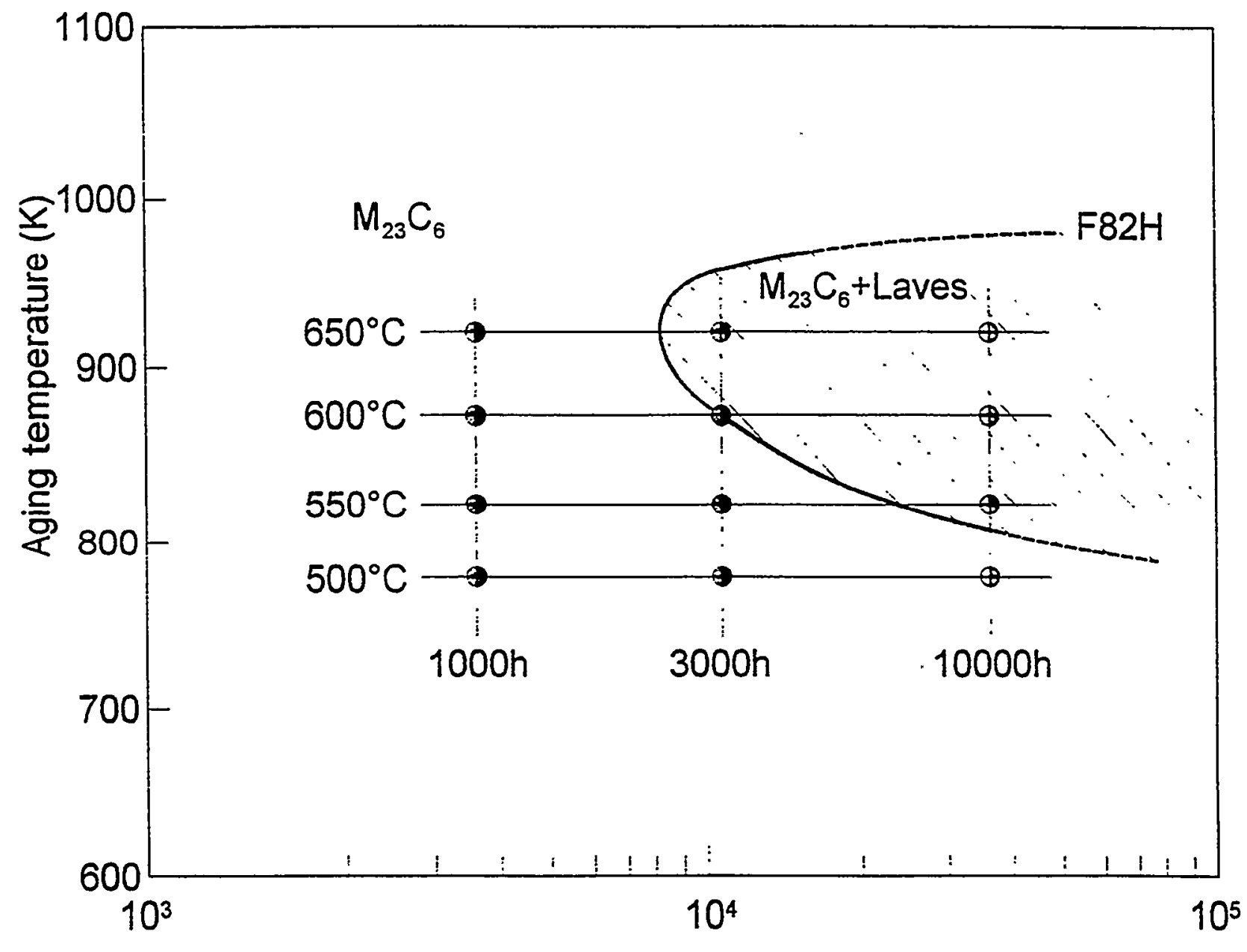

Aging time (ks) 


\section{PROPERTIES OF WELDMENTS (as weld)}

\section{TIG weldments}

Method: Narrow Gap Oscillating Electrode TIG

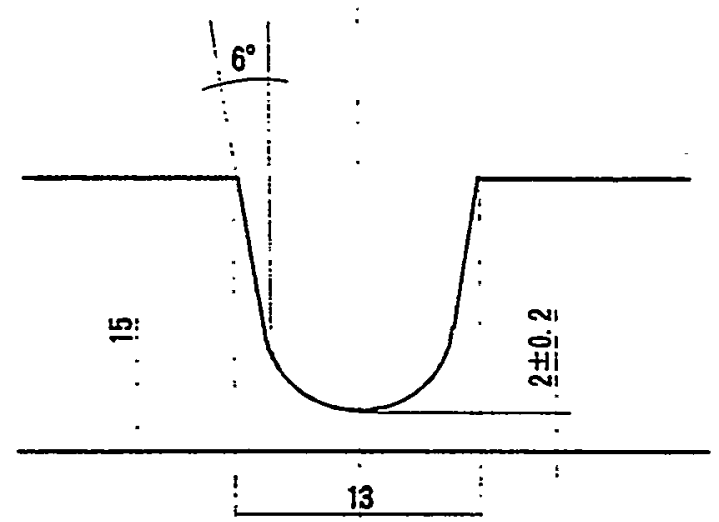

(a) Groove (15 mm)

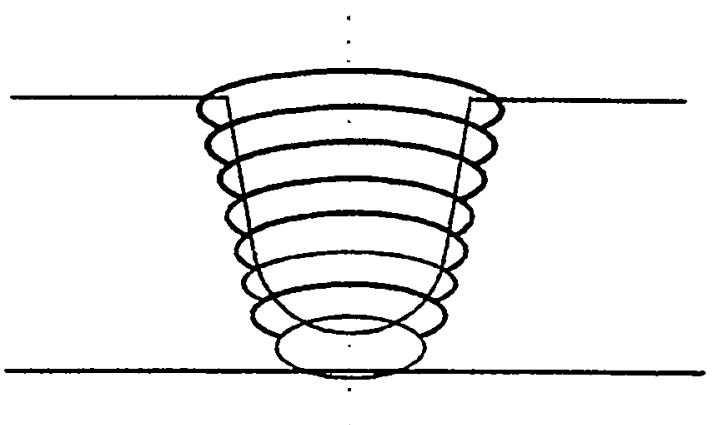

(b) Layers

EB weldments

Method: I-groove welding (without filler metal)

OPost-Welding Heat Treatment (PWHT):

$720^{\circ} \mathrm{C} \times 1 \mathrm{~h}$ for both weldments

0 Thermal aging is in progress

$500-650^{\circ} \mathrm{C} /$ up to $10000 \mathrm{~h}$ 


\section{TIG WELDED JOINT}

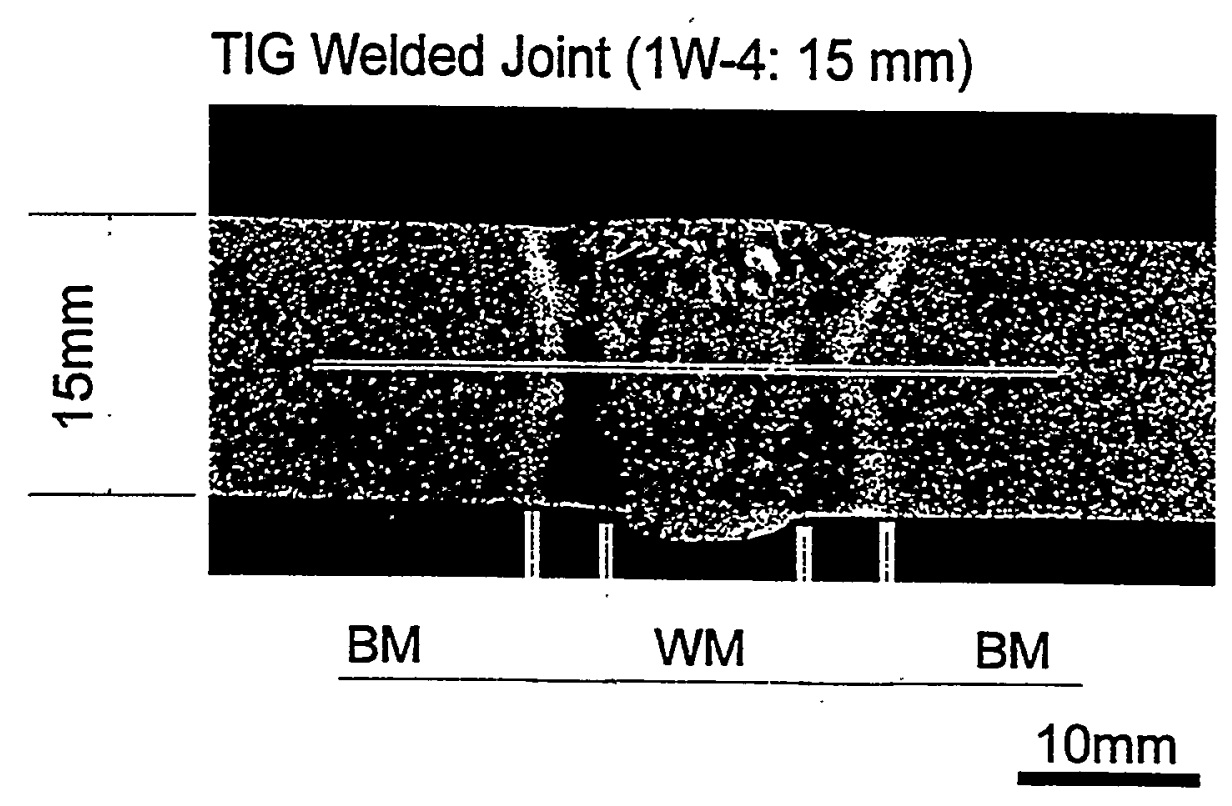

HAZ

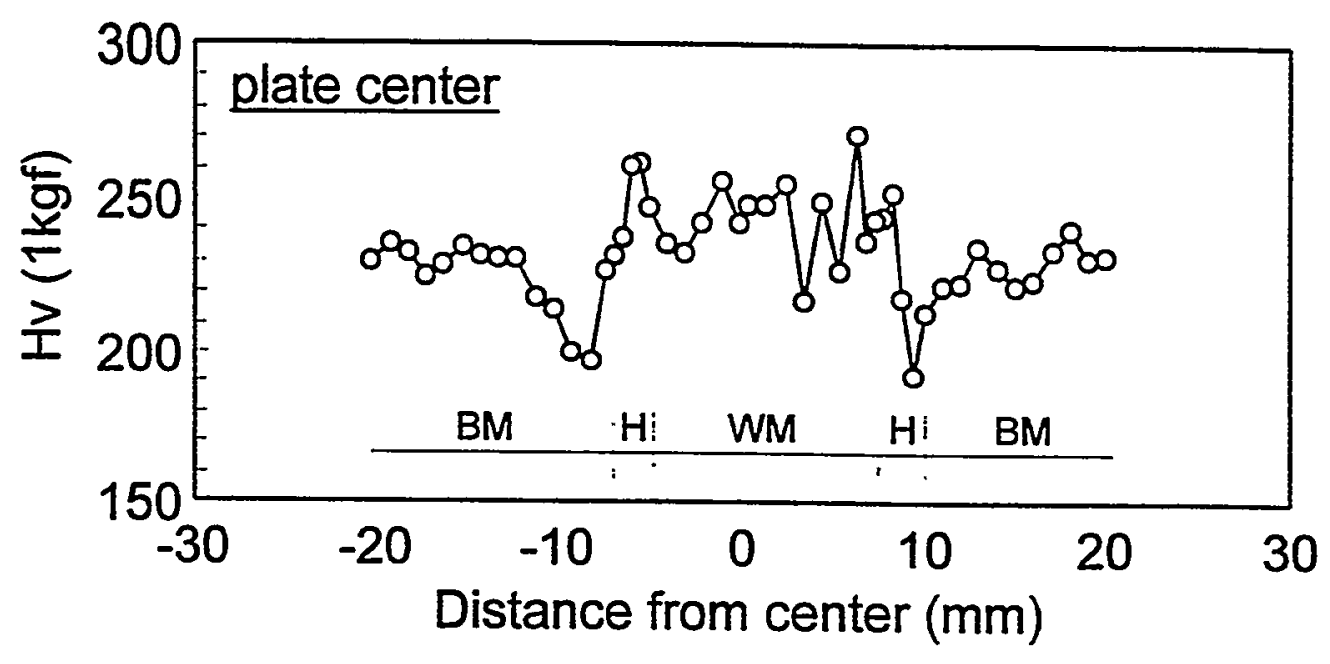




\section{EB WELDED JOINT}

EB Welded Joint (4-21: $15 \mathrm{mmt})$
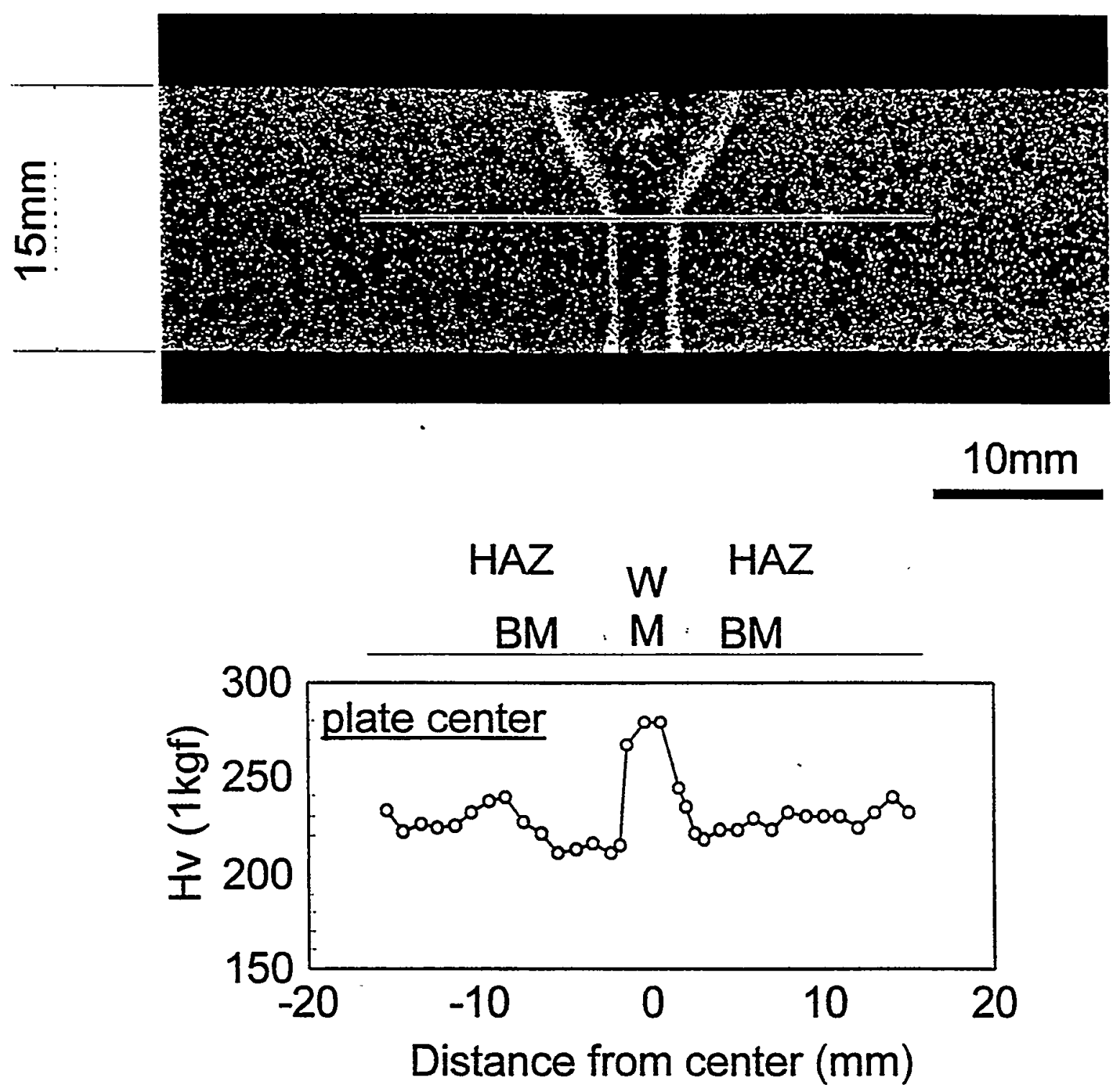


\section{Tensile Properties of F82H IEA heat TIG welded Joint}
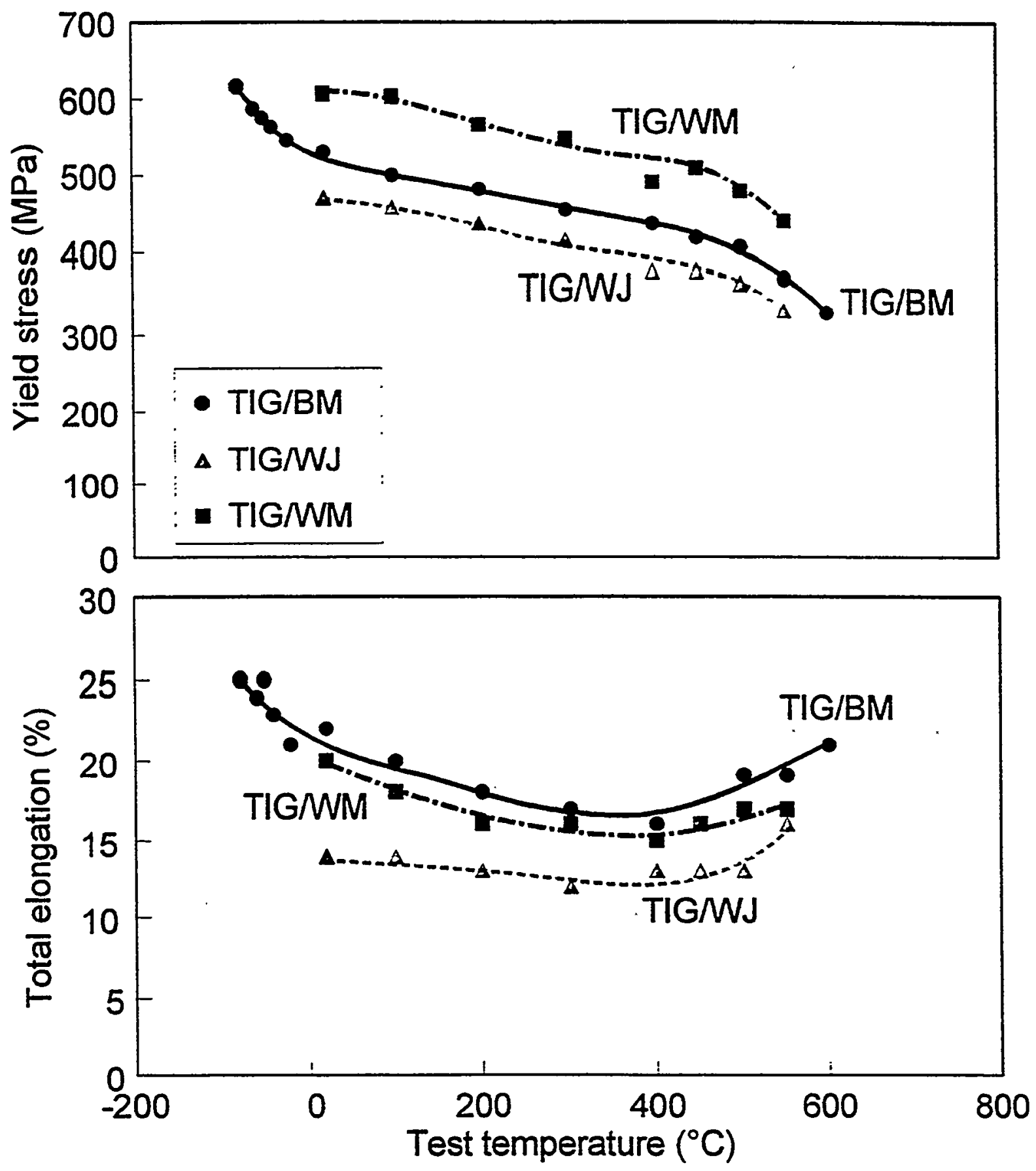
Tensile Properties of F82H IEA heat TIG and EB welded Joint

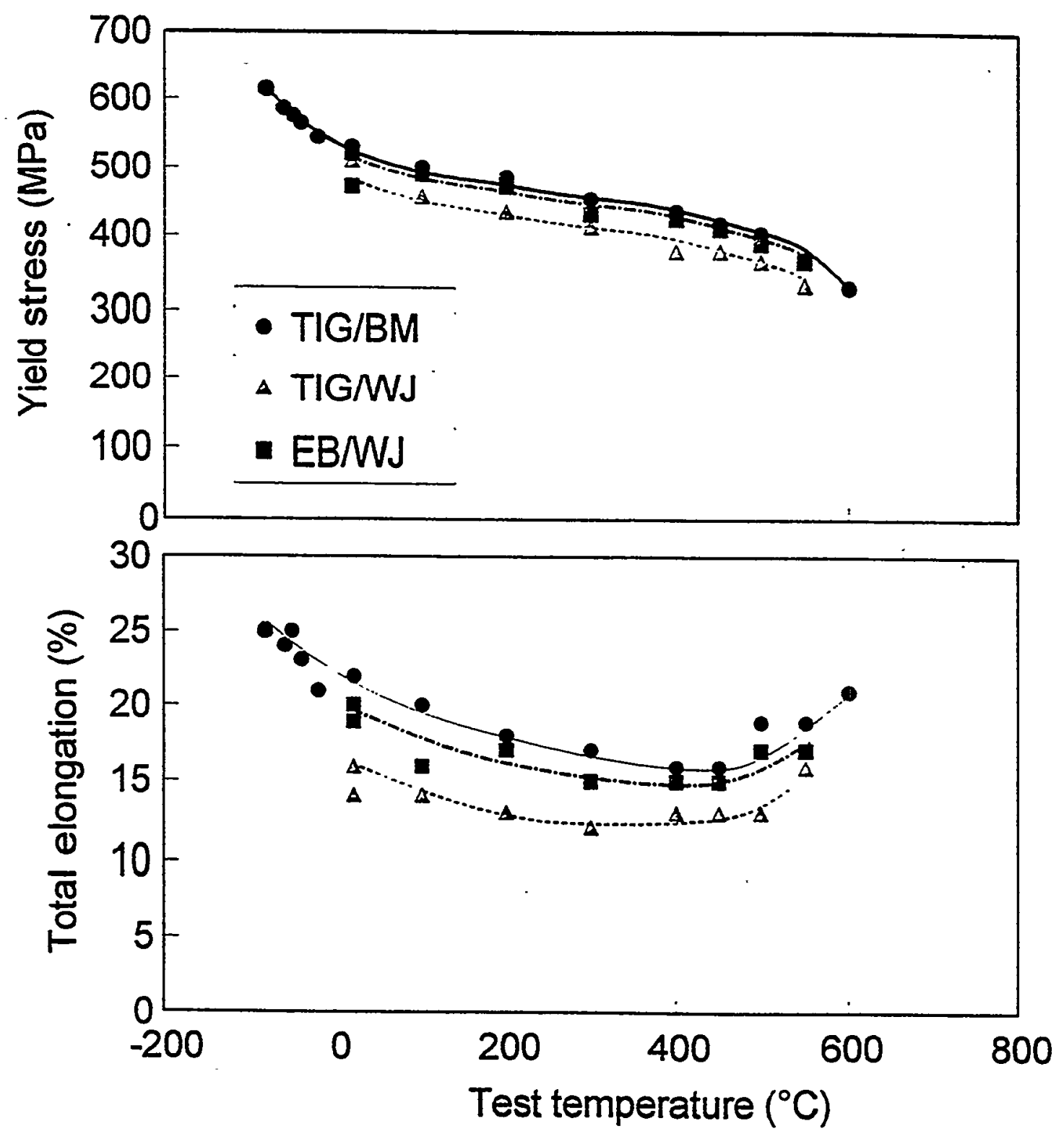


Fracture Toughness of F82H IEA heat (1"CT, RT test)

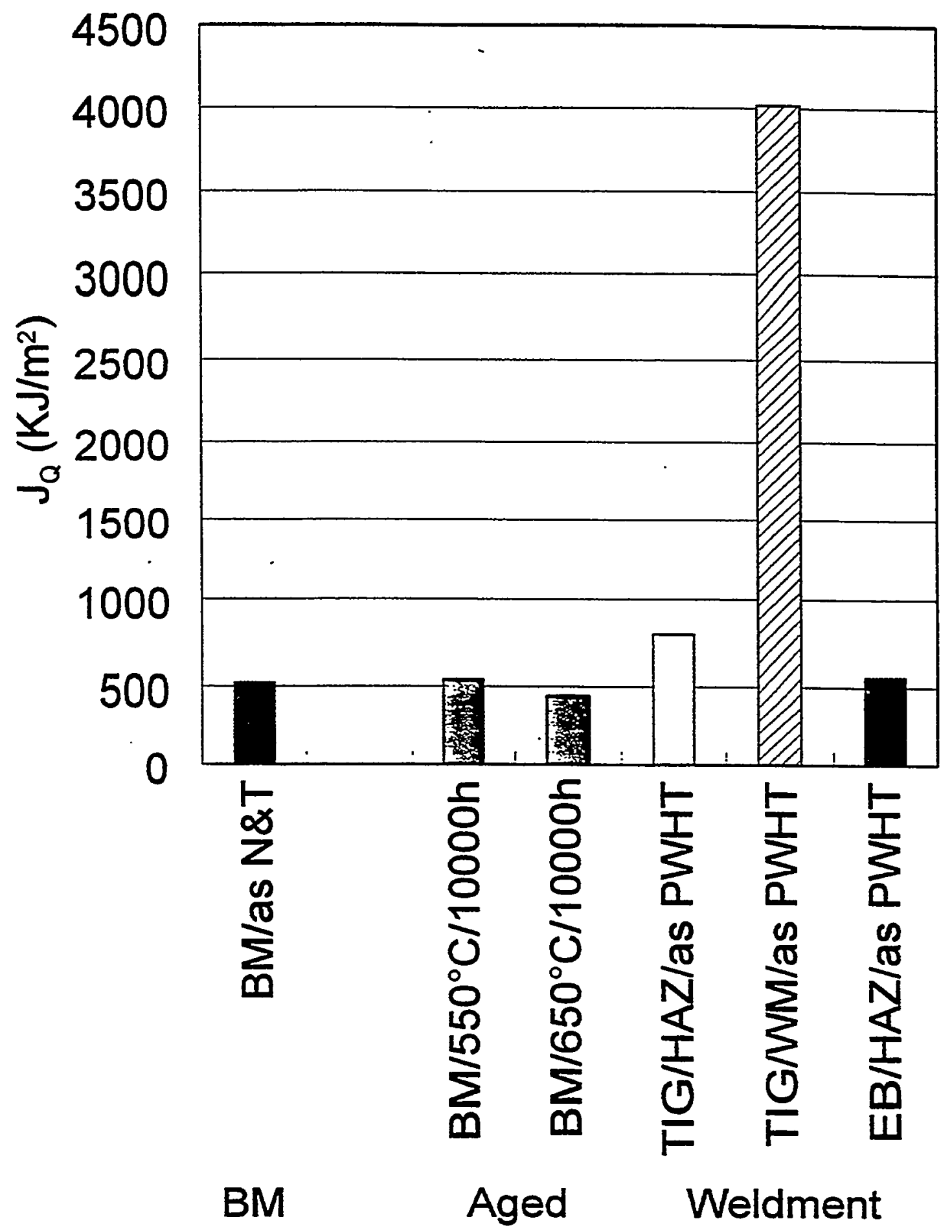




\section{PROPERTIES OF WELDMENTS (as weld)}

Summary

\begin{tabular}{|c|c|c|c|c|c|}
\hline & \multicolumn{2}{|c|}{ TIG } & \multicolumn{2}{|c|}{ EB } \\
\hline & & $15 \mathrm{~mm}$ & $25 \mathrm{~mm}$ & $15 \mathrm{~mm}$ & $25 \mathrm{~mm}$ \\
\hline \multicolumn{2}{|c|}{ WM } & $10-15 \mathrm{~mm}$ & $10-20 \mathrm{~mm}$ & $2-8 \mathrm{~mm}$ & $3-10 \mathrm{~mm}$ \\
\hline \multicolumn{2}{|c|}{ HAZ } & $2-5 \mathrm{~mm}$ & $3-10 \mathrm{~mm}$ & $1-3 \mathrm{~mm}$ & $2-4 \mathrm{~mm}$ \\
\hline \multicolumn{2}{|c|}{ Total } & $20 \mathrm{~mm}$ & $23 \mathrm{~mm}$ & $12 \mathrm{~mm}$ & $14 \mathrm{~mm}$ \\
\hline \multicolumn{2}{|c|}{ Hv max } & 275 & 285 & 280 & 300 \\
\hline \multicolumn{2}{|c|}{ Hv min } & 187 & 200 & 210 & 210 \\
\hline \multirow{6}{*}{ RTMJ } & YS & $510 \mathrm{MPa}$ & $470 \mathrm{MPa}$ & $474 \mathrm{MPa}$ & $520 \mathrm{MPa}$ \\
\hline & UTS & $629 \mathrm{MPa}$ & $605 \mathrm{MPa}$ & $605 \mathrm{MPa}$ & $640 \mathrm{MPa}$ \\
\hline & FS & $1947 \mathrm{MPa}$ & $1475 \mathrm{MPa}$ & $1455 \mathrm{MPa}$ & $1464 \mathrm{MPa}$ \\
\hline & $\mathrm{Eu}$ & $4 \%$ & $4 \%$ & $7 \%$ & $5 \%$ \\
\hline & Et & $16 \%$ & $14 \%$ & $20 \%$ & $19 \%$ \\
\hline & $\mathrm{RA}$ & $82 \%$ & $77 \%$ & $78 \%$ & $77 \%$ \\
\hline \multirow{2}{*}{$R T / J_{Q}$} & HAZ & - & $800 \mathrm{~kJ} / \mathrm{m}^{2}$ & - & $550 \mathrm{~kJ} / \mathrm{m}^{2}$ \\
\hline & WM & & $4020 \mathrm{~kJ} / \mathrm{m}^{2}$ & - & 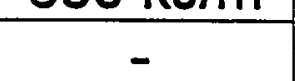 \\
\hline \multirow{2}{*}{ DBTT } & HAZ & $-87^{\circ} \mathrm{C}$ & $-82^{\circ} \mathrm{C}$ & $-44^{\circ} \mathrm{C}$ & $-38^{\circ} \mathrm{C}$ \\
\hline & WM & $12^{\circ} \mathrm{C}$ & $-6^{\circ} \mathrm{C}$ & & \\
\hline
\end{tabular}

- EB weld has almost the same properties as BM

- TIG has hard/brittle WM and annealed HAZ

- Both weld has the same or better fracture toughness than BM at room temperature 


\section{PROGESS IN ROUND-ROBIN TEST}

- SUMMARY -

Base Metal

- Fracture toughness in brittle region was $\sim 90-100 \mathrm{MPam}^{1 / 2}$.

- Reduction of area and fracture stress changes in brittle region.

- Cyclic softening occurs at any temperature in fatigue test.

Aging

- $500^{\circ} \mathrm{C}$ aging has almost no effect till up to $10000 \mathrm{~h}$.

- Tensile strength decreases by high-temp aging.

- Both decrease in USE and shift in DBTT occurs by high-temp aging.

Weldments

- EB weld has almost the same properties as BM.

- TIG has hard/brittle WM and annealed HAZ.

- Both welds have the same or better fracture toughness than BM at room temperature. 
IEA WG Meeting on RAF

October 1-2, 1998

Petten, Nethrelands

\title{
Development of SPS Bonding Procedure for RAF Steel
}

\author{
JAERI A. Hishinuma
}

\section{Background:}

- The RAF steel is the first candidate material for DEMO and beyond.

- Fabrication techniques are important for fusion application as well as material properties themselves.

- HIP (Hot Isostatic Pressing) Bonding is an important techniques for the blanket construction

- And then, development studies for the HIP bonding techniques have been executed.

- Optimum conditions of the HIP bonding for RAM steels are:

$150 \mathrm{MPa} \times 1040{ }^{\circ} \mathrm{C} \times 2 \mathrm{hr}$, (too high, see fig.1)

and tempering condition:

$740{ }^{\circ} \mathrm{C} \times 2 \mathrm{hr}$

- Problems: HIP temperature is too high from a viewpoint of phase instability (fig.1)

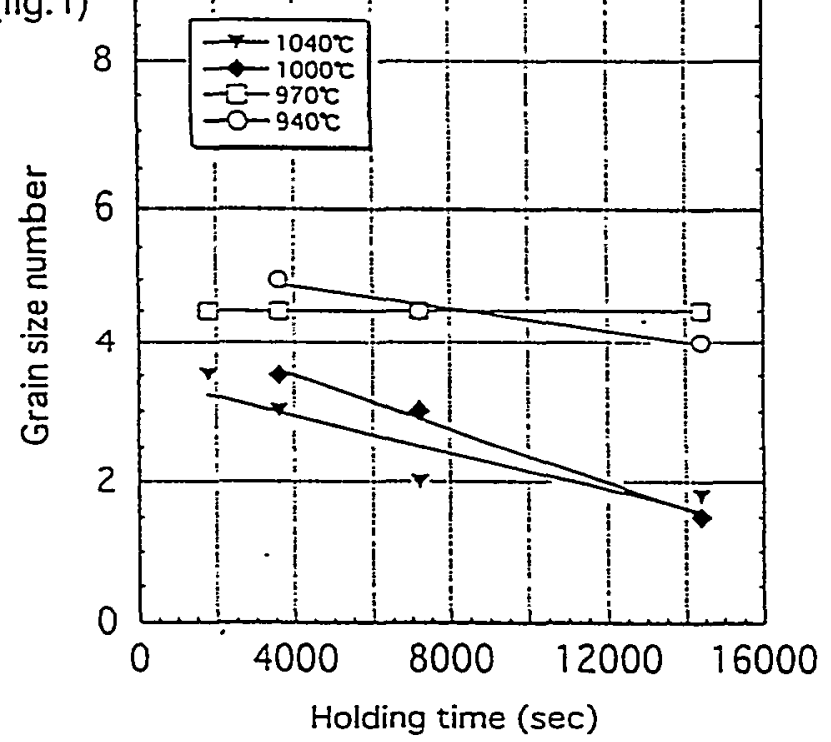

Fig.1 Relation between grain size number and holding time of HIP-equivalent heat treatment 


\section{SPS (Spark Plasma Sintering) Bonding}

\section{Feature of the SPS process}

To make possible sintering and sinter-bonding at low temperature and short periods by charging the intervals between powder particles with electrical energy and effectively applying a high temperature spark plasma generated momentarily.

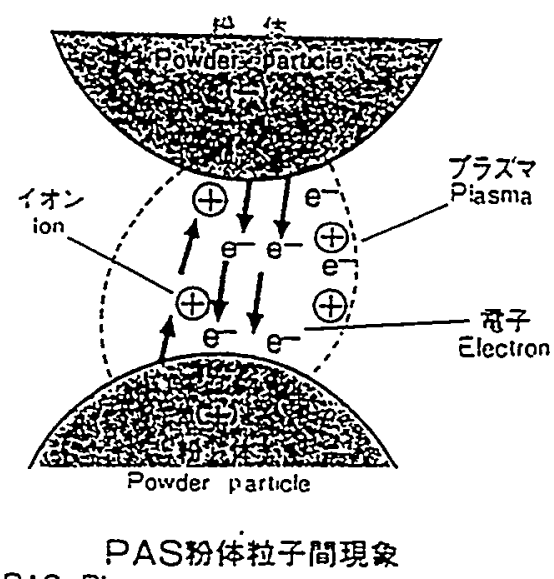

PAS Phenomenon, caused between

Powder paricles

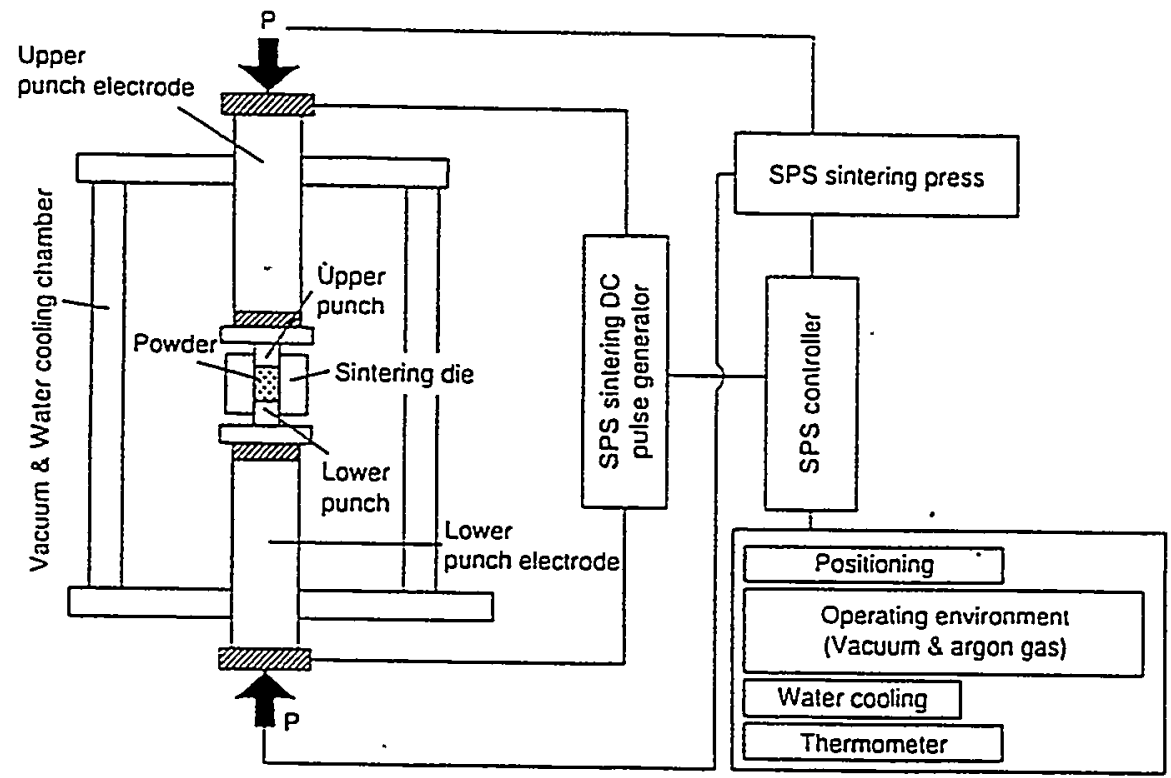

SPS system configuration 


\section{Experiments procedures}

1. Specimens used:

$\mathrm{F} 82 \mathrm{H}$

size: $20 \mathrm{~mm}^{\phi} \times 7 \mathrm{~mm}^{\mathrm{h}}$ or $15 \mathrm{~mm}^{\mathrm{h}}$

roughness:0.2, 16, $40 \mu \mathrm{m}$

2. SPS conditions;

Pressure: 20 - $50 \mathrm{MPa}$

Temperature: $800,850,900^{\circ} \mathrm{C}$

Holding time: $300-3600 \mathrm{sec}$.

Vacuum: $1 \times 10^{-2}$ torr

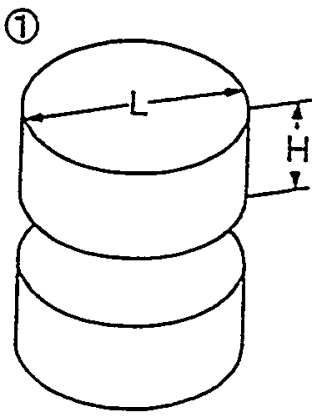

4. Tensile test

As SPS

PSHT(Post SPS-bonding Heat Treatment): $740^{\circ} \mathrm{C} \times 40 \mathrm{~min}$

Tensile specimen: SS-3 type

Temperature: Room temperature $-600^{\circ} \mathrm{C}$

Strain rate: $5 \times 10^{-3} \mathrm{sec}$

Vacuum: $1 \times 10^{-5}$ torr

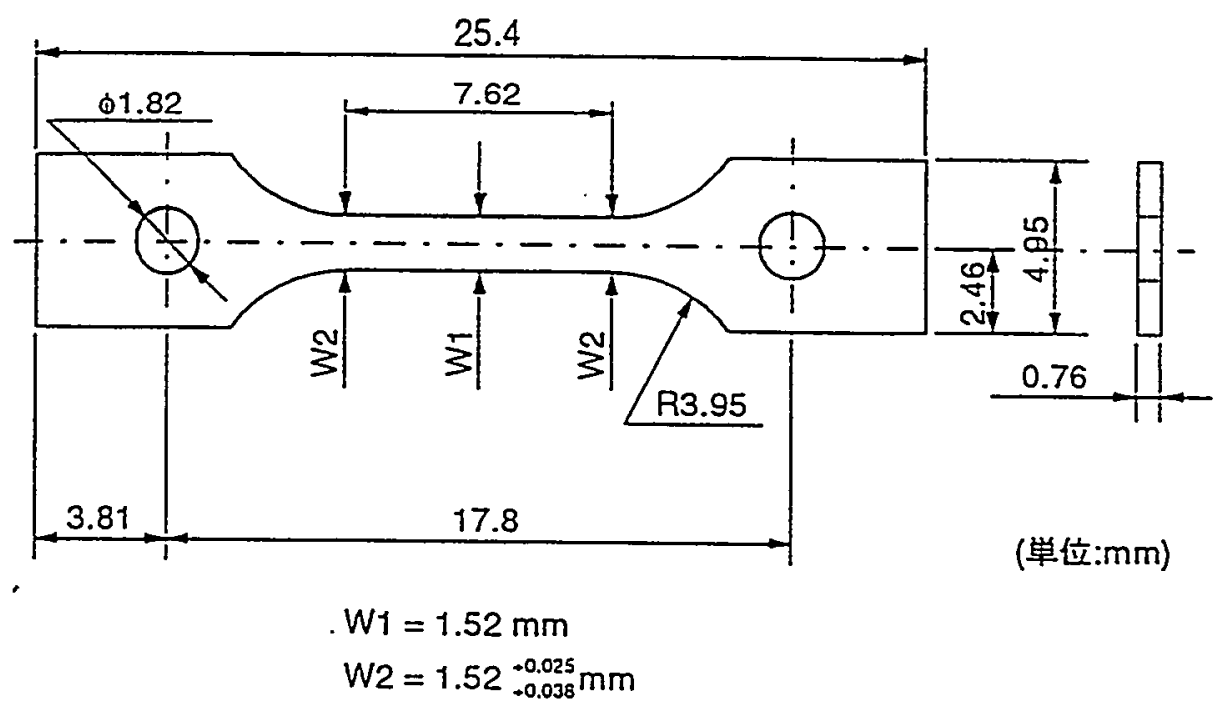



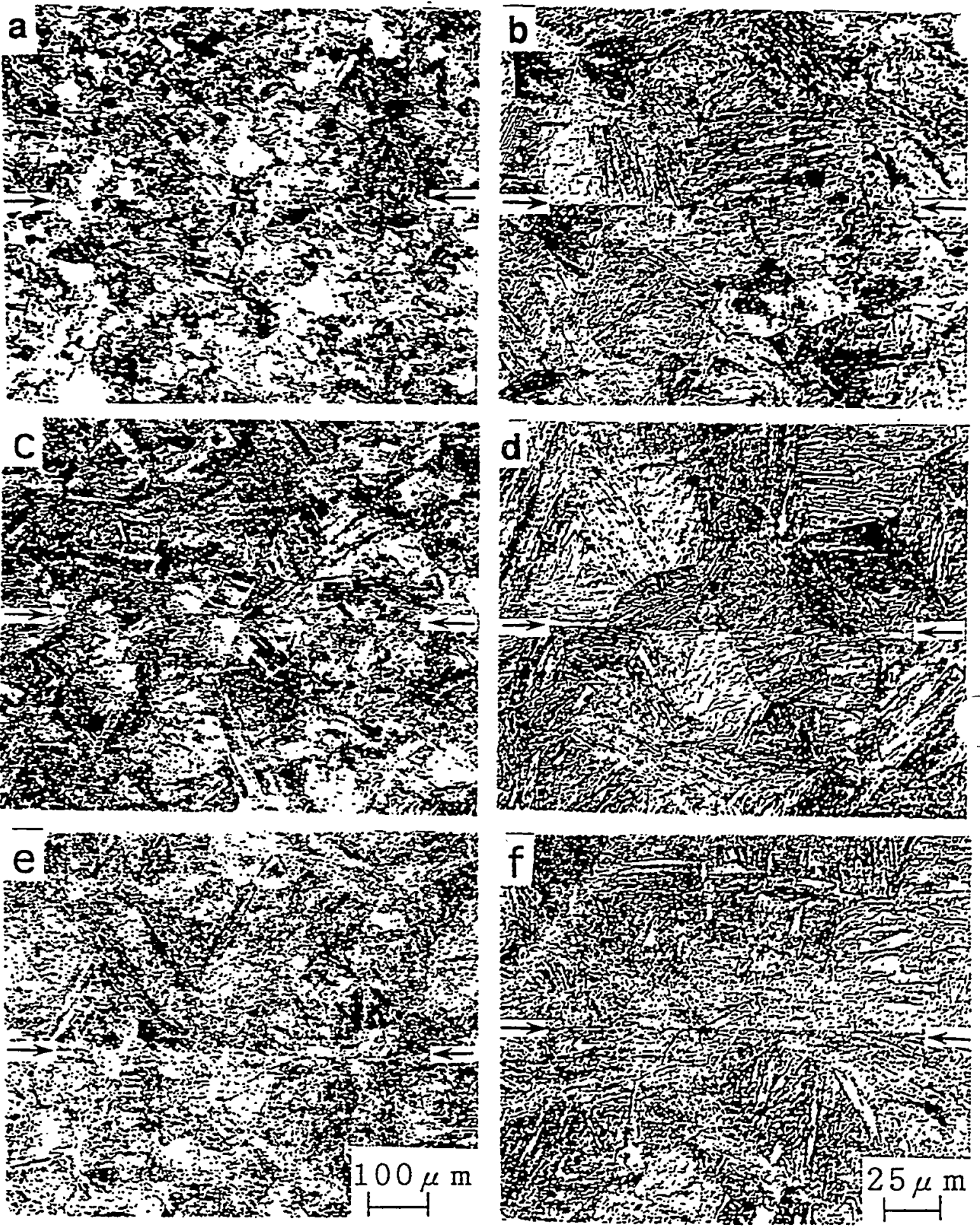

Fig.1 Optical microstructures of the SPS bonding area of F82H bonded under conditions of (a),(b) $800^{\circ} \mathrm{C}$ (c),(d) $850^{\circ} \mathrm{C}$ and (e),(f) $900^{\circ} \mathrm{C}$ for $1 \mathrm{hr}$ under pressure of $20 \mathrm{MPa}$. 

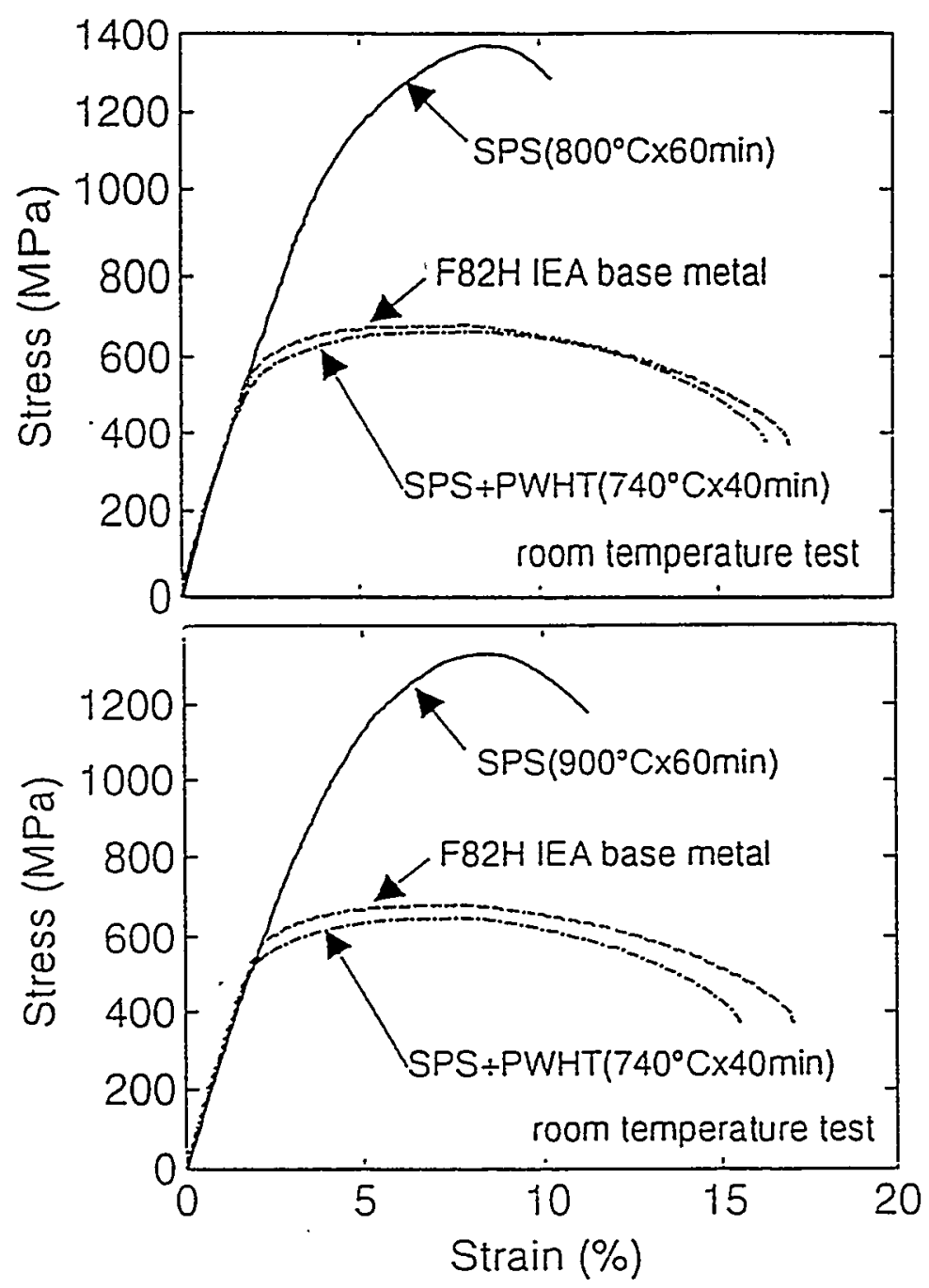

Fig. 2 Stress-str5ain curves tested at room temperature for joints of $\mathrm{F} 82 \mathrm{H}$ SPS-bonded at 800 and $900^{\circ} \mathrm{C}$ for $1 \mathrm{hr}$ under pressure of $20 \mathrm{MPa}$ with that of the base metal.

\section{Summary}

- Preliminary experiments for PSP bonding techniques have been carried out in order to examine a possibility to fusion devices

- PSP bonding techniques can be applicable to fabrication of blanket structure.

- An advantage of the SPS bonding compared with the HIP bonding techniques, is that the bonding can be done at lower temperature and short periods, without changing any microstructures. 


\section{Irradiated Properties and Composition Effects}




\section{PRESENT STATUS \\ OF \\ JAERI IRRADIATION PROGRAM \\ FOR LAM}

K. Shiba/JAERI

IEA Working Group Meeting

on

Reduced Activation Ferritic/Martensitic Steels

1-2, October, 1998

ECN Petten, The Netherlands 


\section{IRRADIATION PROGRAM IN JAERI}

OJapan/US Collaboration

- HFIR target Irradiation

- HFIR RB Irradiation

- LAF research started from Phase 2 (1987-1995)

- Currently Phase 3 (1995-1999)

- Continue to Phase 4 (1999-2005)

OJAERI Domestic Program

- JMTR/JRR-2/JRR-3/JRR-4 Irradiation

- Accelerator Irradiation

(TIARA dual/triple-beam irradiation) 


\section{JAPAN/US COLLABORATION}

\section{HFIR Phase 2 Irradiation}

- Basic Irradiation Behavior on Mechanical Properties/Microstructure

- $\mathrm{F} 82 \mathrm{H} / \mathrm{F} 82 \mathrm{H}+{ }^{10} \mathrm{~B}$ (Helium effects) $/{ }^{54} \mathrm{Fe}$ $\mathrm{F} 82 \mathrm{H}$ (Hydrogen effects)

- Tensile/TEM

- 200/250/300/400/500/600 ${ }^{\circ} \mathrm{C}$ : 2.5-57 dpa

HFIR Phase 3 Irradiation

- Irradiation Behavior on Fracture Mechanism

- F82H IEA heat/F82H+2\%Ni

- Tensile/Charpy/Fracture Toughness/Creep

- 300/500 ${ }^{\circ} \mathrm{C}:$ 8-20 dpa

- Irradiation of 1 capsule has finished. Waiting for PIE.

HFIR Phase 4 Irradiation

- Details are in discussion 


\section{JAERI REACTOR IRRADIATION}

Preliminary Irradiation Data on F82H

- F82/F82H/F82H $+{ }^{10} \mathrm{~B}$

- Tensile/Charpy

- 250-600 ${ }^{\circ} \mathrm{C}$ : 0.05-0.9 dpa

Advanced Research on Irr. Behavior of LAF

- F82H IEA/F82H $+{ }^{10} \mathrm{~B}$

- Tensile/Charpy/Fracture Toughness/Fatigue

- 250-350 ${ }^{\circ} \mathrm{C}: 0.5-2 \mathrm{dpa}$ 


\section{JAERI REACTOR IRRADIATION}

Scheduled JAERI Reactor Irradiation

JRR-3:- $250^{\circ} \mathrm{C} / 2 \mathrm{dpa}$ (Apr.1998 - Mar.2000)

- F82H IEA

- Fatigue/Tensile (irr. effects on fatigue)

JMTR: - $250^{\circ} \mathrm{C} / 1.8 \mathrm{dpa}$ (Apr.1999 - Mar.2000)

- F82H IEA/F82H $+{ }^{10} \mathrm{~B}$

- Tensile/Charpy/Fracture Toughness (correlation between properties)

JRR-3:- $300-400^{\circ} \mathrm{C} / 1 \mathrm{dpa}$ (Apr.1999 - Mar.2000)

- $\mathrm{F} 82 \mathrm{H} / \mathrm{F} 82 \mathrm{H} / \mathrm{IEA} / \mathrm{F} 82 \mathrm{H}+{ }^{10} \mathrm{~B} / \mathrm{F} 82 \mathrm{H}+2 \% \mathrm{Ni}$

- TEM/Tensile

(reliability of helium simulation)

JMTR: - ?? ${ }^{\circ} \mathrm{C} / 1.8 \mathrm{dpa}$ (Apr.2000 - Mar.2001)

- In planning 


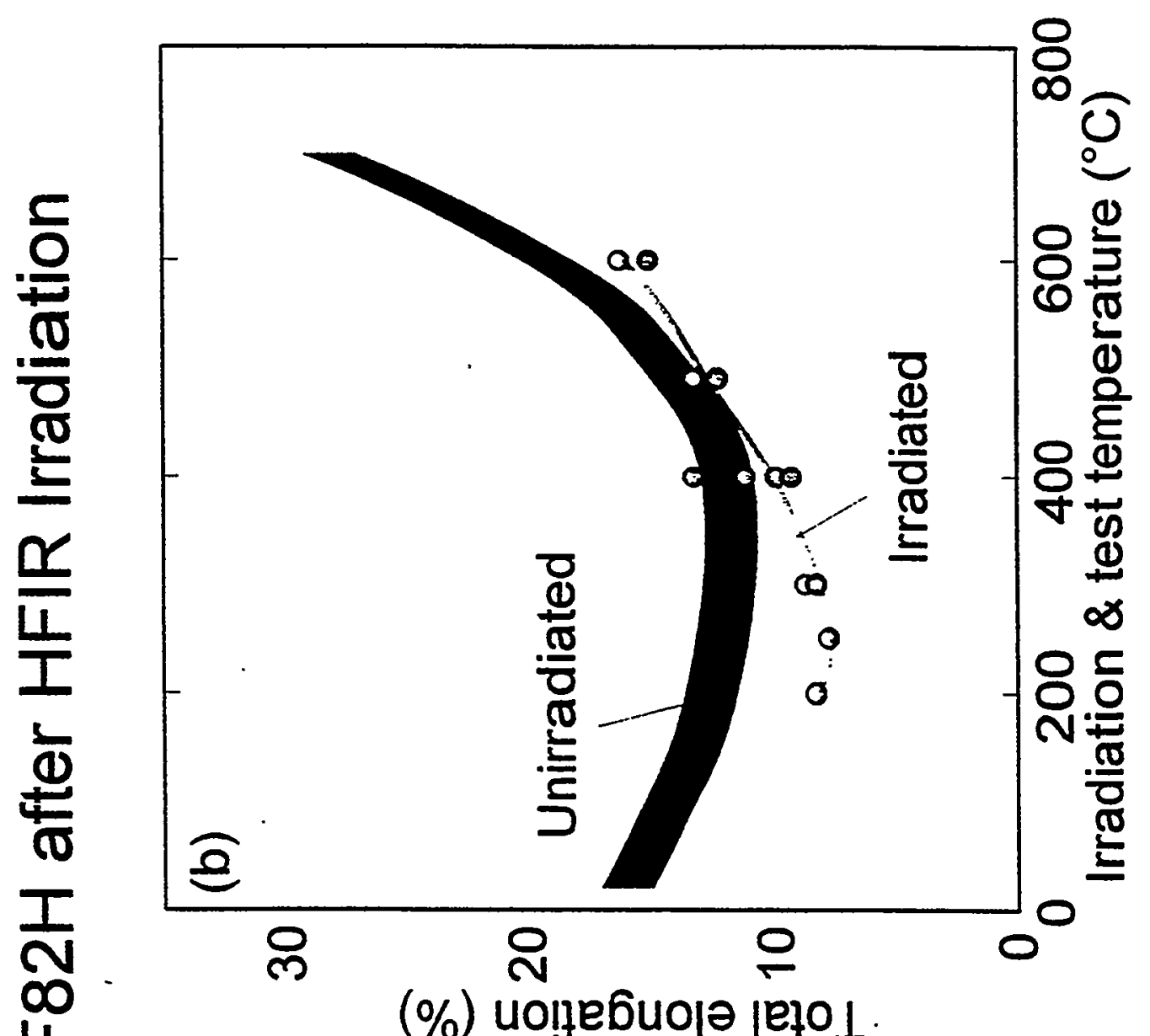

4

$\frac{1}{0}$
0
0
$\frac{1}{0}$
$\frac{0}{0}$
$\frac{2}{0}$
$\frac{0}{0}$
$\frac{5}{10}$

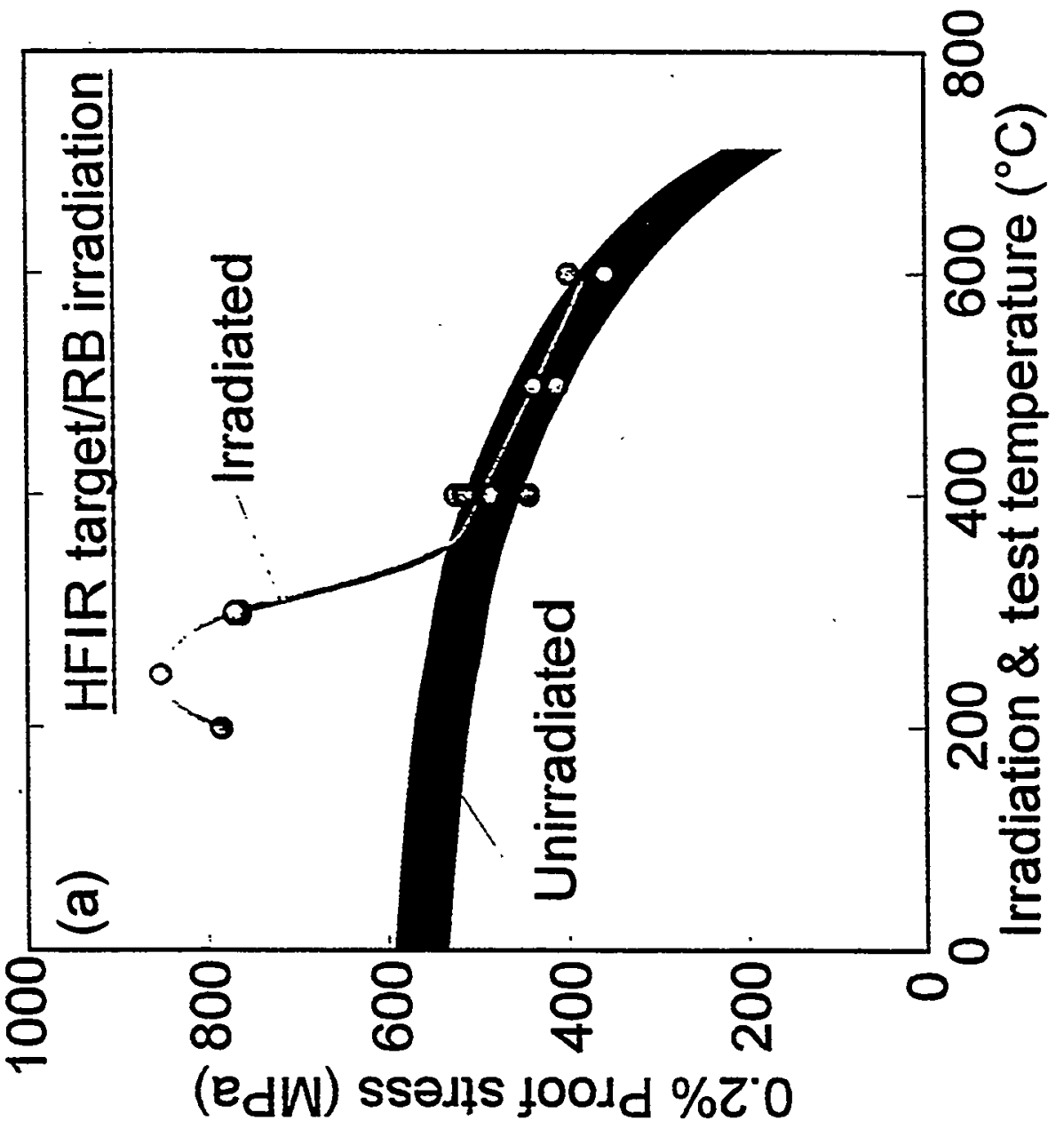




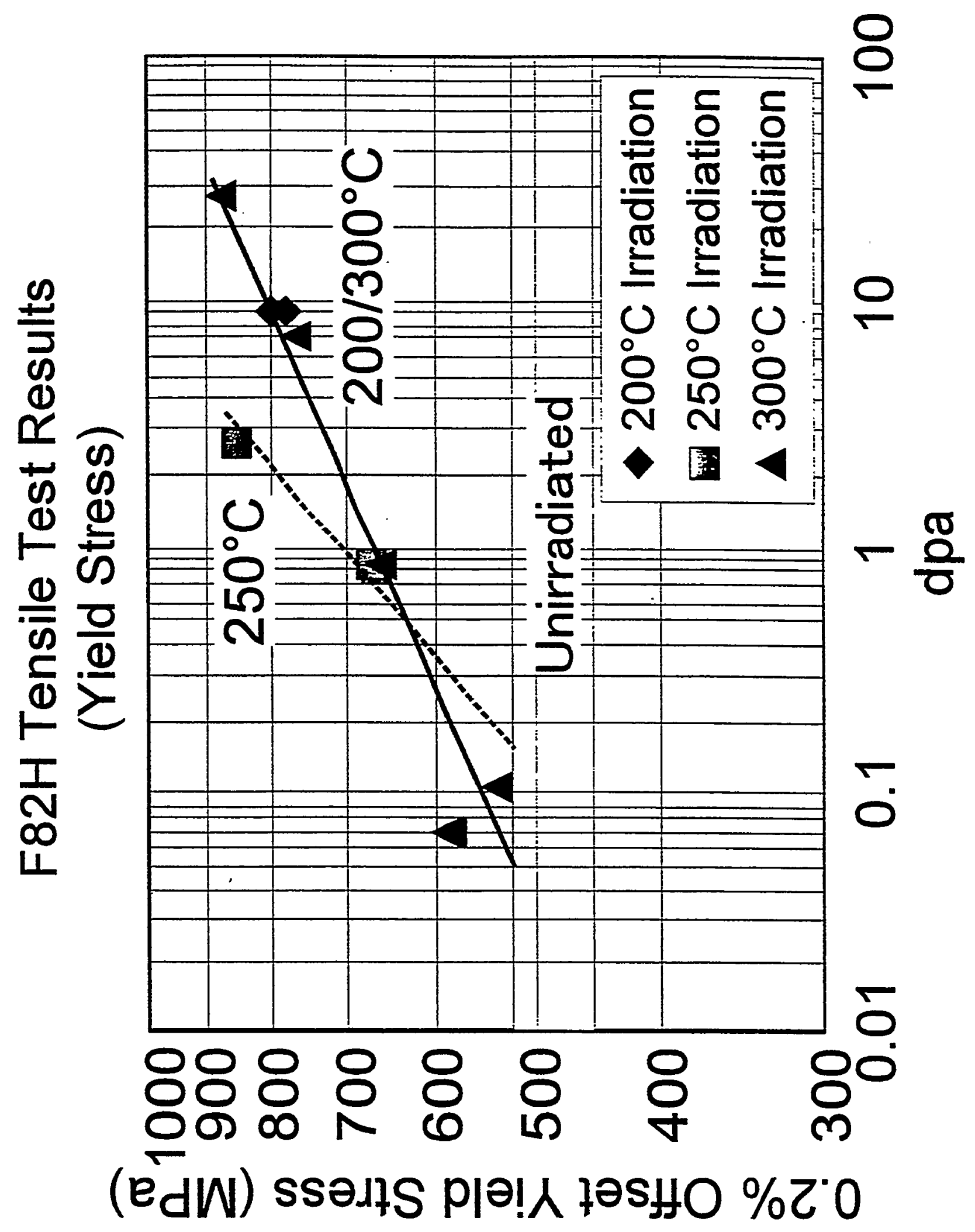




\section{Microstructure of F82H (HFIR Irradiation: $250^{\circ} \mathrm{C} 2.5 \mathrm{dpa}$ )}

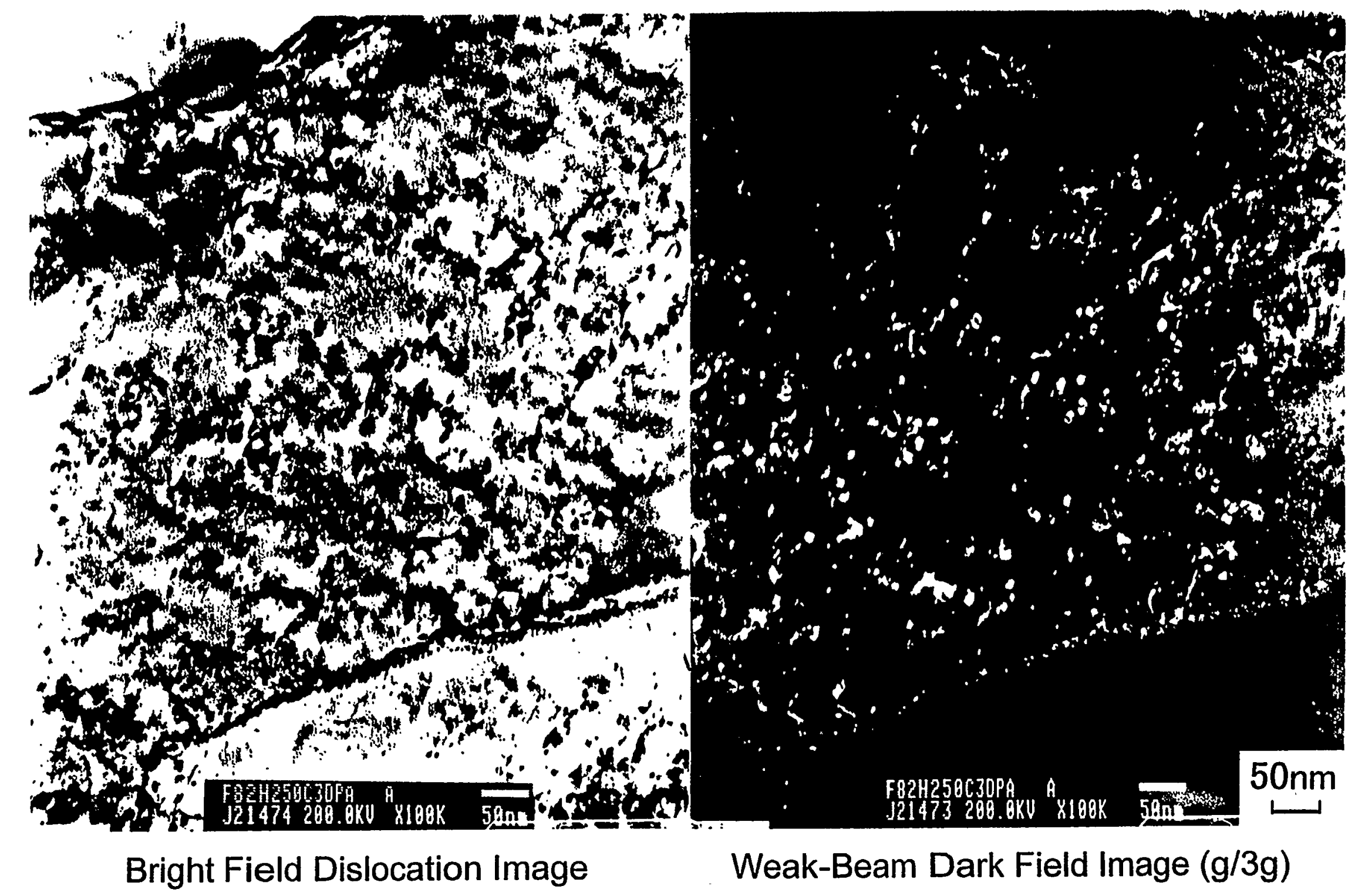




\section{OTHER RESEARCH ON RAF/MS IN JAERI}

\section{AMTEX Program (JFT-2M)}

Research on the Availability of Ferritic/Martensitic Material in Plasma Device

O Computer Simulation and Preliminary Experiment using JFT-2M were conducted in this year.

Subjects:

- Ripple reduction in the ITER

- Availability of ripple-less plasma operation with RAF/Ms vacuum vessel

- Research on the undesirable effects for plasma production, control, etc. 


\section{Advanced Ma terial Tokamak Experiment (AMTEX) Expe rim e $n$ tal $P$ la n

\begin{tabular}{|c|c|c|c|c|c|}
\hline 1998 & 1999 & 2000 & 2001 & 2002 & 2003 \\
\hline \multirow{4}{*}{$\begin{array}{l}\text { Design for } \\
\text { AMTEX and } \\
\text { pre liminary } \\
\text { expe rim ent } \\
\text { (Including } \\
\text { V-alloy } \\
\text { Research) }\end{array}$} & \multirow{2}{*}{\multicolumn{5}{|c|}{$\begin{array}{l}\text { Reduction of to ro idal fie ld ripple } \\
\text { Reactor Physics R\&D is ue: } \\
\text { reduce the ripple los s of high energy particles in a } \\
\text { reactorscenario } \\
\text { Install the ferritic boards on JFT-2M }\end{array}$}} \\
\hline & & & & & \\
\hline & & \multicolumn{4}{|c|}{ Ferritic ste el-plas ma matching test } \\
\hline & & \multicolumn{4}{|c|}{$\begin{array}{l}\text { Reactor Physics R\&D is ue: } \\
\text { verify the validity of ferritic steel as the reactor } \\
\text { structual mate rial from the view-points of } \\
\text { plas ma confinement } \\
\text { install the ferritic boards ins ide the vacuum } \\
\text { vessel(VV), and then replace the VVwith } \\
\text { ferromagnetic } V V\end{array}$} \\
\hline
\end{tabular}




\section{Computational Results[3,5]}

\section{Insertion of Ferrite Board near VV}

Ripple magnitude $(\delta)$ is reduced over the whole plasma region.

The structure of the magnetic field (Bt) is extremely modified. Toroidal mode number $(n)$ of the TF is about twice the number of TFC in the outer region. [3]

$D^{r p}{ }_{c 1} \sim n^{5} \delta^{4.5}$ or $n^{4} \delta^{4}[4]$ $\Rightarrow$ Ripple loss depends on only $\delta$ and $n$.

$D^{r p}{ }_{c l}$ : Diffusion coefficient of ripple trapped particle in collisionless region

$\Rightarrow$ In order to obtain the guide line for reduction of higher mode ripple, $\delta$ is analyzed by Fourier expansion.

JFT-2M

(present)
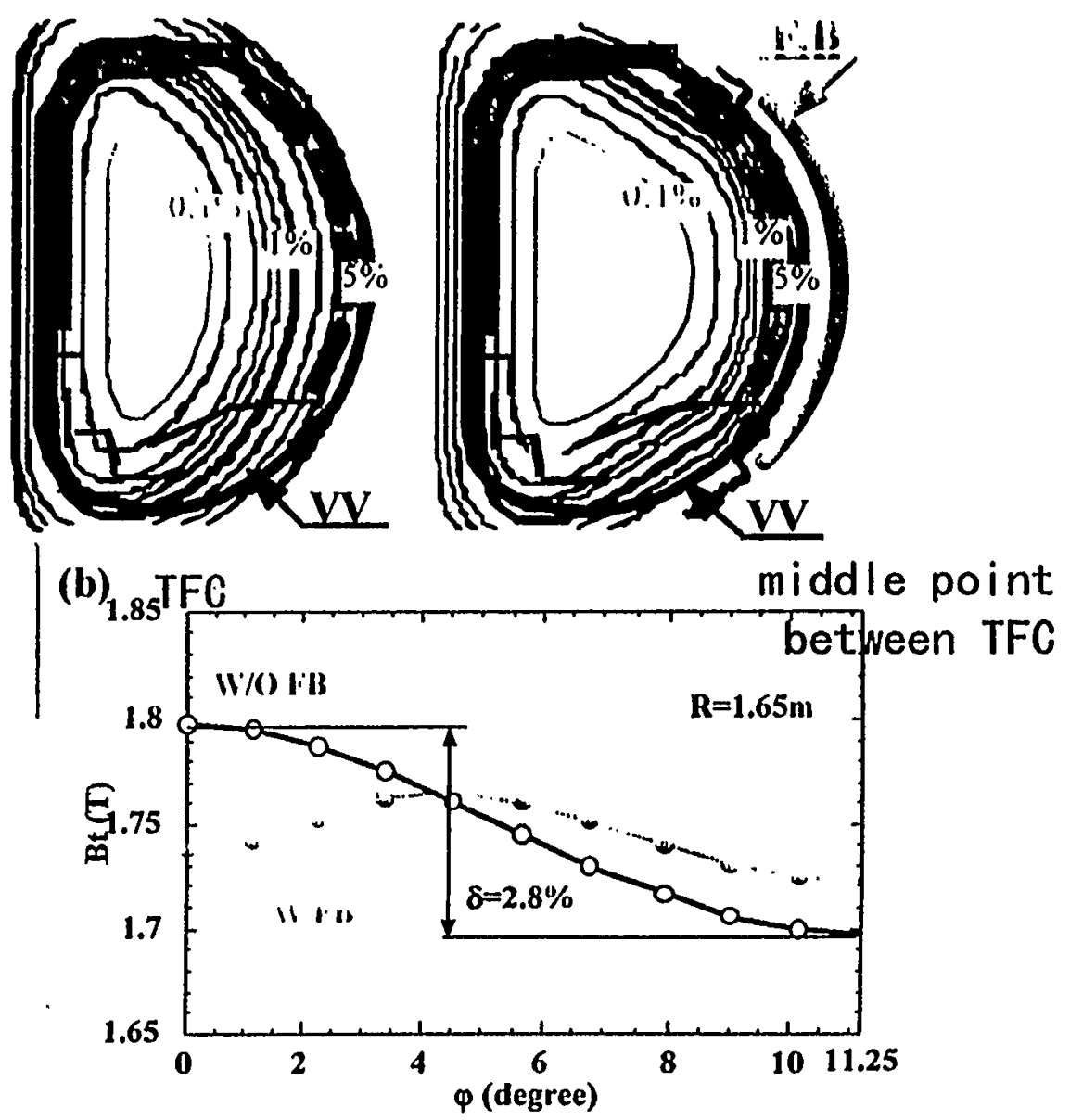

[3] M. Sato, et al., at ICFRM-8, Sendai, Japan, 1997, P2-C060.

[4] P. N. Yushmanov, Reviews of Plasma Physics 16, 1990, p117. 


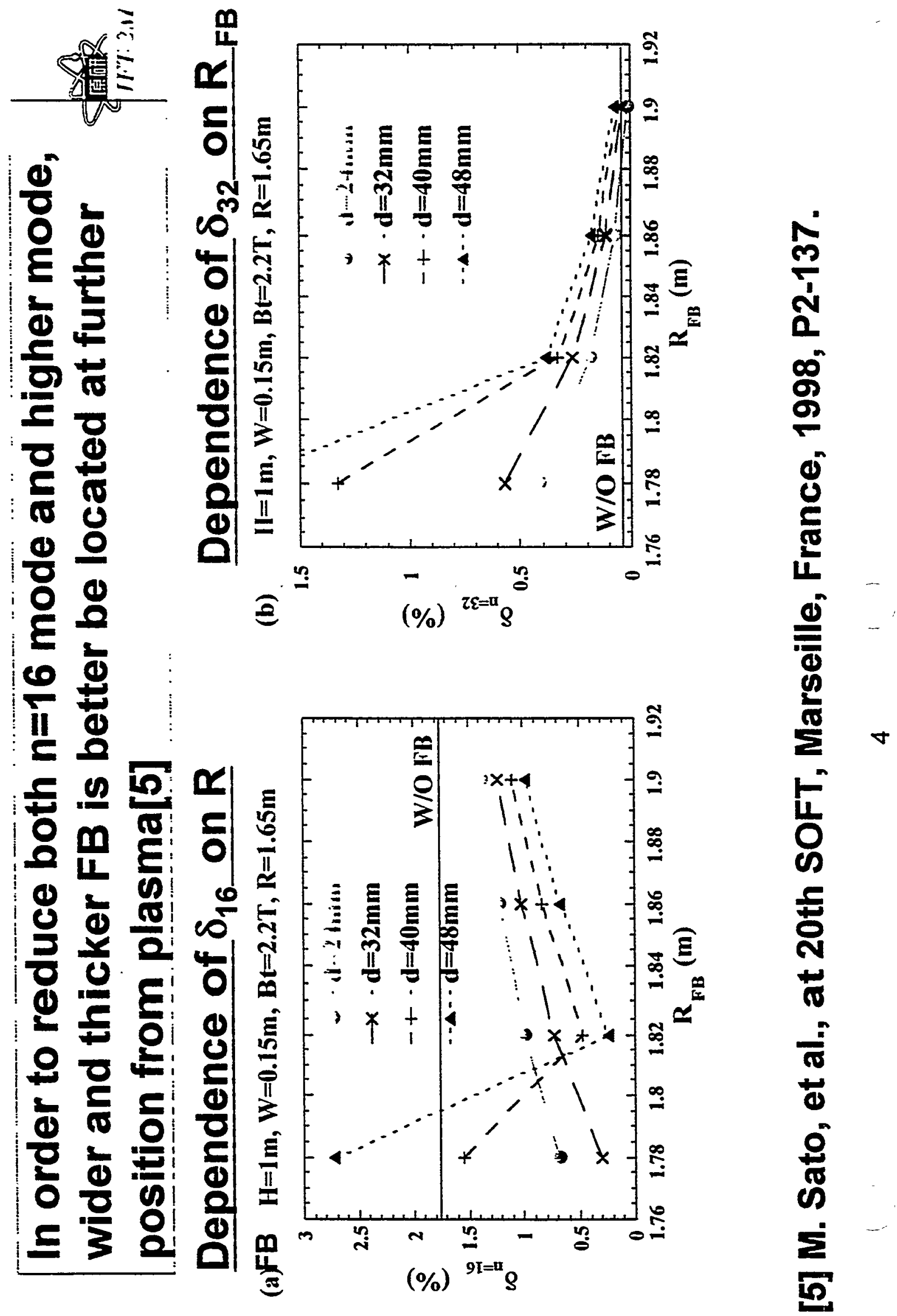




\section{Summary}

Compulational resulu!ts

- In order to reduce fast ion losses

$\rightarrow$ the ripple magnitude $(\delta)$ have to be reduced and its higher toroidal mode number have to be suppressed.

Guideline of the design for reduction of $\delta$ and higher mode (Number of toroidal field coil $=16$ )

$\rightarrow$ Wider and thicker FB is better to be located at further position from plasma.

Prosinest of Forritio slow!

- Even if a good new material will be used for a demo fusion reactor, the ferritic steel have a big possibility to use in the reactor.

$\leftarrow$ Because the ferritic steel will be used for the vacuum vessel and ripple reduction. 


\section{SUMMARY}

_ Japan/US collaboration will be extended to 2005 (HFIR Phase 4).

- Some HFIR Phase 3 capsules has been finished the irradiation and waiting for PIE.

- Several JAERI reactor capsules are planned for fracture mechanics research.

- AMTEX program is conducted to research the availability of RAF/M steels in plasma device. 
ǸG

\title{
Post Irradiation Properties of F82H plate, TIG and EB welds
}

\author{
IEA-RAFM Workshop \\ Petten, 1-2 October 1998
}

E.V. van Osch, M.G. Horsten. J. Rensman, M.I. de Vries ECN Nuclear Research. Petten

tel.+31-224.56.4650, fax 1883,

e-mail vanosch@ecn.nl

$30-\operatorname{sep}-98$

IEA.RAFM. 1.2Ot IYSX, Paten

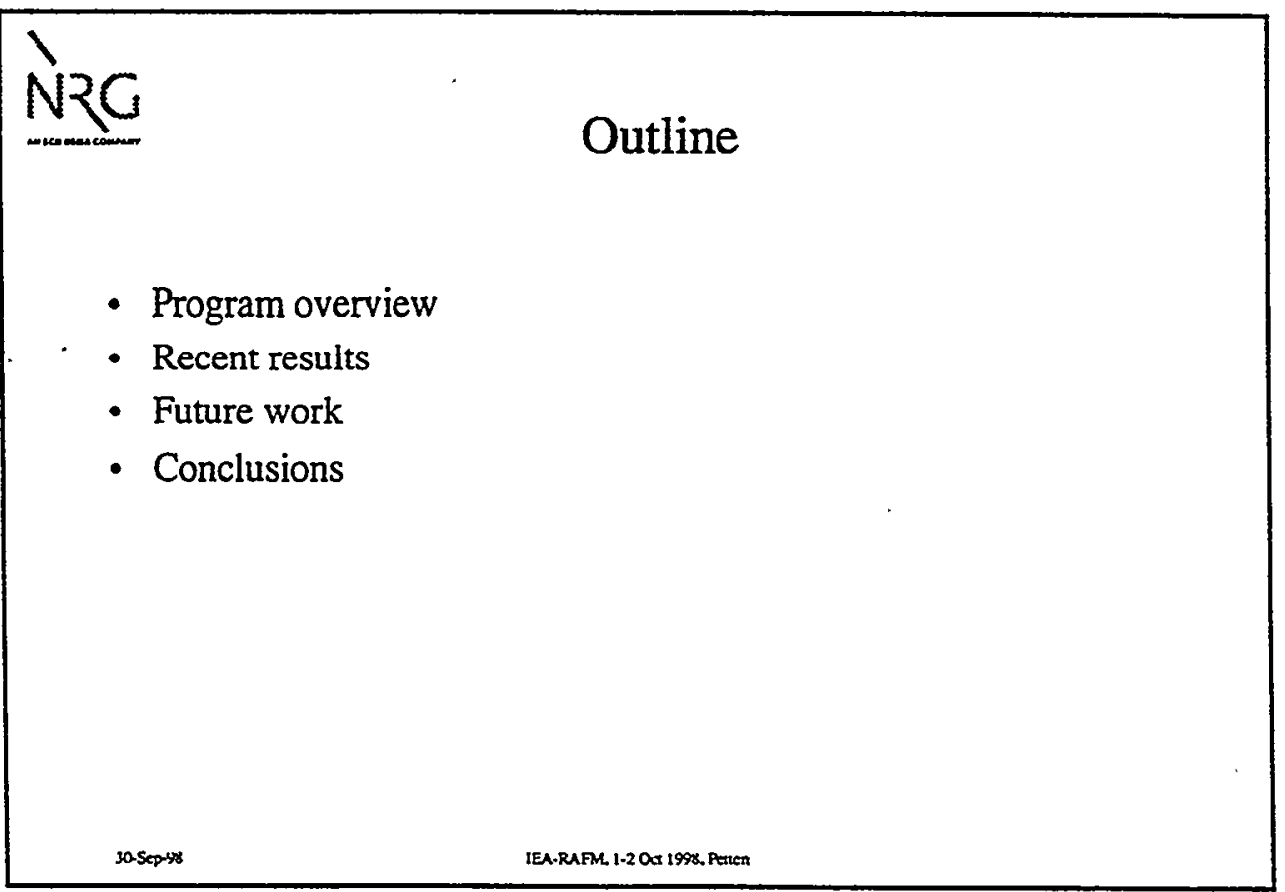


N2G

\section{Status F82H Irradiation Programme}

\begin{tabular}{|c|c|c|c|c|c|}
\hline capsule & $300^{\circ} \mathrm{C}$ & Type & Irradiation & testing & contents \\
\hline ILAS-4 & $2.5 \mathrm{dpa}$ & Tensile & Completed & Completed & Plate \\
\hline ILAS-6 & $2.5 \mathrm{dpa}$ & $\begin{array}{l}\text { Tensile. } \\
\text { LCF }\end{array}$ & Completed & In progress & $\begin{array}{l}\text { EB15, TIG15,EB15-CEA, } \\
\text { T91-EB15-CEA. }\end{array}$ \\
\hline ILAS-7 & 10 dpa & Tensile & In progress & - & EB15, TIG15. \\
\hline ILAS-8 & $2.5 \mathrm{dpa}$ & Tensile & Completed, cooling & - & $\begin{array}{l}\text { EB25, TIG25, EB25- } \\
\text { Schelde }\end{array}$ \\
\hline CHARIOT-2 & $2.5 \mathrm{dpa}$ & CT, KLST & Completed & Completed & Plate \\
\hline CHARIOT-4 & $2.5 \mathrm{dpa}$ & CT. KLST & Completed, cooling & - & T91-EB15-CEA, plate. \\
\hline CHARIOT-5 & $2.5 \mathrm{dpa}$ & CT, KLST & Completed, cooling & - & $\begin{array}{l}\text { TIG15, EB25, EB15, EB15- } \\
\text { CEA }\end{array}$ \\
\hline CHARIOT-6 & 10 dpa & CT. KLST & In progress & - & Plate. \\
\hline CHARIOT -7 & $10 \mathrm{dpa}$ & CT.KLST & In progress & - & TIG15. EB15. EB25. \\
\hline
\end{tabular}

Programme 1998: FTT and Impact tests of reference and 2.5 dpa irradiated, LCF and remainder tensile tests $2.5 \mathrm{dpa}$ irradiated.

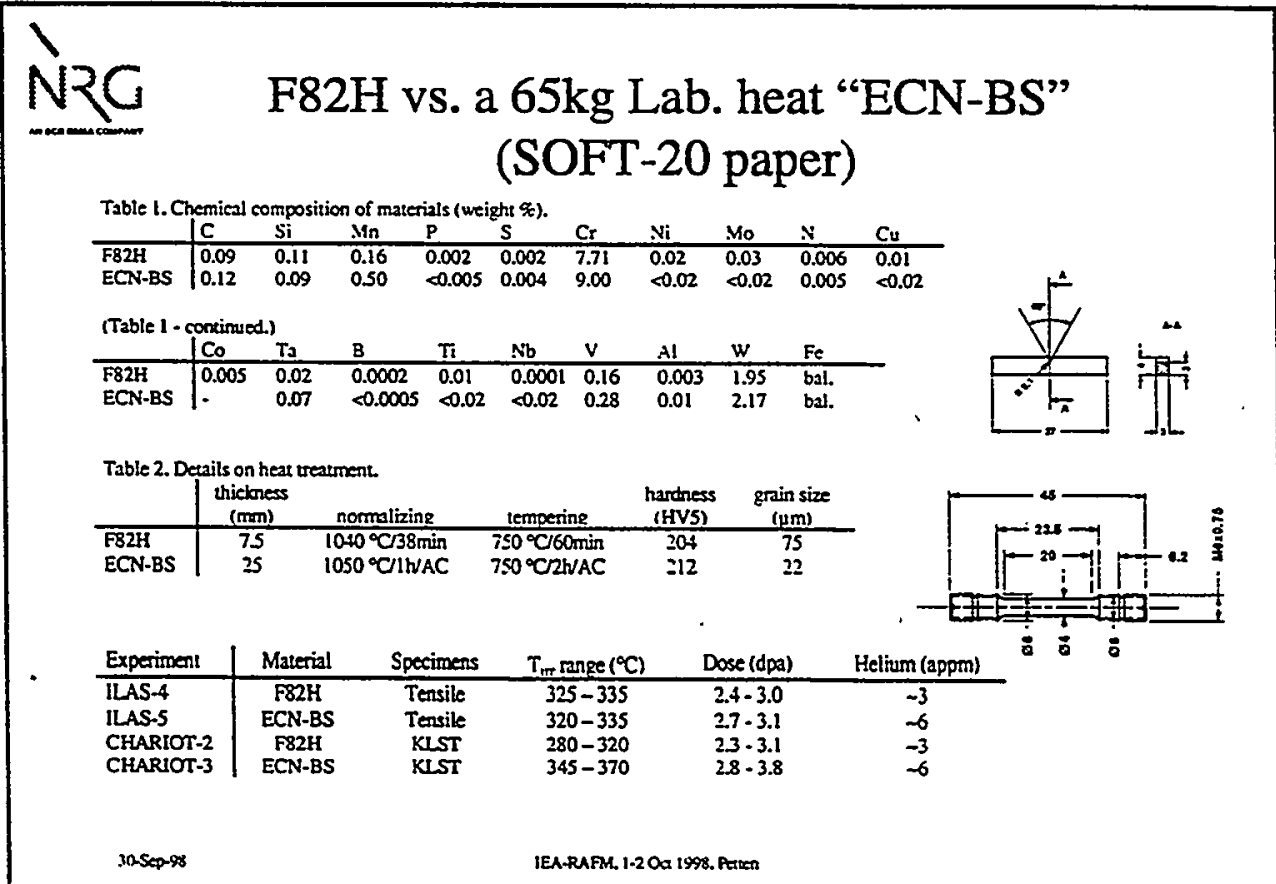



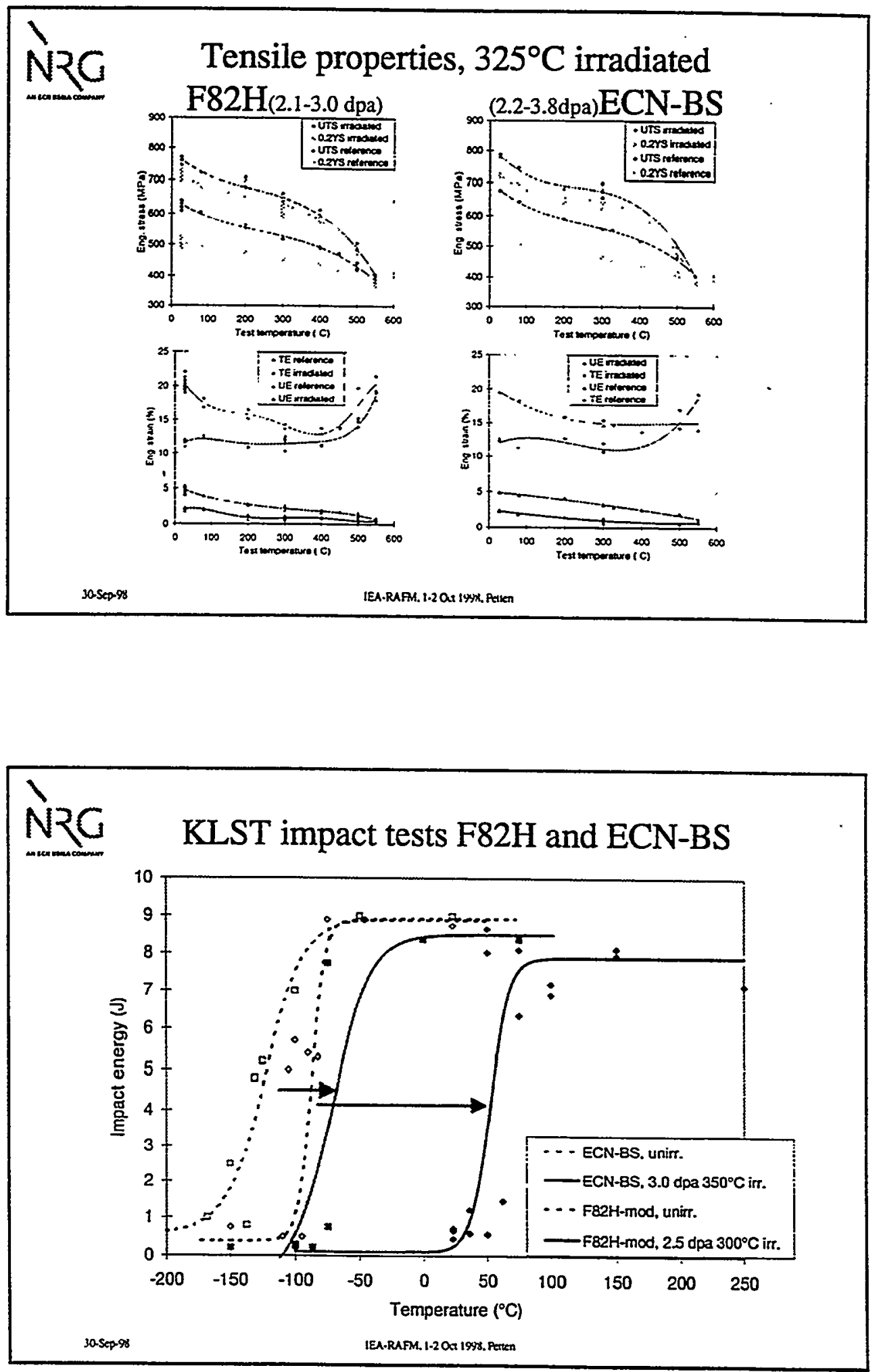


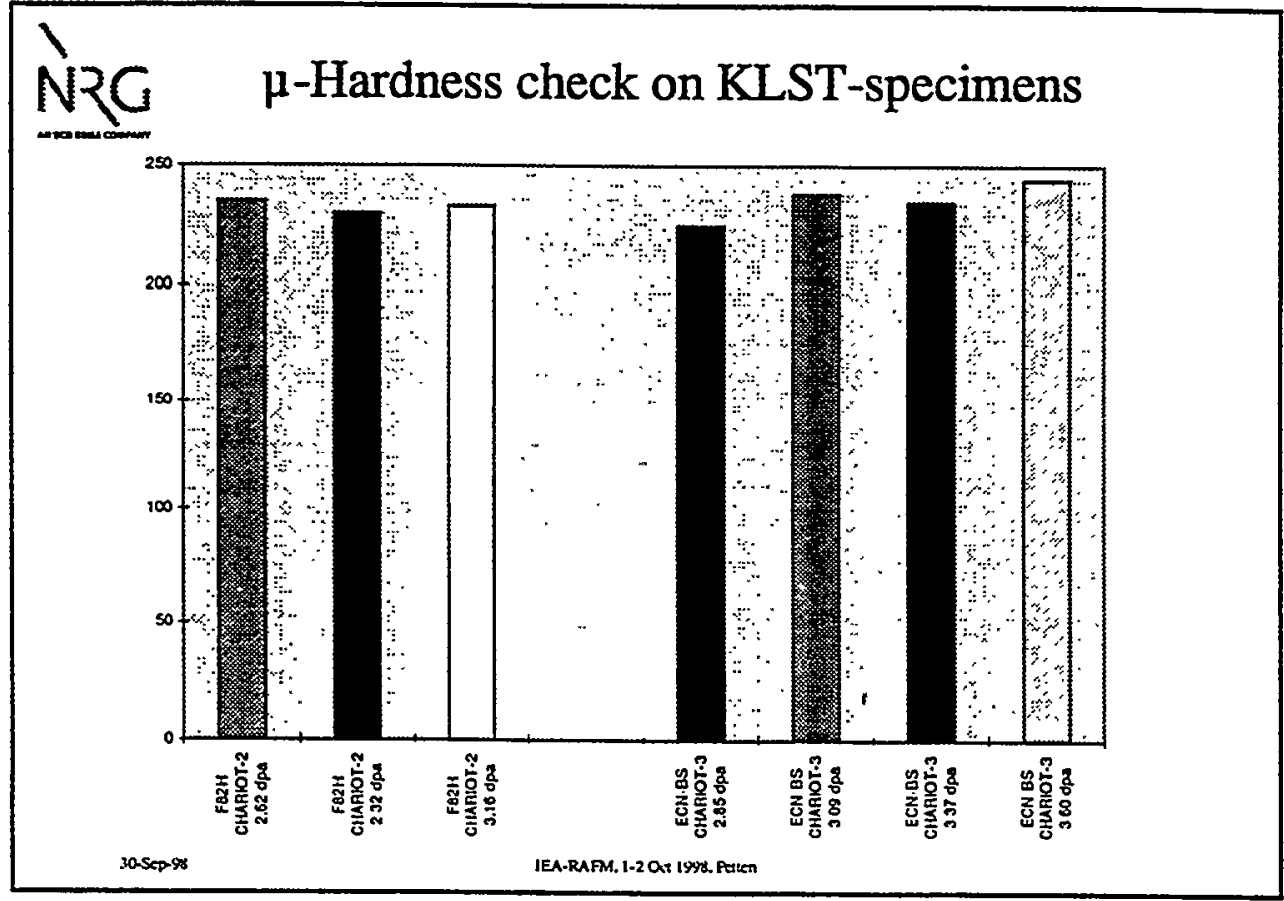

NRG Reference and PI-test Results on welded IEA-
F82H
- Reference tensile tests
- EB15, EB25, TIG15, TIG25, EB15-CEA, T91-EB 15-CEA, EB15-
- Schelde, EB25-Schelde.
- EB15: no significant difference between root and cap
- TIG15: difference: root 10-20 MPa lower YS and UTS than cap, ductility
slightly higher (RA 2-4\%, UE and TE 1 \%)
- EB better than TIG: higher strength, higher ductility.
2.5 dpa Post irradiation (ILAS-6) tensile tests
- EB15, TIG15, EB15-CEA, T91-EB15-CEA: in progress
- preliminary results: weldments (ILAS-6) show significant higher
irradiation hardening than 7.5 mm plate (ILAS-4)
3ascoses



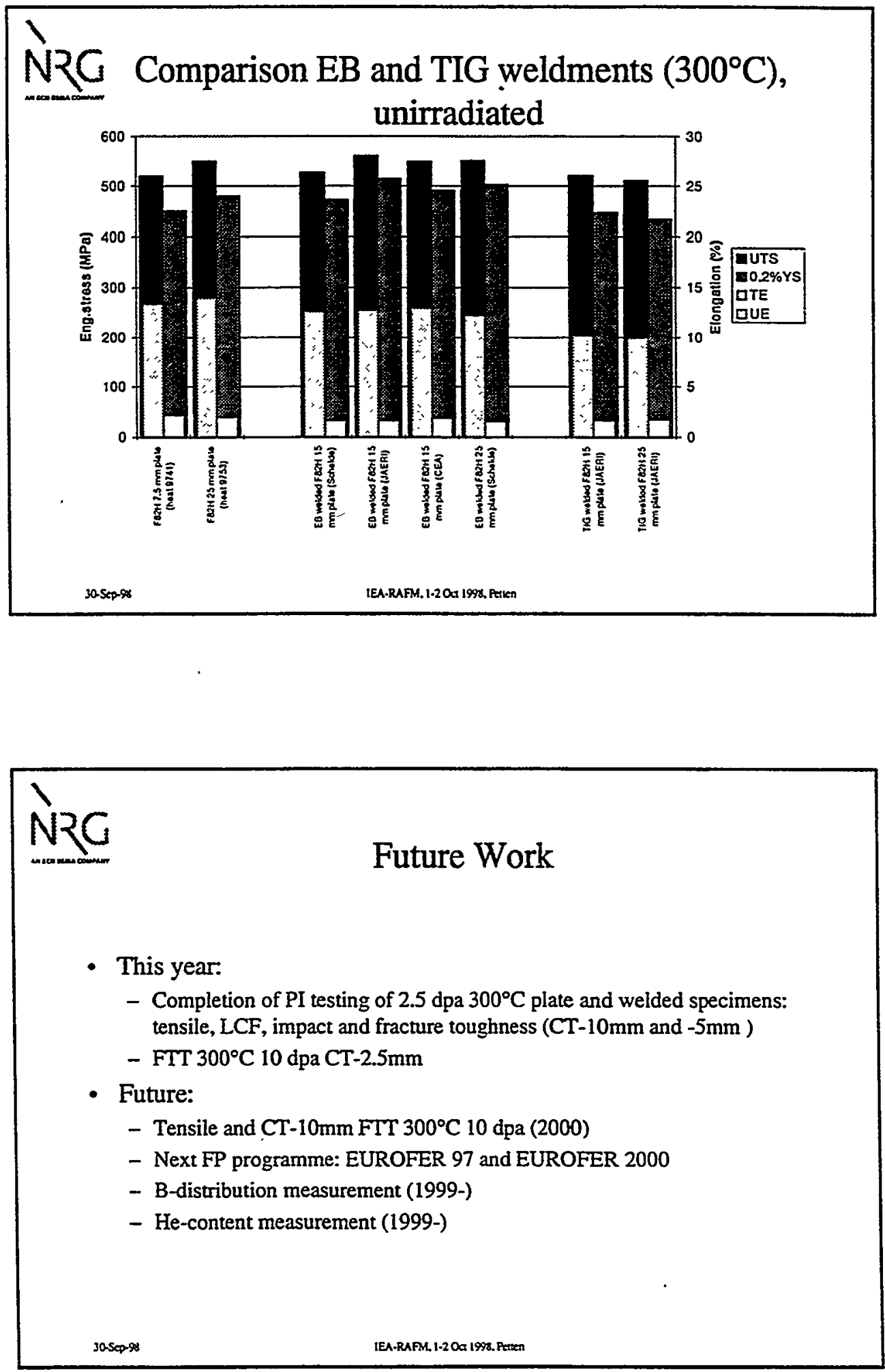


\section{Conclusions-1 (programmatic)}

- Testing of $2.5 \mathrm{dpa} 300^{\circ} \mathrm{C}$ irradiated $\mathrm{F} 82 \mathrm{H}$ specimens (plate and EB and TIG-weldments) will be completed by the end of 1998.

- FTT of $10 \mathrm{dpa} 300^{\circ} \mathrm{C}$ irradiated $2.5 \mathrm{~mm}$ CT's will be available by the end of this year.

- Bulk of $10 \mathrm{dpa} 300^{\circ} \mathrm{C}$ F82H data will be generated in 2000 .

- The next EU Programme (1999-2002) will focus on EUROFER

- Comparison of F82H and ECN-BS seem to show non-hardening embrittlement: F82H larger DBTT-shift, however same level of hardness.

- Preliminary results of ILAS-6 irradiation seem to show strong sensitivity of $\mathrm{F} 82 \mathrm{H}$ hardening to irradiation temperature: $40^{\circ}$ lower irr. temperature yields up to $100 \mathrm{MPa}$ more hardening! 


\section{Japanese University Program of RAFS R \& D for Fusion Reactor}

A. Kohyama and A. Kimura

Institute of Advanced Energy

Kyoto University

IEA RAF Workshop

Oct.1-2, 1998 ECN, Petten, Netherland 


\section{Blanket Structure of FFHR}

I.A.E, Kyoto University

\section{Radiation}

shield

$P_{n}=1.5 \mathrm{MW} / \mathrm{m}^{2}$

$\mathrm{Nd}=450 \mathrm{dpa} / 30 \mathrm{y}$
Self-cooled

$T$ breeder

$T B R>1.2$
5 order
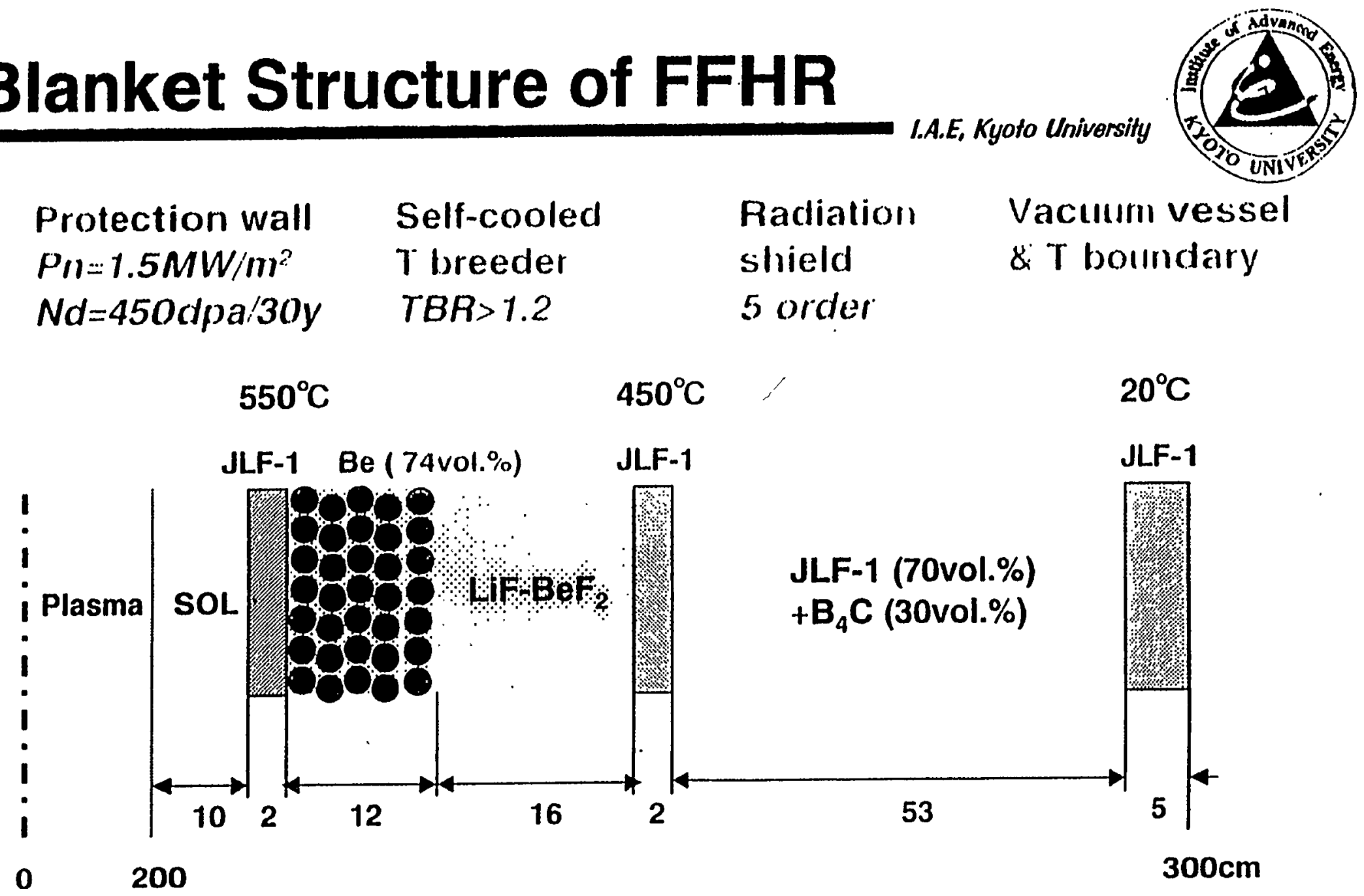


\section{Material Issues for FFHR}

OReduced Activation Ferritic Steels; JLF-1

1. High Temperature Strength $\left(\mathrm{T}>500^{\circ} \mathrm{C}\right)$

2. Compatibility with FLiBe

3. High resistance to Neutron Irradiation

4. Ferro-magnetism

\#1: Operation temperature of Flibe cooling system is recommended to be higher than $500^{\circ} \mathrm{C}$.

\#2: Corrosion tests of JLF-1 in FliBe is going on.

\#3: Microstructural examination of a RAF steel revealed that void swelling was still less than $1 \%$ after neutron irradiation to 200dpa.

\#4: Investigations on the effects of magnetism are strongly demanded.

fer. CREST (CRIPEI, U. Tokyo, Kyoto U. KHI) water coilinis: 


\section{Accomplishments in Monbusho/DOE Collaboration (1)}

1. Tensile data up to $60 \mathrm{dpa}$ at temperatures between 365 and $600^{\circ} \mathrm{C}$

1) hardening and softening

2) saturation of hardening at around $10 \mathrm{dpa}$

3) no saturation of softening at 30dpa

a) mechanism of irradiation hardening and softening (1-cluster)

b) superiority of martensitic single phase steels (9Cr-2W)

c) an addition of $\mathrm{Al}$ to improve high temperature strength

2. Swelling data and TEM examinations up to $70 \mathrm{dpa}$ at $420^{\circ} \mathrm{C}$

1) swelling peak temperature; $420^{\circ} \mathrm{C}$

2) incubation period (35-50dpa)

3) swelling rate (35-70dpa); 0.013\%/dpa

4) irradiation up to $200 \mathrm{dpa} ; 0.6 \%$

a) no large effects of 30wtppm of $B$ addition

b) incubation period appears to be correlated with the changes in the precipitation morphology

c) relation between irradiation hardening and swelling (Tirr dependence) 


\section{Accomplishments in}

\section{Monbusho/DOE Collaboration (3)}

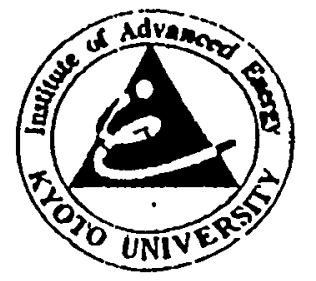

5. He related experiments

1) B addition; no enhancement of DBTT shift (FFTF/MOTA)

2) 480atppmHe implantation; no enhancement of irradiation hardening and embrittlement

3) $\mathrm{Ni}$ addition; large irradiation hardening below $170^{\circ} \mathrm{C}$

a) He suppress the growth of $V$ and I-cluster

b) He stabilized thermally $V$ and I-clster

c) He-V or He-I cluster does not influence the irradiation hardening

d) Ni-Iclusters has strong potential of irradiation hardening

e) controlling irradiation temperature is strongly demanded for this type of experiments 


\section{Current Irradiation Research in JUPITER and Domestic University Program (1)}

1. HFIR-MFE-RB-11J,12J;

- Thermal Neutron Shielded, 300 and $500^{\circ} \mathrm{C}$;

Materials; JLF-1, mod.-JLF-1, F82H

Specimen type; PT, CVN1.5, SS-Tensile, DCT, TEM

1) low temperature irradiation effects; toughness, creep

(also in ATR; 200 and $300^{\circ} \mathrm{C}$ )

2) fracture toughness measurements

3) effects of $\mathrm{Ni}$ addition

2. HFIF-MAE-HBB.14J;

- Unshielded, 300, 500, 800.0

Materials; JLF-1, mod.JLF-1, F82H

Specimen type; PT, CVN1.5, SS-Tensile, DCT, TEM

1) transmutation effects

2) ODS steel $\left(800^{\circ} \mathrm{C}\right)$ 


\section{Current Irradiation Research in JUPITER and Domestic University Program(2)}

3 HFIR-MFE-RB-13J;

- Thermal Neutron Shielded,

"Varying 7 emperature; $\left.200 / 3500^{\prime \prime} \mathrm{C}\left(350^{\circ} \mathrm{C}()^{\prime}\right), 30(1) / \mathrm{b00}\right) 0^{\circ} \mathrm{C}\left(500^{\circ} \mathrm{C}\right)$

Materials; JLF-1, mod.-JLF-1, F82H

Specimen type; CVN1.5, SS-Tensile, TEM

1) factors controlling irradiation hardening $\left(200 / 350^{\circ} \mathrm{C}\right)$

V-cluster, I-cluster, precipitation behavior

2) stability of defect clusters $\left(300 / 500^{\circ} \mathrm{C}\right)$

4 JOYO (Japarn)-Marico;

$\cdot 370-600^{\circ} \mathrm{C}, 5-20 \mathrm{dpa}$

Materials; JLF-1, mod.JLF-1, F82H

Specimen type; CVN1.5, SS-Tensile, SS-Fatigue, TEM

1) post-irradiation fatigue tests, specimen size dependence

2) . DBTT/ Fatigue/ Hv/ Swelling correlation 


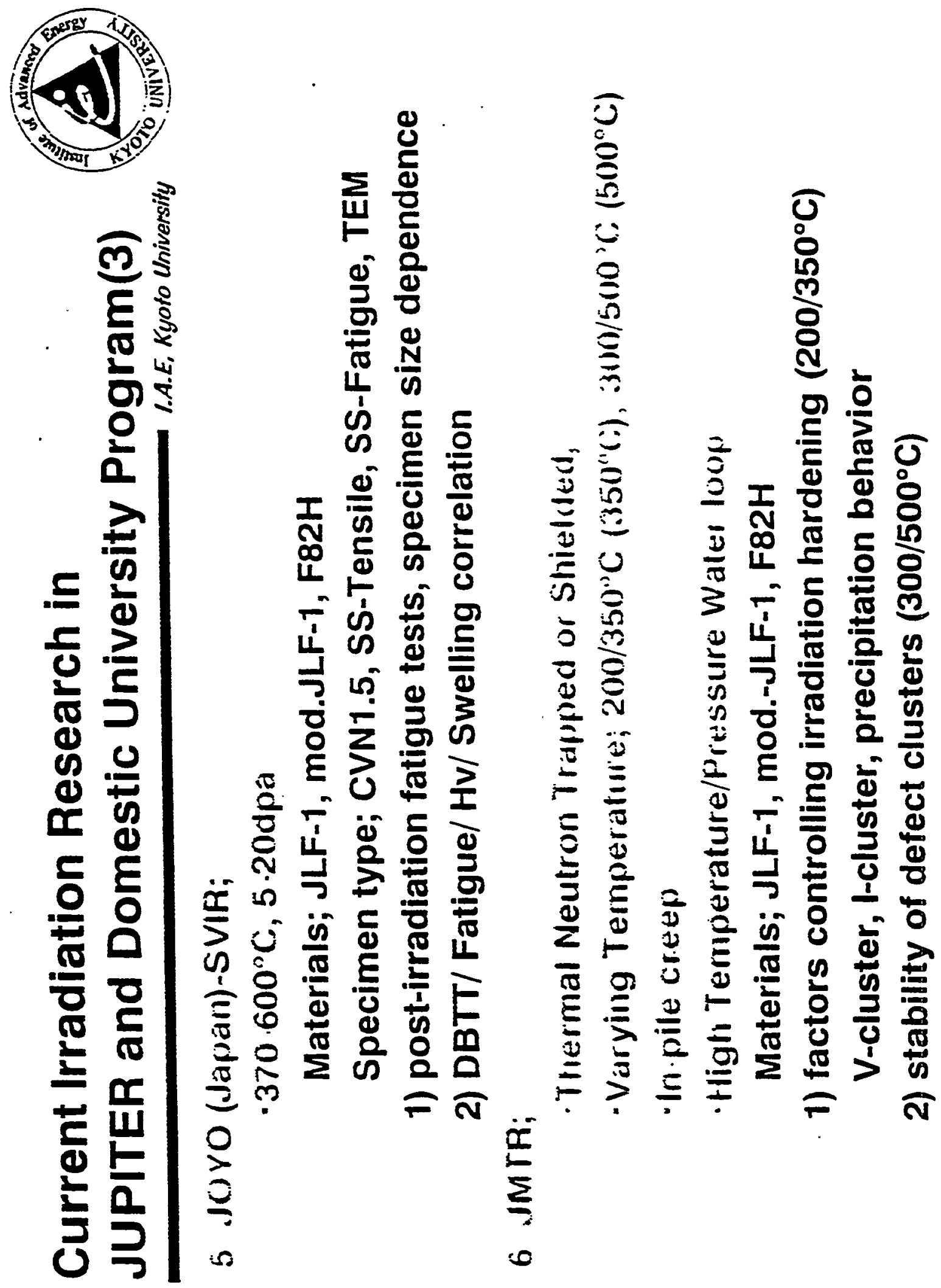


JU:

Jor FOSIOK HATERIALs HFIR

In-situ electrical resistivity

High dpa Irrad. (with thermal shield) Vary. Temp. Exp. High dpa Irrad. (no-thermal shleld) High dpa Irrad. (with thermal shield)

PTP Mater. Irrad. In-situ thermal conductivity

\section{HFBR}

In-situ electrical resistivity ATR

Mater. Irrad.

Precisely Temperature Controlling Experiment
The Monbusho/US-DOE Collaboration on fusion Materials

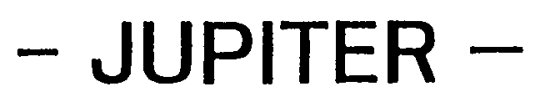

Ver.15:9/1/98

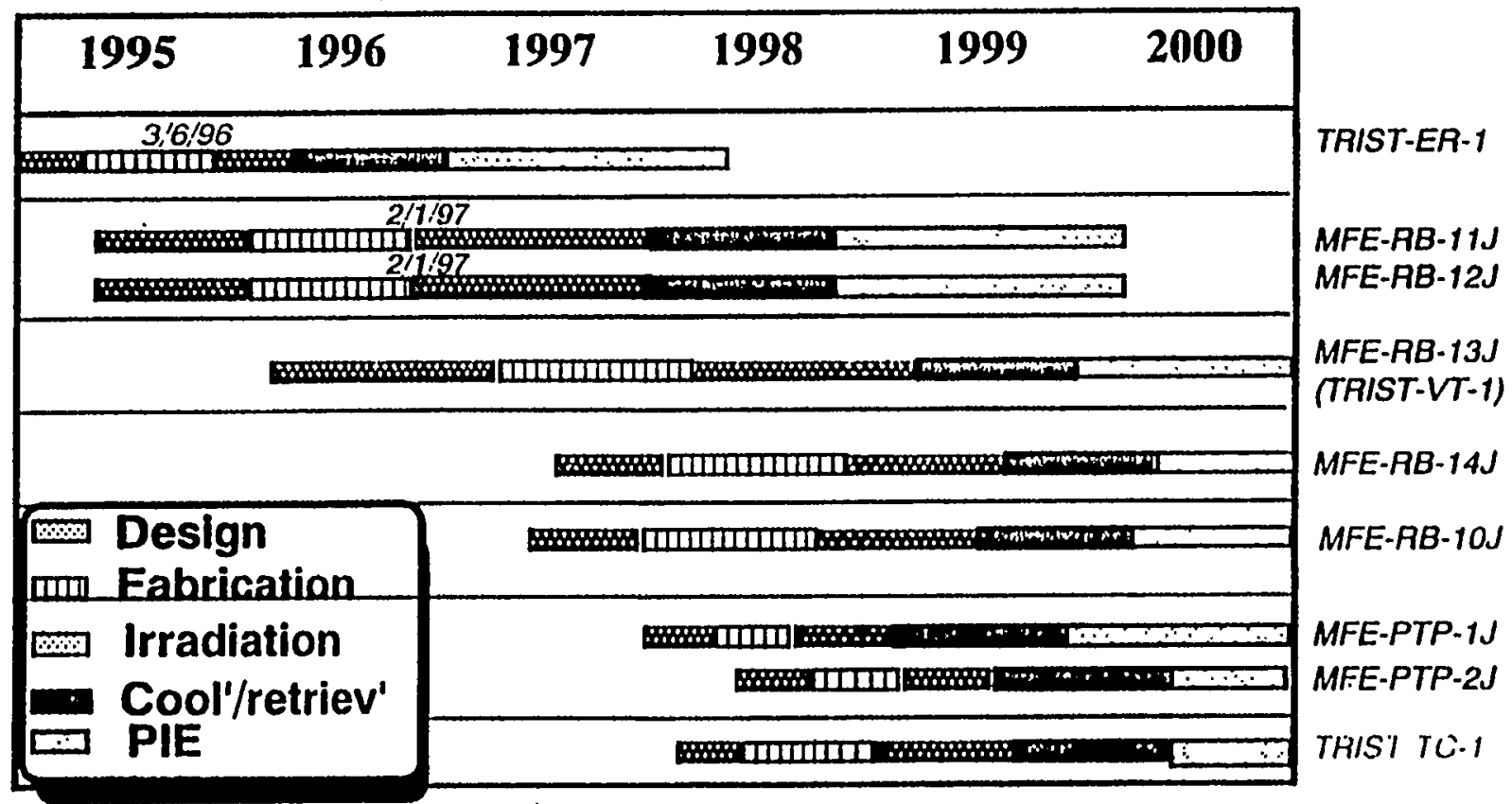

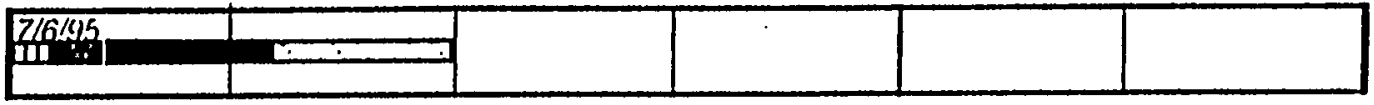

ISEC-3

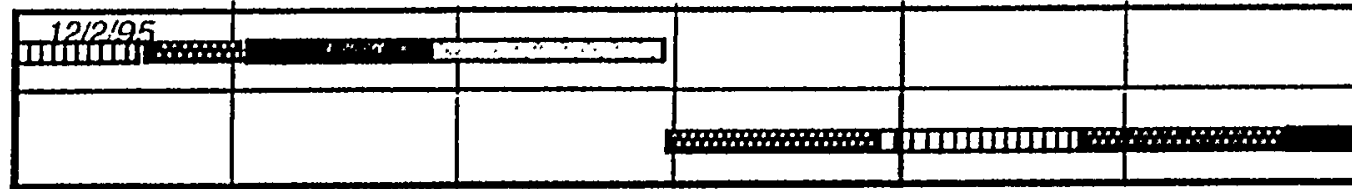

P3-A1 ATRIITV-1 


\section{Future Planning of Irradiation Research (1)}

1. Continuous effort to builı irradiation data base

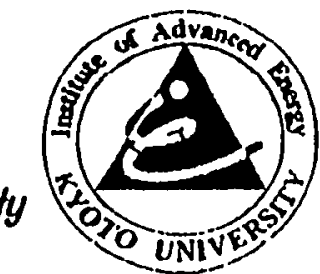

1) temperature dependence

2) dose dependence

3) irradiation effects on material system component

- structure gradient, temperature and stress gradient

- compatibility (including $\mathrm{H}$ and $\mathrm{He}$ )

2. Modeling material performance under inradiation

1) understanding physics of radiation effects

2) bridging over the gaps between basic research and engineering

- International collaboration is strongly demanded JUPITER-II (2001-2006) -planning stage- 


\section{Future Planning of Irradiation Research (2)}

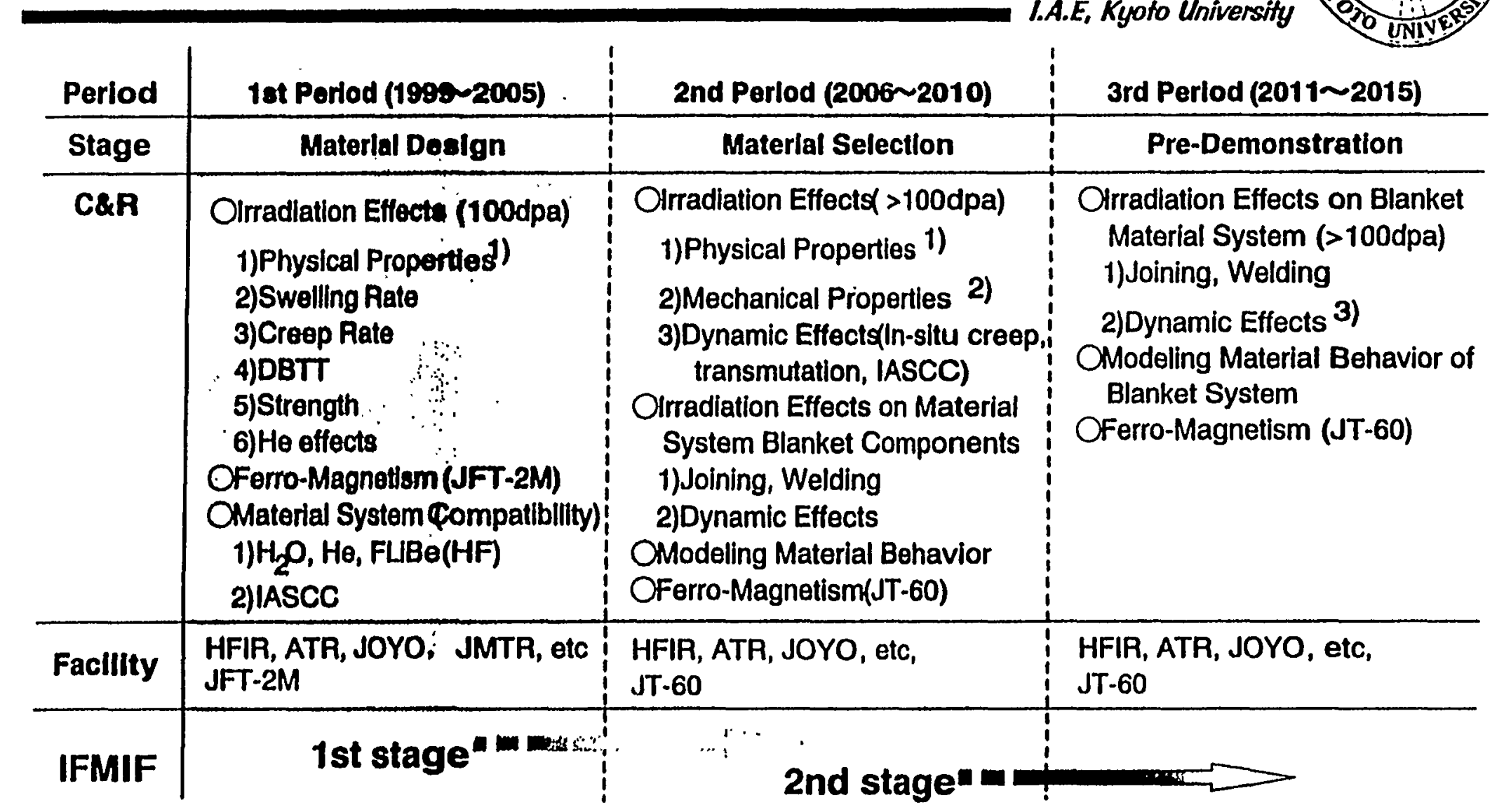

1)Thermal conductivity. Thermal expansion, Heat capacity, Magnetic/electric properties 2)elasticity, tensile, impact, fracture toughness, fatigue, dimensional stability

3)IASCC, creep, fatigue, creep/fatigue 


\section{Application of Multiple Beams to Energy Materials Research}

Instifute' of Advarketed Energy

Kyoto linwersity
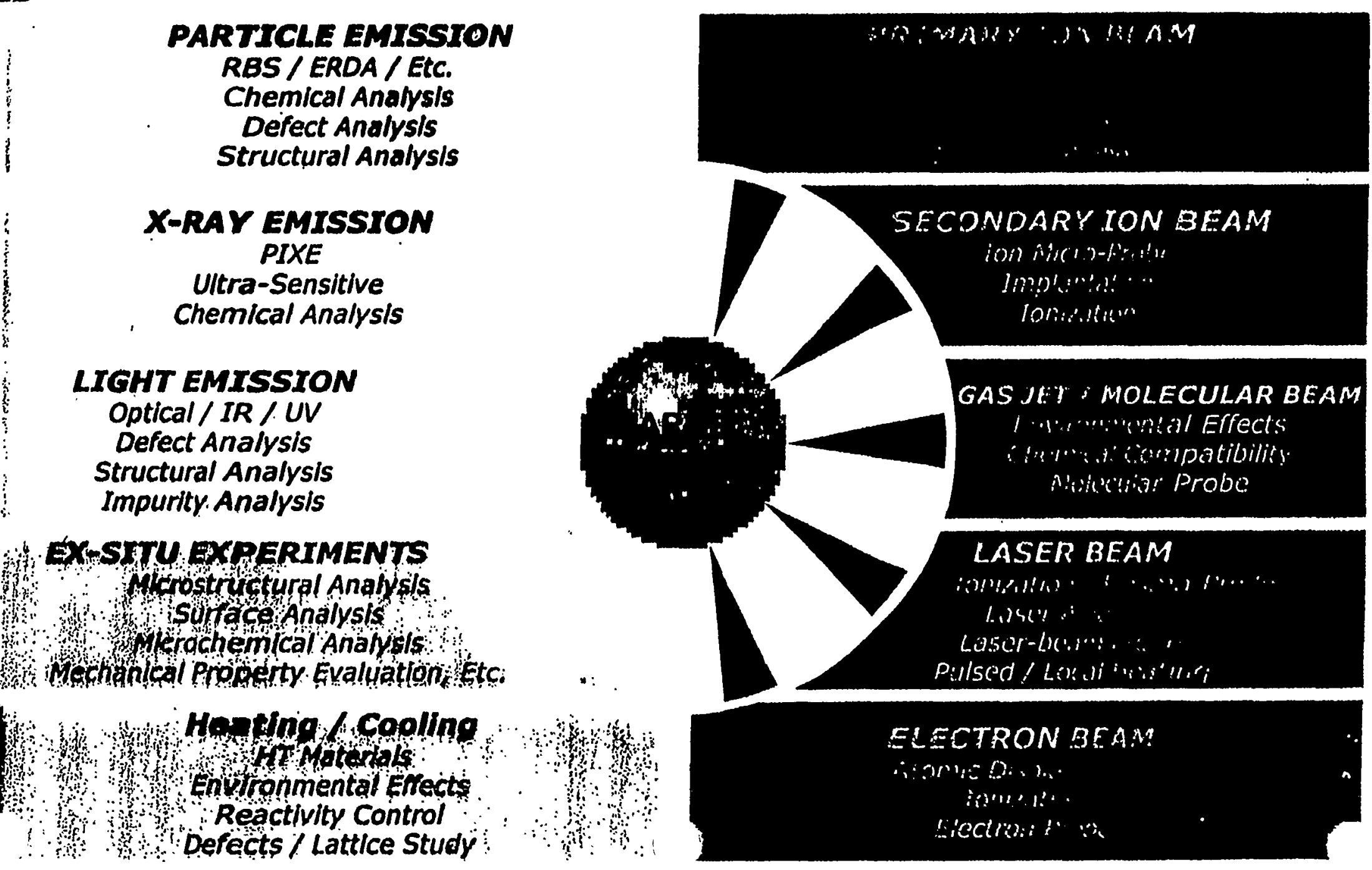
Specification of DUET Dual-Beam Facility at IAE Kyoto Univ.

Institufe of Advanced Energy

Kyoto llniversity

\begin{tabular}{|c|c|c|}
\hline & $\begin{array}{c}\text { DUET } \\
\text { Kyoto University }\end{array}$ & $\begin{array}{c}\text { HIT } \\
\text { University of Tokyo }\end{array}$ \\
\hline Location & Uji, Kyoto, Japan & Tokai, Ibaragi, Japan \\
\hline Year built & 1999 & 1983 \\
\hline Primary accelerator & 1.7MV Tandetron & 1MV Tandetron \\
\hline Secondary accelerator & 1MV Singletron & 3.75MV Van de Graaff \\
\hline Primary beam & $\begin{array}{c}\text { 6.8MeV Ni } 5 \mu \mathrm{A} \\
6.8 \mathrm{MeV} \text { Si } 40 \mu \mathrm{A} \\
5.1 \mathrm{MeV} \mathrm{He} 2 \mu \mathrm{A} \\
\text { Etc. }\end{array}$ & $4 \mathrm{MeV} \mathrm{Ni} 1.5 \mu \mathrm{A}$ \\
\hline Secondary beam & $\begin{array}{c}1 \mathrm{MeV} \mathrm{H} 75 \mu \mathrm{A} \\
1 \mathrm{MeV} \mathrm{He} 15 \mu \mathrm{A} \\
\end{array}$ & $\begin{array}{r}1 \mathrm{MeV} H 50 \mu \mathrm{A} \\
1 \mathrm{MeV} \mathrm{He} 10 \mu \mathrm{A} \\
\end{array}$ \\
\hline Target temperature & $4 \sim 2073 K$ & $300 \sim 973 K$ \\
\hline Target environment & Vacuum, $\mathrm{He}, \mathrm{O}_{2}$, etc. & Vacuum \\
\hline $\begin{array}{l}\text { In-beam experiments } \\
\text { (*Planned for FY1999) }\end{array}$ & $\begin{array}{c}\text { RBS, ERDA, PIXE*, } \\
\text { Optical/IR /UV } \\
\text { spectrometry*, } \\
\text { Photoluminescence*, } \\
\text { Laser ablation* } \\
\end{array}$ & $\begin{array}{l}\text { RBS, Optical/IR/UV } \\
\text { spectrometry }\end{array}$ \\
\hline
\end{tabular}


$\|\mid k\|\|,\|_{2} h \|$

(1) $(1,1), 1,2$ (U), s

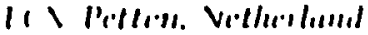

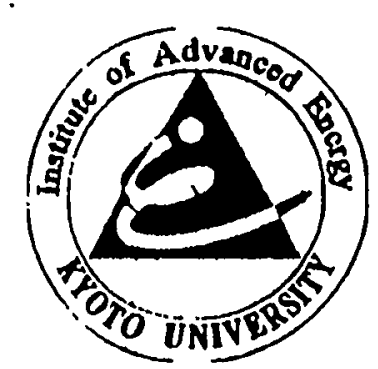

\section{Ferritic Isotopic Tailoring (FIST) Experiment}

- present status and future plan -

Institute of Advanced Energy

Kyoto University

S. Ohnuki, Y. Kohno+, A. Kohyama+t

K. Shiba* and A. Hishinuma*

Hokkaido University, +: Univ. of Tokyo

+t: Kyoto University, *: JAERI

collaborators:

Kimura, Y. Katoh, T. Shibayama,

H. Takahashi, T. Muroga, A. Nishimura,

D.S. Gelles and M.L. Hamilton

Kohyama@iae.kyoto-u.ac.jp

http://infosrv.iae.kyoto-u.ac.jp 


\section{Background and Objective}

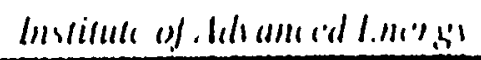

The Past:

kivorn l'nirerevill

- isotope tailored F82H disks were irradiated in HFIR

- these discs were provided to JUPITER

- FIST experiment was proposed at JUPITER WS (8/21-23/97)

Present Status: (FIST-I)

- F82H discs were shipped to PNNL 5/98

- SP tests finished at PNNL (by S. Ohnuki, M.L. Hamilton ) 7/98

- TEM inspection method was established and TEM inspection done (by S. Ohnuki, D.S. Gelles) 7/98

- Gas Analysis finished 8/98

Objectiv'c:

- Io simulate thefusion environment effects of hydrogen and helium on ferritic alloys

FIST-1 is the first step for go/no go decision of FIST-2 and beyond 


\section{Outline of the FIST Experiment}

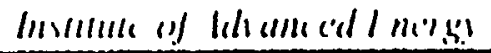

Use Fe isotope tailoring to produce $\mathrm{H}$ and $\mathrm{He}$ in HFIR

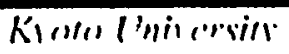
$\mathrm{Fe}(54)$ at $\$ 20 \mathrm{~K} / \mathrm{gm}$ and $\mathrm{Fe}(56)$ at $\$ 2 \mathrm{~K} / \mathrm{gm}$ for comparison with natural $\mathrm{Fe}$ Alloy classes of interest:

JLF-1, (ODS ferritic, Fe-9Cr), F82H (supplied from JAERI)

Issues:

Cost (dependent on smallest melt size possible),

Cost of materials: Japan/(US?) Alloy production: Japan

Irradiation in HFIR

PIE/ gas analysis: PNNL or IMR-Tohoku

Schedule (probably for FY2000)

A Scope

(1) Isotope-tailored specimen:

JLF1 + Fe54 or Fe56(B10), ODS ferrite + Fe54 or Fe56(B10)

Model $9 \mathrm{Cr}+\mathrm{Fe} 54$ or Fe56(B10)

(2) Specimen fabrication

Isotopes: ORNL Fabrication: Japan

(3) Irradlation

HFIR (RB or PTP)

$300,500 \mathrm{C}(+400,600 \mathrm{C}) \quad 5 \mathrm{dpa}, 10 \mathrm{dpa}$

(4) Specimen type \& mass

TEM specimen (TEM \& shear punch/Disk Bend test) : $\quad 0.01 \mathrm{gm}$

TN: tensile specimen(SS-J):

Charpy(1.5mm):

$0.10 \mathrm{gm}$

$0.35 \mathrm{gm}$ 


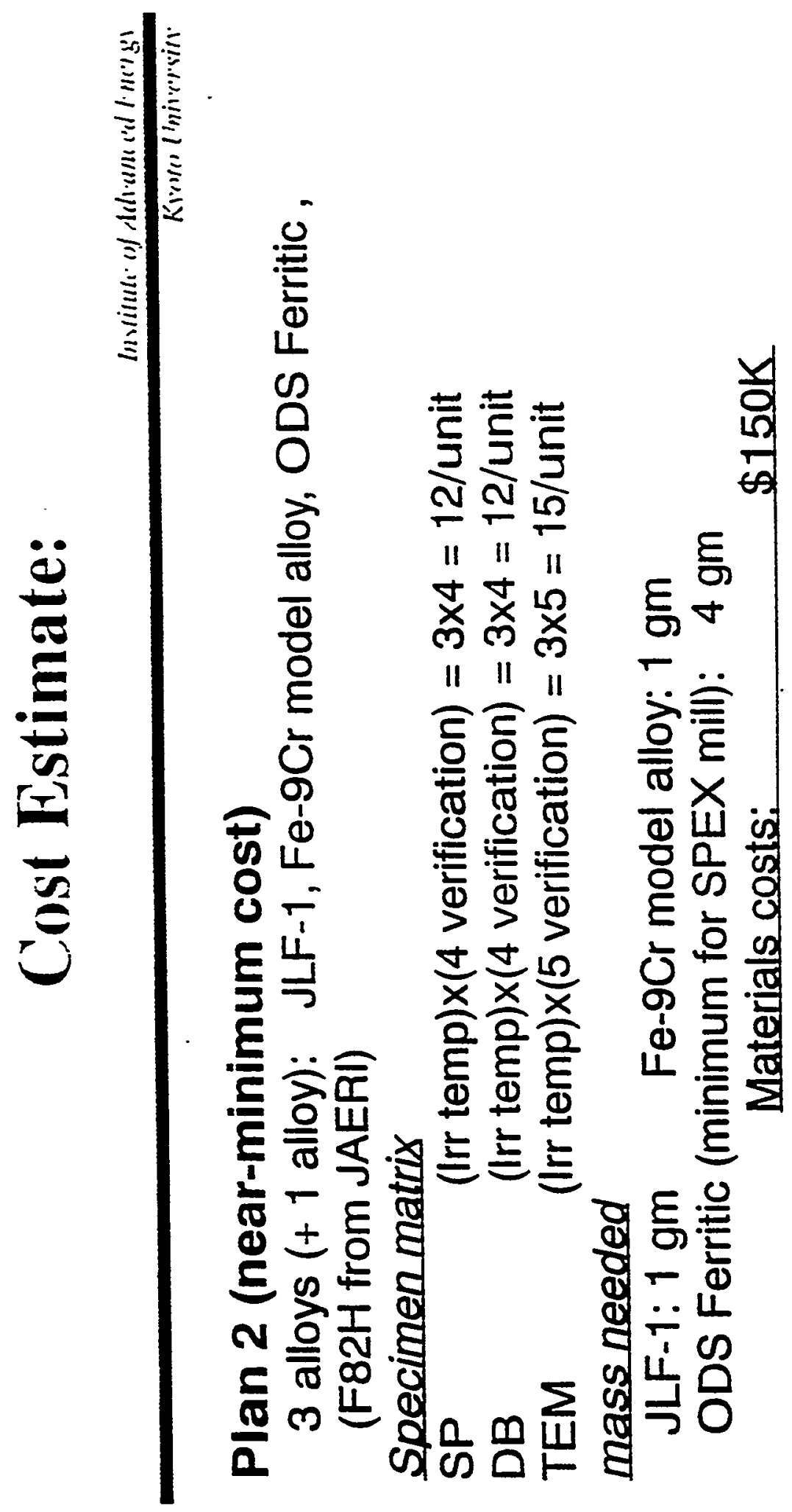




\section{Cost Estimate - 1-}

Invilute ol dell am od l-nerg!

Kiomro linirersiry.

$\therefore$ alloys: JLF.1, Fe.9C, model alloy, OOS Ferritic (F82H tu(m, JAERl)

Specimen matrix

Charpy 6 specimens/unit

Tensile (@RT, @Irr temp)x(2 strain rates)x(2 verification $)+(2$ extra $)=2 \times 2 \times 2+2=$

10/unit

SP

DB

(@RT, @Irr temp)x(4 verification $)+(2$ extra $)=2 \times 4+2=10 /$ unit

TEM

(@RT, @Irr temp)x(4 verification) + (2 extra) $=2 \times 4+2=6 /$ unit

mass needed

JLF-1 including toughness $\quad 20 \mathrm{gm}$

Fe-9Cr model alloy $8 \mathrm{gm}$

ODS Ferritic $8 \mathrm{gm}$

Total mass: $20+8+8=36 \mathrm{gm}$ (Assumed yield rate of $70 \%: 50 \mathrm{gm}$ needed)

Materials costs:

Fe54

Fe56

$\$ 20 \mathrm{~K} / \mathrm{gm} \times 50 \mathrm{gm}=\$ 1 \mathrm{M}(120 \mathrm{MY})$

$\$ 1 \mathrm{~K} / \mathrm{gm} \times 50 \mathrm{gm}=\$ 50 \mathrm{~K}(6 \mathrm{MH}) \quad$ Natural Fe: negligible

Total \$1.05M 


\section{Cost Estimate -2-}

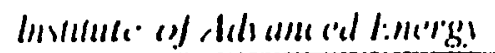

Kinno l'minersin

Alloy fabrication cost:

Nippon Steel Corporation estimate $\$ 50 K-\$ 100 K$

PIE cost: negligibly small compared to isotope cost

+ Gas analysis $\mathrm{H}, \mathrm{He} \sim \$ 2 \mathrm{~K} /$ sample $\$ 20 \mathrm{~K}$ for 10 samples

+ Charpy

Tests at Oarai

+ DB

Tests at Oarai

+ Tensile (total 30 specimens) $\rightarrow$ MATRON 1 or 2 weeks $\$ 5 \mathrm{~K}-\$ 10 \mathrm{~K}$

$+\mathbf{S P}$

Tests at Oarai or PNNL(unknown)

+ TEM total 32 specimens

Total PIE cost

less than \$1K (?)

$\sim$ \$200K

TotalExperimental Cost:

$\sim \$ 1.25 \mathrm{M}$ 


\section{F82H IAE}

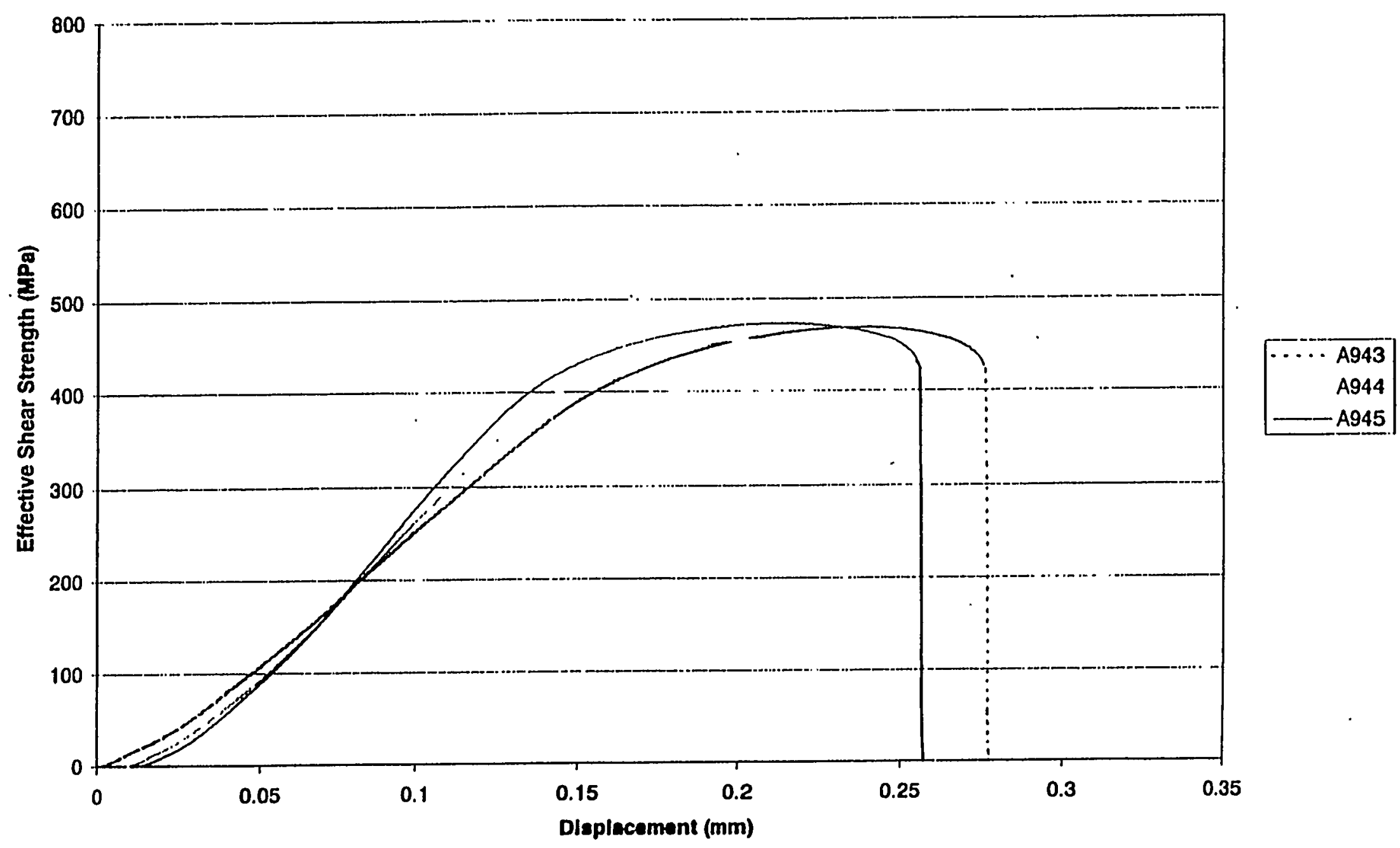




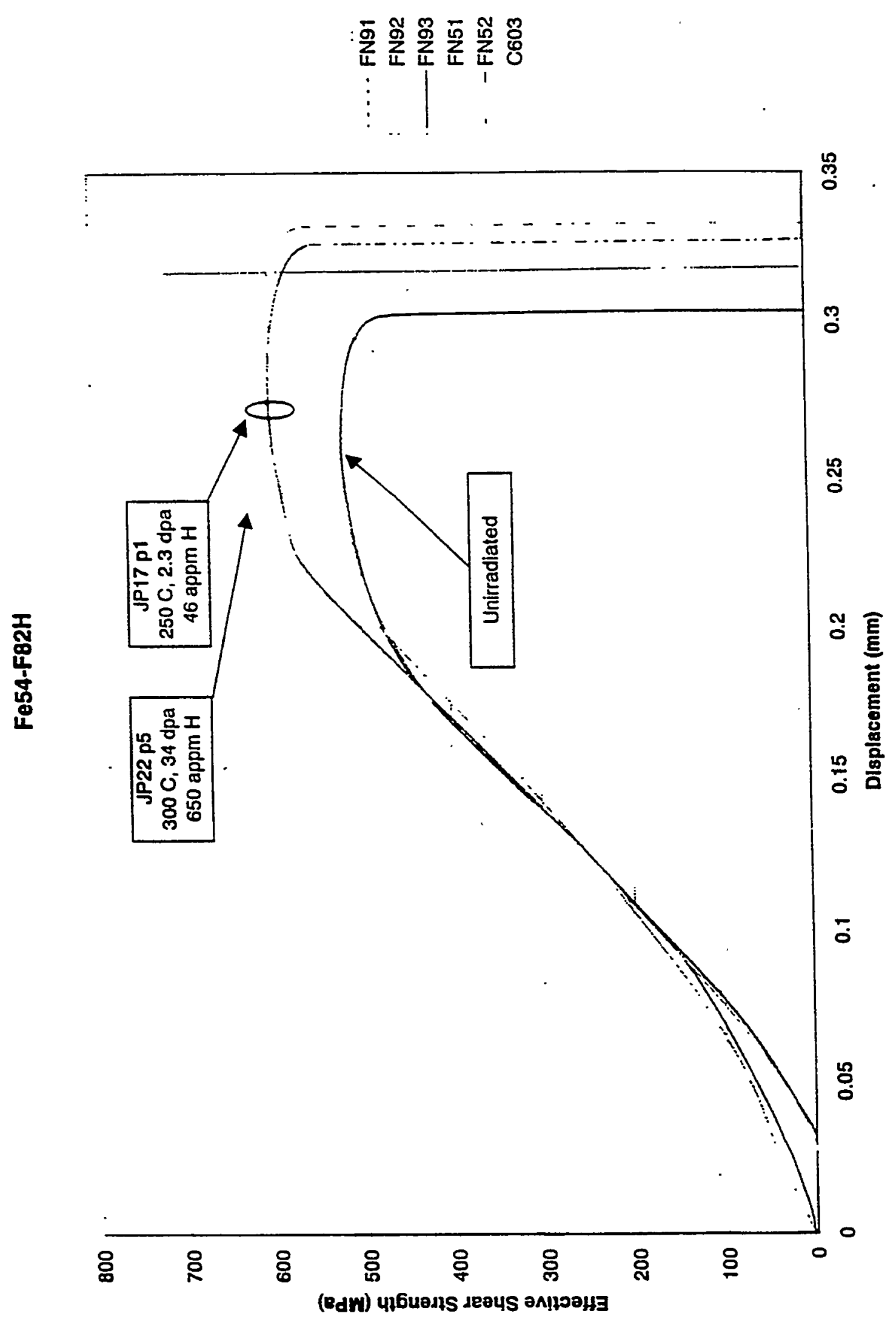




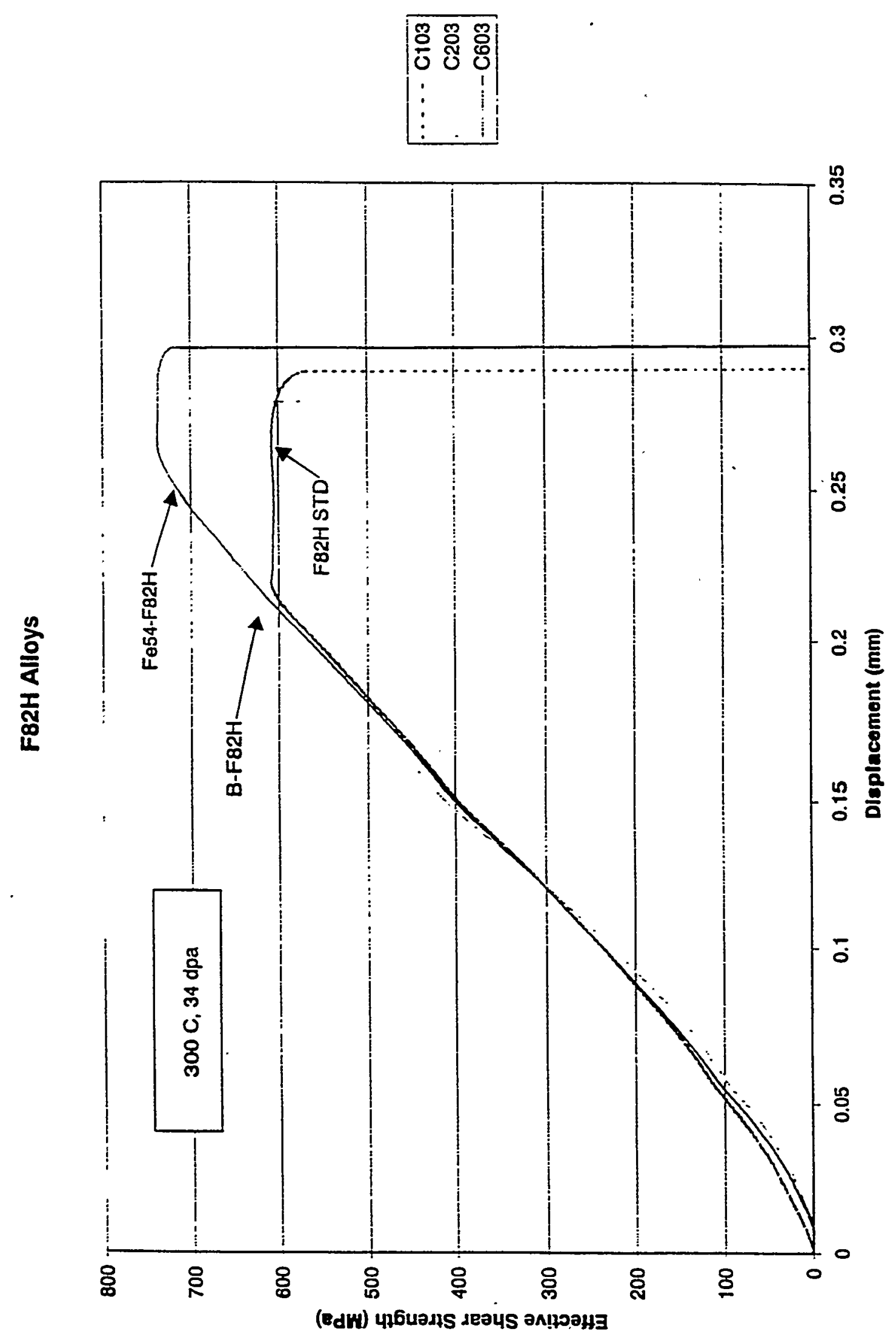




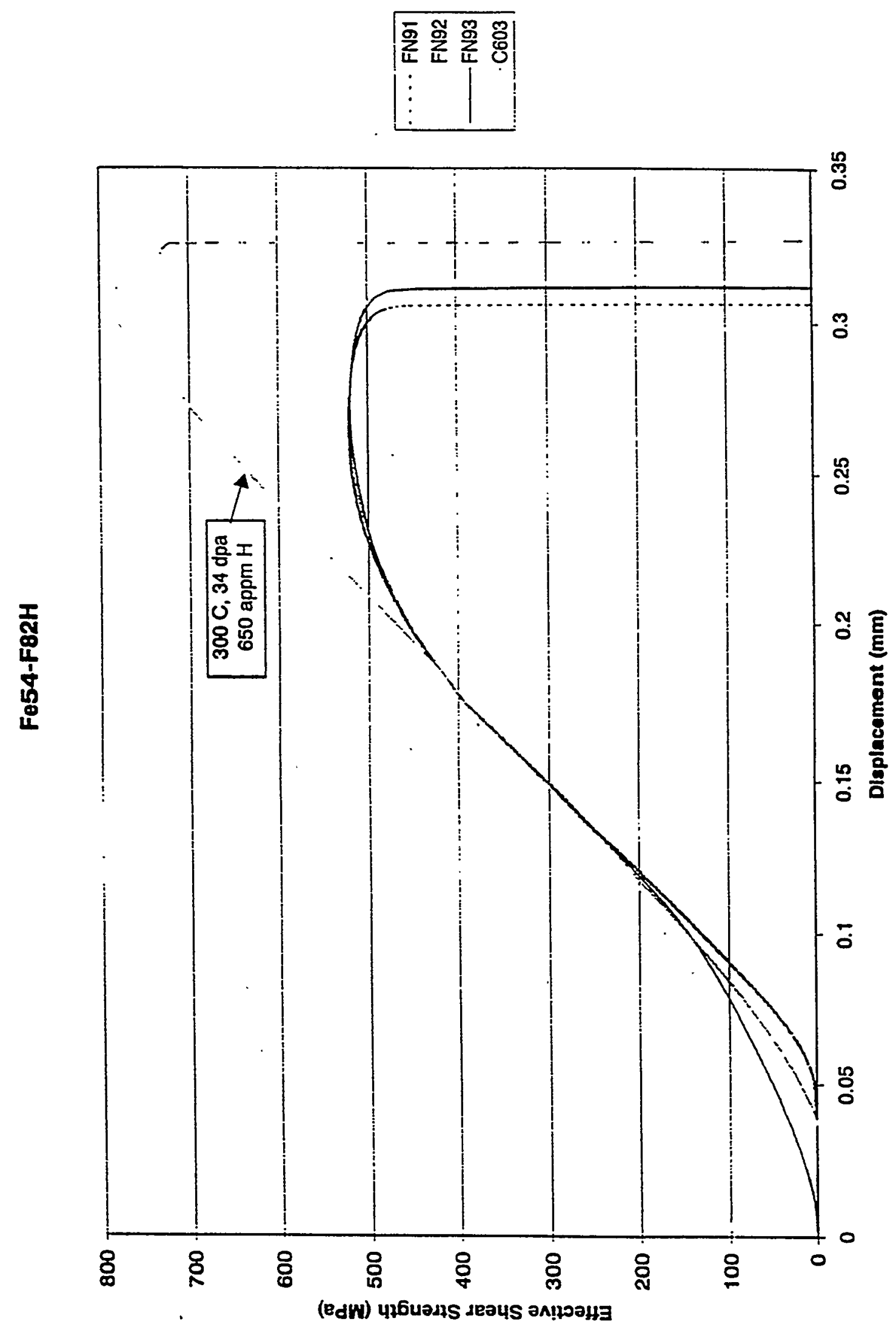




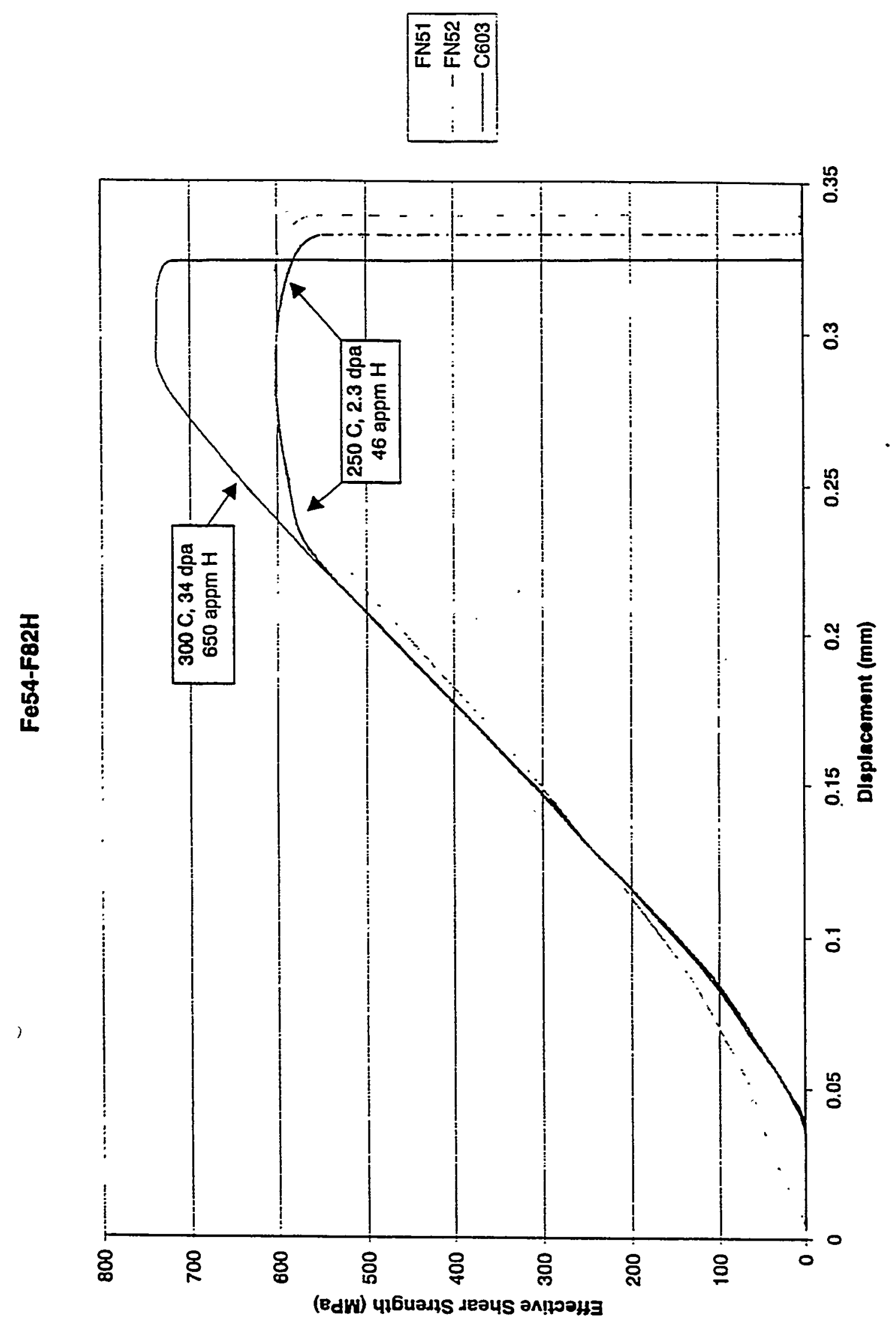




\section{P'reliminary Results on fils'r-1 Microstructural Inspection}

F82H-Fe54 (300C, 34dpa) 650appmH

Voids: small size $(\sim 5 \mathrm{~nm})$, low number density

Dislocation: high density

F82H-B (300C, 34dpa) 320appmHe + Li

Cavities: on sub-boundaries

Dislocation: medium density

F82H STD (300C, 34dpa)

Cavities: no

Dislocation: medium density

F82H-Fe54 (250C, 2.3dpa) 46appmH

Cavities: no

Dislocation: medium density 


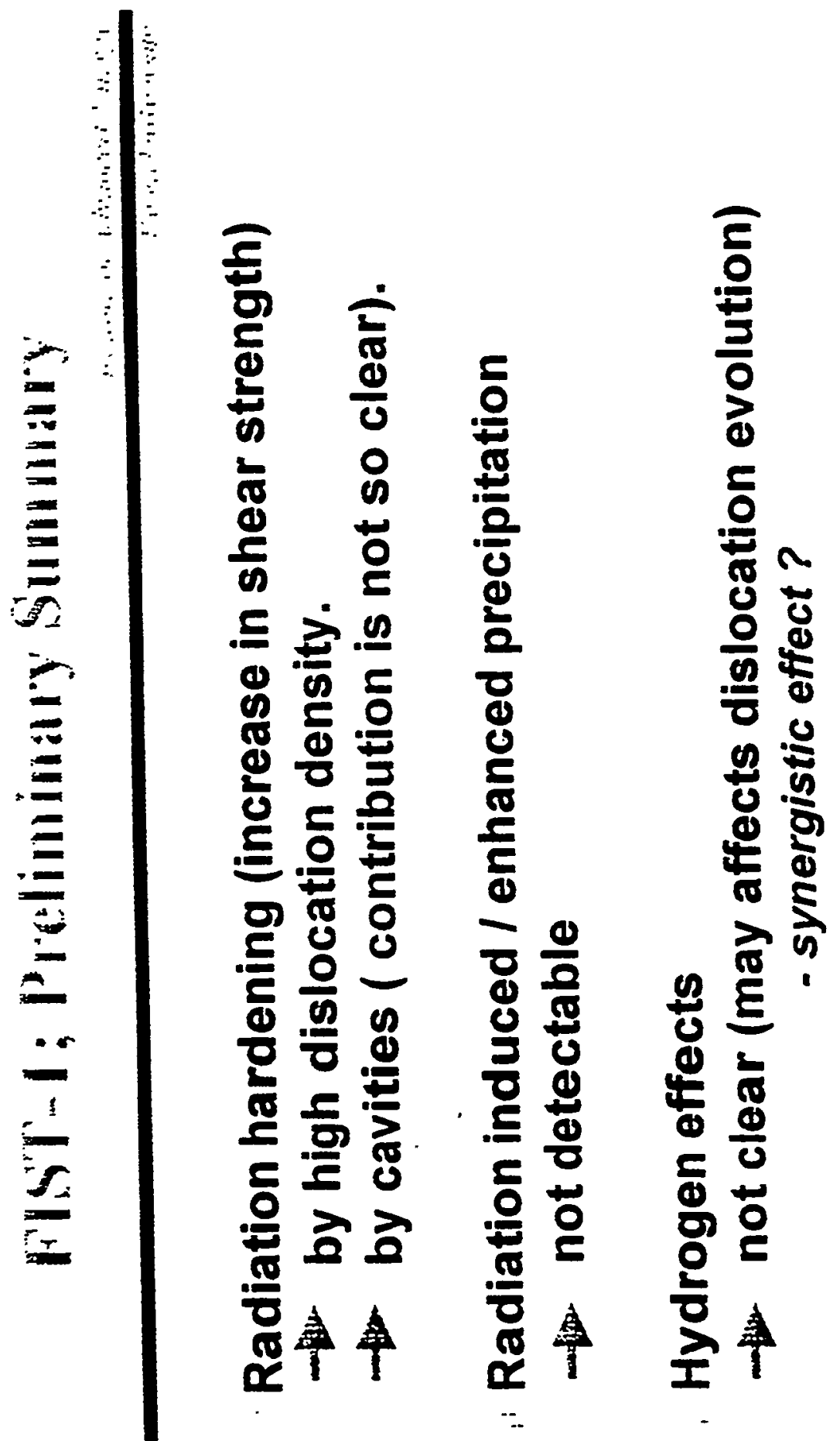




\section{EFFECT OF TANTALUM ON PROPERTIES OF 9Cr-2WVTa STEEL}

\section{R. L. Klueh}

\section{Oak Ridge National Laboratory \\ IEA Working Group Meeting on \\ Ferritic/Martensitic Steels}

Petten, The Netherlands, October 1-2, 1998 


\section{Cr-2WVTa STEEL HAS EXCELLENT STRENGTH AND IMPACT PROPERTIES}

- 9Cr-2WVTa steel: Fe-9Cr-2W-0.25V-0.07Ta-0.1C (wt. \%)

- Steel is used in normalized and tempered condition

- Steel has extra low Charpy transition temperature prior to irradiation: $-88^{\circ} \mathrm{C}(1 / 3$-size Charpy specimen)

- DBTT after irradiation to $28 \mathrm{dpa}$ at $365^{\circ} \mathrm{C}$ in FFTF was extremely low at $-56^{\circ} \mathrm{C}$ (a shift in DBTT of only $32^{\circ} \mathrm{C}$ )

- The shift in DBTT is less than for other steels for similar conditions in FFTF and HFR 


\section{BEHAVIOR OF 9Cr-2WVTa STEEL IS IMPROVEMENT OVER SANDVIK HT9}

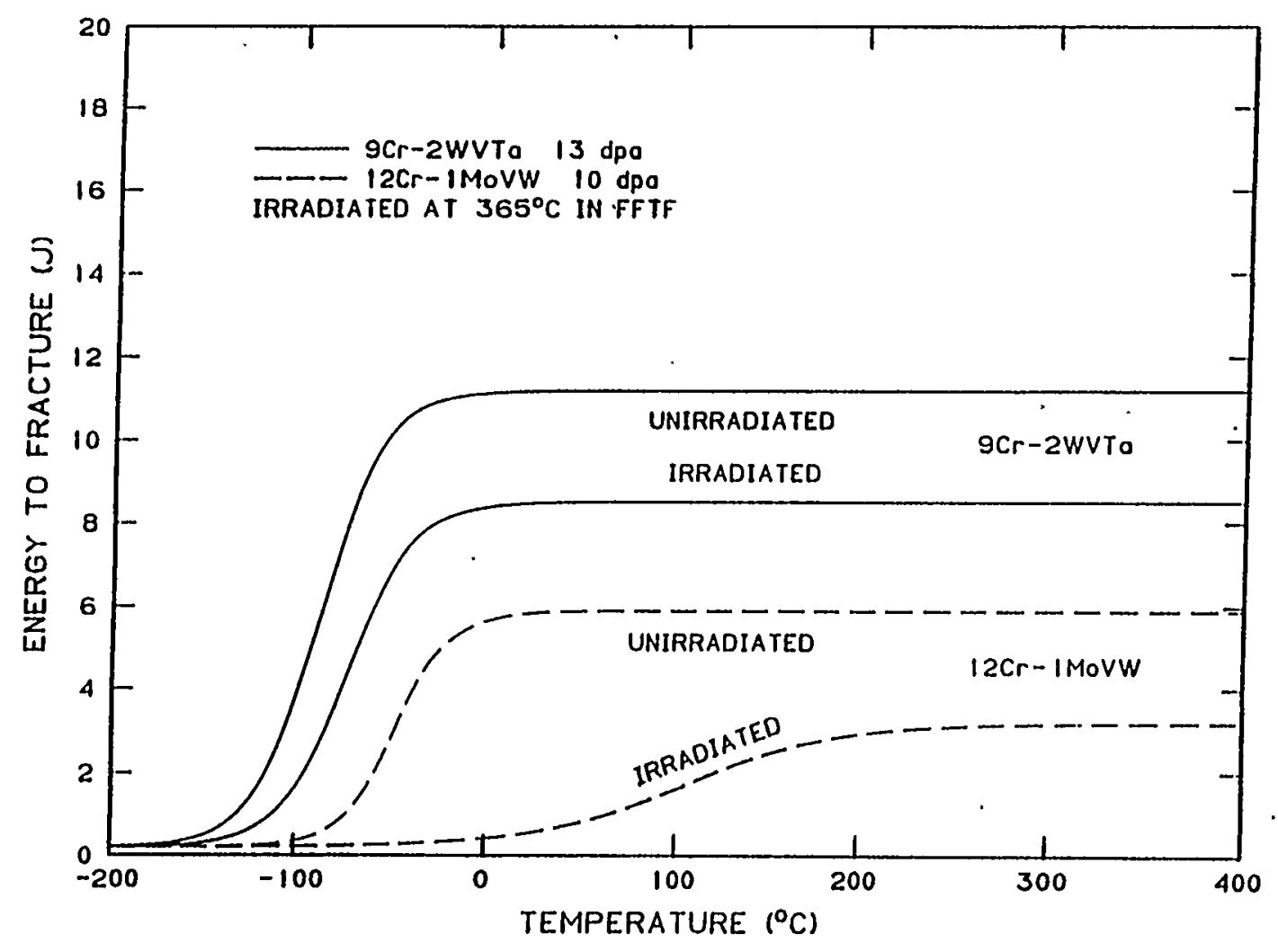

- HT9 was first martensitic steel in U.S. fusion program

- Properties are improved before and after irradiation 


\section{Cr-2WVTa HAS SUPERIOR PROPERTIES TO OTHER STEELS IRRADIATED IN HFR}

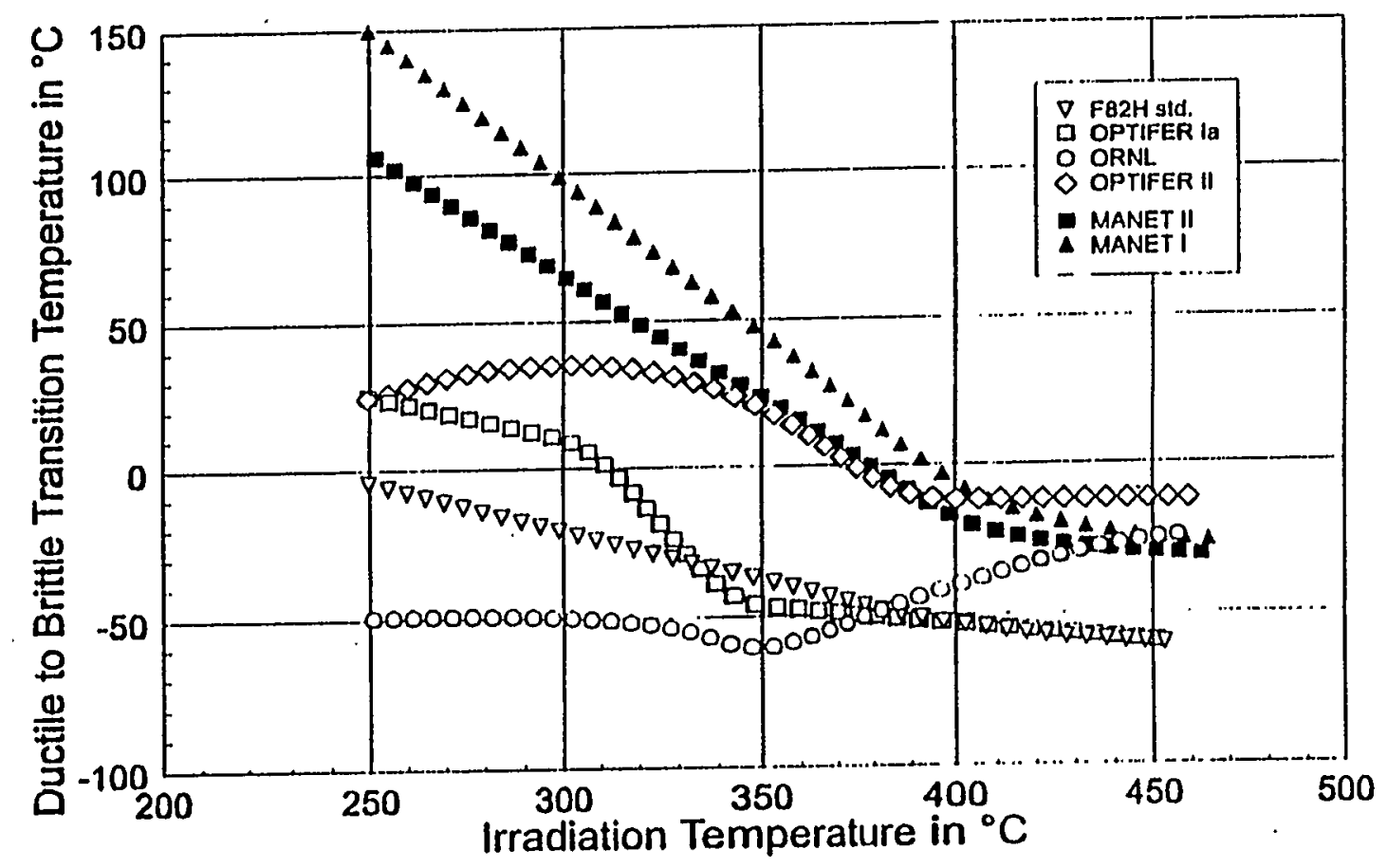

- Irradiation in HFR to $0.8 \mathrm{dpa}$ at $250-450^{\circ} \mathrm{C}$

- Shifts in transition temperature of $20-30^{\circ} \mathrm{C}$ for $9 \mathrm{Cr}-2 \mathrm{WVTa}$

- DBTT and shift in DBTT for 9Cr-2WVTa steel increases $\mathrm{W}^{\circ} \mathrm{h}$ irradiation temperature from $^{\circ} 350$ to $450^{\circ} \mathrm{C}$ 


\section{Cr-2WVTa STEEL SHOWS DIFFERENT BEHAVIOR FROM MOST OTHER STEELS}

- Inverse temperature effect

- For most steels, DBTT and shift in DBTT decreases with irradiation temperature because irradiation hardening decreases with irradiation temperature

DBTT for 9Cr-2WVTa increased with temperature

- Increase between 350 and $450^{\circ} \mathrm{C}$ in HFR irradiation

- Increase between 365 and $393^{\circ} \mathrm{C}$ in FFTF irradiation

- Increase in DBTT with fluence

-DBTT for 9Cr-2WVTa increases with fluence in FFTF at $365^{\circ} \mathrm{C}$; for most other steels it saturates with fluence 


\section{Cr-2WVTa STEEL HAS SIMILARITIES AND DIFFERENCES WITH 9Cr-2WV}

- Steels are the same except for the $0.07 \% \mathrm{Ta}$

- Two steels have much in common

- Similarities in microstructure before and after irradiation

- Similar strengths before and after irradiation

- Steels have different Charpy properties

- 9Cr-2WVTa has better Charpy properties than $9 \mathrm{Cr}-2 \mathrm{WW}$ before and after irradiation

- 9Cr-2WV steel behaves like most steels during irradiation

- No inverse temperature effect

- DBTT saturates with fluence 


\section{MICROSTRUCTURES OF 9Cr-2WVTa AND 9Cr-2WV HAVE FEW DIFFERENCES}

- Similarities before and after irradiation in FFTF:

- similar dislocation structures and lath size - similar precipitate structures $\left(\mathrm{M}_{23} \mathrm{C}_{6}\right.$ and $\left.\mathrm{MC}\right)$

- similar dislocation loop structure formed during irradiation $\left(40-100 \mathrm{~nm}\right.$ at $\left.3 \times 10^{19} \mathrm{~m}^{-3}\right)$

- Differences before and after irradiation:

-9Cr-2WVTa has smaller prior-austenite grain size

- small portion of tantalum is in MC precipitate

-Atom probe indicates $>90 \% \mathrm{Ta}$ is in solution before irradiation 


\section{Cr2WVTa and 9Cr-2WV STEELS HAVE SIMILAR PRECIPITATE STRUCTURES}

Steel

Ppt

Before Irradiation

Density Av Diam

$$
\left(\mathrm{m}^{-3}\right)
$$

(nm)

9Cr-2WV

$\mathrm{M}_{23} \mathrm{C}_{6}$

MC

9Cr-2WVTa $\mathrm{M}_{23} \mathrm{C}_{6}$

MC
$1.2 \times 10^{18}$

$4.5 \times 10^{19}$

$5.9 \times 10^{19}$

125

54

136

$7.5 \times 10^{18}$

29
After Irradiation

Density Av Diam

$\left(\mathrm{m}^{-3}\right)$

$3.2 \times 10^{19}$

(nm)

160

60

$1.1 \times 10^{18}$

$4.1 \times 10^{19}$

143

$5.6 \times 10^{18}$

36 


\section{Cr-2WVTa and 9Cr-2WV STEELS HAVE SIMILAR TENSILE PROPERTIES}

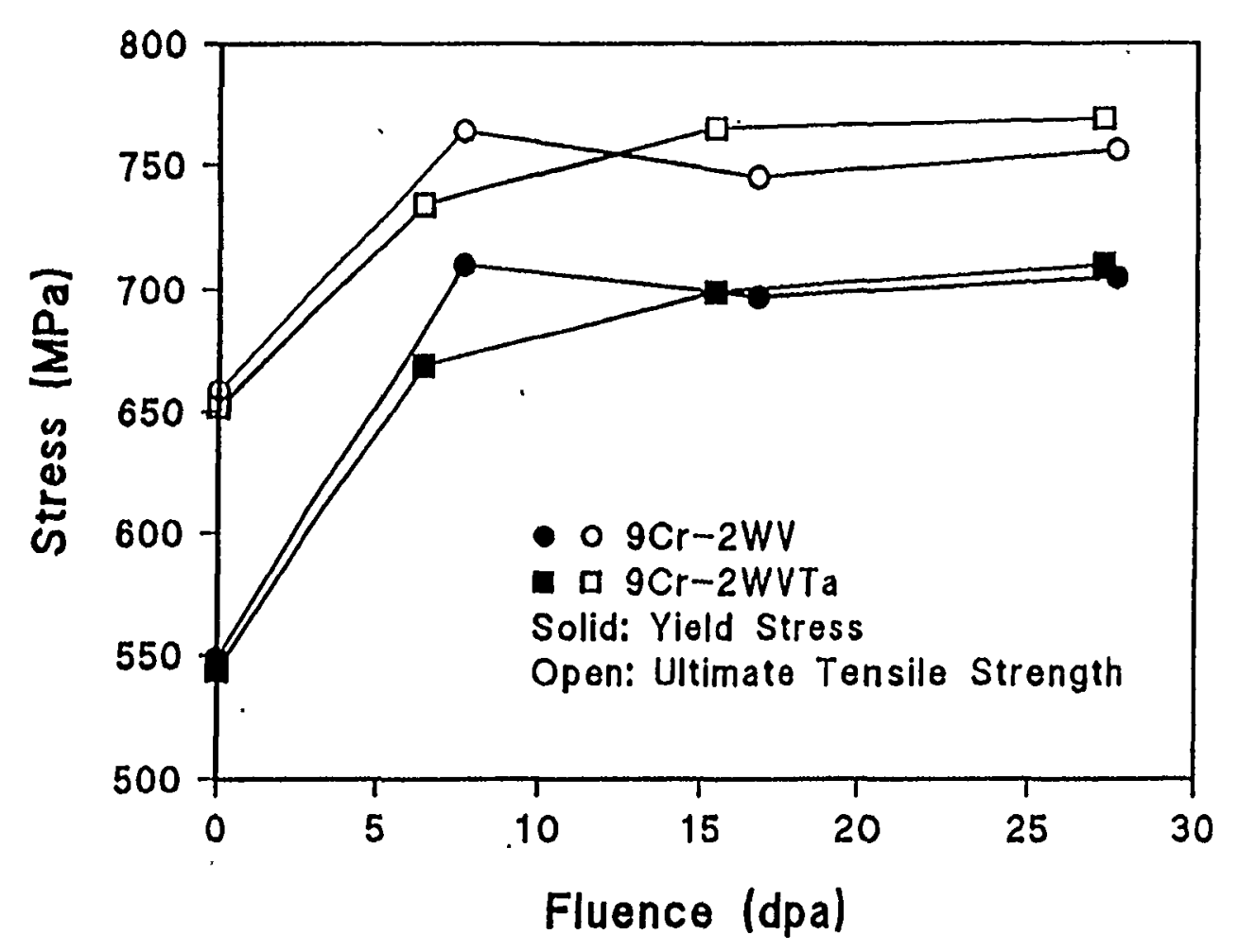

- Properties of steels are similar from 0-28 dpa at $356^{\circ} \mathrm{C}$

- Hardening saturates with fluence 


\section{CHARPY PROPERTIES OF 9Cr-2WVTa AND 9Cr-2WV STEELS ARE DIFFERENT}

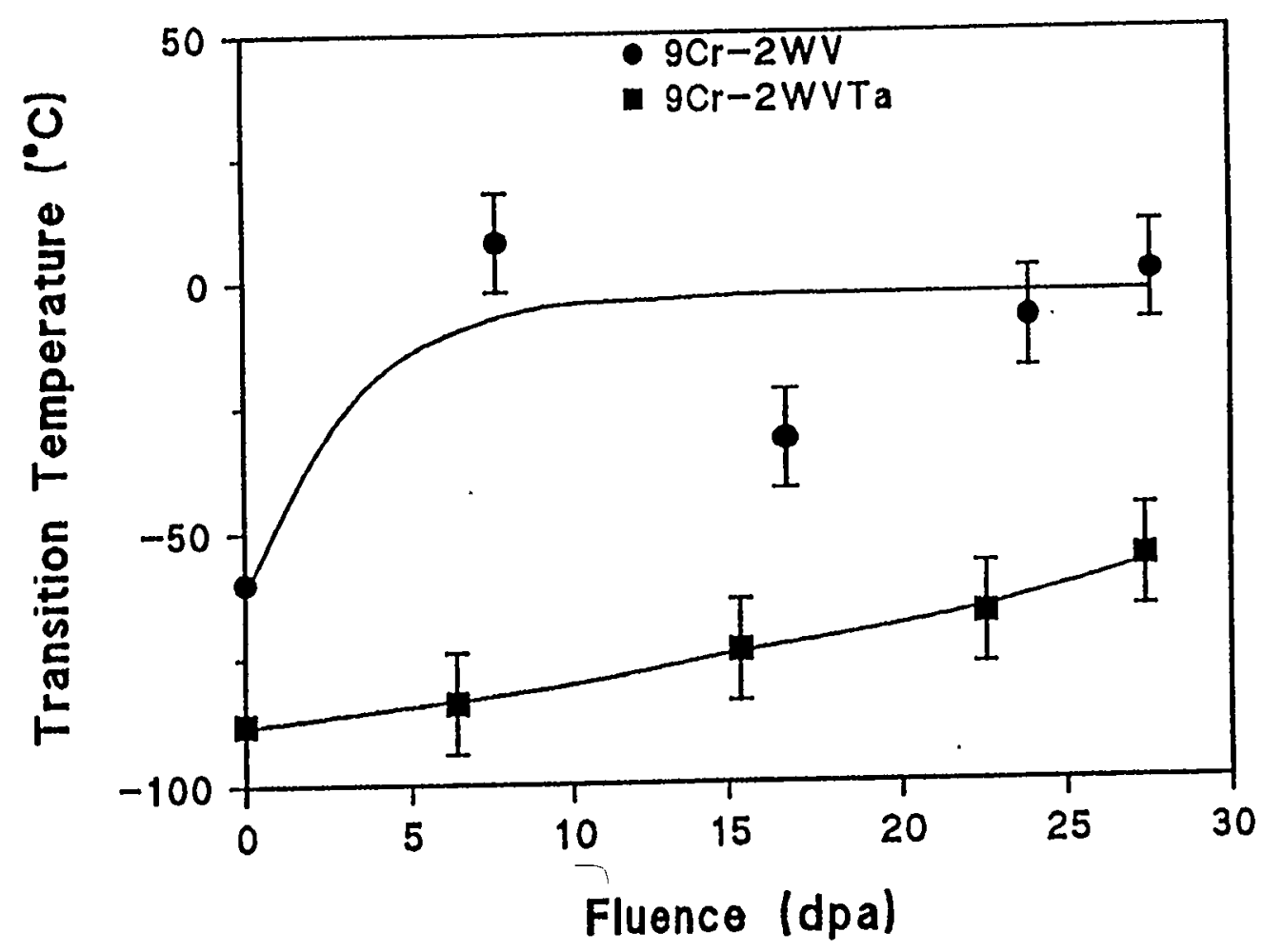

- Transition temperature saturates with fluence for $9 \mathrm{Cr}-2 \mathrm{WV}$ but not for 9Cr-2WVTa irradiated in FFTF to $28 \mathrm{dpa}$ 


\section{TANTALUM IN SOLUTION AFFECTS CHARPY PROPERTIES OF 9Cr-2WVTa}

- Difference in prior-austenite grain size could explain difference in the normalized-and-tempered Charpy impact properties of $9 \mathrm{Cr}-2 \mathrm{WV}$ and $9 \mathrm{Cr}-2 \mathrm{WVTa}$

- The prior-austenite grain size cannot explain the inverse temperature effect and fluence effect for $9 \mathrm{Cr}-2 \mathrm{WVTa}$

- Similar lath sizes and precipitate characteristics in 9Cr$2 \mathrm{WV}$ and $9 \mathrm{Cr}-2 \mathrm{WVTa}$ before and after irradiation means these cannot explain difference in irradiation effects

- Tantalum in solution must favorably affect Charpy impact properties of 9Cr-2WVTa steel 


\section{HYPOTHESIS: TANTALUM AFFECTS FLOW STRESS OR FRACTURE STRESS}

- Strength of 9Cr-2WVTa and $9 \mathrm{Cr}-2 \mathrm{WV}$ steels is not substantially different before and after irradiation

- Tantalum effect on fracture stress or flow stress can explain differences in $9 \mathrm{Cr}-$ 2WVTa and 9Cr-2WV

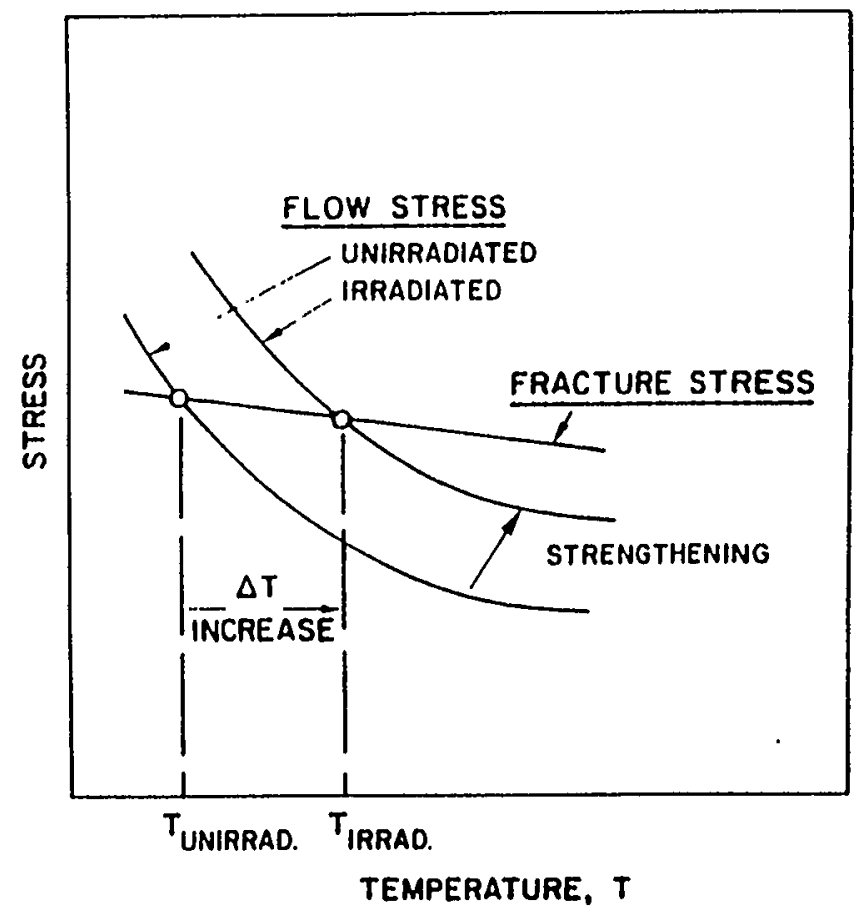




\section{TANTALUM PRECIPITATION CAN EXPLAIN IRRADIATION BEHAVIOR OF 9Cr-2WVTa}

- If tantalum precipitates during irradiation, tantalum removal from solution would lower the fracture stress or change the flow stress

- Precipitation depends on diffusion, and irradiation at higher. temperatures (for a given fluence) will accelerate loss of tantalum and produce the inverse temperature effect observed in HFR and FFTF

- At lower temperatures, higher fluences (longer times) are required for precipitation, which causes the observed continuous increase in DBTT with increasing fluence

- Loss of tantalum from solution still needs to be verified 


\section{Cr-2WVTa MAINTAINS SUPERIOR PROPERTIES DESPITE LOSS OF TANTALUM}

- Once tantalum is lost from solution, matrix composition and microstructure (except for prior austenite grain size) will be similar for the 9Cr-2WV and 9Cr-2WVTa steels

- This should result in similar DBTT shifts for the steels

- After 14 dpa in FFTF at $393^{\circ} \mathrm{C}$, similar shifts were observed for two irradiations of each steel: 46 and $32^{\circ} \mathrm{C}$ vs. 35 and $43^{\circ} \mathrm{C}$ for the $9 \mathrm{Cr}-2 \mathrm{WV}$ and $9 \mathrm{Cr}-2 \mathrm{WVTa}$; respectively.

- 9Cr-2WVTa still had lower DBTT because of lower value before irradiation due to smaller prior-austenite grain size 


\section{OTHER TANTALUM-CONTAINING STEELS SHOULD SHOW SIMILAR BEHAVIOR}

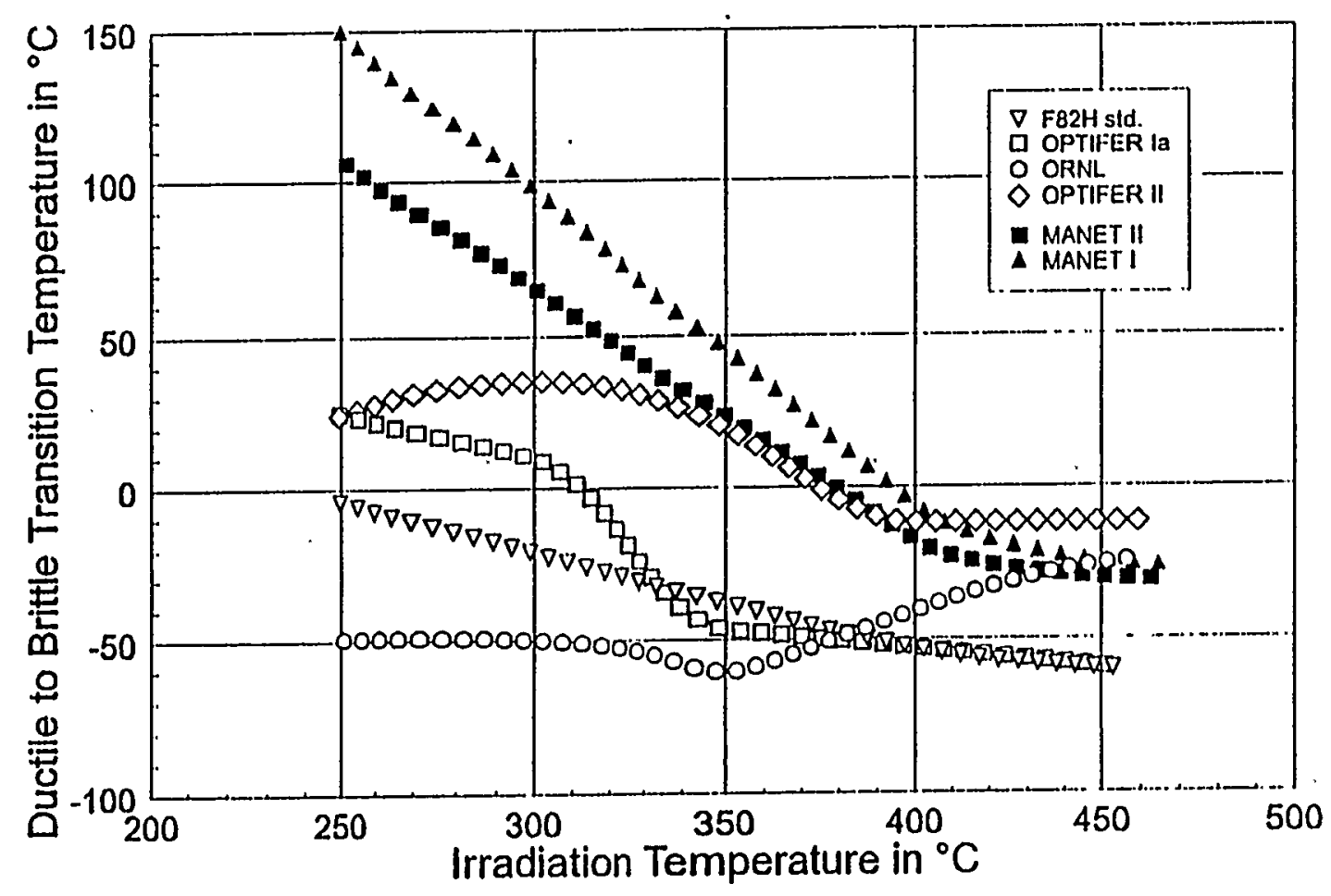

- F82H and OPTIFER-Ia contain tantalum

- Effect of tantalum loss on other steels not evident after $0.8 \mathrm{dpa}$ in HFR as for the 9Cr-2WVTa 


\section{F82H AND OPTIFER-Ia SHOW INVERSE TEMPERATURE EFFECT AFTER 2.4 dpa}
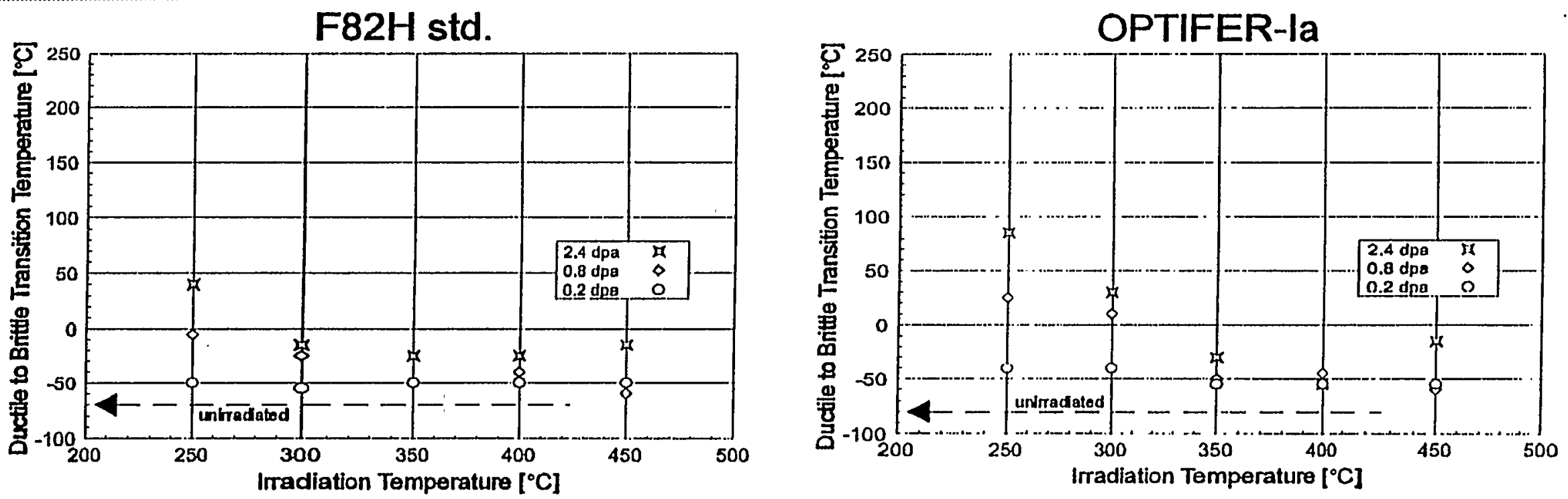

\section{ORNL 3791}

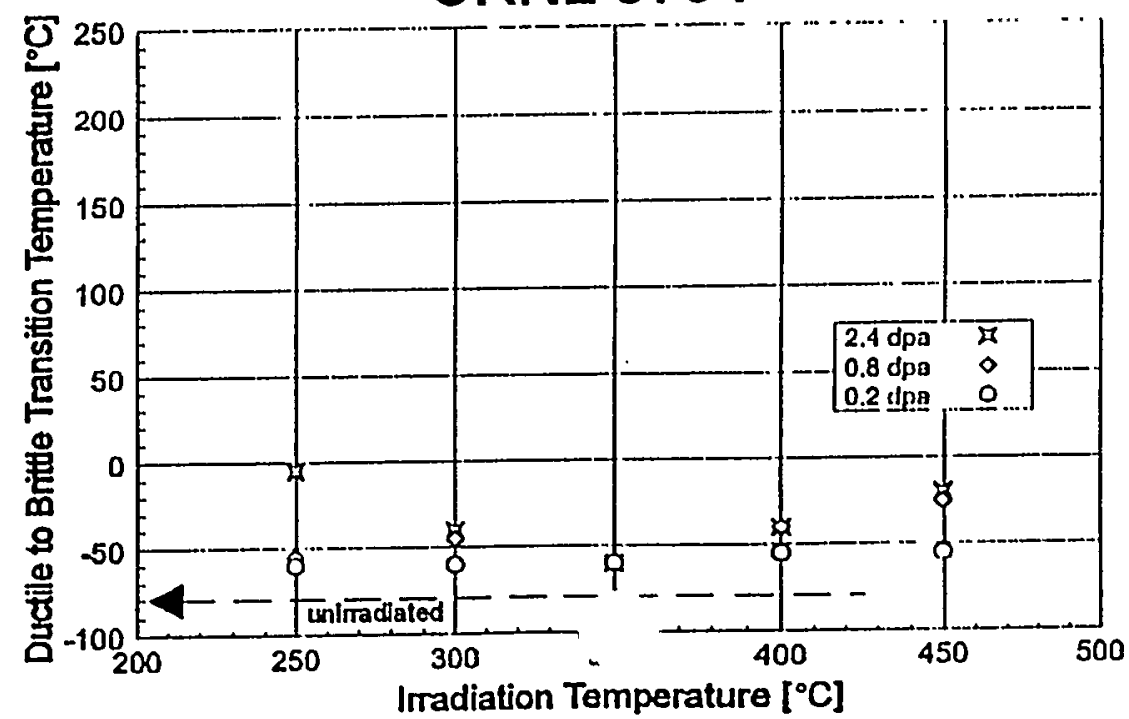




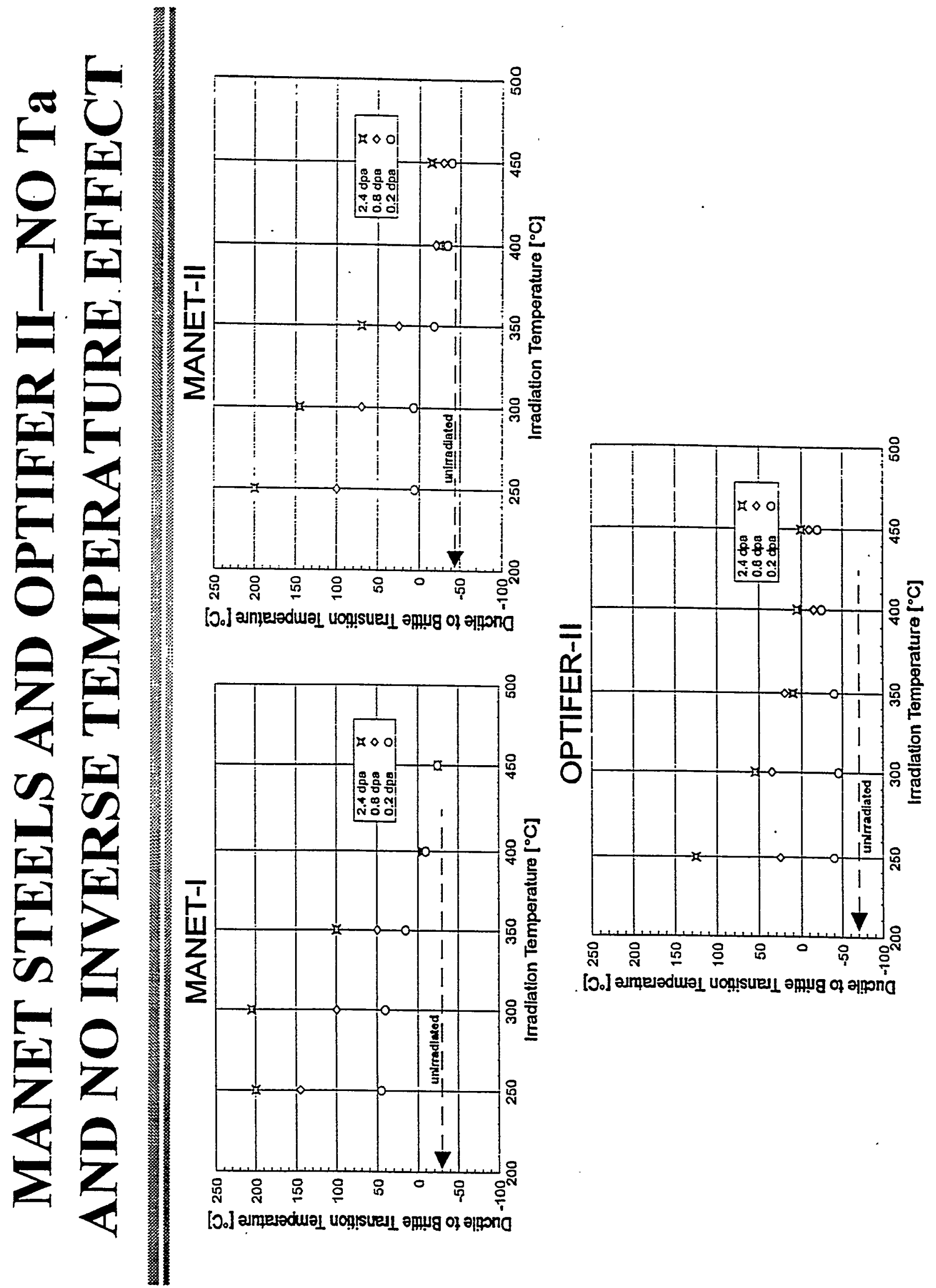




\section{SUMMARY AND CONCLUSIONS}

- Excellent irradiation resistance of 9Cr-2WVTa steel is due to smaller prior-austenite grain size and tantalum in solution, which can affect fracture stress or flow stress

- Irradiation resistance due to tantalum in solution is lost during irradiation; change is attributed to precipitation of tantalum, which still needs to be verified

- Similar effect is observed in other tantalum-containing steels (F82H and OPTIFER-Ia)

- Irradiation resistance of steels without tantalum should be improved by proper heat treatment to change prioraי'stenite grain size 


\section{ODS Alloys and \\ Alloy Development}




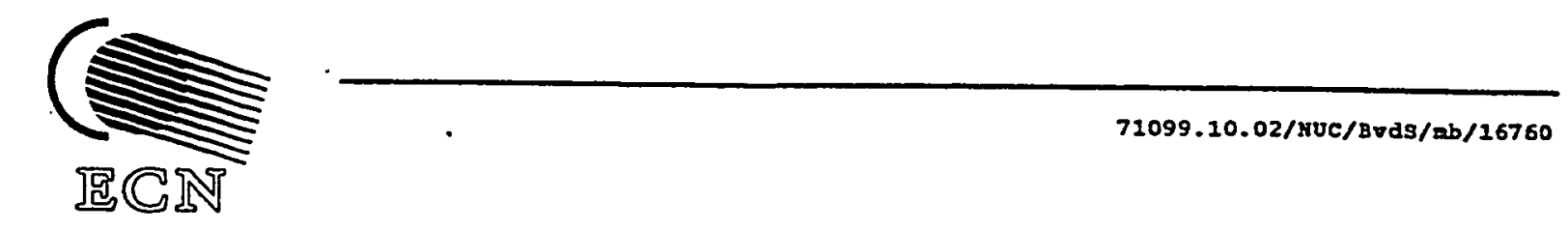

\section{OXIDE DISPERSION STRENGTHENED RAFM STEELS REVIEW AND PROSPECTS}

\section{Presentation at IEA RAFM Workshop Petten, 1 October 1998}

\section{B. van der Schaaf}

NRG, Westerduinweg 3, P.O. Box 25, 1755 ZG Petten, The Netherlands tel. +31224564665 e-mail: vanderschaaf@ecn.nl , fax: +31 224563490 


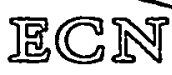

OVERVIEW

- Objective ODS steels for blankets

- Investigated materials

- Testing parameters

- Results

- Conclusion

- Prospects 
古@N

\section{OBJECTIVE ODS STEELS FOR BLANKETS}

-High creep strength at temperatures of $875 \mathrm{~K}$ or higher

-Suppress helium bubble effects

-Low swelling

- Low irradiation creep

-Component manufacturing easy

- Cost in line with improvement 


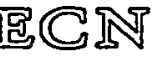

ORIGINAL OBJECTIVE OF ODS STEEL

-Develop LMFBR canning material for up to 200 dpa with maximum temperatures in the range $875-975 \mathrm{~K}$

- Resist creep deformation caused by internal fuel pin pressure build-up

- Strike the balance between:

-Fabricability of cans typically 0.3 $\mathrm{mm}$ wall thickness and $6 \mathrm{~mm}$ diameter

-Acceptable mechanical properties under high temperature high stress condition 


\section{总戉}

Typical ODS steel chemical compositions

\begin{tabular}{|l|l|l|l|l|l|l|l|l|}
\hline ID & $\mathrm{C}$ & $\mathrm{Cr}$ & $\mathrm{Ti}$ & $\mathrm{Mo}$ & $\mathrm{W}$ & $\mathrm{Ti}_{2} \mathrm{O}_{3}$ & $\mathrm{Y}_{2} \mathrm{O}_{3}$ & Origin \\
\hline DT02 & $<0.08$ & 13 & 3.5 & 1.5 & - & 2 & - & SCK \\
DY05 & $<0.08$ & 13 & 3.5 & 1.5 & - & - & 0.5 & SCK \\
\hline DT2203Y5 & na & 13 & 2.2 & 1.5 & - & 0.9 & 0.5 & SCK \\
\hline CEA-A & na & 14 & 1 & 0.3 & - & - & 0.25 & CEA \\
EM10+Y & na & 9 & - & 1.0 & - & - & na & CEA \\
\hline SUKO01 & 0.013 & 11 & 0.4 & - & 2.6 & - & 0.52 & PRNFD \\
SUK002 & 0.031 & 13.7 & - & - & 3.0 & 0.17 & 0.22 & PRNFD \\
KO01 & 0.19 & 106 & 0.5 & - & 2.3 & 0.93 & - & PRNFD \\
\hline MA956 & 0.019 & 19.1 & 0.34 & na & - & - & 0.48 & PNL \\
MA957 & na & 13.1 & 0.95 & 0.27 & - & 0.95 & 0.25 & PNL \\
\hline
\end{tabular}




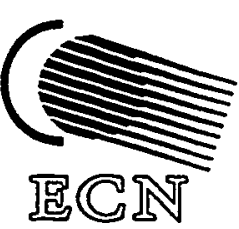

TYPICAL POWDER SIZES

Elemental

Pre-alloyed

Yttrium oxide*

Yttrium oxide
$100-150 \mu \mathrm{m}$

$<150 \mu \mathrm{m}$

$<44 \mu \mathrm{m}$

$<20 \mathrm{~nm}$

Inert atmosphere essential

- vacuum

- inert gas

* Depending on process 


\section{SCK FABRICATION ROUTE}

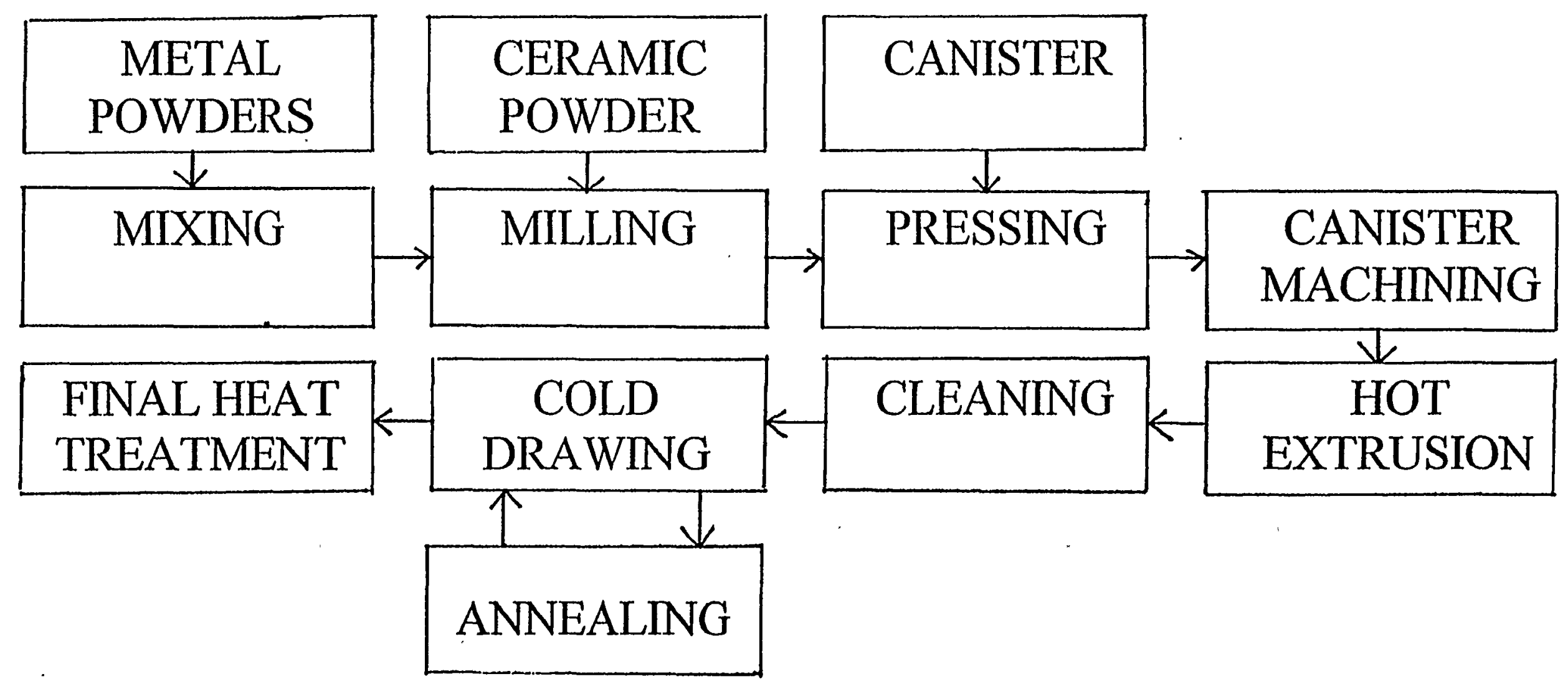


焉包

POST-IRRADIATION

TESTING

PARAMETERS

-Irradiations

Electrons $\leq 20 \mathrm{dpa}$

Fast Fission Neutrons $\leq 200$ dpa

- Mechanical testing

- Thermal creep : $\leq 10000 \mathrm{hrs}$

- Irradiation creep : $\leq 925 \mathrm{~K}$

- Tensile

- Charpy energy

- Microstructure

Maximum dose $200 \mathrm{dpa}$

- Swelling

Maximum dose $200 \mathrm{dpa}$ 


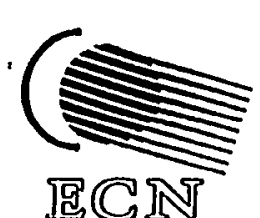

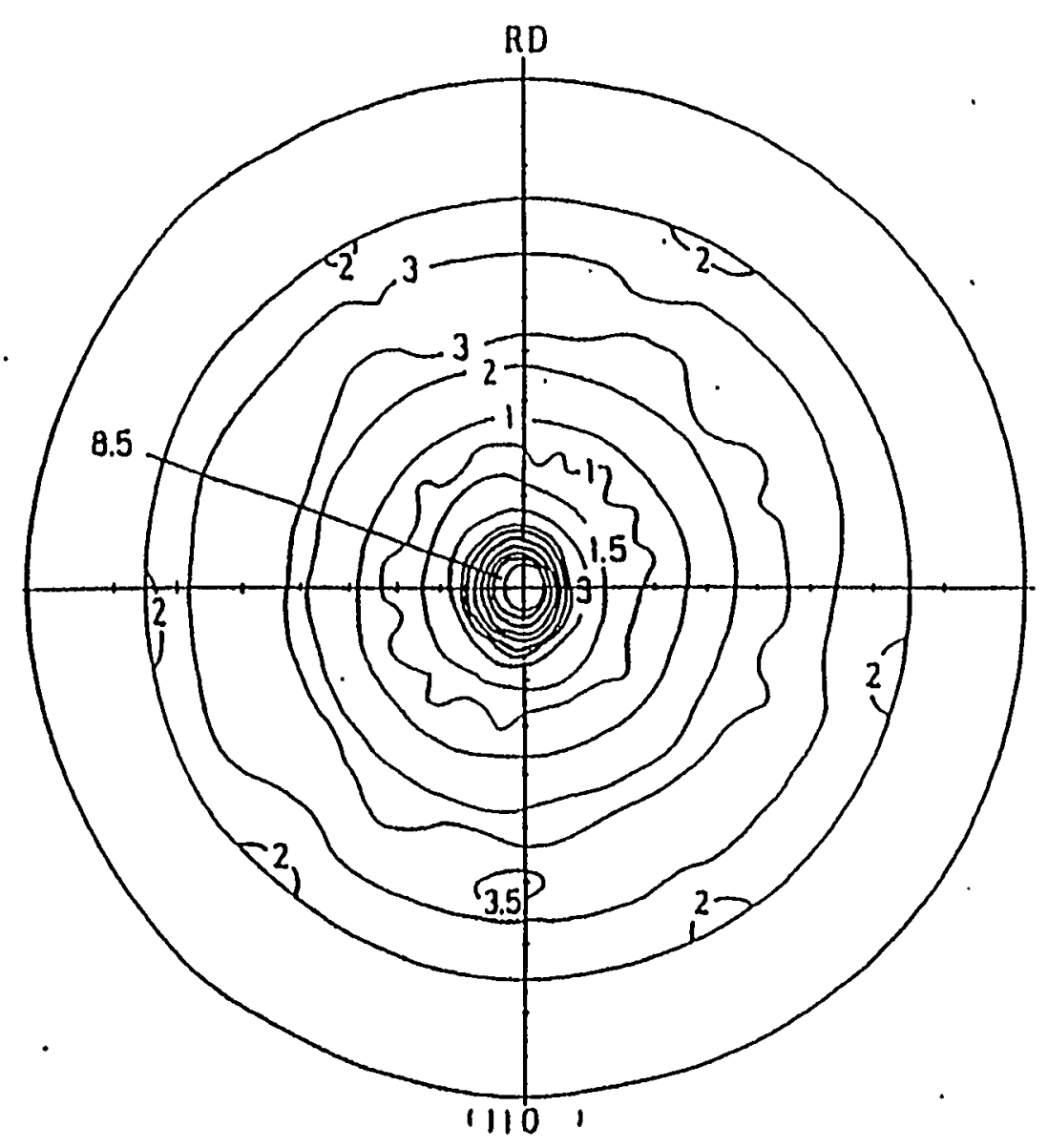

ODS Ferritic Steel (No.4)

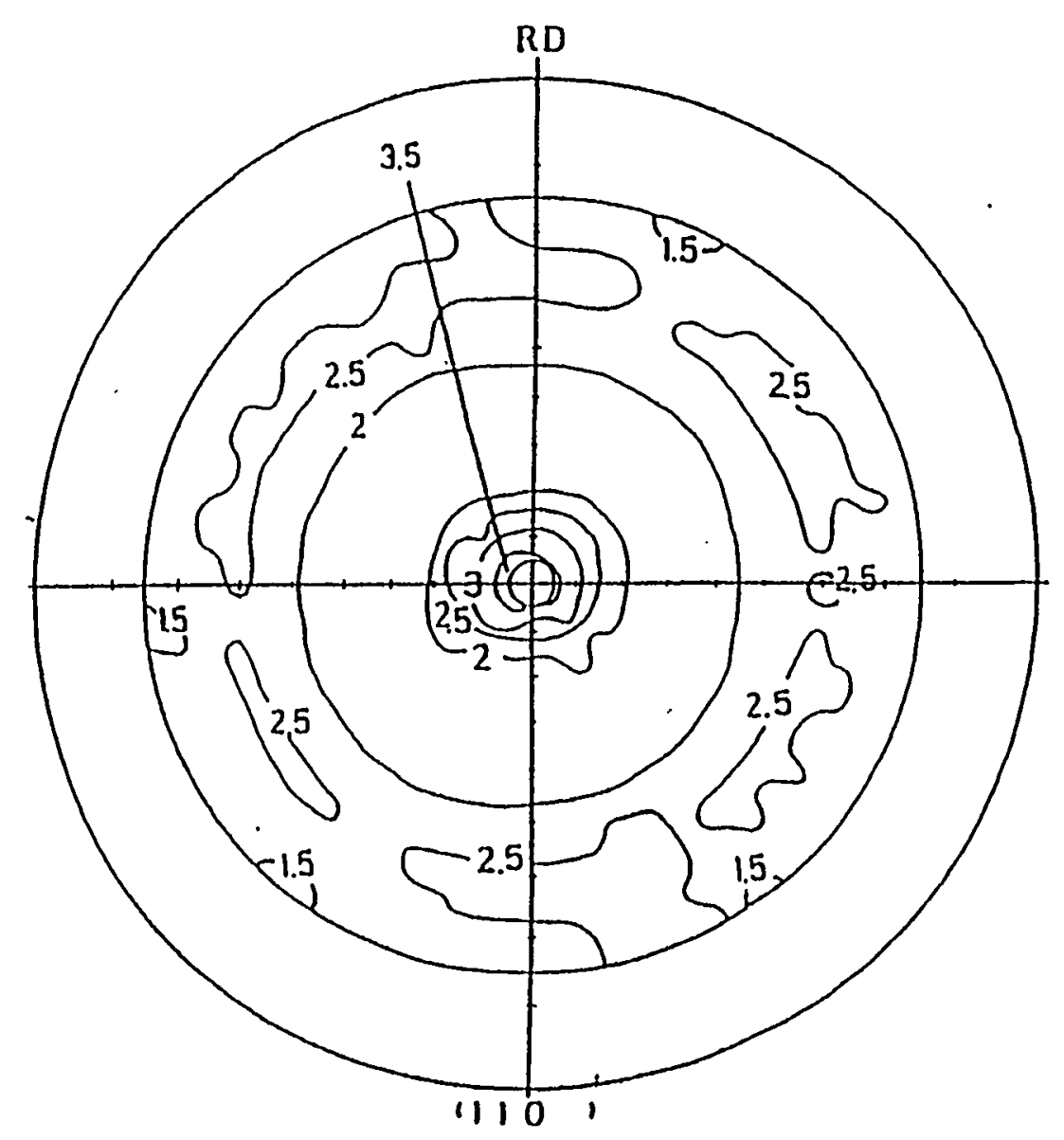

ODS Martensitic Steel (No.16) 

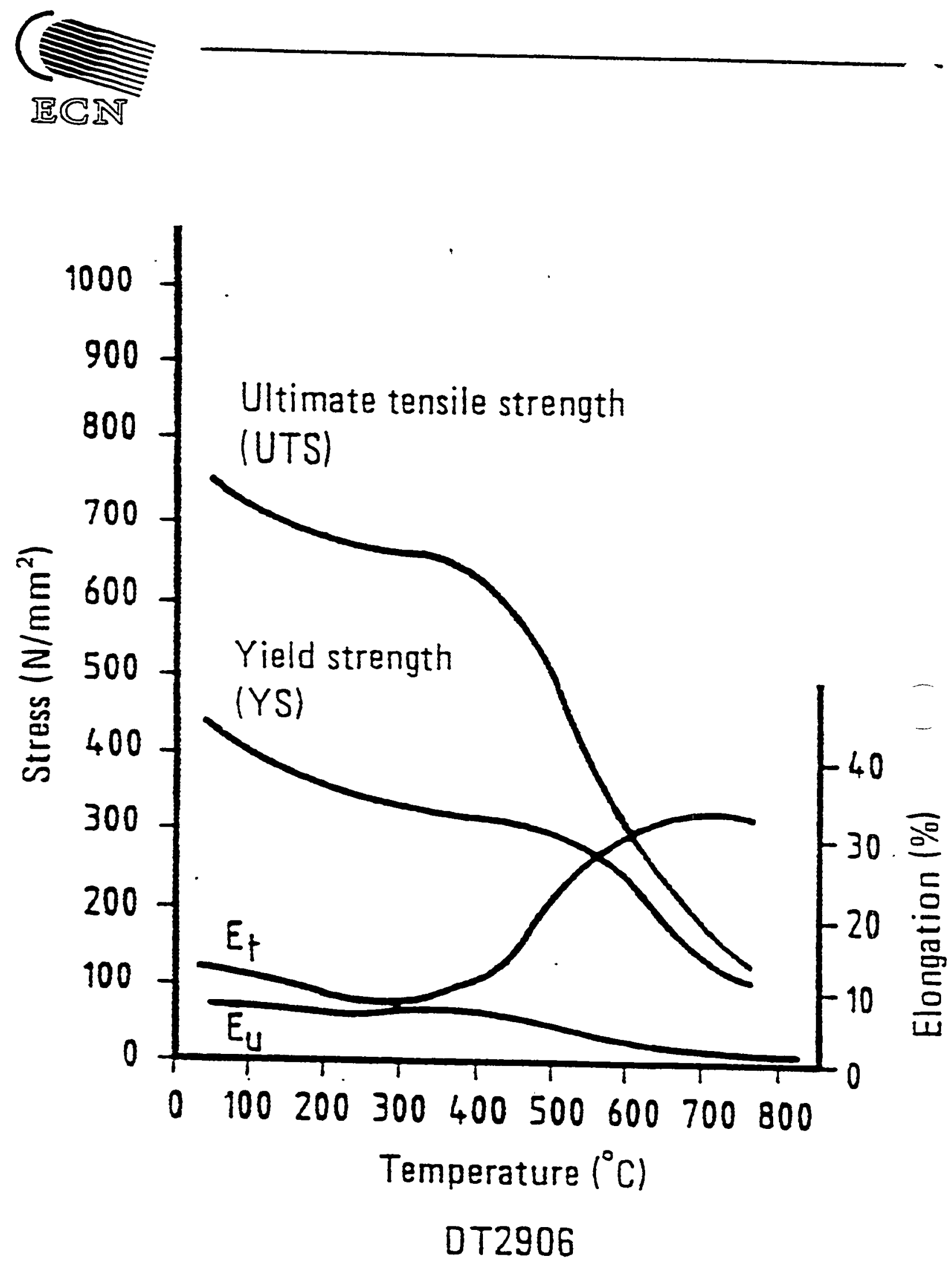

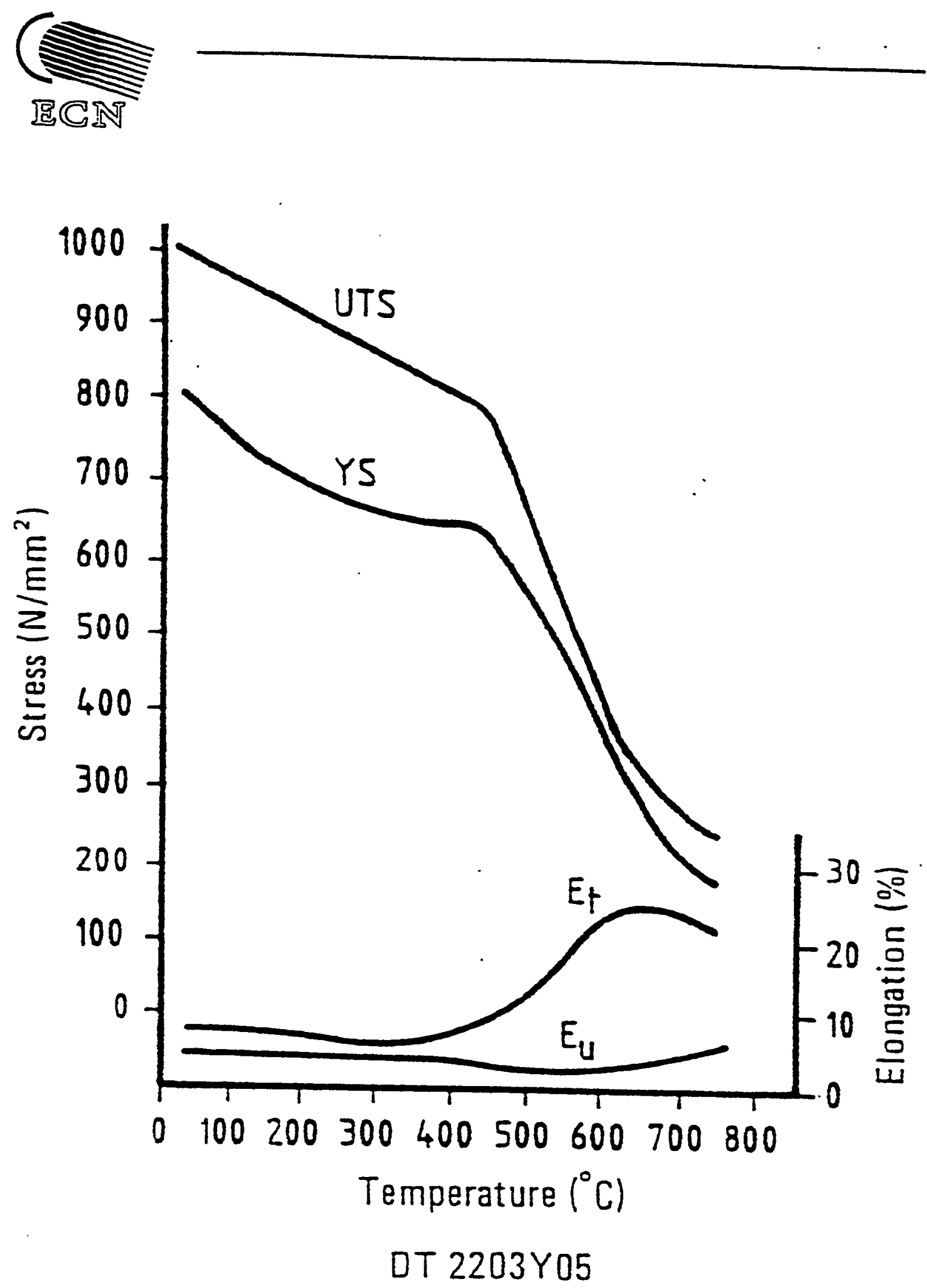


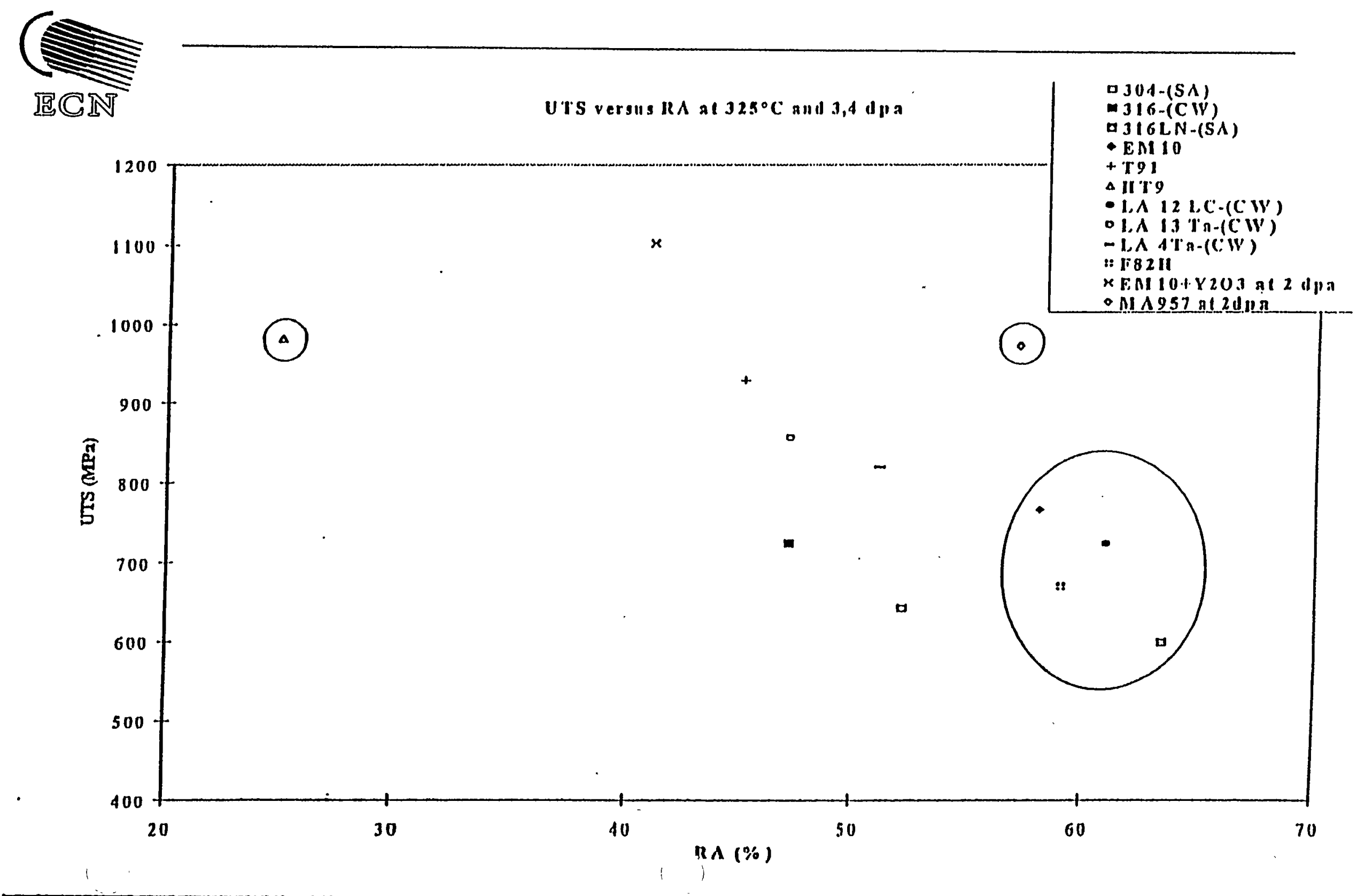




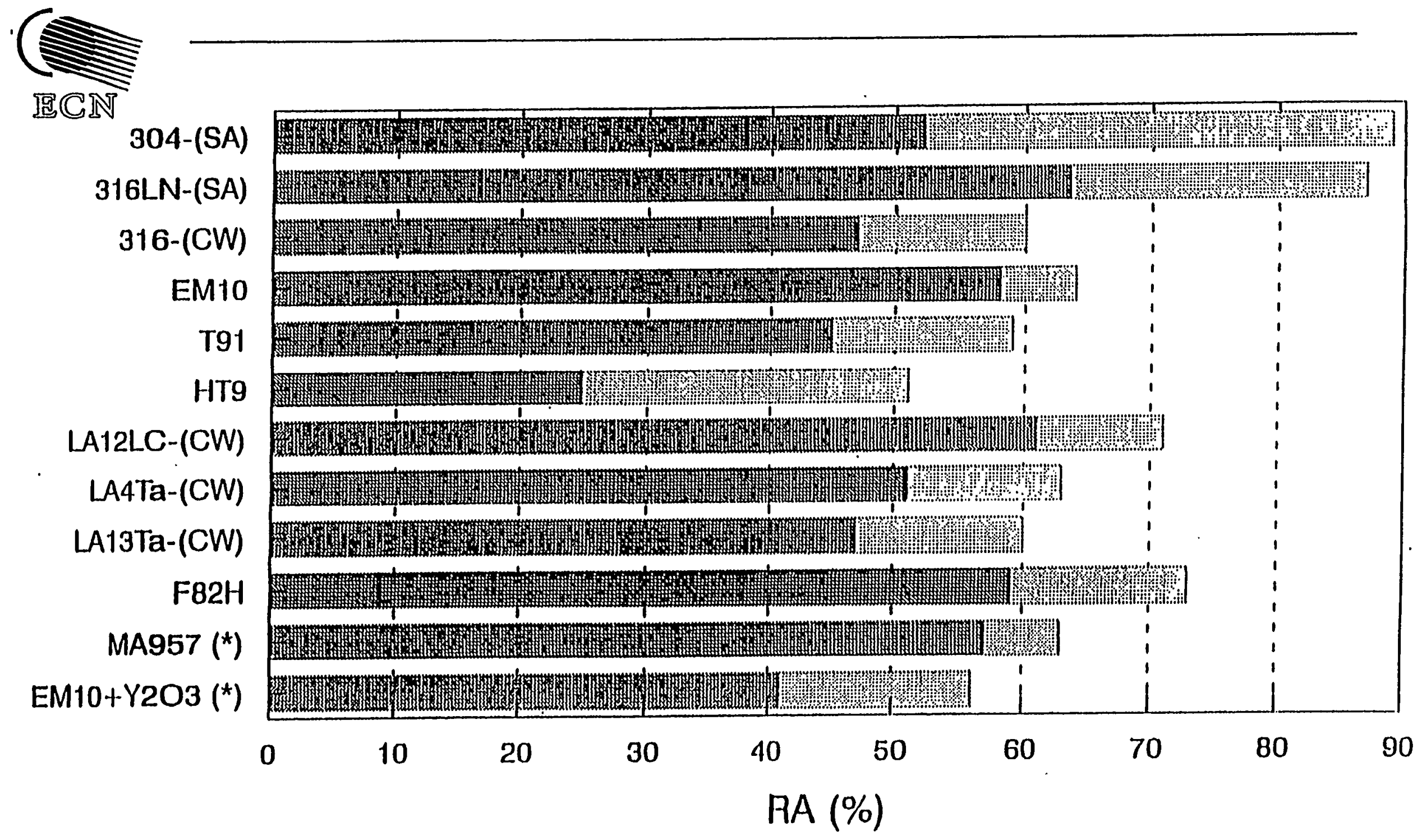

(n⿴囗十) 3.4 dpa $170 \mathrm{dpa}$ 


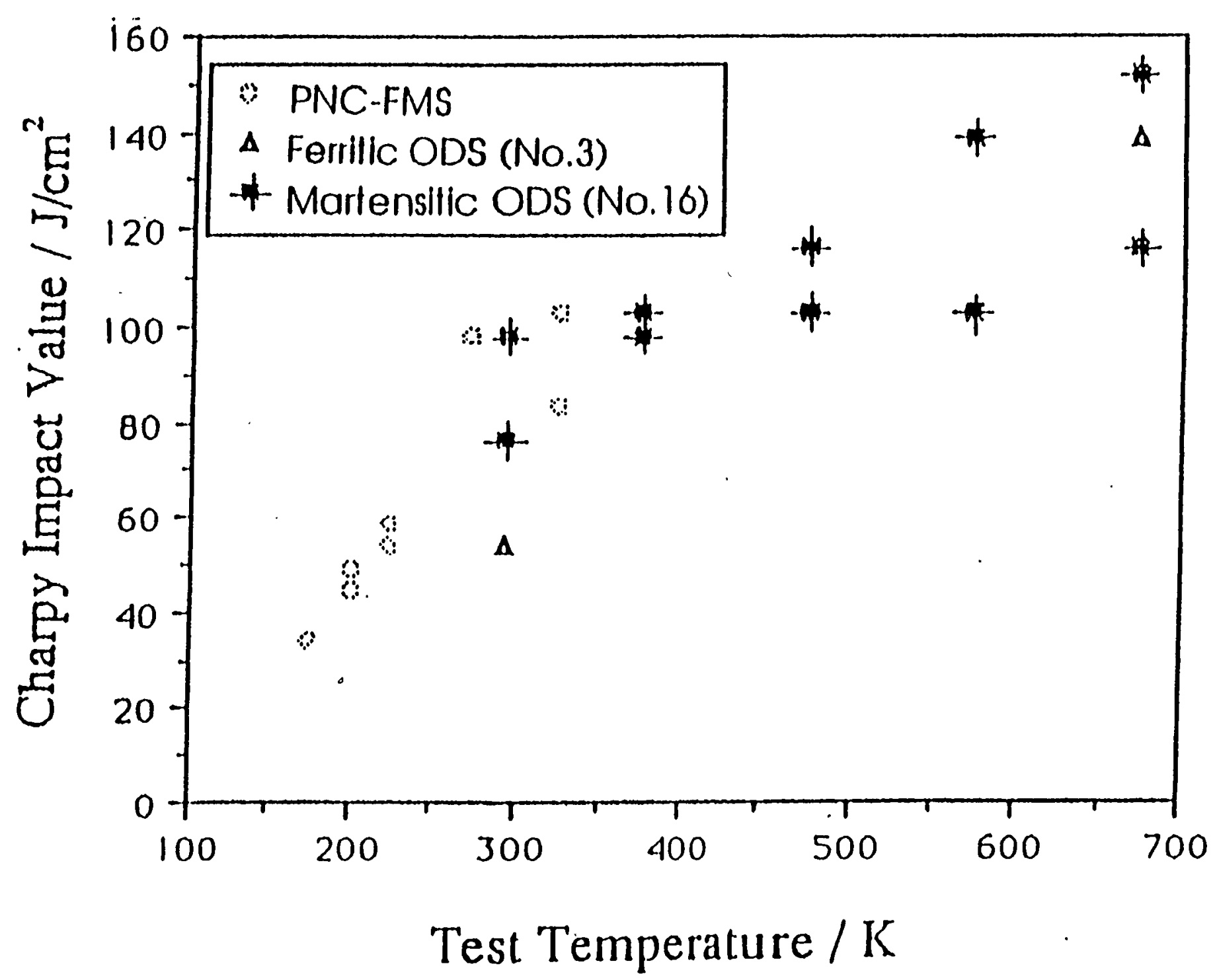




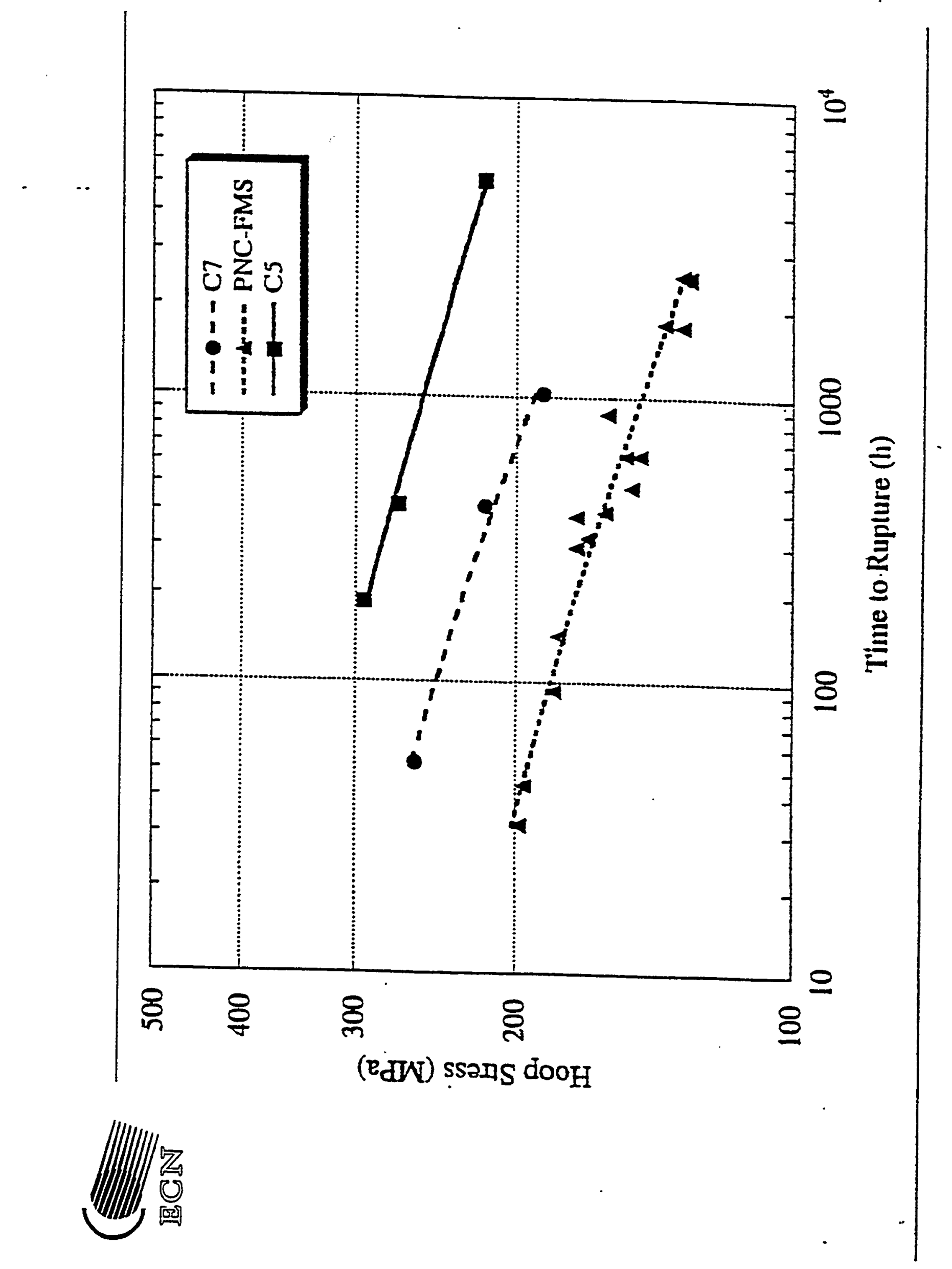




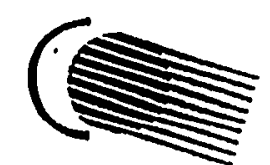

逸书

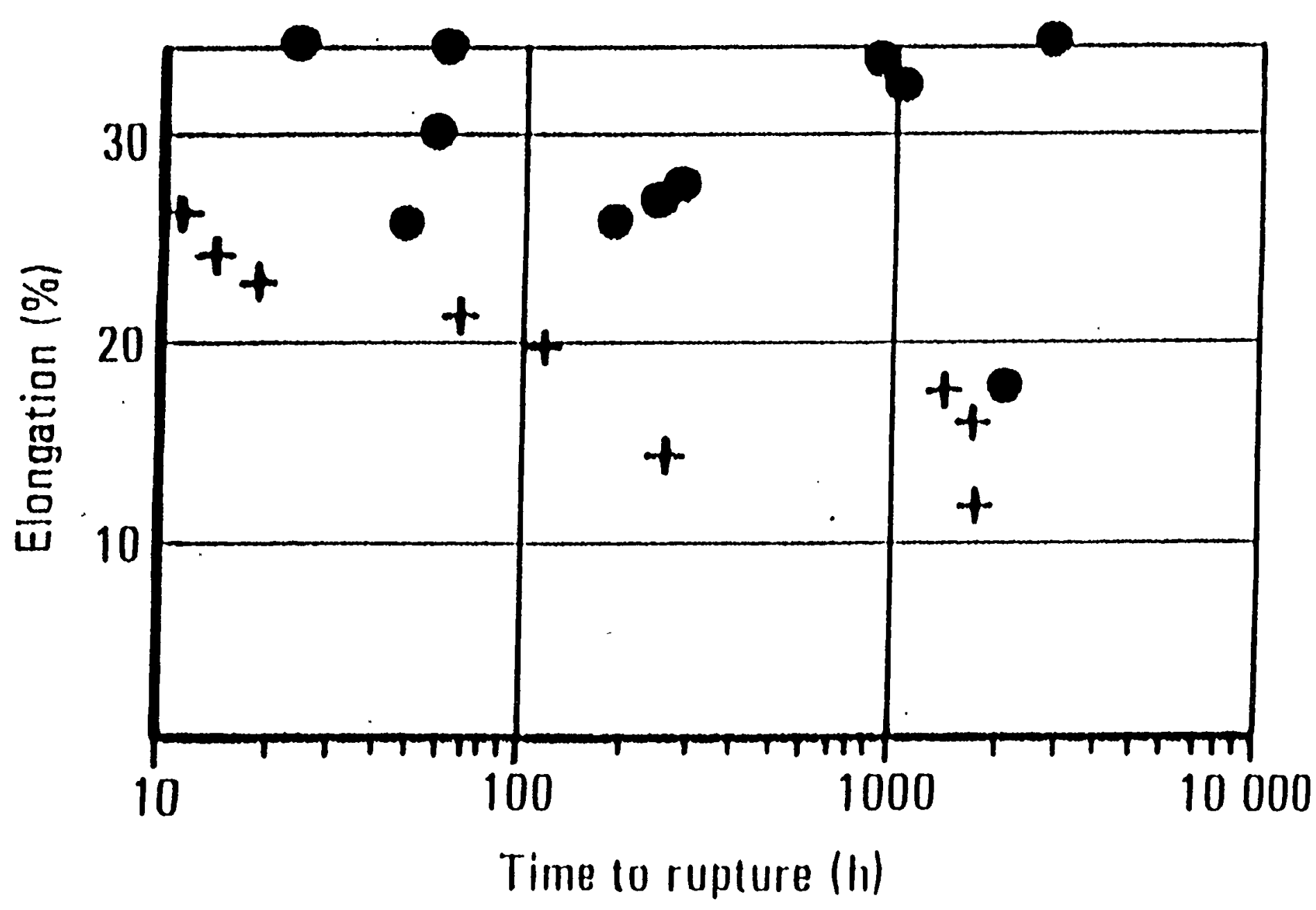




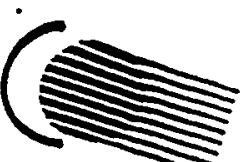

]러요

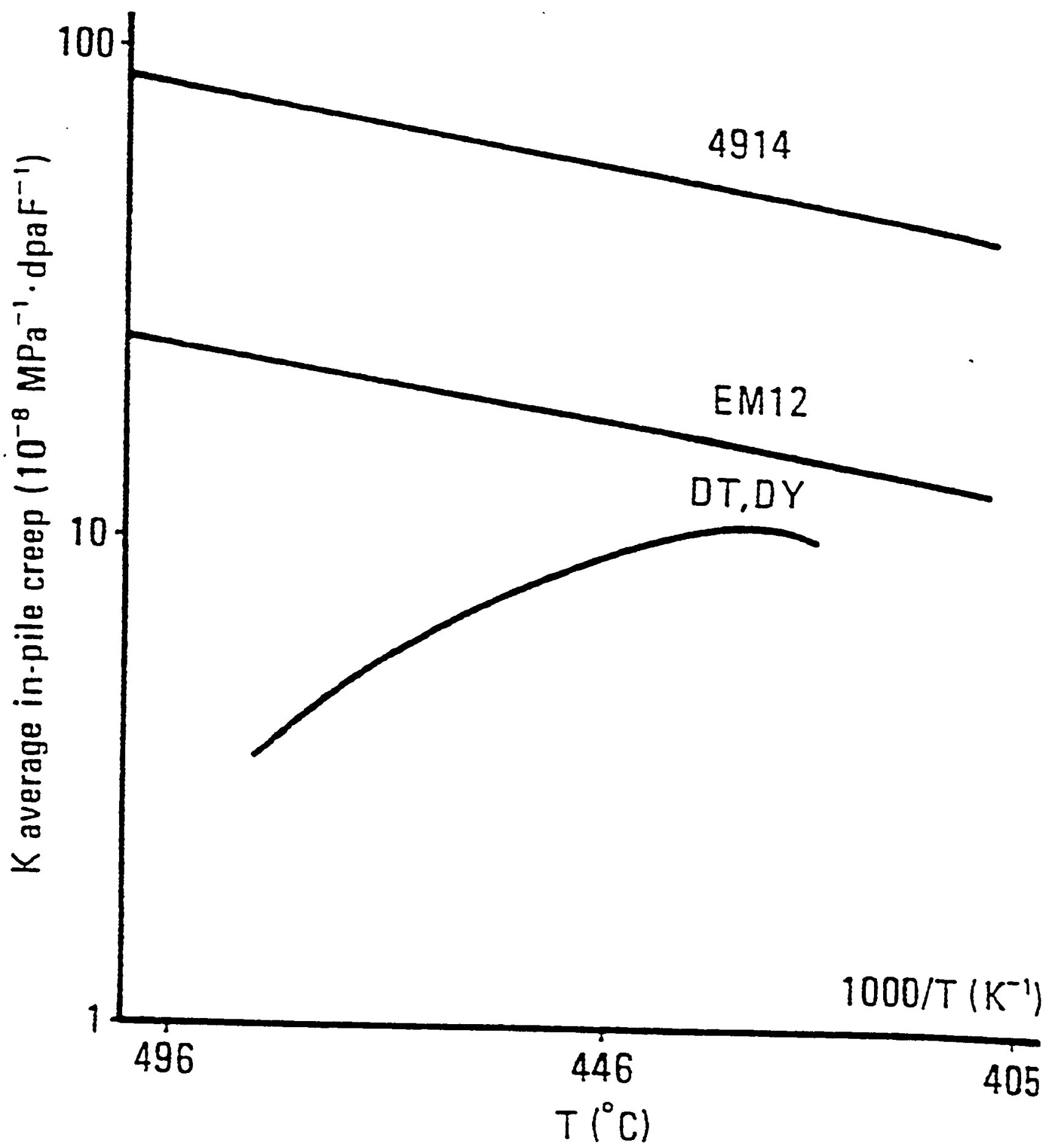


胥CN

CONCLUSION

- Oxide dispersion strengthening improves conventional $\mathrm{Cr}$ steels properties

- Helium effects seem to be suppressed by the ODS microstructure

- Most information is strongly oriented to LMFBR fuel canning

- Severe anisotropy observed

- Thick-walled parts or large diameter tube experience is not available in open literature

-In open literature no information is available yet on ODS RAFM steel 
IG@N

\section{PROSPECT}

- Conventional Cr steels with ODS have properties promising for ODS RAFM high temperature application under high neutron loads

- ODS potential in RAFM is:

-Operating temperature increase to $900 \mathrm{~K}$

-Helium effects reduction

-Fabrication route must be developed for ODS RAFM blanket "heavy sections"

- Joining of ODS RAFM steel must be addressed in an early stage

- Too early to decide on which $\mathrm{Ti}_{2} \mathrm{O}_{3} / \mathrm{Y}_{2} \mathrm{O}_{3}$ mixture to apply 
咆爬

\section{DEVELOPMENT PRIORITIES}

-Assure that the conventional fabrication routes produce acceptable ODS RAFM properties improvement

- Find a manufacturing route for thick walled (10-20 mm) ODS RAFM parts with homogeneous, isotropic properties

-Develop joining methods resulting in acceptable properties

-Keep corrosion properties in the required range 


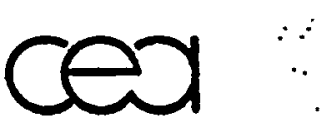

IEA WORSHOP ON REDUCED-ACTIVATION F/M STEELS ECN, Petten, The Netherlands, 1-2 October 1998

\title{
DEVELOPMENT OF $9 \mathrm{Cr}$ FERRITIC- MARTENSITIC STEELS STRENGTHENED BY OXIDE DISPERSION
}

\author{
V. LAMBARD, A. ALAMO \\ Commissariat à l'Energie Atomique \\ CEA-Saclay, SRMA \\ Metallurgical Resarch Lab. \\ 91191 Gif-sur-Yvette, FRANCE
}




\section{Oxide Dispersion Strengthened «ODS» ferritic allovs}

\section{MECHANICAL ALLOYING Attrition}

Metallic powders + oxide powder(s)

\section{POWDER COMPACTION Extrusion, HIP}

Metallic matrix with a homogeneous distribution of oxide particles (average size $15 \mathrm{~nm}$ )

High temperature strength Resistant to environmental degradation

High dimensional stability under irradiation (swelling, in-pile creep) (1) 


\section{ODS FERRITIC STEELS}

\section{Distribution of $\mathrm{Y}_{2} \mathrm{O}_{3}$ Oxide Particles}

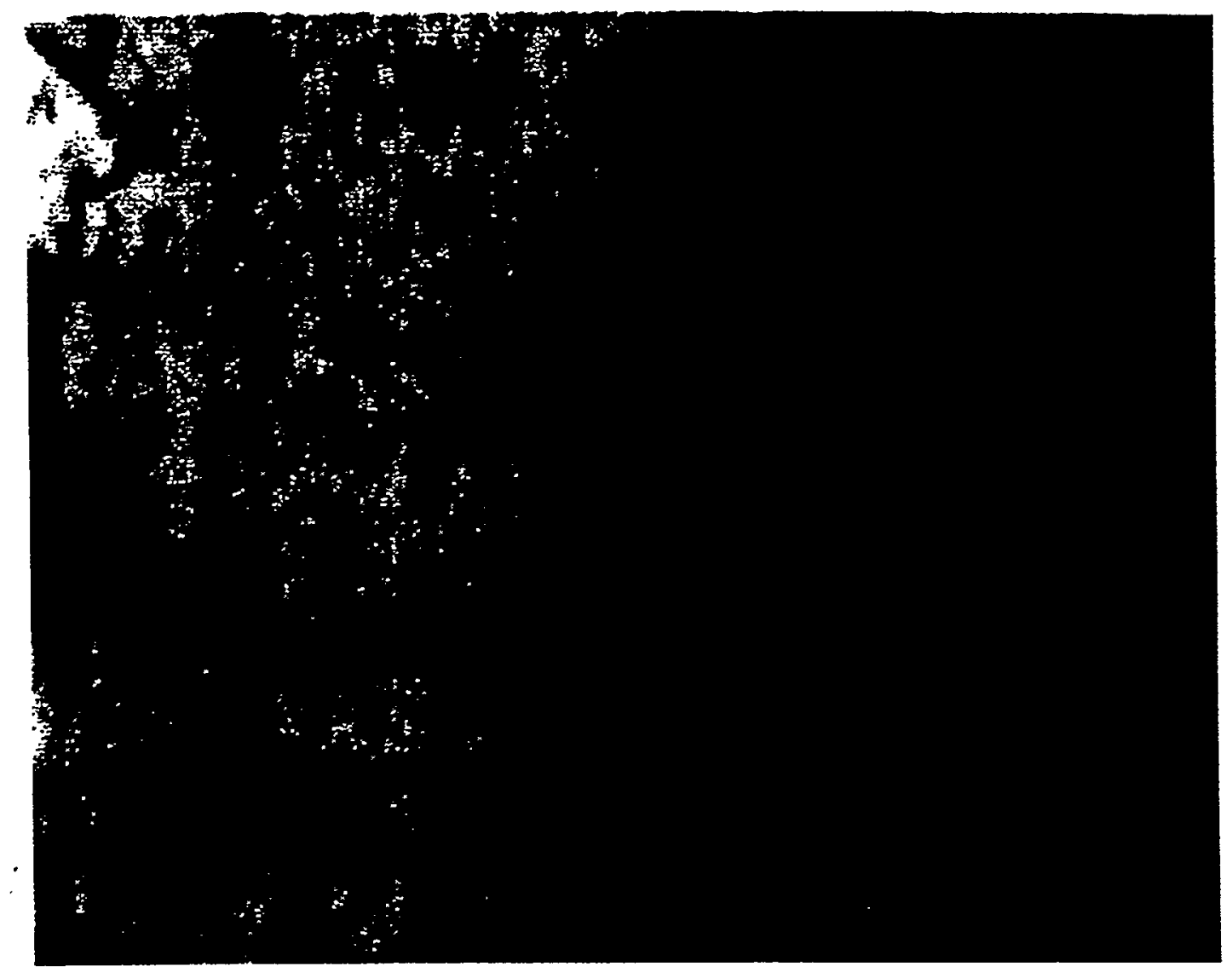

\section{$100 \mathrm{~nm}$}

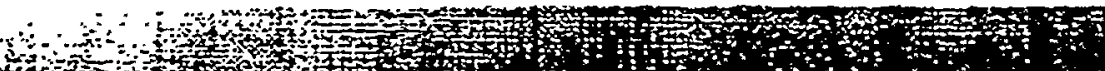

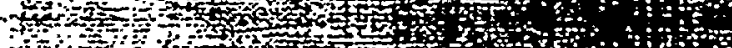




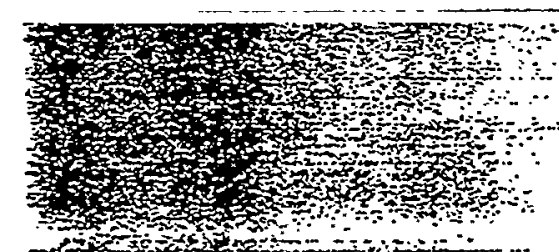

\section{ced}

\section{ODS FERRITIC STEELS}
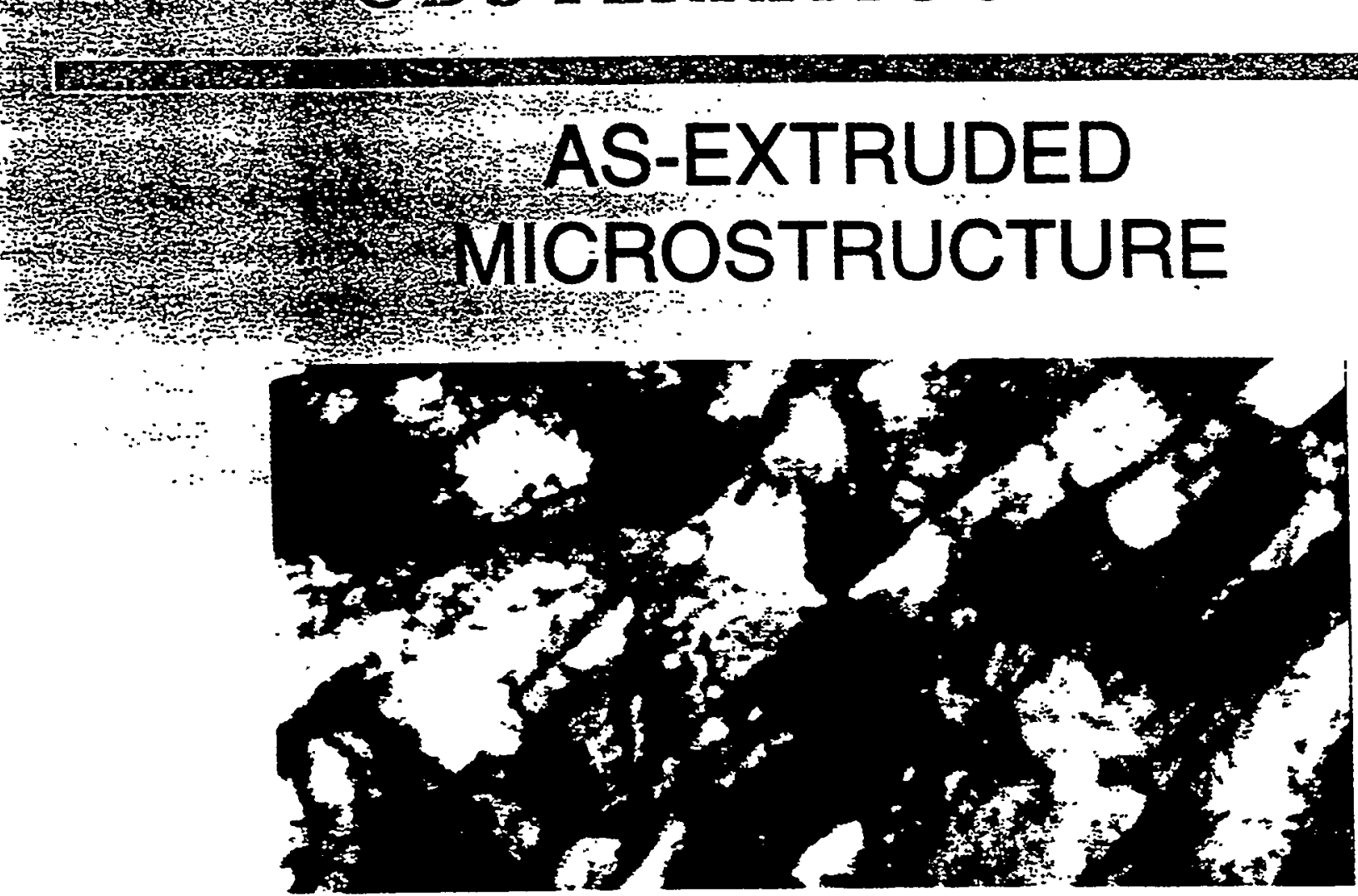

Longitudinal Section

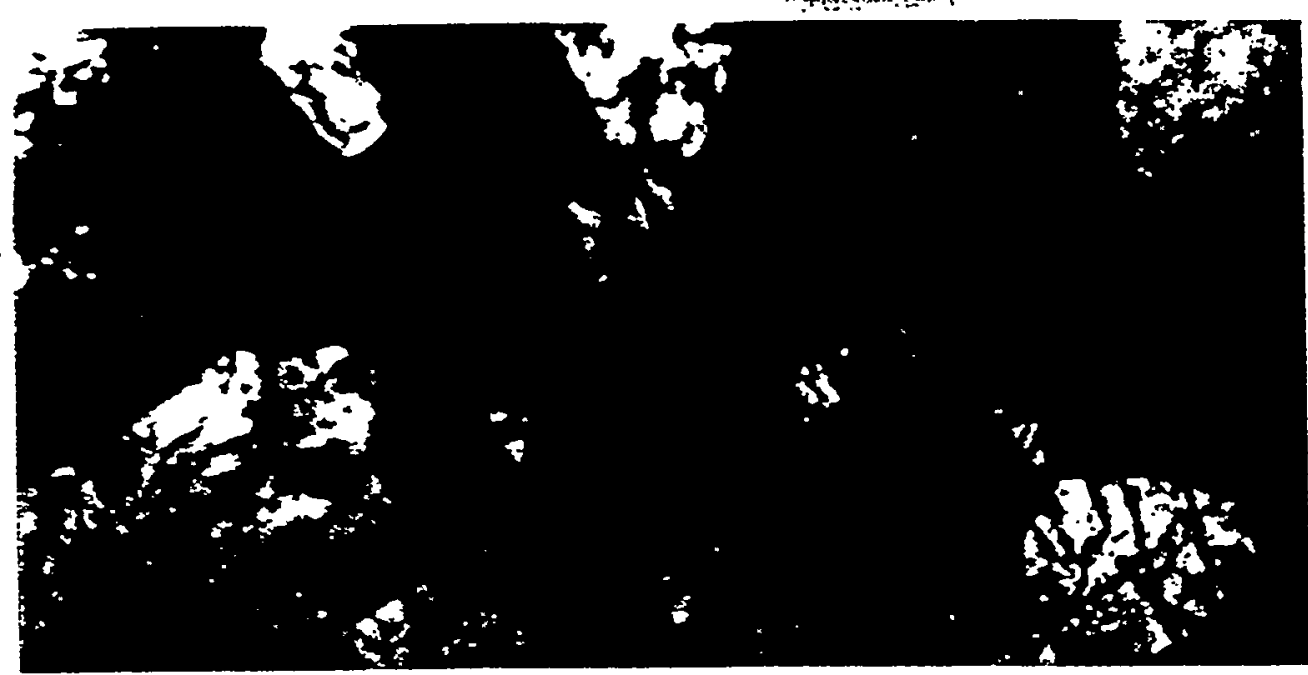

in:

Transverse Cross-section

$500 \mathrm{~nm}$ 


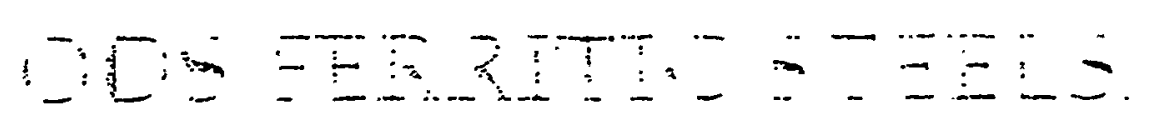

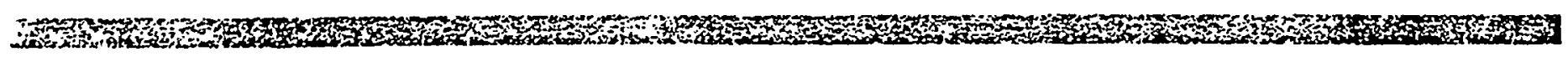
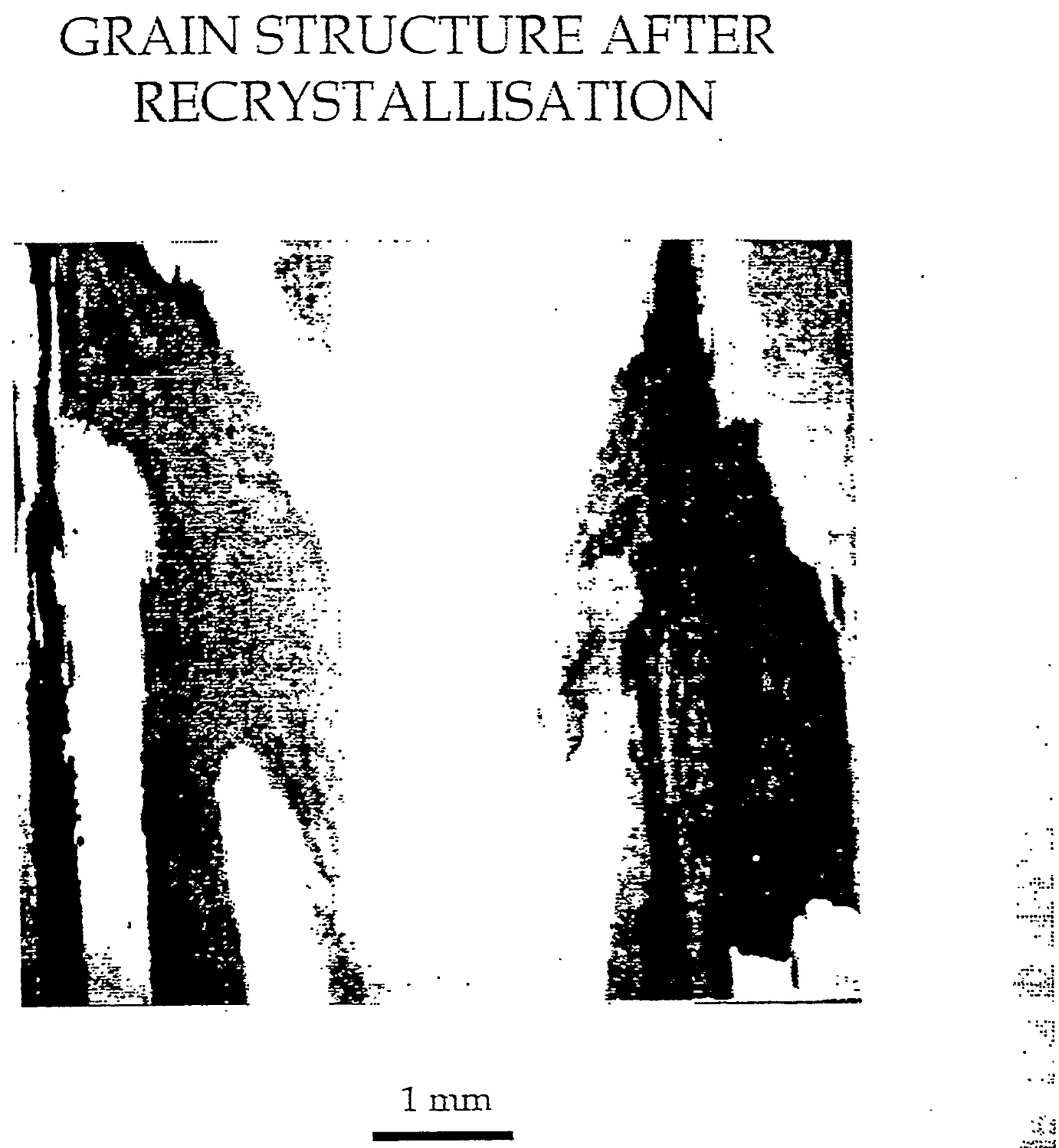


\title{
ODS FERRITIC STEELS
}

\section{Main characteristics :}

- Elongated grains:

\author{
d about $500 \mathrm{~nm}$, \\ aspect ratio: $20-50$
}

- High texture

- Anisotropic properties

- Grain size of recrystallised structure :

\author{
$\mathrm{d}>\mathbf{1} \mathrm{mm}$
}

- Recrystallisation temperature :

$$
\mathrm{T}>1300^{\circ} \mathrm{C}
$$

- Low ductility 


\section{ODS FERRITIC STEELS}

\section{RECRISTALLISATION TEMPERATURES}
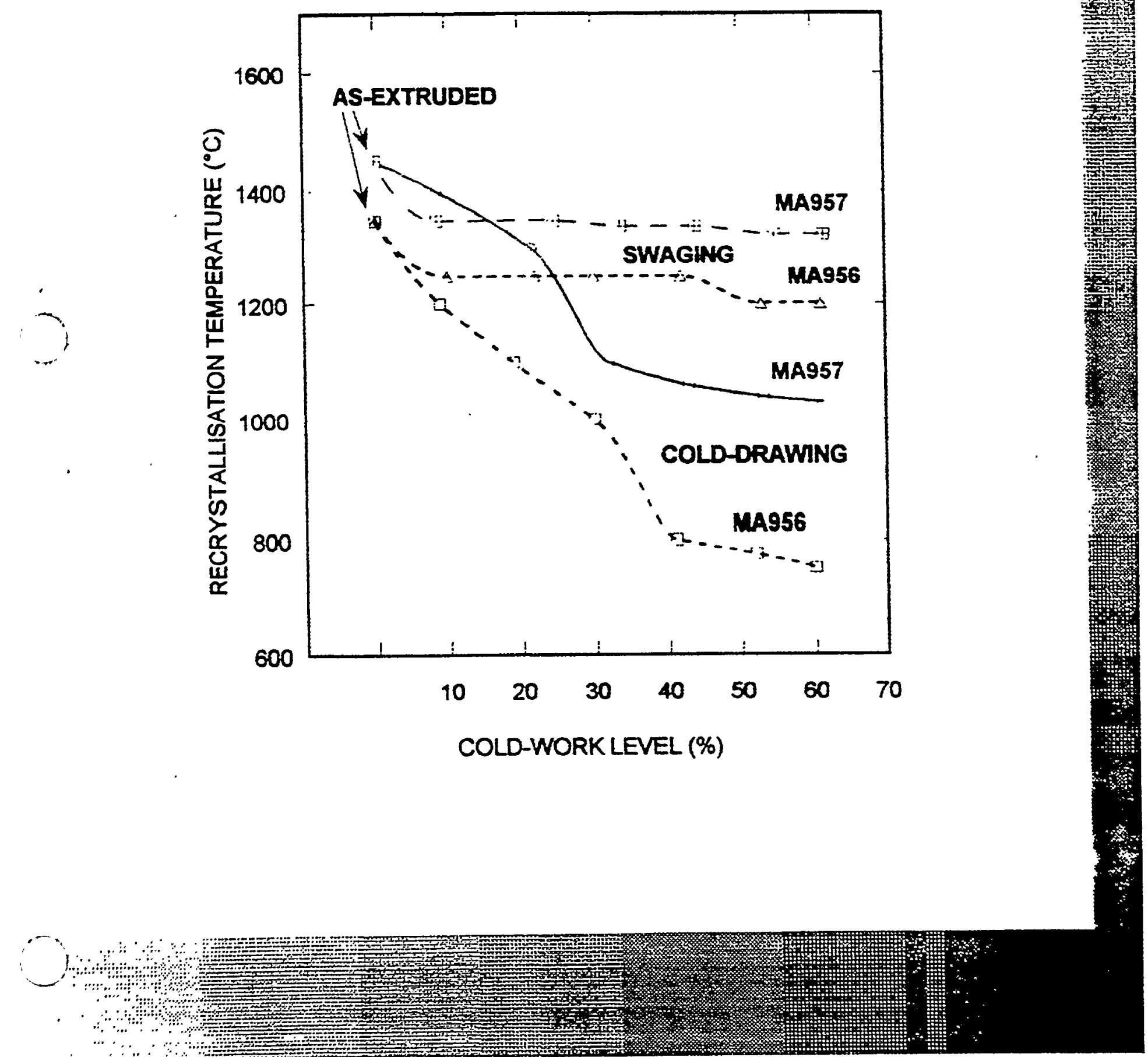


\section{ODS FERRITIC STEELS}

\section{MA957}

\section{(Fe $-14 \mathrm{Cr}-1 \mathrm{Ti}-0.3 \mathrm{Mo}-0.25$ Y2O3) \\ Intermediate grain size structure \\ (cold-drawing and recrystallisation)}

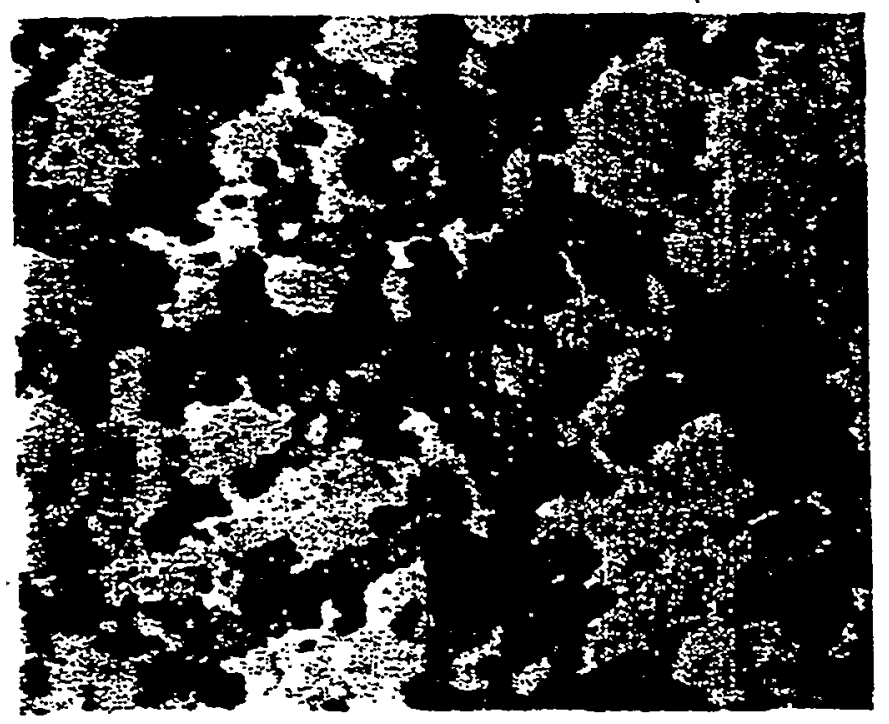

Cross-section

\section{Longitudinal section}

$100 \mu \mathrm{m}$

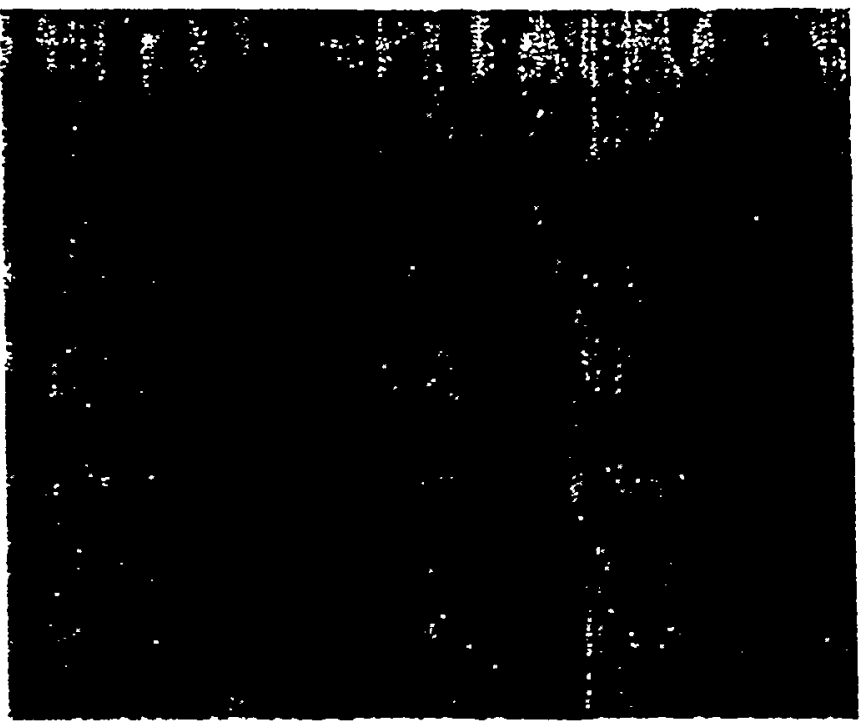




\section{ODS FERRITIC STEELS}

\section{Creep behaviour}

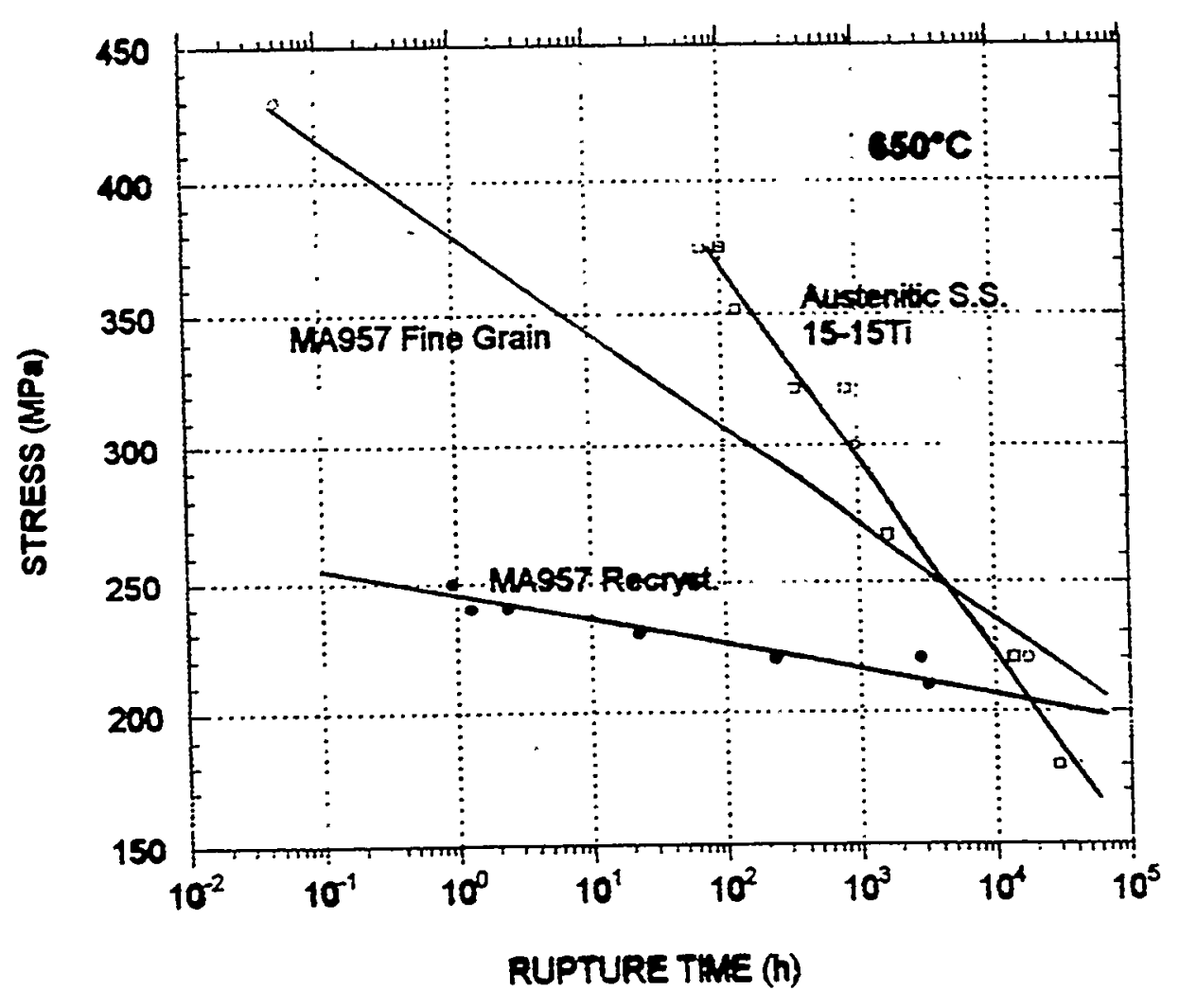

\section{Ageing behaviour}

\section{Precipitation of intermetallic phases $\chi$ phase $(\mathrm{Cr}, \mathrm{Ti})$ \\ Laves phase (Mo, $\mathrm{Ti} ; \mathrm{Cr}$ ) \\ $\alpha$ ' phase (Cr-rich, under irradiation)}

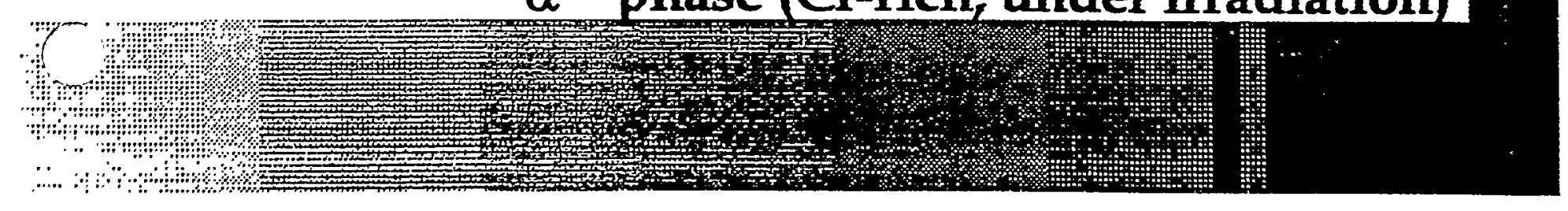




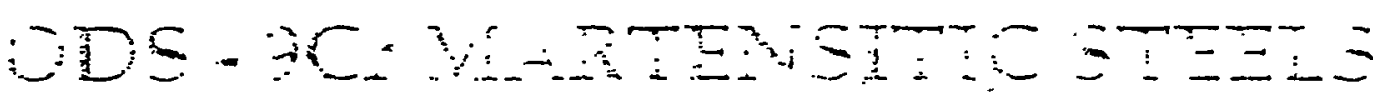

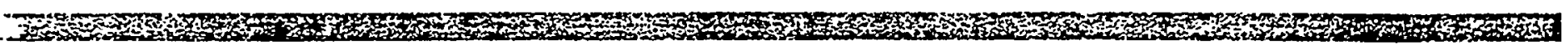
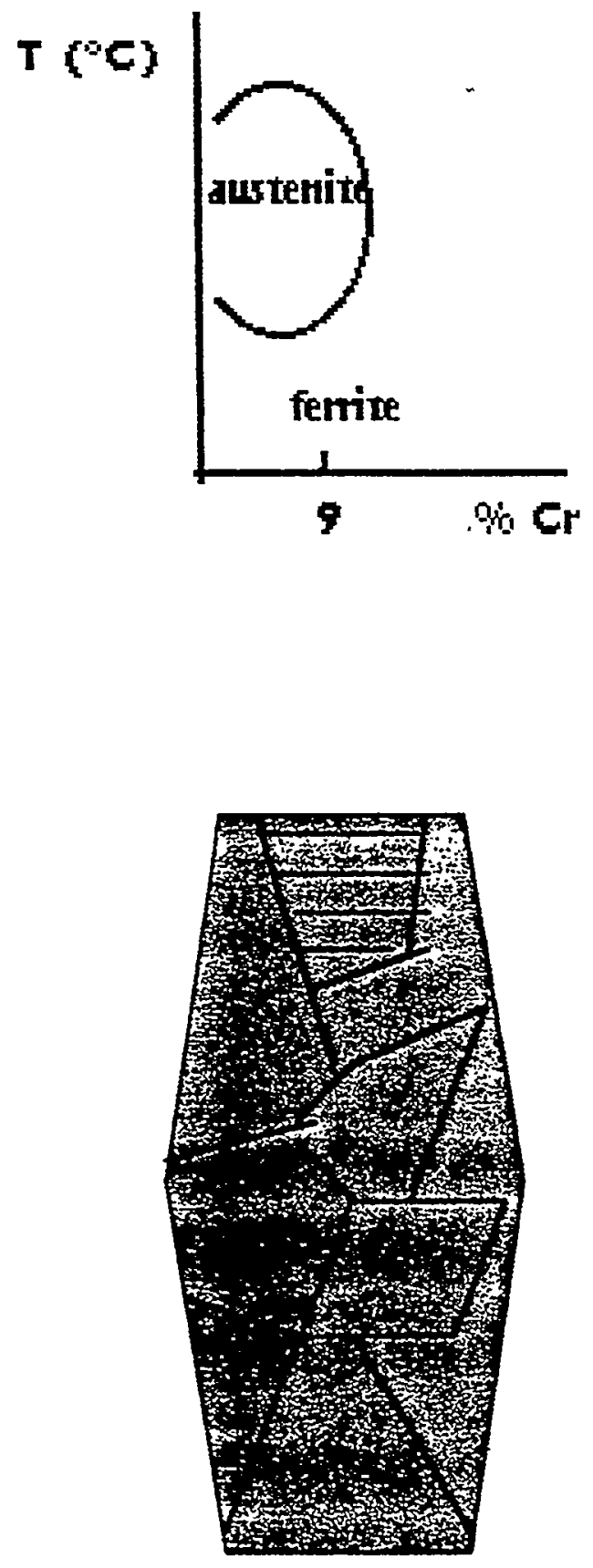

\section{Fast cooling: austenite $=>$ martensite} Several variants/ grain

$\therefore$ ,

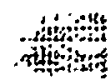




\section{ODS F/M STEELS \\ Why a 9Cr-ODS steel ? \\ To avoid embrittlement by intermetallic \\ Control of chemical composition} phase precipitation.

To decrease anisotropic properties of the fully ferritic materials.

$$
\begin{gathered}
\\
\text { ODS - 9Cr MARTENSITIC ALLOY } \\
9 \mathrm{Cr}-\mathrm{Mo}+\mathrm{Y}_{2} \mathrm{O}_{3} \\
9 \mathrm{Cr}-\mathrm{W}+\mathrm{Y}_{2} \mathrm{O}_{3}
\end{gathered}
$$

Martensitic transformation could decrease anisotropy.

$\mathrm{Y}_{2} \mathrm{O} 3$ dispersion assures good creep properties. 9Cr martensitic matrix have a good stability under ageing and irradiation. 


\section{ODS - 9Cr MARTENSITIC STEELS}

\section{OXIDE PARTICLE DISTRIBUTION}

(determined on carbon extraction replica by TEM from about 1000 particles for each material)

Frequency- size diagrams :

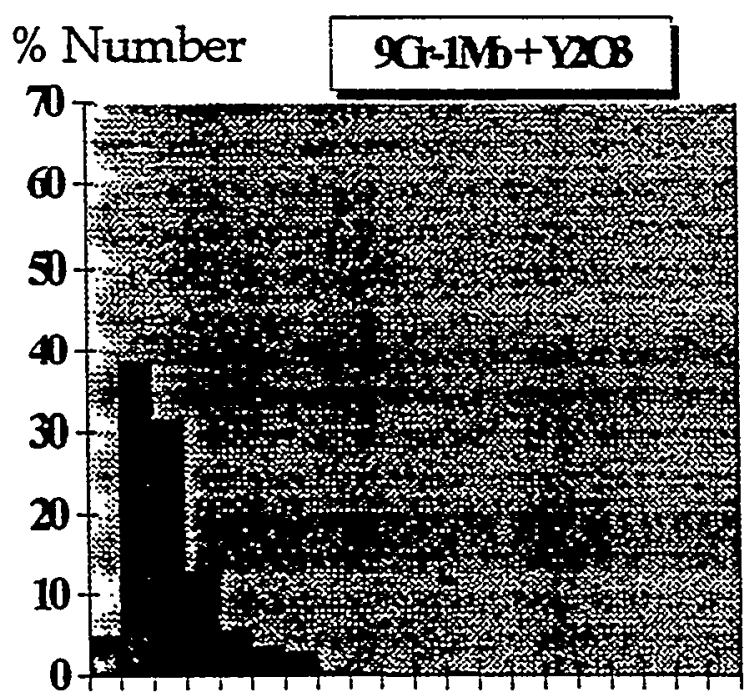

5152535455065758595 Chamer(m)

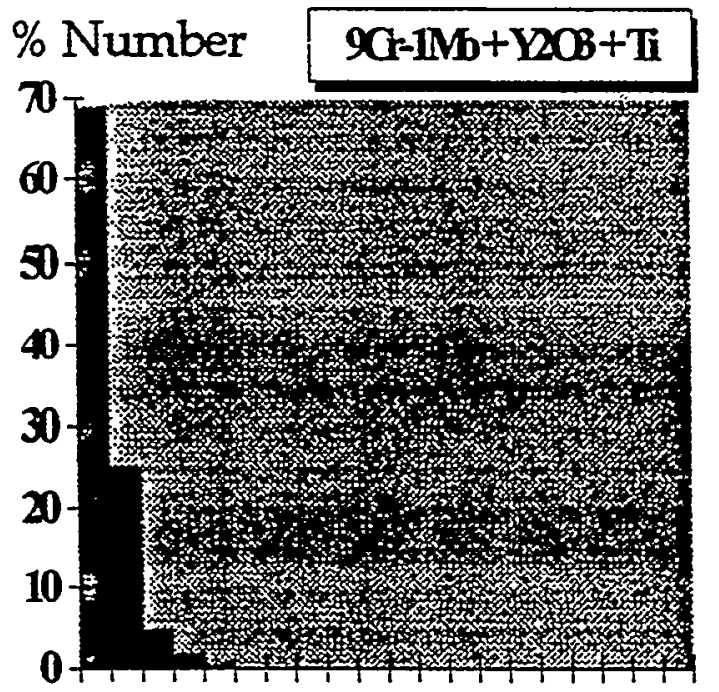

515253545565758595

Deanetrin)

\section{Chemical composition (at $\%$ ):}

$$
\begin{array}{ll}
9 \mathrm{Cr}-1 \mathrm{Mo}+\mathrm{Y}_{2} \mathrm{O}_{3}: & 55 \mathrm{Y}-34 \mathrm{Si}-11 \mathrm{Mn} \\
9 \mathrm{Cr}-1 \mathrm{Mo}+\mathrm{Y}_{2} \mathrm{O}_{3}+\mathrm{Ti} & 50 \mathrm{Y}-50 \mathrm{Ti}
\end{array}
$$




\section{ODS - 9CH STEELS}

\section{Equiaxed grain structure}

\section{Martensitic matrix}

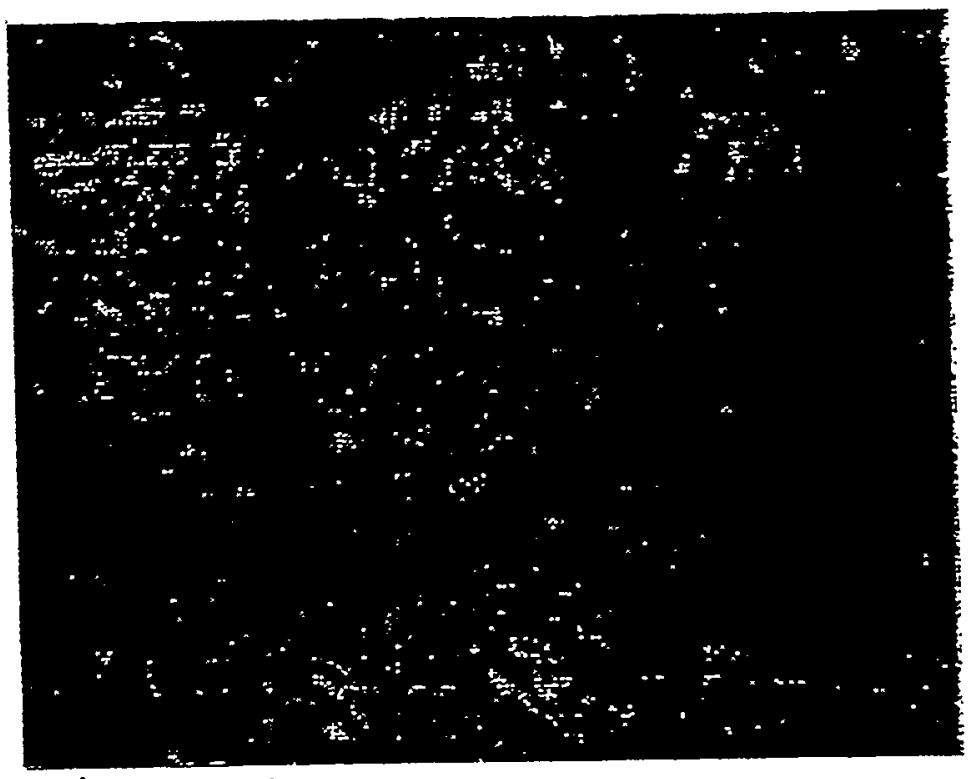

\section{Ferritic matrix}

Longitudinal sections

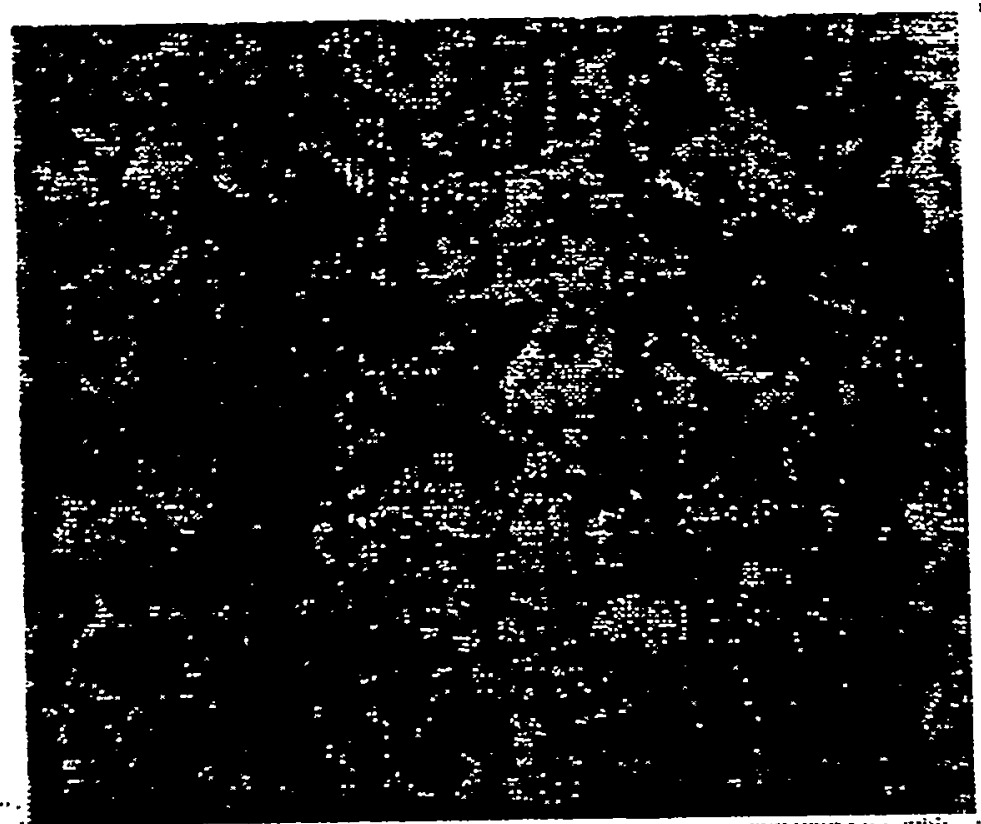

$10 \mu \mathrm{m}$ 


\section{cen \\ ODS - 9Cr MARTENSITIC STEELS}

Effects of Heat Treatments

Austenitisation :

No changes in hardness and grain growth in the range $1000-1250^{\circ} \mathrm{C}$.

Tempering :

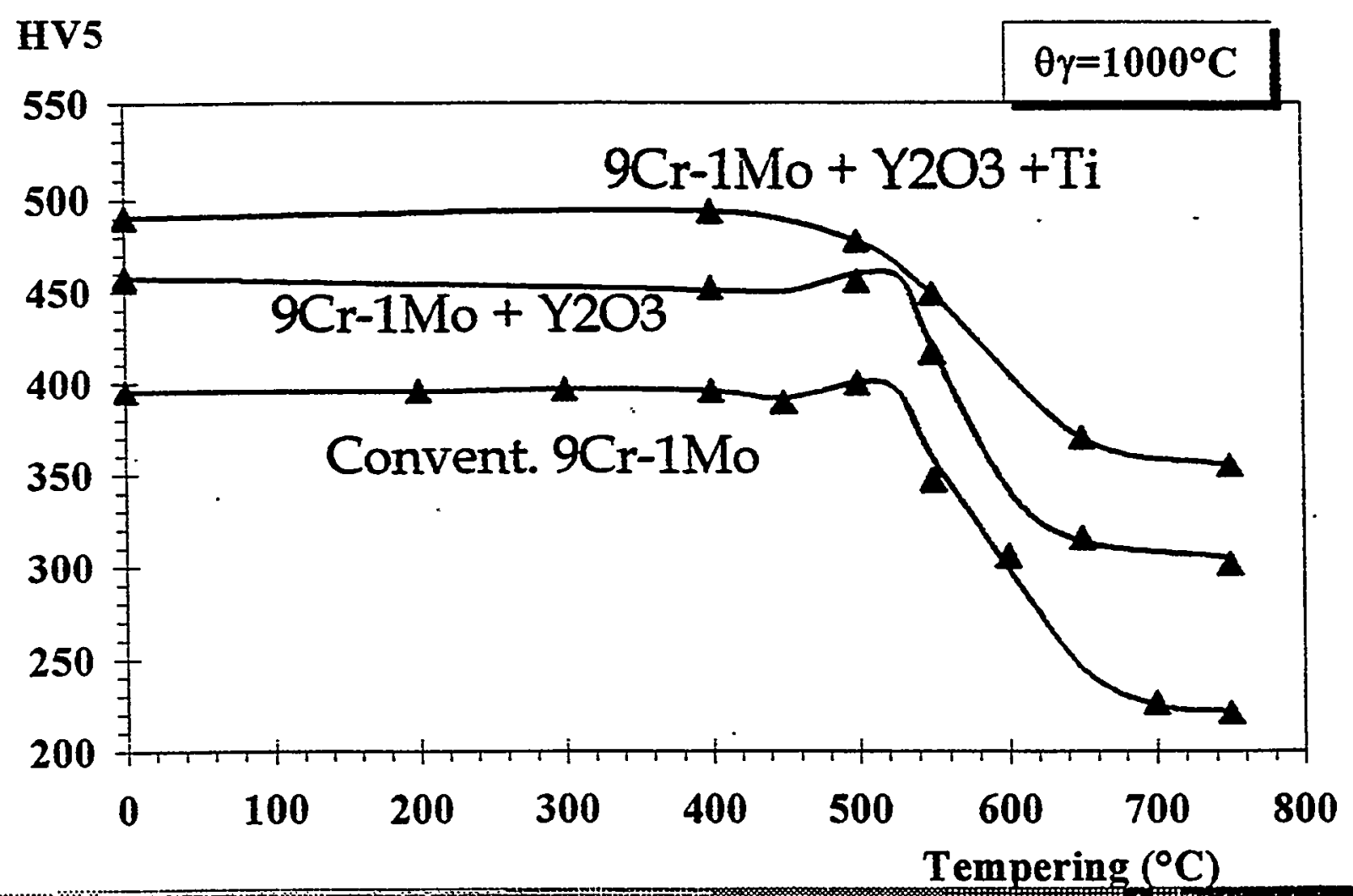




\section{cen \\ ODS - 9Cr MARTENSITIC STEELS}

\section{TENSILE PROPERTIES}

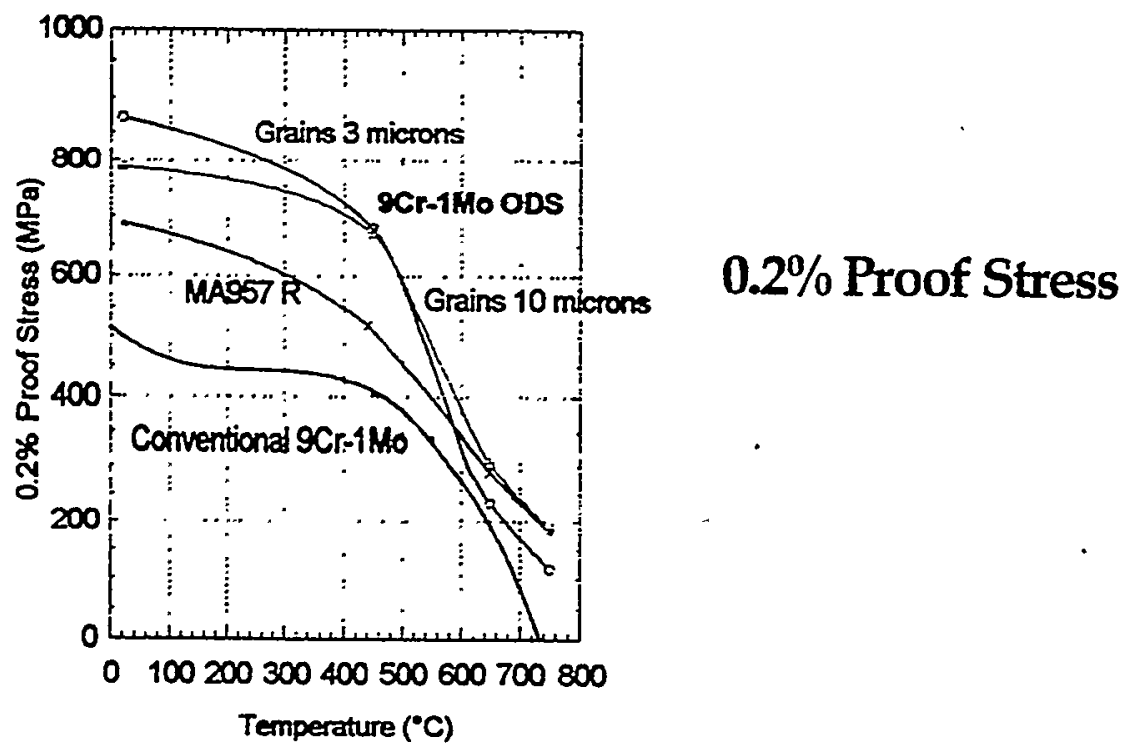

Reduction in area

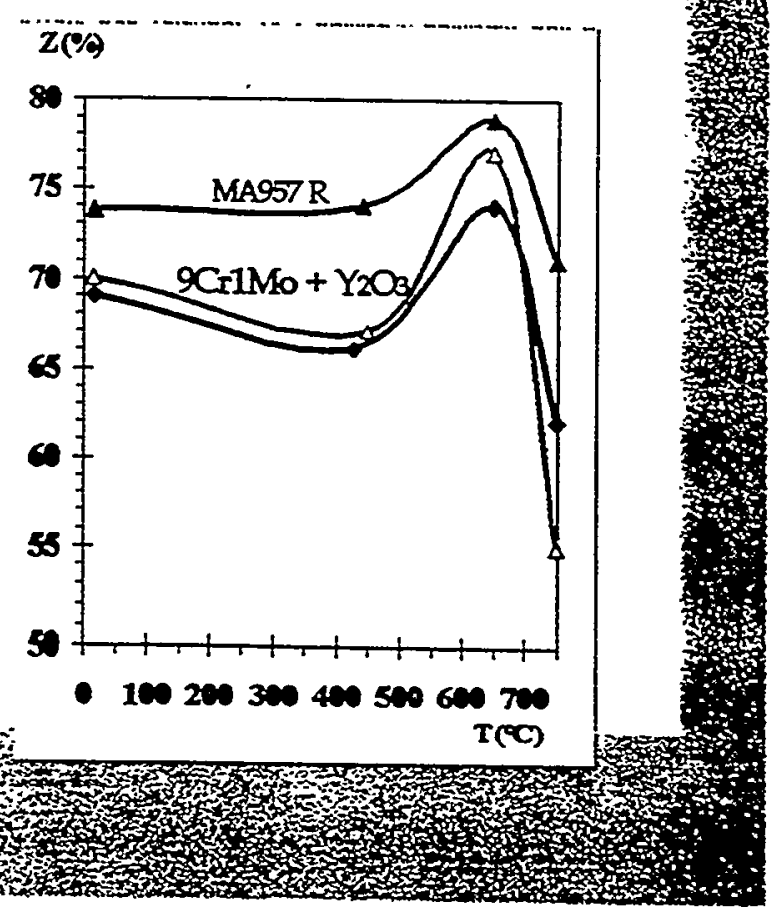


IEA WG Meeting on RAF

October 1-2, 1998

Petten, Nethrelands

\title{
Preliminary Studies for ODS/RAF Development
}

\author{
JAERI A. Hishinuma
}

\section{Background:}

- RAM Fe-(7-9) Cr ferritic srteels are the first candidate alloy for DEMO and beyond.

- However, the design window of the RAM for fusion reactors is not enough, (For example, see Fig.1)

- High temperature strength is required from viewpoints of

1) Safety under off-normal condition (i.e. $>600 \mathrm{MPa}$ at $600^{\circ} \mathrm{C}$ )

2) Application for other coolant system such as liquid metals or Hegas.

- ODS/RAM is one of the candidates for

1) Overlay materials for RAM (in near future)

2) Alternative materials of current candidate RAM (in future)

- Another advantage - good irradiation resistance (see Fig.2)

\section{Manufacturing process of ODS/RAM}

Raw powder of $\mathrm{Fe}-8 \mathrm{Cr}$ alloy and other elements: $<150 \mu \mathrm{m}$

Mechanical alloying: 10D type Attrition Mill,

Weight ratio : powder :ball $=1: 15$.

Rotating speed: $220 \mathrm{rpm}$

Rotating time: $48 \mathrm{hr}$

Hot extrusion: $1050^{\circ} \mathrm{C}$, extrusion ratio $=-7$

Heat treatments: $1050^{\circ} \mathrm{C} / 1 \mathrm{hr} / \mathrm{AC} \rightarrow 750^{\circ} \mathrm{C} / 1 \mathrm{hr} / \mathrm{AC}$ 


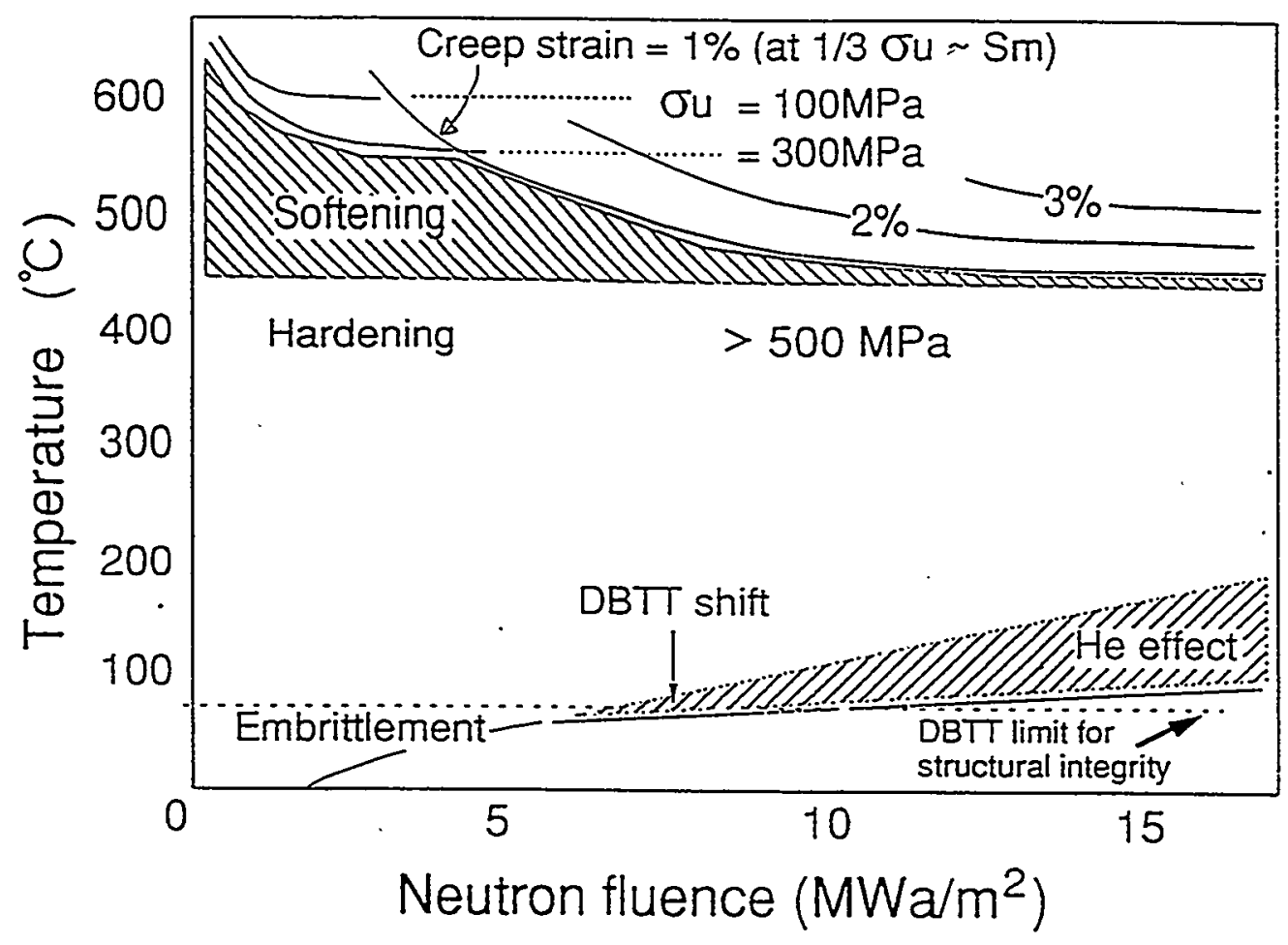

Fig. Service condition for F82H (estimated)

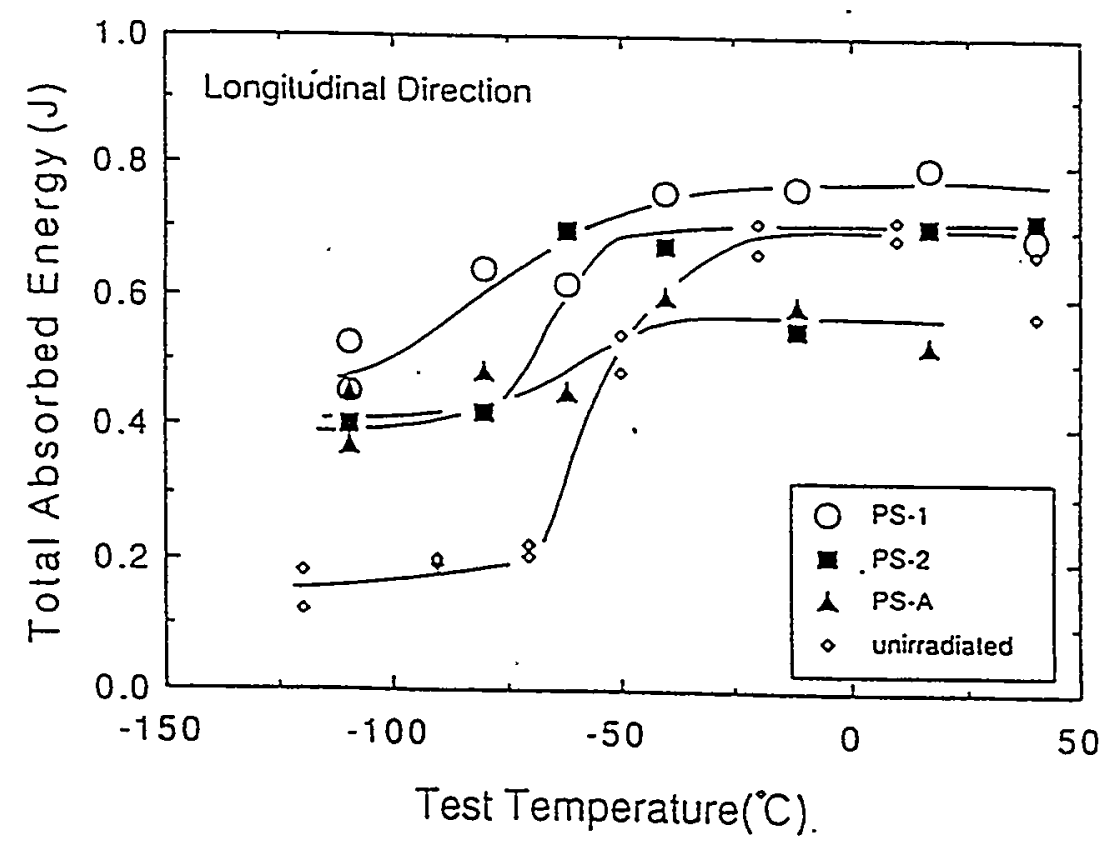


Table 1 Chemical compositions of ODS/RAM steels.

\begin{tabular}{|c|c|c|c|c|c|c|c|c|c|c|c|c|}
\hline 你号 元菜 & $\mathrm{C}$ & $\mathrm{Si}$ & $\mathrm{Mn}$ & $P$ & $\mathrm{~S}$ & $\mathrm{Ni}$ & $\mathrm{Cr}$ & W & $\mathrm{Ti}$ & $Y$ & 0 & $\mathrm{~N}$ \\
\hline G 91 & 0.12 & 0.006 & $<0.005$ & 0.001 & 0.003 & 0.052 & 7.99 & 0.096 & 0.092 & $\begin{array}{c}0.088 \\
(0.11)\end{array}$ & 0.12 & 0.0094 \\
\hline G92 & 0.12 & $<0.005$ & $<0.005$ & 0.001 & 0.003 & 0.012 & 7.94 & $<0.005$ & 0.093 & $\begin{array}{c}0.15 \\
(0.19)\end{array}$ & 0.13 & 0.0082 \\
\hline G 93 & 0.12 & $<0.005$ & $<0.005$ & 0.001 & 0.002 & $<0.005$ & 7.92 & $<0.005$ & 0.093 & $\begin{array}{c}0.23 \\
(0.29)\end{array}$ & 0.16 & 0.0088 \\
\hline G 94 & 0.12 & $<0.005$ & $<0.005$ & 0.001 & 0.002 & $<0.005$ & 7.92 & $<0.005$ & 0.18 & $\begin{array}{c}0.24 \\
(0.30)\end{array}$ & 0.15 & 0.0090 \\
\hline G95 & 0.12 & $<0.005$ & $<0.005$ & 0.001 & 0.002 & $<0.005$ & 7.88 & $<0.005$ & 0.27 & $\begin{array}{c}0.23 \\
(0.29)\end{array}$ & 0.15 & 0.0091 \\
\hline G 96 & 0.12 & $<0.005$ & $<0.005$ & 0.002 & 0.003 & $<0.005$ & 7.89 & 1.74 & 0.28 & $\begin{array}{c}0.23 \\
(0.29)\end{array}$ & 0.15 & 0.0096 \\
\hline
\end{tabular}

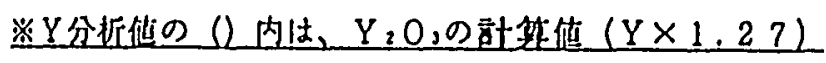




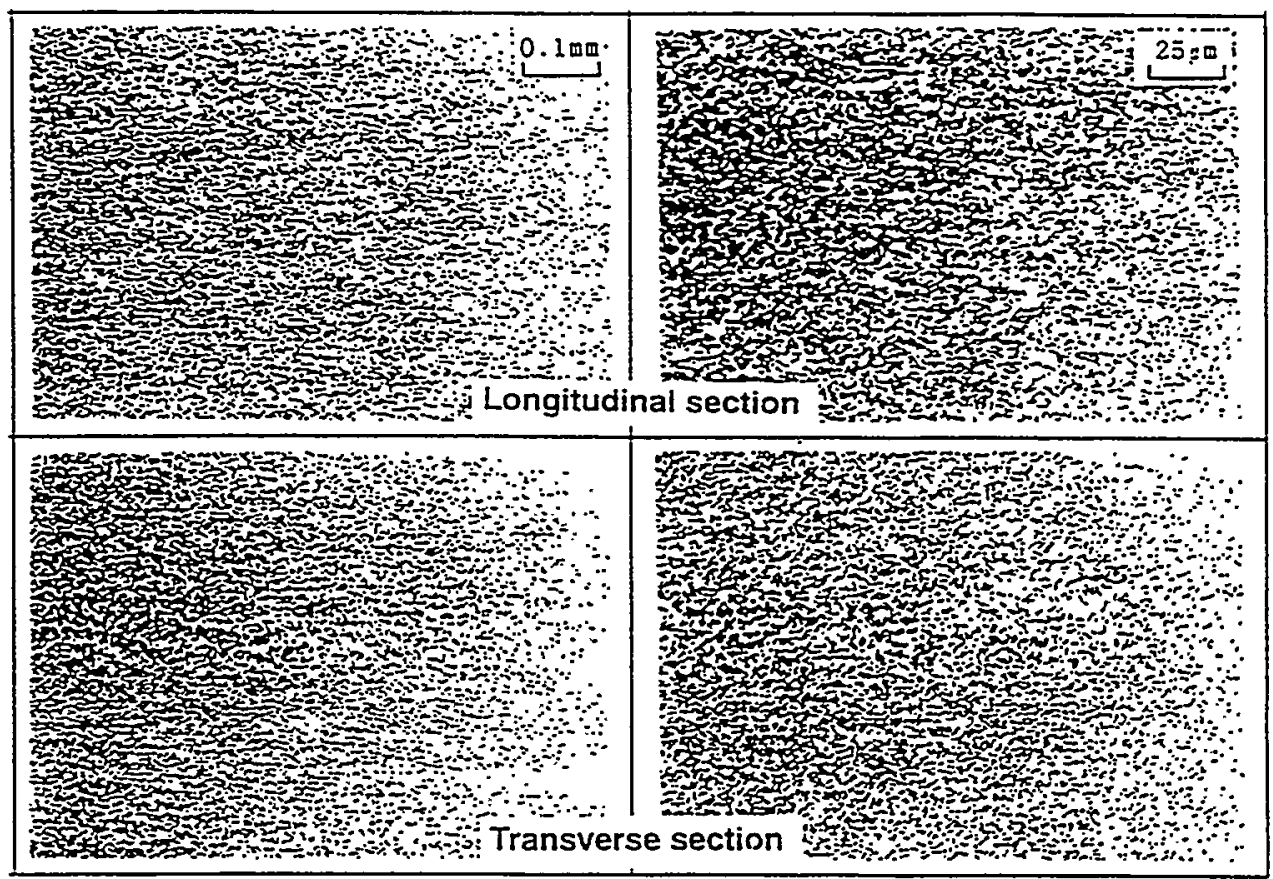

Fig.1 Optical microstructure of $\mathrm{G} 96$ (Fe-0.12C-8Cr-2W-0.3Ti-0.3 $\mathrm{Y}_{2} \mathrm{O}_{3}$ ) after annealing at $1050^{\circ} \mathrm{C}$ for $1 \mathrm{hr}$ and tempering at $750^{\circ} \mathrm{C}$ for $1 \mathrm{hr}$.
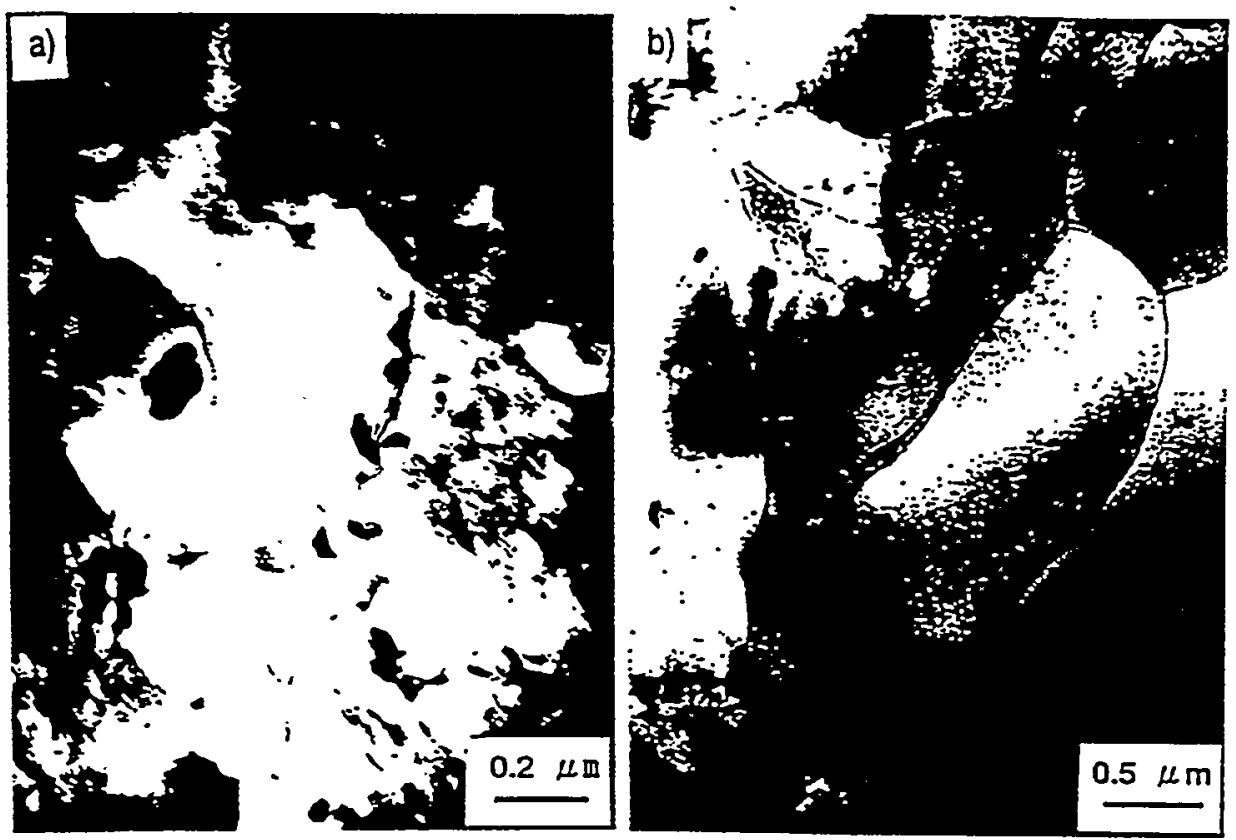

Fig. 2 TEM microstructure of $\mathrm{G} 96$ (Fe-0.12C-8Cr-2W-0.3Ti-0.3 $\mathrm{Y}_{2} \mathrm{O}_{3}$ ) after annealing at $1050^{\circ} \mathrm{C}$ for $1 \mathrm{hr}$ and tempering at $750^{\circ} \mathrm{C}$ for $1 \mathrm{hr}$. 


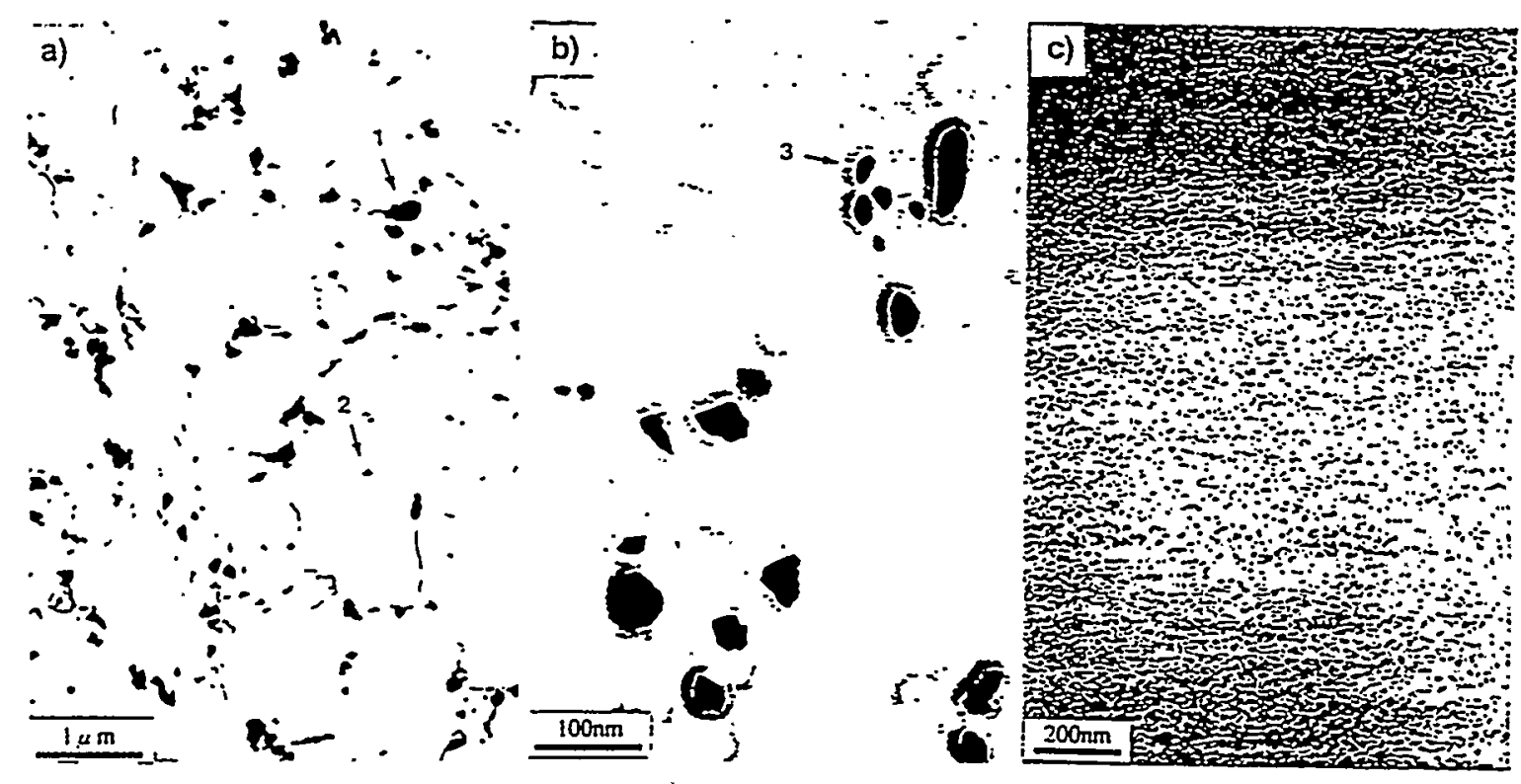

Fig. 3 TEM microstructure of dispersed particles of G96 (Fe-0.12C-8C $\mathrm{C}-2 \mathrm{~W}-0.3 \mathrm{TT}-0.3 \mathrm{Y}_{2} \mathrm{O}$ after annealing at $1050^{\circ} \mathrm{C}$ for $1 \mathrm{hr}$ and tempering at $750^{\circ} \mathrm{C}$ for thr by extraction carbo replica methods.

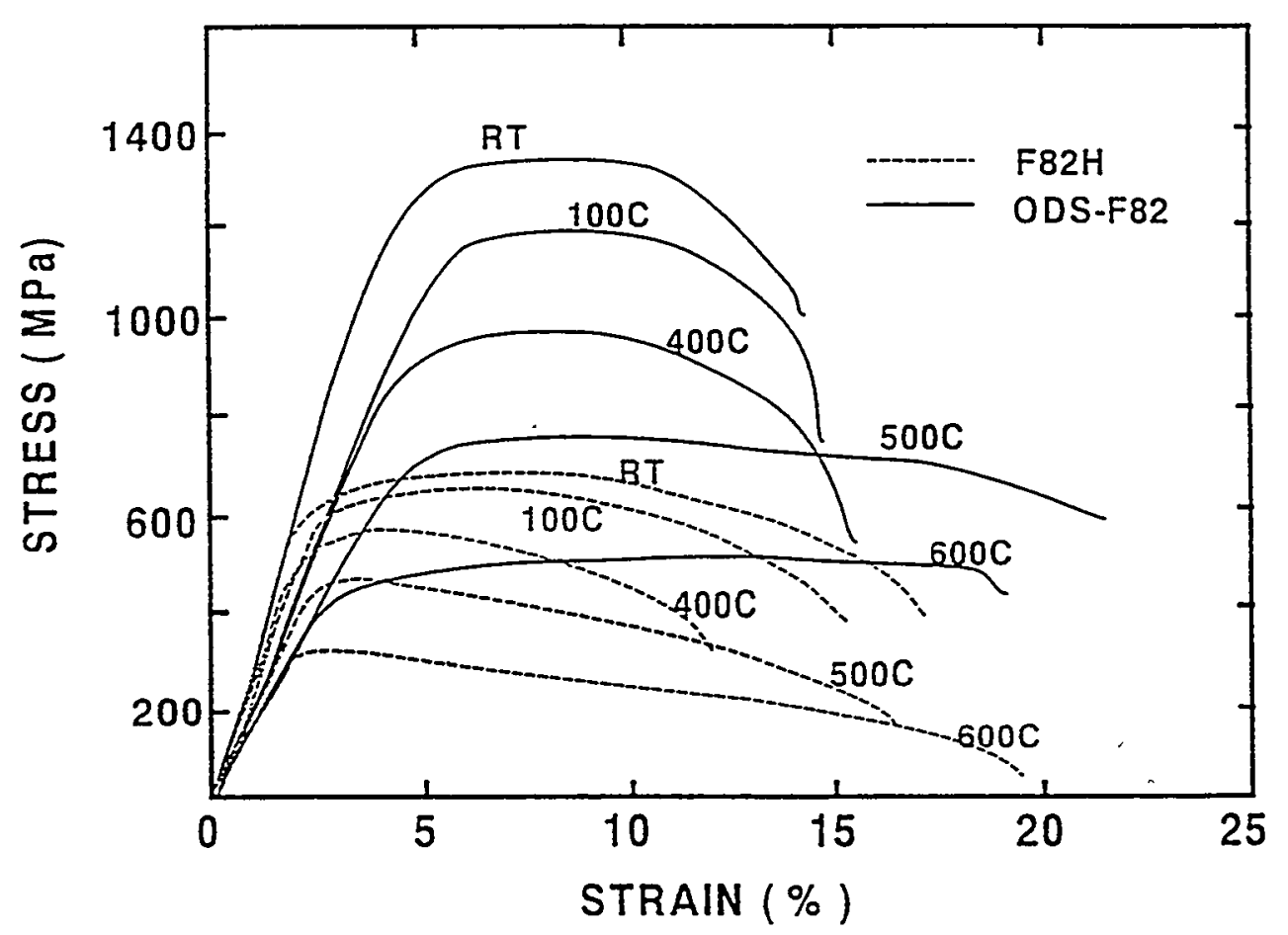

Fig. 4 Stress-strain curves of ODS/RAM G96 (Fe-0.12C-8Cr-2W-0.3Ti-0.3Y $\mathrm{O}_{3}$ ) . and RAM F82H at room temperature to $600^{\circ} \mathrm{C}$. 


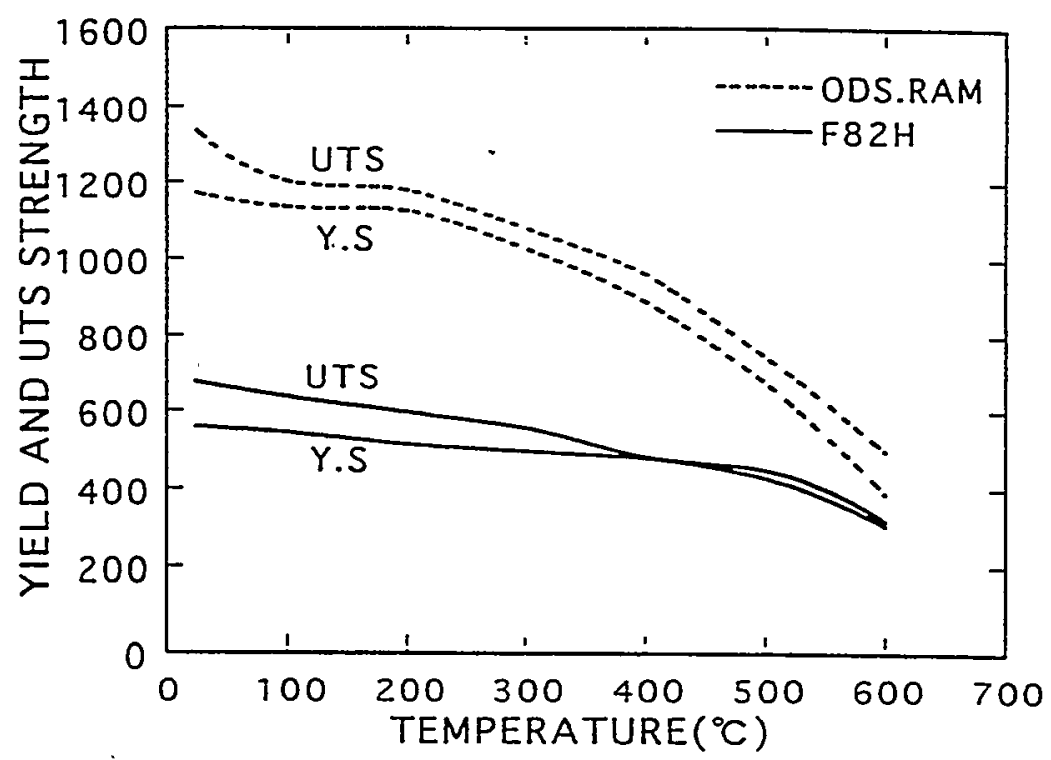

Fig.5 Temperature dependence of $Y S$ and UTS for ODS/RAM G96 ( $\mathrm{Fe}-0.12 \mathrm{C}-8 \mathrm{Cr}-2 \mathrm{~W}-0.3 \mathrm{Ti}-0.3 \mathrm{Y}_{2} \mathrm{O}_{3}$ ) and RAM F82H.
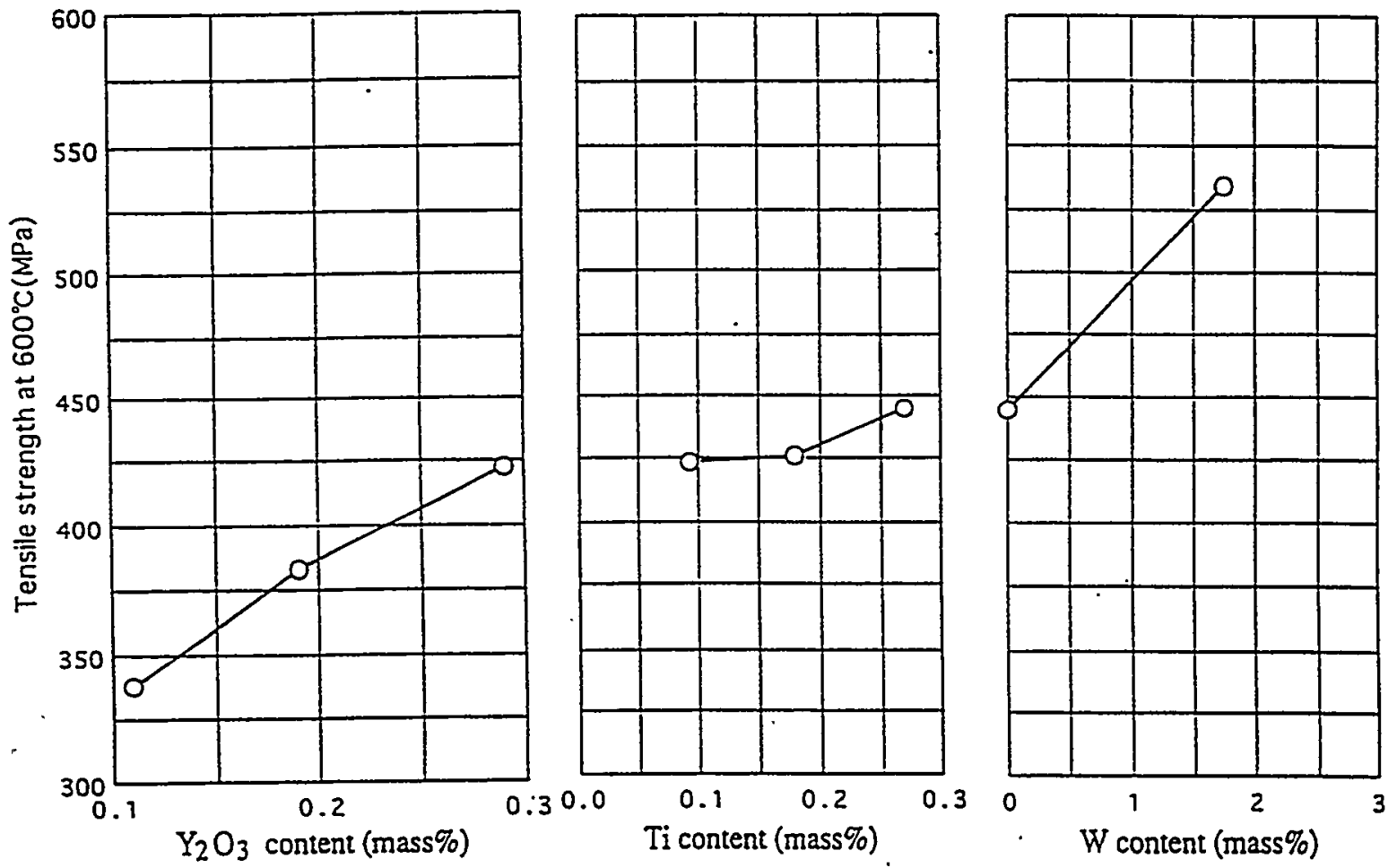

Fig. $6 \mathrm{Y}_{2} \mathrm{O}_{3}, \mathrm{Ti}$ and $\mathrm{W}$ contents dependence on fractured area transition temperature(FATT) of ODS/RAM 


\title{
Integrating Modeling, Experiment and Data Base Development: A Mechanism Based Approach to Developing Advanced Ferritic Steels for Fusion Applications
}

\author{
G. R. Odette \\ University of California Santa Barbara
}

IEA Workshop on Reduced Activation Ferritic Martensitic Steels

October 1-2, 1998

Petten Netherlands

Research Sponsored by the US Department of Energy and the US Nuclear

Regulatory Commission 


\section{Outline}

Recommendations of the Fusion Energy Systems Advisory Committee (FESAC) Review Panel on the Structural Materials Program (C. Baker and S. Harkness, Co-Chairs)

Recommendations on creating a knowledge base by the integration of modeling, experiment, engineering data compilations and structural integrity assessment methods

Roadmapping and an example from UCSB studies of RPV embrittlement

A brief update on some recent progress UCSB studies of FM steels 


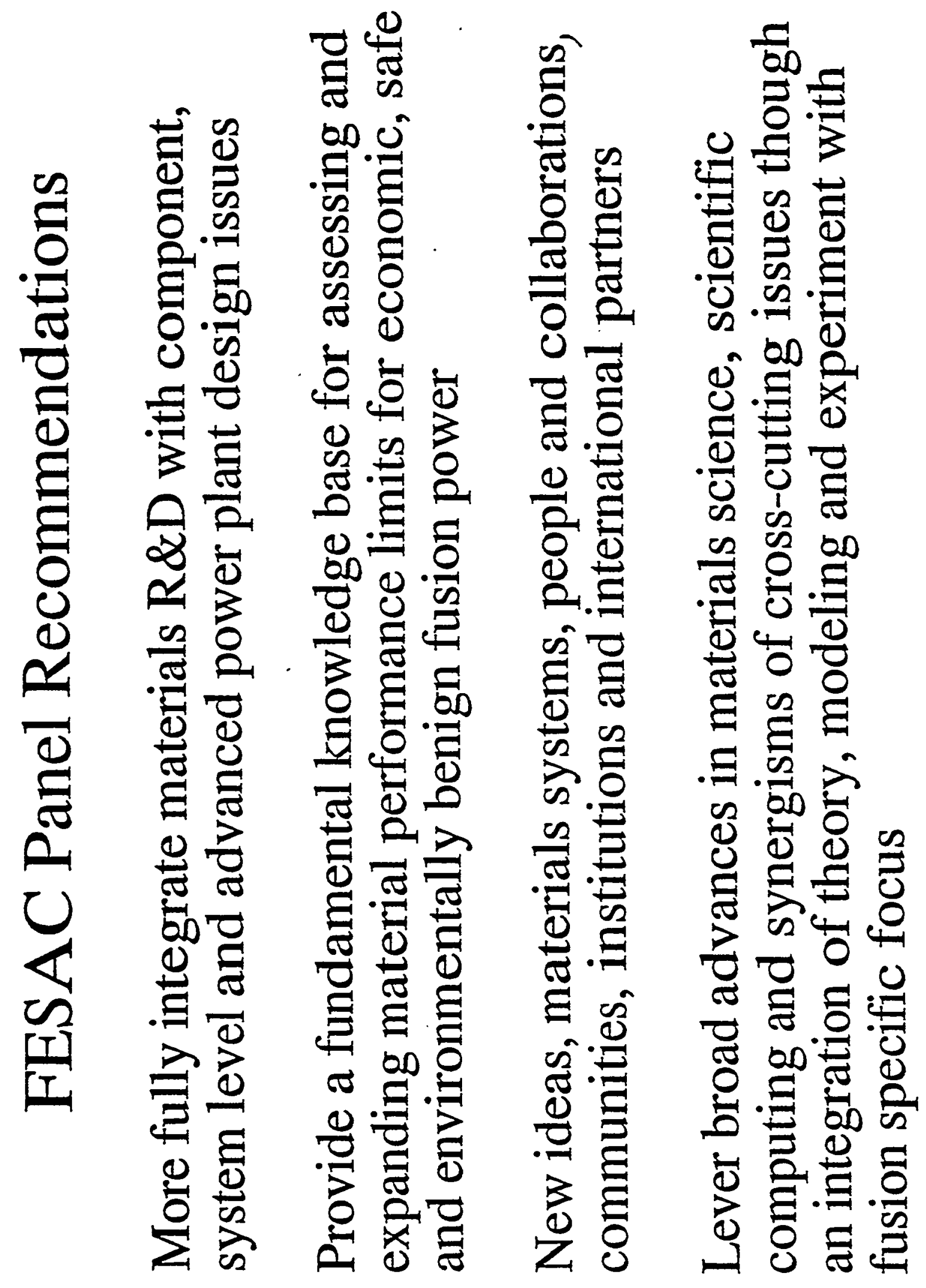


- Pursue fusion energy materials science and technology as a partner in the international effort.

- It is very important to coordinate the U.S. efifort with others based on the complexity of the materials challenge and the level of resources available in the U.S.

\section{Balance of the Proaram}

Based on the information on the U.S. and International Fusion Materials Programs that is included as Appendix 1, the Committee considered the appropriate balance between current programmatic elements in the context of the suggested goal and objectives and reached the following conclusions.

- The fusion materials program would benefit from an increase in the fraction of research related to the modeling of materiais behavior.

- The fusion materials program should maintain a focus on key issues related to in-vessel structures in a D-T fueled reactor, with significant emphasis on irradiation experiments. However, the fraction of research related to basic understanding of materials performance in the fusion environment should be increased. This increase is motivated by increased capability and sophistication of computer modeling, the need to make the most effective use of expensive and difficult-to-obtain materials data, and the desire to form stronger connections with the greater scientific community. This basic research should develop mechanistic, micro-structurally based models of irradiation effects on material properties. Such semi-empirical models can be used to evaluate, correlate and extrapolate engineering data; and to provide insight on pathways to improved materials. The modeling approaches should include direct simulation methods, like those based on molecular dynamics and Monte Carlo techniques, which are rapidly developing to link macro and meso size scales with rigorous treatments of key phenomena that occur at the atomic scale.

- In reccgnition of the new direction in the fusion energy sciences program, additional emphasis should be placed on developing the knowledge base for fusion materials.

- The fusion materiais research program should emphasize: a) innovative experiments to address key common and long-term issues; b) assessment of information to provide the best estimates of stress-temperaturedisplacements per atoms (dpa) -corrosion limits that can be systematically refined and improved with continuing research; c) increasingly reliable property predictions; and d) mechanism based approaches to improved materials. In the near and intermediate term, development of this knowledge base will primarily rely on intermediate dose fission reactor irradiation experiments coupled with an expanded basic research and 


\section{What is Modeling}

Not simply theoretical equations, but includes:

basic mechanism and controlled variable experiments on microstructures/properties focussed on key issues

fundamental laws of thermodynamics, kinetics, structure-property relations, micromechanics and macromechanics -- quantified in submodels

integrated models of embrittlement

statistical fits to the engineering data base 
modeling efiort. This efior should be coupled with the recommenced increased eiforts to develop micro-mecinanical predictive models.

- An increase in involvement with integrated component modeling would be beneficial to the fusion materials program.

- There is a great opportunity to develop and appiy modern computational toois to engineering design, analysis and simulation of in-vessei components. Such studies would benefit the materials research program by sharpening the understanding of performance requirements and promoting the development of advanced design methods needed to assure structural integrity without undue conservatism. These studies will also lead to major improvements in the engineering science underlying advanced in-vessel designs. While the fusion materials program should not take the lead in these studies, it shouid increase its participation in these activities.

An increased emphasis should be placed on resolving the key feasibility issues raised by each materials systems in conjunction with other parts of the Fusion Program. Examples for each of the three materials systems which are currently under some level of development include:

Ferritic Steels

- The suitability of the application of ferromagnetic materials for invessel components in magnetic confinement devices (in collaboration with researchers outside the materials program and with some emphasis on Tokamaks). This should include an analysis of the perturbations of the device's magnetic fields and issues of dynamic control of the plasma.

- The effect of irradiation, including the influence of helium, on fracture toughness, ductility and constitutive properies to better determine periormance constraints over the temperature range of interest.

- Alloy development and design concepts resulting in higher, maximum operating temperatures in a fusion irradiation environment.

- Vanadium Alloys

- The viability of electric insulators for candidate liquid metal/vanadium systems and the suitability of coolants other than liquid lithium and possible designs to accommodate the chemical reactivity of lithium (in collaboration with researchers outside the materials program). 
- The effect of irradiation, including the influence of helium, on fracture toughness, ductility and constitutive properties to better determine periormance constraints over the temperature range of interest.

* High temperature limitations with particular emphasis on the role of helium in creep and creep rupture and alloy development strategies to expand these limits.

- $\quad$ SiC/SiC Composites

- Structural joining methods and identification of properties and methods for designing thermal-mechanically loaded, inherently brittle structures.

- Irradiation effects on thermal conductivity, the stability of fibers and fiber coatings, including the effects of transmutations.

- Coatings and claddings to provide adequate hermiticity for helium coolants.

- Opportunities for new and innovative approaches to fusion materials research should be expanded.

- The motivation for new approaches is illustrated by Table 1, which shows the results of initial calculations done for the APEX study. The allowable neutron loads are sensitive functions of the assumed fraction of the charged particle energy that reaches the first wall, the thickness of the wall, the coolant temperature and the results from detailed analysis of the specific design. While, therefore, the absolute estimates of allowable neutron wall loadings would be expected to change in other studies, the general trends would be expected to be maintained. The preiiminary APEX calculations shown in Table 1 indicate the possible limitations for any of the current candidate materials in allowable neutron wall loading relative to the level of $7 \mathrm{MW} / \mathrm{m}^{2}$, which is viewed as an attractive design target. Future inclusion of materials which had previously been eliminated based on long term activation level considerations alone may open up the system design window. These exploratory efforts should consider the needs of innovative magnetic confinement concepts as well as the needs of inerial fusion energy.

- Therefore the introduction of new ideas and people to the fusion materials program should be encouraged. Modest increases in funding and a yearly opportunity for competitive, peer reviewed proposals are possible mechanisms to support such renewal. Innovative approaches to promoting sustained and mutually beneficial collaborations between laboratories, universities and industry should be developed. 


\begin{tabular}{|c|c|}
\hline \multicolumn{2}{|c|}{$\begin{array}{c}\text { Tabie I" } \\
\text { Peak Wall Load Limits for "Dry" First Wall }\end{array}$} \\
\hline Material (Interface Temperature) & $\begin{array}{l}\text { Allowable Peak Neutron Wall } \\
\text { Loading }\left(\mathrm{MW} / \mathrm{m}^{2}\right)\end{array}$ \\
\hline Ferritic Steel $\left(500^{\circ} \mathrm{C}\right)$ & 1.5 \\
\hline $\mathrm{V}-\mathrm{Cr}-\mathrm{Ti}\left(600^{\circ} \mathrm{C}\right)$ & 3.2 \\
\hline $\mathrm{SiC}-\mathrm{SiC}\left(700^{\circ} \mathrm{C}\right)$ & 2.5 \\
\hline $\begin{array}{l}\text { Oxide-Dispersion Strengthened } \\
\text { Ferritic (ODS) }\left(600^{\circ} \mathrm{C}\right)\end{array}$ & 2.6 \\
\hline $\mathrm{Nb} I \mathrm{Zr}\left(700^{\circ} \mathrm{C}\right)$ & 6.6 \\
\hline Tungsten $\left(800^{\circ} \mathrm{C}\right)$ & 8.8 \\
\hline $\begin{array}{l}\text { TZM }\left(800^{\circ} \mathrm{C}\right) \\
\text { T11 }\left(800^{\circ} \mathrm{C}\right)\end{array}$ & $\begin{array}{c}13 \\
11.6\end{array}$ \\
\hline
\end{tabular}

- Peer review should be expanded along with the ușe of well-defined measures of quality and progress towards program goals.

- Standards of quality, performance and progress towards program goals should be more fully developed and utilized at both universities and national laboratories. These metrics should be used to facilitate an ordering of programmatic priorities toward the more promising new developments in material systems. Periodic expert peer reviews in the context of these metrics should be supported. The review panels should include leading materials scientists from outside the program.

\section{Specialized Facilities for Materials Research and Development}

As part of the overall charge, the panel was asked to review the program efforts aimed at a fusion neutron source test facility including US involvement in the international fusion material irradiation facility (IFMIF). The Panel also considered the general topic of specialized neutron irradiation facilities for materials research and development.

The fusion environment in which the materials of the in-vessel system have to function is complex and arguably more challenging than faced by any other potential power generation concept.

*Information taken from "Exploring Novel High Power Density Concepts for Attractive Fusion Reactors," APEX Studies, M. Abdou, et al.

19. 


\section{Issues}

Clearly need to know basic constitutive and failure properties for design and operation

Changes in key properties in fusion environments function of many variables and variable combinations

$$
\Delta \mathrm{P}=\mathrm{f}(\phi, \phi(\mathrm{E}), \phi \mathrm{t}, \mathrm{T}, \mathrm{comp} . / \mathrm{imp} ., \mathrm{TMT}, \ldots . .)
$$

No fusion experimental facility, reactor experiments are expensive and time consuming, and higher temperature studies will require higher $\phi t$ irradiations - must extrapolate based on models and mechanism experiments

Not only radiation effects 


\section{'Roadmapping'}

Realistically identify what the future product and buyer

Identify the existing and new needed constituents to meet objectives (people, tools, materials, support resources, organizational structure, ...)

Provide plans about how various elements fit together (a building and not just bricks)

Methods for assessment and modification of roadmap (knowledge based flexibility of paramount importance)

Enhanced ways for people to interact, cooperate and contribute 


\section{RPV Embrittlement 'Roadmap'}

\section{Products:}

$\Delta \mathrm{T}_{\mathrm{cvn}, \mathrm{k}}=\mathrm{f}(\mathrm{Cu}, \mathrm{Ni}, \mathrm{P}, \mathrm{HT}, \phi \mathrm{t}, \phi, \mathrm{T}, \ldots .$.$) physically based,$ calibrated to engineering data base for regulations

aid in technical judgments

early warnings of potential technical surprises

improved methods 


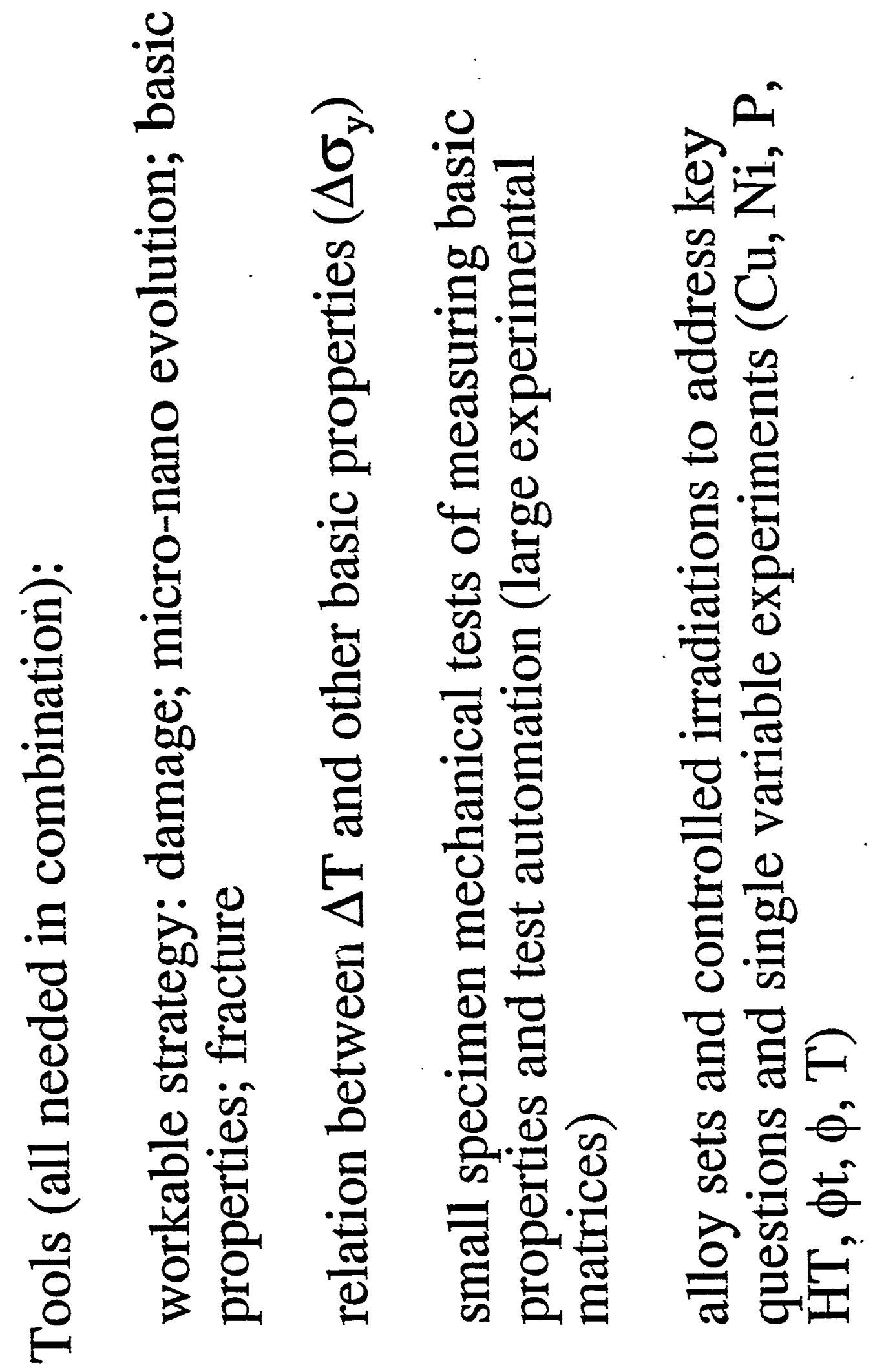


Tools (cont.):

microstructural characterization methods (SANS, AP, FEGSTEM, PIA)

verification and ties to 'real' steels and environments (surveillance samples and PREDB)

modeling (defect production, transport and fate, thermodynamic, kinetic, $\mathrm{MD}, \mathrm{MC}$, structure property, macromechanical, micromechanical) - key role of cascades \& thermodynamics in multiconstituent alloys

integration and multiscale-physics model of $\Delta T=f(\ldots .$.

statistical fits to the data base 


\section{Assessments and Modifications:}

verification experiments and tests and data fits (theory led observation in many cases)

learn from mistakes, exploit new opportunities (IVAR delay, piggyback experiment)

develop new capabilities while meeting nearer term objectives (CM-FR)

new initiatives (RPV Master Curve) 


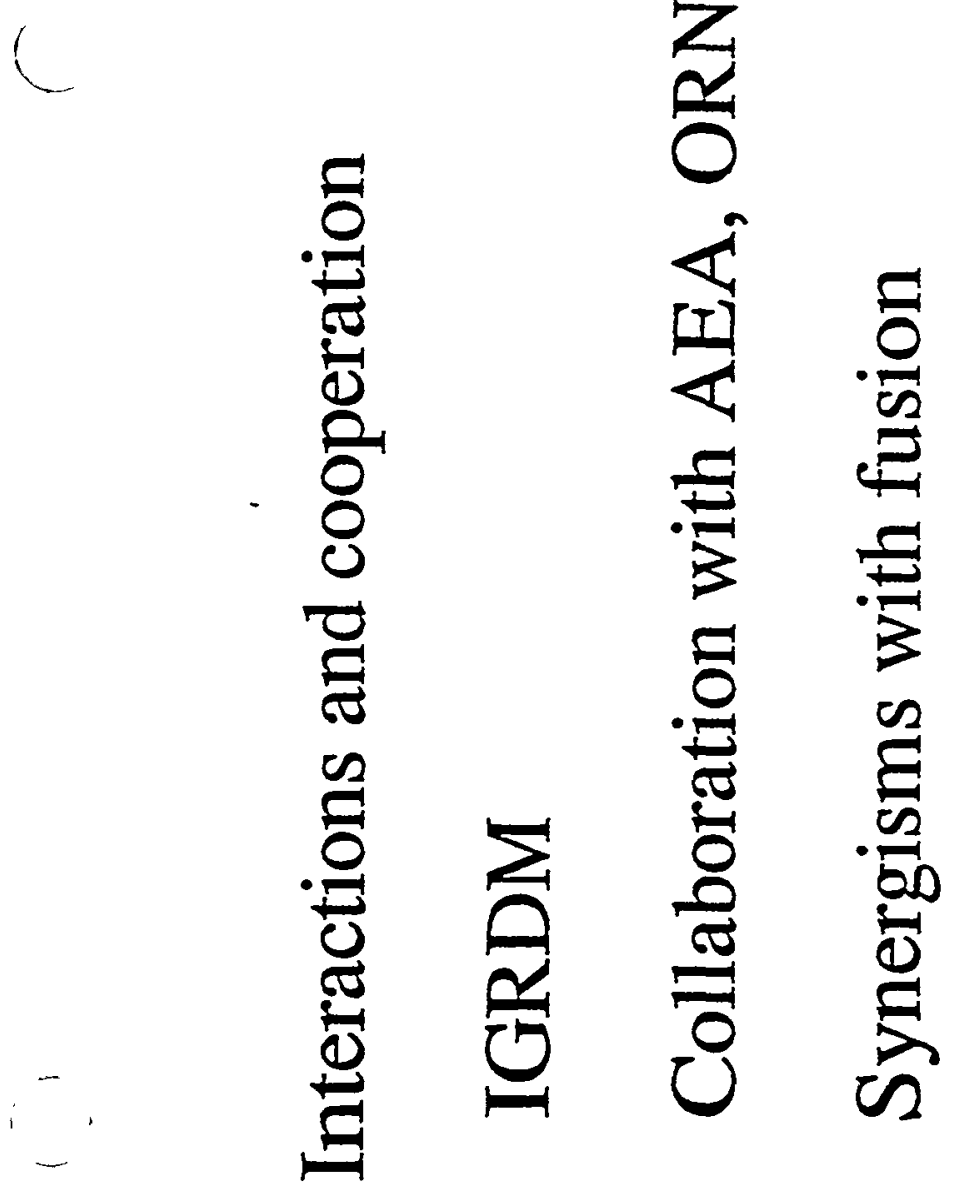




\section{Pressure Vessel Embrittlement}

- Exposure to neutrons embrittles pressure vessel steels (in both charpy and fracture toughness tests) as manifested by transition temperature increases $(\Delta T)$ and upper shelf decreases ( $\Delta U S E)$

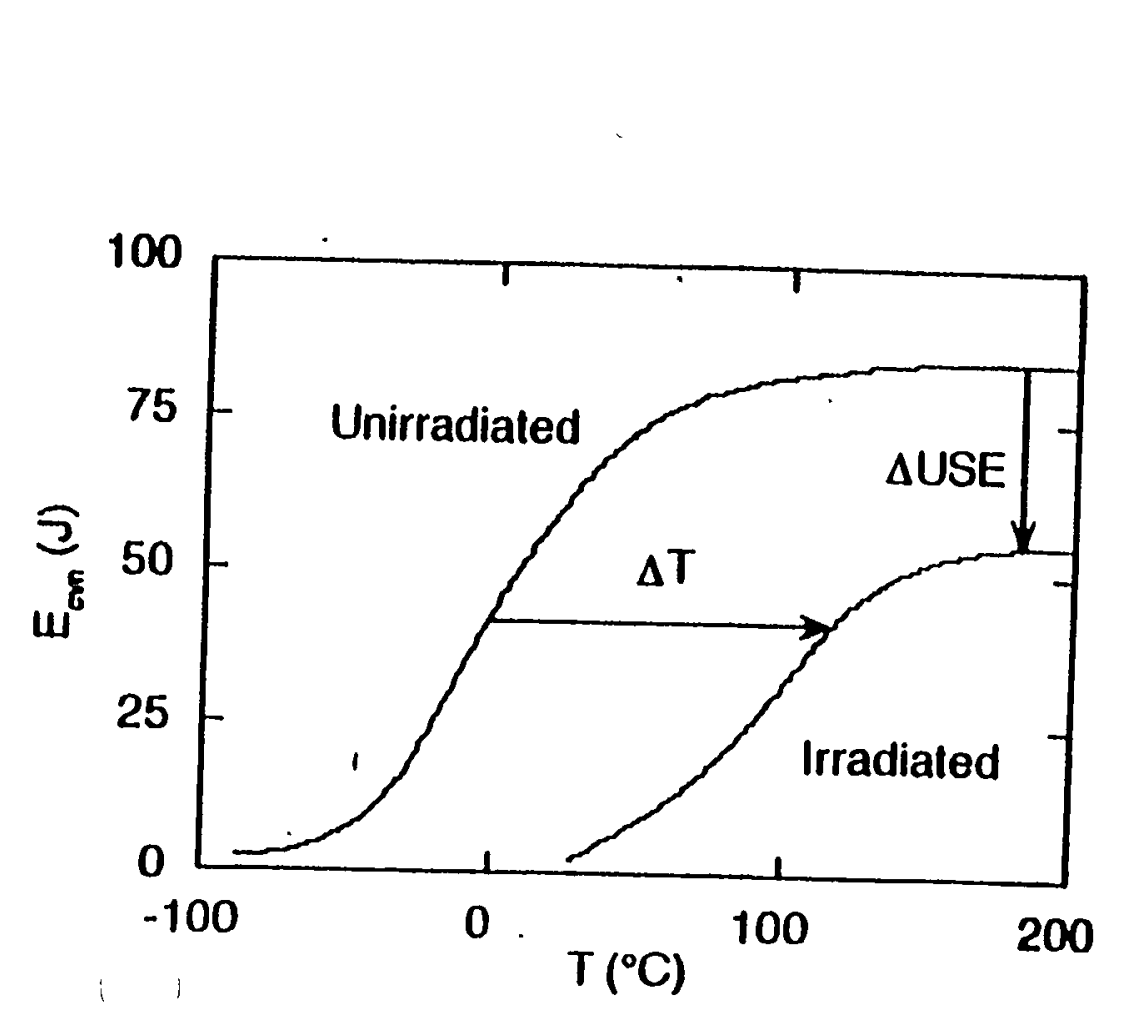

Alloys

- Q\&T Mn-Mo-(...) platcs, forgings and welds

\section{Variables}

- $\mathrm{Cu}(<0.5 \%), \mathrm{Ni}(<1.5 \%), \mathrm{P}(<0.03 \%)$

- Microstructure and hcal licalment

- Irrad. temp. $\left(T \approx 255-310^{\circ} \mathrm{C}\right)$

- Fluence $\left(\phi 1<6 \times 10^{23} \mathrm{n} / \mathrm{m}^{2}\right)$

- Flux $\left(\phi \approx 10^{12}-10^{1.5} \mathrm{n} / \mathrm{m}^{2}-\mathrm{s}\right.$; tests ill $\left.\phi \approx 10^{16}-10^{18} \mathrm{n} / \mathrm{m}^{2}-\mathrm{s}\right)$

Synergistic Interactions 


\section{Multiscale Modeling of Neutron Irradiation Embrittlement}

\section{Models}

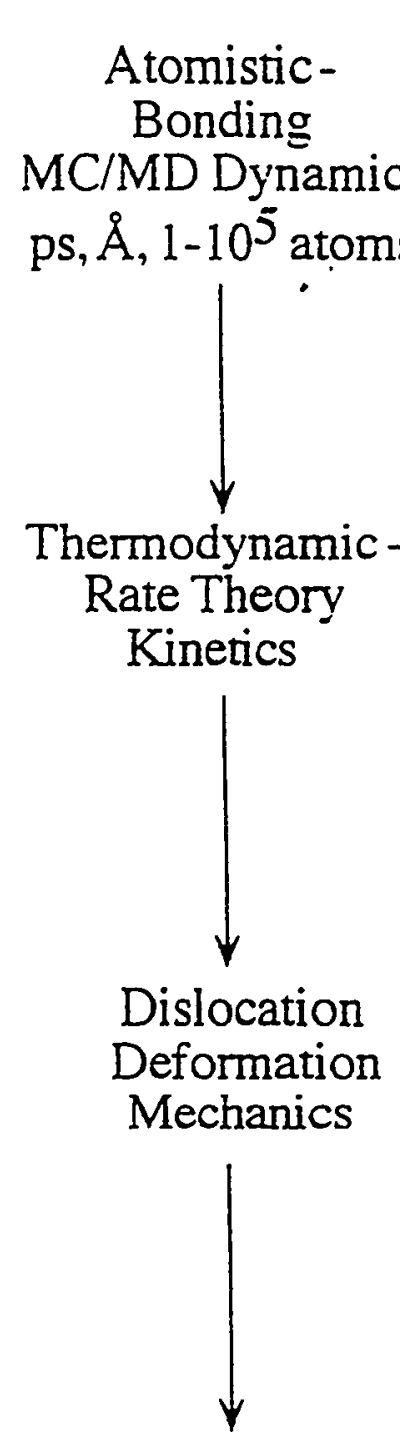

Macroconstitutive Local

FractureMechanics

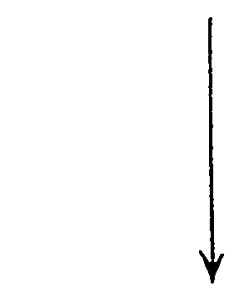

IndependentPhysical lalidation of Engineering Database (Gs, m, $10^{31}$ atoms)
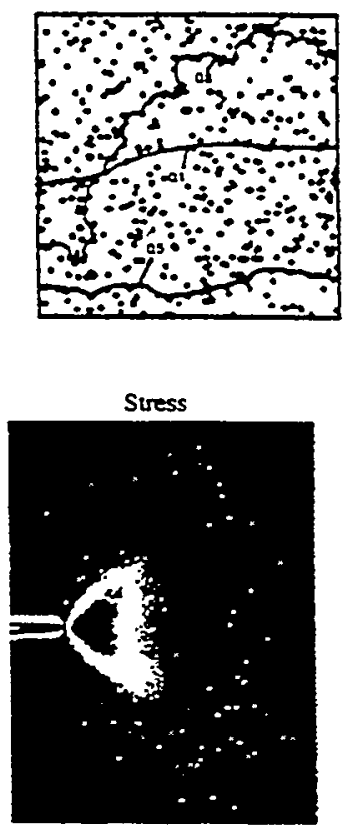

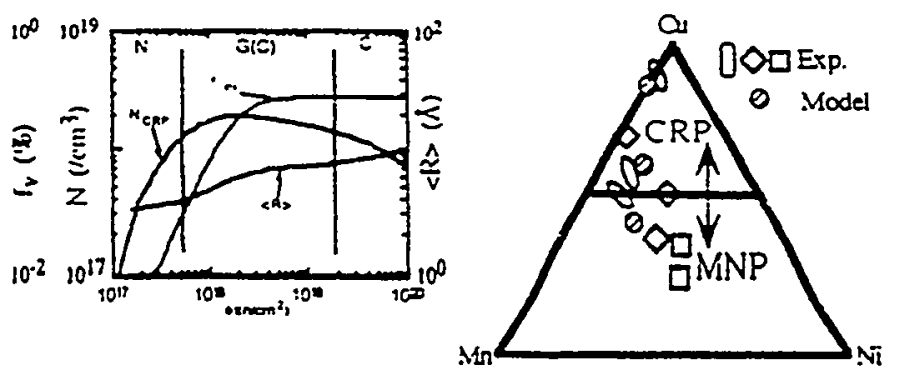

Experiments
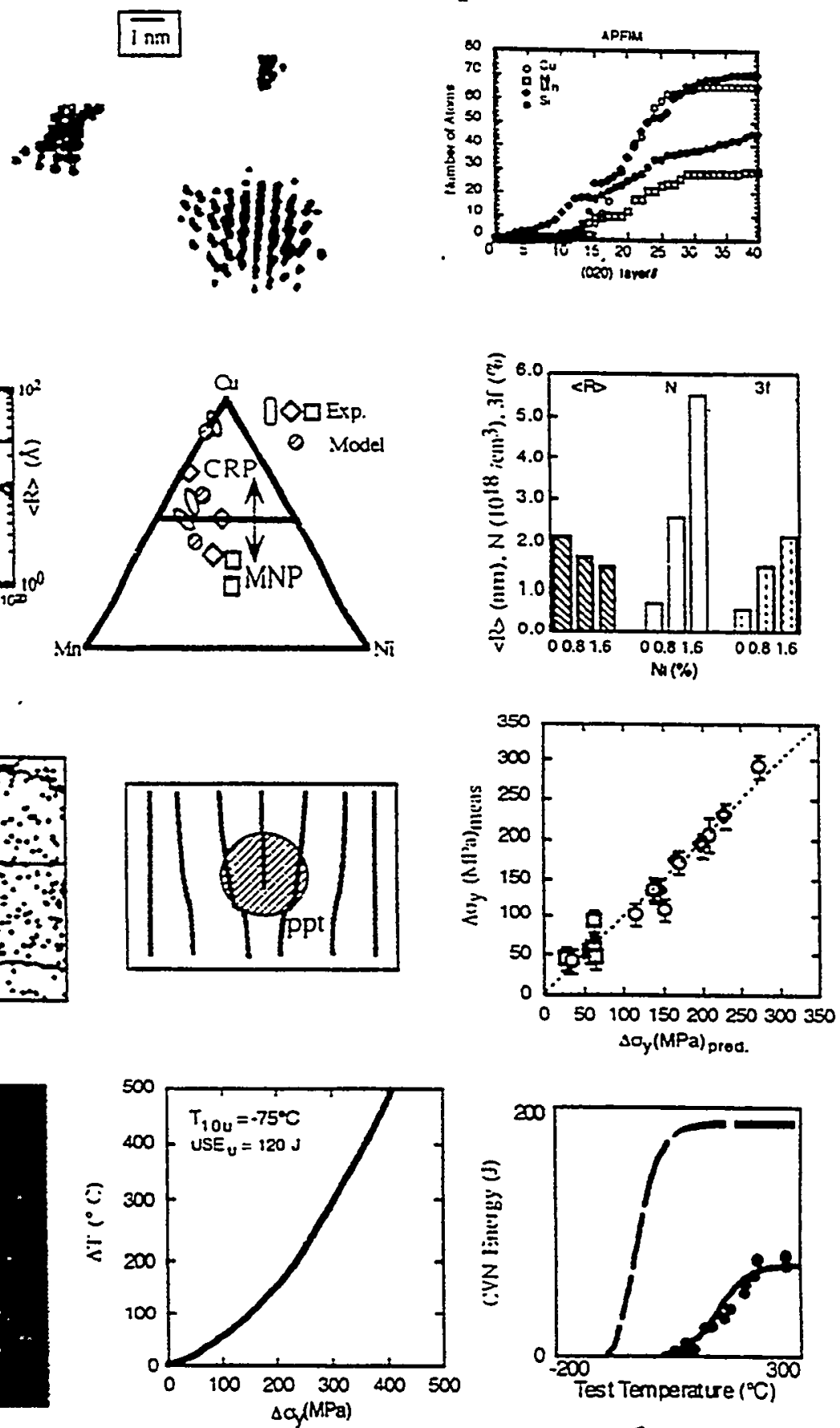

- sus
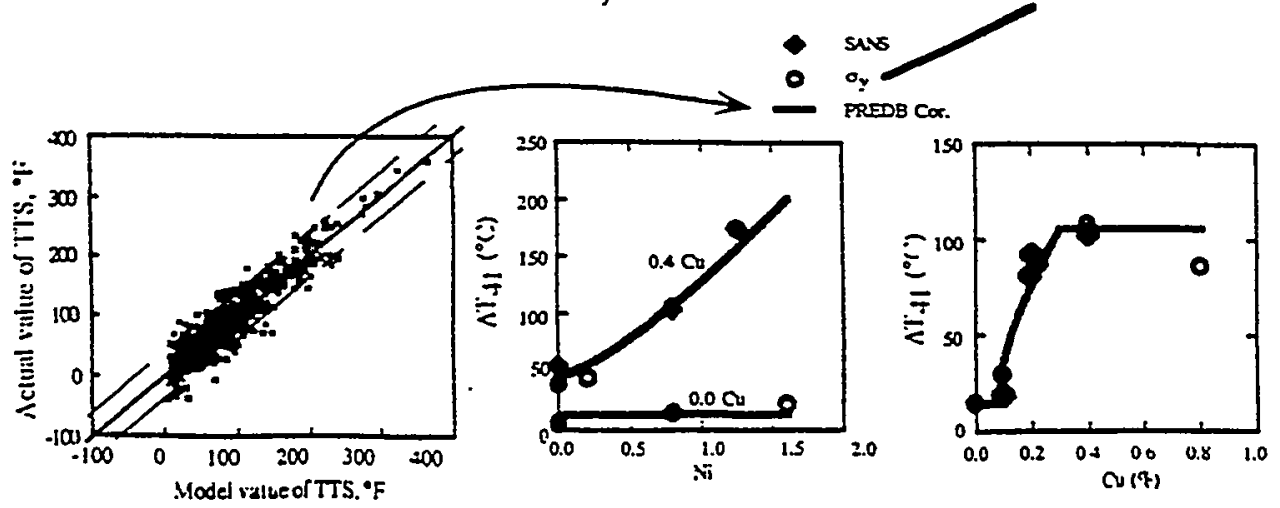


\section{Microstructural Characterization Methods}
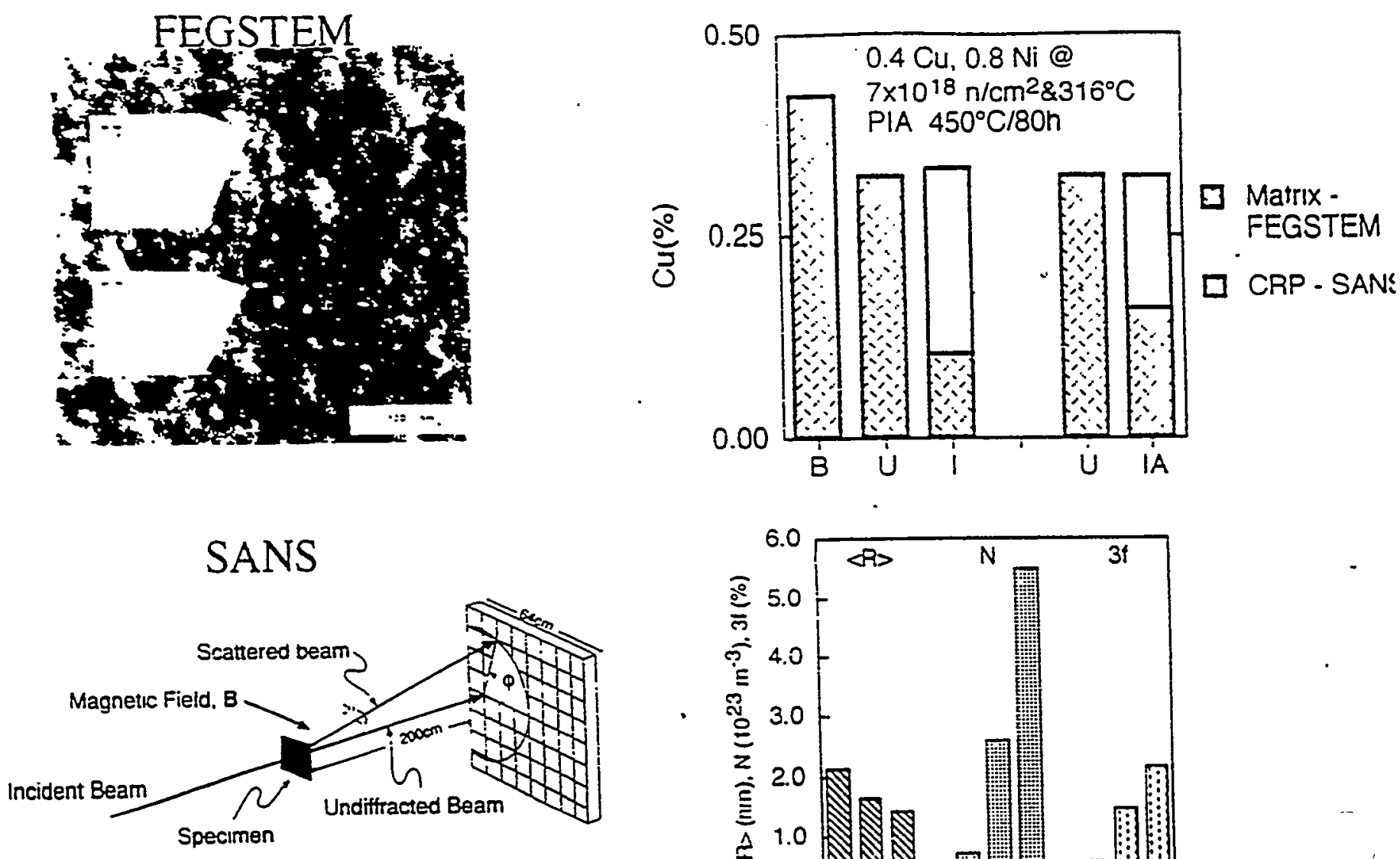

APFIM
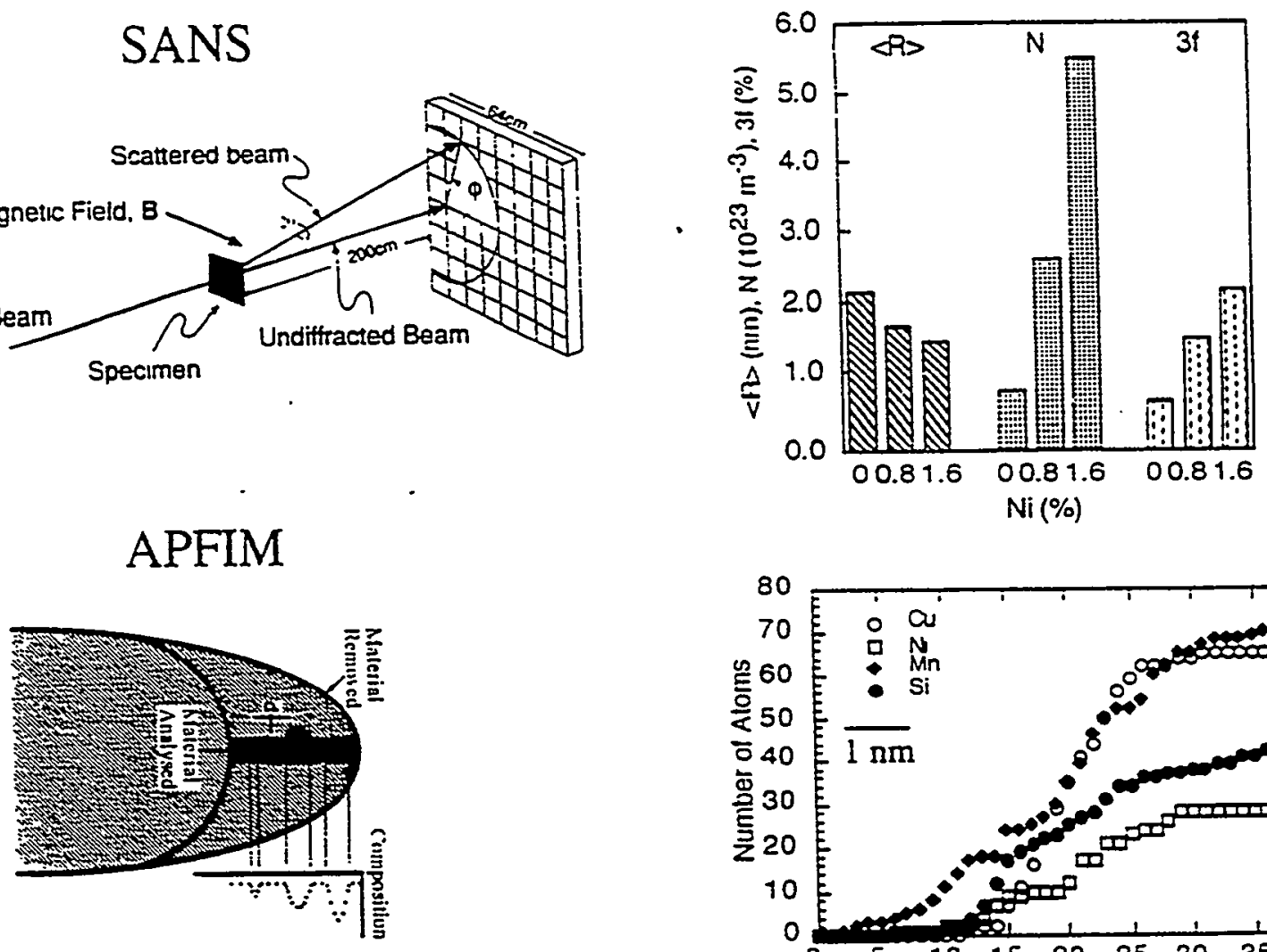

PIA: $\mathrm{T}_{\mathrm{i}}+0$ to $60^{\circ} \mathrm{C}$
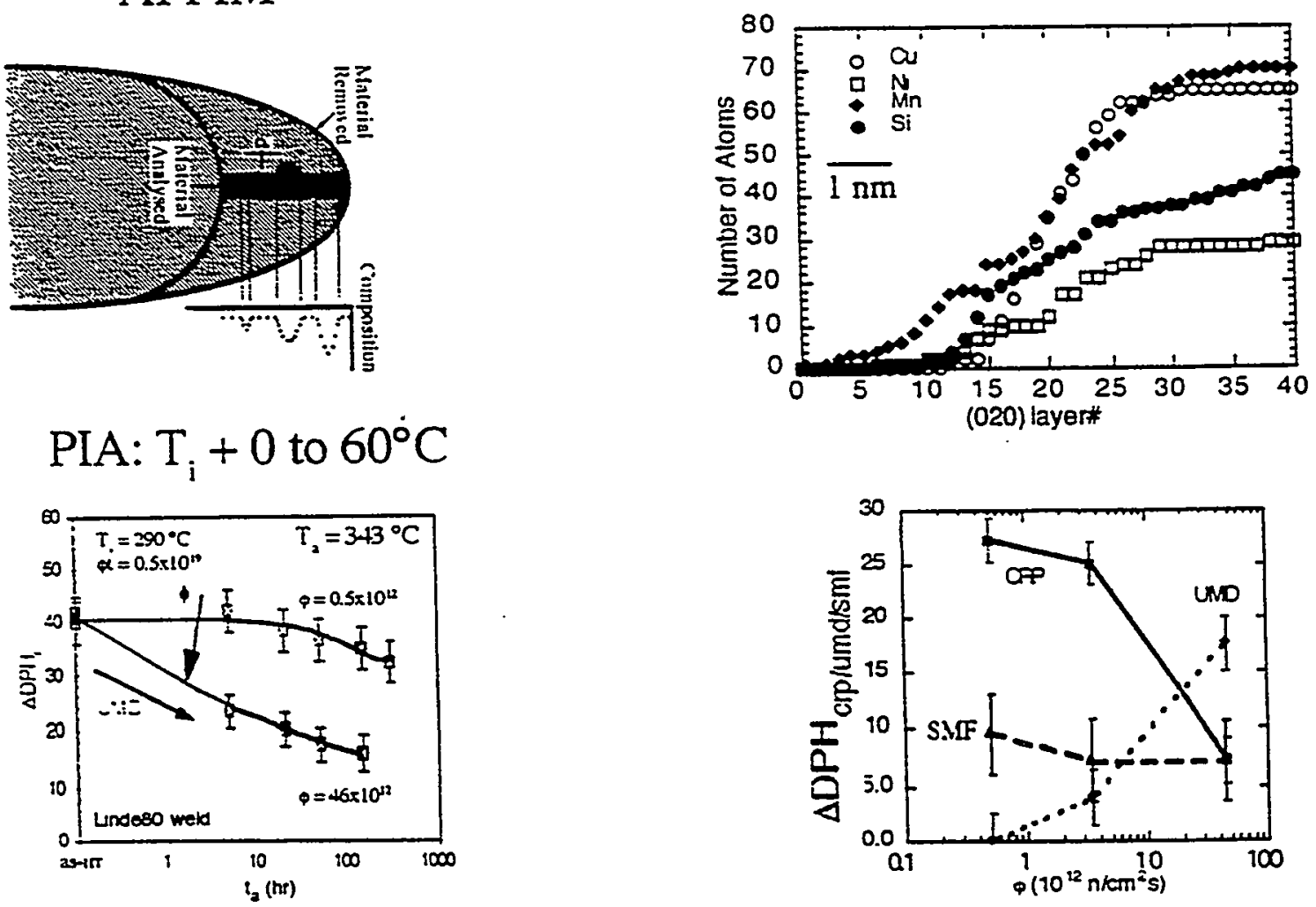

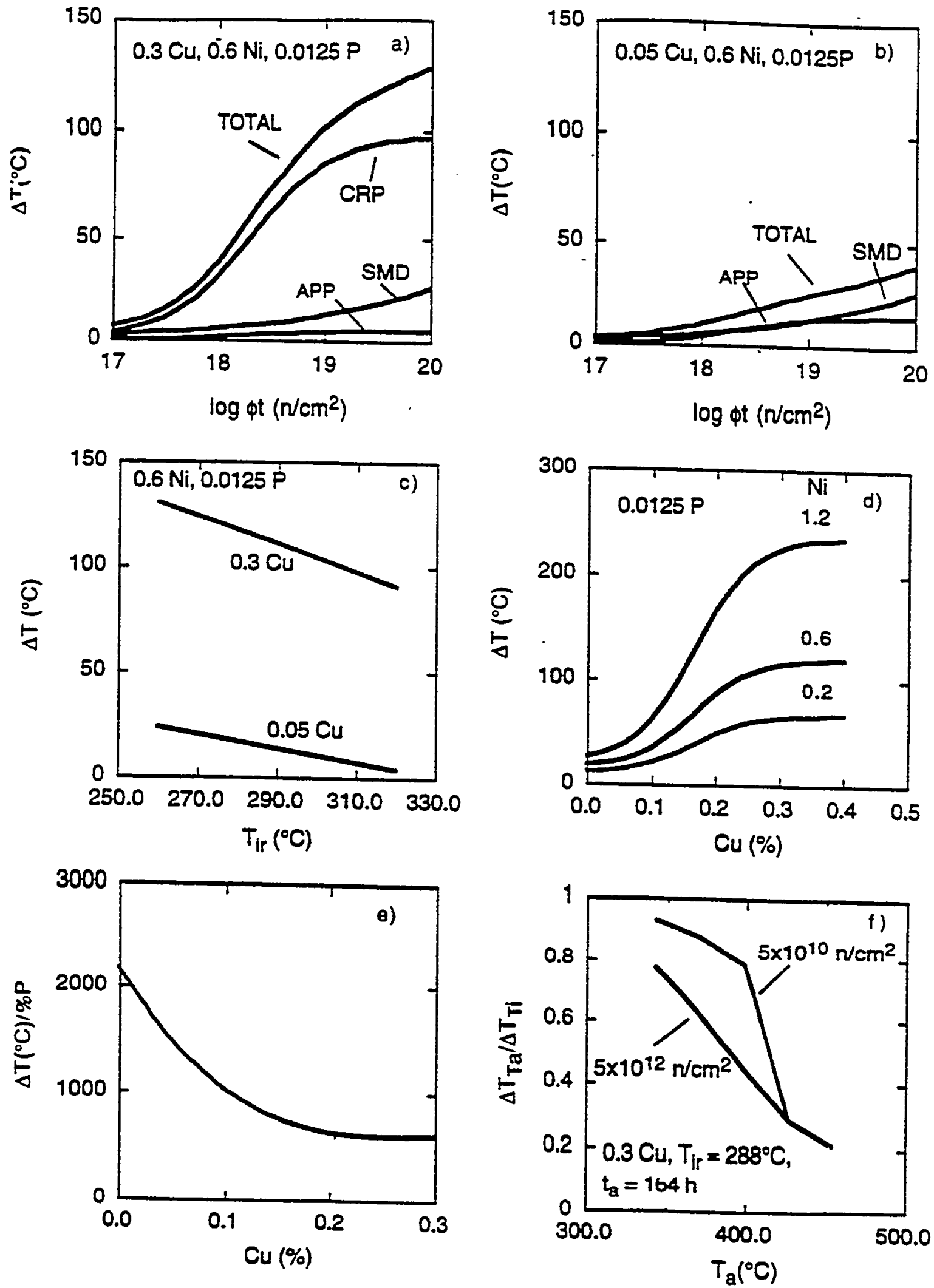

Figure 19 Trendis in embrittlement with key irradiation and PIA variabies - see text for discussion. 


\section{Fracture and Crack Blunting}
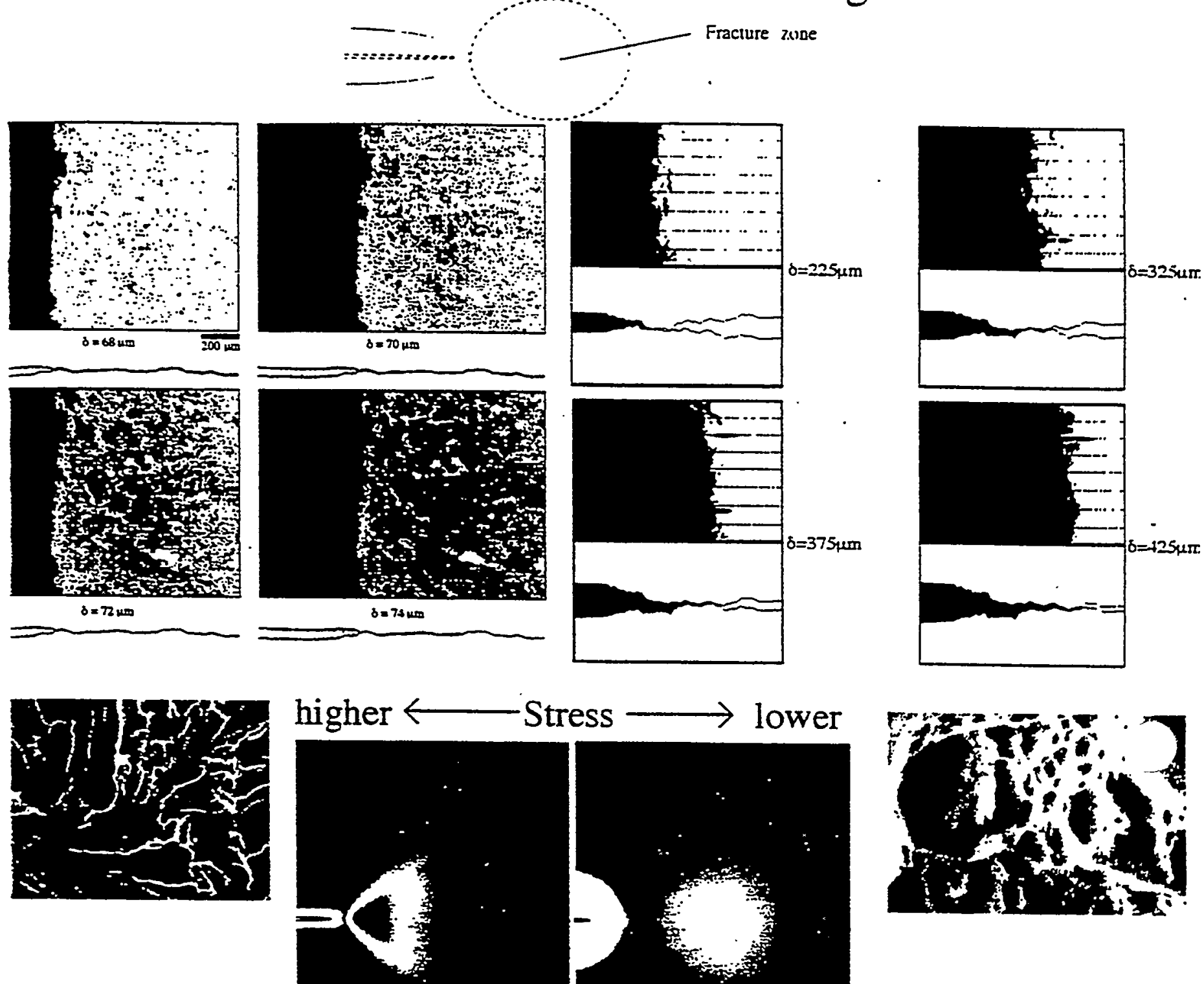

Brittle
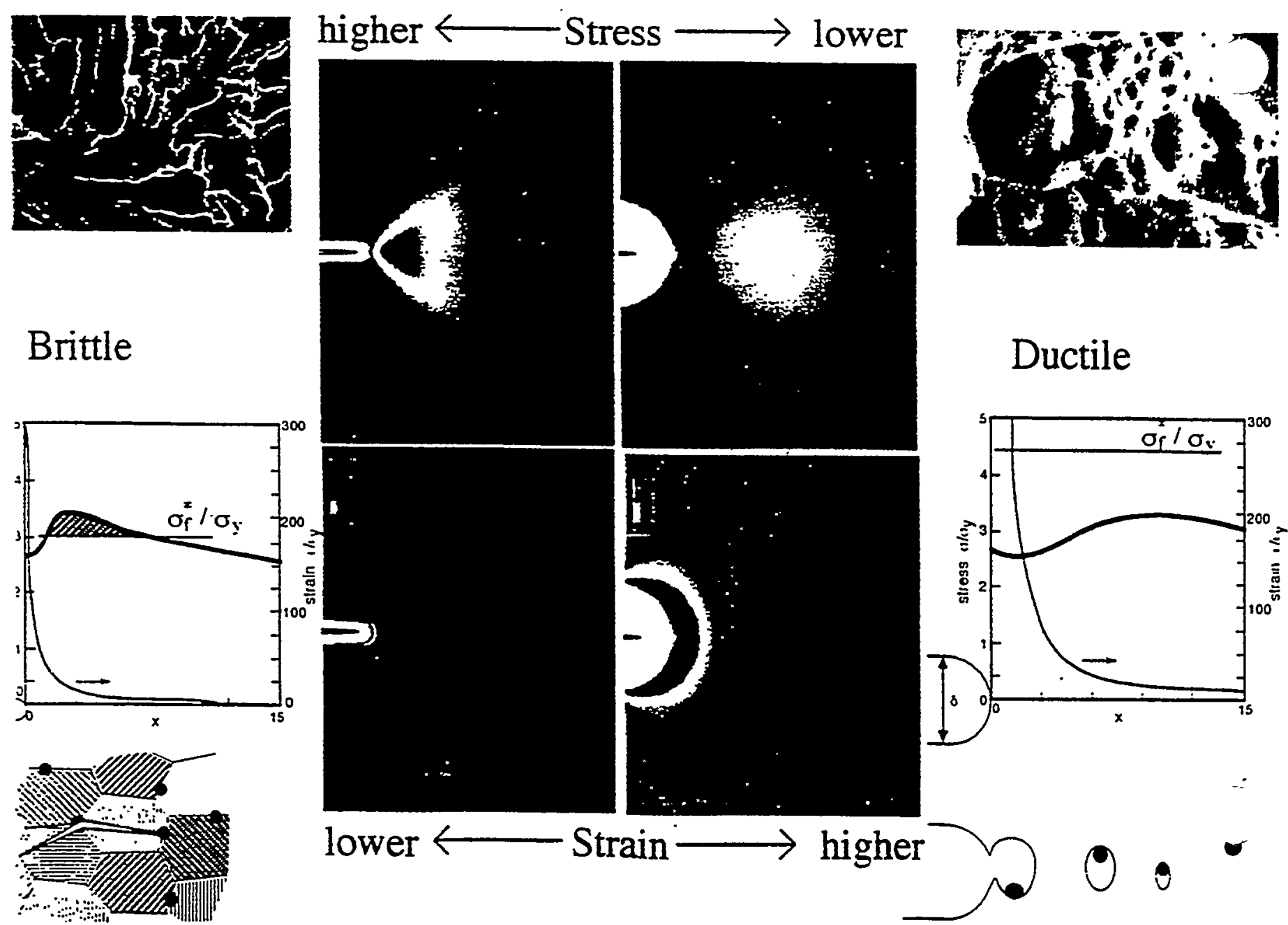

\section{Ductile}
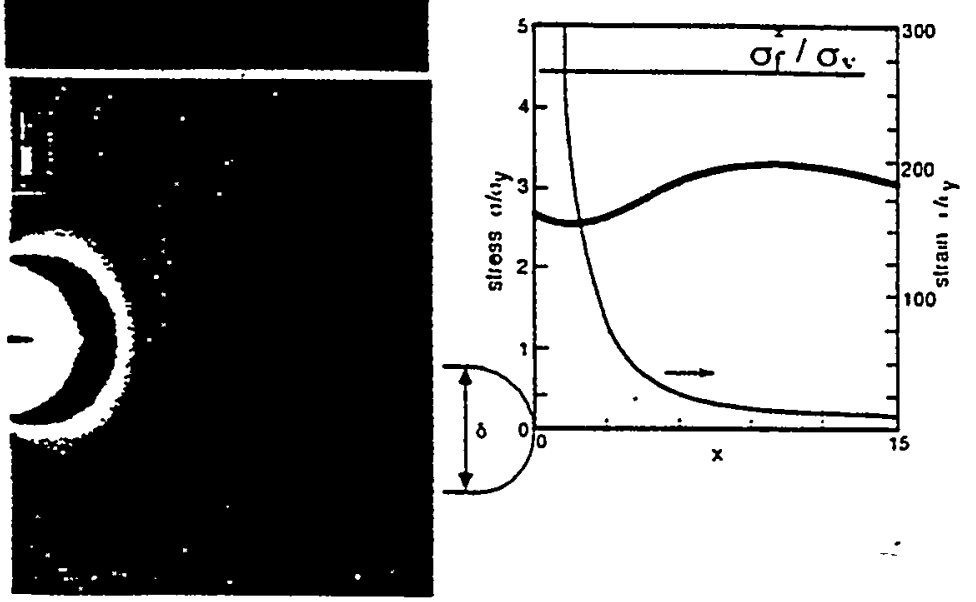

lower 


\section{Progress on $\mathrm{MC}$}

New method for direct constraint corrections

Additional data and new analysis of unirradiated F-82H

Appications of the equivalent yield stress model (EYSM)

Initial testing of unirradiated T91

Collaboration with ECN Petten in combining unirradiated -irradiated data in first evaluation of the MC-shift procedure

Collaboration with ECN Petten and CRRP/PSI in ongoing and future irradiations 


\section{Constraint Correction F82H}

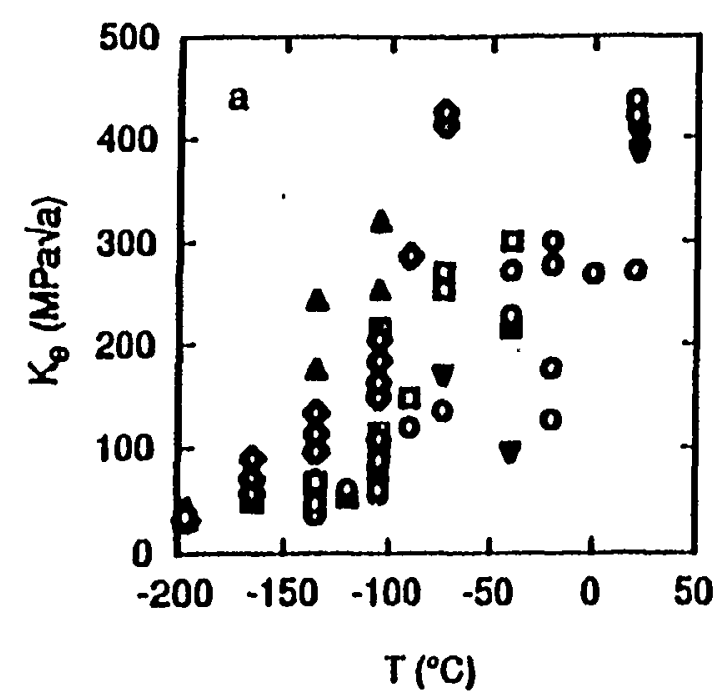

\begin{tabular}{ll} 
- & $10 / .5-\mathrm{B}$ \\
- & $10 / .1$ \\
D & $3.3 / .5$ \\
- & $10 / .1$ \\
- & $30 / .5$ \\
\hline & $M C / .75$
\end{tabular}

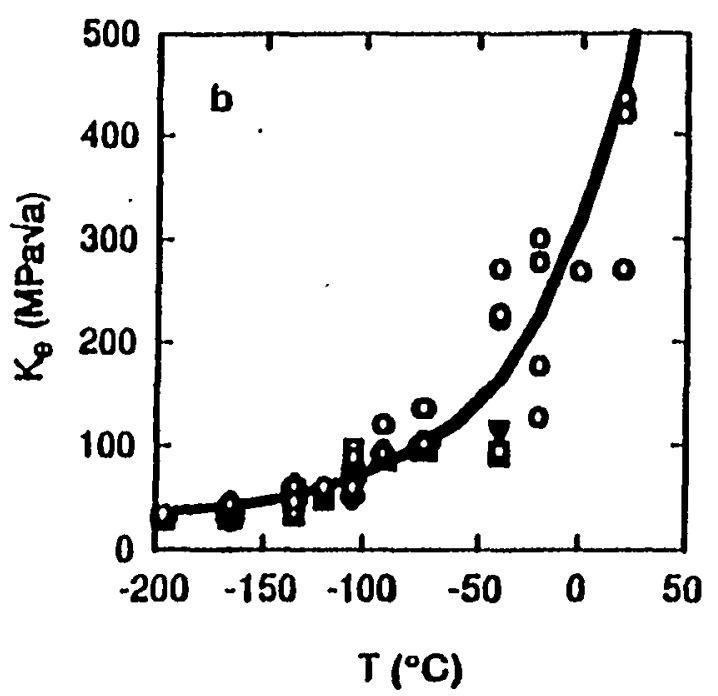

a) Static $K_{e}(T)$ data for F-82II from pre-cracked Charpy-type specimens with $W=3.3$ and $10 \mathrm{~mm}$ and $a / W=0.1$ and 0.5 and from a bend bar with $W=30$ $\mathrm{mm}$ and $\mathrm{a} / \mathrm{W}=0.5$. b) $\mathrm{K}_{\mathrm{c}}(\mathrm{T})$ data constraint corrected (diamonds) to a common reference geometry $a / W=0.5, W=10 \mathrm{~mm}$ shown by the open circles (no correction). 


\section{MC- $\Delta$ T -- FMS}

Two $\mathrm{K}_{\mathrm{e}}(\mathrm{T}) \mathrm{MC}$ using shifts $\left(-55\right.$ to $+140^{\circ} \mathrm{C}$ ) for various sizes, crack geometry, loading rate and irradiation (ECN data $2.5 \mathrm{dpa}$ at $300^{\circ} \mathrm{C}$ )
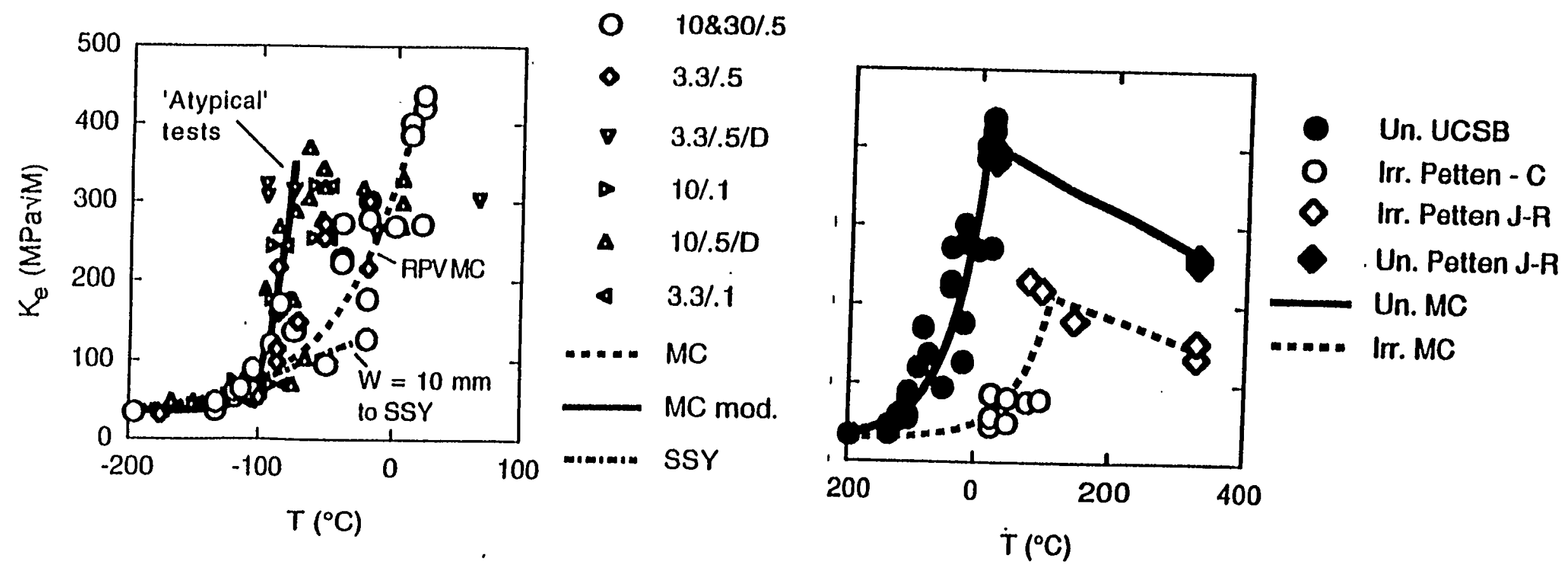


\section{$\mathrm{MC}-\Delta \mathrm{T}(\mathrm{T} 91)$}

Static $\mathrm{K}_{\mathrm{e}}\left(\approx \mathrm{K}_{\mathrm{Jc}}<200 \mathrm{MPa} \sqrt{\mathrm{m}}\right)$ for $0.2 \mathrm{~T}$ and 0.5T CT specimens -- the unirradiated cleavage intitiation $0.2 \mathrm{~T}$ data (UCSB) is constraint corrected and combined with unirradiated $(\mathrm{J}-\mathrm{R})$ and irradiated $\left(\mathrm{K}_{\mathrm{q}}\right)$ data to estimate shift of $\approx 145^{\circ} \mathrm{C}$
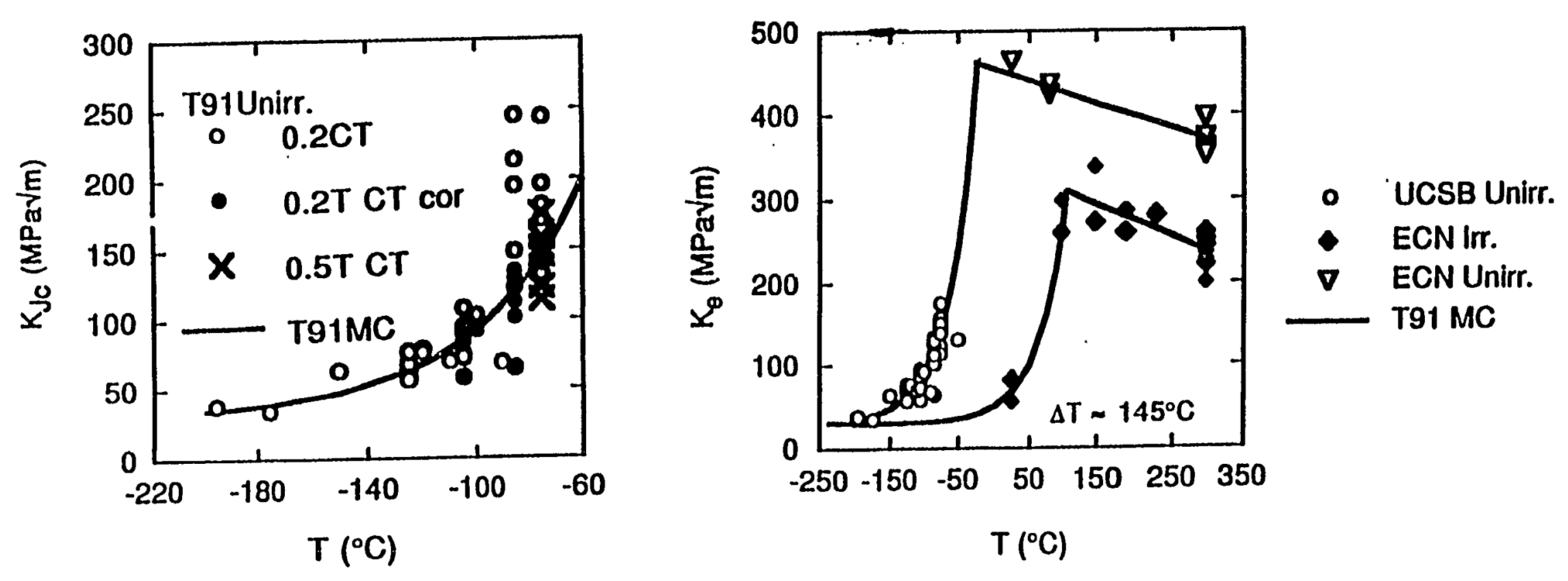


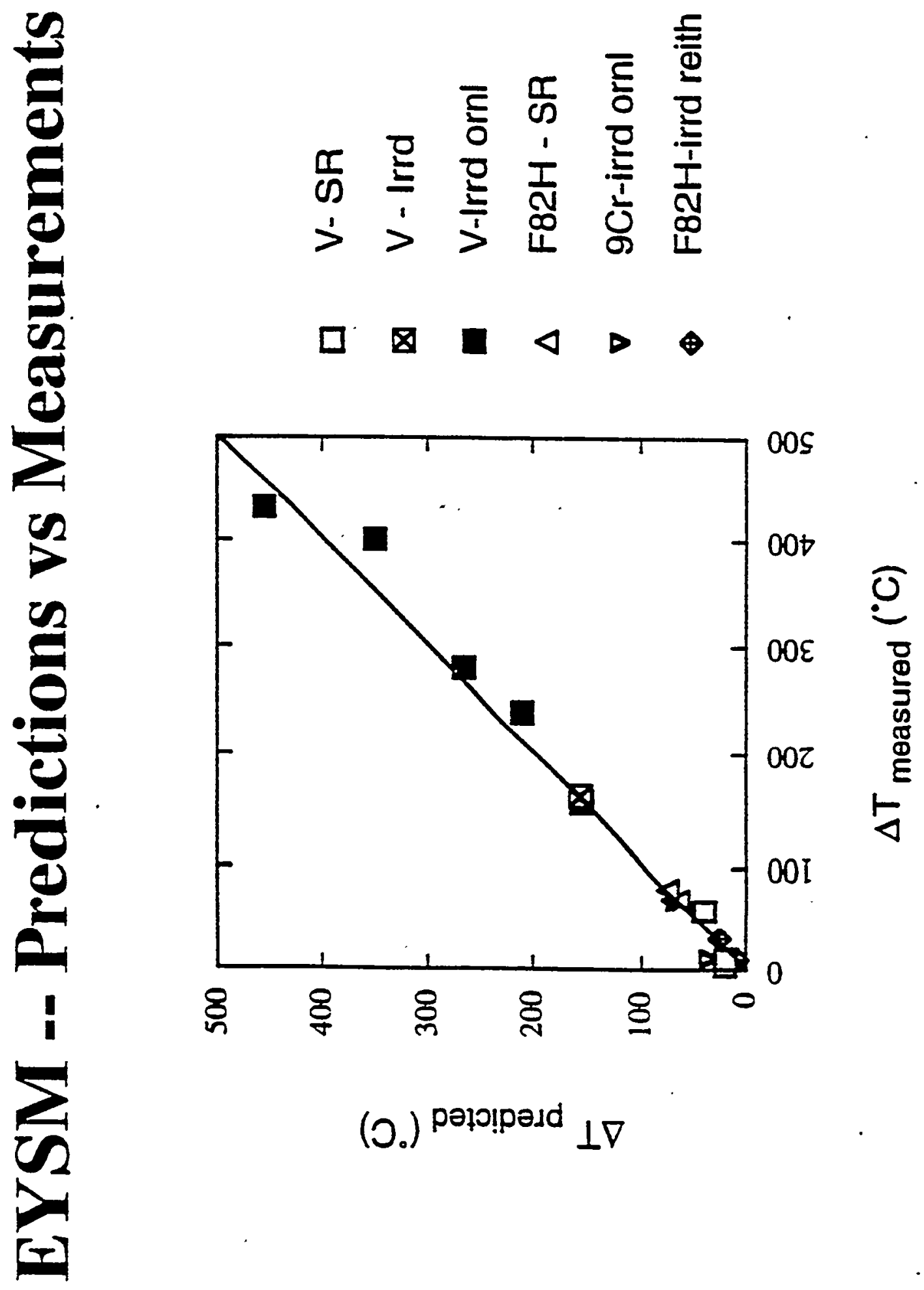




\section{Collaboration ECN Petten}

\section{UCSB and PNL F-82H samples in Petten High Flux Reactor 'CHARIOT' irradiation matrix}

\begin{tabular}{|l|l|l|l|}
\hline \multicolumn{2}{|c|}{ CHARIOT-2 } & \multicolumn{1}{|l|}{ CHARIOT-4 } & \multicolumn{1}{|c|}{ CHARIOT-6 } \\
\hline $\begin{array}{l}\text { Irradiation Dose and } \\
\text { Temperature }\end{array}$ & $\begin{array}{l}2.5 \mathrm{dpa}, 300^{\circ} \mathrm{C} \\
\text { (out) }\end{array}$ & $\begin{array}{l}2.5 \mathrm{dpa}, 300^{\circ} \mathrm{C} \\
\text { (out) }\end{array}$ & $\begin{array}{l}10 \mathrm{dpa}, 300^{\circ} \mathrm{C} \\
\text { (out '99-2000) }\end{array}$ \\
\hline Contents & $\begin{array}{l}\text { 10mm CT } \\
\text { KLST } \\
\text { TEM discs }\end{array}$ & $\begin{array}{l}10 \mathrm{~mm} \mathrm{CT} \\
\text { KLST }\end{array}$ & $\begin{array}{l}10 \mathrm{~mm} \mathrm{CT} \\
\text { KLST } \\
\text { TEM discs }\end{array}$ \\
\hline ECN & $\begin{array}{l}\text { TEM discs } \\
\text { mini-tensiles }\end{array}$ & $\begin{array}{l}\text { TEM discs } \\
\text { mini-tensiles }\end{array}$ \\
\hline PNL & SANS coupons & PMC & $\begin{array}{l}\text { PMC } \\
\text { SANS coupons }\end{array}$ \\
\hline
\end{tabular}




\section{Collaboration CRRP/PSI}

Data in support of MC- $\Delta \mathrm{T}$ for F82H -- MAster Curve Experiments (MACE) in the Hungarian Research Reactor ( $\sim 35 \mathrm{~mm}$ diameter by $170 \mathrm{~mm})$, with first dose $\sim 0.5-5$ $\mathrm{dpa}, \sim 150^{\circ} \mathrm{C}, 250^{\circ} \mathrm{C}, 350^{\circ} \mathrm{C}$ starting $1 / 99$

\begin{tabular}{|c|c|c|}
\hline Qty & Specimen & Post-irradiation test \\
\hline 6 & $0.4 \mathrm{~T} \mathrm{CT}$ 's, $\mathrm{a} / \mathrm{W} \sim 0.5$ & $\mathrm{~K}(\mathrm{~T})$-- static \\
\hline 30 & $0.2 \mathrm{~T}$ CT's, $20 \% \mathrm{SG}, \mathrm{a} / \mathrm{W} \sim 0.5$ & $\mathrm{~K}(\mathrm{~T})$-- static \\
\hline 13 & $0.1 \mathrm{~T}$ 3PB's, a/W 0.5 & $\mathrm{~K}(\mathrm{~T})$-- static \\
\hline 13 & $0.1 \mathrm{~T}$ 3PB's, a/W 0.1 & $\mathrm{~K}(\mathrm{~T})$-- static \\
\hline 19 & $\mathrm{MCVN}, \mathrm{a} / \mathrm{W} \sim 0.5$ & $\mathrm{~K}(\mathrm{~T})$-- dynamic \\
\hline 40 & Minitensiles & $\sigma=\sigma(\varepsilon, \dot{\varepsilon}, \mathrm{T})$ \\
\hline 4 & SANS coupons & \\
\hline 10 & TEM discs & \\
\hline
\end{tabular}




\section{Collaborators at UCSB}

Takuya Yamamoto (Tohoku U.) -- Deformation and fracture modeling and experiments (1 year)

Yoshiharu Murase (NRIM) -- creep-fatigue interaction; fracture and failure processes (1 year)

Philippe Spatig (CRPP/PSI) -- Deformation and fracture modeling and experiments MACE experiments 


\section{Other}

Properties and mobility of SIA and IC in BCC alloys

Cascade vacancy clustering and complex formation

Superposition of net strength contributions from multiple hardening sources

Indentation methods to assess yield/post yield constitutive properties

Constitutive equations for $\mathrm{F} 82 \mathrm{H}$ and T91

- Partially supported by US NRC 

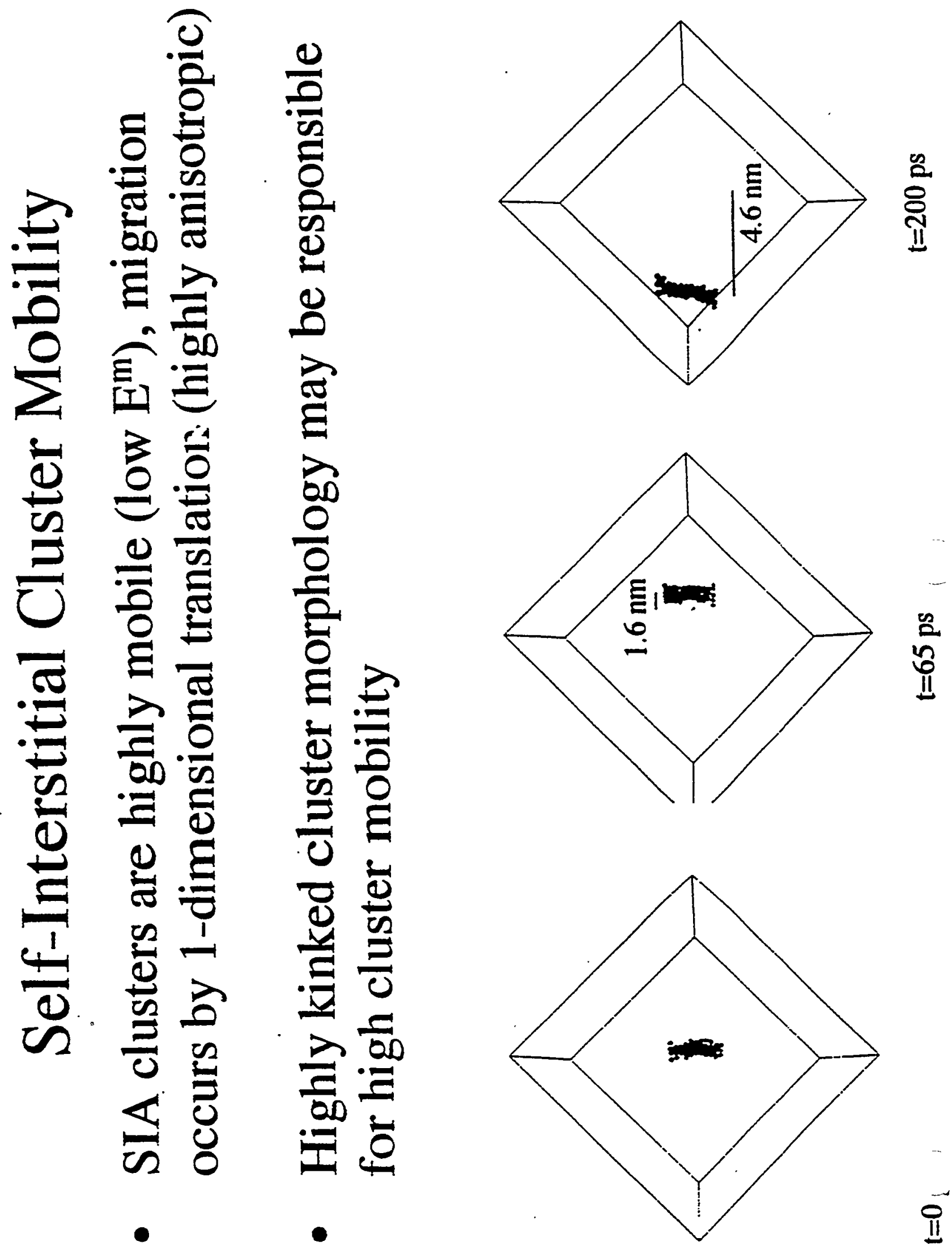


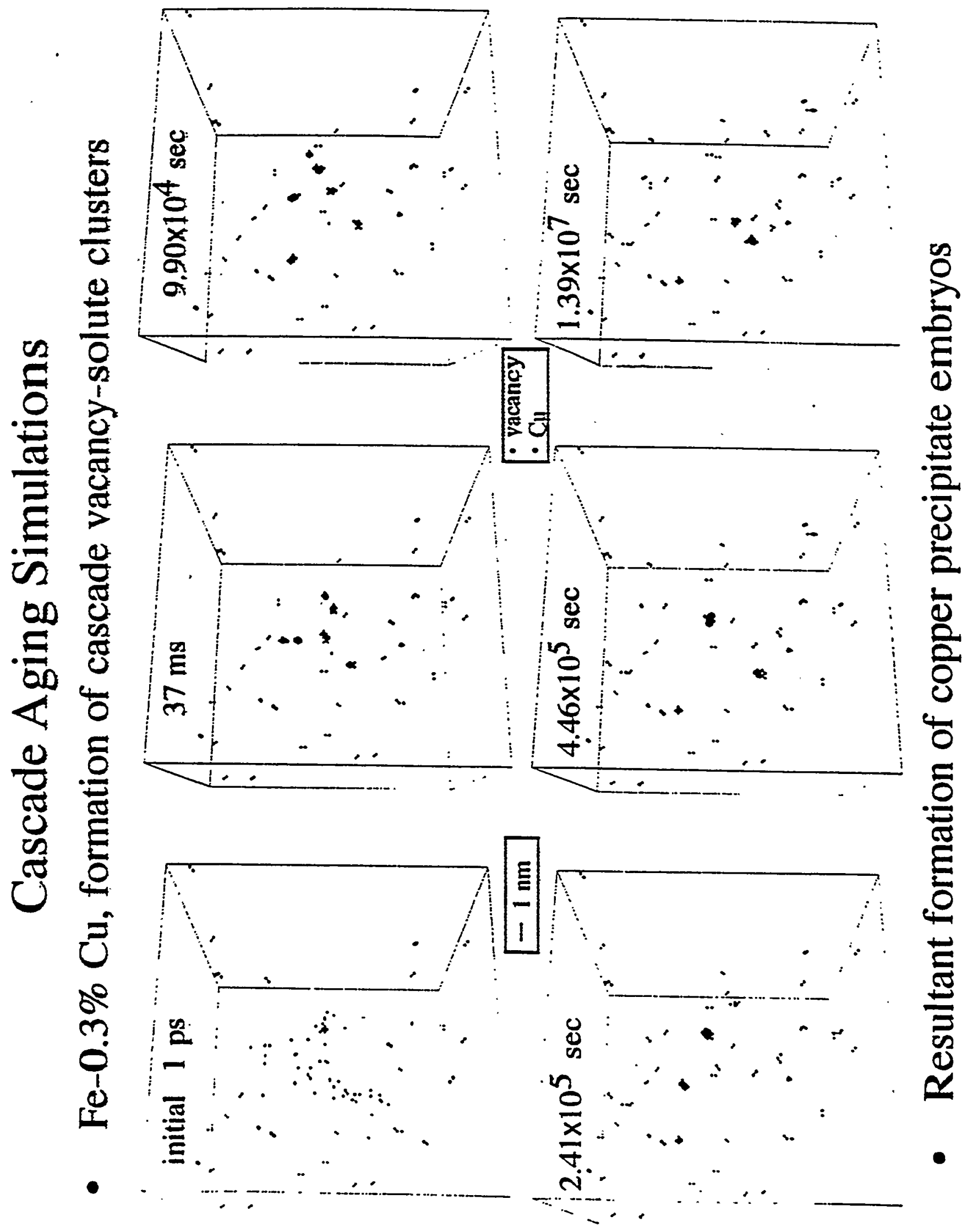




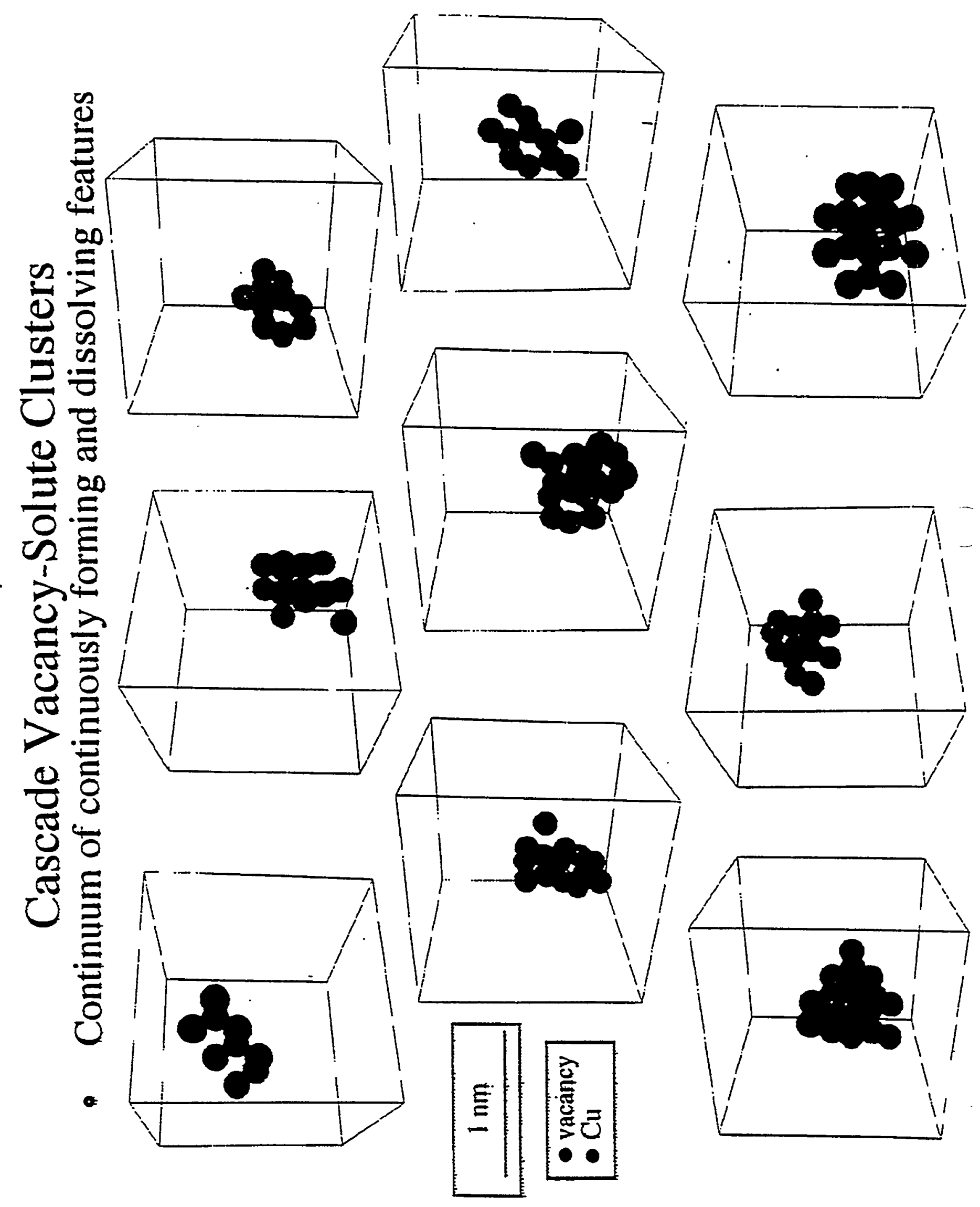




\section{Superposition Rules}

Superposition parameter $\mathrm{S}$ interpolates from LS to RSS

$$
\sigma_{\mathrm{t}}=\mathrm{S}\left(\sigma_{1}+\sigma_{2}\right)+(1-\mathrm{S}) \sqrt{\sigma_{1^{2}+\sigma_{2}^{2}}}
$$

$S$ determined from (Foreman-Makin type) computer models of dislocation-strong $\left(\alpha_{S}\right) /$ weak $\left(\alpha_{w}\right)$ obstacle interaction

$$
\mathrm{S} \sim \alpha_{\mathrm{s}}-\alpha_{\mathrm{w}}\left(4.3-2.4 \alpha_{\mathrm{s}}\right)
$$

Predicted strengthening in RPV steels agrees with empirical values; decrease in $\mathrm{S}$ during PIA enhances recovery
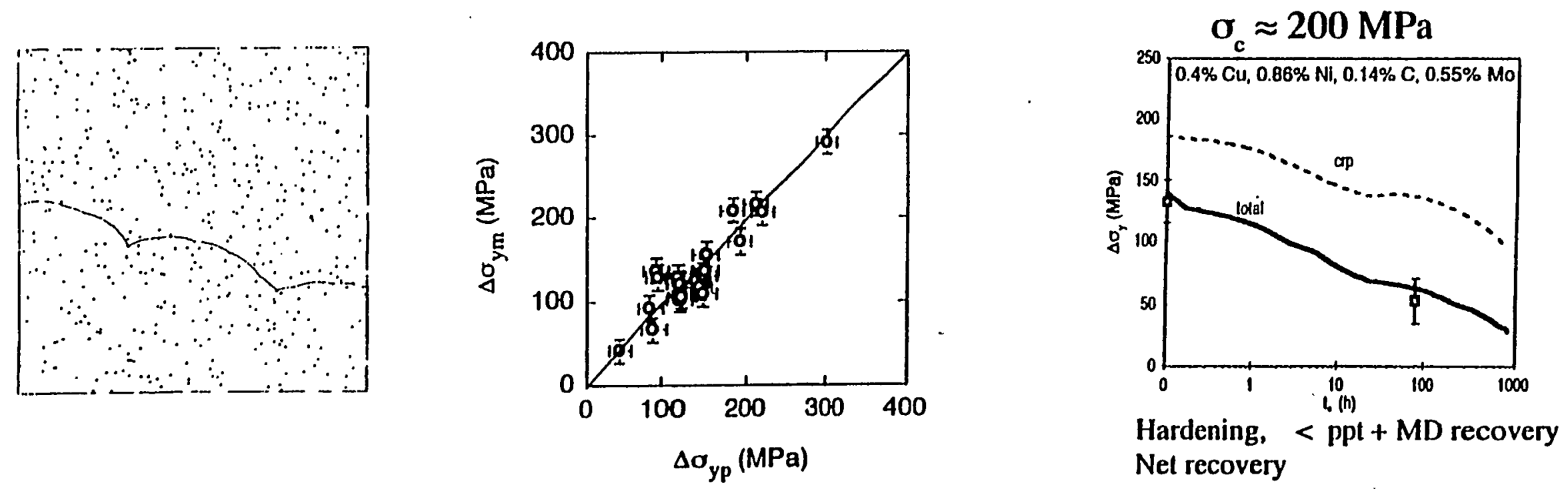

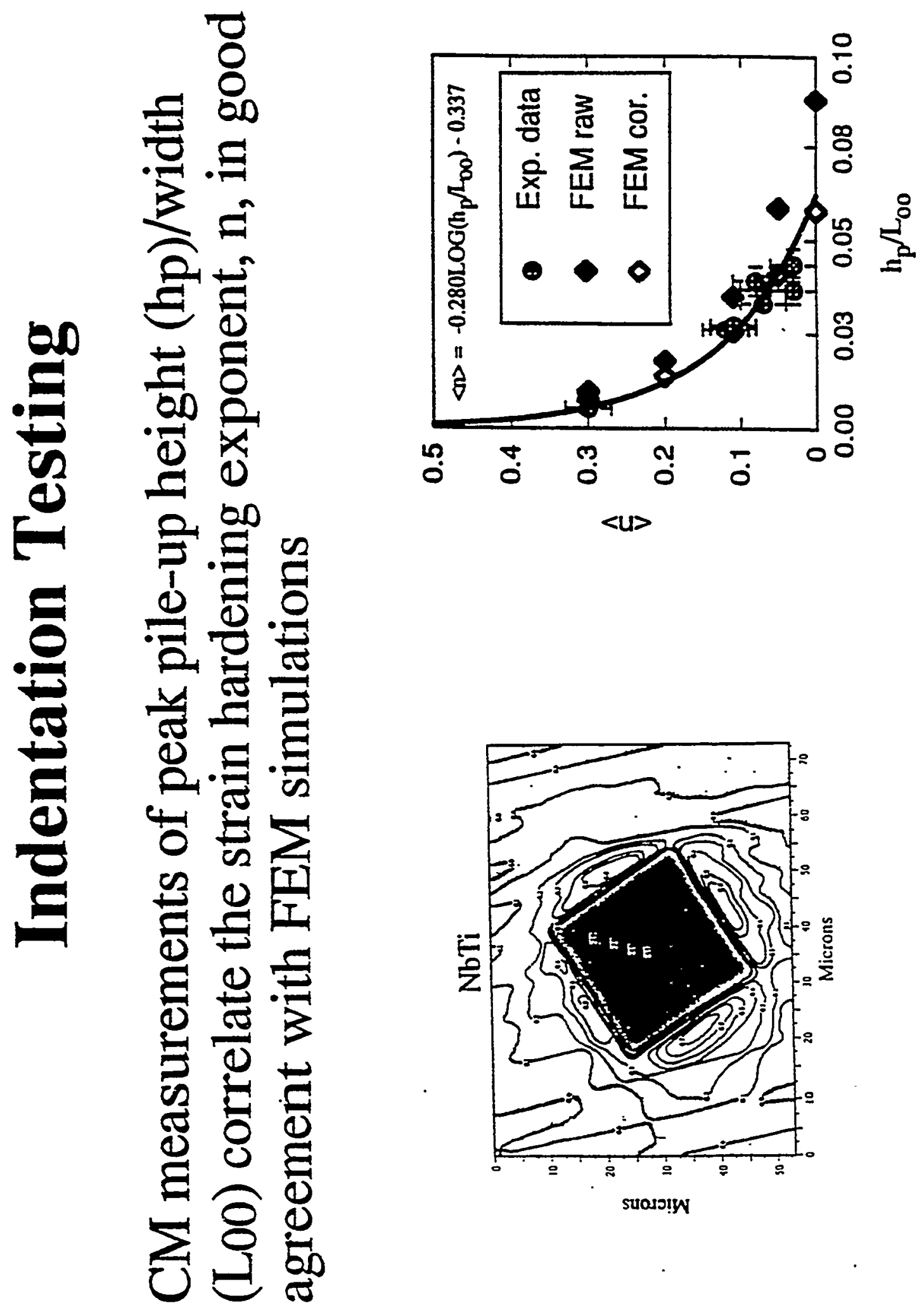


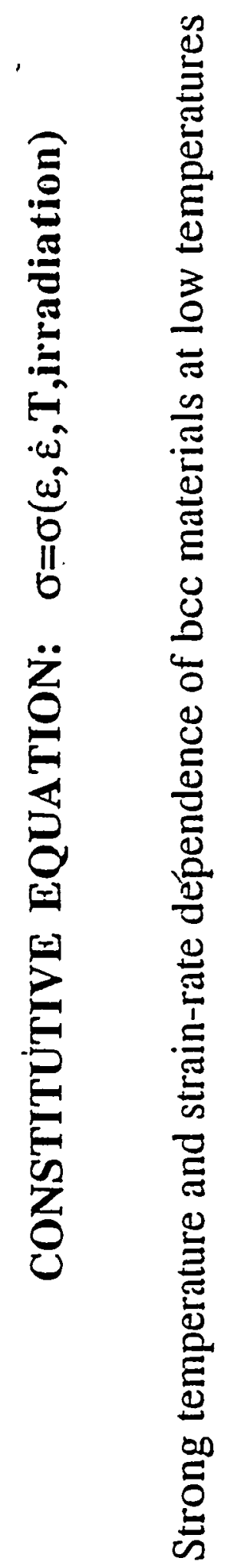

토ำ
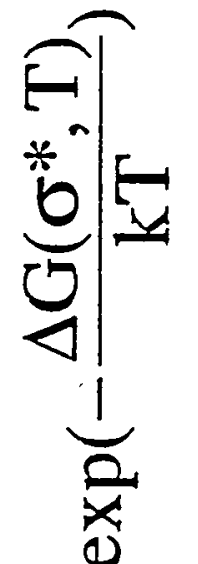

(1)

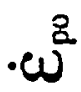

II

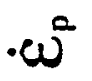

$\stackrel{3}{0}$

$\frac{\pi}{0}$

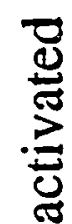

를

$\underline{2}$

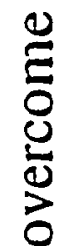

용

$\frac{0}{0}$

更

क्ष

$\stackrel{5}{0}$

ज्ञ
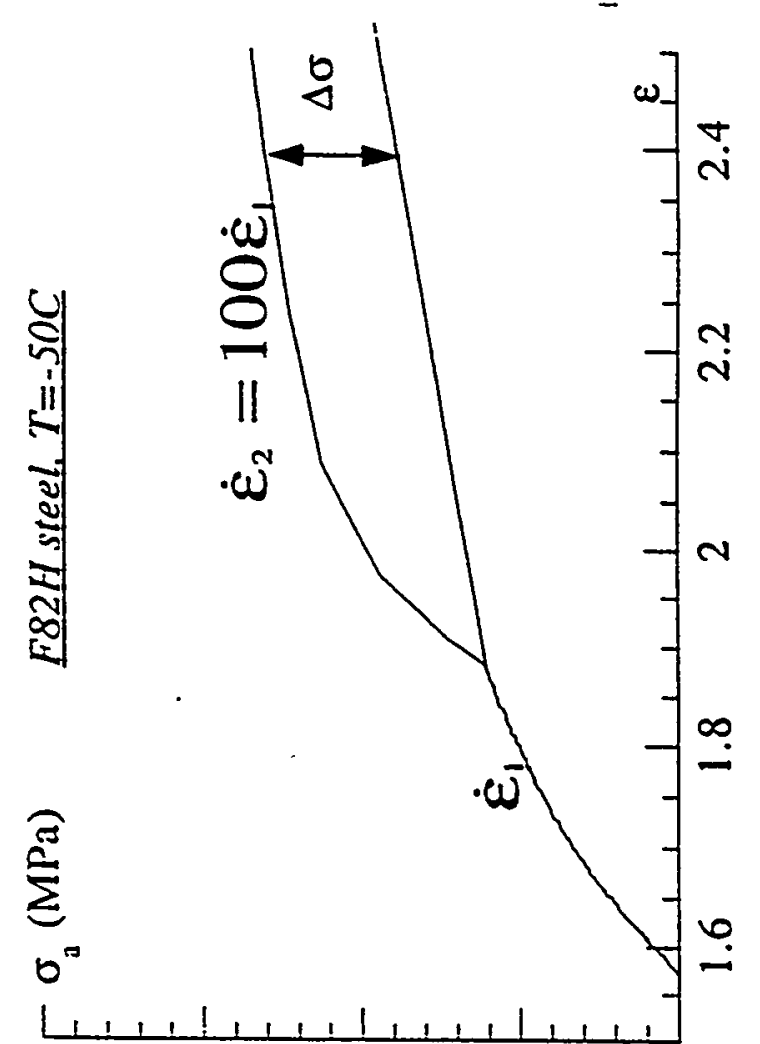

8 ஜ

응

$\stackrel{0}{ \pm}$

.2

$\checkmark$ 


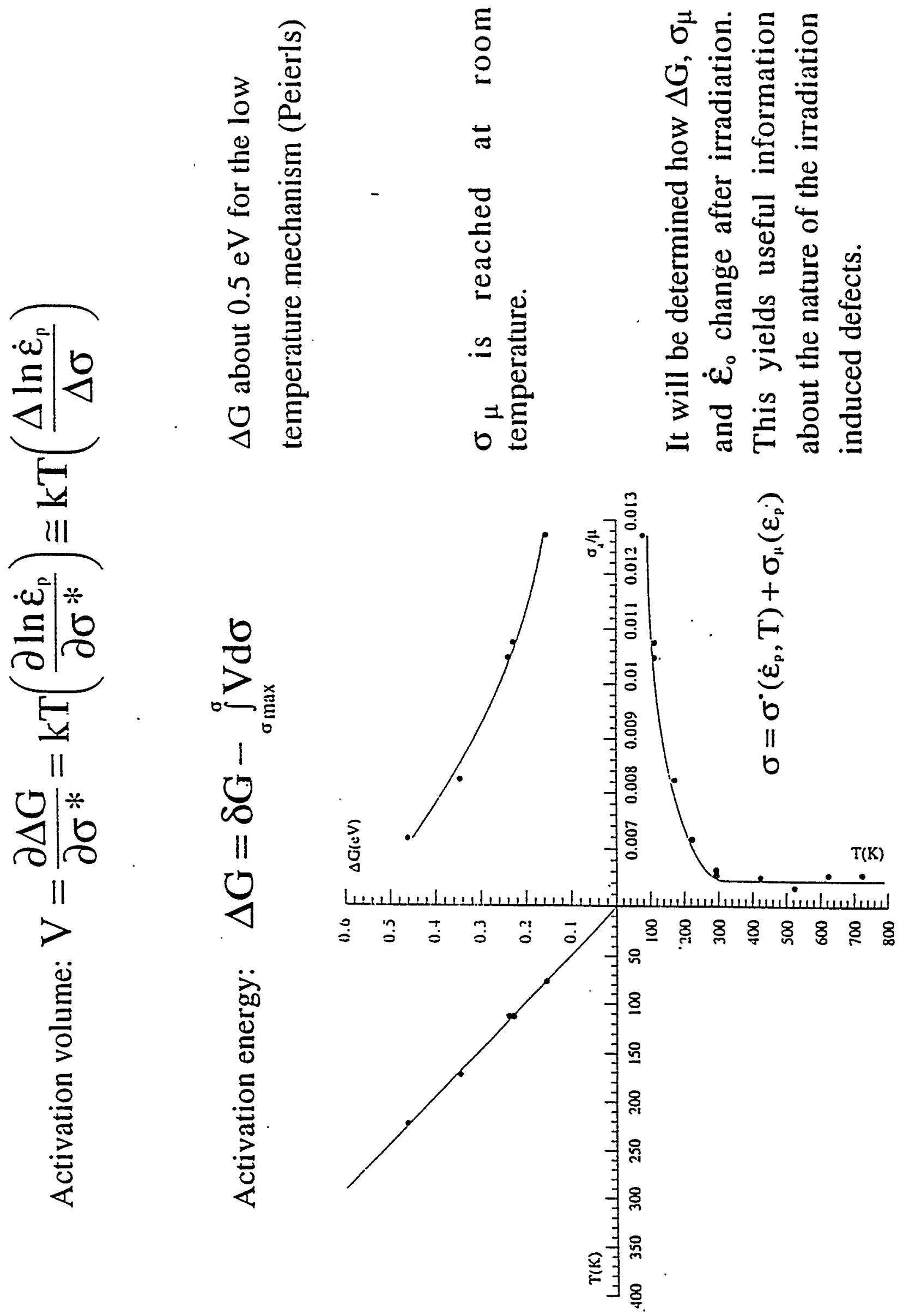




\section{Helium Effects Studies}


ELECTRON MICROSCOPY OF RAF STEELS WITH HELIUM

$R$. Schäublin

CRPP - Fusion technology Materials group EFL

5232 Villigen - PSI 
- MATERIALS:

$$
\begin{aligned}
& \text { F82H }=3-755 \mathrm{CH}-2 \mathrm{H} \\
& \text { OPTIMAXA E - } 9.0 \text { Or . IN }
\end{aligned}
$$

- IRRADIATIONS:

$$
\begin{aligned}
& D 590^{\circ} \mathrm{MeV} \text { protens. (PSI) } \\
& \text { F82H }\left[\begin{array}{l}
0,5 \text { dpa @ } 250^{\circ} \mathrm{C} \\
1,7 \text { dpa @ } 40^{\circ} \mathrm{C}
\end{array}\right. \\
& D \text { ncutrons (Petten) } \\
& \text { F82H - 2,5 dpe @ 250 C } \\
& \text { OPIIMAXA - 2,5 dpe@ } 250^{\circ} \mathrm{C}
\end{aligned}
$$


FSH

Material characterization:

TEM - Dislocation type and density

- Carbide composition and size distribution

- Grain / lath boundary chemistry

Comparison between:

- Unirradiated

- Unirradiated deformed

- Irradiated to 0,5 dea 


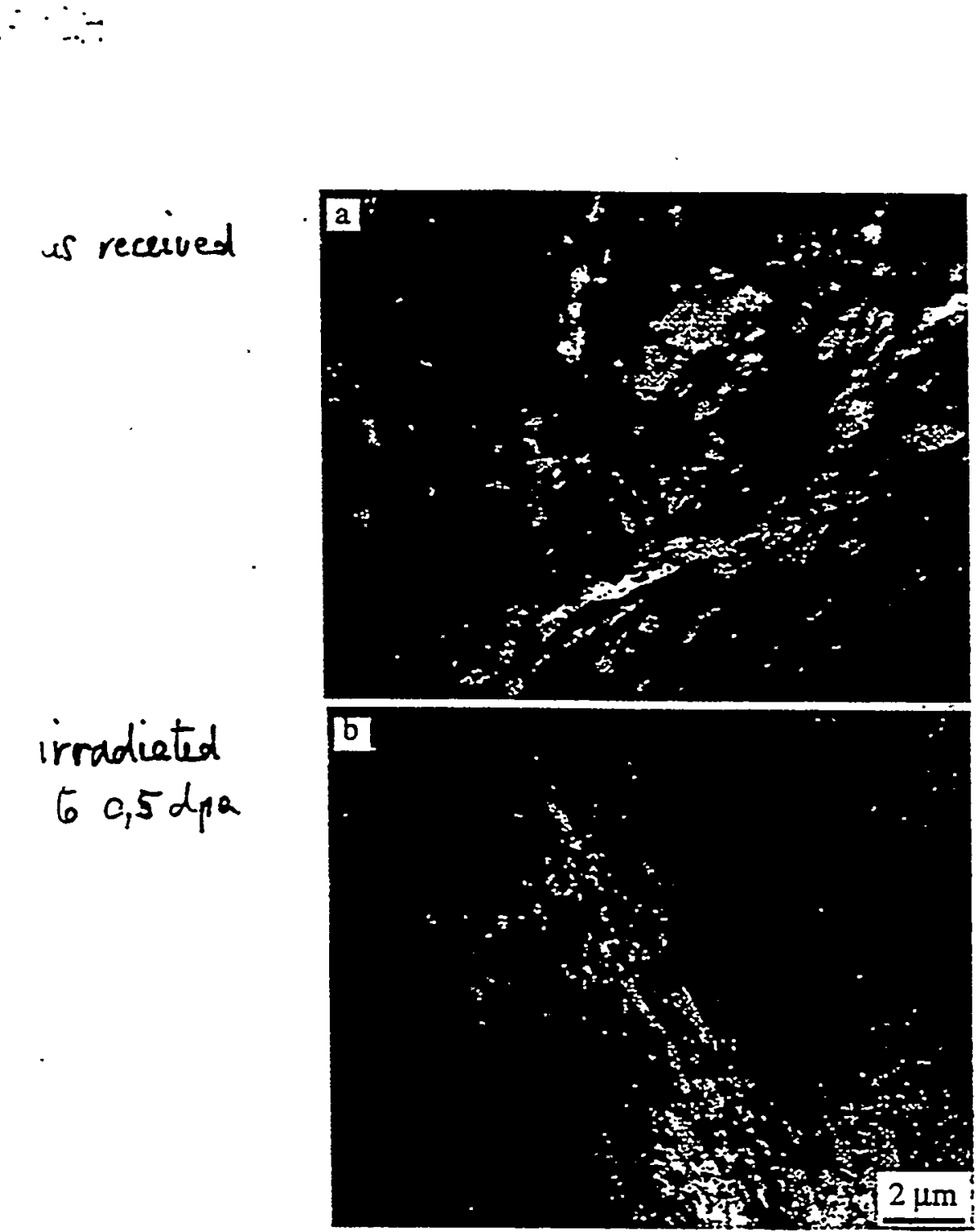

Fig. 1 
= ¿-

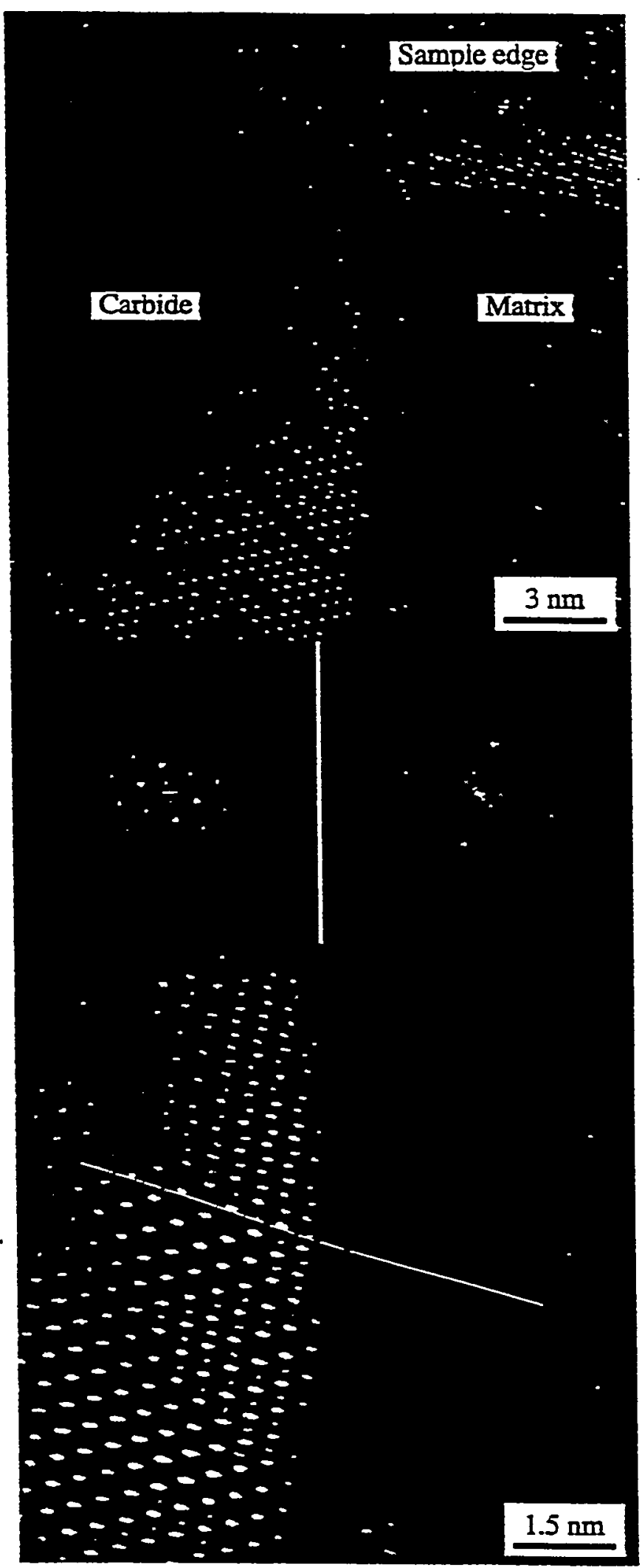

CM300 - FEG PTME - EDEI 
F82.4

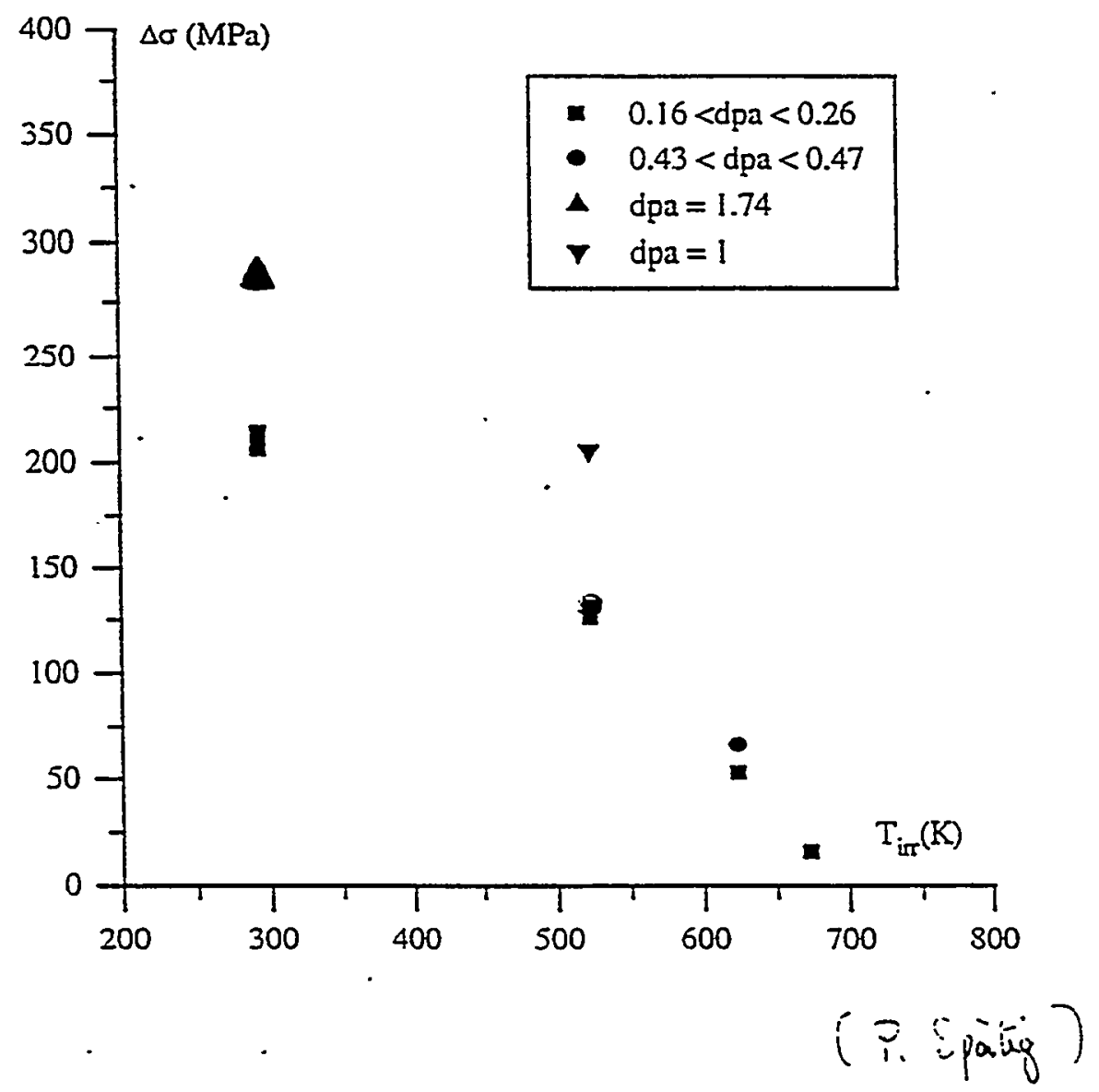

Finure 3 

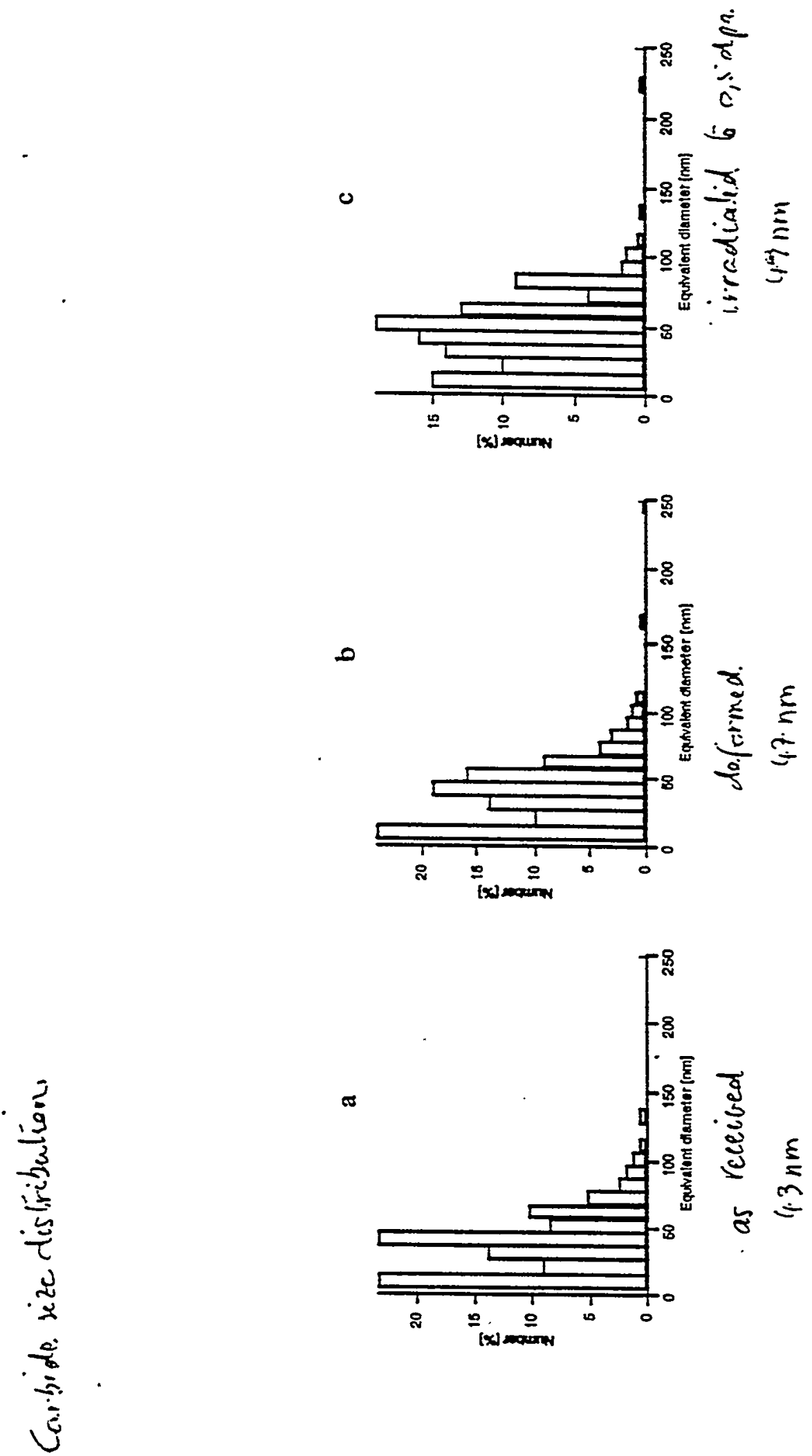

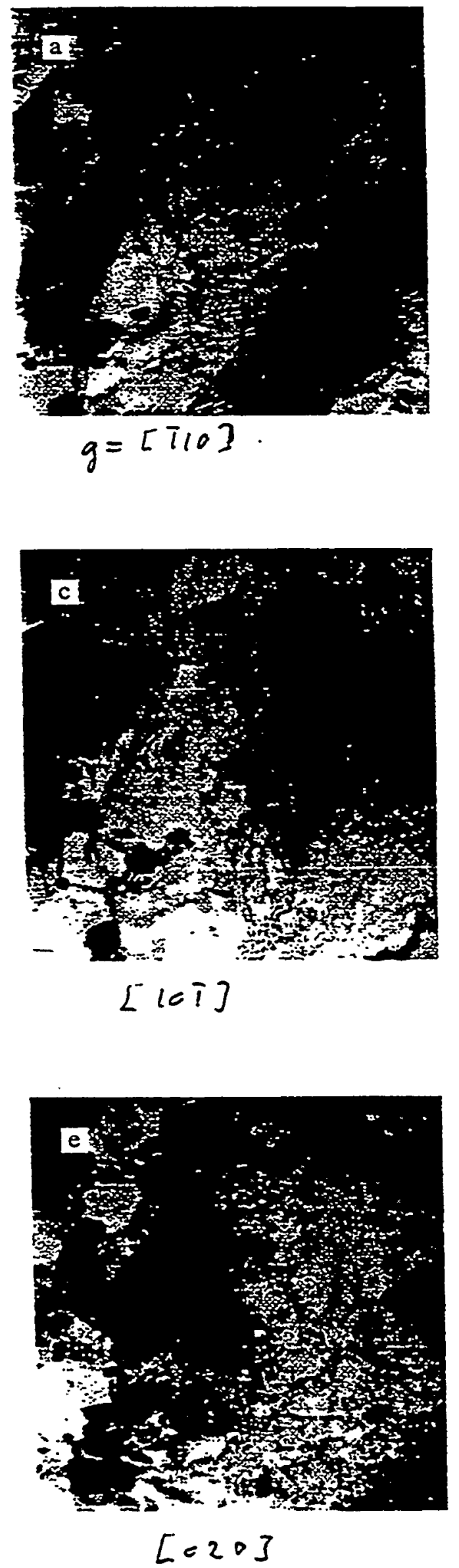
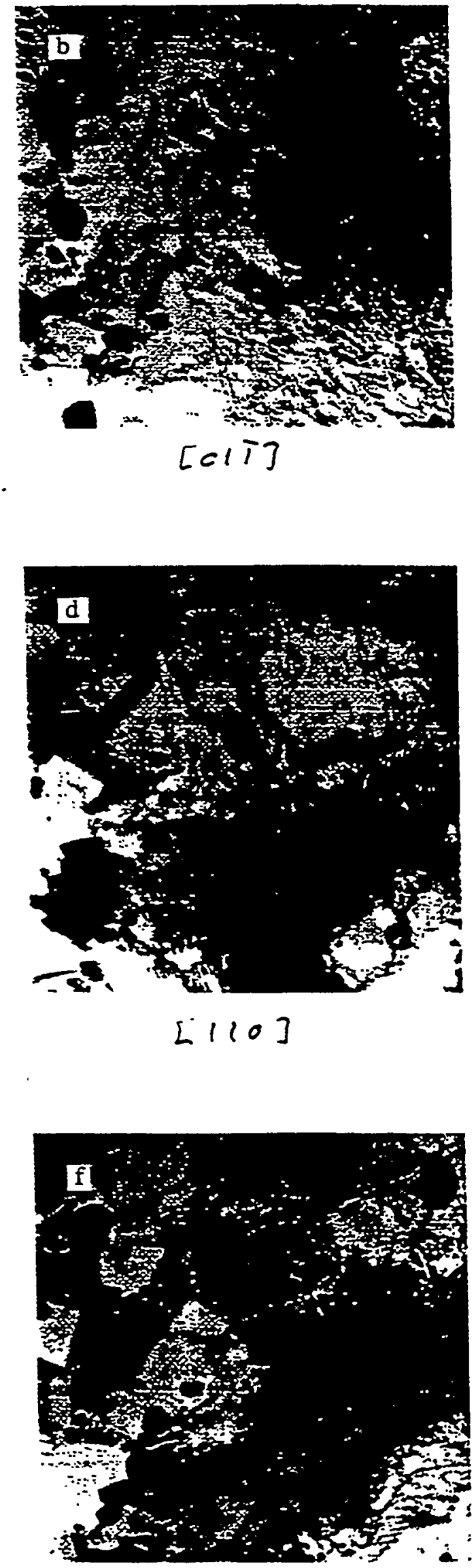

[200] 
$=82$

- Carbide compoition: $M_{23} C_{6}$

$$
\begin{aligned}
& M: \quad 60,69 \mathrm{Cr} \\
& 28,89 \mathrm{Fe} \\
& 6,39 \mathrm{~W} \\
& T_{a}, V \text { and } \mathrm{Ti}_{i}
\end{aligned}
$$

- Dirbcations :

- Burgers vecter : $\left.\frac{1}{c} a_{c}<111\right\rangle$

- Dominent serew character

- Disbcetion density:

$$
\begin{array}{ll}
\text { as received } & 0,86 \times 10^{10} \mathrm{~cm}^{-2} \\
\text { deformed } & 1,0 \times 10^{10} \mathrm{~cm}^{-2}
\end{array}
$$

irradiated 0,sdpa $0,9 \times 10^{10} \mathrm{~cm}^{-2}$

- No visuble defeets in irradieted materieal 
F2:-

as resecived

Ciemisai, maps ving

\section{EFTEM}

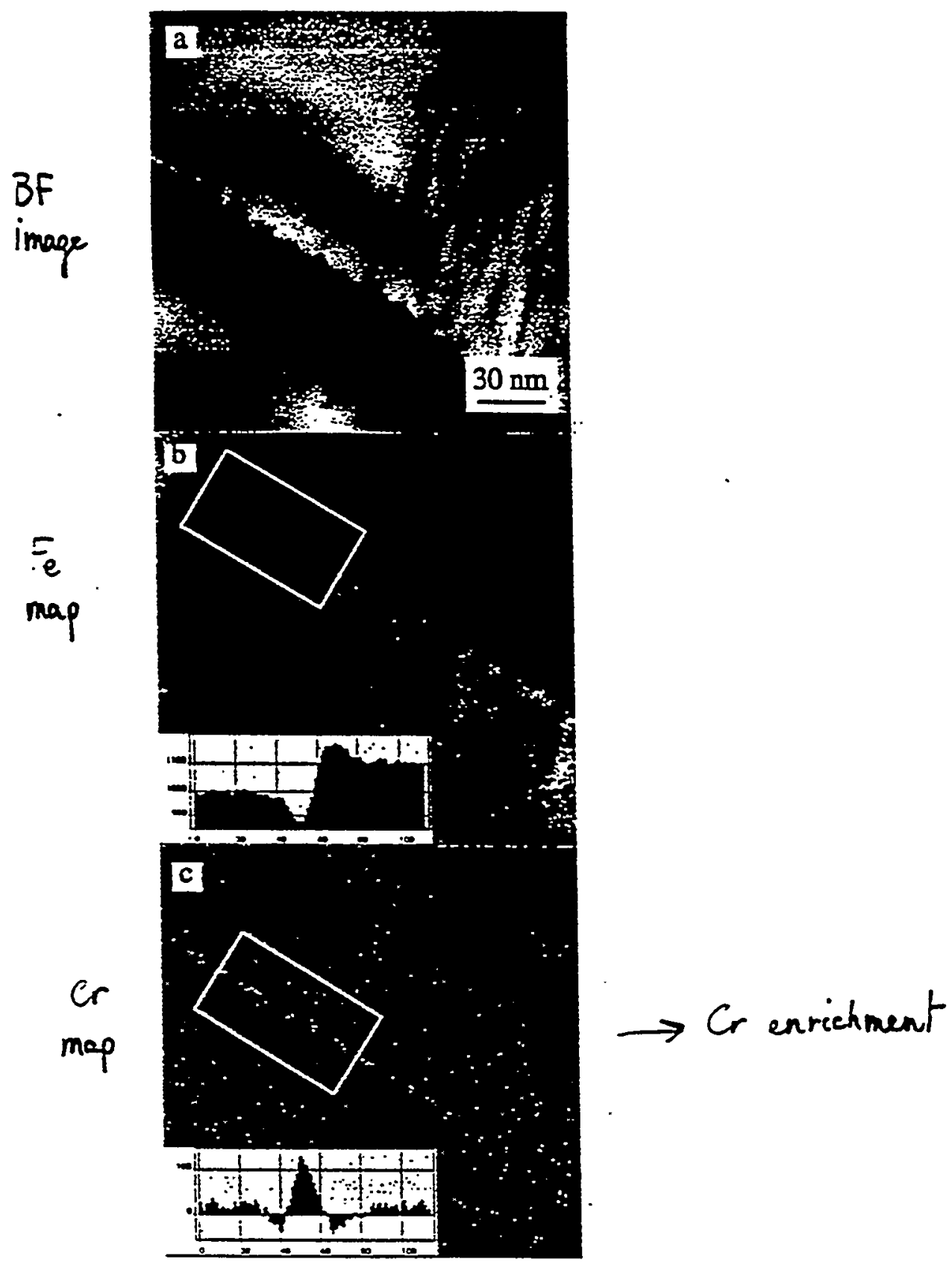

Fig. 2 
$\mathrm{F} 2 \mathrm{HH}$

irradiated

6 0,5 dye

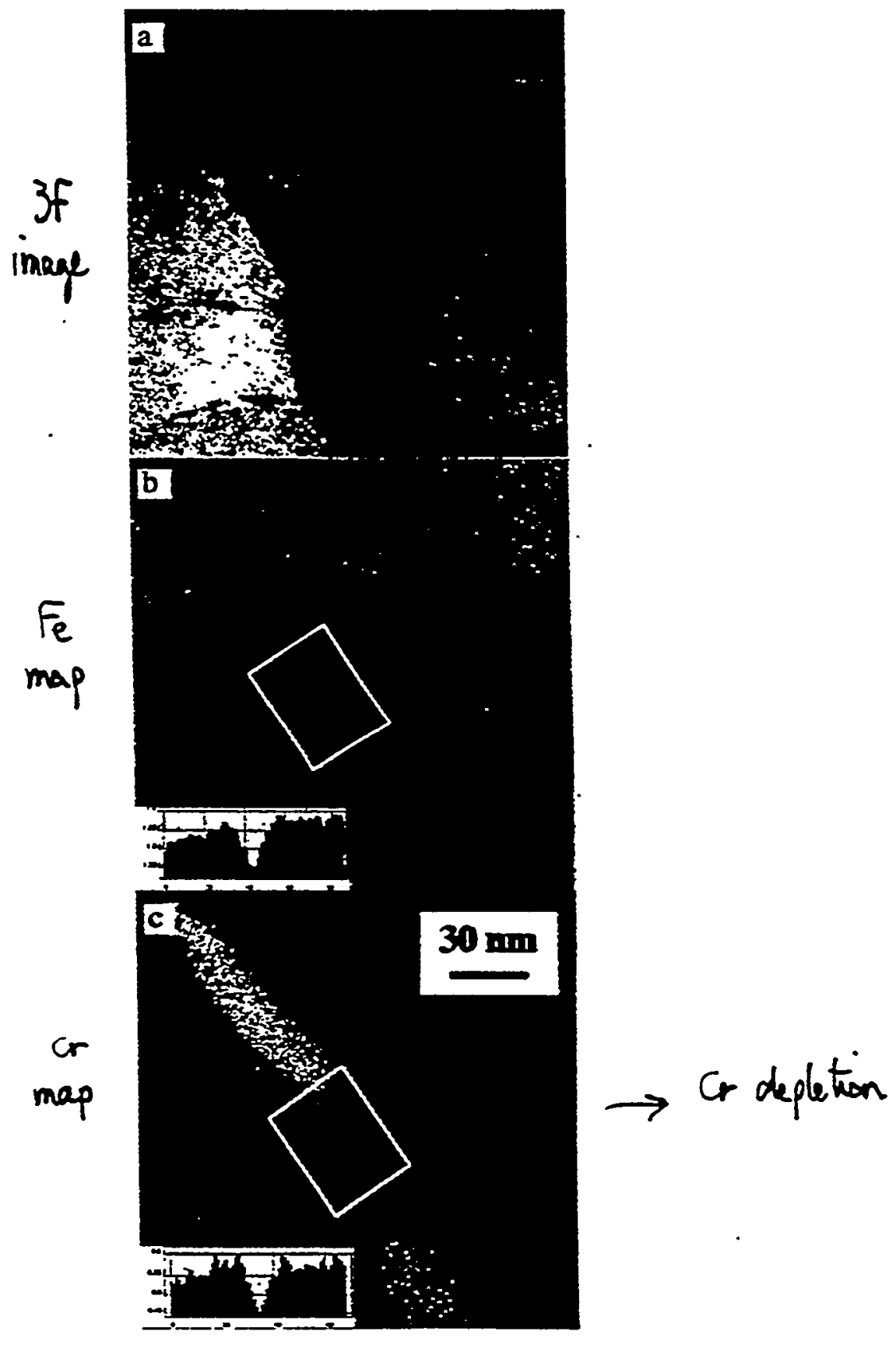

Fig. 3 
$=\widehat{8} 2 \mathrm{r}:$

irradiated

to 1,7 dpe

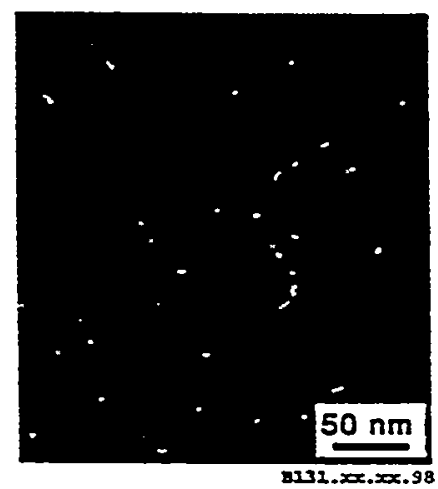

WB-TEM

- Dislocations $1 / 2 a_{0}<111>$

- Black dots in BF 
Frort

is reecived

$$
\text { In-sita tearing }
$$
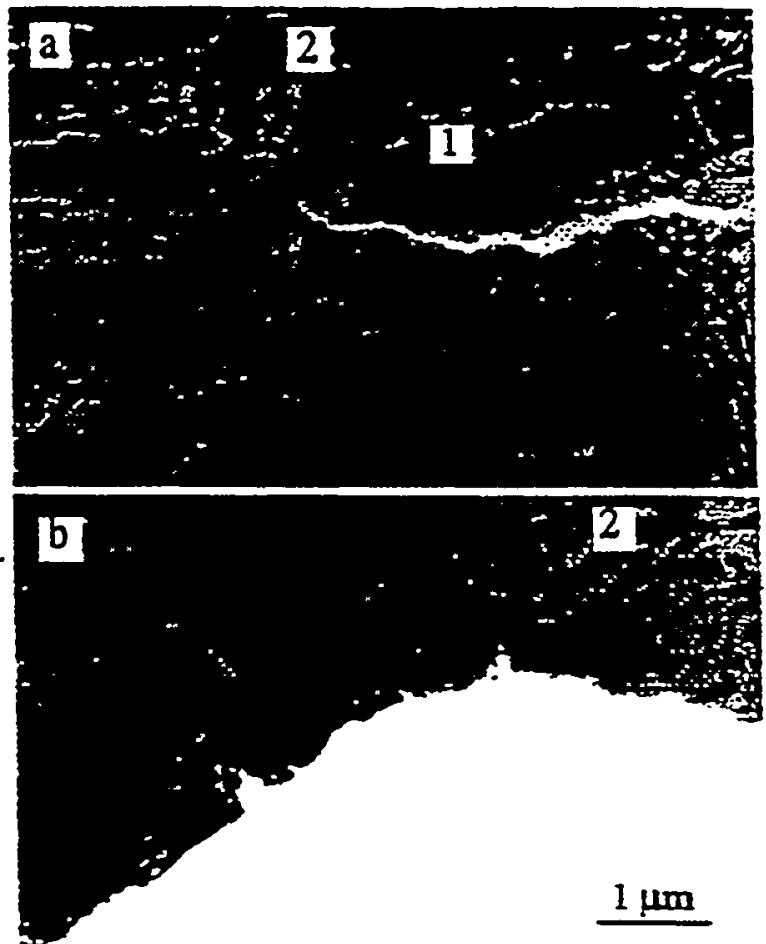

Fig. 4 
SUMmary

- No differences in microstructure! defects between as received, deformed and irradiated to 0,5 da

- Difference in boundary chemistry after irradiation Invisible defects? 


\section{OPTIMAX A 2.5 dpa, neutrons, $250^{\circ} \mathrm{C}$}

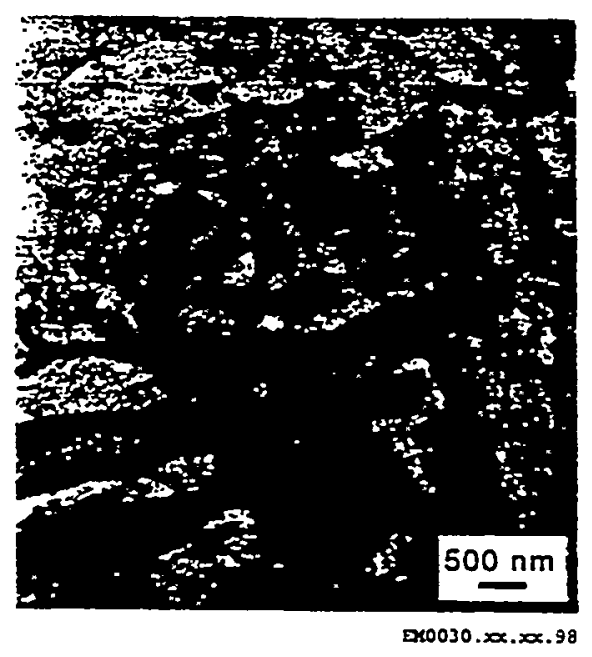

General view in BF-TEM 
OPTIMAX A 2.5 dpa, neutrons, $250^{\circ} \mathrm{C}$

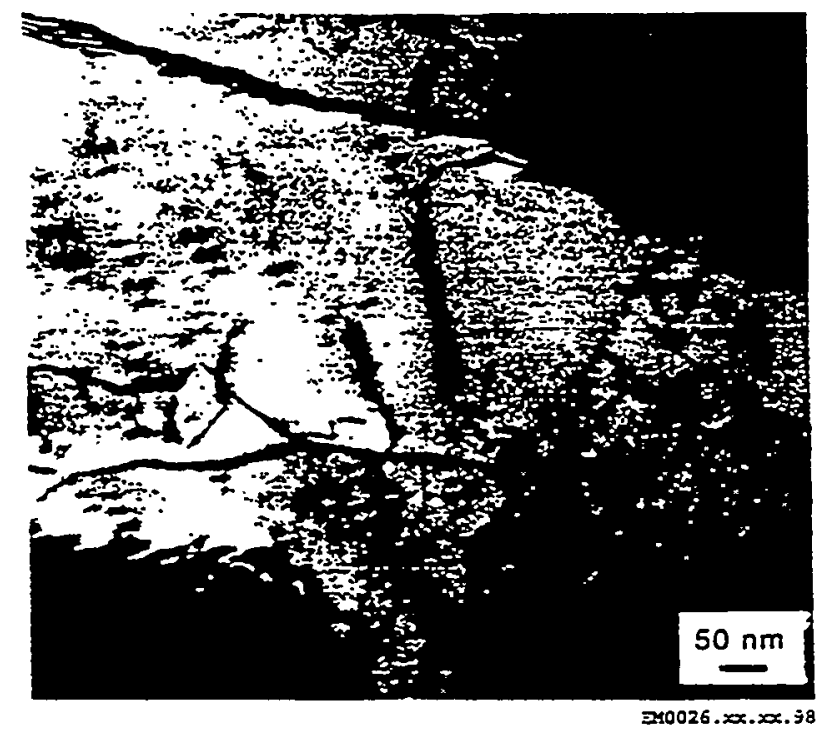

General view in BF-TEM

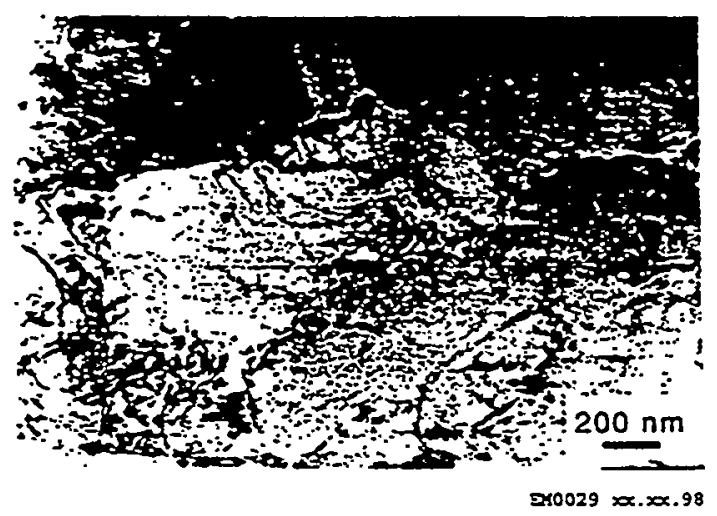

General view in BF-TEM 


\section{OPTIMAX A $2.5 \mathrm{dpa}$, neutrons, $250^{\circ} \mathrm{C}$}

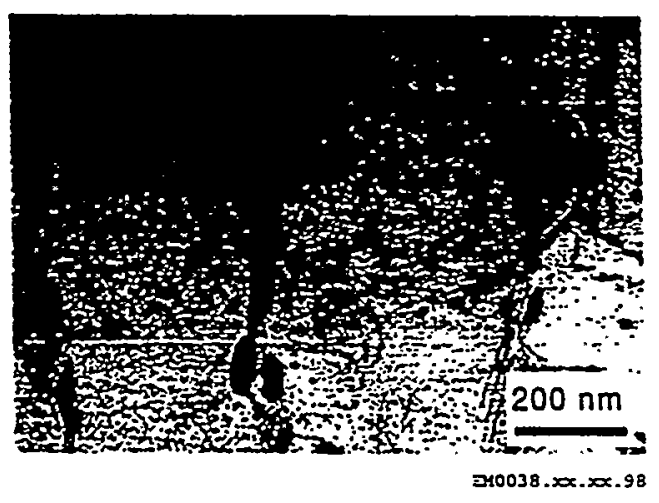

Defocussed BF-TEM

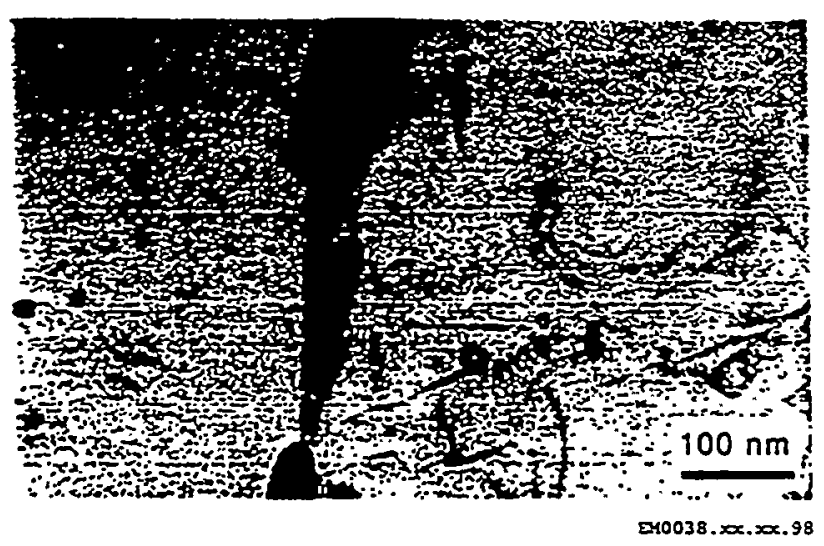

Defocussed BF-TEM

Facetted bubbles

Bubble facets on $\{011\}$ planes 
SUMMARY

opizmax A: No vieble defects

- Cocritier f Faceted Density: $2 \times 10^{20} \mathrm{~m}^{-3} \quad$ (heghest) Sige: $9 \mathrm{~nm}$

F.82H $\therefore \quad \therefore$ Bleck dots. (bops)

Density: $3 \times 10^{21} \mathrm{~m}^{-3}$

Sige: $3 \mathrm{~nm}$

- No cavities 
F82H 2.5 dpa, neutrons, $250^{\circ} \mathrm{C}$

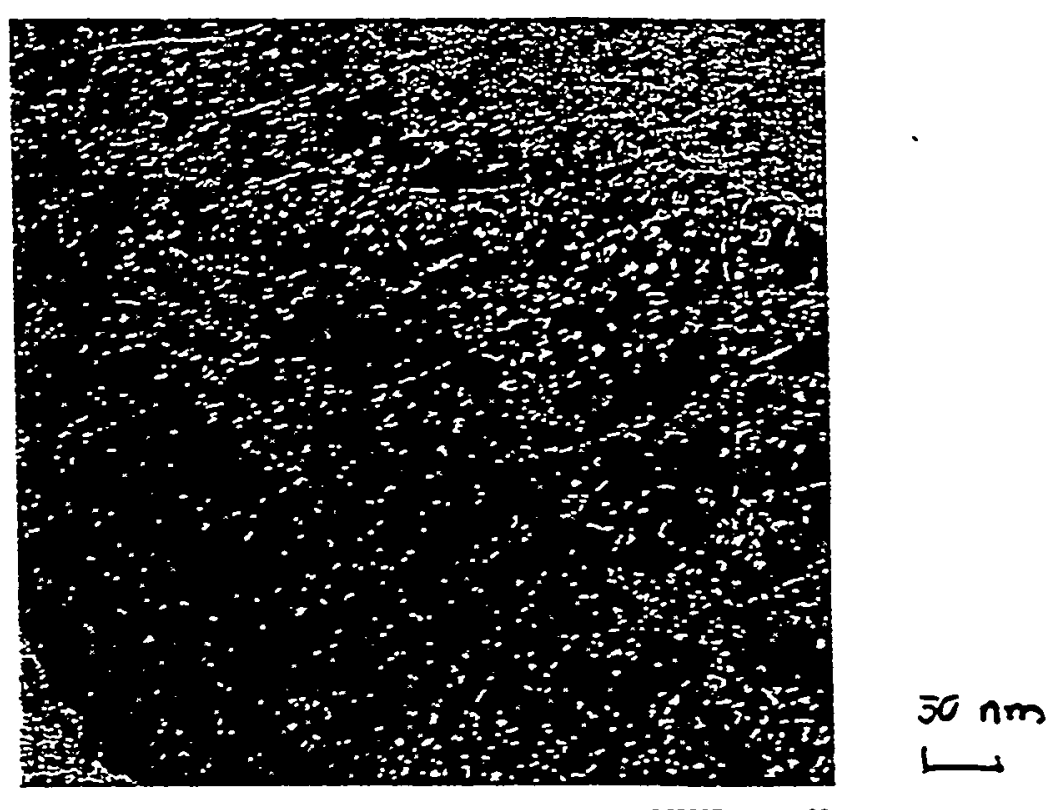

iVB image

$\rightarrow$ Defects (loope," black dofr")

$\rightarrow$ no He bubbles nor curities 


\section{IDENTIFICATION of "SMALL" JEFECTS in a TEM TYP \\ The simulation approach: MD simulation $\rightarrow$ semple muctislice imoge simulation $\rightarrow$ image in TEM}

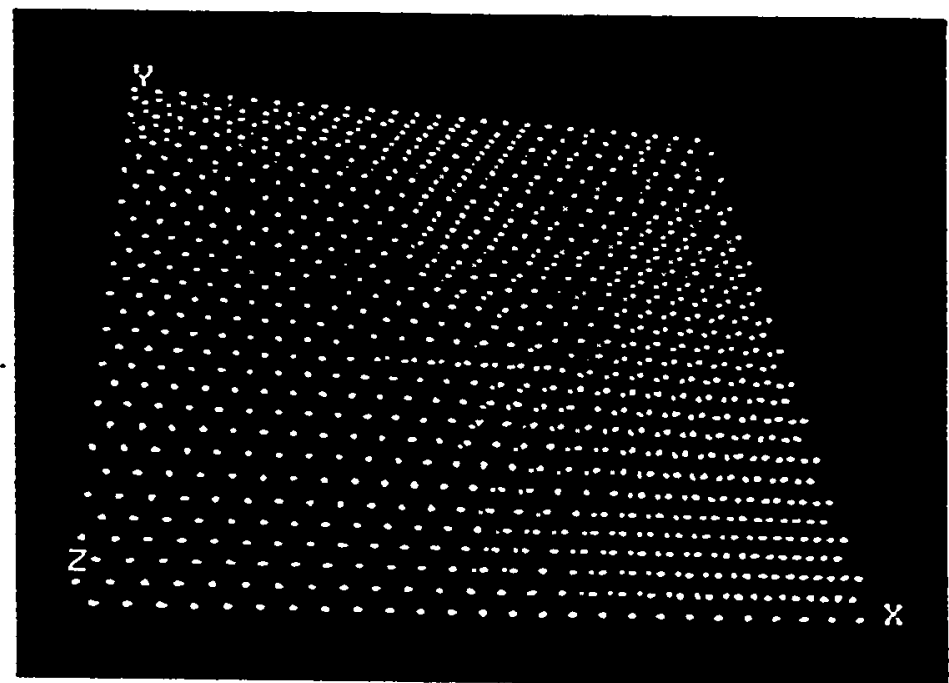

Figure 1 : View of a (111) plane curted in the MD sample containing the $2 \mathrm{~nm}$ SFT

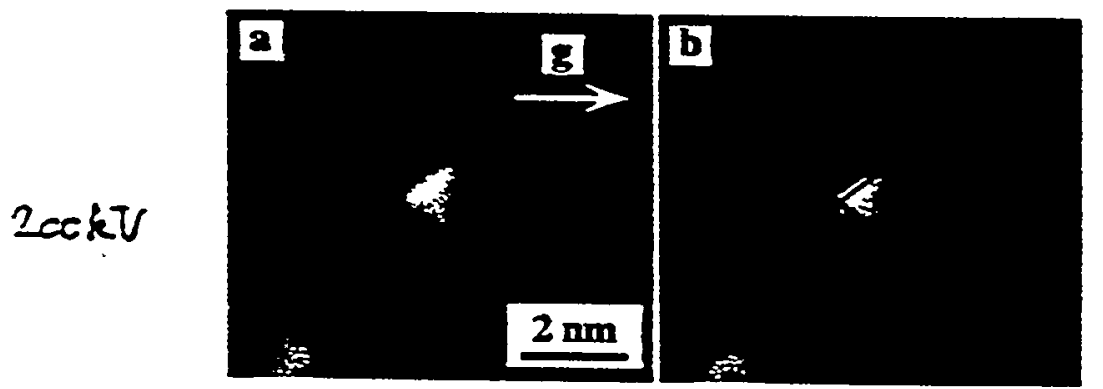

$$
\text { (Y. Dai, ?SI) }
$$

Figure 2 : a) Experimental weak beam $g(6 g), g=(200)$ of an SFT. b) Same as a) but with the wire frame of a $2 \mathrm{~nm}$ SFT

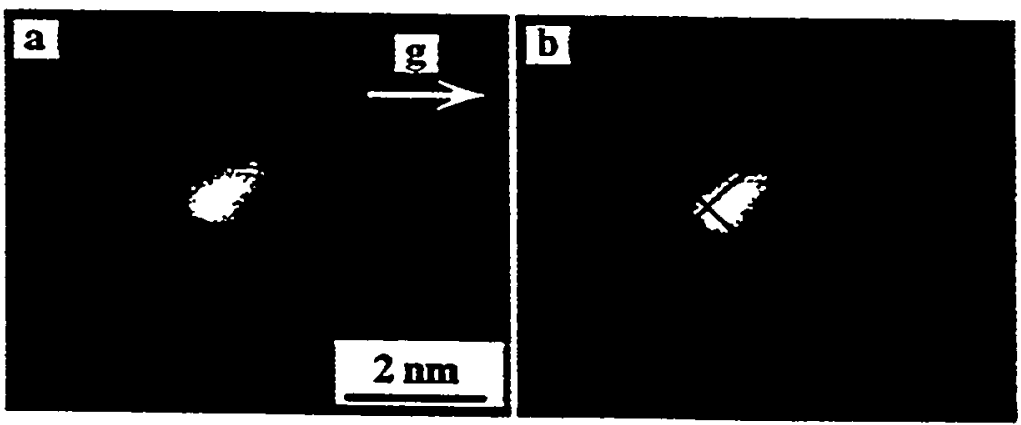

Figure 3 : a) Simulated weak beam $g(6 z), g=(200)$ of the MD simulated $2 \mathrm{~nm}$ SFT. b) Same as a) but with the wire frame of the MD simulated SFT.

$\Rightarrow 20 \%$ defference between inage sige and obyeer size 


\section{Dislocation loops in Al}

Diameters of the loop:

$\sim 0.5 \mathrm{~nm} \rightarrow 8$ interstitials $(0.57 \mathrm{~nm})$

$\sim 1.0 \mathrm{~nm}->19$ interstitials $(1.14 \mathrm{~nm})$

$-2.0 \mathrm{~nm}->37$ interstitials $(1.71 \mathrm{~nm})$
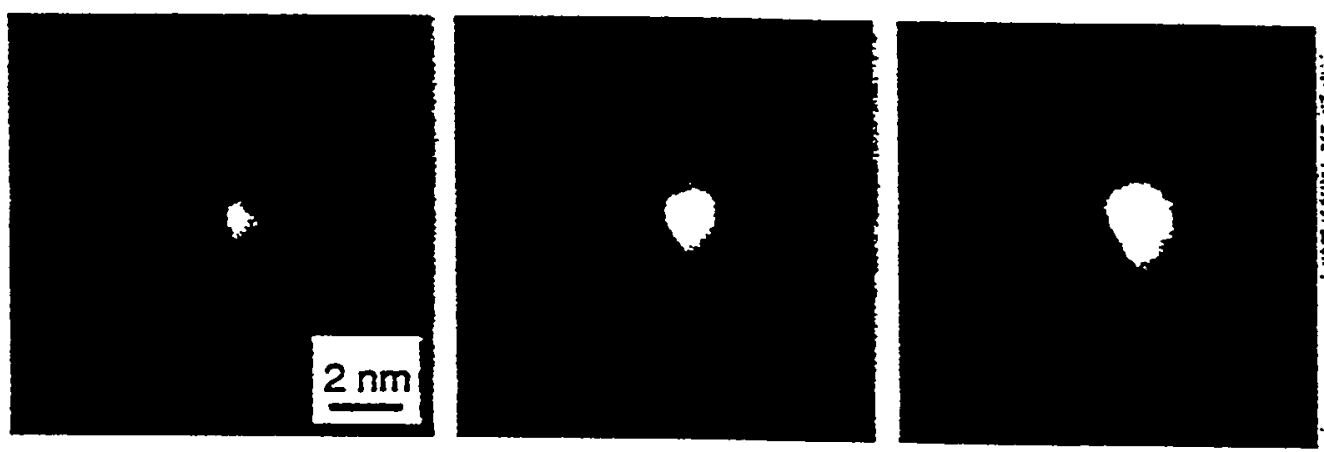

$2 \infty k v$

$\Rightarrow$ Difference image 6 chject $\nearrow$ when abject size $\downarrow$ 


\section{TENSILE AND CHARPY PROPERTIES \\ OF B-DOPED F82H AFTER IRRADIATION}

K. Shiba/JAERI

IEA Working Group Meeting on

Reduced Activation Ferritic/Martensitic Steels

1-2, October, 1998

ECN Petten, The Netherlands 


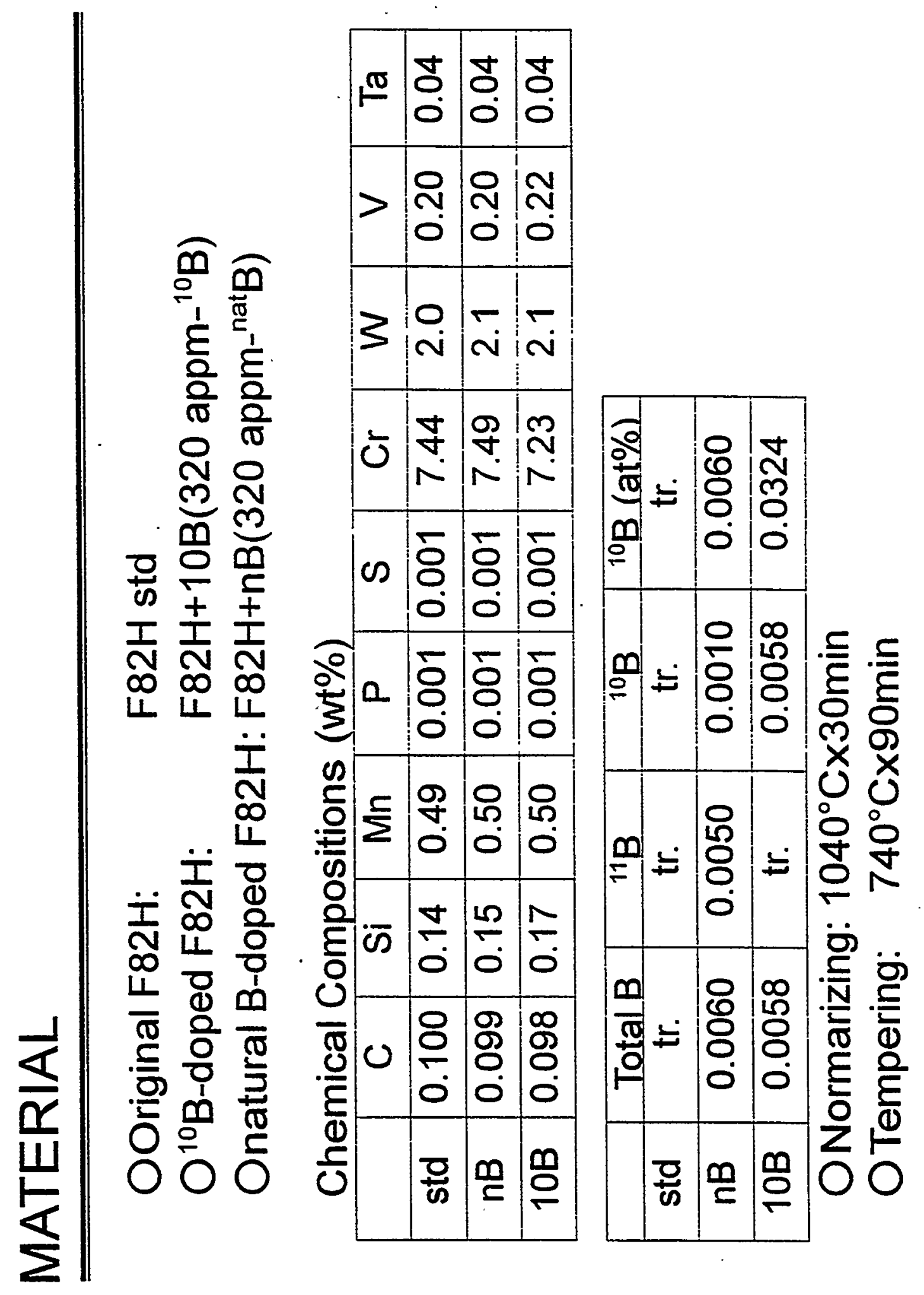




\section{IRRADIATION MATRIX OF F82H AND B-DOPED F82H}
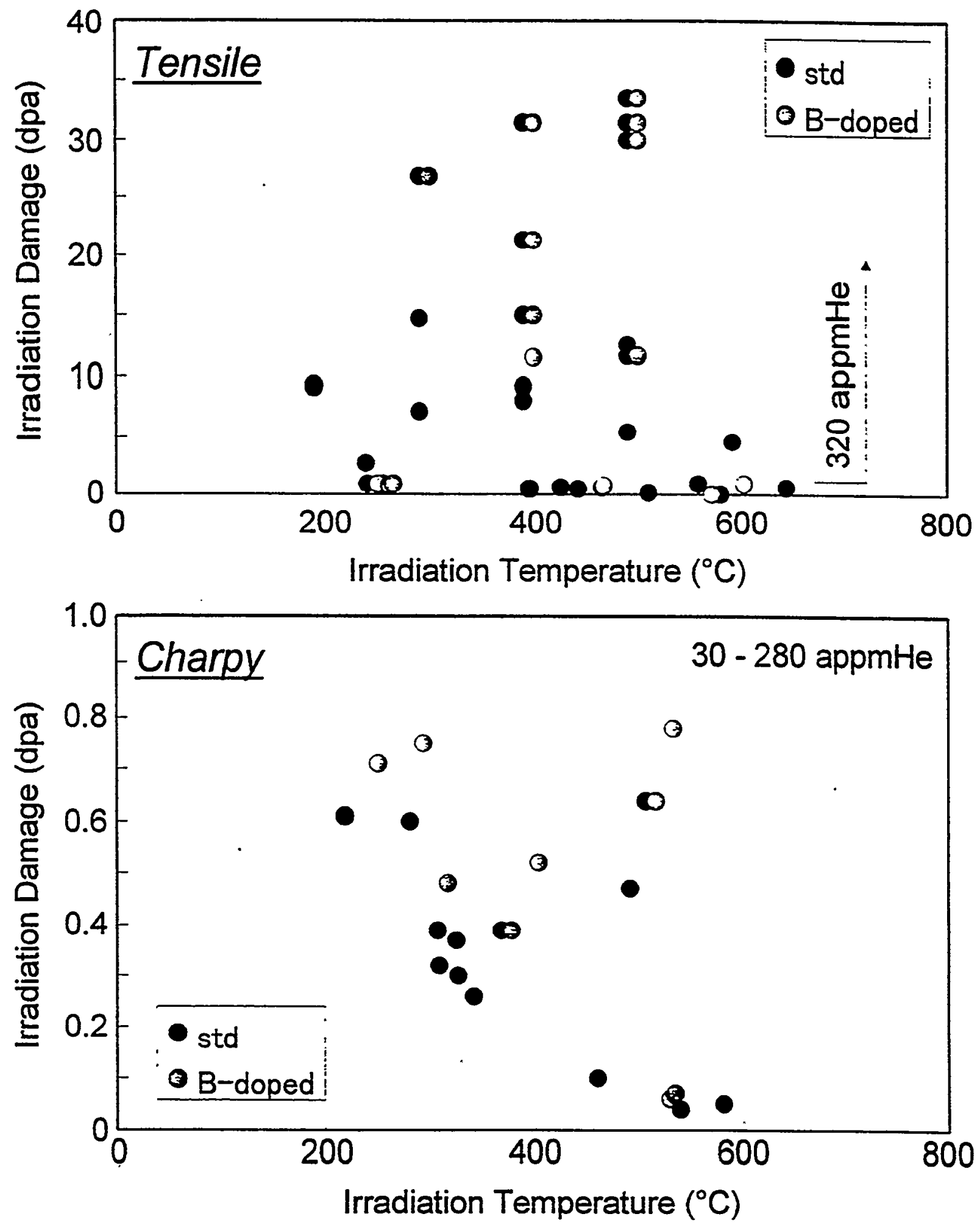


\section{SPECIMENS}

OHFIR Irradiation

- SS-3 Sheet Tensile

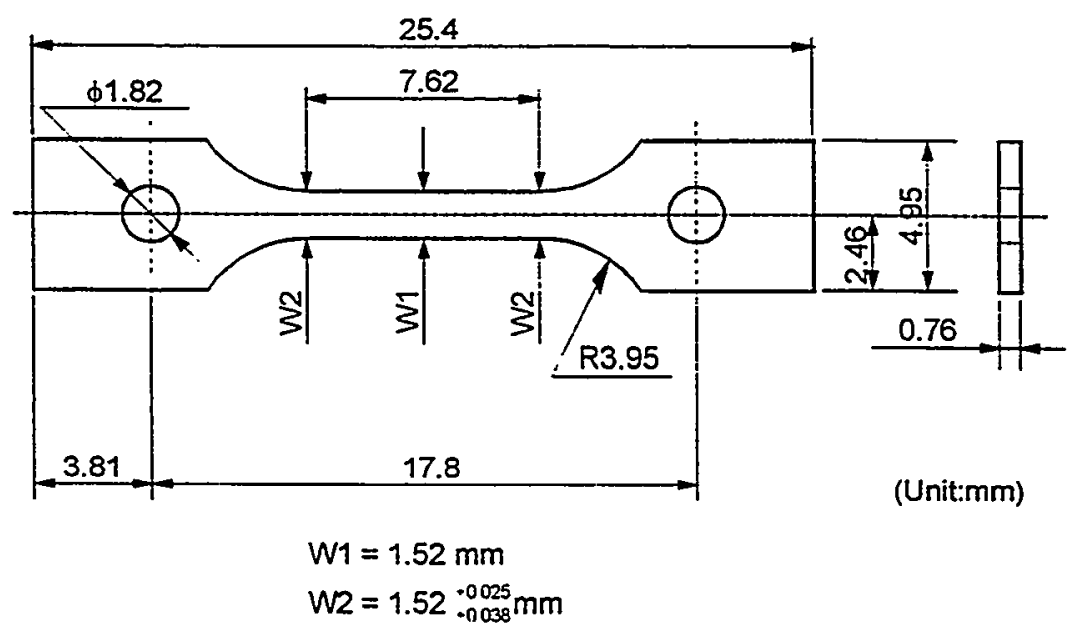

\section{OJMTR/JRR-2 Irradiation}

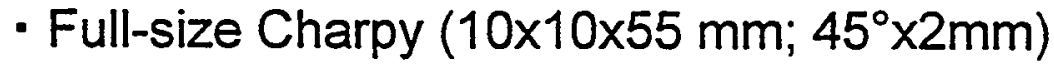

- Round-bar Tensile ( $\phi 4 \times 20 \mathrm{~mm})$

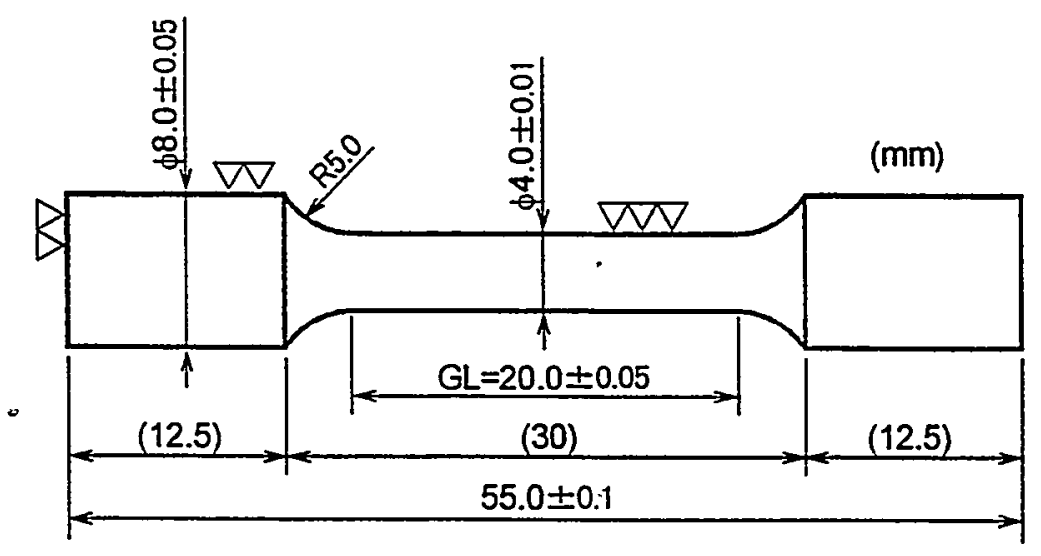




\section{Tensile Test Resuts of $\mathrm{F} 82 \mathrm{H}$ with and without ${ }^{10} \mathrm{~B}$-doping}
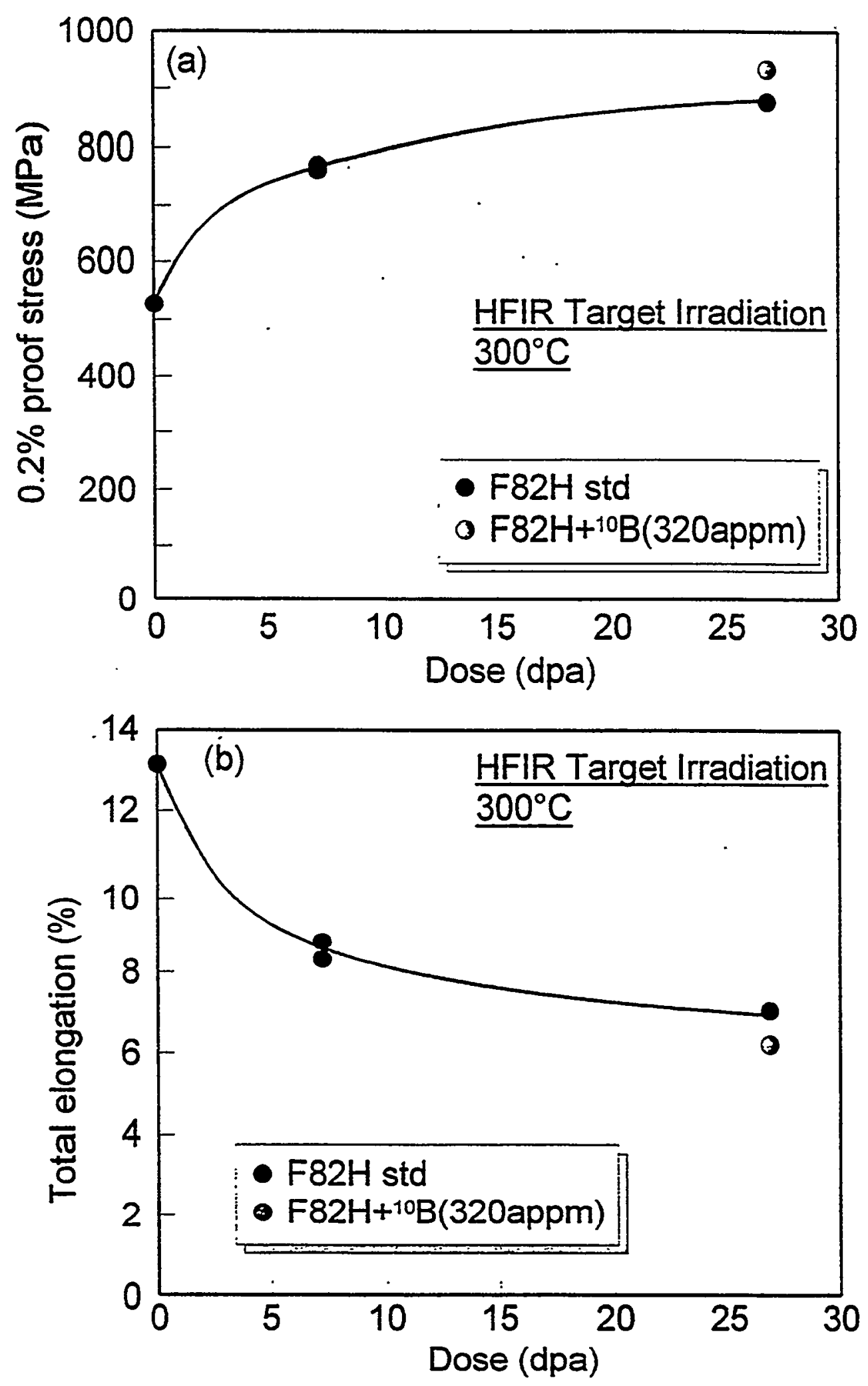


\section{Tensile Test Resuts of $\mathrm{F} 82 \mathrm{H}$ with and without ${ }^{10} \mathrm{~B}$-doping}
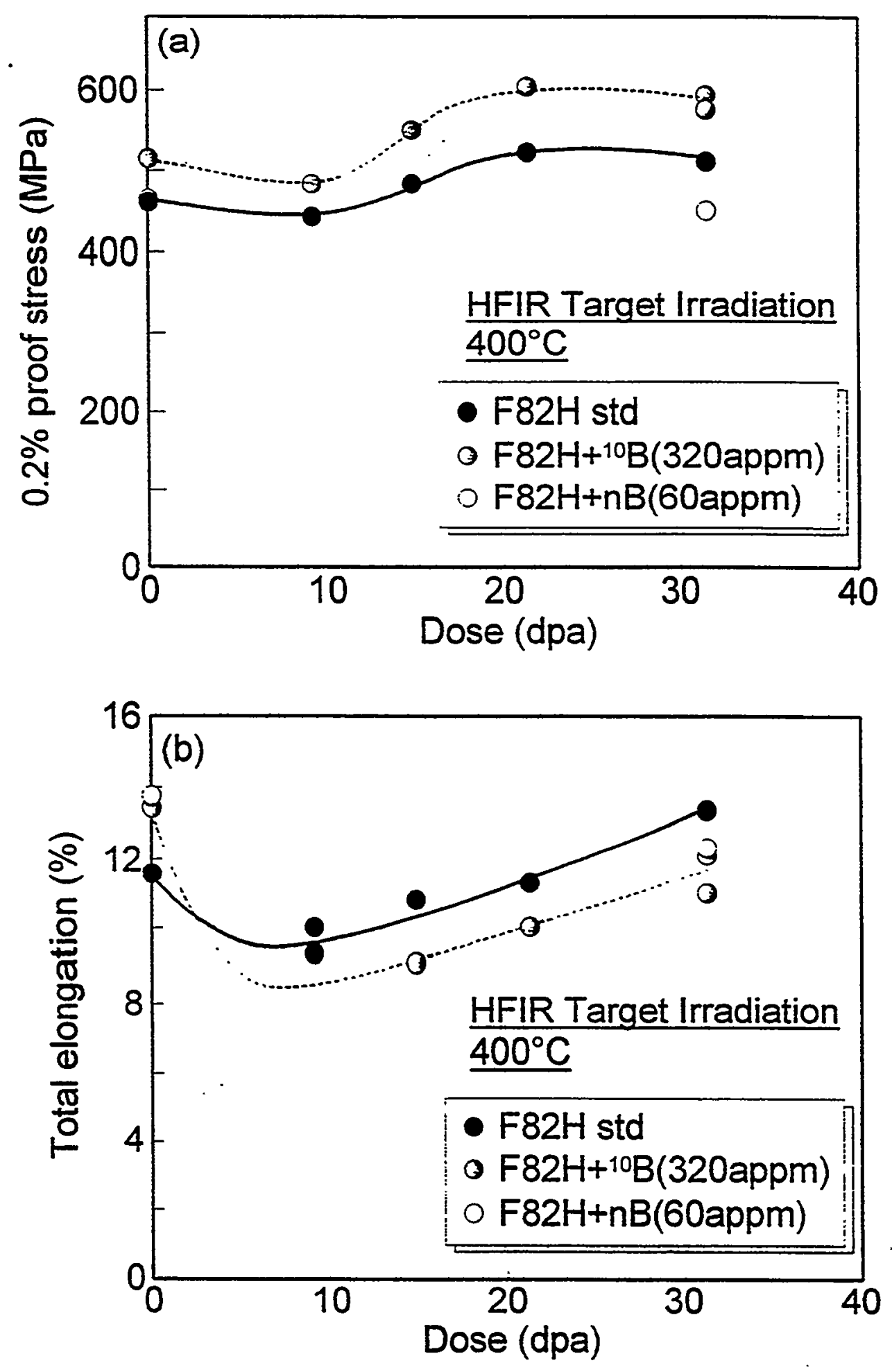


\section{Tensile Test Resuts of F82H with and without ${ }^{10} \mathrm{~B}$-doping}
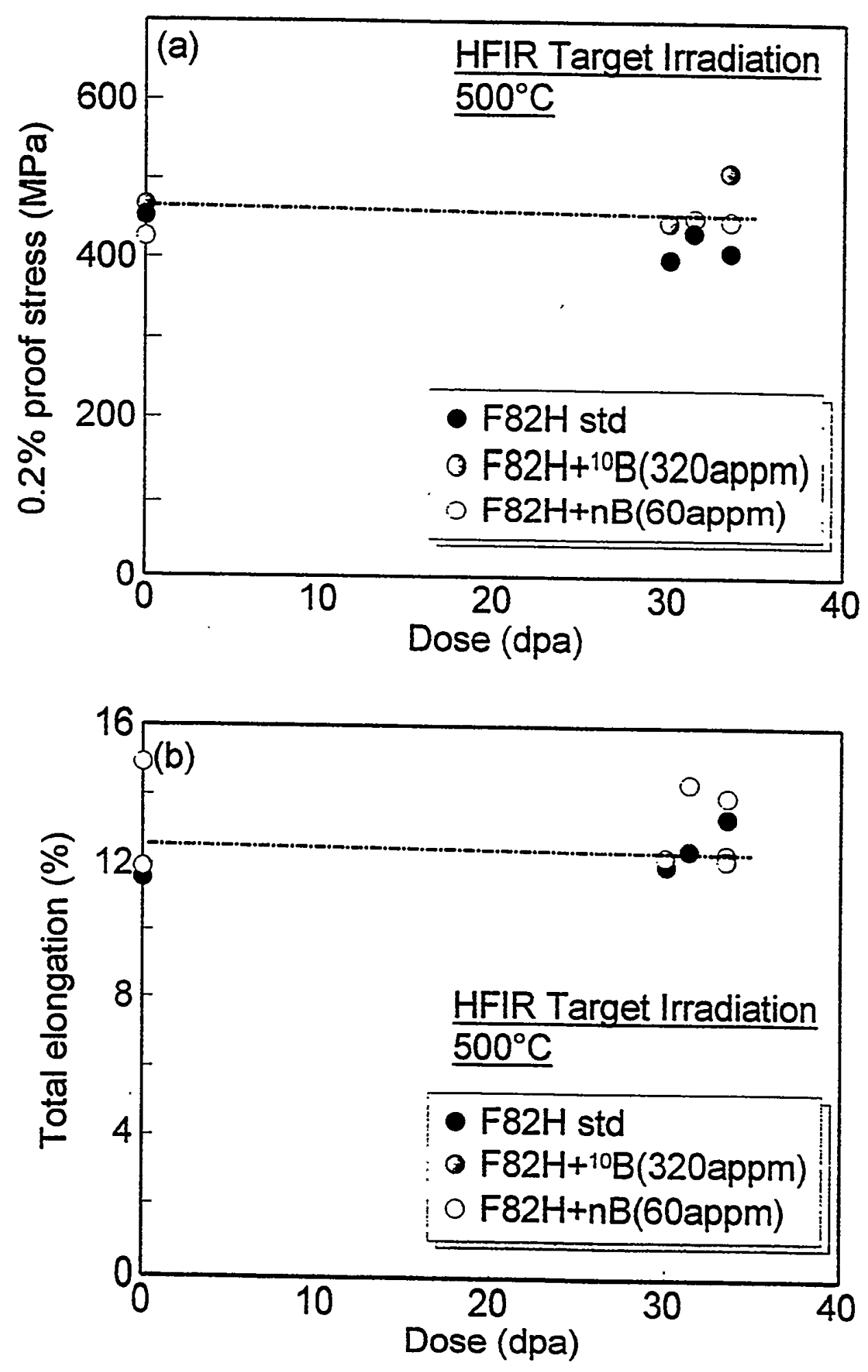


\section{TENSILE PROPERTIES OF F82H AND B-DOPED F82H}
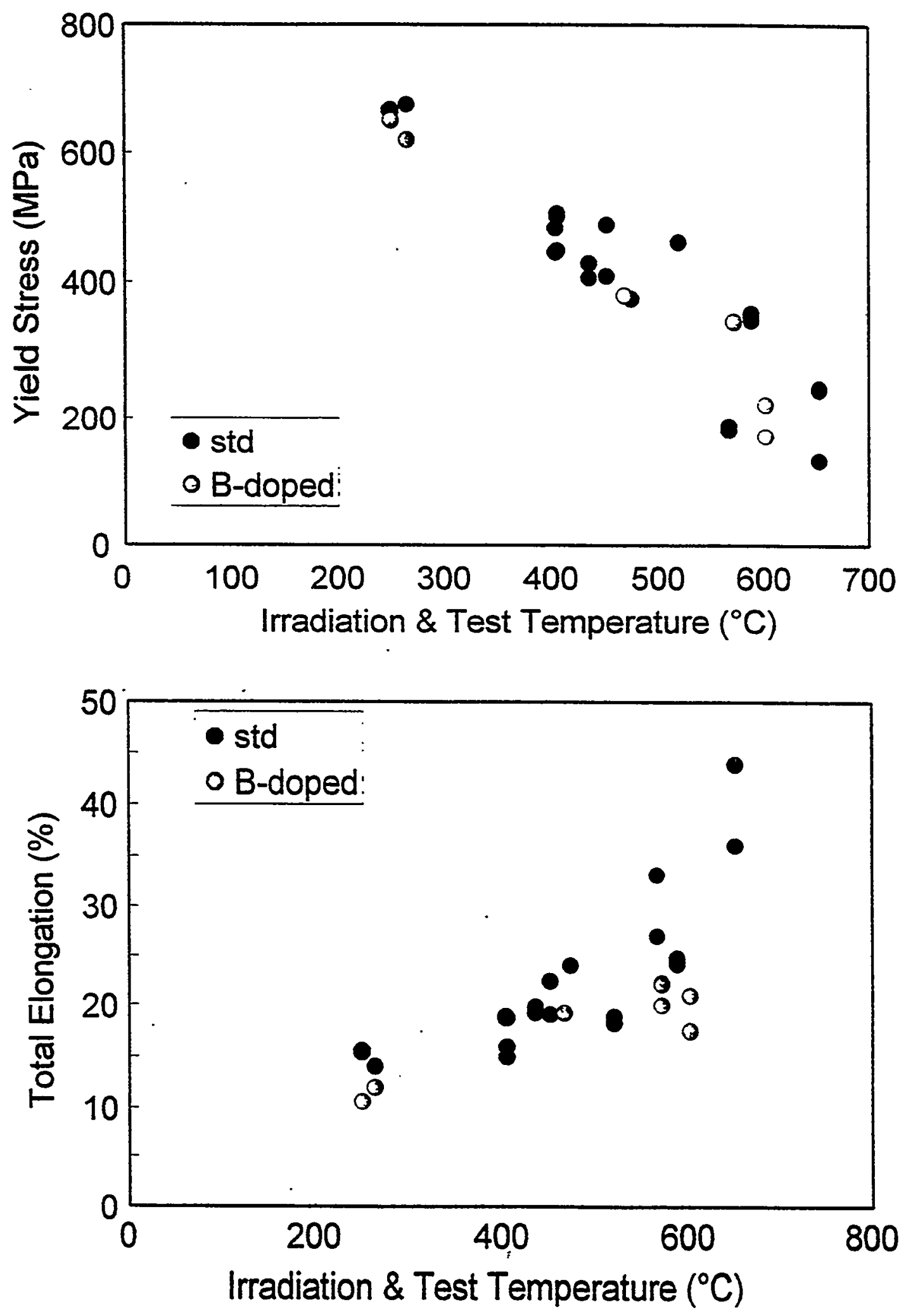


\section{TENSILE PROPERTIES OF F82H AND B-DOPED F82H}

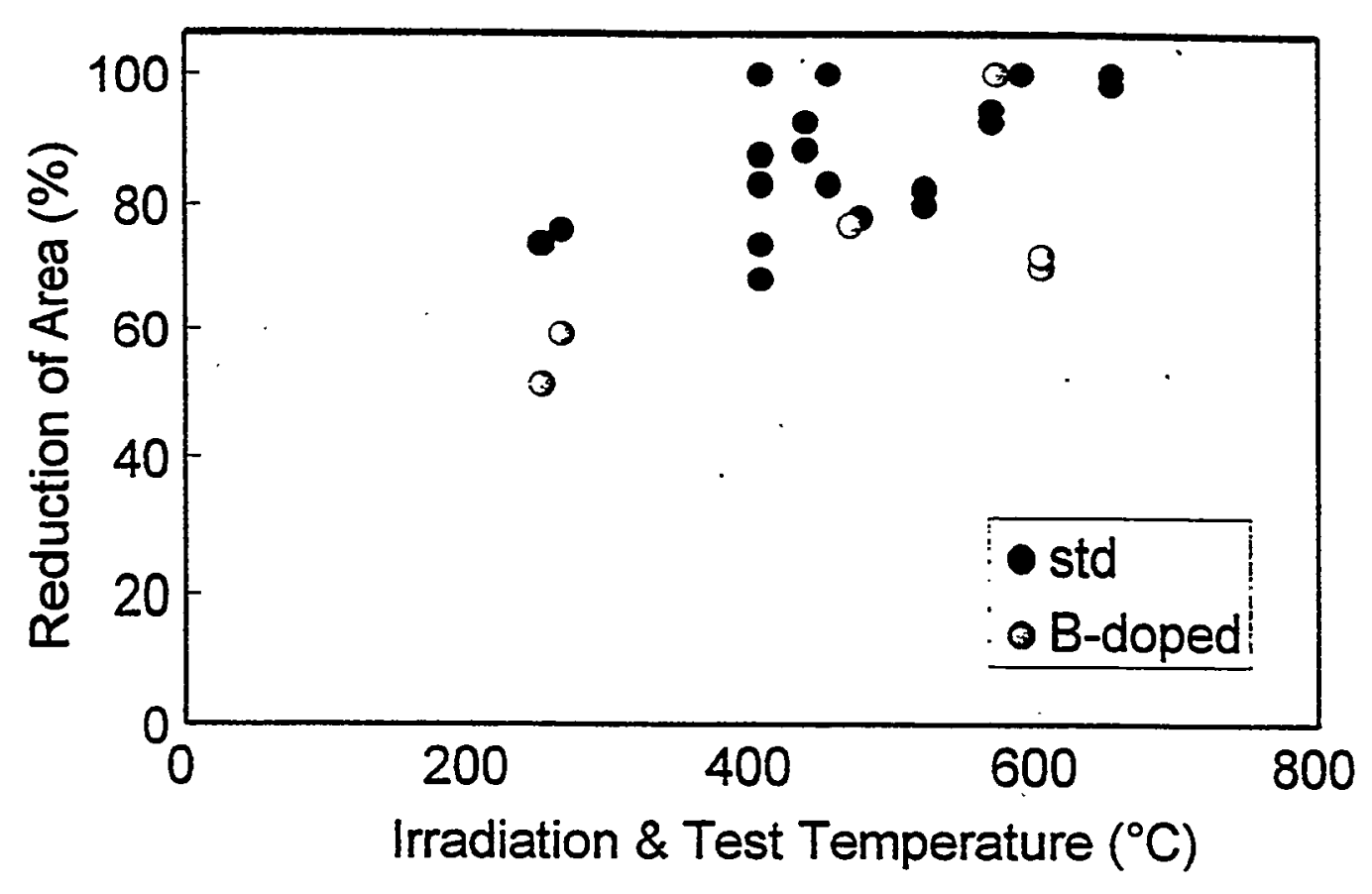




\section{TENSILE PROPERTIES OF F82H AND B-DOPED F82H}
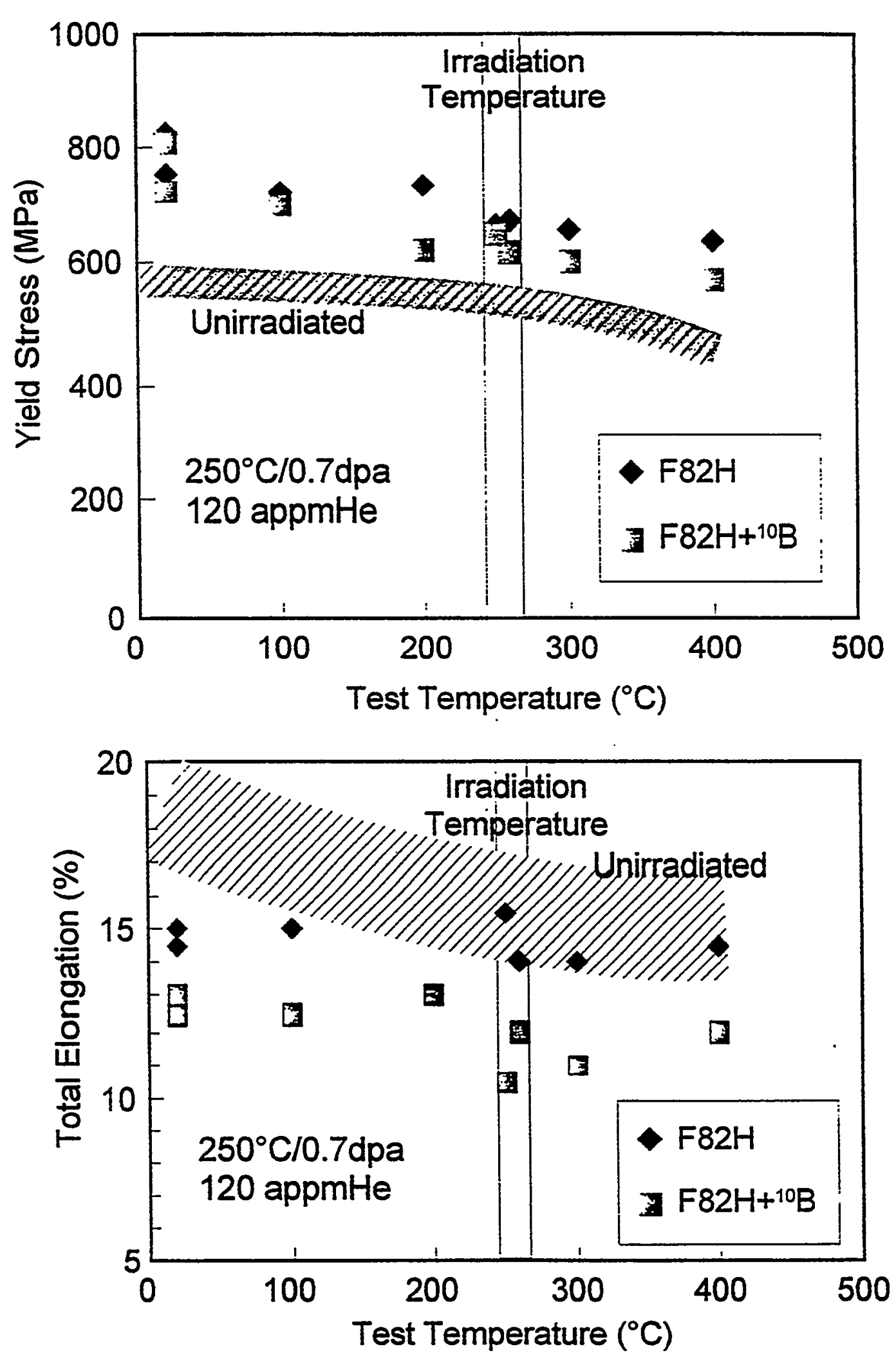


\section{TENSILE PROPERTIES OF F82H AND B-DOPED F82H}
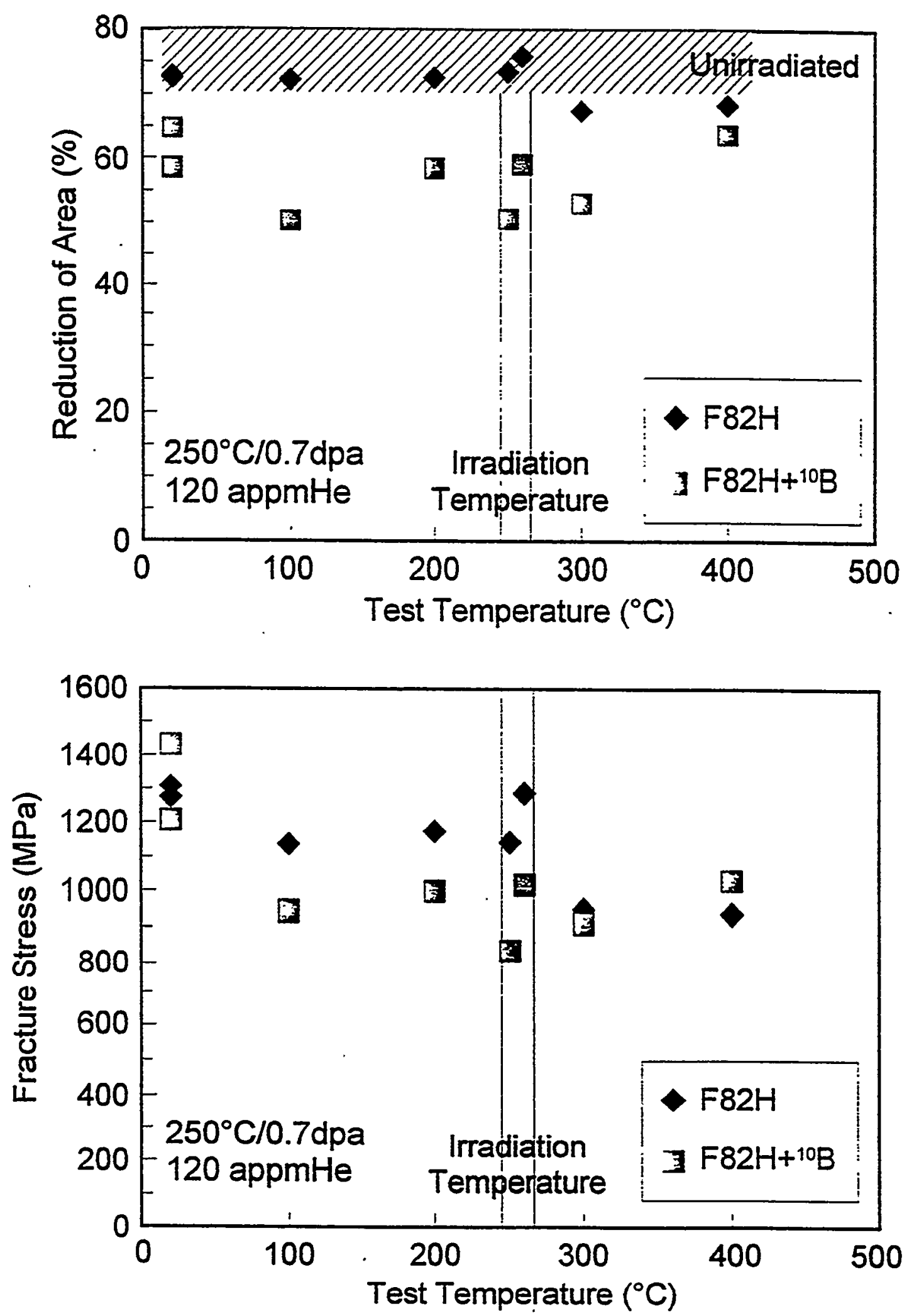


\section{Charpy Impact Test Resuts of $\mathrm{F} 82 \mathrm{H}$ with and without ${ }^{10} \mathrm{~B}$-doping}

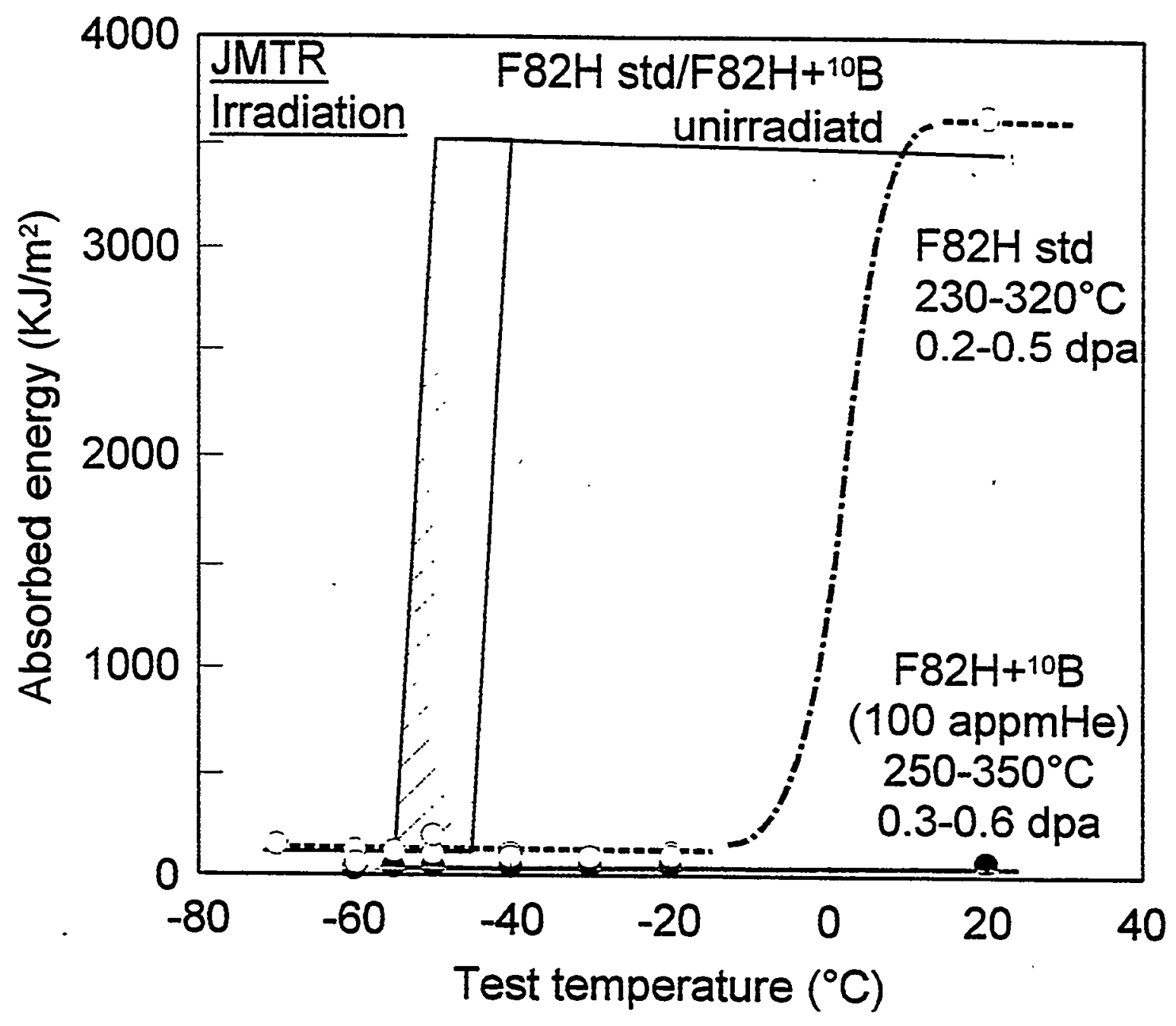




\section{Charpy Properties of $\mathrm{F} 82 \mathrm{H}$ and $\mathrm{F} 82 \mathrm{H}+10 \mathrm{~B}$ $\left(400^{\circ} \mathrm{C} / 0.4 \mathrm{dpa}\right.$ Irradiation)}
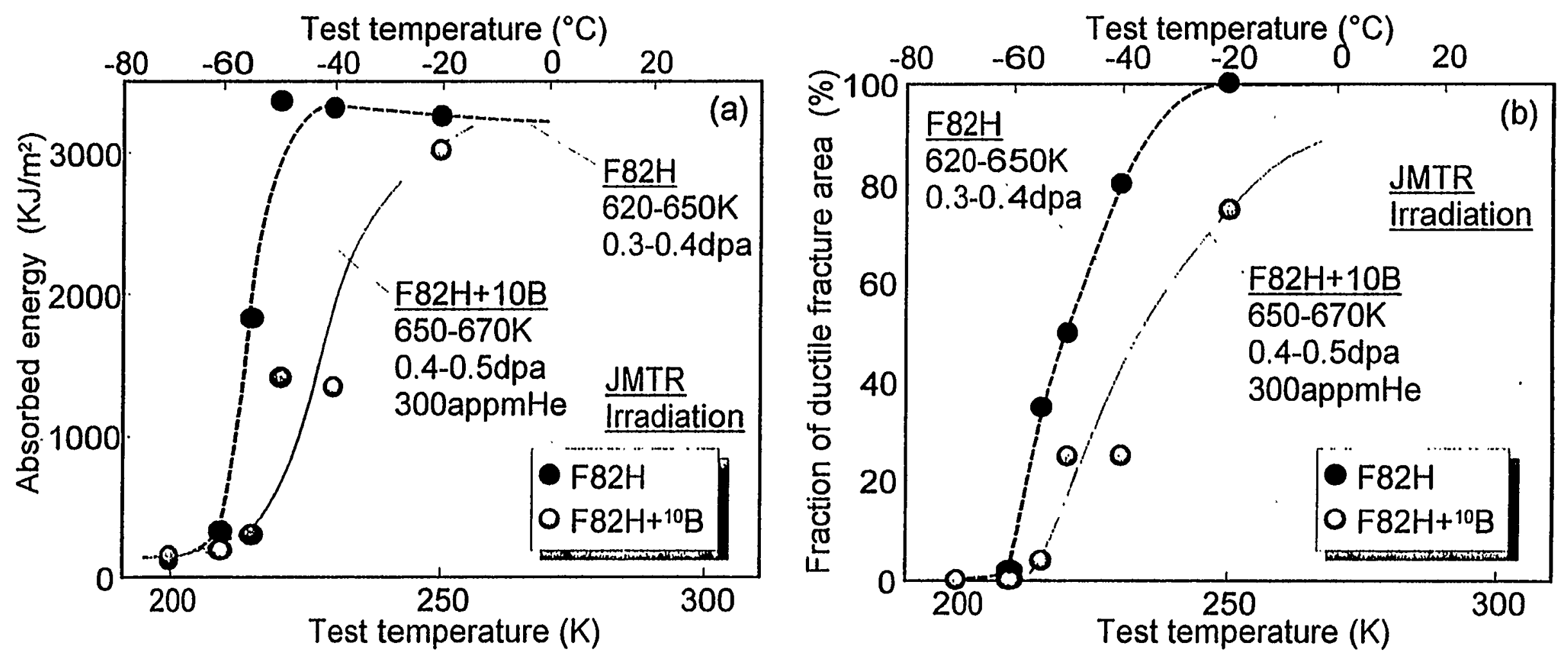


\section{Charpy Properties of $\mathrm{F} 82 \mathrm{H}$ and $\mathrm{F} 82 \mathrm{H}+10 \mathrm{~B}$ (400-530 ${ }^{\circ} \mathrm{C}$ Irradiation)}
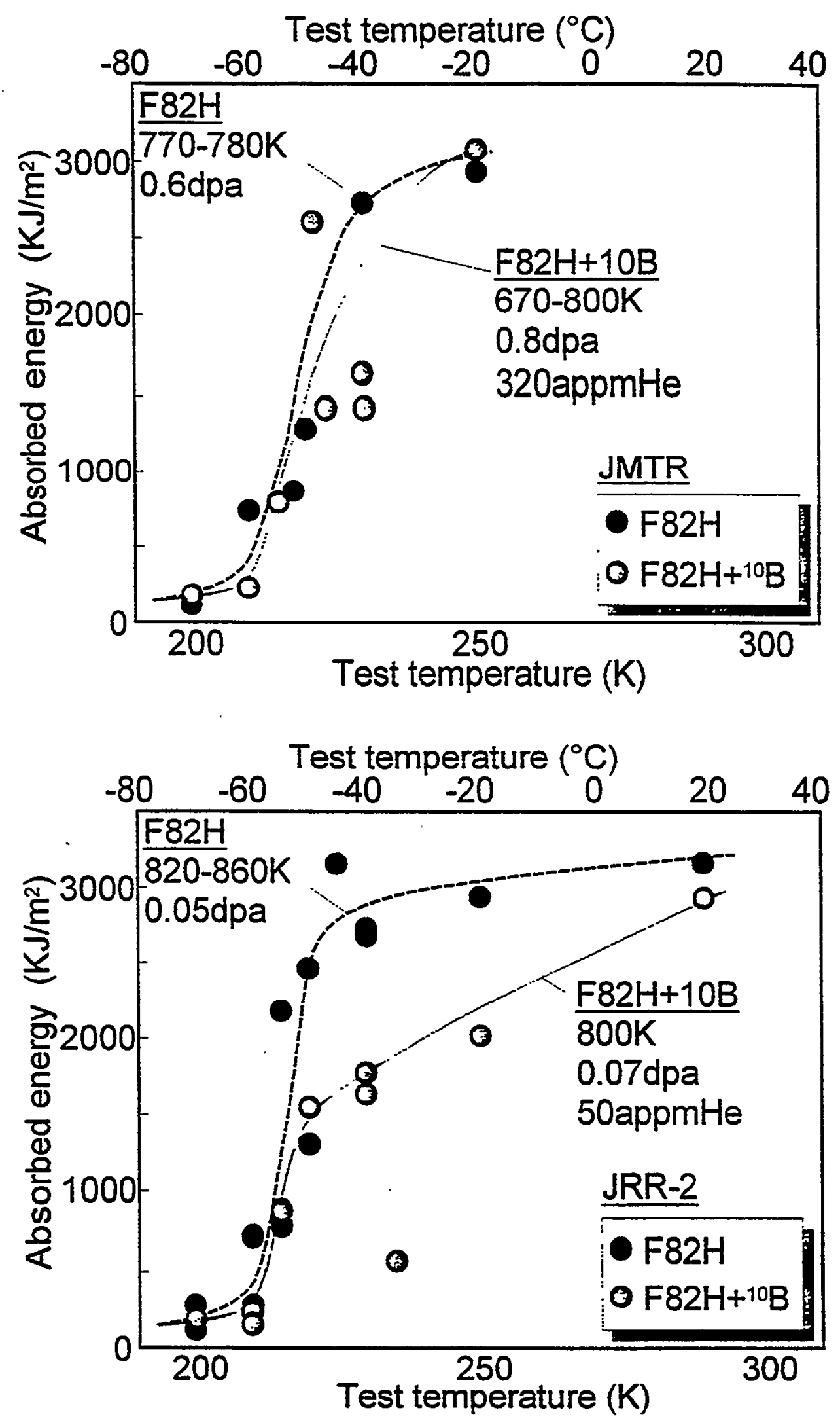


\section{SUMMARY OF MICROSTRUCTURE}

\begin{tabular}{|c|c|c|c|c|}
\hline \multicolumn{2}{|c|}{} & \multicolumn{2}{|c|}{$\mathrm{F} 82 \mathrm{H} \mathrm{org}$} & $\mathrm{F} 82 \mathrm{H}+10 \mathrm{~B}$ \\
\cline { 2 - 5 } & $250^{\circ} \mathrm{C}$ & $300^{\circ} \mathrm{C}$ & $300^{\circ} \mathrm{C}$ \\
\hline \multirow{2}{*}{$\begin{array}{c}\text { Dislocation } \\
\text { Loops }\end{array}$} & $\begin{array}{c}\text { Number } \\
\text { Density }\end{array}$ & $2.5 \mathrm{dpa}$ & $57 \mathrm{dpa}$ & $\begin{array}{c}57 \mathrm{dpa} \\
320 \mathrm{appmHe}\end{array}$ \\
\cline { 2 - 5 } & $\begin{array}{c}\text { Average } \\
\text { Size }\end{array}$ & $8 \mathrm{~nm}$ & $8 \mathrm{~nm}$ & $11 \mathrm{~nm}$ \\
\hline \multirow{2}{*}{ Cavities } & $\begin{array}{c}\text { Number } \\
\text { Density }\end{array}$ & - & - & $2 \times 10^{22} \mathrm{~m}^{-3}$ \\
\cline { 2 - 5 } & $\begin{array}{c}\text { Average } \\
\text { Size }\end{array}$ & - & - & $3 \times 10^{22} \mathrm{~m}^{-3}$ \\
\hline
\end{tabular}




\section{OBSERBED PHENPMENA}

Tensile Properties

- Small increase in strength and slightly lower elongation. $\left(400^{\circ} \mathrm{C}\right)$

- Less elongation at high temperature above $550^{\circ} \mathrm{C}$.

- Less ductility (elongation and RA) at $250^{\circ} \mathrm{C}$, but no difference in strength.

Charpy Properties

- DBTT shift to above RT occurred by $250-350^{\circ} \mathrm{C}$ irradiation to 0.5 dpa (120 appmHe).

- Wider transient temperature range appeared by irradiation above $400^{\circ} \mathrm{C}(>50$ appmHe), however, USE stayed the same revel of boron-free alloy. 

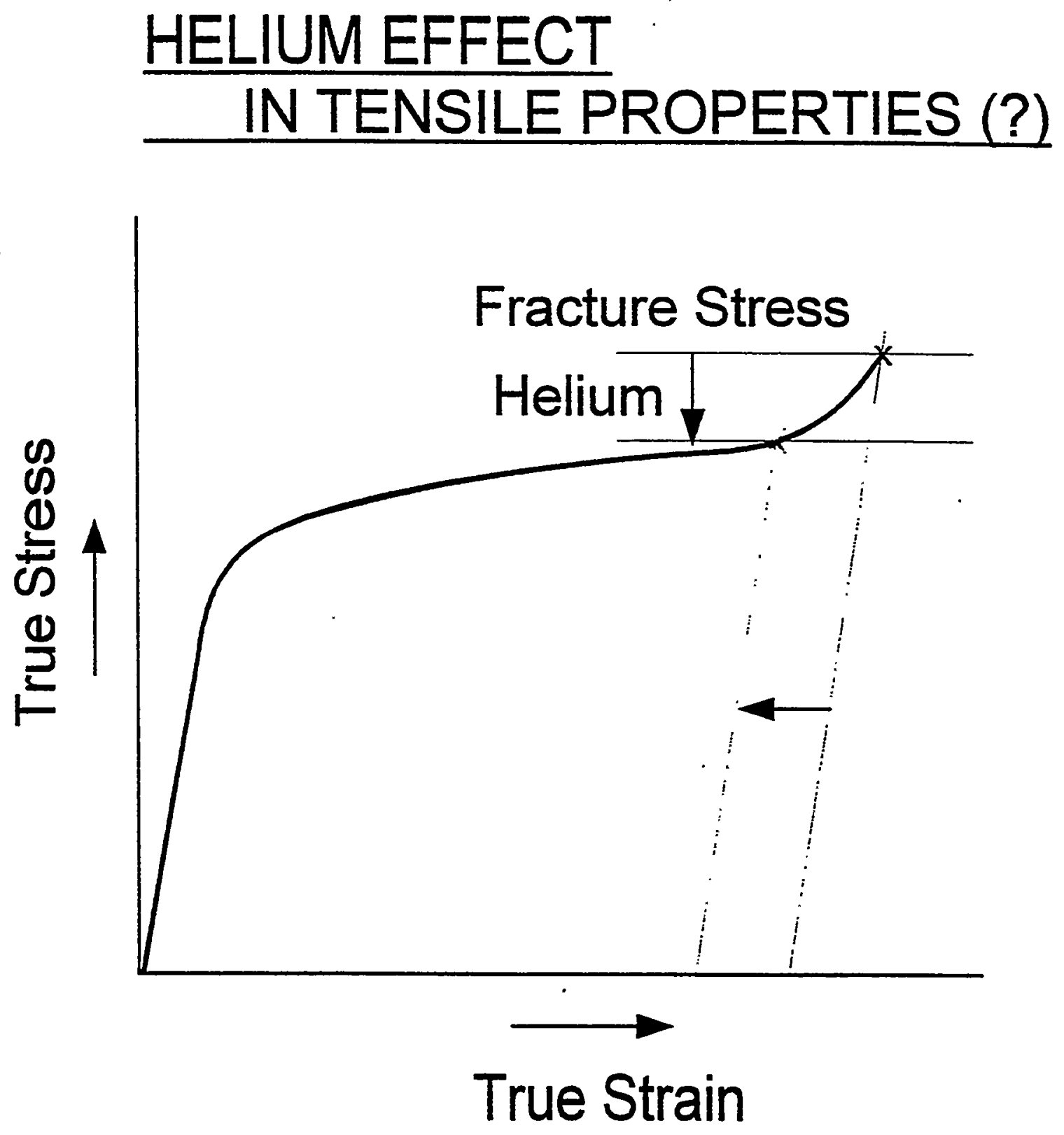


\section{Fracture Mechanism (?)}
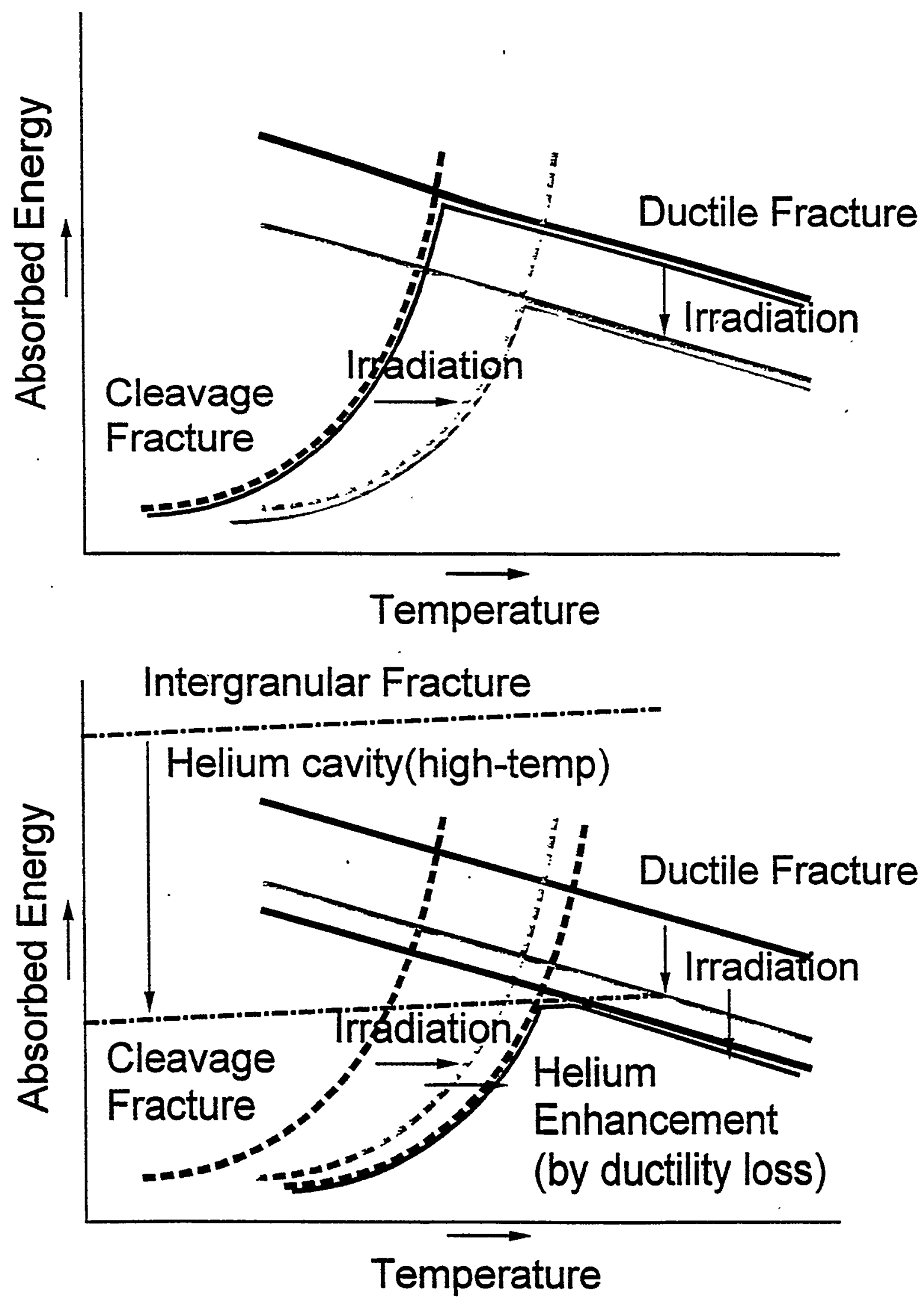


\section{SUMMARY}

- Helium effects on tensile properties appear as low elongation and reduction of area, however, it does not seem critical.

- Helium caused some shift in transition behavior and DBTT in Charpy properties, but did not decrease USE above $400^{\circ} \mathrm{C}$.

- Boron doped specimens had almost the same microstructure as boron-free alloy, except for small cavities after $300^{\circ} \mathrm{C}$ irradiation to $57 \mathrm{dpa}$. 
IEA Workshop on Reduced-Activation Ferritic/Martensitic Steels

\section{He - Effects on Mechanical Properties and Microstructure of RAFM Steels after Irradiation}

E. Materna-Morris, A. Möslang, R. Lindau, M. Rieth, K. Ehrlich, Forschungszentrum Karlsruhe Institut für Materialforschung I + II 
- Dual Beam He implantation

- HFR irradiation

Mechanical tests:

Tensile tests

Impact tests

Fracture and microstructural analysis 


\section{Experimental}

\section{Chemical composition (wt\%):}

\begin{tabular}{|c|c|c|c|c|c|c|c|c|c|c|c|c|c|}
\hline & ory & $w_{1}$ & $n$ & $\mathrm{~N} N$ & TA & $0 \%$ & $\mathrm{~N}$ & $\mathrm{~B}$ & S! & $\mathrm{NIO}$ & $M$ & $\mathrm{NB}, W \mathrm{~W}$ & ren \\
\hline 62.H/y & 7.73 & 2.06 & 0.083 & 0.003 & 0.018 & 0.092 & 0.188 & $<0.002$ & 0.00 & 0.032 & 0.0053 & $0.006 \quad 0.010$ & bal \\
\hline $821 \mathrm{ngd}$ & 7.7 & 1.95 & 0.16 & 0.008 & 0.009 & 0.088 & 0.162 & 0.0002 & 0.11 & 0.021 & 0.004 & 0.00020 .01 & bal \\
\hline WANETH & 10.8 & & 0.76 & 0.023 & & 0.13 & 0.22 & 0.0085 & 0.14 & 0.66 & 0.59 & 0.14 & bal \\
\hline
\end{tabular}

Alpha-particle cyclotron irradiations:

\begin{tabular}{|c|c|c|c|}
\hline & Cherpy V & Tensile & Fatlguen \\
\hline Hrad temporature ( $\mathrm{C})$ & 250 & $60-550^{*}$ & $250^{*}$ \\
\hline 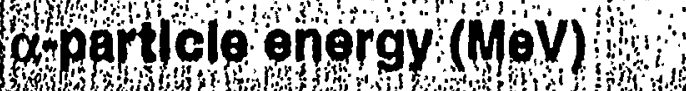 & $0-104$ & $0-60$ & $0-104$ \\
\hline Damage rate $(d p a s)$ ) & $(4.5-7.5) \times 10^{-7}$ & $(1.4-1.8) \times 10^{-6}$ & $(4.5-7.5) \times 10^{-7}$ \\
\hline 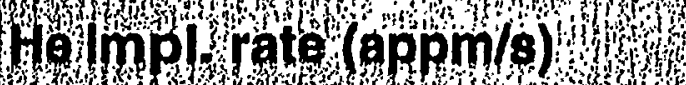 & $(6-10) \times 10^{-4}$ & $(2.5-3.0) \times 10^{-3}$ & $(6-10) \times 10^{-4}$ \\
\hline Damagedose $(\mathrm{dpa})$ & 0.22 & 0.30 & 0.30 \\
\hline Hercancentration (appm) & 300 & 500 & 400 \\
\hline
\end{tabular}

*Irradiation temperature equal test temperature 


\section{Tensile Properties F82H-mod not irradiated}

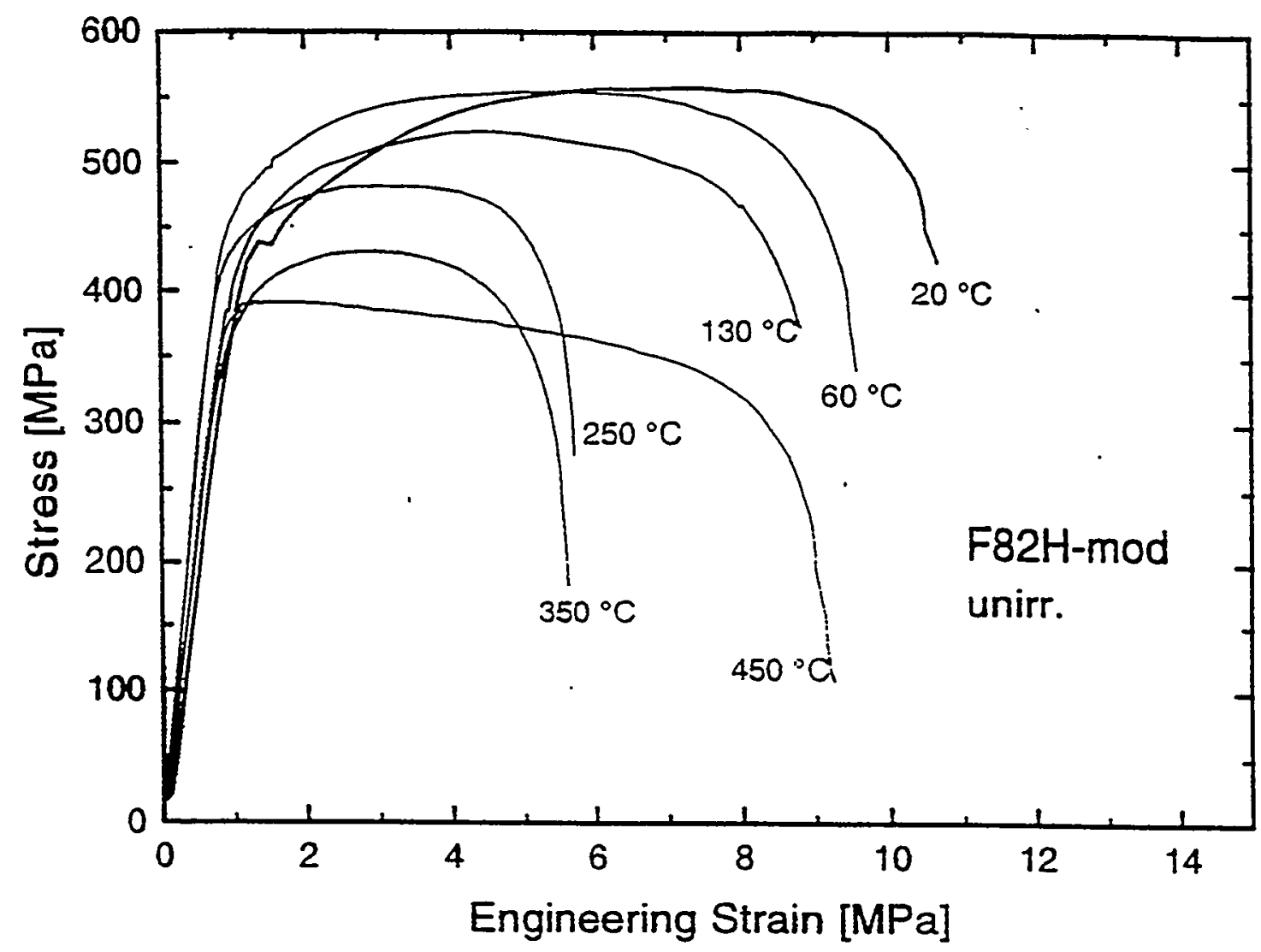

Experimental conditions:

Sheet tensile specimens (gauge volume: $7.0 \mathrm{~mm} \times 2.0 \mathrm{~mm} \times 0.20 \mathrm{~mm}$ ) Small strain rate $\left(1.2 \times 10^{-4} \mathrm{~s}^{-1}\right)$

Standard heat treatment $\left(1040^{\circ} \mathrm{C} / 0.5 \mathrm{~h}+750^{\circ} \mathrm{C} / 2 \mathrm{~h}\right)$

Vacuum furnace

\section{Main results:}

- Compared to MANET steel much lower yield strength and ultimate tensile strength

- In contrast to MANET steel no dynamic strain aging

- Temperature dependent microstructural recovery similar to conventional 9-12 CrMoVNb steels 


\section{Helium and damage effects}
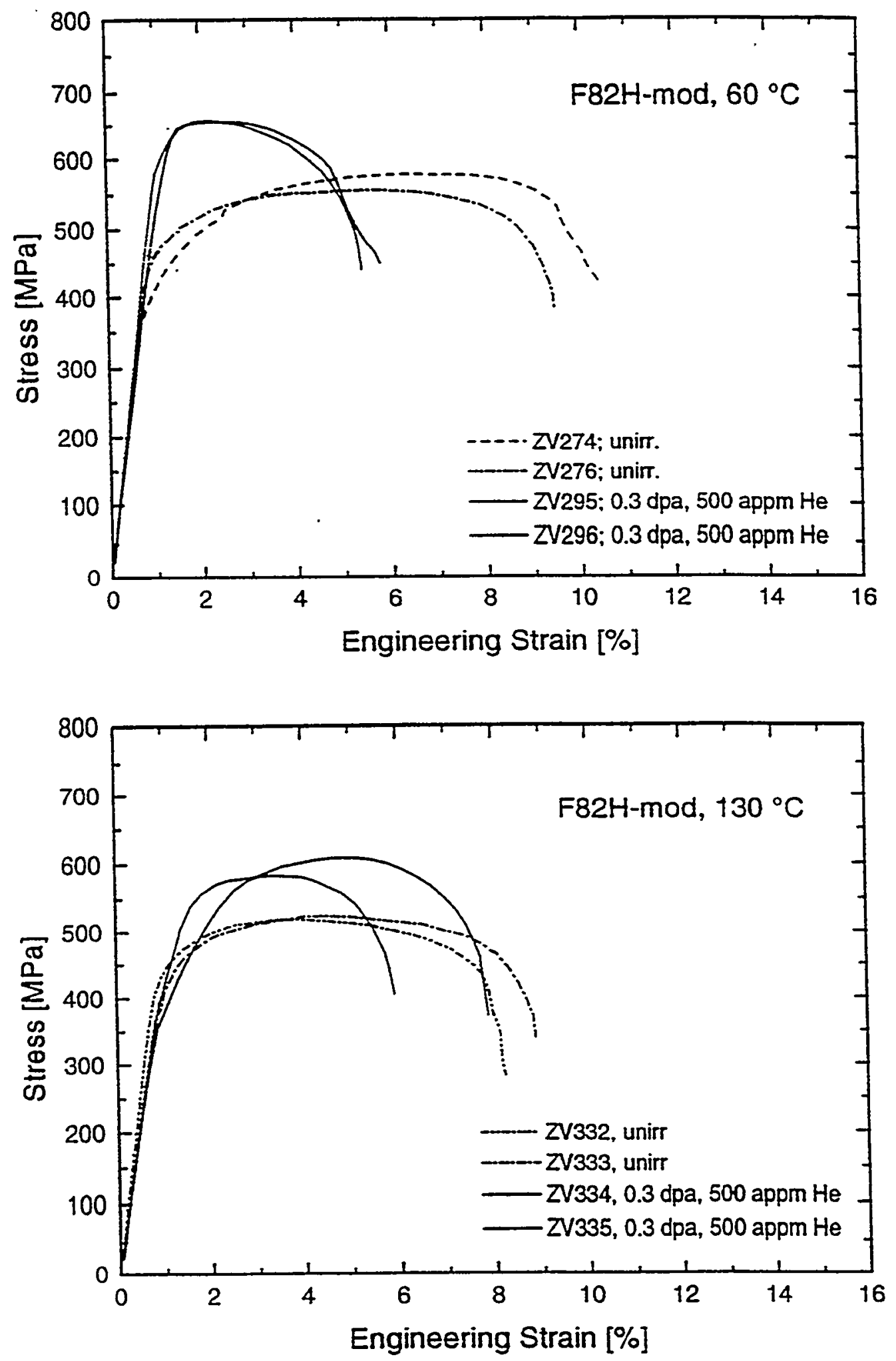


\section{Tensile properties \\ Helium and damage effects}
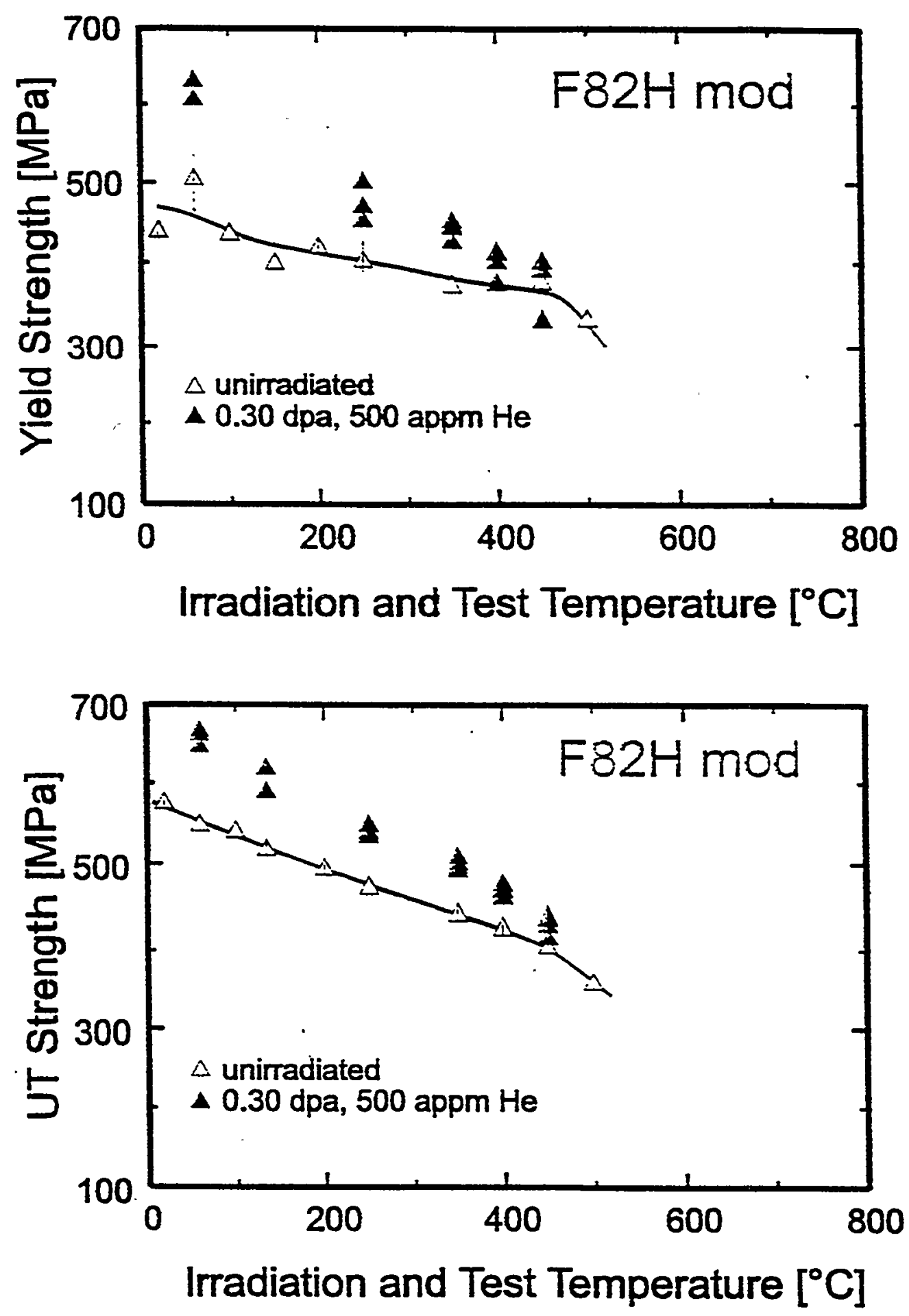


\section{Tensile properties Helium and damage effects}
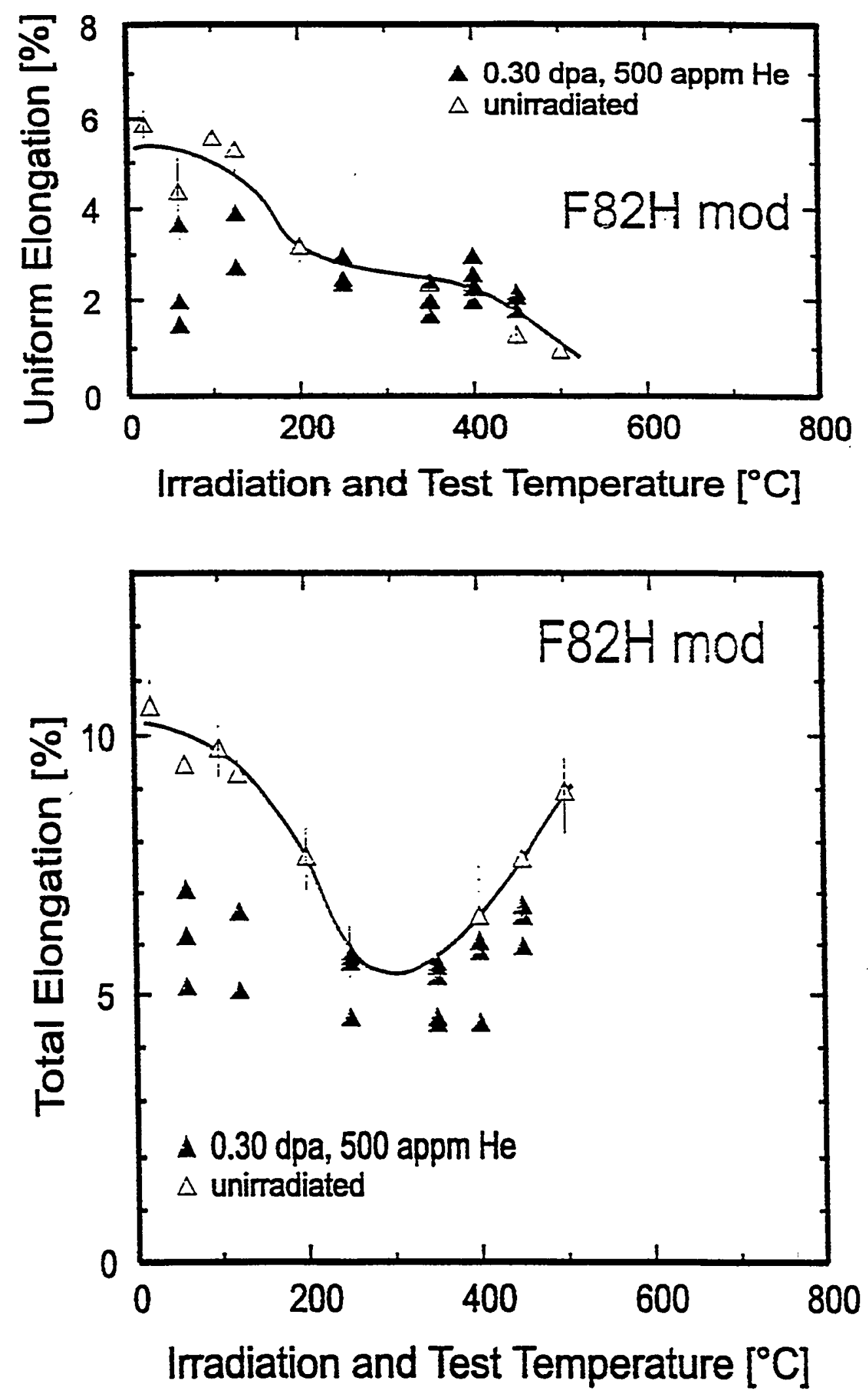


\section{Forschungszentrum Karisruhe Technik und Umwelt}

\section{SEM - Micrographs of F82H Tensile Specimen}

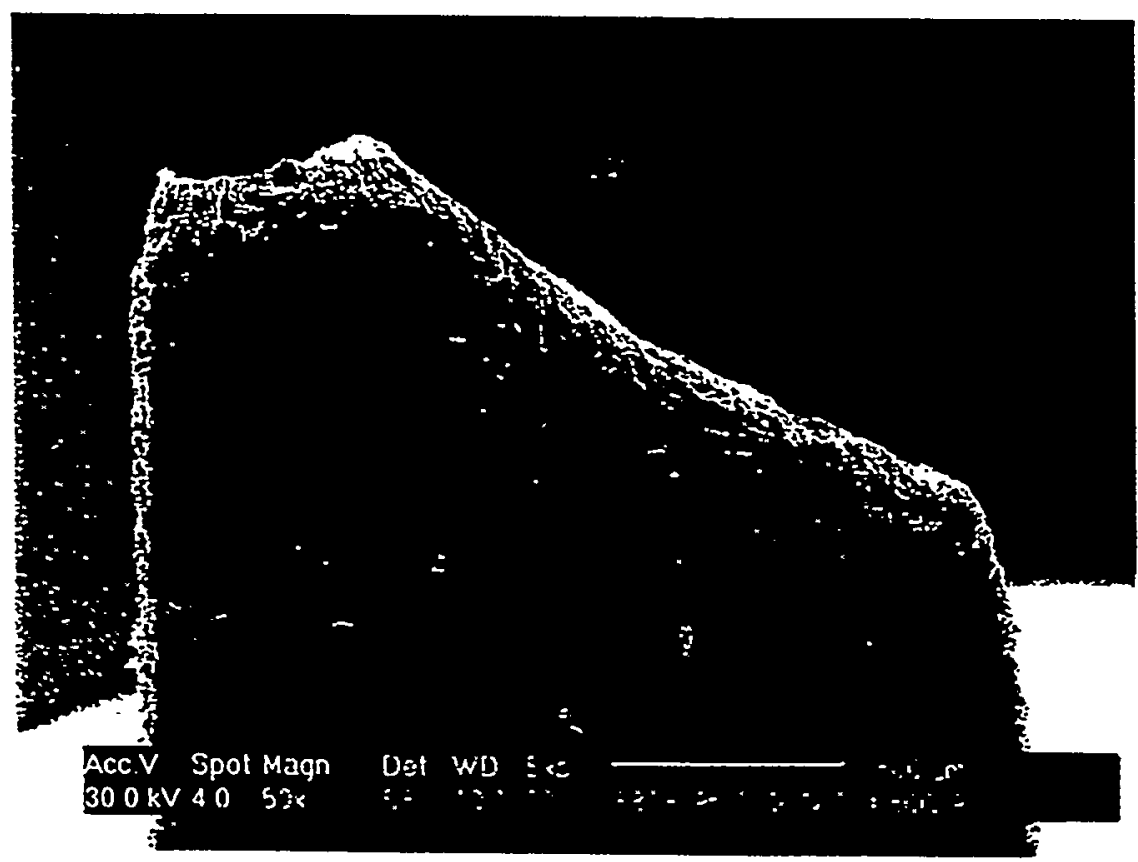

$\mathrm{T}_{\text {test }}=60^{\circ} \mathrm{C}$

side view

tilt angle: $0^{\circ}$

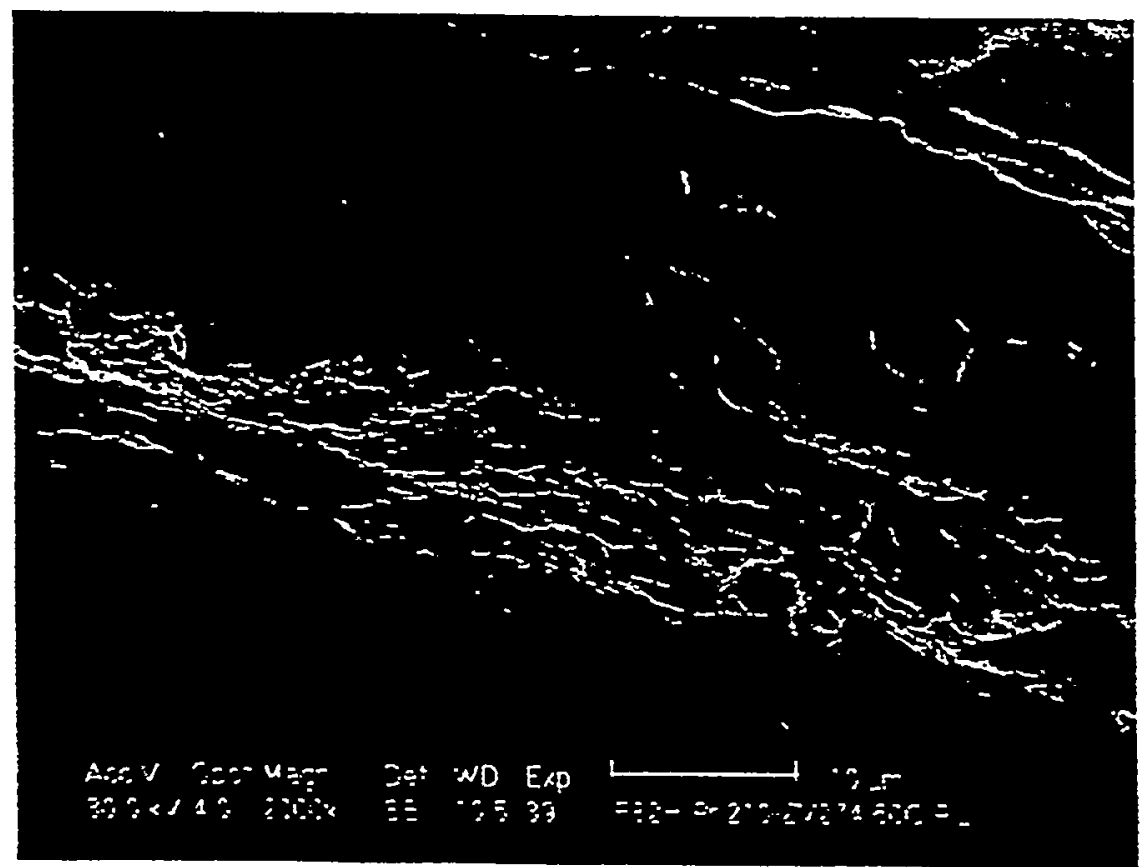

$T_{\text {test }}=60^{\circ} \mathrm{C}$

side view

tilt angle: $30^{\circ}$ 


\section{Forschungszentrum Karisruhe Technik und Umwelt}

\section{TEM -Micrographs of F82H Charpy Specimen after Dual-Beam Irradiation}

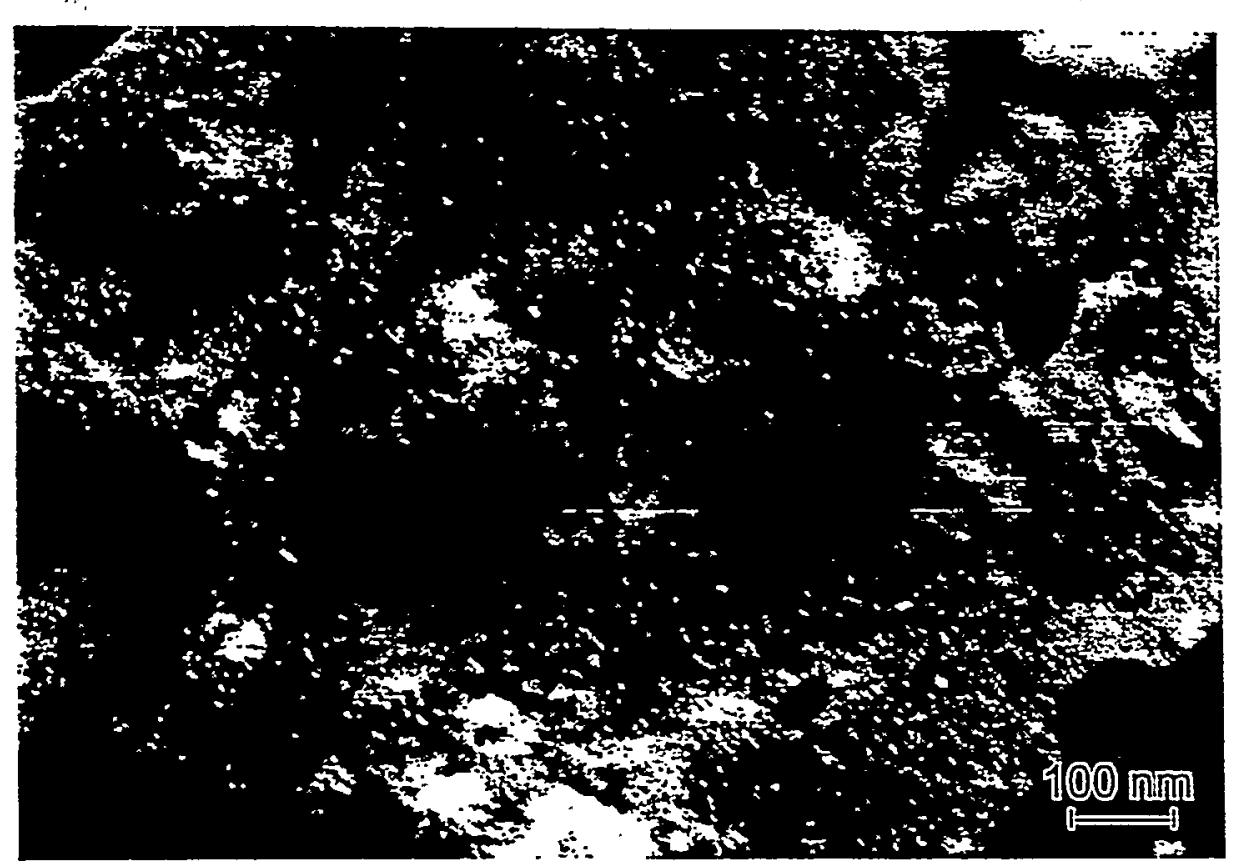

300 appm He

$0.2 \mathrm{dpa}$

$T_{\text {irr }}=250^{\circ} \mathrm{C}$

high

disl. density

$\mathrm{d}_{\mathrm{b}}=10.5 \mathrm{~nm}$

$\rho_{b}=1.5 \times 10^{22} \mathrm{~m}^{-3}$

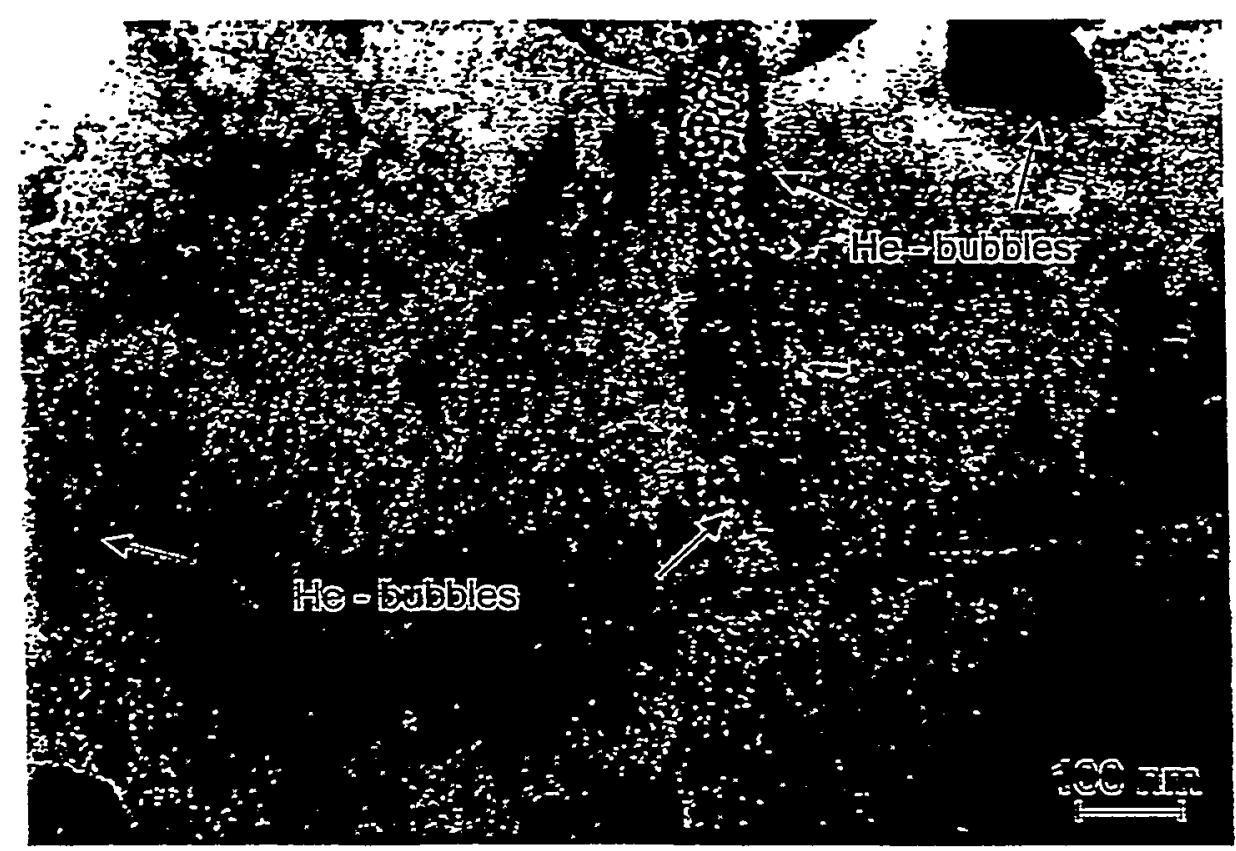

low

disl. density

$d_{\mathrm{o}}=2-5 \mathrm{~nm}$

$\rho_{\mathrm{b}}=3.7 \times 10^{22} \mathrm{~m}^{-3}$ 


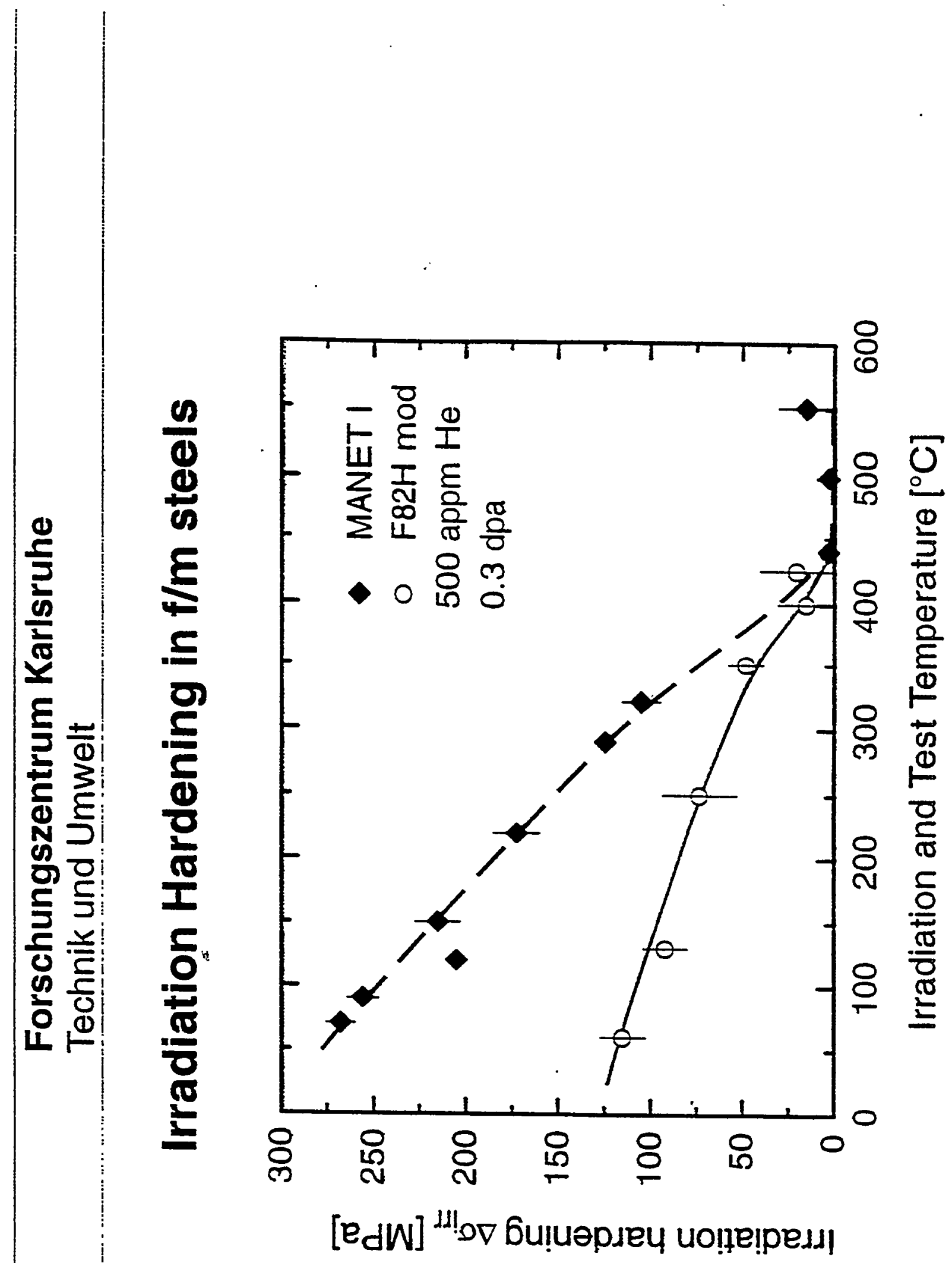




\section{Tensile Properties}

Irradiation hardening $\Delta \sigma$ of $\mathrm{Fe}$ and $\mathrm{F} 82 \mathrm{H}$-mod for different , irradiation sources and He/dpa ratios

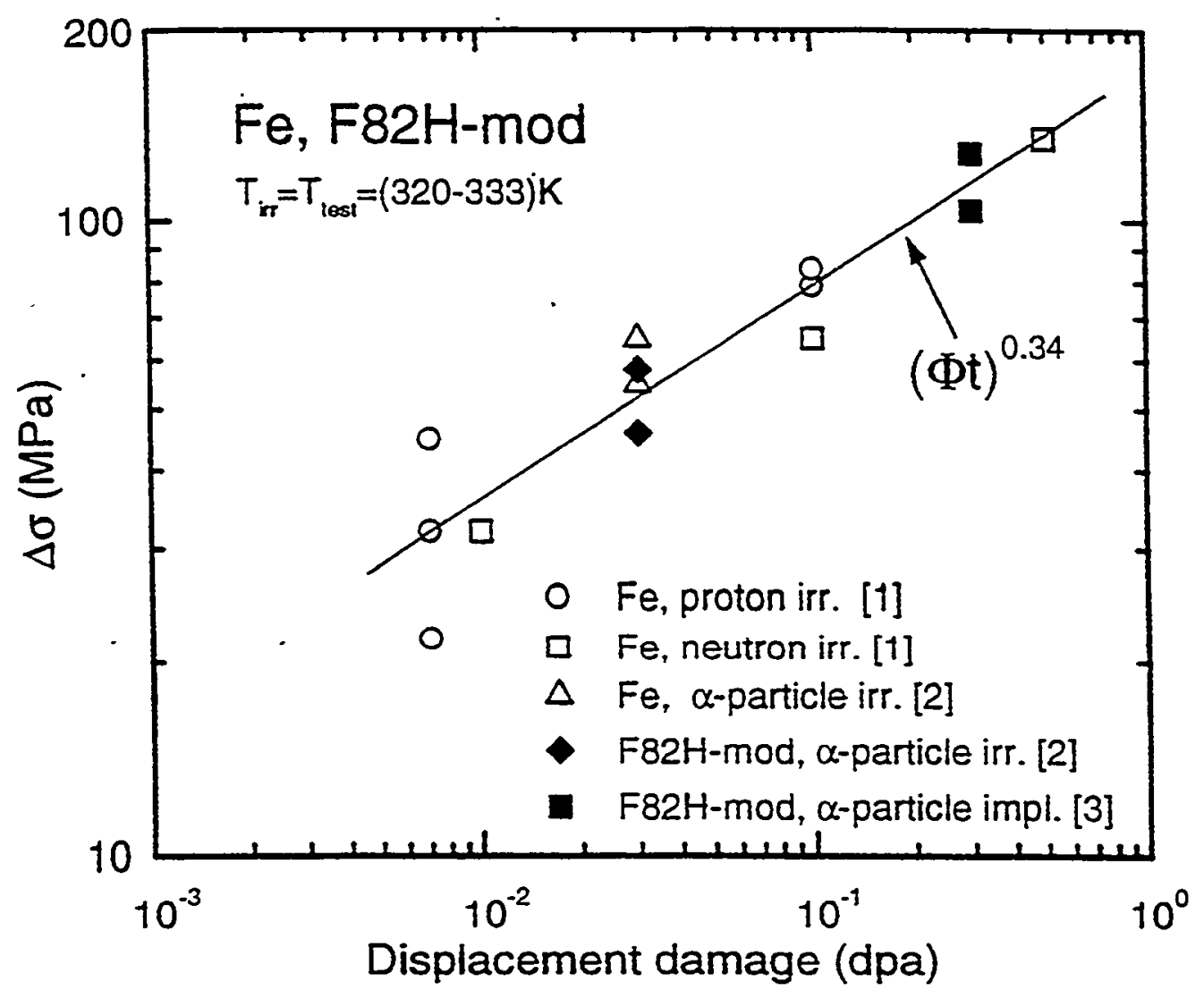

[1] Y. Chen. P. Spātig and M. Victoria (ICFRM-8) $590 \mathrm{MeV}$ proton irradiation from PIREX facility at $320 \mathrm{~K}$;

average damage rate $=1.3 \times 10^{-6} \mathrm{dpa} / \mathrm{s} ; \mathrm{He} /$ damage rate $\approx 80 \mathrm{appm} \mathrm{He/dpa}$.

[2] A. Möslang $75 \mathrm{MeV} \alpha$-particle irradiation from Dual Beam Facility at $333 \mathrm{~K}$; damage rate $=1.1 \times 10^{-7} \mathrm{dpa} / \mathrm{s} ; \mathrm{He} /$ damage rate $\approx 20$ appm He/dpa; The $\mathrm{Fe}$ and $\mathrm{F} 82 \mathrm{H}$-mod specimens were irradiated together.

[3] R. Lindau*, E. Materna-Morris*, A. Möslang*, D. Preininger*, M. Rieth* and H.D. Röhrig (ICFRM-8)

$(0-60) \mathrm{MeV} \alpha$-particle irradiation from Dual Beam Facility at $333 \mathrm{~K}$; damage rate $=1.6 \times 10^{-6} \mathrm{dpa} / \mathrm{s} ;$ He/damage rate $\approx 1670 \mathrm{appm}$ He/dpa 


\section{DPA dependency of the}

irradiation induced $\triangle$ DBTT shift

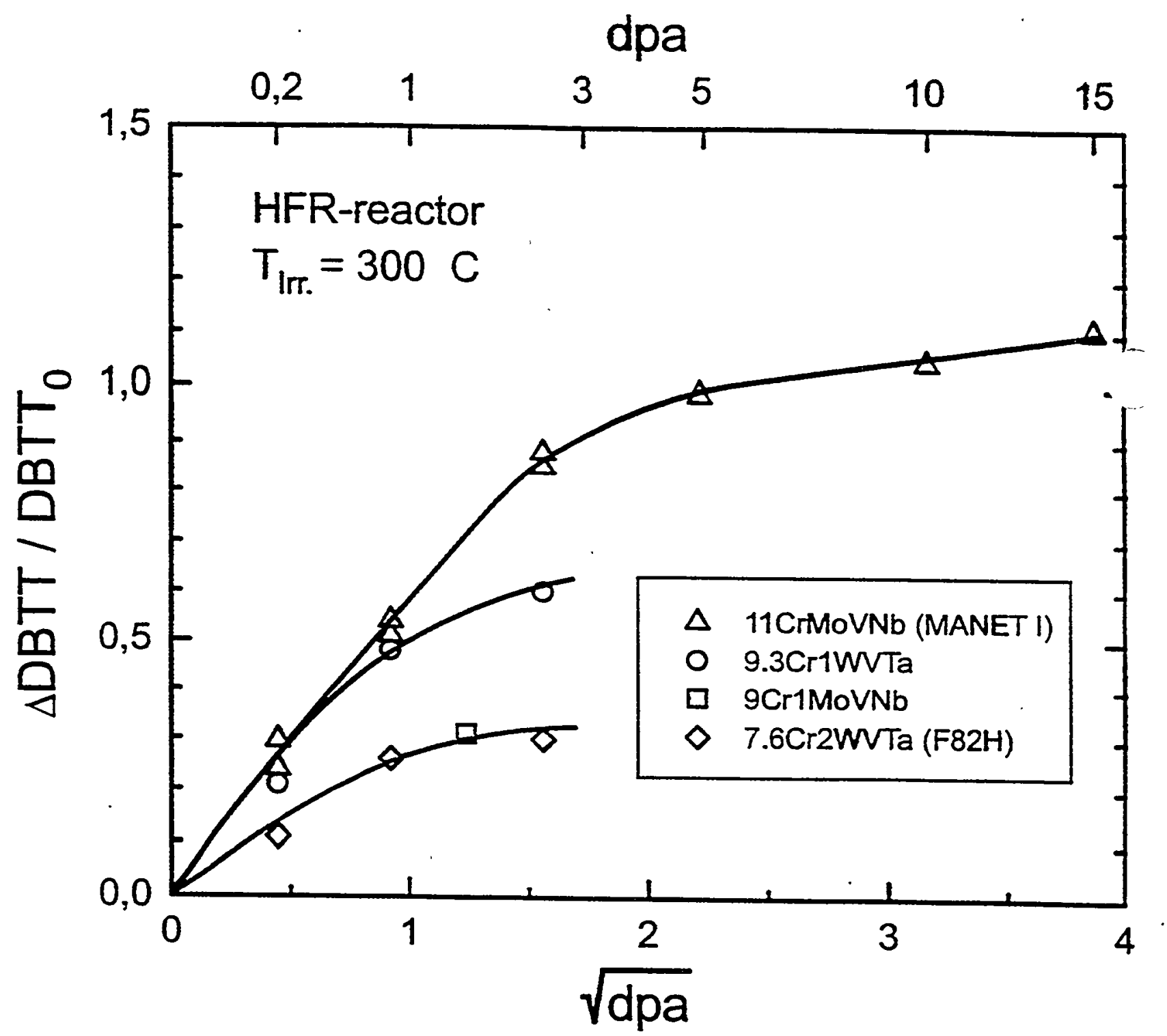



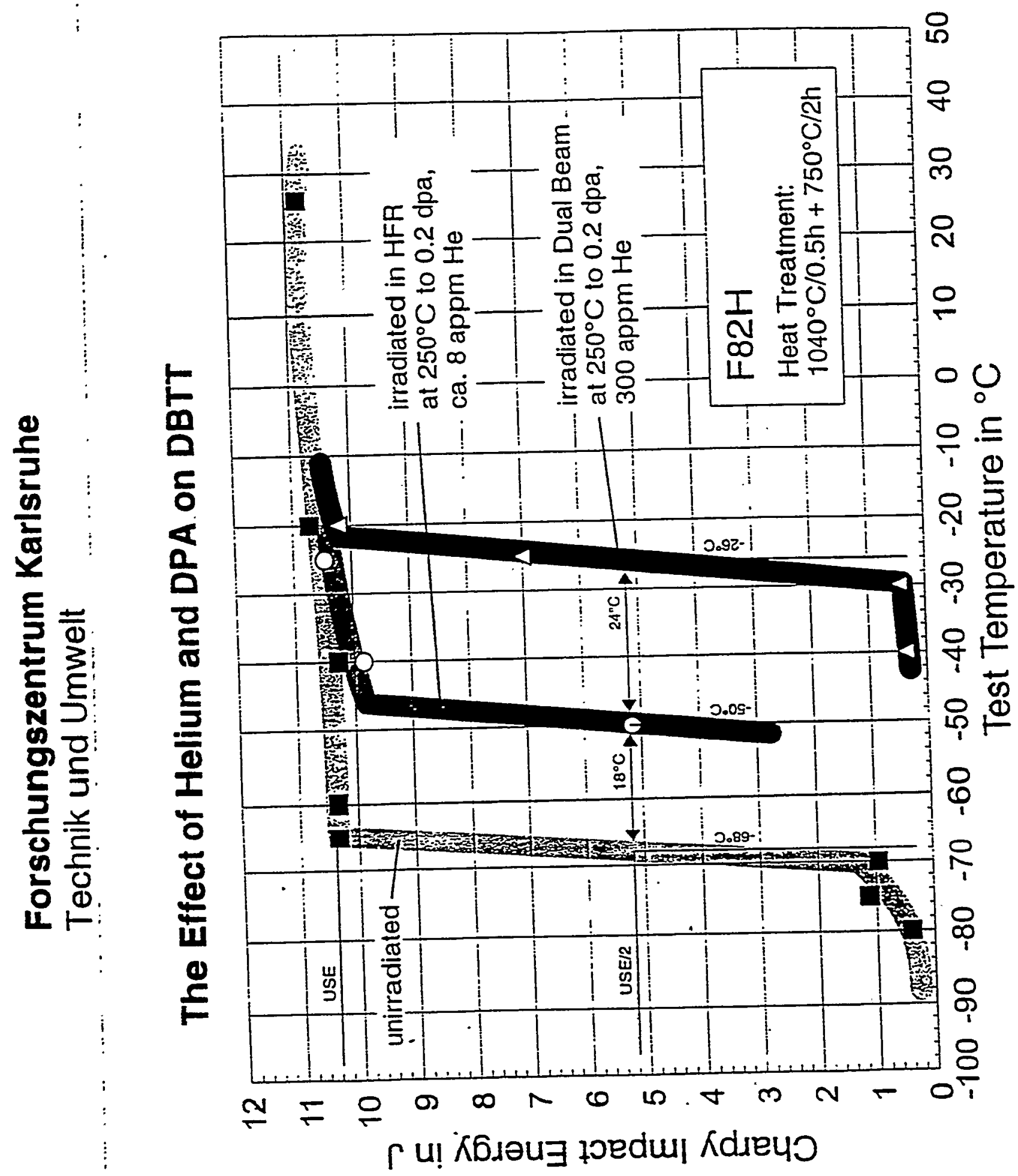


\section{Impact properties Helium and damage effects}

Depending on the He/dpa ratio, either He or dpa damage has the main contribution to $\triangle D B T T$ :

Low dpa level $\left(0.2 \mathrm{dpa}, 250^{\circ} \mathrm{C}\right)$ :

F82H: $\quad 8$ appm He . HFR irrad.: $\triangle D B T T=18^{\circ} \mathrm{C}$

F82H: $\quad 300$ appm He Implantation: $\triangle D B T T=44^{\circ} \mathrm{C}$

$\rightarrow$ He dominates embrittlement

Medium dpa level ( $0.8 \mathrm{dpa}, 250^{\circ} \mathrm{C}$, HFR-irrad.)

MANET-1: $\quad 70$ appm He

OPTIFEA II: $\quad 60$ appm He

F82H

ORNL 3791: <10 appm He $\triangle D B T T=145^{\circ} \mathrm{C}$ $\triangle D B T T=95^{\circ} \mathrm{C}$ $\triangle D B T T=60^{\circ} \mathrm{C}$ $\triangle D B T T=25^{\circ} \mathrm{C}$

$\rightarrow$ The He contribution to $\triangle D B T T$ cannot be determined quantitatively, because $\mathrm{He}$ and alloying effects $(\mathrm{B}, \mathrm{Ni}$ doping) can hardly be separated.

Microstructure at $250^{\circ} \mathrm{C}$ :

-He-bubbles are observed both after cyclotron implantation and reactor irradiation

Non-homogeneous bubble size distribution $(\approx 10 \mathrm{~nm}$ in disloc. networks, $\quad \leq 2-3 \mathrm{~nm}$ in matrix)

\section{Phenomenological embrittlement model}

- Correlation between dynamic quasi cleavage fracture and irradiation induced changes of strength and fracture stress. Micro-mechanical and microstructural details are not yet completely included.

- It predicts that the relative shift $\triangle D B T T / D B T T_{0}$ should be basically proportional to $\sqrt{d p a}$ and to $\sqrt{c_{H e}}$. 


\section{Forschungszentrum Karlsruhe Technik und Umwelt}

HFR-irradiation: $0,8 d p a, 250 \ldots 450^{\circ} \mathrm{C}$ Chemical compositions of the alloys

\begin{tabular}{|l|c|c|c|c|c|}
\hline & MANET II & OPTIFER la & OPTIFER II & F82H & ORNL 3791 \\
\hline $\mathrm{Cr}$ & 9.94 & 9.3 & 9.5 & 7.73 & 8.9 \\
\hline $\mathrm{C}$ & 0.1 & 0.10 & 0.125 & 0.092 & 0.11 \\
\hline $\mathrm{N}$ & 0.023 & 0.0155 & 0.0159 & 0.0027 & 0.0215 \\
\hline $\mathrm{Mn}$ & 0.79 & 0.50 & 0.49 & 0.083 & 0.44 \\
\hline $\mathrm{P}$ & $<0.006$ & 0.0046 & 0.0043 & 0.003 & 0.015 \\
\hline $\mathrm{S}$ & $<0.007$ & 0.005 & 0.002 & 0.003 & 0.008 \\
\hline $\mathrm{V}$ & 0.22 & 0.26 & 0.28 & 0.189 & 0.23 \\
\hline $\mathrm{B}$ & 0.007 & 0.0061 & 0.0059 & 0.003 & $<0.001$ \\
\hline $\mathrm{Si}$ & 0.14 & 0.06 & 0.038 & 0.09 & 0.21 \\
\hline $\mathrm{Ni}$ & 0.66 & 0.005 & 0.005 & 0.032 & $<0.01$ \\
\hline $\mathrm{Mo}$ & 0.59 & 0.005 & 0.005 & 0.0053 & 0.01 \\
\hline $\mathrm{Al}$ & $<0.2$ & 0.008 & 0.008 & 0.01 & 0.017 \\
\hline $\mathrm{Co}$ & $<0.2$ & & & 0.0024 & 0.012 \\
\hline $\mathrm{Cu}$ & $<0.01$ & 0.035 & 0.007 & 0.0059 & 0.03 \\
\hline $\mathrm{Nb}$ & 0.14 & 0.009 & 0.009 & 0.0057 & \\
\hline $\mathrm{Zr}$ & 0.034 & & & & $<0.001$ \\
\hline $\mathrm{Ti}$ & & $\cdot$ & & 0.0104 & $<0.01$ \\
\hline $\mathrm{W}$ & & 0.96 & 0.006 & 2.06 & 2.01 \\
\hline $\mathrm{Ta}$ & & 0.066 & 0.018 & 0.018 & 0.06 \\
\hline $\mathrm{Ge}$ & & & 1.2 & & \\
\hline $\mathrm{Ce}$ & & $<0.001$ & $<0.001$ & & \\
\hline $\mathrm{Fe}$ & $\mathrm{bai}$ & $\mathrm{bal}$ & $\mathrm{bal}$ & $\mathrm{bal}$ & $\mathrm{bal}$ \\
\hline
\end{tabular}




\section{Forschungszentrum Karlsruhe Technik und Umwelt}

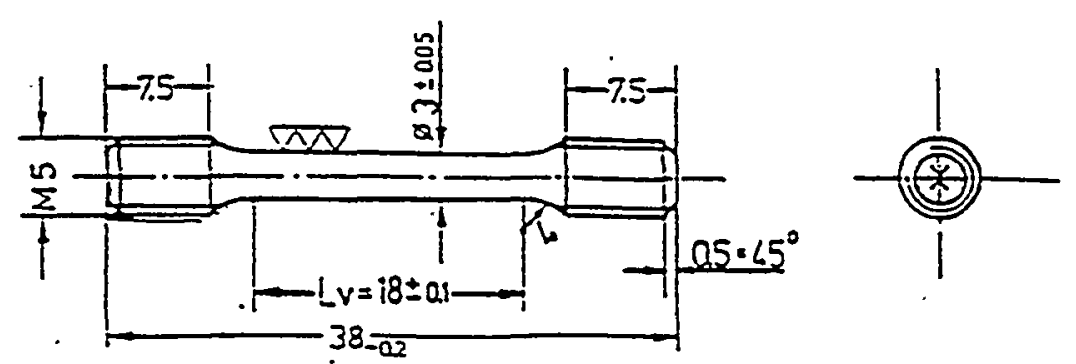

Tensile specimen

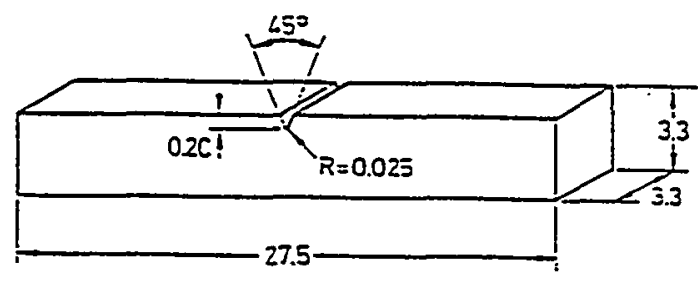

Charpy specimen 
Table $2-$ Heat treatments, grain size and hardness of the alloys

\begin{tabular}{|c|c|c|c|}
\hline Heat & Heat treatment & $\begin{array}{l}\text { ASTM- } \\
\text { grain size }\end{array}$ & $\begin{array}{l}\text { Hardness } \\
\text { HV } 0.4\end{array}$ \\
\hline OPTIFER Ia & $1075^{\circ} \mathrm{C} 30 \mathrm{~min}+780^{\circ} \mathrm{C} 2 \mathrm{~h}$ & 6.5 & 199 \\
\hline OPTIFER II & $950^{\circ} \mathrm{C} 2 \mathrm{~h}+780^{\circ} \mathrm{C} 2 \mathrm{~h}$ & 7.5 & 206 \\
\hline $\mathrm{F} 82 \mathrm{H}$ & $1040^{\circ} \mathrm{C} 30 \mathrm{~min}+750^{\circ} \mathrm{C} 2 \mathrm{~h}$ & 6.5 & 227 \\
\hline ORNL 3791 & $1050^{\circ} \mathrm{C} 30 \mathrm{~min}+750^{\circ} \mathrm{C} 2 \mathrm{~h}$ & 8.5 & 245 \\
\hline MANET II & $965^{\circ} \mathrm{C} 2 \mathrm{~h}+1075^{\circ} \mathrm{C} 30 \min +750^{\circ} \mathrm{C} 2 \mathrm{~h}$ & 7.5 & 274 \\
\hline
\end{tabular}




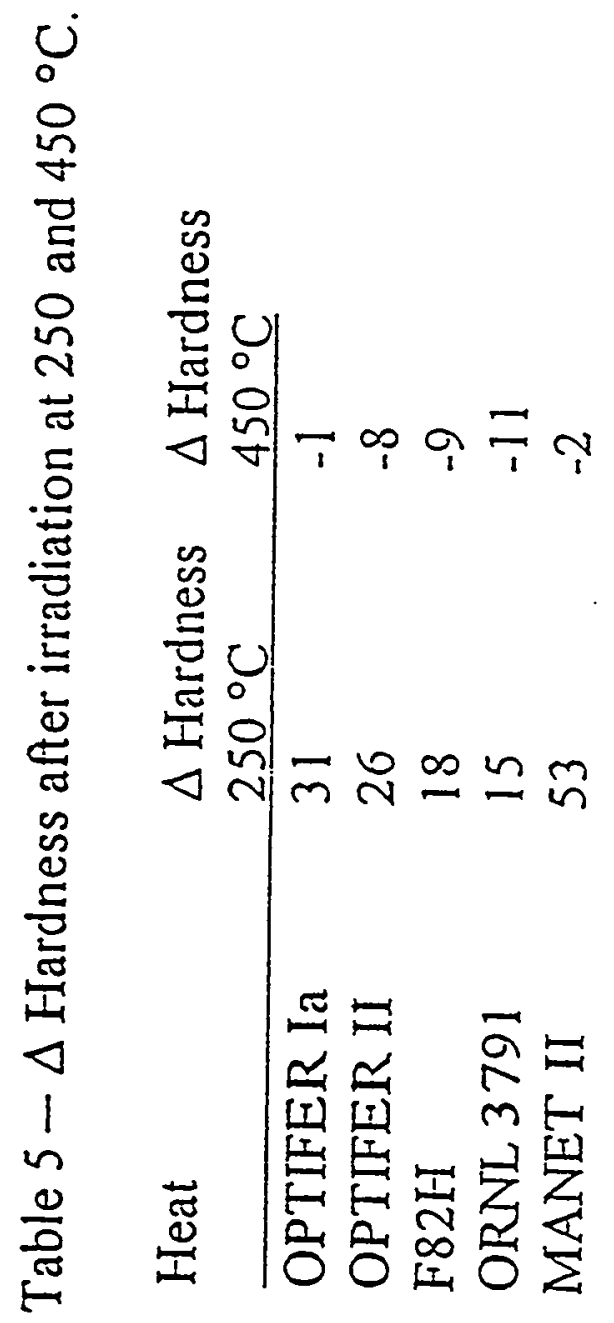


$\mathrm{DBTT}_{\text {unirradiated }}-\mathrm{DBTT} \mathrm{T}_{\text {irradiated }}$

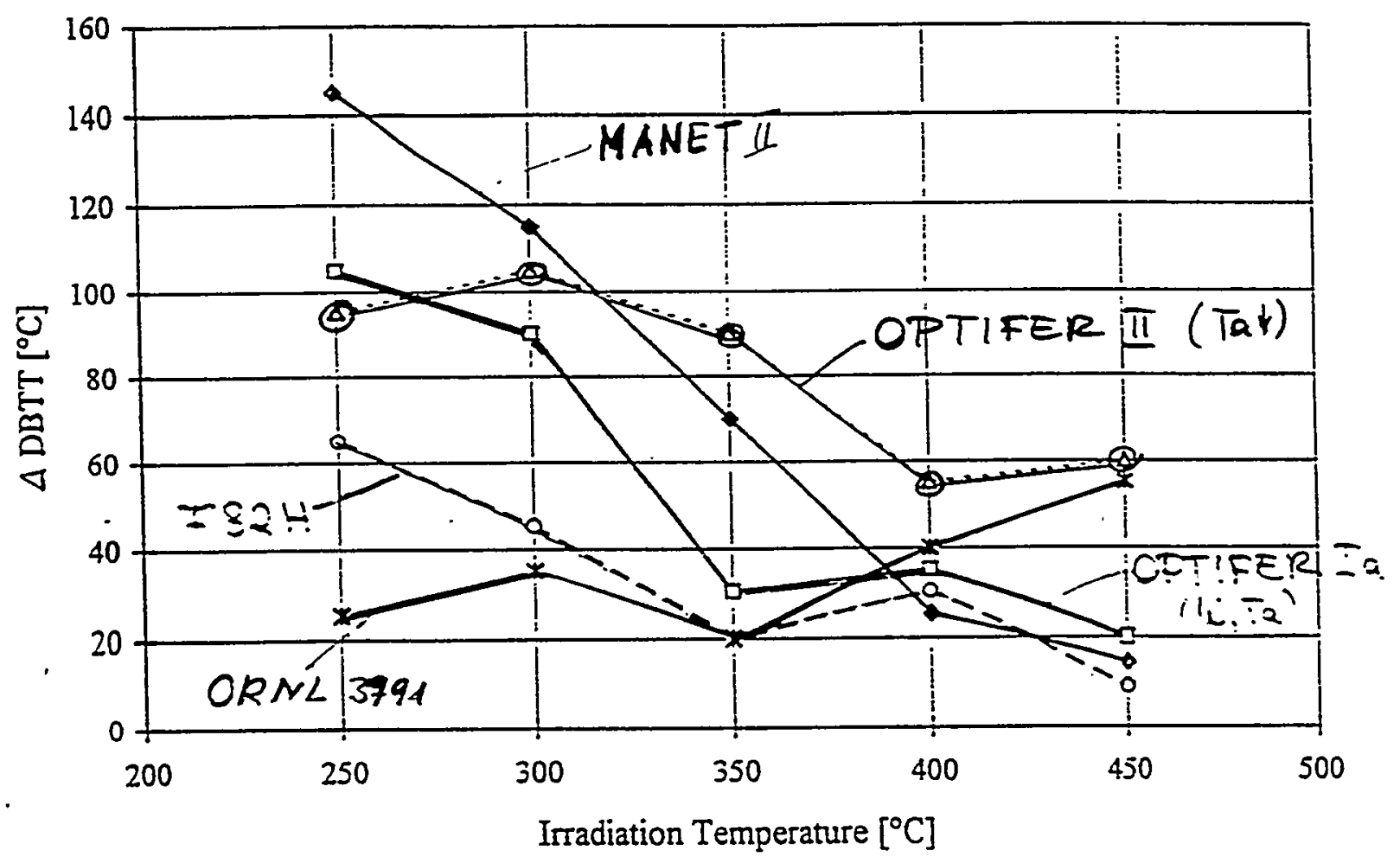




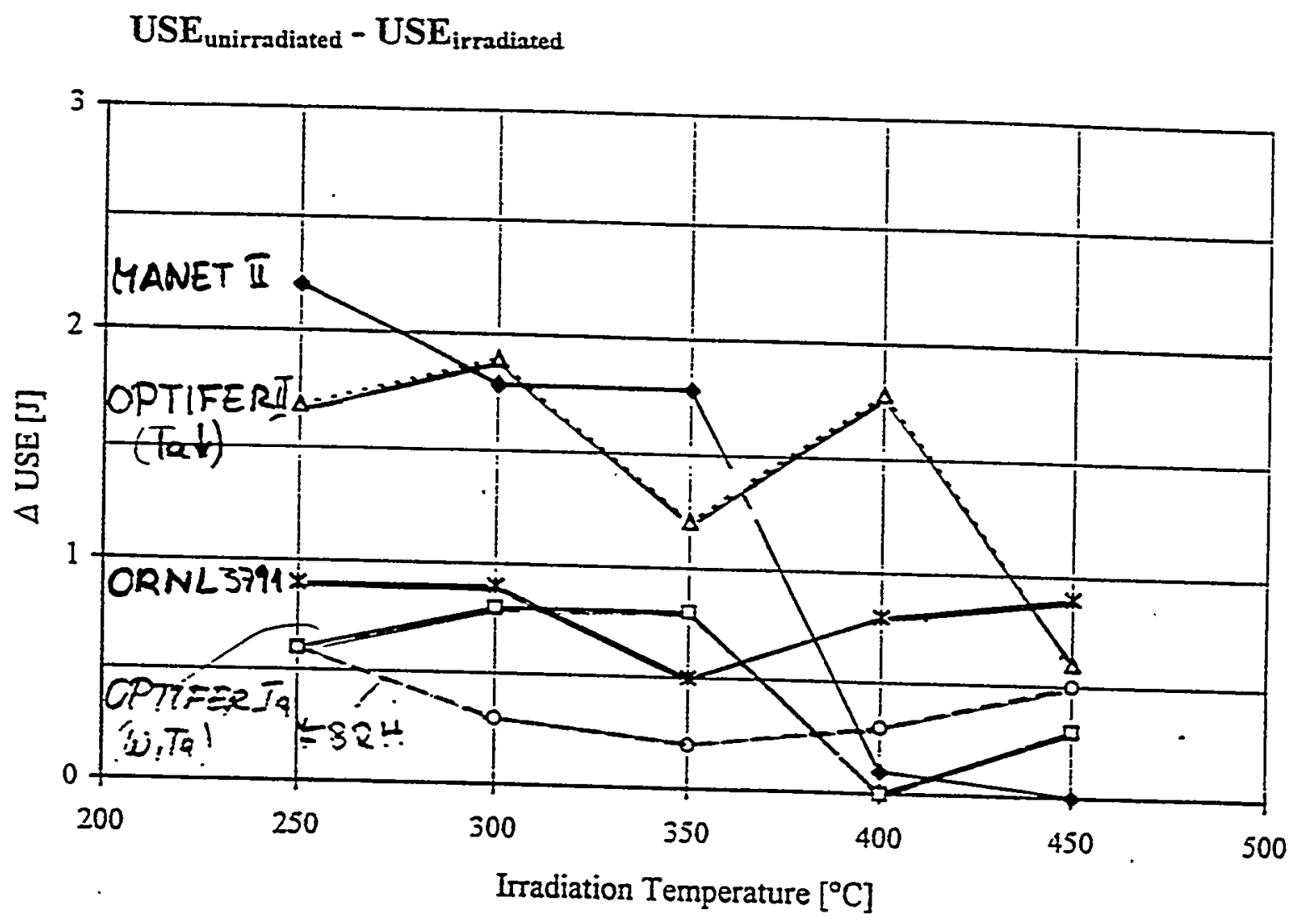


Table 8 - Calculated helium content after complete ${ }^{10} \mathrm{~B}$ burn-up irradiation temperature and the $\Delta$-values of the mechanical properties after $\mathrm{T}_{\text {irad. }}=250^{\circ} \mathrm{C}$.

\begin{tabular}{lllllll} 
& OPTIFER Ia & OPTIFER II & F82H & ORNL 3791 & MANET II \\
\hline max. He & 60 appm & 60 appm & $<20$ appm & $<10 \mathrm{appm}$ & $70 \mathrm{appm}$ \\
$\Delta \mathrm{DBTT}$ & 105 & 95 & 65 & 25 & 145 \\
$\Delta \mathrm{R}_{\mathrm{p} 0.2}$ & 83 & 22 & 39 & - & - \\
$\Delta \mathrm{R}_{\mathrm{m}}$ & -7 & -62 & -35 & - & - \\
$\Delta \mathrm{A}$ & -7.5 & -9.2 & -6.2 & - & - \\
\hline
\end{tabular}


Figure 12 - The DBTT shift depending on the He content.

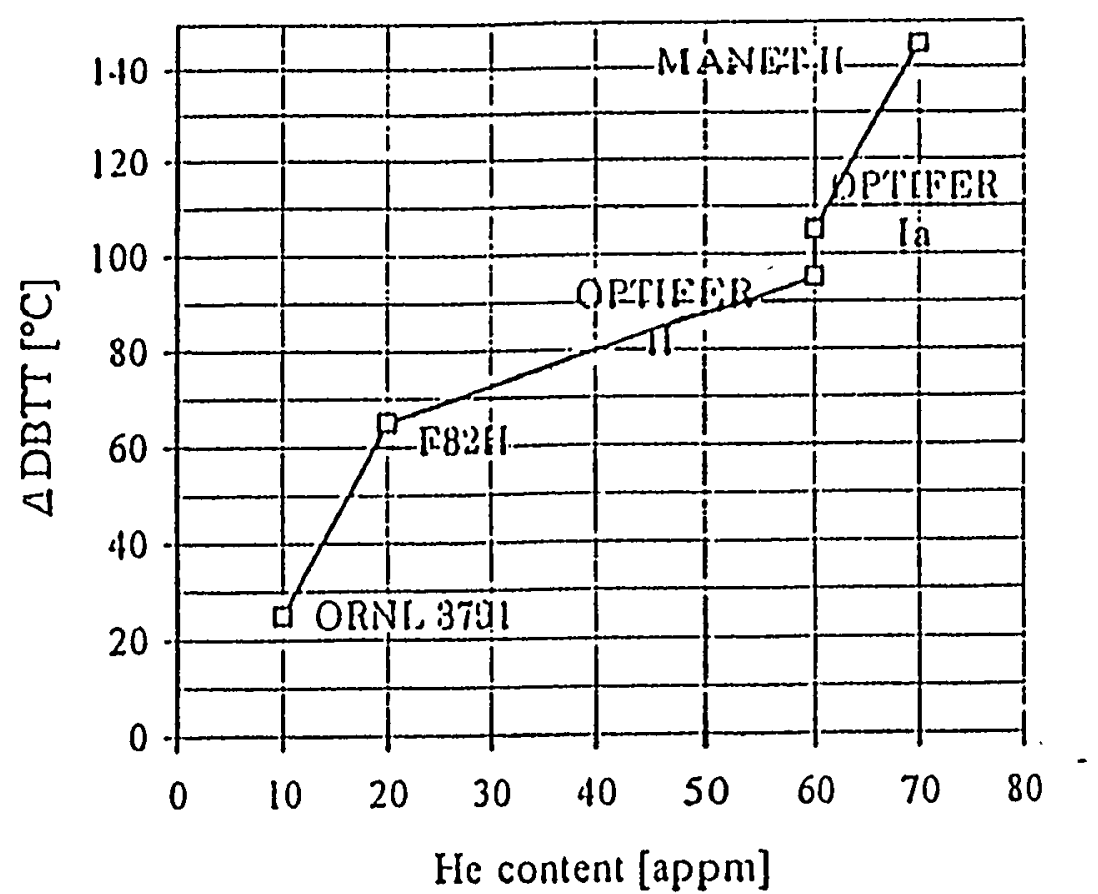




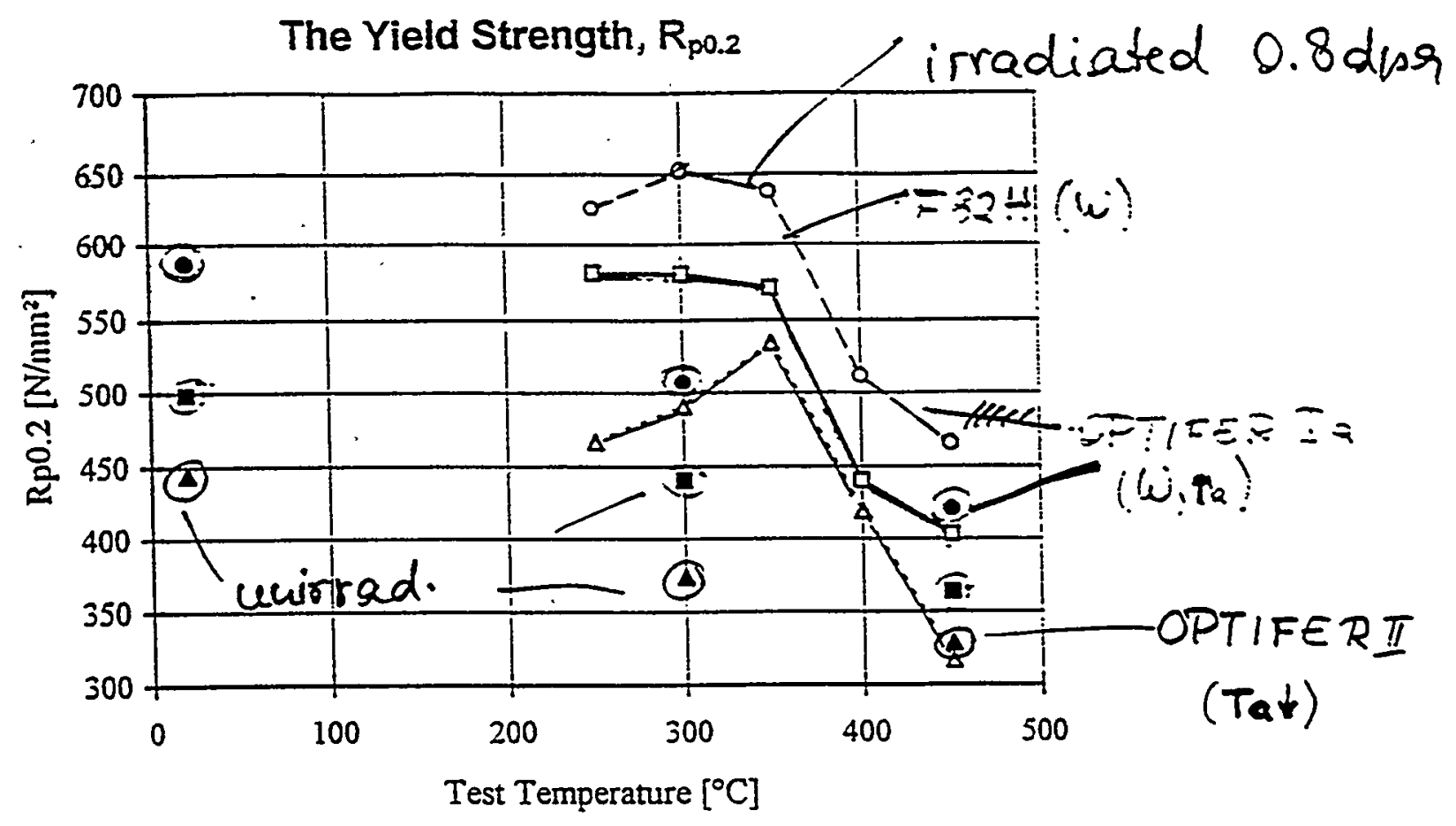

The Tensile Strength, $R_{m}$

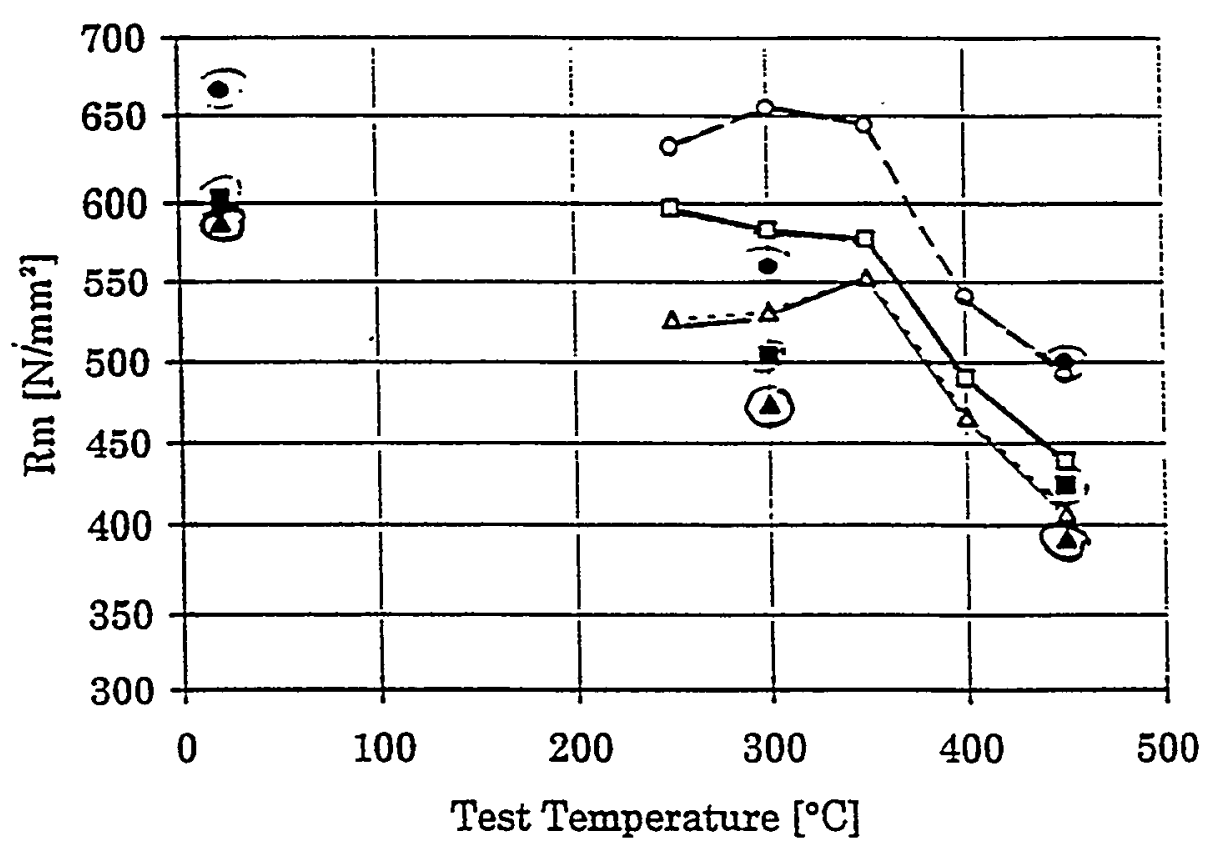

The Total Elongation, A

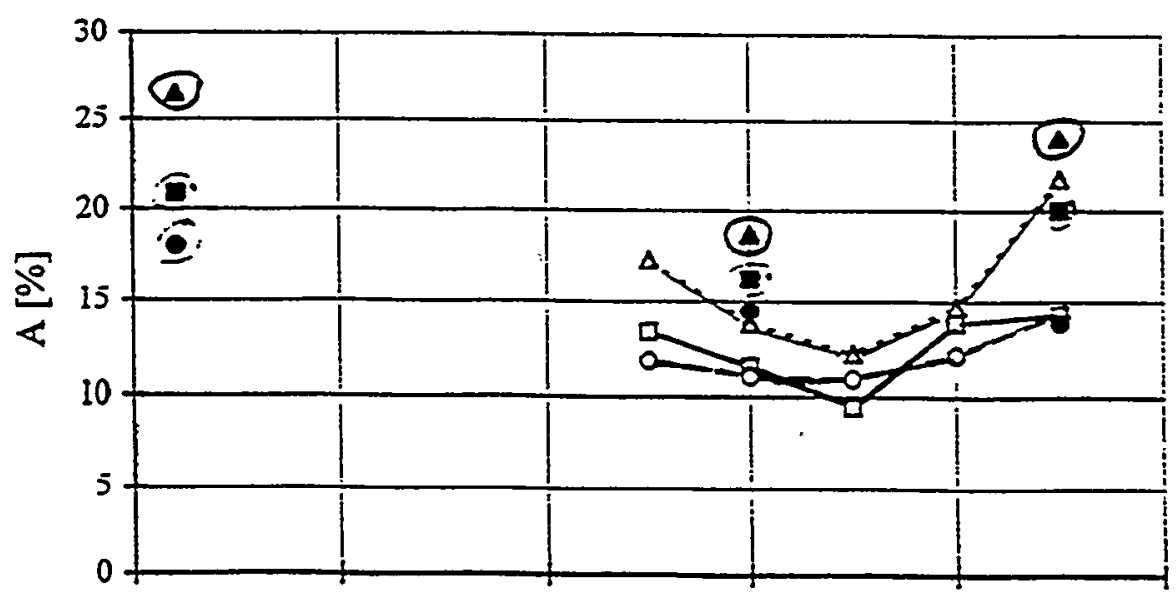




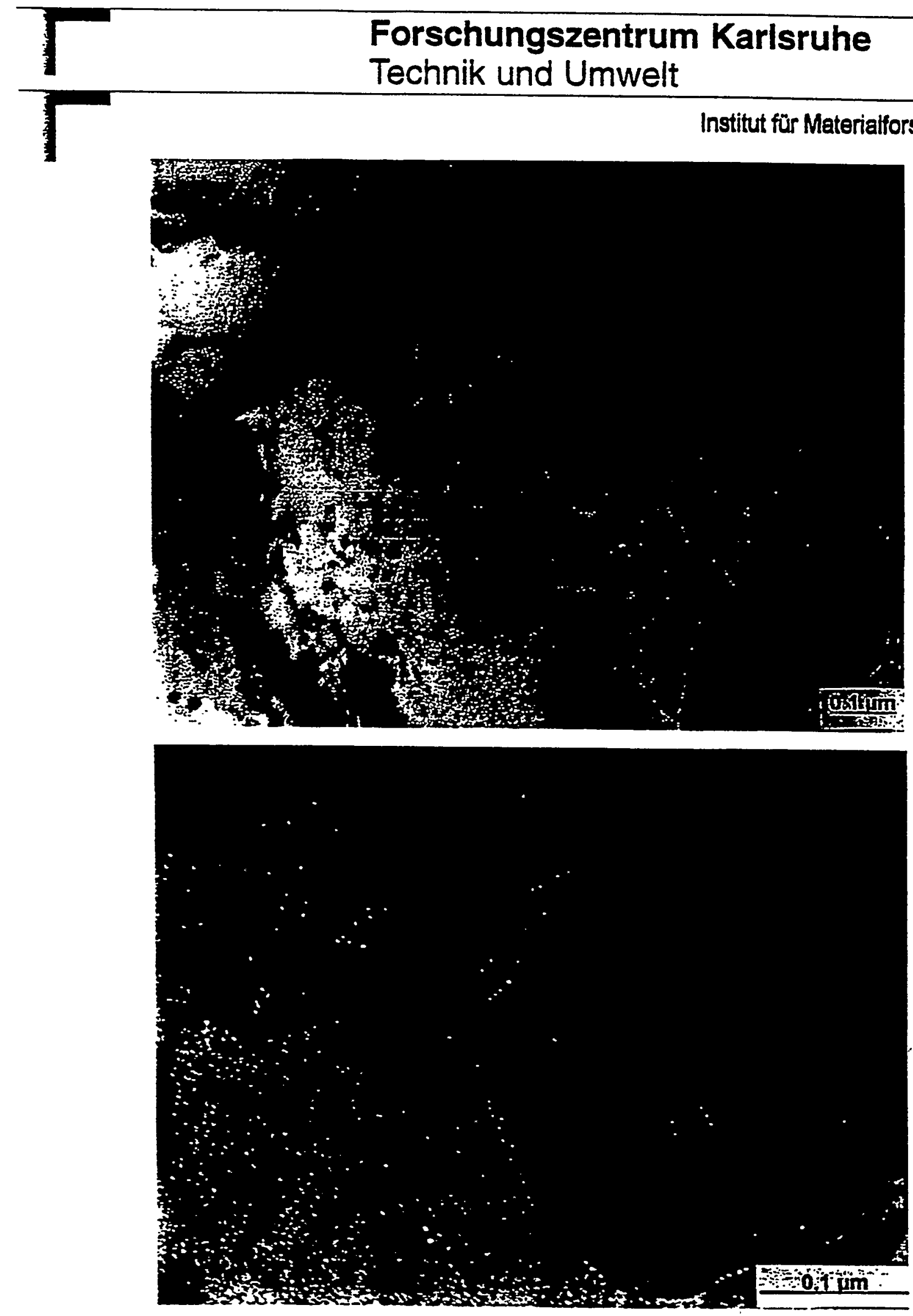

ENenomenorm aros 

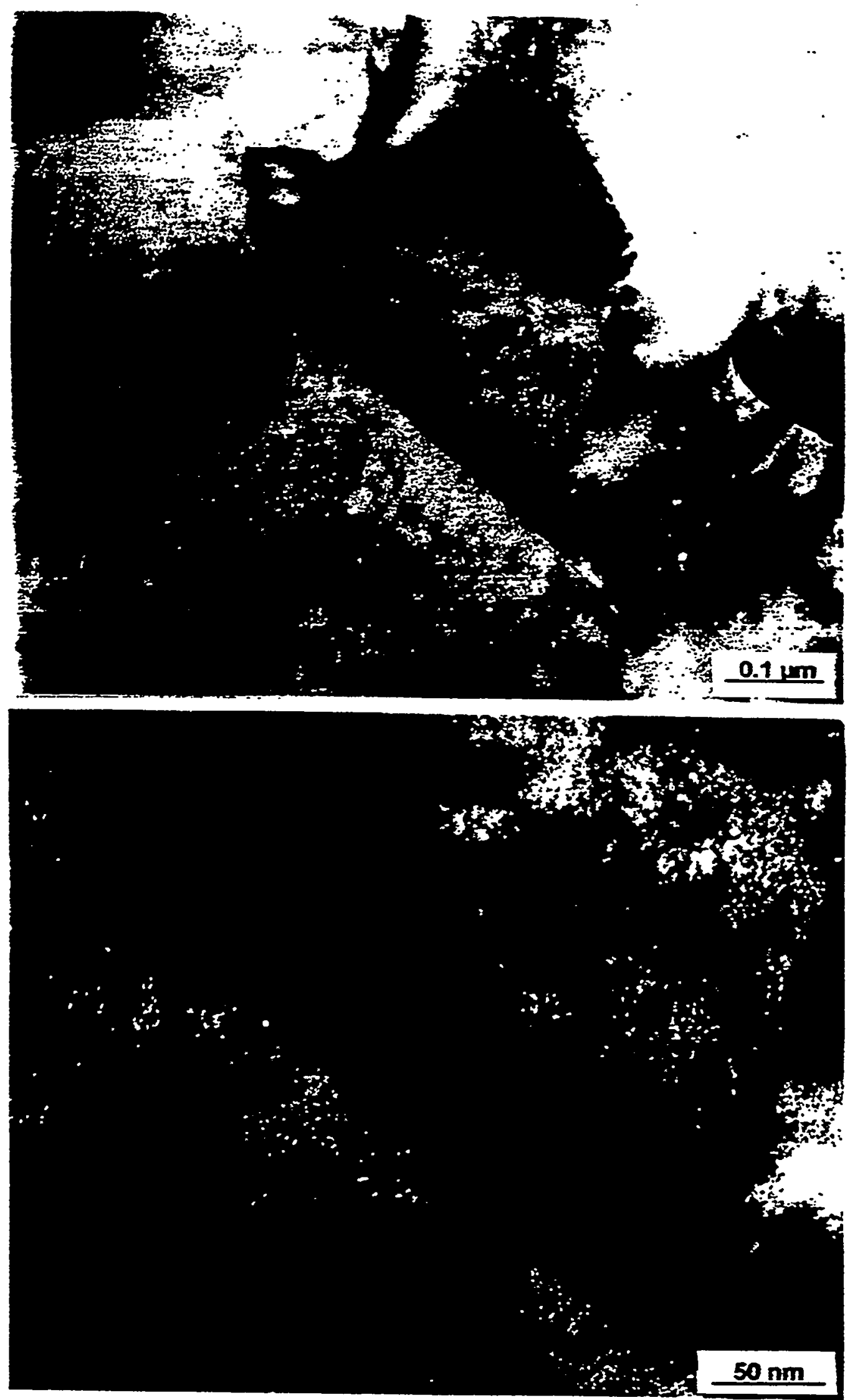

OPTIFER H: $0.8 \mathrm{dpa}, T_{\text {Ir. }}=450^{\circ} \mathrm{C}$ 


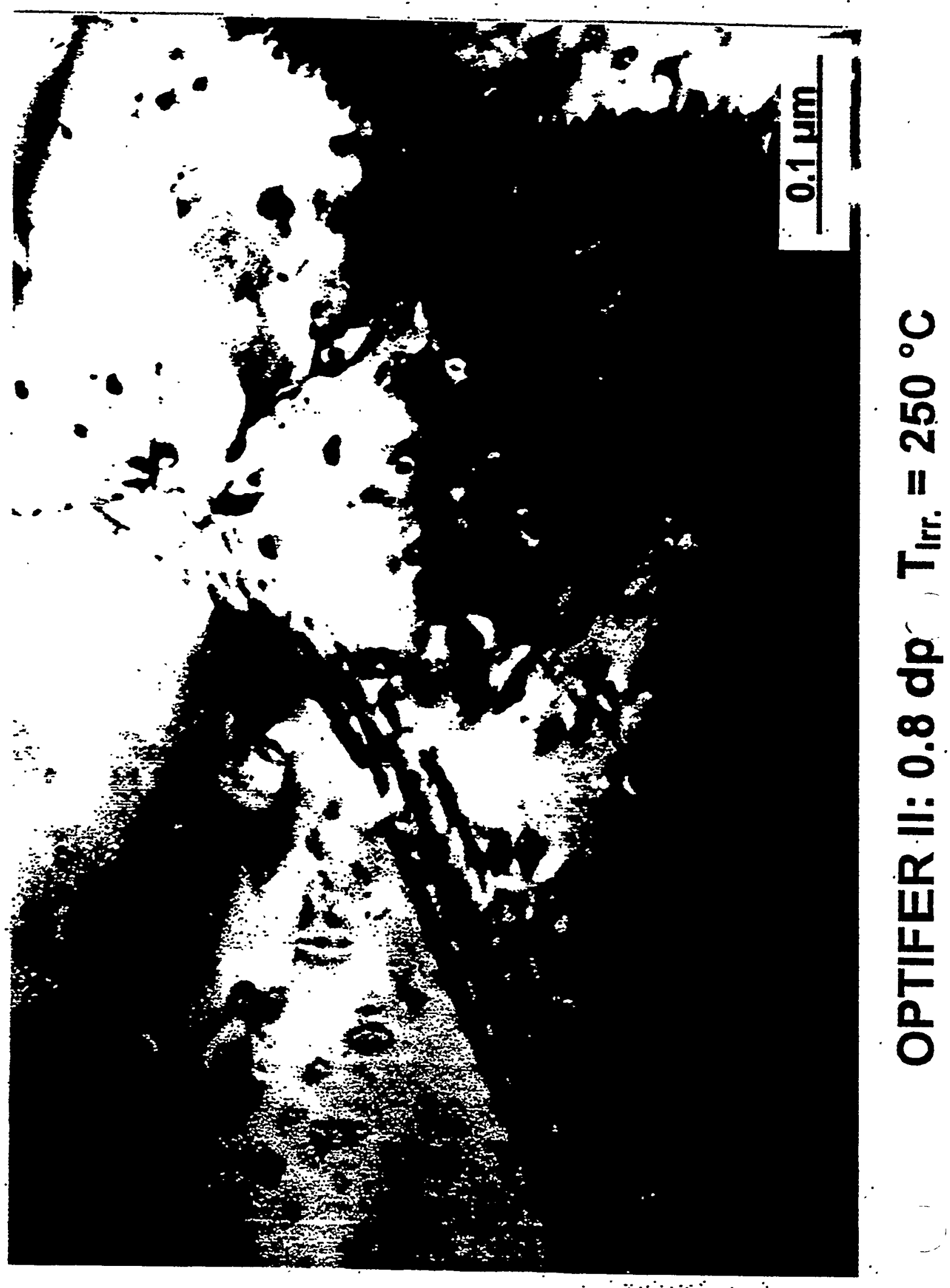




\section{Forschungszentrum Karisruhe \\ Technik und Umwelt}

Quantitative analysis of the irradiation effects at $250^{\circ} \mathrm{C}$.

\begin{tabular}{|c|c|c|c|c|c|}
\hline Heat & $\begin{array}{c}\text { He- } \\
\text { bubbles } \\
\text { diameter } \\
{[\mathrm{nm}]}\end{array}$ & $\begin{array}{c}\text { He- } \\
\text { bubbles } \\
\text { density } \\
{\left[\mathrm{cm}^{-3}\right]}\end{array}$ & $\begin{array}{c}\text { He- } \\
\text { bubbles } \\
\text { location }\end{array}$ & $\begin{array}{c}\text { Density of } \\
\text { loops } \\
{\left[\mathrm{cm}^{-3}\right]}\end{array}$ & $\begin{array}{c}\text { density of } \\
\alpha^{*} \\
{\left[\mathrm{~cm}^{-3}\right]}\end{array}$ \\
\hline OPTIFER Ia & 2 & $1.5 \times 10^{16}$ & $\mathrm{D}, \mathrm{H}$ & $5.9 \times 10^{15}$ & $1.0 \times 10^{16}$ \\
\hline OPTIFER II & 3 & $3.8 \times 10^{18}$ & D & $2.6 \times 10^{17}$ & $2.1 \times 10^{15}$ \\
\hline F82H & 1.5 & $1.4 \times 10^{17 *}$ & $\mathrm{H}, \mathrm{S}, \mathrm{P}$ & $1.9 \times 10^{16}$ & $7.5 \times 10^{15}$ \\
\hline ORNL 3791 & 2 & $1.1 \times 10^{15 *}$ & D, P & $1.0 \times 10^{15}$ & $3.5 \times 10^{10}$ \\
\hline MANET II & 2 & $3.6 \times 10^{16}$ & D, S, P & $1.4 \times 10^{15}$ & $3.8 \times 10^{15}$ \\
\hline
\end{tabular}

Quantitative analysis of the irradiation effects at $450^{\circ} \mathrm{C}$.

\begin{tabular}{|c|c|c|c|c|c|}
\hline Heat & $\begin{array}{c}\text { He- } \\
\text { bubbles } \\
\text { diameter } \\
{[\mathrm{nm}]}\end{array}$ & $\begin{array}{c}\text { He-bubbles } \\
\text { density } \\
{\left[\mathrm{cm}^{-3}\right]}\end{array}$ & $\begin{array}{c}\text { He- } \\
\text { bubbles } \\
\text { location }\end{array}$ & $\begin{array}{c}\text { Density of } \\
\text { loops } \\
{\left[\mathrm{cm}^{-3}\right]}\end{array}$ & $\begin{array}{c}\text { Density of } \\
\alpha(+) \\
{\left[\mathrm{cm}^{-3}\right]}\end{array}$ \\
\hline OPTIFER Ia & 2 & $5.7 \times 10^{i 5}$ & D, S, P & - & - \\
\hline OPTIFER II & 6 & $1.2 \times 10^{15}$ & D, S & - & - \\
\hline F82H & 3 & $3.7 \times 10^{16 *}$ & H, D & - & $1.6 \times 10^{16}$ \\
\hline ORNL 3791 & 3 & $4.6 \times 10^{15 *}$ & D & - & - \\
\hline MANET II & 4 & $1.1 \times 10^{16}$ & D, P & - & - \\
\hline
\end{tabular}

$\mathrm{D}=$ Dislocations, $\mathrm{S}=$ Sub-grain boundaries, $\mathrm{P}=$ Precipitates,

$H=$ Homogenous distributed, ${ }^{*}=$ Maximum concentration 

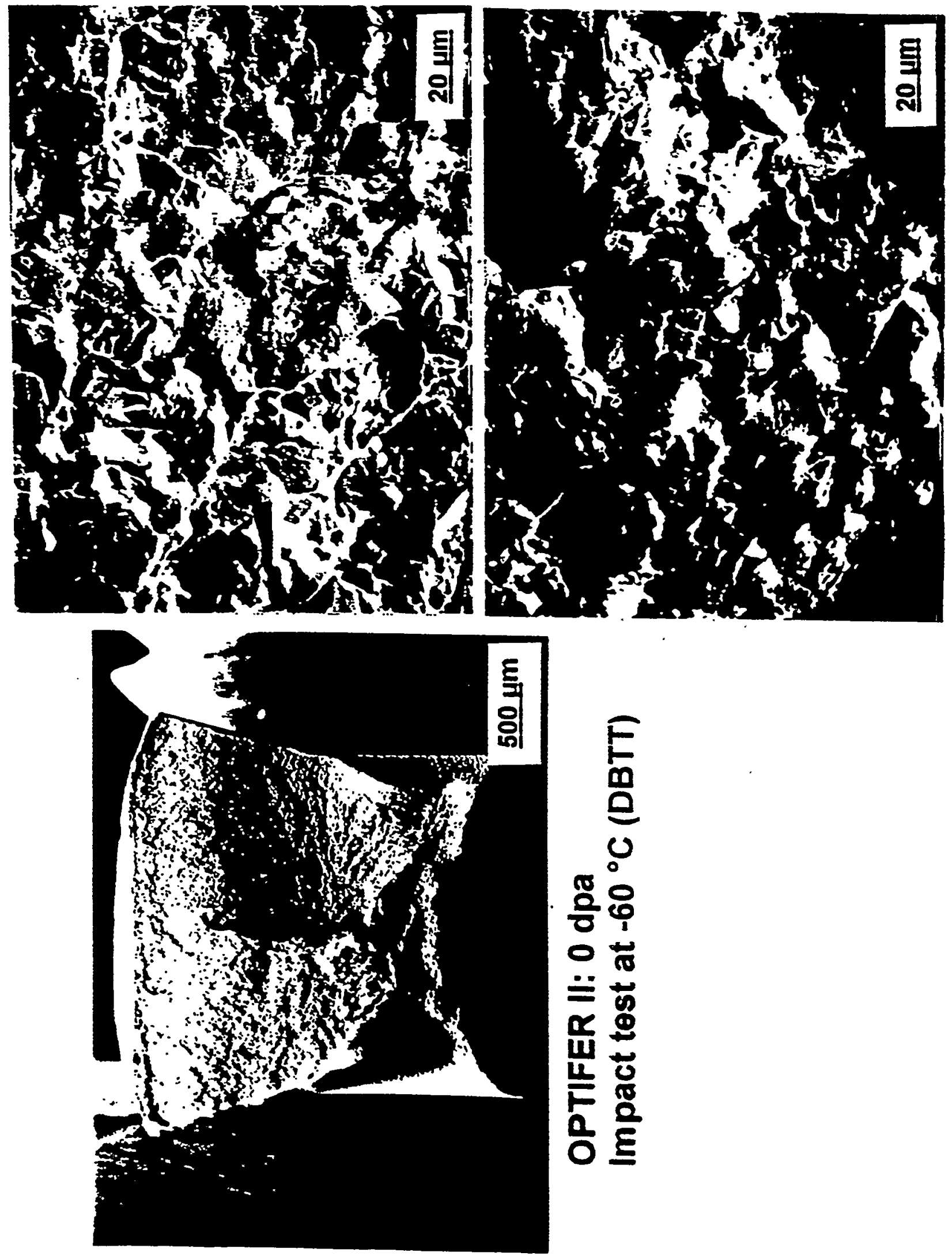

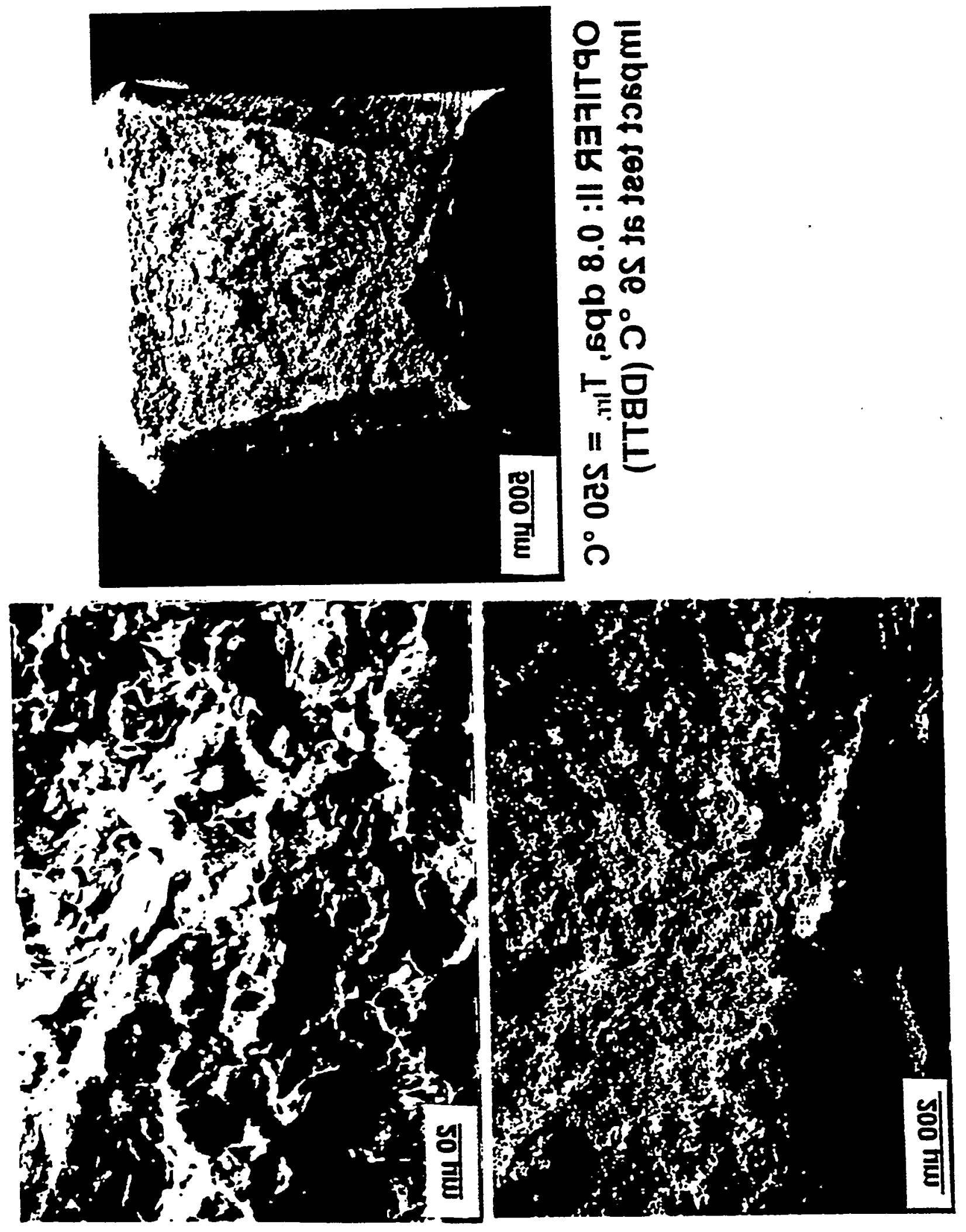


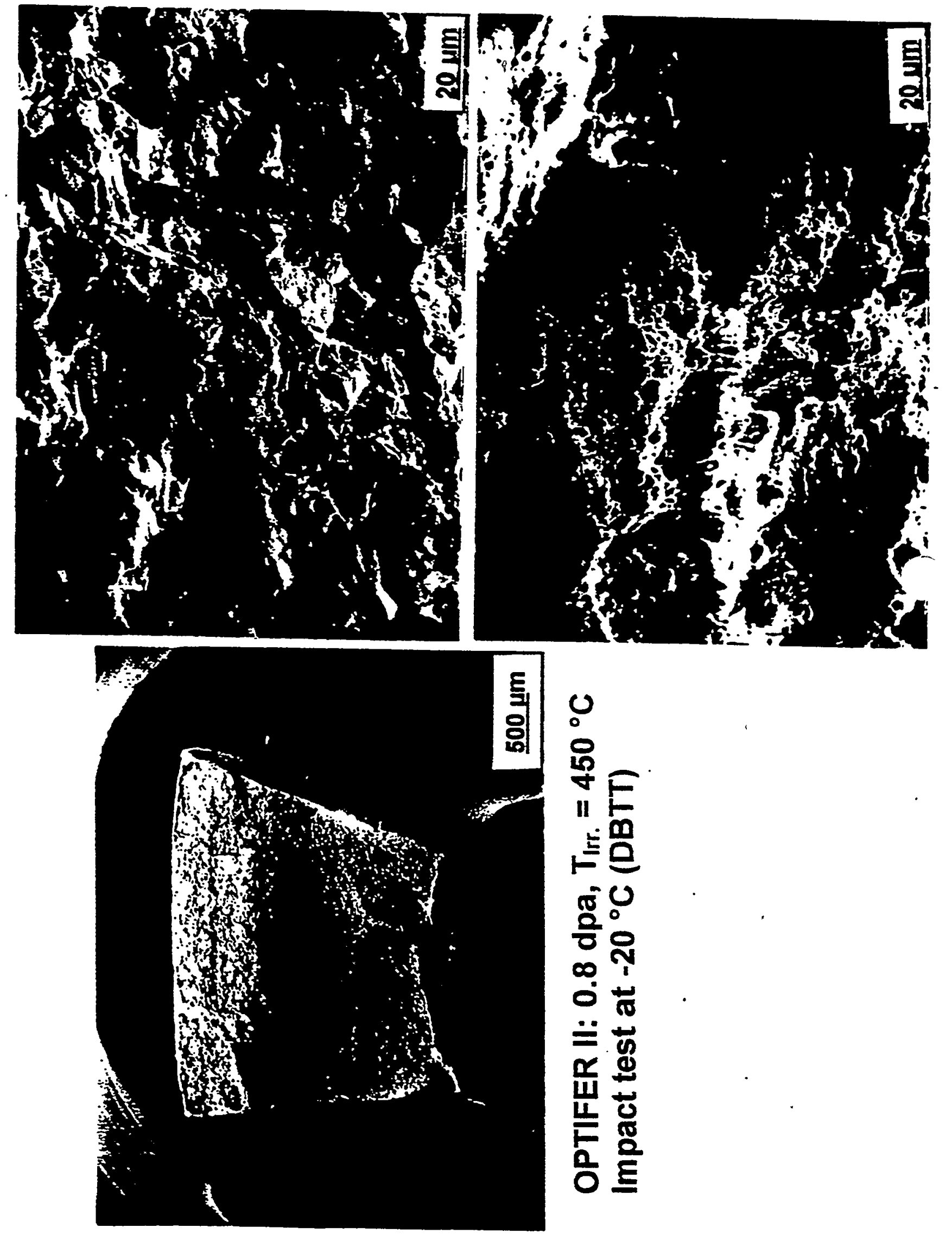



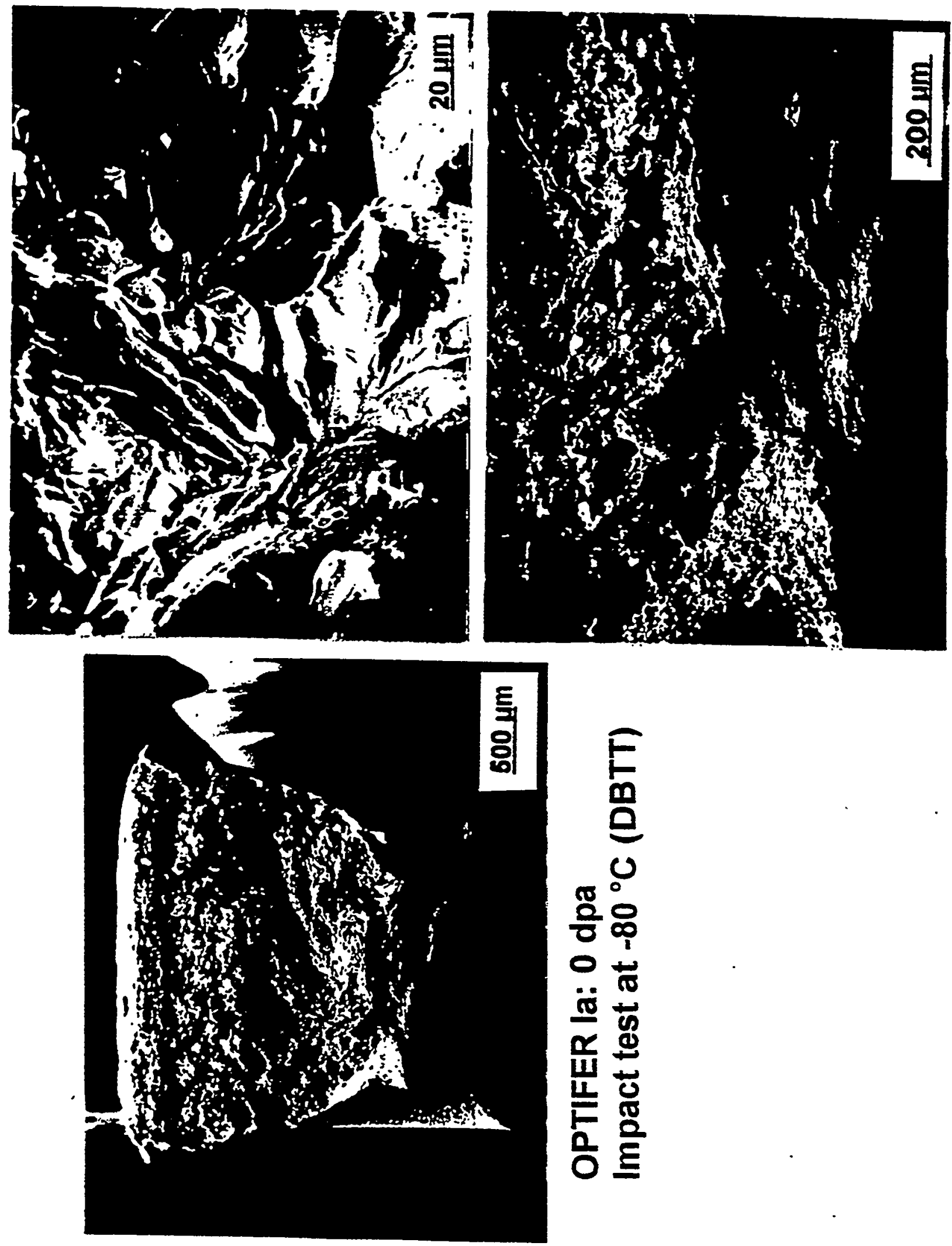


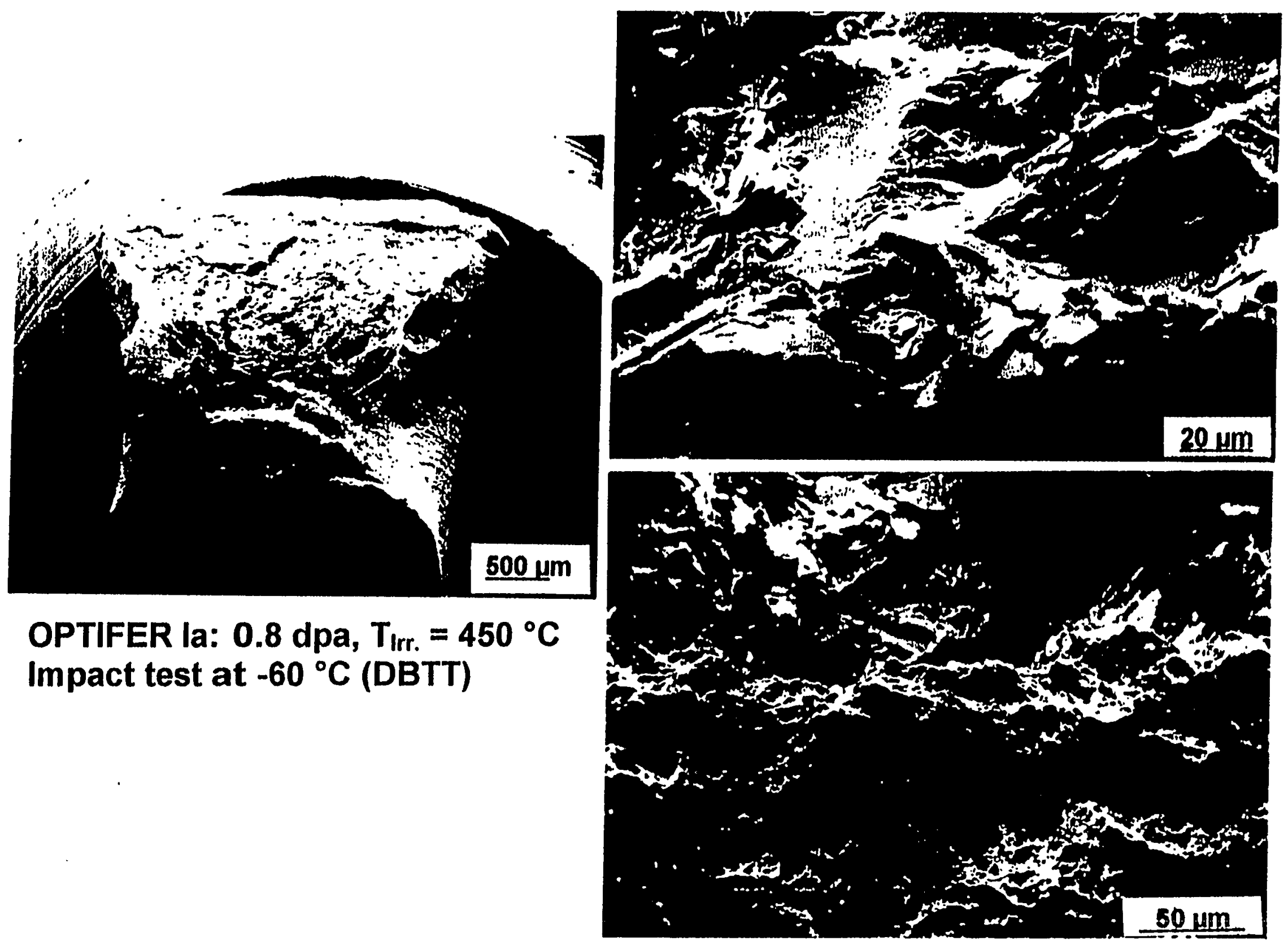



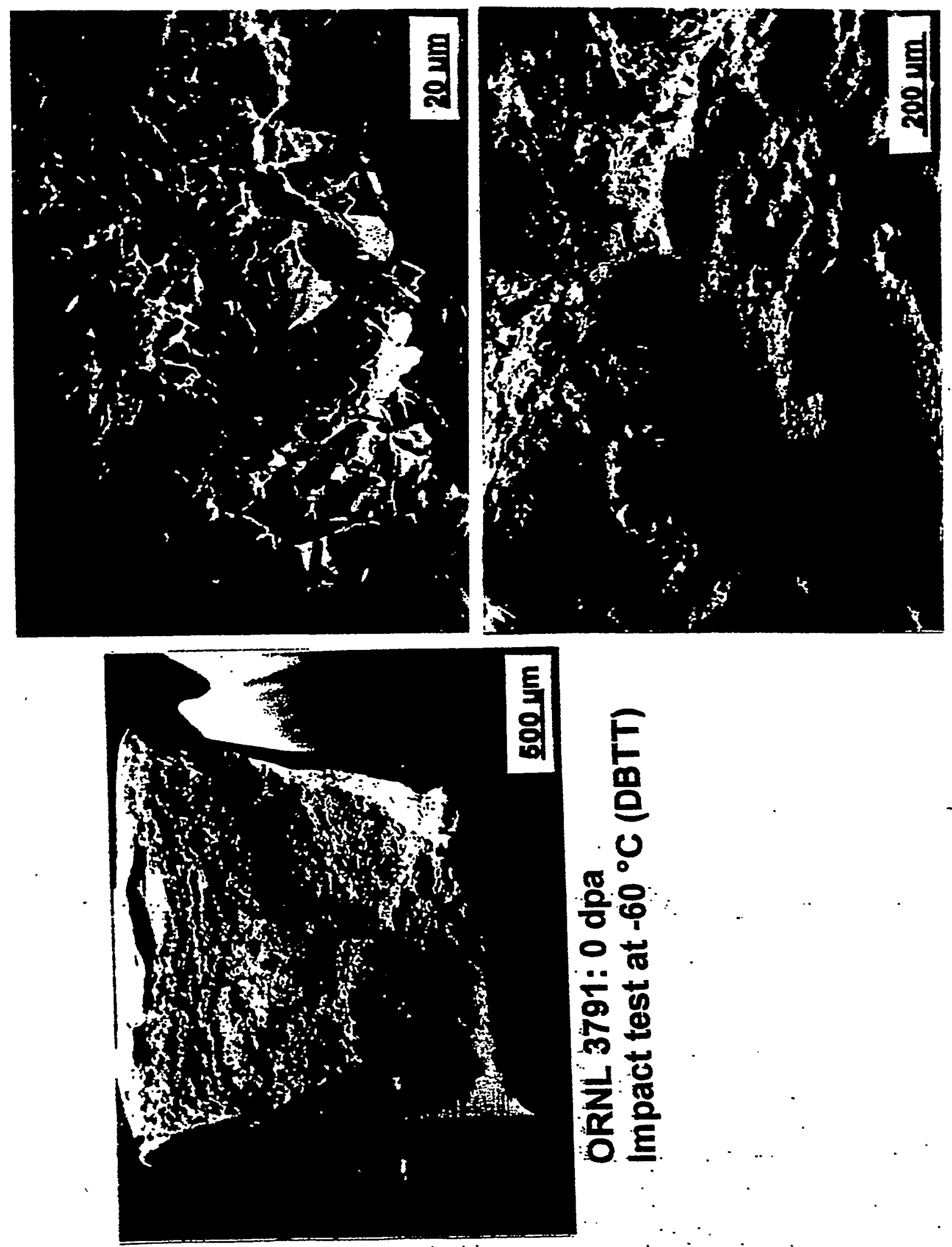

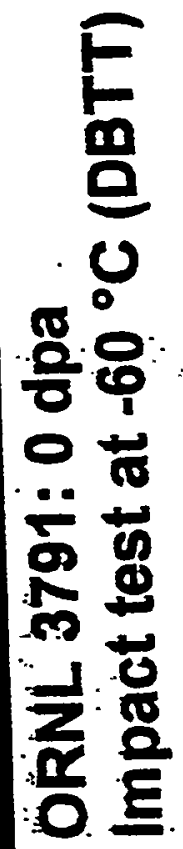



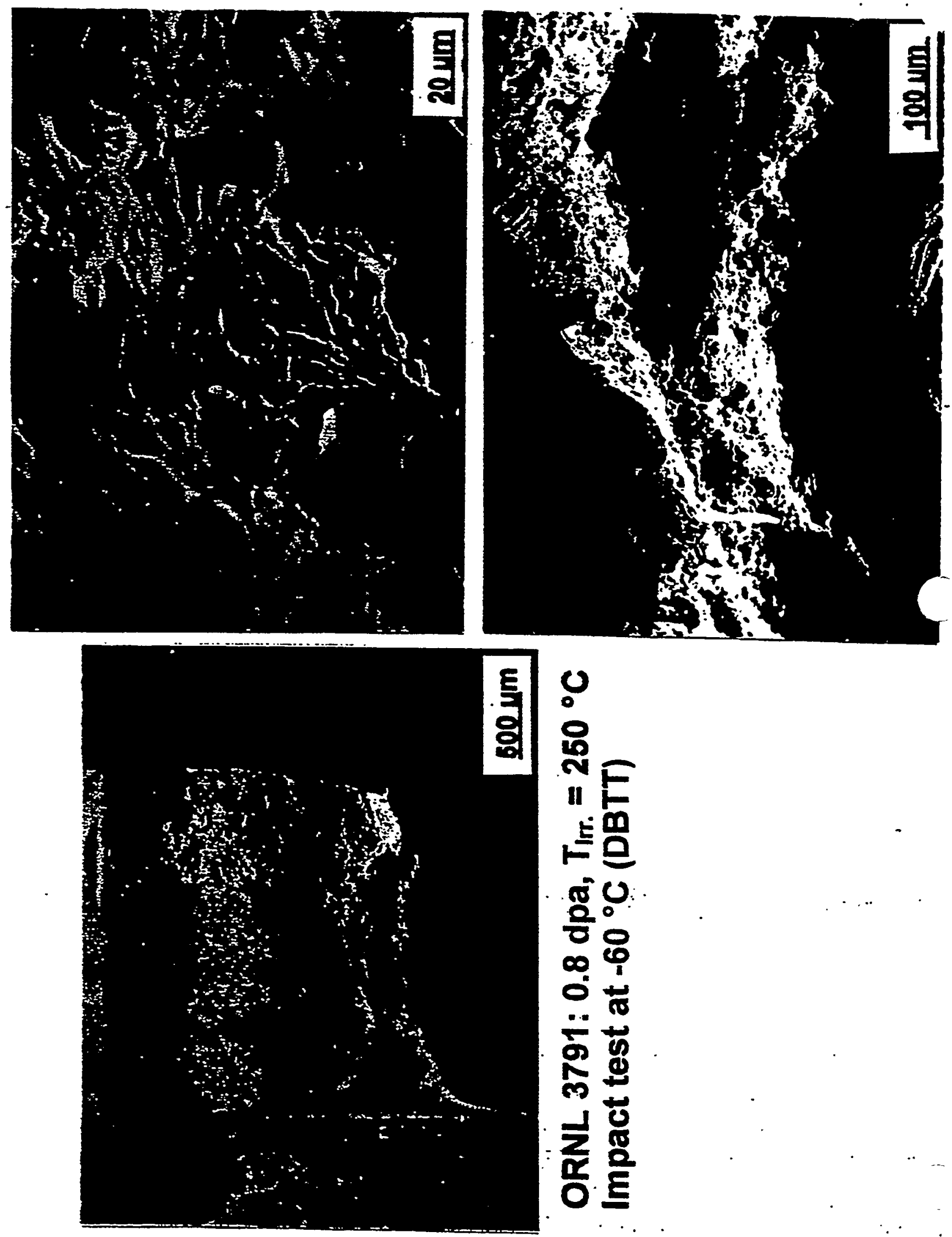


\section{References:}

Nuclear Fusion Projekt Annual Report of the Association Forschungszentrum Karlsruhe/EURATOM, October 1996 - September 1997; Report FZKA 6050, EUR 18156 EN, December 1997, ISSN 0947-8620.

Lindau R., Materna-Morris E., Möslang A., Preininger D., Rieth M., Röhrig H.D.: Influnce of Helium on Impact Properties of Reduced Activation Ferritic-Martensitic Cr-Steels, ICFRM-8, Oct. 26 - 31, 1997, Sendai, Japan.

Rieth M., Daffener B., and Röhrig H.D.:Embrittlement Behaviour of Different International Low Activation Alloys after Neutron Irradiation, ICFRM-8, Oct. 26 - 31, 1997, Sendai, Japan

Materna-Morris E. I., Rieth M. and Ehrlich K.:Mechanical Properties and Microstructure of HFR-Irradiated Ferritic/Martensitic Low-Activation Alloys. Will be published in: Effects of Radiation on Materials, 19th Int. Symp., ASTM STP 1366, M. L. Hamilton, A. S. Kumar, S. T. Rosinski and M.L. Grossbeck, Eds., American Society for Testing and Materials, 1999 


\title{
Further Assessment of Helium Induced Embrittlement in RAMS
}

\author{
A. Kimura \\ Institute of Advanced Energy \\ Kyoto University
}

IEA Workshop on Reduced Activation Ferritic/Martensitic Steels

Oct.1-2, 1998 ECN, Petten, Netherlands 


\section{Back Ground (1)}

I.A.E, Kyoto University

1) $9 \mathrm{Cr}-2 \mathrm{~W}$ martensitic steels are much more highly resistant to

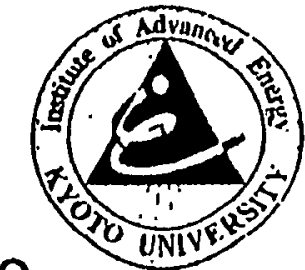
irradiation-induced degradation than the other candidates, which results from the large? calparilly of the matensillc; structure trapping point defects.

2) It is considered that the trapping eff:!; works on transmulation helium, resulting in the high resistance to the helium embrittlement in martensitic steels, since the martensitic structure prevents helium atoms from segregating at a crack nucleation site, such as grain boundary or in the matrix.

3) However, the results of isotope tailoring experiments utilizing addition of nickel or boron-10 suggested sever helium embrittlement of the martensitic steels. Helium implantation experiment of $\mathrm{F} 82 \mathrm{H}$ also showed a larger shift of DBTT than neutron irradiated one. 


\section{Back Ground (2)}

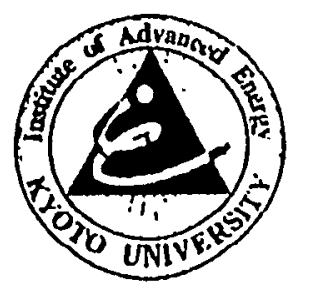

4) In contrast, the DBTT shift of a $9 \mathrm{Cr}-2 \mathrm{~W}$ steel implanted with 120at.ppm of helium by cyclotron was explained in terms of displacement damage, suggesting no helium embrittlement of the steel. Furthermore, irradiation hardening of martensitic steel was three times larger in the $1 \% \mathrm{Ni}$ added steel than without the addition when irradiated at temperatures less than $170^{\circ} \mathrm{C}$ up to only $0.15 \mathrm{dpa}$. 


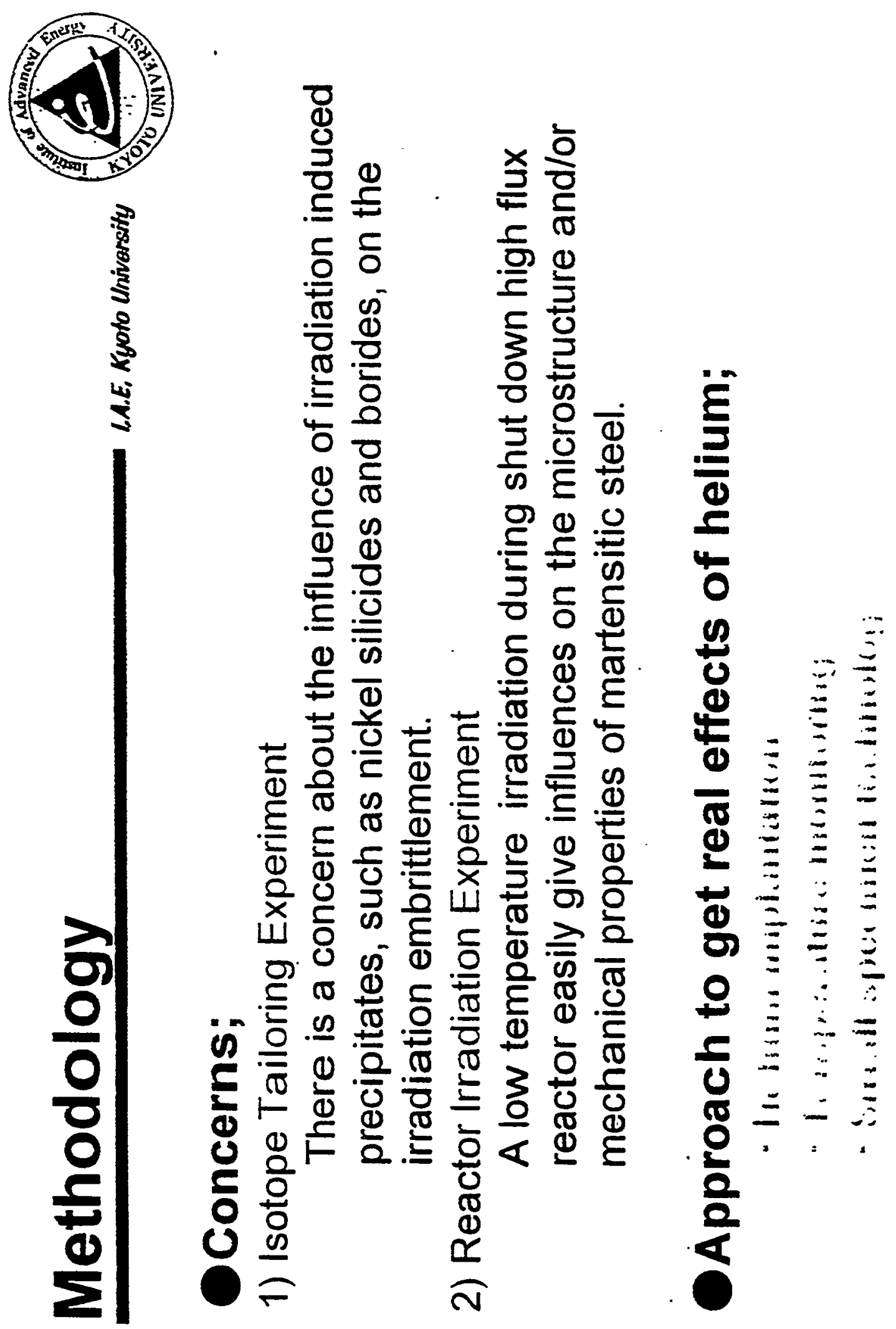




\section{Experimental}

\section{Material}

RAMS; 9Cr-2W-V,Ta,Ti, B

(0) SP test

Test Temp.: RT $\sim 77 \mathrm{~K}$

Cross Head Speed: $0.2 \mathrm{~mm} / \mathrm{min}$

(O) SEM, TEM Observation

(O) Annealing

Temp.: RT $\sim 873 \mathrm{~K}$

Period: 1hour

Vac.: $5.0 \times 10^{-7}$ torr

(0) Vickers Hardness Measurement

Loading weight: $200 \mathrm{~g}$

() Positron Life Time Measurement
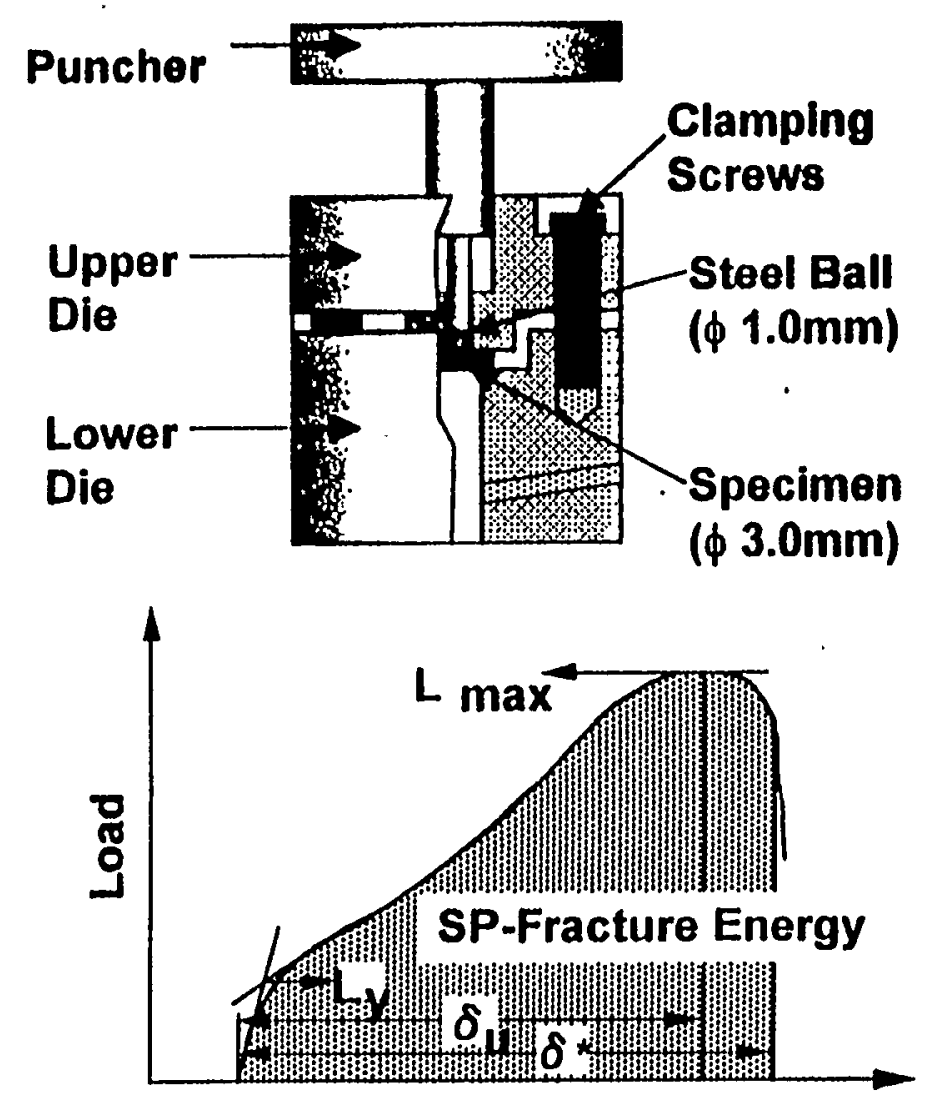

Deflection

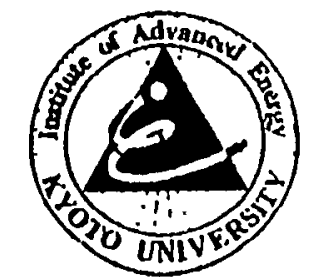




\section{Hardness Measurement}

I.A.E, Kyoto Univarsity
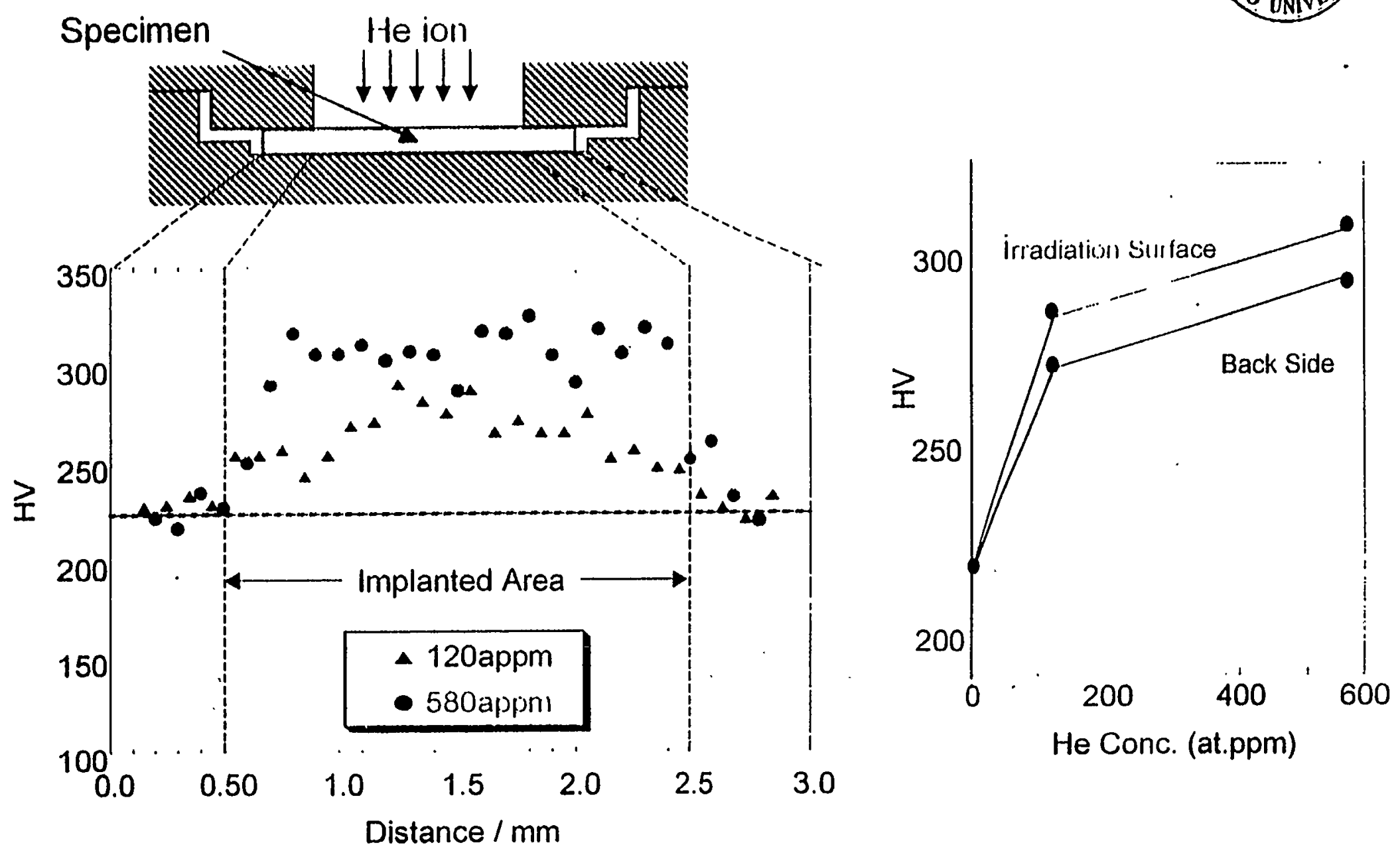


\section{He Implantation}

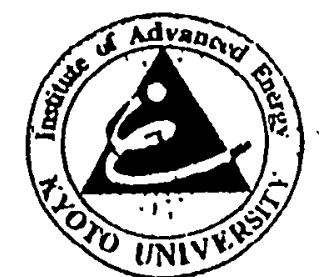

Cyclotron Irradiation

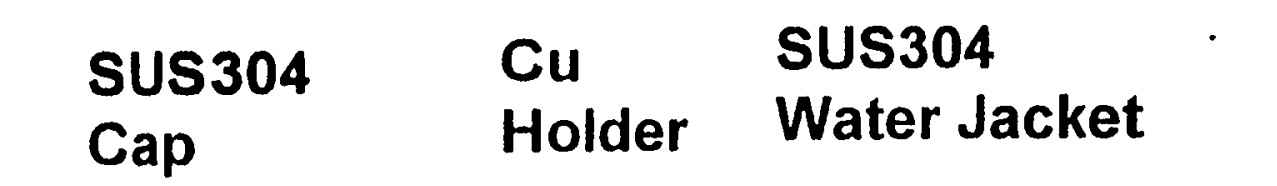

1) Beam; $36 \mathrm{MeV}-\alpha$

2) Degrader

3) Temperature; $<150^{\circ} \mathrm{C}$

4) He conc.;

120, 580at.ppm

5) Displ. Damage;

$0.048,0.232 \mathrm{dpa}$

6) Range; $0.23 \mathrm{~mm}$

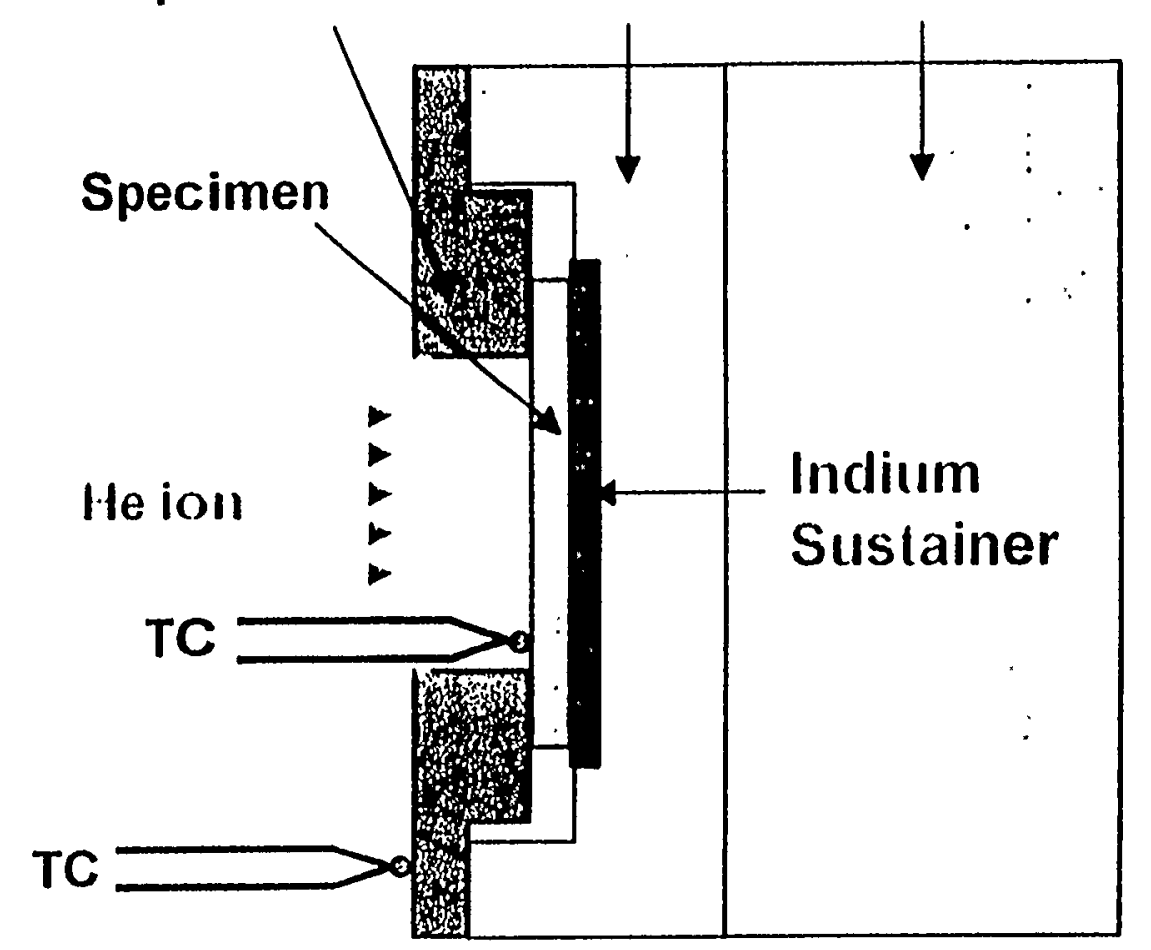




\section{SP Energy - Temperature}

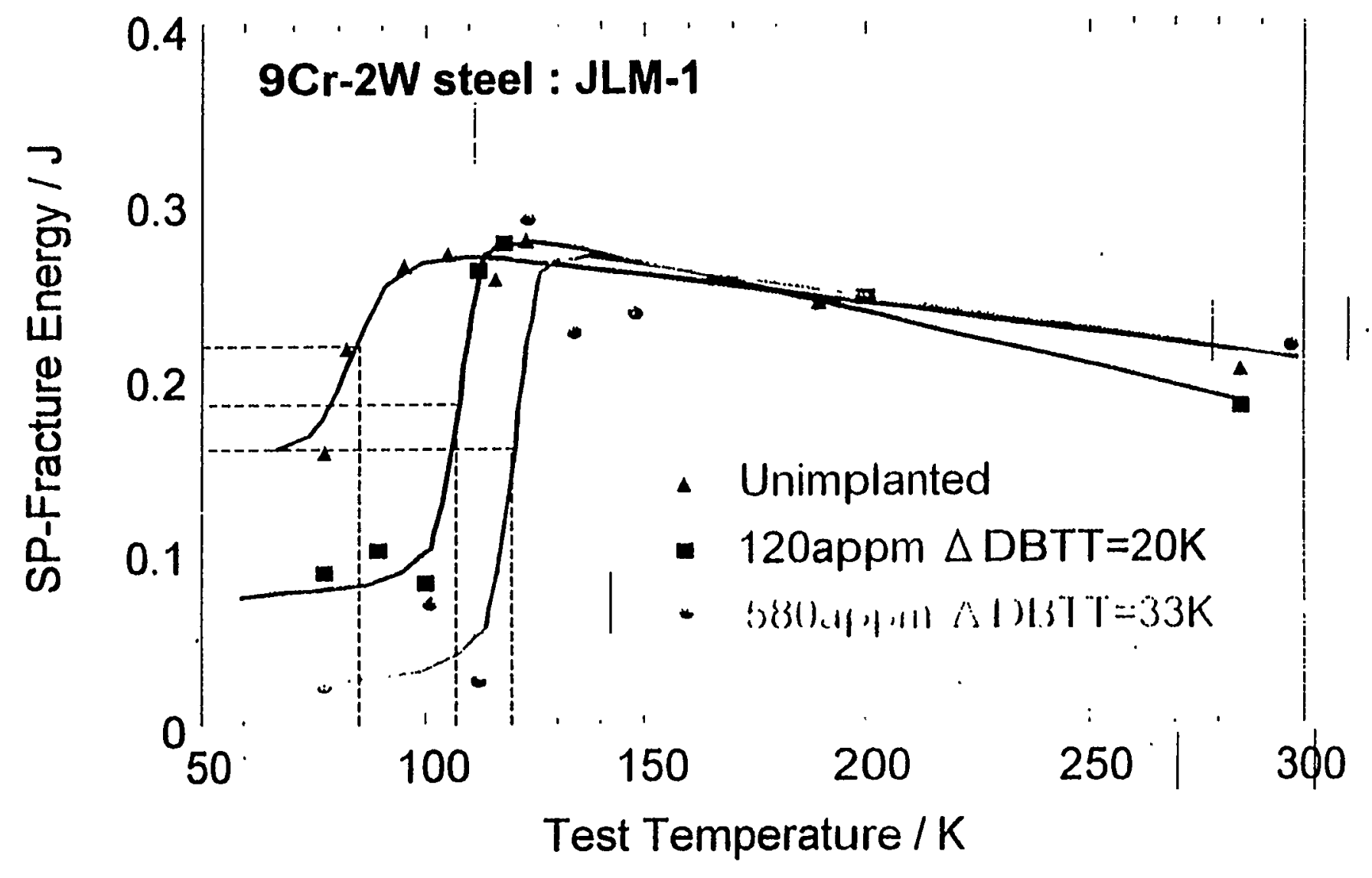




\section{SEM fractograph}
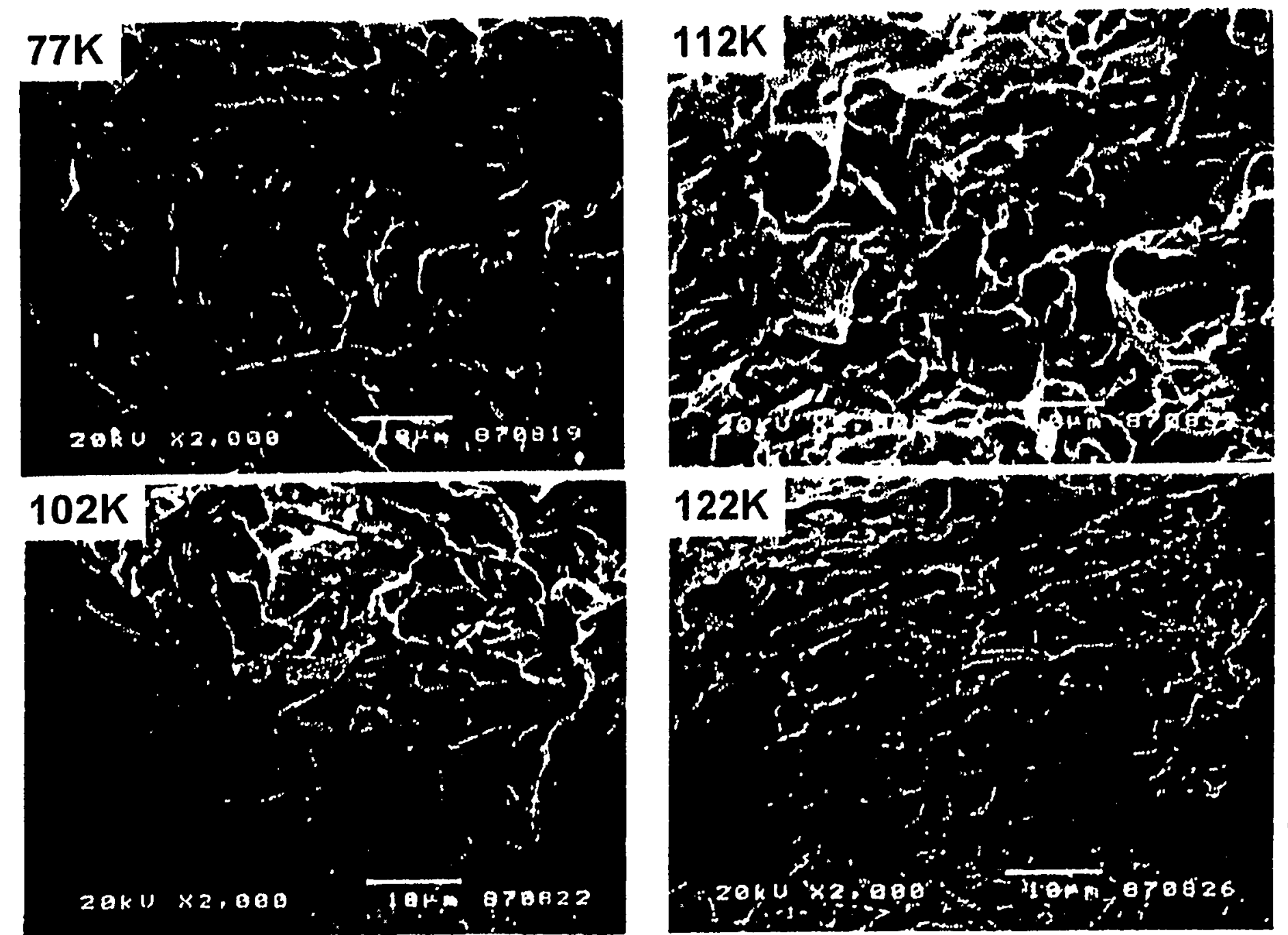

$\overline{10 \mu \mathrm{m}}$ 


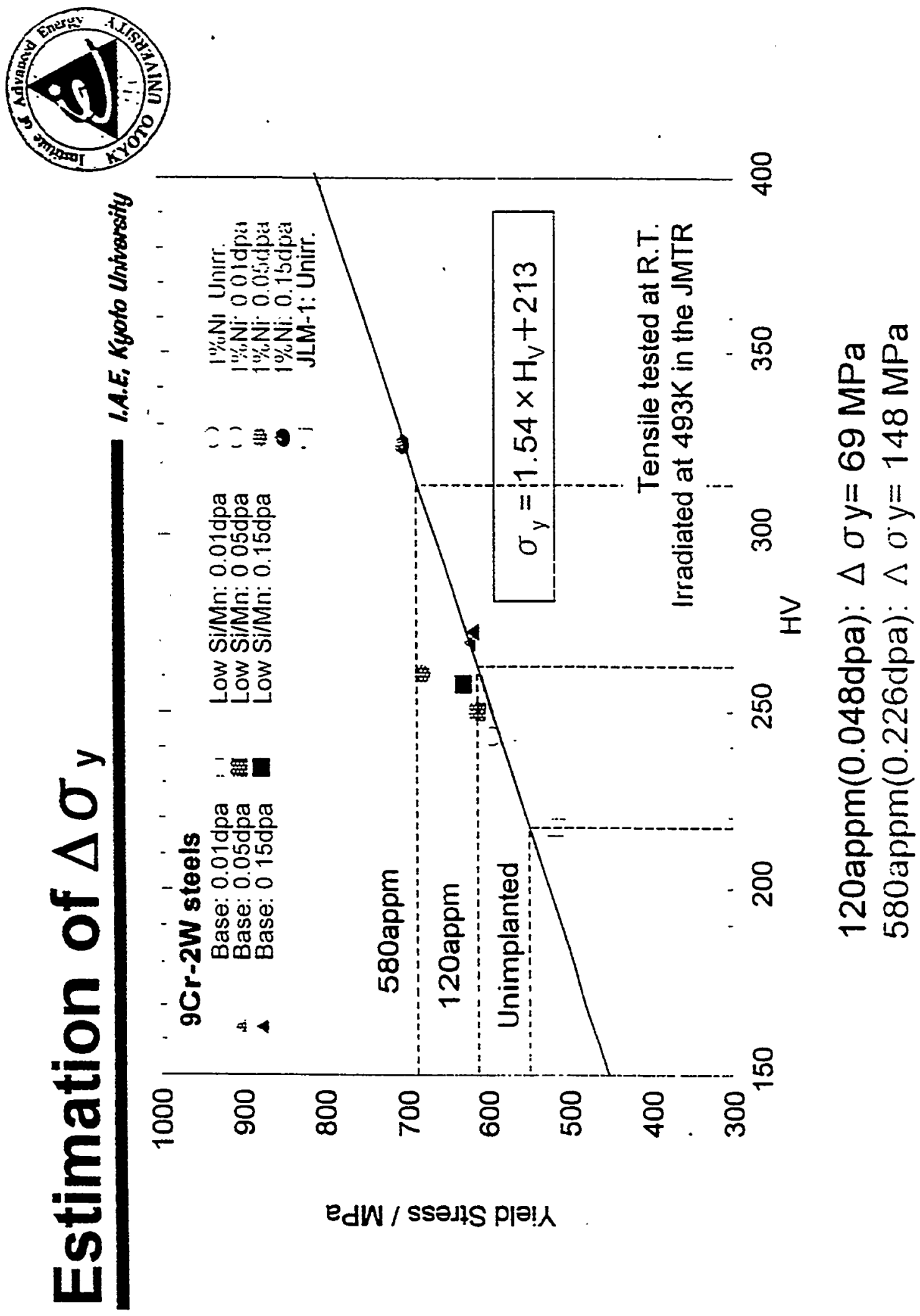




\section{Dose dependence of $\Delta \sigma y$}
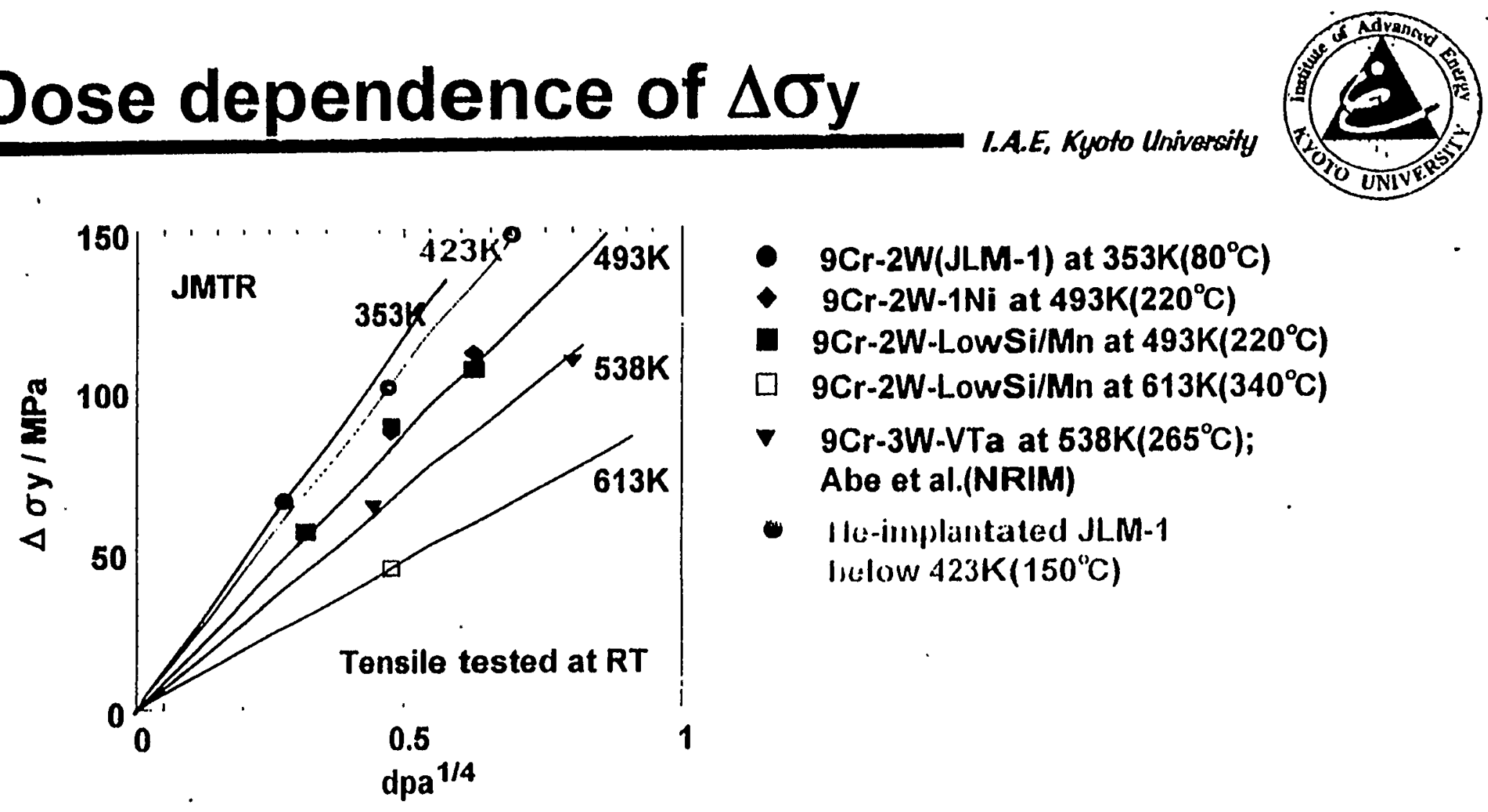

- $9 \mathrm{Cr}-2 \mathrm{~W}(\mathrm{JLM}-1)$ at $353 \mathrm{~K}\left(80^{\circ} \mathrm{C}\right)$

- $9 \mathrm{Cr}-2 \mathrm{~W}-1 \mathrm{Ni}$ at $493 \mathrm{~K}\left(220^{\circ} \mathrm{C}\right)$

- 9Cr-2W-LowSi/Mn at $493 \mathrm{~K}\left(220^{\circ} \mathrm{C}\right)$

- $9 \mathrm{Cr}-2 \mathrm{~W}-$ LowSi/Mn at $613 \mathrm{~K}\left(340^{\circ} \mathrm{C}\right)$

> $9 \mathrm{Cr}-3 \mathrm{~W}-\mathrm{VTa}$ at $538 \mathrm{~K}\left(265^{\circ} \mathrm{C}\right)$; Abe et al.(NRIM)

- I/c-implantated JLM-1 liclow $423 \mathrm{~K}\left(150^{\circ} \mathrm{C}\right)$

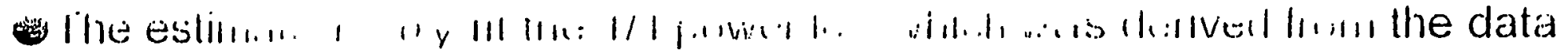

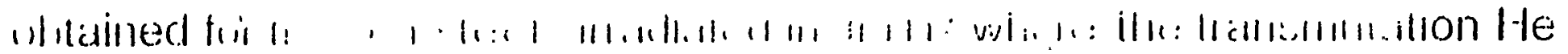

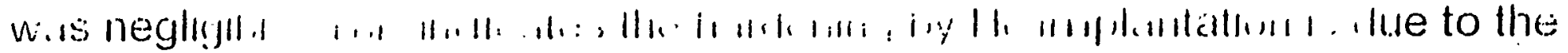

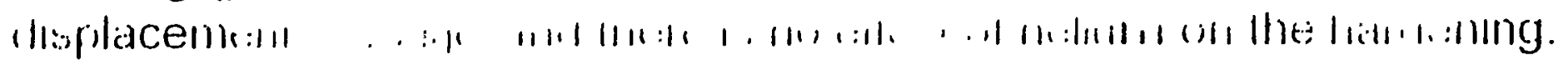




\section{Estimation of $\triangle \mathrm{DBTT}$ - CVN10}

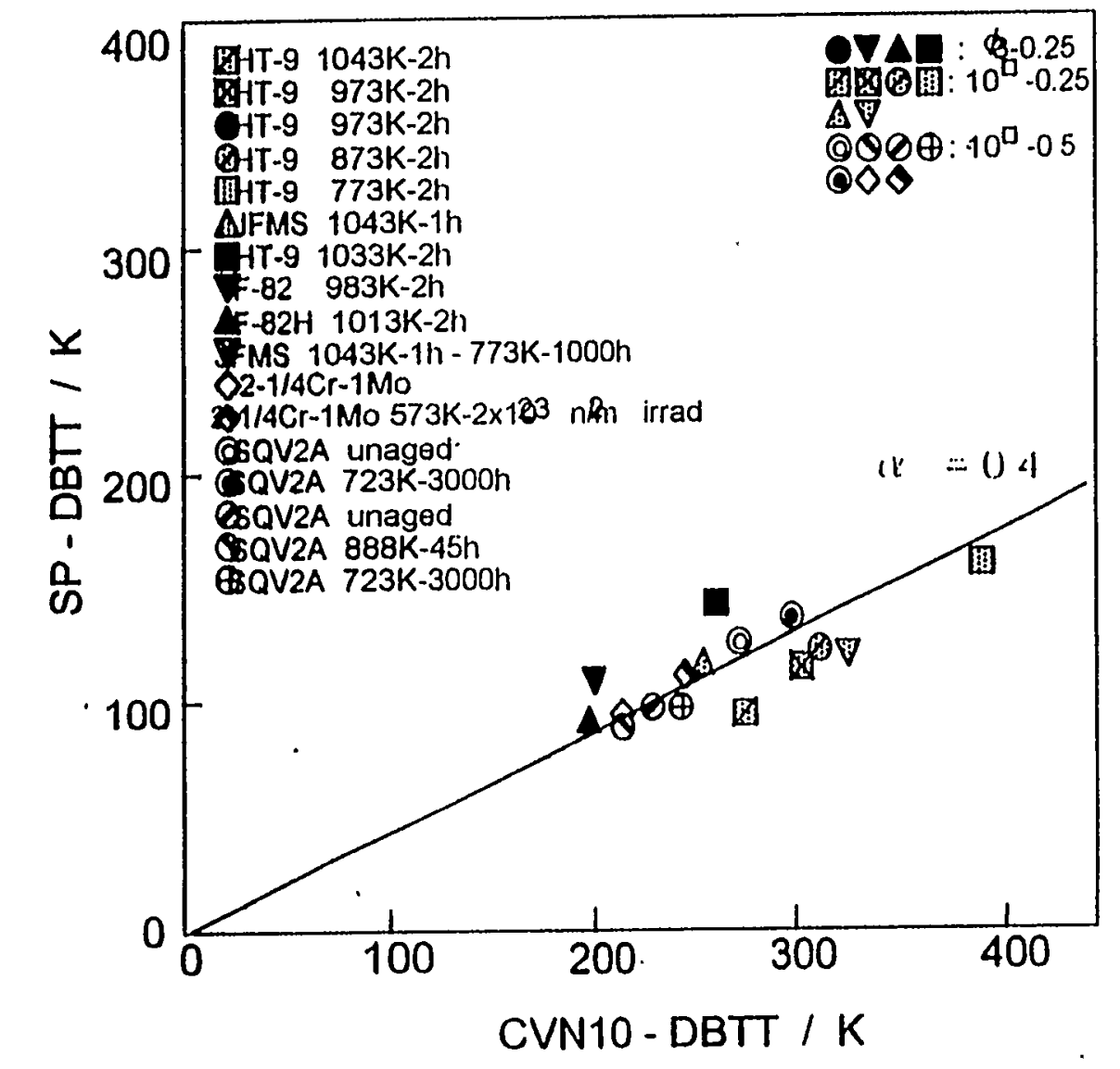

SP-DBTT(K)

$=0.4 \times$ CVN10 - DBTT(K)

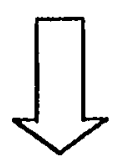

120appm : $\triangle \mathrm{DBTT}-\mathrm{CVN10}=50 \mathrm{~K}$

58()appm: : IDBTT-CVN10 $=83 \mathrm{~K}$ 


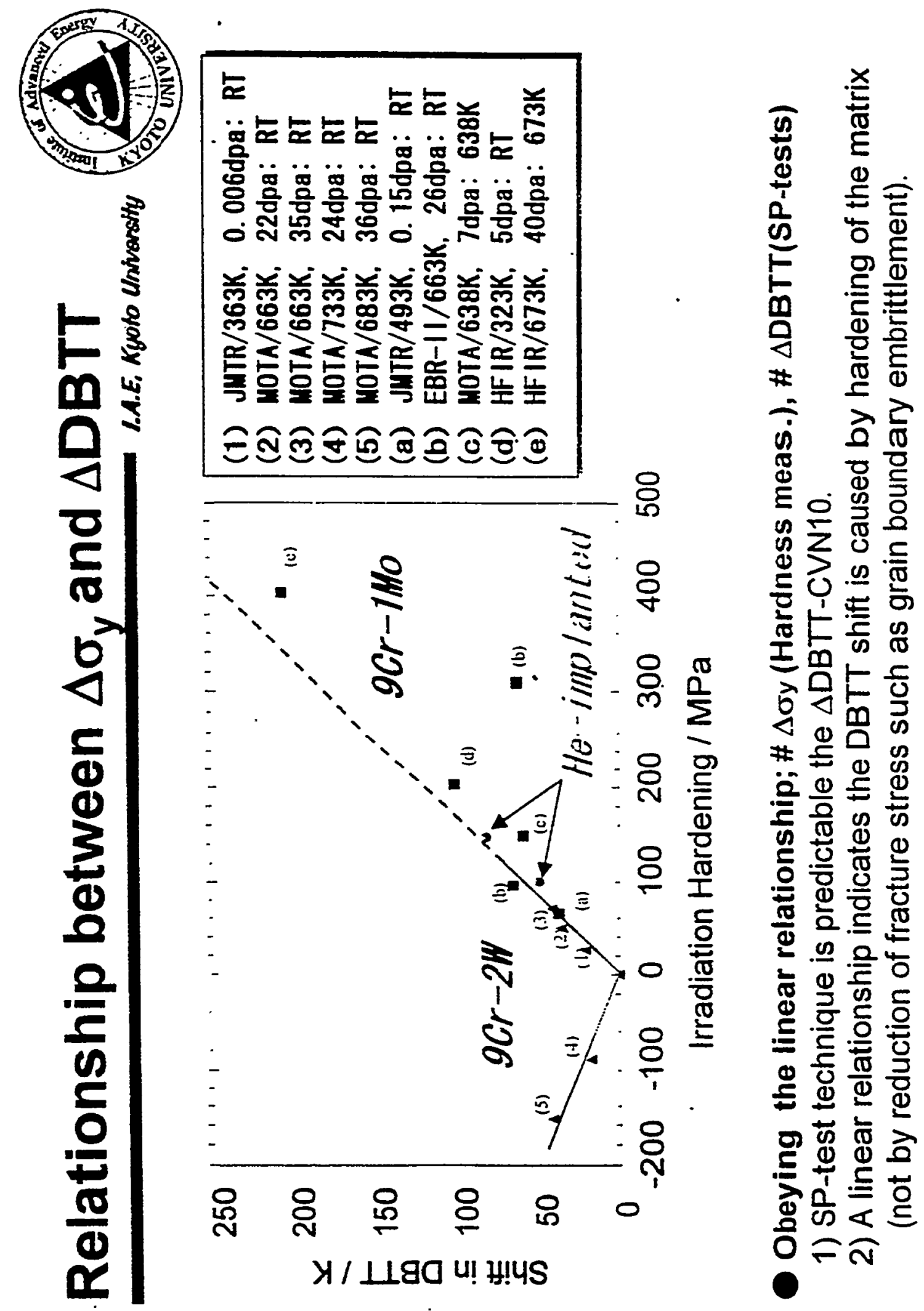




\section{Annealing Behavior (Hv)}

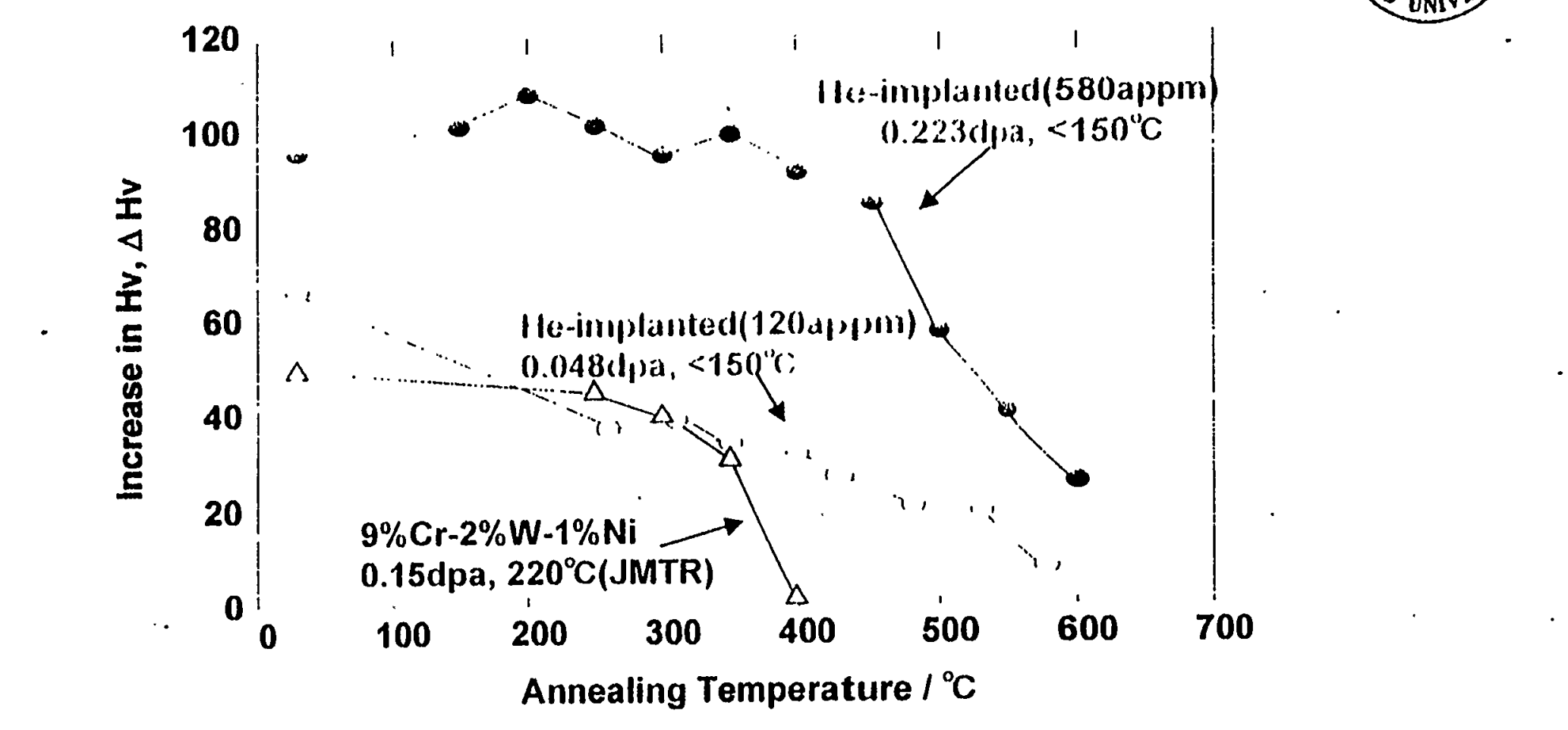

I.A.E, Kyoto Univorsity

He implantation reduced the recovery of irradiation hardening, suggesting that the thermal stability of I-clusters was increased by helium.

There is a possibility that helium retard the recovery of dislocation structure and reduces the thermal creep rate. 


\section{TEM micrograph}

\section{He-implanted up to 580appm}
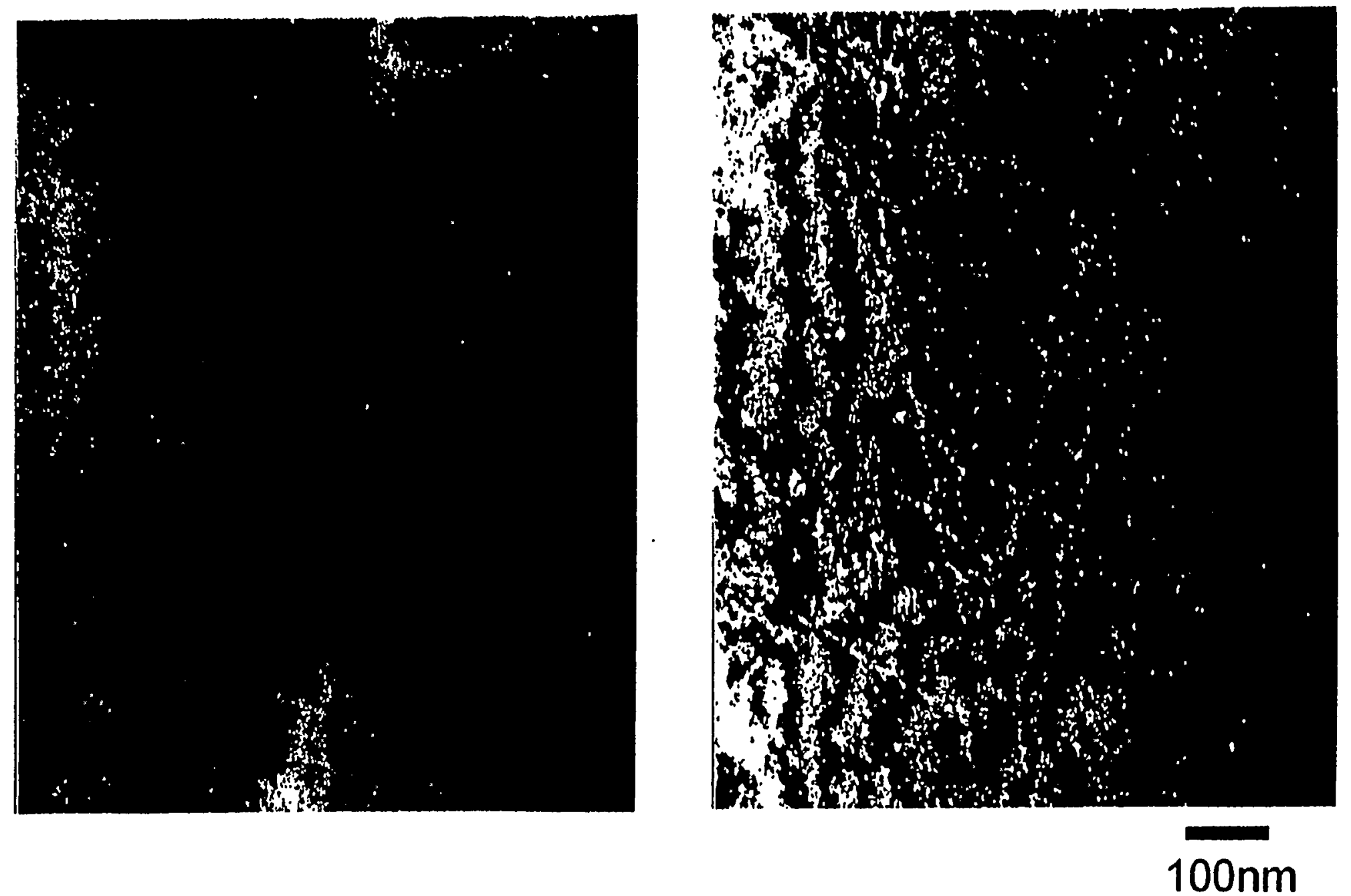


\section{TEM micrograph}

Post-implantation annealing up to $873 \mathrm{~K}$
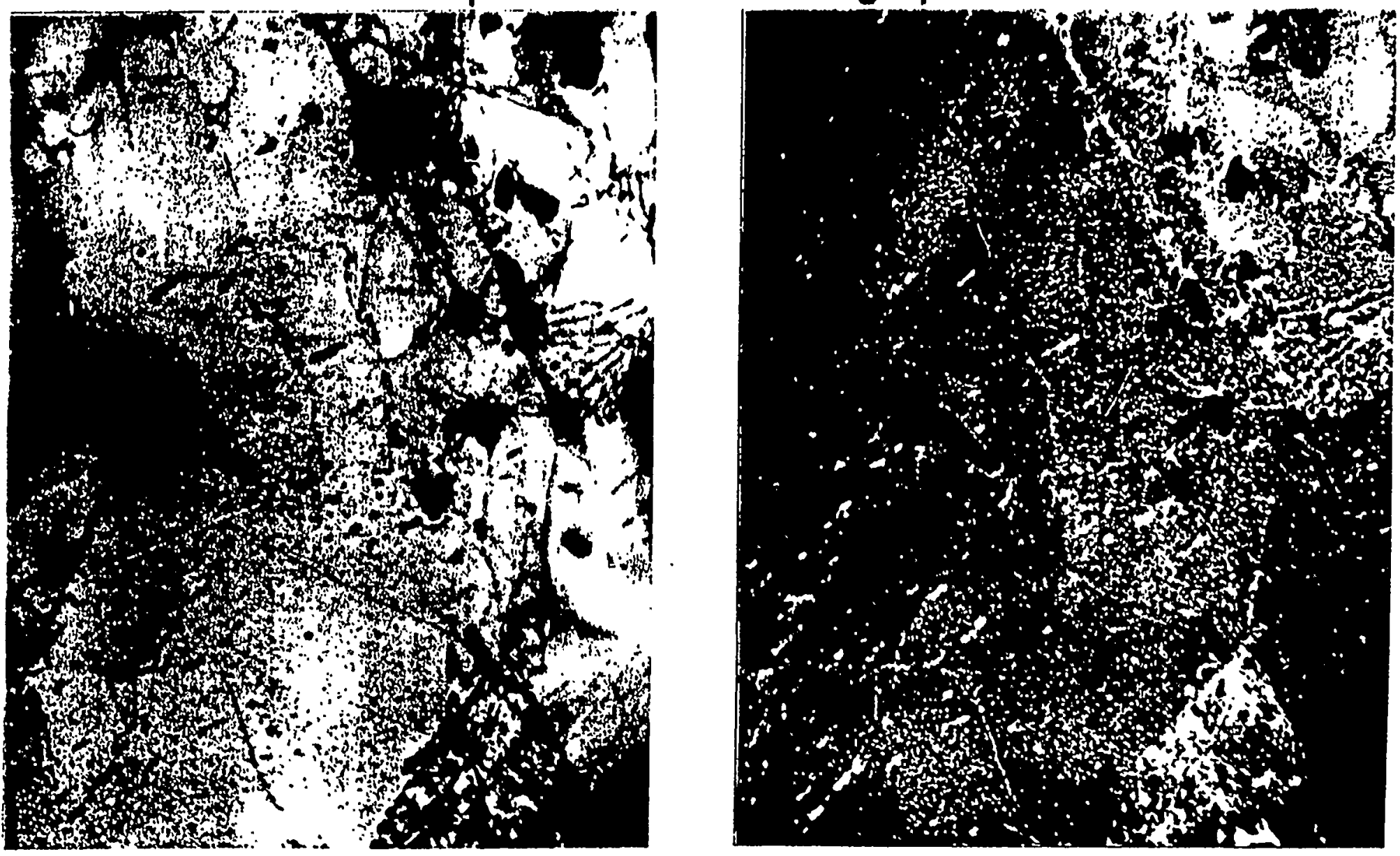

$200 \mathrm{~nm}$ 


\section{PAS Measurement}

I.A.E, Kyoło University

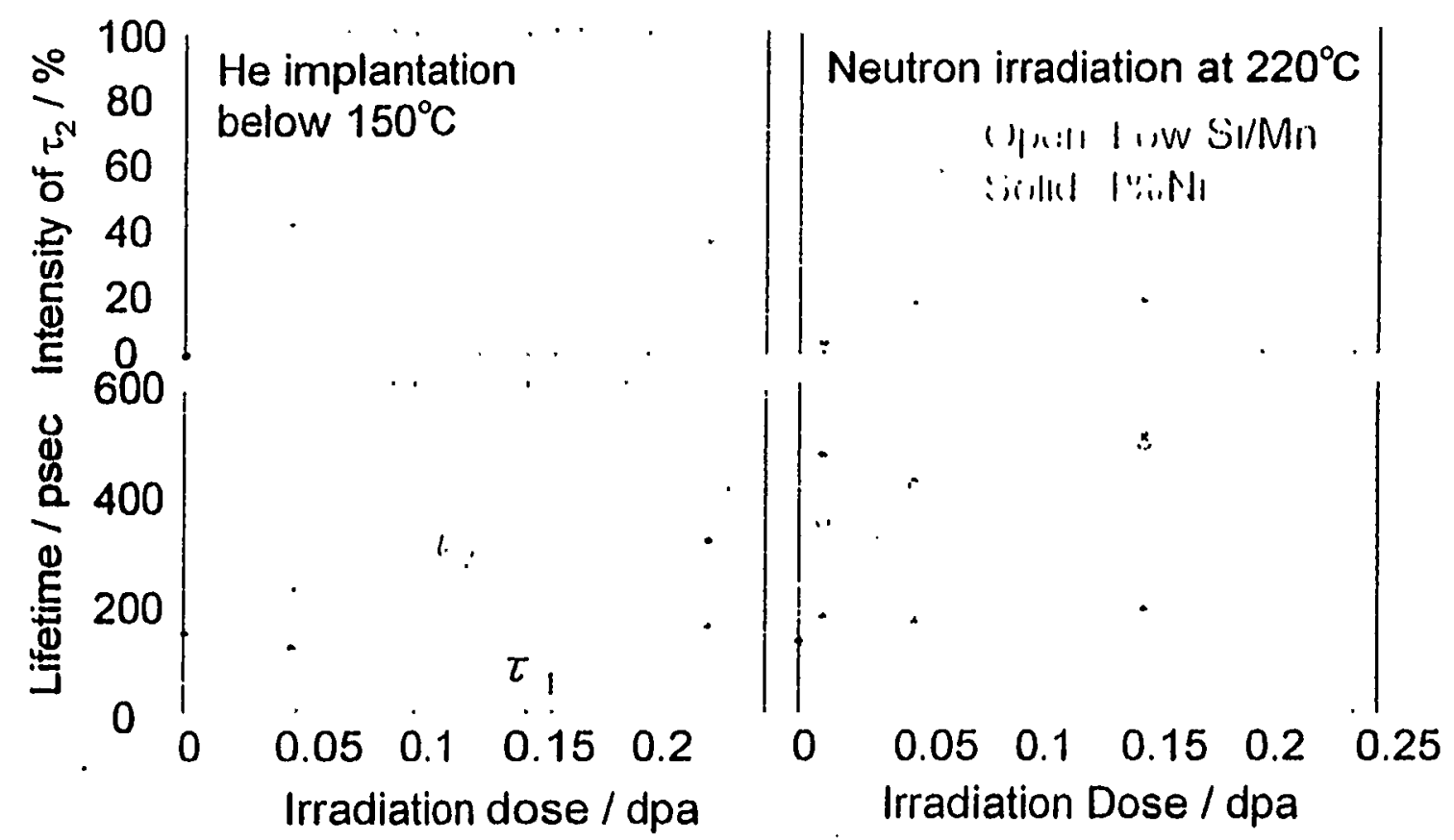

UNIVY.

OHe implantation reduced the size of microvoids and increased their density. (suppress the vacancy migration, resultantly the growth of microvoids) 


\section{Annealing Behavior (PAS)}

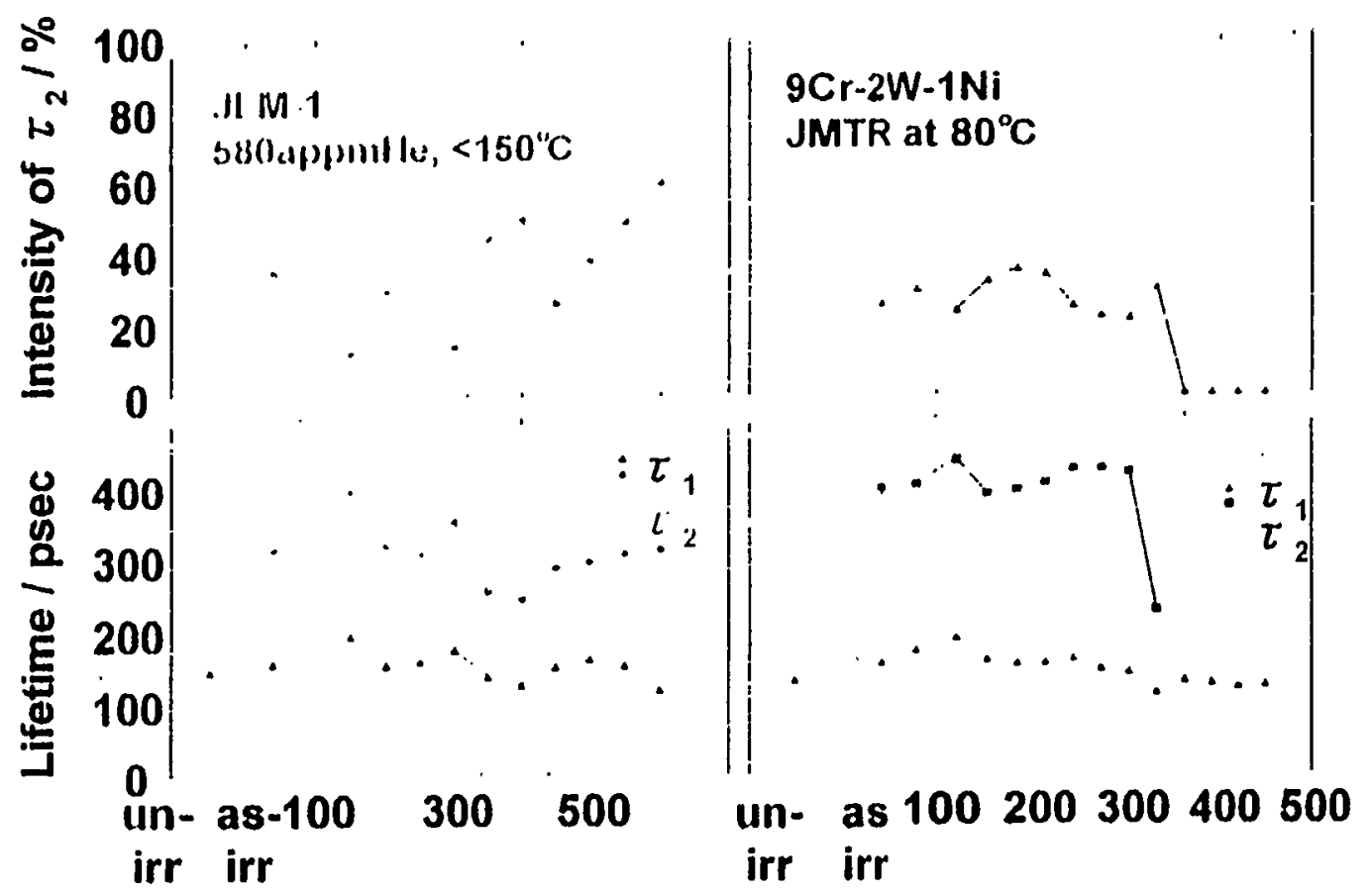

Annealing Temperature $/{ }^{\circ} \mathrm{C}$

No decrease in the intensity of the longer life time component, $\tau 2$, was observed in the $9 \mathrm{Cr}$ steel implanted with He following the annealing to $600^{\circ} \mathrm{C}$. He stabilized the microvoids. 


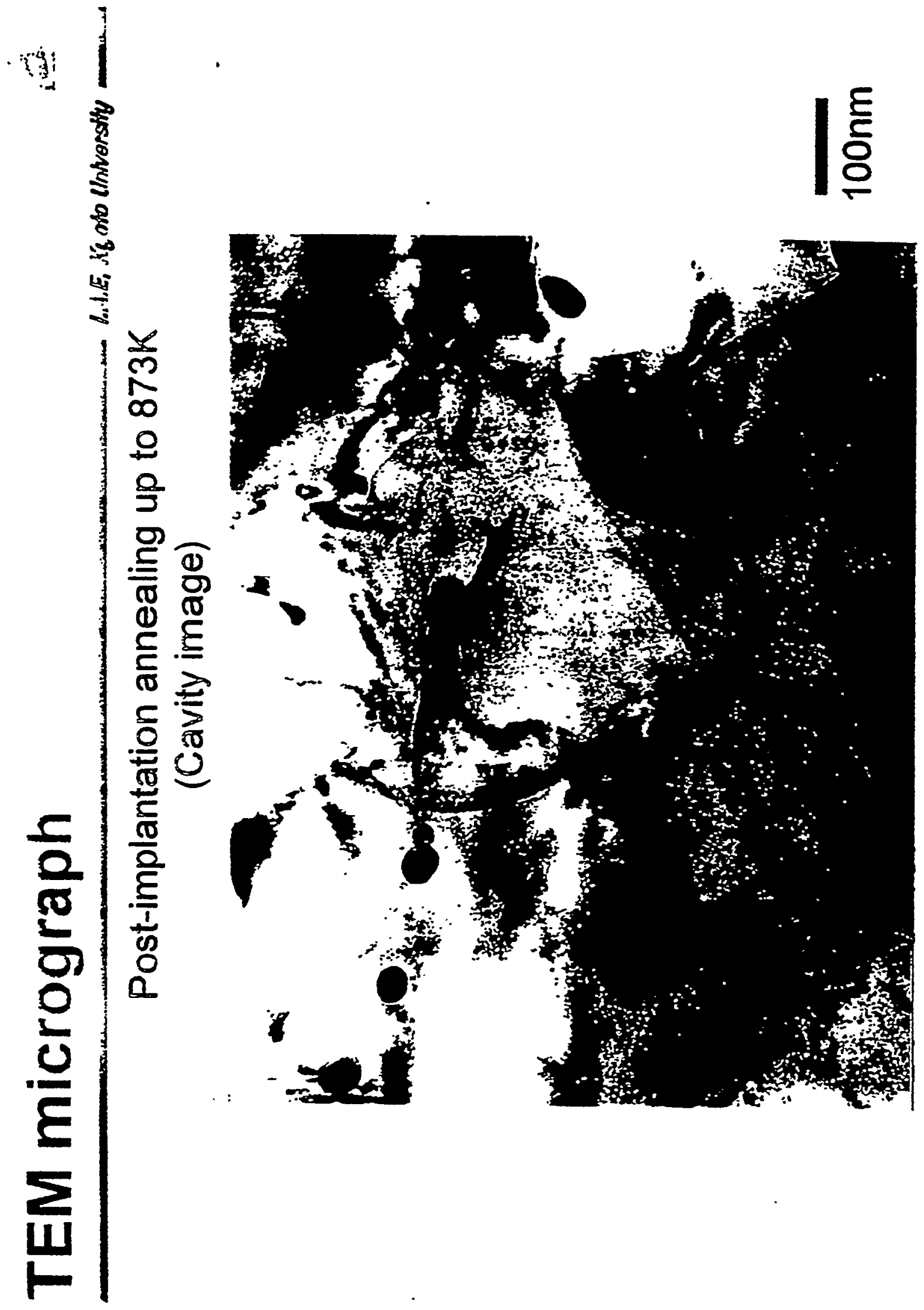




\section{Conclusion}

Effects of He implantation (580atppm) on DBTT

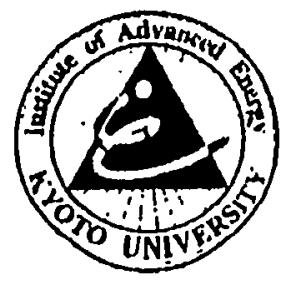

- Fracture mode; CLEAVAGE

- $\triangle \mathrm{DBTT}(\mathrm{CVN}-10) ; 83 \mathrm{~K}$ (explained in terms of hardening mechanism)

- $\triangle \sigma y(\Delta H v=90) ; 150 M P a$ (explained in terms of DISPLACEMENT DAMAGE)

- Linear relationship; $\triangle \mathrm{DBTT}$ and $\Delta \sigma y$ (validity of SP technique

to estimate $\triangle \mathrm{DBTT}(\mathrm{CVN}-10)$

- Effects of He implantation (580atppm) on microstructure

- V, I-cluster;

1) He reduced the size of V-clusters but increased the density

2) He increased the thermal stability of V-clusters

- Hardening mechanism; good relation to the I-cluster behavior

Thus, 580atppintle does NOT enhance the irradiation hardening and/or embrittlement of RAFS which consists of martensitic structure containing a number of trapping site for He atoms.

ONext step is to find out the critical lle concentration to cause $\mathrm{He}$ induced embrittlement. 


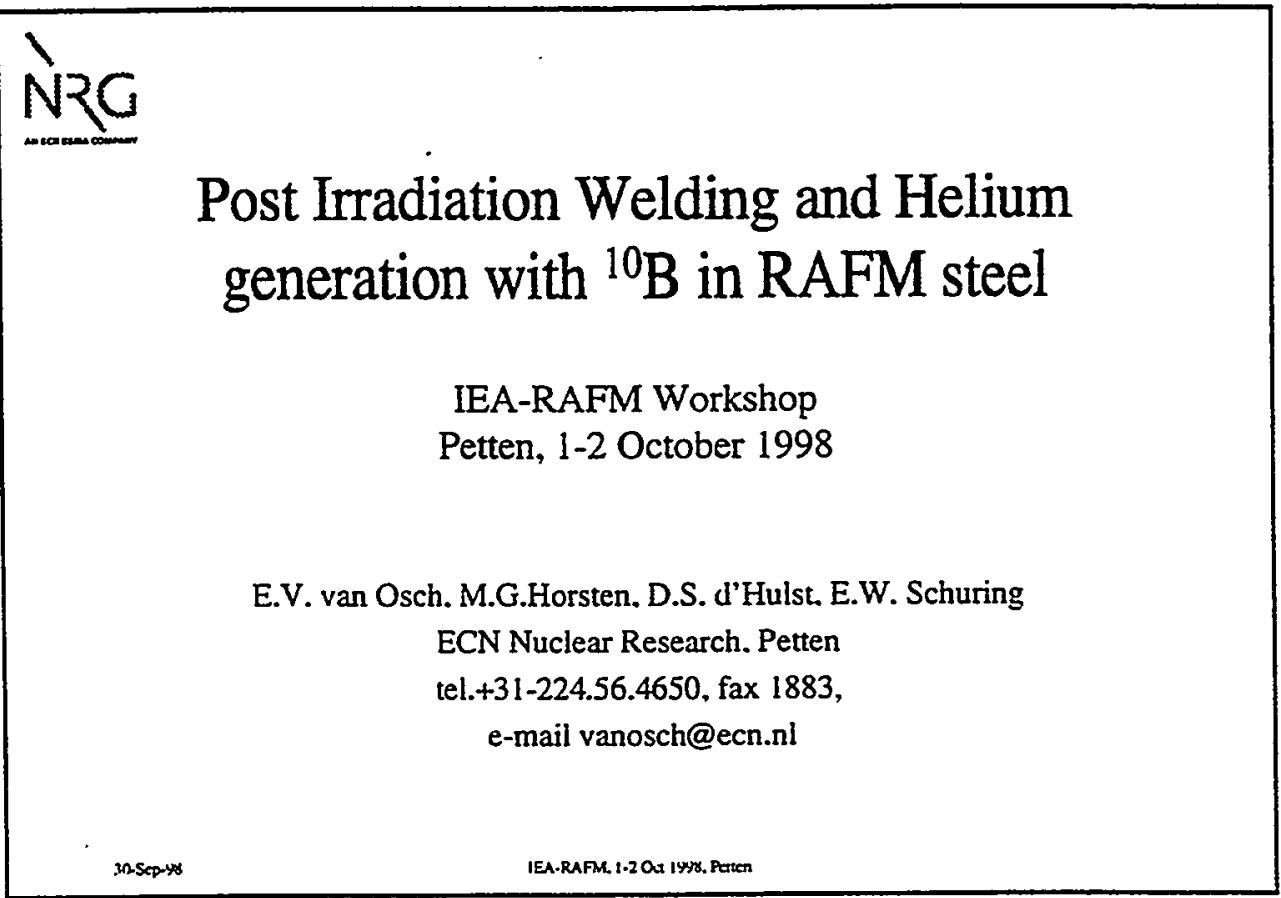

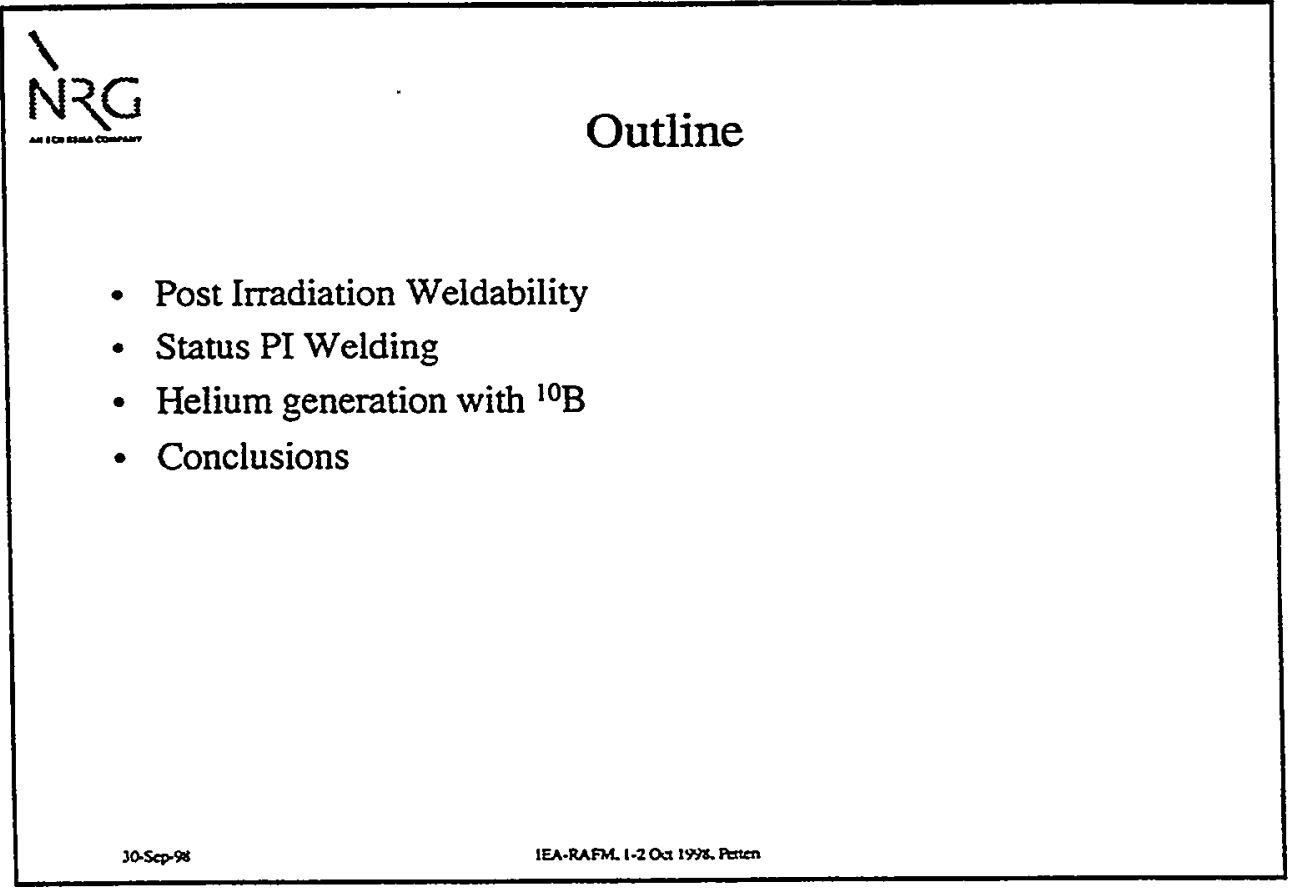




\section{Post Irradiation Weldability: Why?}

- In view of Repair/Replacement of Irradiated FM components

- Austenitic steels (TTER/BWR/PWR): Weld Cracking due to Hebubble coalescence and growth on Grain Boundaries during Cool-down; cracking threshold $\sim 1$ appm He.

- Mitigation strategies:

- reduce amount helium

- low heat input welding methods: e.g. Laser welding

- reduce/compensate thermal stresses

- FM-steels expected to have much higher He-tolerance

- DEMO He-levels up to 100 's appm

\section{Status Welding of Irradiated F82H}

- Irradiated F82H available: 2 dpa ( 5 appm $\mathrm{He})$ 1, 3 and $5 \mathrm{~mm}$ plates and limited amount $2.5 \mathrm{dpa} 1 \mathrm{~mm}$ plates.

- First 1,3 and 5mm TIG welded heterogeous joints (irr. to unirr) succesful, no external defects (SEM)

- 1 and $3 \mathrm{~mm}$ plates: single pass, no filler wire

- $5 \mathrm{~mm}$ plate: Y-groove, 4-6 passes

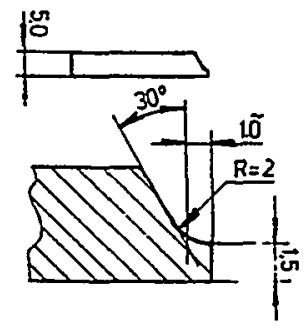



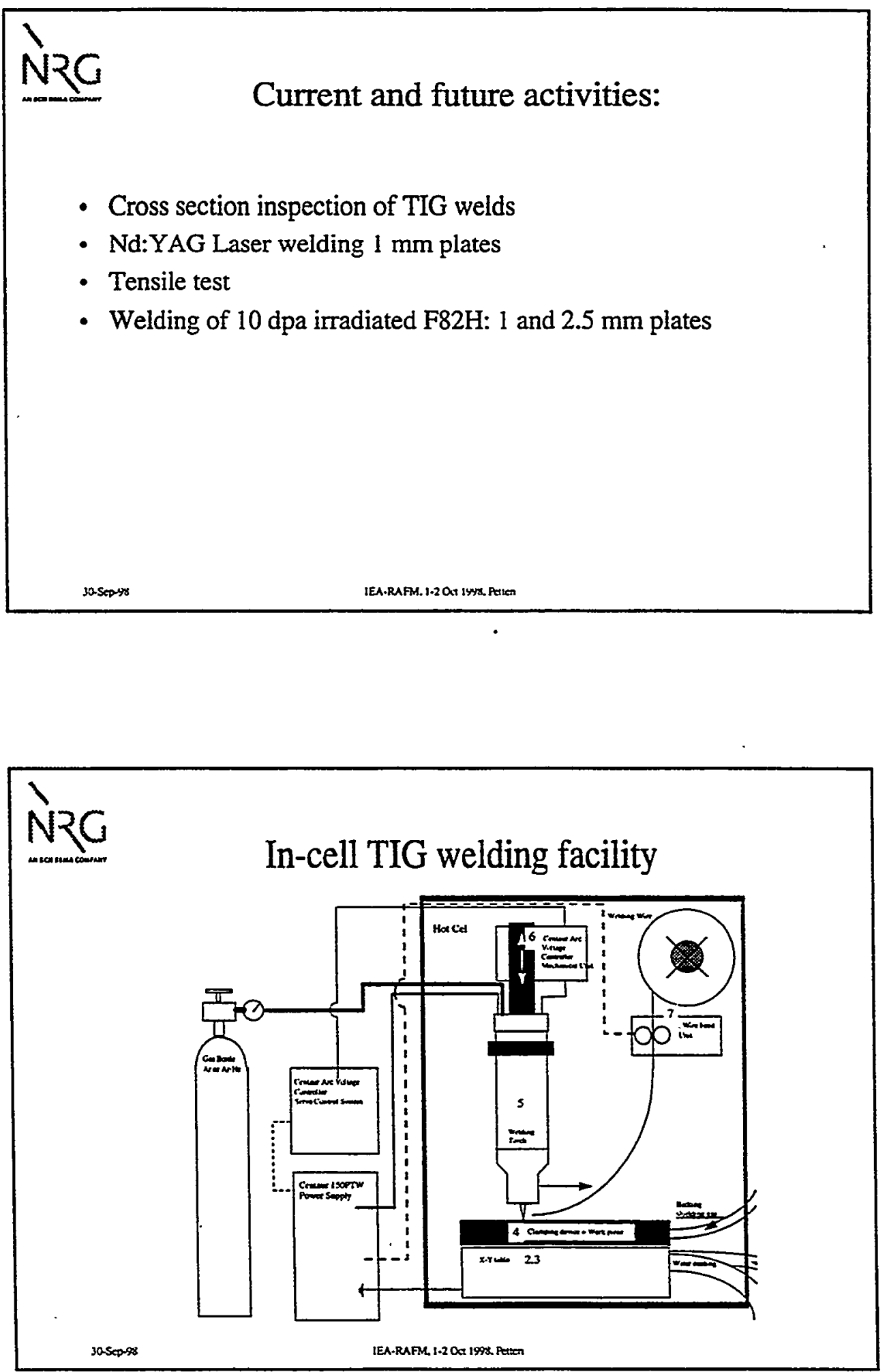

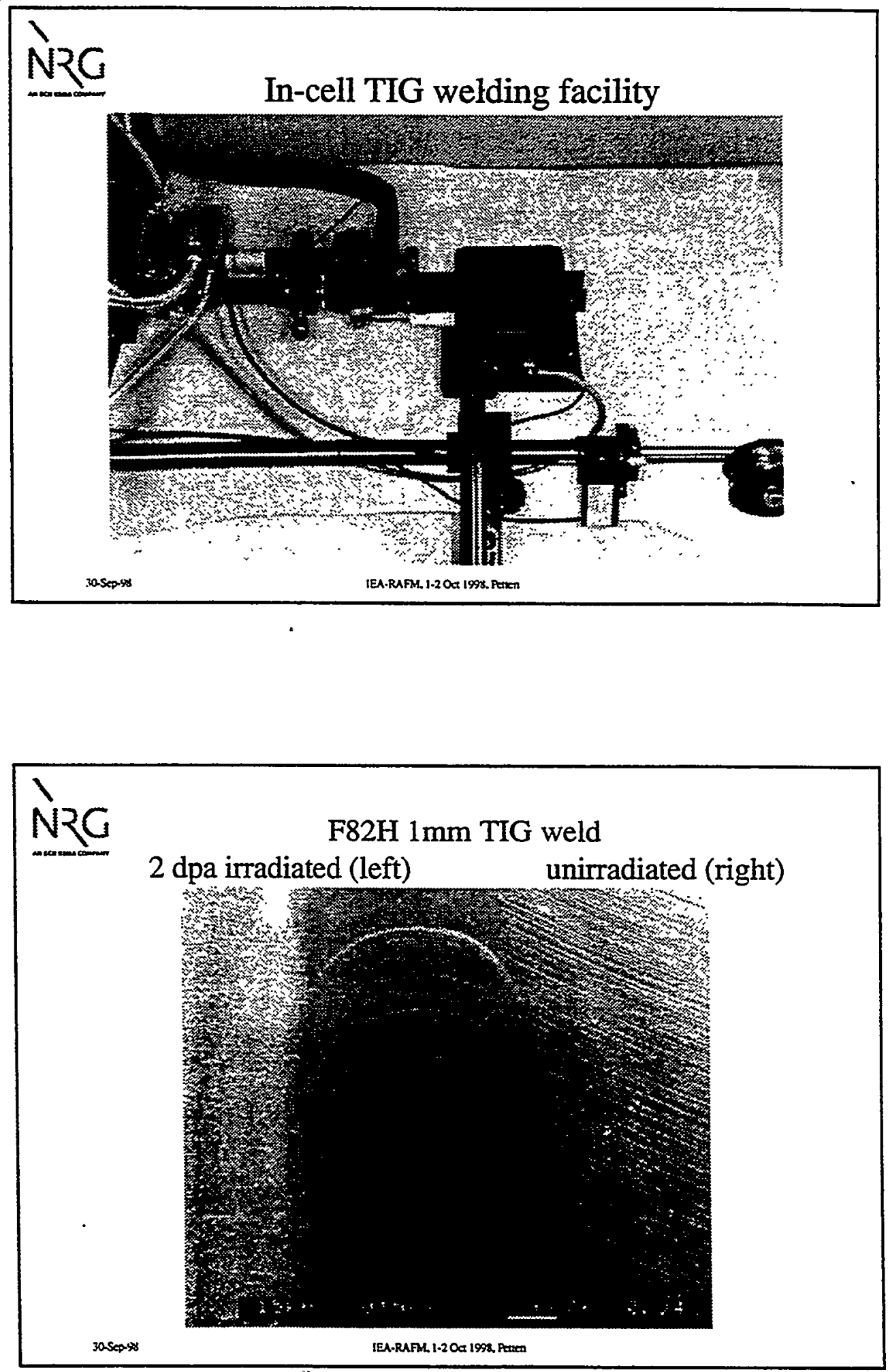


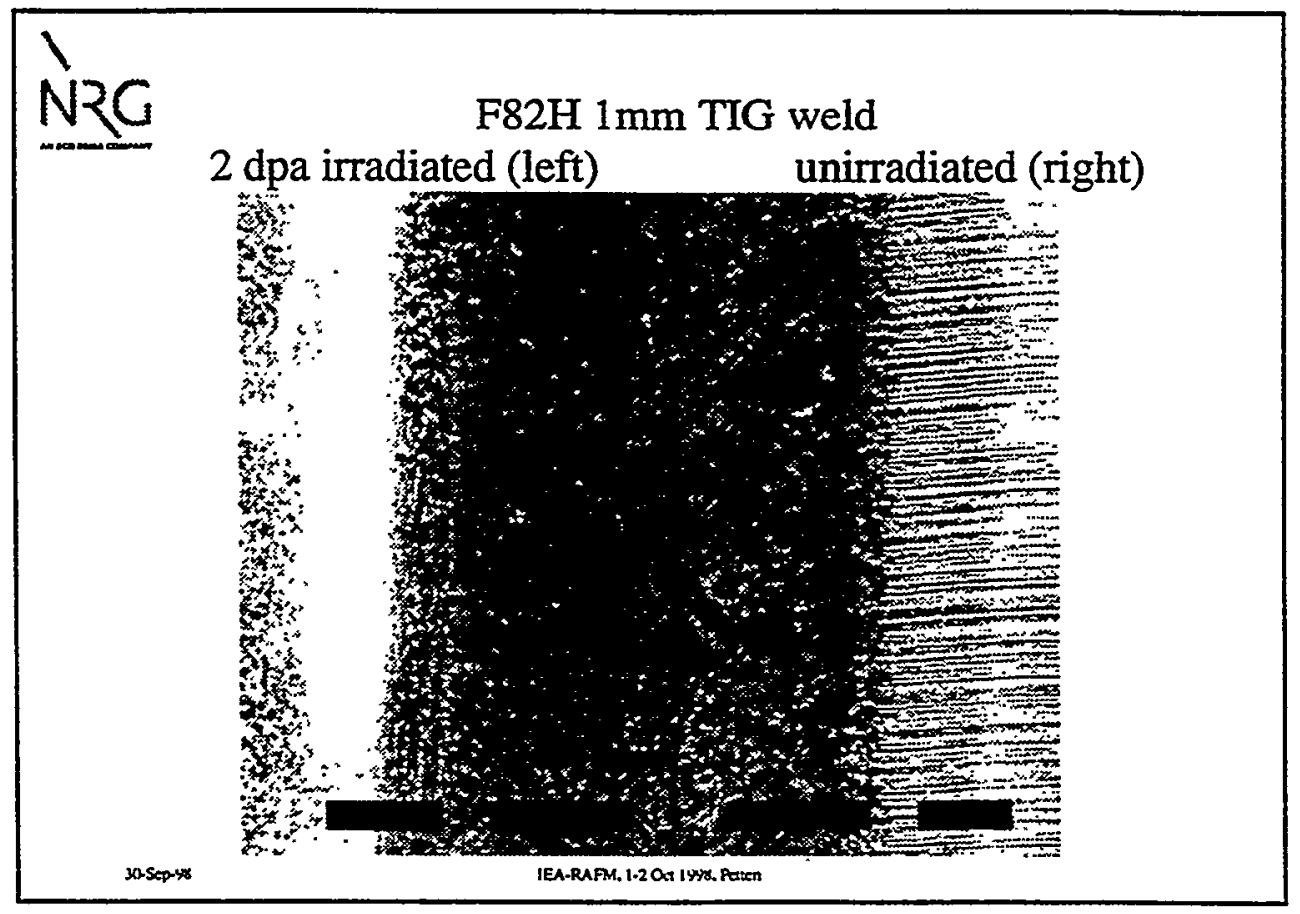

\section{Helium generation with ${ }^{10} \mathrm{~B}$}

- Ordered $365 \mathrm{~kg}$ lab. heats:

- one identical to "ECN-BS" $\rightarrow$ up to 10 appm He due to "B

- one with 50 wppm ${ }^{10} \mathrm{~B}$ addition $\rightarrow 250$ appm $\mathrm{He}$

- one with 50 wppm "B addition $\rightarrow 10$ appm $\mathrm{He}$ 


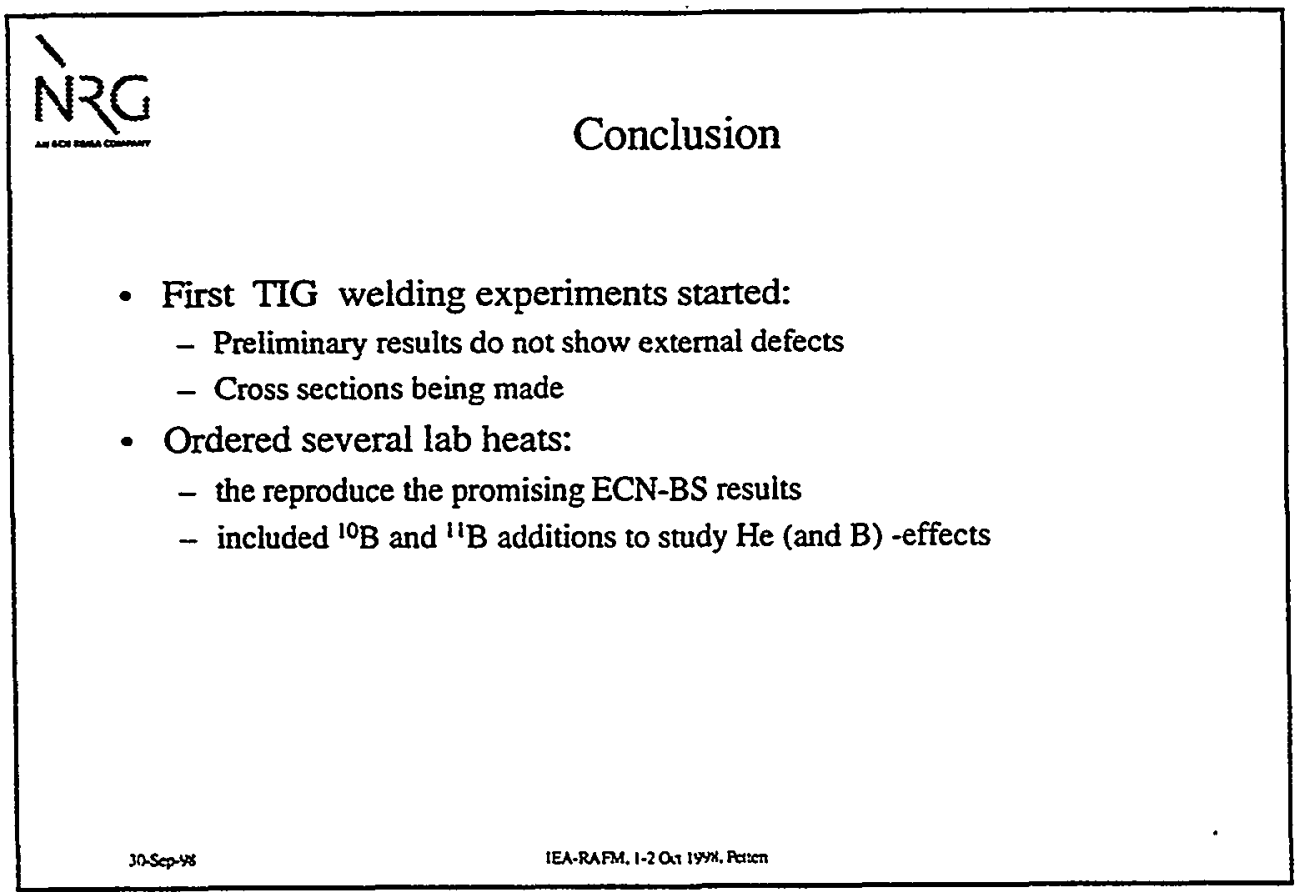




\section{Strategy for Development of Ferritic/Martensitic steels}




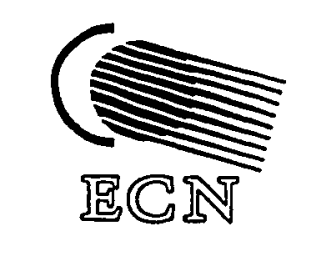

\section{EU STRATEGY FOR RAFM DEVELOPMENT}

\section{Presentation at IEA RAFM Workshop Petten, 2 October 1998}

\section{B. van der Schaaf}

NRG, Westerduinweg 3, P.O. Box 25, 1755 ZG Petten, The Netherlands tel. +31 224564665

e-mail: vanderschaaf@ecn.nl fax: +31224563490 


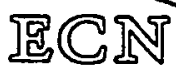

OVERVIEW

1

- Main goals

- Milestones period 1998 - 2009

- Major subjects

- Time schedule 


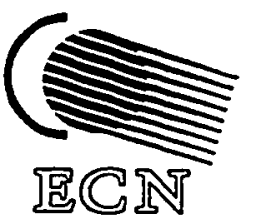

MAIN GOALS

-Engineering RAFM data for ITER test module, ITM, in 2004

-Manufacturing procedures for ITM, RAFM parts in 2004

-DEMO relevant design data in 2009

- High temperature ODS RAFM basis established in 2009

-In case of ODS RAFM success demo relevant design data in 2015 
ICN

MIIESTONES STRUCTURAL MATERIALS SUBPROJECT

Version: 14 July 1998

Author: B. van der Schaaf

\begin{tabular}{|c|c|c|c|}
\hline $\begin{array}{l}\text { Mile- } \\
\text { stone }\end{array}$ & $\begin{array}{l}\text { Time } \\
\text { Scale }\end{array}$ & $\begin{array}{l}\text { Items available at milestone } \\
\text { date }\end{array}$ & $\begin{array}{l}\text { EBP project } \\
\text { phase }\end{array}$ \\
\hline 4 & $98 / 11$ & $\begin{array}{l}\text { - Phase IA neutron } \\
\text { irradiation } \\
\text { - Character irradiated welds } \\
\text { - F82 characterization } \\
\text { - Screening test alloys } \\
\text { complete }\end{array}$ & $\begin{array}{l}\text { Selection ITM } \\
\text { Primary } \\
\text { candidate Alloy. }\end{array}$ \\
\hline 5 & $99 / 06$ & $\begin{array}{l}\text { - Preliminary ITM design } \\
\text { rules } \\
\text { - Data Base Evaluation } \\
\text { - Corrosion data for design }\end{array}$ & $\begin{array}{l}\text { First version } \\
\text { ITM design rules } \\
\text { and data base. }\end{array}$ \\
\hline 6 & $00 / 06$ & $\begin{array}{l}\text { - Evaluation data } \\
\text { EUROFER97 } \\
\text { - Specification } \\
\text { EUROFER2000 } \\
\text { - Phase II neutron irradiation } \\
\text { - Compatibility } \\
\end{array}$ & $\begin{array}{l}\text { Selection ITM } \\
\text { structural steel } \\
\text { specification and } \\
\text { primary radiation } \\
\text { effect limits. }\end{array}$ \\
\hline 7 & $01 / 05$ & $\begin{array}{l}\text { - ITM design rules } \\
\text { - Data Base evaluation } \\
\text { - Compatibility }\end{array}$ & $\begin{array}{l}\text { Second version } \\
\text { ITM design rules } \\
\text { and data base. } \\
\end{array}$ \\
\hline 8 & $02 / 03$ & $\begin{array}{l}\text { - Qualification and } \\
\text { Fabrication processes } \\
\text { - Metallurgical and } \\
\text { Mechanical Character } \\
\end{array}$ & $\begin{array}{l}\text { Confirmation of } \\
\text { materials and } \\
\text { fabrication } \\
\text { feasibility. }\end{array}$ \\
\hline 9 & $02 / 12$ & $\begin{array}{l}\text { - Irradiation Performance } \\
\text { - Compatibility } \\
\text { - ITM rules and data base }\end{array}$ & $\begin{array}{l}\text { Rules and data } \\
\text { base available for } \\
\text { detailed ITM } \\
\text { design }\end{array}$ \\
\hline
\end{tabular}


蛋訨

MILESTONES STRUCTURAL MATERIALS SUBPROJECT

Project period: 2003 - 2009

\begin{tabular}{|c|c|c|c|}
\hline $\begin{array}{l}\text { Mile- } \\
\text { stone }\end{array}$ & $\begin{array}{l}\text { Time } \\
\text { Scale }\end{array}$ & $\begin{array}{l}\text { Items available at milestone } \\
\text { date }\end{array}$ & Project phase \\
\hline 11 & $03 / 09$ & $\begin{array}{l}\text { - Screening ODS RAFM } \\
\text { - Manufacturing ODS } \\
\text { RAFM }\end{array}$ & $\begin{array}{l}\text { Advanced HT } \\
\text { RAFM }\end{array}$ \\
\hline 12 & $04 / 09$ & $\begin{array}{l}\text { - RAFM design data } \\
\text { verification } \\
\text { - Definition additions }\end{array}$ & $\begin{array}{l}\text { Consolidation } \\
\text { ITM design }\end{array}$ \\
\hline 10 & $03 / 06$ & $\begin{array}{l}\text { - Materials for } 150 \mathrm{dpa} \\
\text { irradiations available } \\
\text { - Rigs and reactor choice }\end{array}$ & $\begin{array}{l}\text { High dose } \\
\text { RAFM } \\
\text { properties for } \\
\text { DEMO }\end{array}$ \\
\hline 13 & $05 / 06$ & $\begin{array}{l}\text { - Choice reference ODS } \\
\text { RAFM alloy } \\
\text { - Joining, manufacturing } \\
\text { routes } \\
\text { - Assessment data } \\
\end{array}$ & $\begin{array}{l}\text { HT RAFM } \\
\text { engineering data }\end{array}$ \\
\hline 14 & $06 / 06$ & $\begin{array}{l}\text { - High dose RAFM PIE } \\
\text { - Design limits }\end{array}$ & $\begin{array}{l}\text { High dose } \\
\text { RAFM data for } \\
\text { DEMO }\end{array}$ \\
\hline 15 & $0.7 / 09$ & $\begin{array}{l}\text { - Assessment ODS RAFM } \\
\text { potential } \\
\text { - Decision on continuation }\end{array}$ & $\begin{array}{l}\text { Advanced HT } \\
\text { RAFM } \\
\text { development }\end{array}$ \\
\hline 16 & $09 / 09$ & $\begin{array}{l}\text { - Engineering data } 150 \mathrm{dpa} \\
\text { validated }\end{array}$ & $\begin{array}{l}\text { High dose } \\
\text { RAFM data for } \\
\text { DEMO }\end{array}$ \\
\hline
\end{tabular}


g모요

EBP, STRUCTURAL MATERIALS PROGRAM

\begin{tabular}{|l|l|}
\hline WP & TASK \\
\hline SM 1 & Irradiation Performance \\
\hline SM 2 & $\begin{array}{l}\text { Metallurgical and Mechanical } \\
\text { Characterization }\end{array}$ \\
\hline SM 3 & $\begin{array}{l}\text { Compatibility with Liquids and } \\
\text { Hydrogen }\end{array}$ \\
\hline SM 4 & $\begin{array}{l}\text { Qualification of Fabrication } \\
\text { Processes }\end{array}$ \\
\hline SM 5 & $\begin{array}{l}\text { Rules for Design, Fabrication and } \\
\text { Inspection }\end{array}$ \\
\hline SM 6 & Qualification for DEMO \\
\hline
\end{tabular}

Version: 980925

Author : B. van der Schaaf 


\section{Fusion Materials Programs in Japan}

Specialist Group Organized under Fusion Council of JAEC

A brief Summary of the Draft Report as of 24th July, 1998

- A specialist group organized under The Japanese Atomic Energy Commission (JAEC) has been discussed on how could $R \& D$ of the first-wall structural materials be carried out timely and efficiently. A draft report of this activitywas provided in June, 1998.

- The draft report will be subjected to discussion at the Fusion Council audited by the public and then, will be opened to the public reviewing for a period of time.

- The report is expected to be finalized by Atomic Energy Commission in September, 1998.

\section{Summary of Report (draft as of July, 1998)}

Strategy of Fusion Materiais Development

- First Wall Structural Materials -

Definition of evolutionary stages for the class of materials

- Exploratory/Development Stage

- Optimization/Nerification Stage

- Engineering Application Stage

Candidate materials and their strategic priority - Three candidate material categories are selected and priorization is made from view points of their technical maturity and potential properties

- The first priority category : prime candidate material

Reduced activation ferritic steels

Current stage; Optimization / Verification Stage

Current tasks; Establishing database for base line properties and selecting the final candidate specification

- The secondary priority materials category: advanced materials 
Vanadium alloys, and $\mathrm{SiC} / \mathrm{SiC}$ composites

Current stage; Exploratory/Development Stage

Strategy for development procedure

- Development studies for the three candidate materials have been carried out in parallel and will be continued up to the point where a definitive conclusion for candidacy is shown, while the current priority is now given to the reduced activation ferritic steels.

- The potential suitability of the three categories of materials are checkand reviewed evaluated every five years, in terms of technical maturity and their established properties regarding the requirements of the systems to be applied.

- The strategic goal for DEMO reactors is set around the year 2015 after the third evaluating stage.

General comments

- A high energy- and high flux neutron source with adequate test capacity (e.g. (FMIF) is indispensable for the development and evaluation of candidate materials, with conventional supplementary test means being utilized.

- Technical review / evaluation of the three candidate materials is continually necessary in order to invest funding and manpower efficiently.

- Importance of close collaborative domestic activities among national institutes, universities, and the industrial firms in parallel with international cooperation, is emphasized for achieving the mission. JAERI is expected to serve as a core organization for the domestic activities. 
STRUCTURAL MATERIALS DEVELOPMENT FOR FUSION REACTOR

\begin{tabular}{|c|c|c|c|c|}
\hline \multirow{2}{*}{ Reactor Development } & \multicolumn{4}{|c|}{ ITER Construction } \\
\hline & & & DEMO Design & ing Construction \\
\hline \multirow{2}{*}{ Check and Review } & C\&R 1 (2005) & C\&R $2(2010)$ & C\&R 3 (2015) & (2030) \\
\hline & (Approval of PCF) & $\begin{array}{l}\text { (Evaluation of } V \text { alloy } \\
\& \mathrm{SiC} / \mathrm{SiC} \text { composile) }\end{array}$ & $\begin{array}{l}\text { (Materials Selection } \\
\text { for DEMO) }\end{array}$ & \\
\hline $\begin{array}{l}\text { Reduced Activation } \\
\text { Ferritic Steel (RAF) }\end{array}$ & 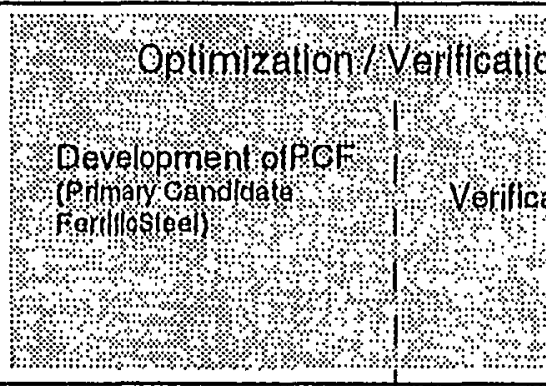 & 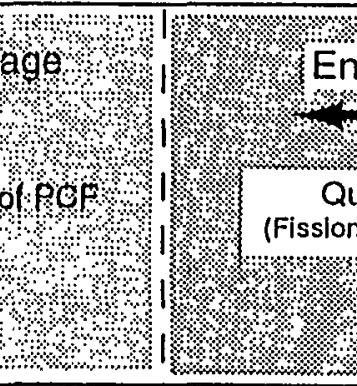 & 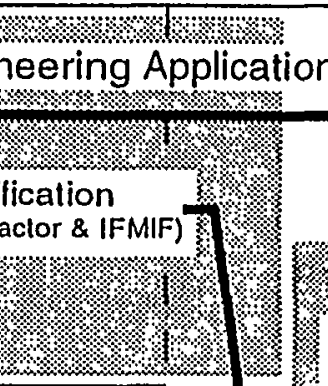 & $\begin{array}{l}\text { Stage } \\
\text { Selection of } \\
\text { the DEMO }\end{array}$ \\
\hline Vanadium Alloy & $\begin{array}{ll}\text { Exploratory I } & 1 \\
\text { Developmental } & 1 \\
\text { Stage } & 1 \\
& \end{array}$ & 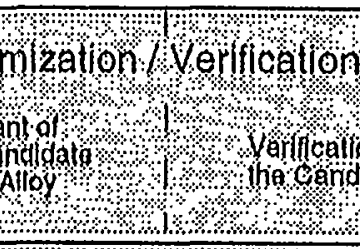 & (age, & $\begin{array}{l}\text { struclural malerial } \\
\text { Dalabase } \\
\text { development } \\
\text { with a Neutron } \\
\text { source (eg. IFMIF) }\end{array}$ \\
\hline SiC/SiC Composite & 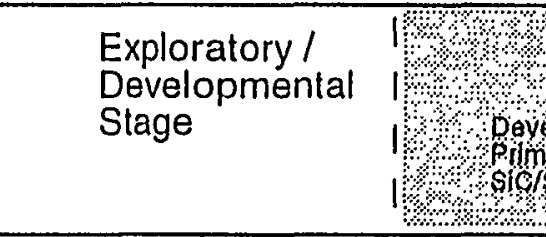 & 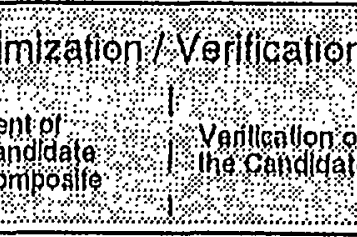 & 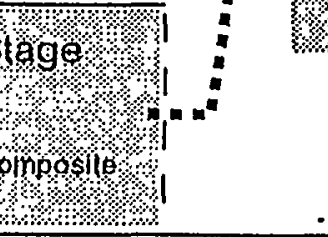 & (1) \\
\hline
\end{tabular}




\title{
U.S. Department of Energy Fusion Energy Science Program and the Fusion Materials Program
}

\author{
F. W. Wiffen
}

Office of Fusion Energy Sciences

U.S. Department of Energy

IEA Ferritic Steels Workshop

Petten, The Netherlands

October 1-2, 1998 


\section{U.S. Fusion Energy Sciences Program Mission}

"Acquire the knowledge base needed

for an economically and environmentally

attractive fusion energy source." 


\section{Technology Program}

Transition Highlights

o ITER tasks have been the focus through FY 1998

o In FY 1999, will begin transition to broad portfolio of activities serving the domestic program and our needs for international collaborations

o Transition will be completed in FY 2000; Technology Program will emphasize enabling technologies for plasma experiments, domestically and internationally 


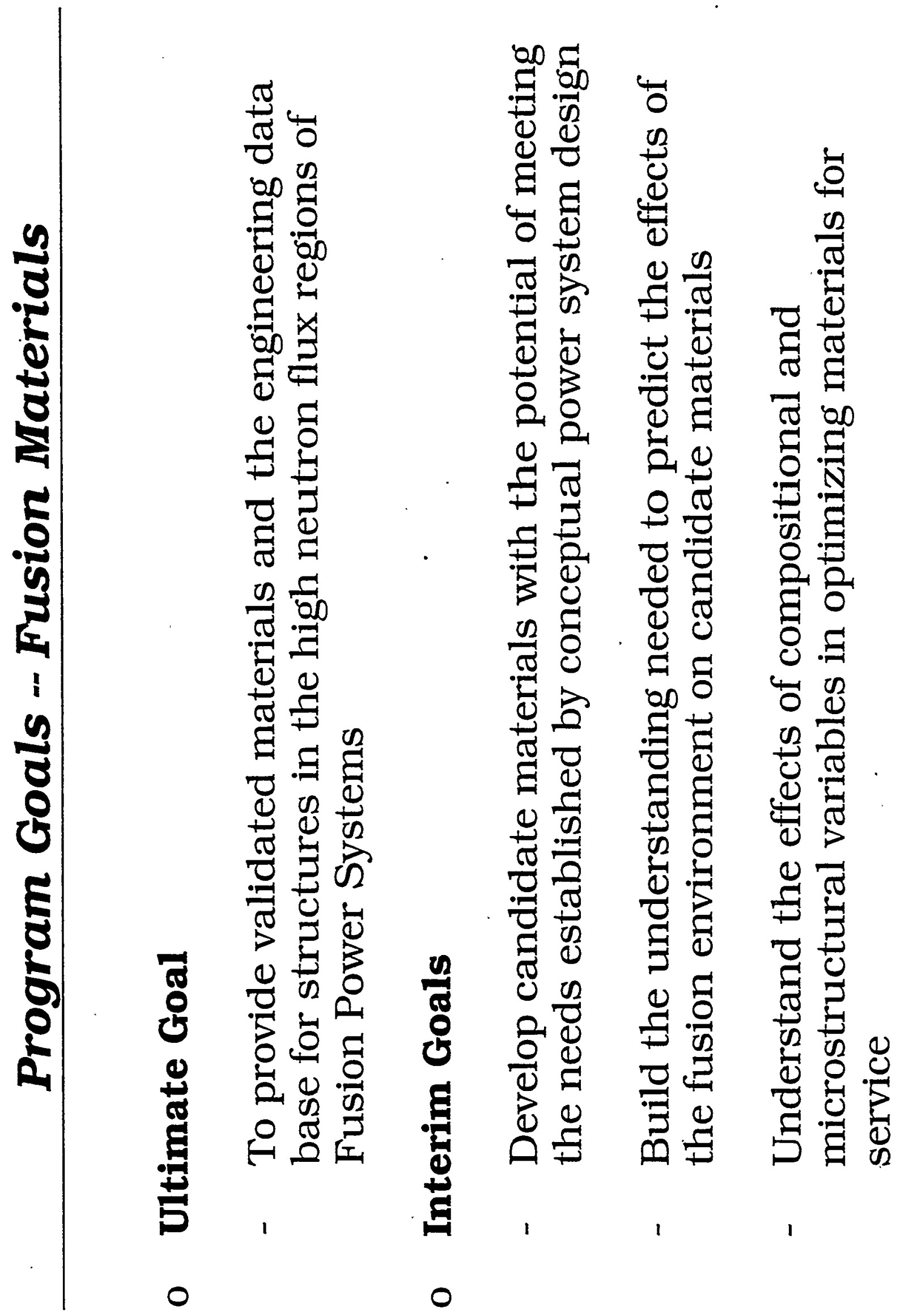




\section{General Approach -- Fusion Materials}

o Include only materials systems that show promise for application in high temperature service and meet low activation criteria

o Focus on questions of feasibility for fusion use and critical issues for acceptable service lifetime

- Concentrate on aspects unique to fusion applications

- The fusion chemical environment

- Neutron irradiation effects in the D-T fusion spectrum

o Balance efforts among candidates for promising design concepts, especially related to choice of coolants

o Develop understanding at the controlling mechanisms level, so that results can be used to interpolate/extrapolate from available data base 


\section{Fusion Energy Sciences FY 1999 Budget}

\section{Program Elements}

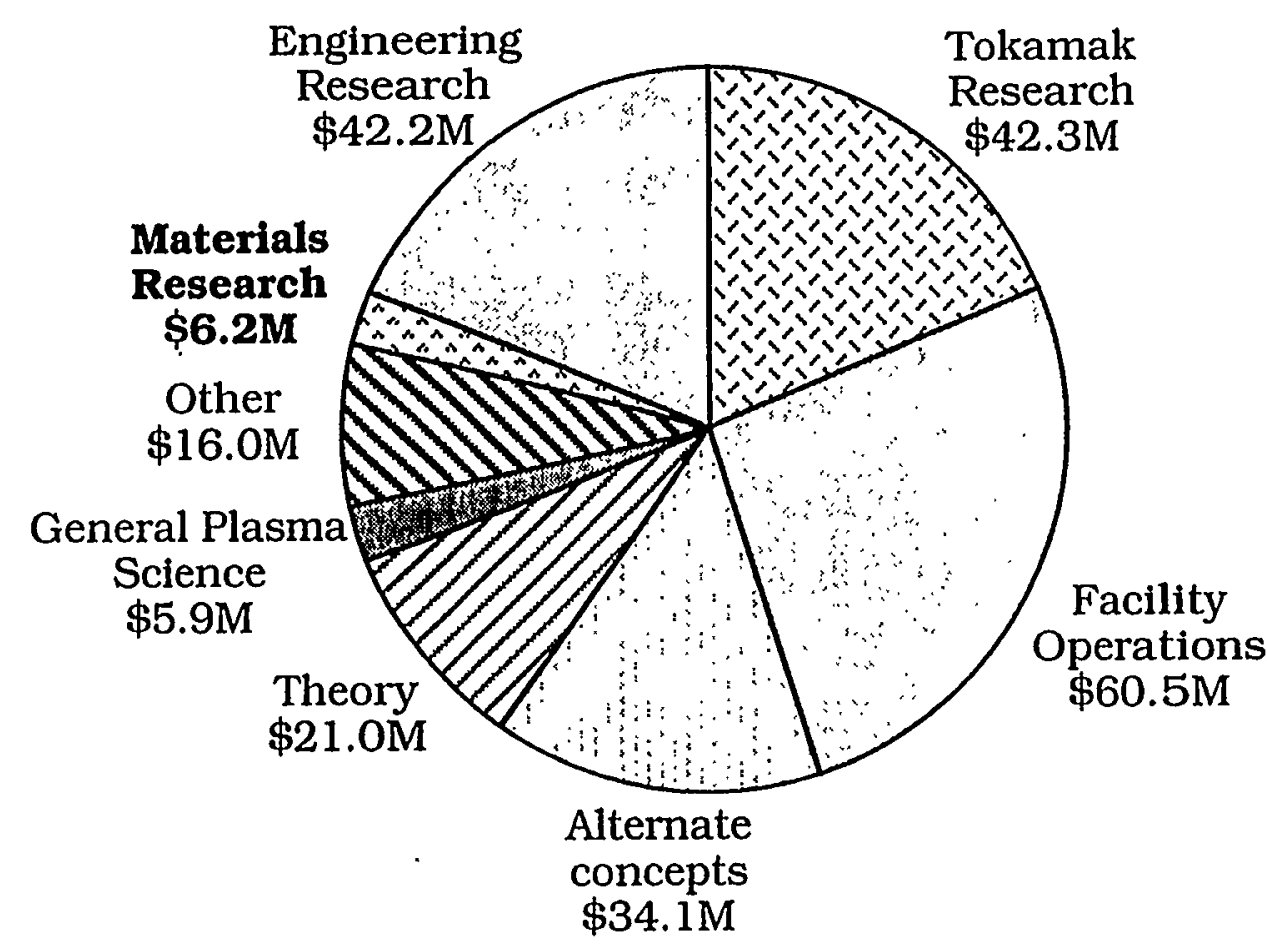

$\$ 228.2$ Million

\section{Materials Research Categories}

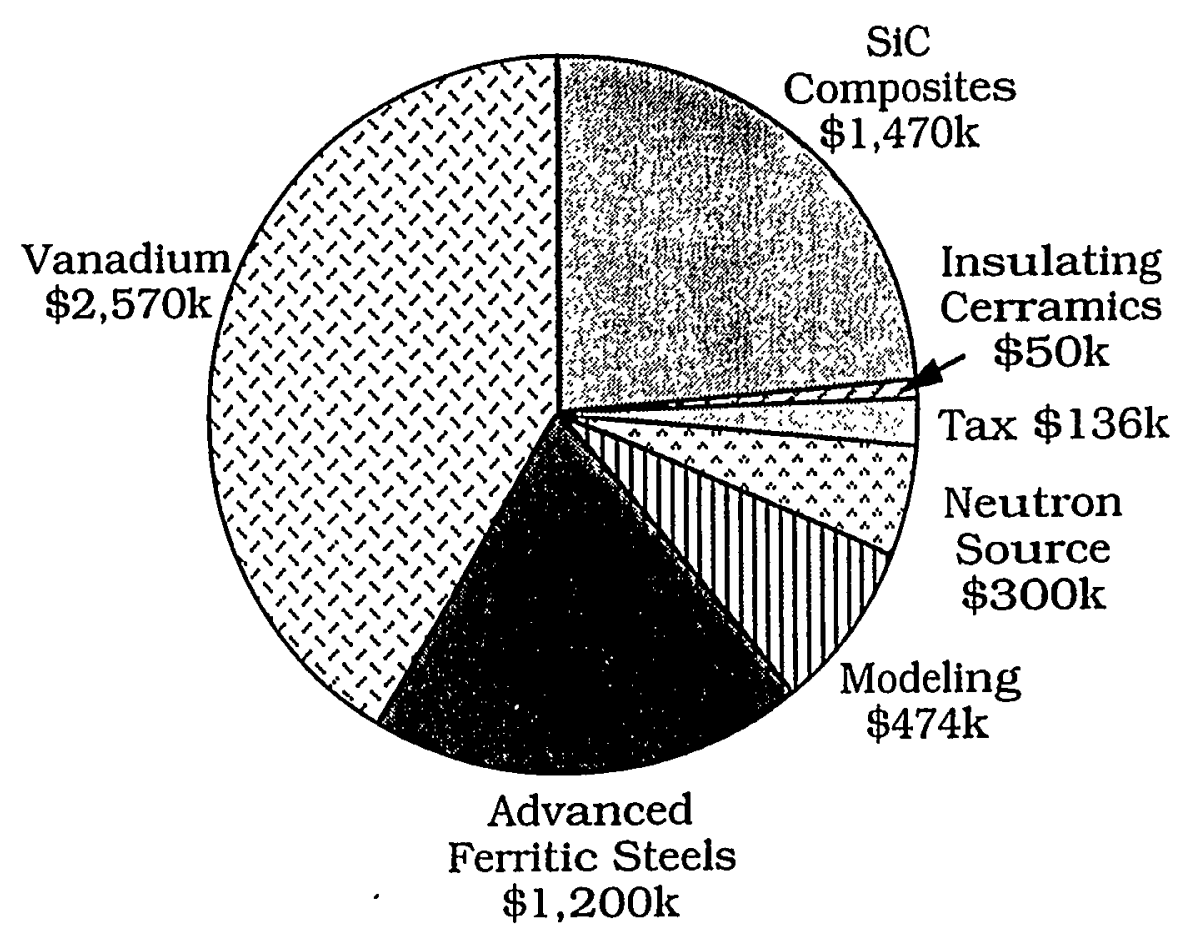

$\$ 6.2$ Million 


\section{Materials Program}

(\$ in Millions)

Budget Summary

$\frac{\text { FY 1998* }}{8.3} \quad \frac{\text { FY } 1999}{7.0}$

Goal:

o Explore innovations in materials technologies needed in the long term to advance fusion science and to achieve fusion's potential as an attractive energy source

- Focus on low-activation structural materials (vanadium alloys, ferritic steels, and silicon carbide composites) for high power density fusion devices

- Small complementary efforts on non-structural materials research is conducted under Fusion Technologies (coolants, insulators, coatings and tritium breeders) and Plasma Technologies (plasma facing materials)

*ITER activities are included 


\section{Fusion Materials Program}

The Modeling Component of the Fusion Materials Program will be expanded

Goal is to examine what is being done now, and to define what is needed in a comprehensive modeling program

Changes will be made to assure that the most important elements of the modeling activity are in place 


\section{Performance Targets for Fusion Reactor Structural Materials}

o Develop materials that will support economically attractive fusion power reactor designs

- Fabricability, weldability, joining technology for field assembly

- Compatibility with operating environment (coolant, tritium breeder, ambient atmosphere, special purpose coatings, hydrogen plasma)

- Adequate performance design window (operating temperature limits, stress limits, irradiation damage)

- Reliability and maintainability

o Develop materials that will achieve the potential of fusion as an environmentally attractive energy source

- Materials not requiring long term geological disposal

- Potential for recycle of materials to minimize environmental impact

o Develop materials that will achieve the potential of fusion as a safe energy source from the viewpoint of the worker and the public

- Acceptable levels of heat from radioactive decay and chemical reactions

- $\quad$ Limited dispersability of radioactivity

- $\quad$ Acceptable BHP (needs to be defined) 


\section{Technological Challenges to Establish Feasibility}

Advanced Ferritic Steels

o Establish that ferromagnetic structural materials are acceptable for MFE concepts

o Establish the potential design window for advanced ferritic steels -- focus on the role of displacement damage concurrent with helium production on low temperature fracture behavior

o Determine the effects of nuclear transmutations (e.g., production of $\mathrm{He}$, burnout of $\mathrm{W}$ and $\mathrm{Ta}$ )

o Resolve system specific compatibility issues (e.g., $\mathrm{T}$ barrier for He or PbLi coolants) 


\section{Fusion Materials Program}

Issues with the use of ferromagnetic steels in magnetically confined fusion systems require a more complete evaluation

1. The materials program (R. L. Klueh) has surveyed the information available worldwide

2. Experiments in Japan need to be monitored -- HT-2 and JFT2M structures using ferritic steel components

3. Complete analysis can only be done by the fusion physics/design analysis teams, not by the materials community 


\section{Technological Challenges for the Materials Development Step}

Advanced Ferritic Steels

Explore oxide dispersion strengthening as an approach to improving the high temperature strength of martensitic steels while maintaining acceptable low temperature toughness characteristics 


\section{Focus on Research in the Advanced Materials Program}

Advanced Ferritic Steels

o Carry out research to determine and understand the effects of displacement damage concurrent with helium on the low temperature fracture behavior of $\mathrm{F} 82 \mathrm{H}$ and closely related compositions

o Explore oxide dispersion strengthening as an approach to improving the high temperature strength of martensitic steels while maintaining acceptable low temperature toughness characteristics 


\section{Technology Program}

Transition Highlights

o Virtual Laboratory for Technology (VLT) is a mechanism for organizing and integrating work of many institutions

- Coordinating program elements

- Advocacy and representation

- Governance with an external Program Advisory Committee and an internal Coordinating Committee 

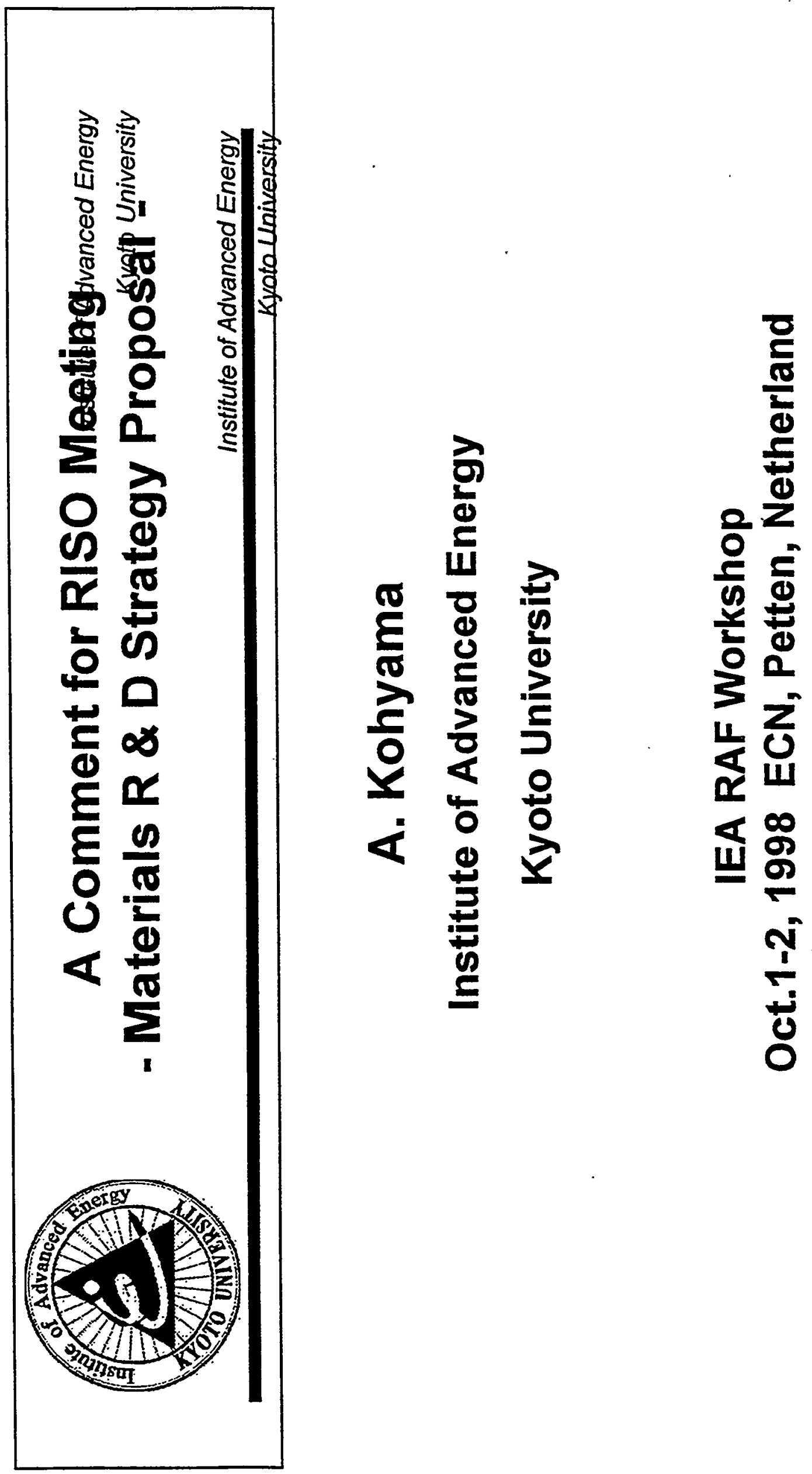


\section{What is the definition of Strategy ? \\ - what are we (they) trying to do at RISO? -}

Institute of Advanced Energy

Kyoto University

Strategy meeting is not a place for a simple information exchange

In military usage, a distinction is made between STRATEGY and

TACTICS. STRATEGY is the utilization, during both peace and war, of all of a nation's forces, through large-scale, long-range planning and development, to ensure security or victory. TACTICS deals with the use and deployment of troops in actual combat.

(from Random House dictionary)

Q1: for security or victory?

Q1-1: are we in peace or in war?

Q2: do you discuss TACTICS ?

Q2-1: what is the goal ?

Q2-2: where do you fight?

Q2-3: how do you fight? 


\section{Where does the Japanese fight and what for?}

Institute of Advanced Energy

Kyoto University

1: Fusion is one of the energy options for the future (in Japan)

- a back-up or insurance to the other options Make it an important option

(ITER special committee report: Japan Domestic) or to keep the position

2: Fusion Research has three major areas (under MOE, STA)

- fusion engineering is still a supporting activity Make it the major one

3: Fusion engineering is under restructuring (toward MOE+STA)

- materials comm. has a strong influence

Restru. Under materials' leadership

4: Materials research has to be more emphasized

- goal of materials $R$ \& $D$ is not clear a clear strategy will make the way
Get a better financial situation within F.E. or even within materials

5: Materials community is under strong criticisms

- should be involved other F.E. activities

- materials $R$ \& $D$ activity should be down sized

Try not to be killed 


\section{Japanese National Strategy n Materials $R$ \& $D$ for $D E M O$}

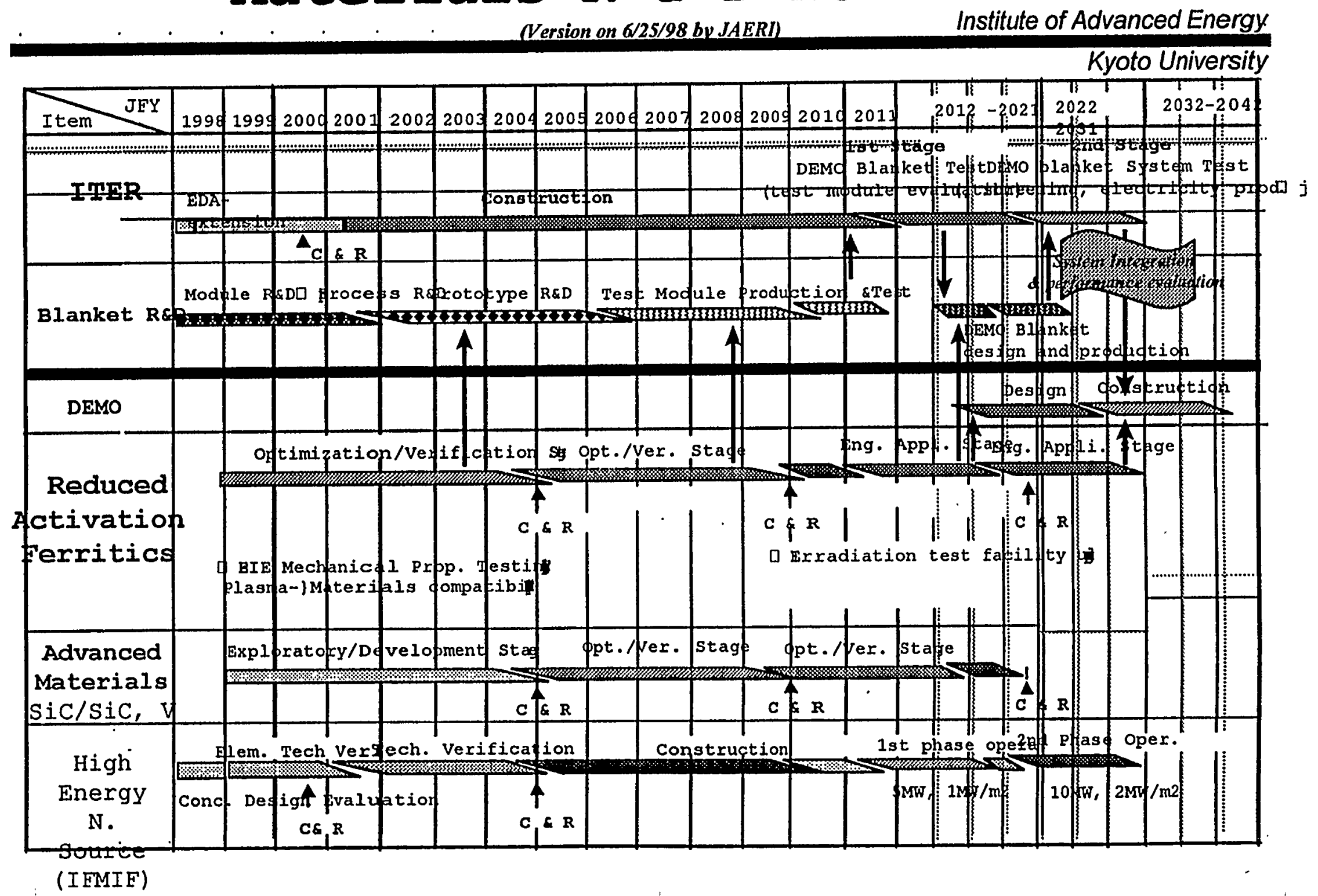




\section{What is the real situation of RAFs ? - what do you wan to do ? -}

Institute of Advanced Energy

1: Can RAFs become materials for power reactor?

Kyoto University

- need ODS options with strong will

Strategy with a clear goal of materials R\&D

2: Can RAFs become candidate materials for ITER ?

- RAFs activity should lead fusion engineering Strategy with a clear goal of materials R\&D

3: Can RAFs keep their major position ?

- RAFs have been the most matured materials Have to create a new phase

- what is the next step? after the current IEA large heats activities(bottom line for Japan)

4: Can RAFs maintain their activity ?

- not likely to be survived as a part of Strategy with a clear goal fusion energy research, as it it

- to move to energy science research or materials science research is an option 


\section{EXTERNAL DISTRIBUTION}

1. K. Abe

2. A. Alamo

3. V. M. Chernov

4. W. Dietz

5. K. Ehrlich

6. D. S. Gelles

7. D. R. Harries

8. A. Hishinuma

9. M. G. Horsten

10. D. Jackson

11. S. Jitsukawa

12. R. H. Jones

13. A. Kimura

14. A. Kohyama

15. T. Kondo

16. E. Materna-Morris

17. R. Odette

18. G. Phillips

19. P. Ruatto

20. R. Schaublin

21. K. Shiba

22. D. L. Smith

23. F. A. Tavassoli

24. B. van der Schaaf

25. E. V. van Osch

26. M. Victoria

27. F. W. Wiffen

28. Z. Xu

29. I. J. Zatz

30. L. P. Zavialsky

31-32. Office of Scientific and Technical Information (2)

\section{INTERNAL DISTRIBUTION}

33. E. E. Bloom

34-37. R. L. Klueh (4)

38. A. F. Rowcliffe

39-40. Central Research Library (2)

41. Document Reference Library

42. Laboratory Records, ORNL-RC

43. ORNL Patent Section 